\title{
Structural Performance of Steel Reinforced Recycled Concrete
}

\section{Members}

\author{
by \\ GHOLAMREZA FATHIFAZL, B.Sc., M.Sc
}

A thesis submitted to the Faculty of Graduate Studies and Research

in partial fulfillment of the Requirements for the Degree of

\section{Doctor of Philosophy}

\author{
Department of Civil and Environmental Engineering \\ Carleton University \\ Ottawa, Ontario, Canada \\ January 2008
}

The Doctor of Philosophy in Civil Engineering is a joint program with the University of Ottawa, administered by the Ottawa-Carleton Institute for Civil Engineering 


$\begin{array}{ll}\begin{array}{l}\text { Library and } \\ \text { Archives Canada }\end{array} & \begin{array}{l}\text { Bibliothèque et } \\ \text { Archives Canada }\end{array} \\ \begin{array}{l}\text { Published Heritage } \\ \text { Branch }\end{array} & \begin{array}{l}\text { Direction du } \\ \text { Patrimoine de l'édition }\end{array} \\ \begin{array}{l}\text { 395 Wellington Street } \\ \text { Ottawa ON K1A 0N4 }\end{array} & \begin{array}{l}\text { 395, rue Wellington } \\ \text { Ottawa ON K1A ON4 }\end{array} \\ \text { Canada } & \begin{array}{l}\text { Canada } \\ \end{array}\end{array}$

Yourfile Votre référence ISBN: 978-0-494-36783-4 Ourfile Notre référence ISBN: 978-0-494-36783-4

NOTICE:

The author has granted a nonexclusive license allowing Library and Archives Canada to reproduce, publish, archive, preserve, conserve, communicate to the public by telecommunication or on the Internet, loan, distribute and sell theses worldwide, for commercial or noncommercial purposes, in microform, paper, electronic and/or any other formats.

The author retains copyright ownership and moral rights in this thesis. Neither the thesis nor substantial extracts from it may be printed or otherwise reproduced without the author's permission.
AVIS:

L'auteur a accordé une licence non exclusive permettant à la Bibliothèque et Archives Canada de reproduire, publier, archiver, sauvegarder, conserver, transmettre au public par télécommunication ou par l'Internet, prêter, distribuer et vendre des thèses partout dans le monde, à des fins commerciales ou autres, sur support microforme, papier, électronique et/ou autres formats.

L'auteur conserve la propriété du droit d'auteur et des droits moraux qui protège cette thèse. $\mathrm{Ni}$ la thèse ni des extraits substantiels de celle-ci ne doivent être imprimés ou autrement reproduits sans son autorisation.
In compliance with the Canadian

Privacy Act some supporting forms may have been removed from this thesis.

While these forms may be included in the document page count, their removal does not represent any loss of content from the thesis.
Conformément à la loi canadienne sur la protection de la vie privée, quelques formulaires secondaires ont été enlevés de cette thèse.

Bien que ces formulaires aient inclus dans la pagination, il n'y aura aucun contenu manquant.

\section{Canadä}




\begin{abstract}
Concrete constitutes a large portion of the construction and demolition waste produced by the construction industry. Up until very recently, no regulations existed concerning the production and disposal of this waste. In the last couple of decades, however, there has emerged greater emphasis on sustainability and so-called green practices, and consequently recycled concrete aggregates (RCA) has been considered as an alternative construction material to conventional natural aggregates. Due to the many previously reported short and long term inferiorities of concrete made with RCA, compared to conventional concrete, the construction industry has been reluctant to use it, especially in structural applications. To overcome this problem, the results of a comprehensive study are presented in this thesis investigating the structural performance of steel reinforced recycled aggregate concrete (RAC) members. The research program was planned to fill the identified gaps in the existing literature on the subject and to provide data which could help remove the fundamental barriers to the use of RAC as structural-grade concrete in the construction industry. The current study focuses on the short- and longterm behaviour of RAC as a new material and on the flexural and shear behaviour of reinforced RAC beams. It is demonstrated theoretically and verified experimentally that RAC is not inherently inferior, but its reported inferiority emanates from using improper concrete mix proportioning methods. Therefore, a new concrete mix proportioning method is developed and verified experimentally. It is also demonstrated that methods of structural design developed for conventional concrete could be used to design RAC structures, provided the mechanical properties of RAC are duly accounted for.
\end{abstract}




\section{ACKNOWLEDGEMENTS}

Before starting my $\mathrm{PhD}$ program, I did not think that so many people would be involved in my research. Over the years, those that I have worked with have continuously helped me and taught me team work. Now, I am a great believer in "one and one equals three", and deeply value team work. So, perhaps the pages of this thesis can be considered as letters of "thanks" to the many individuals who have helped me bring it to completion.

I gratefully acknowledge the technical and financial support of Public Works and Government Services Canada and of Natural Resources Canada. I would like to express my deepest appreciation to my supervisor, Professor A. Ghani Razaqpur, who continually and convincingly conveyed a spirit of adventure with regard to research and scholarship. Without his guidance, persistent help, and encouragement this thesis would not have been possible. In addition, I also would like to express my appreciation to Professor O. Burkan Isgor, Dr. Benoit Fournier and Dr. Simon Foo for their invaluable suggestions and help during all stages of this study. I would also like to thank my friend, Abdelgadir Abbas, with whom I have had the pleasure of collaborating and sharing the joy of researching this topic. The large portion of the testing programme presented in this thesis was carried out at CANMET Material Technology Laboratories in Ottawa. Therefore, I would like to express my heartfelt gratitude to the CANMET staff, Mr. Raymond Chevrier, Mr. Alain Bilodeau, Dr. Nabil Bouzoubaa, Dr. Pierre Nkinambubanzi, Mr. Steve Michaud and Mr. Vince Deacon. I also need to thank Mr. Joe Pezze of ETS (NRCAN) who provided expertise during the creep and bond test setup preparations. I am very grateful to $\mathrm{Mr}$. Denis Jacob from ETS (NRCAN) for his great help during preparation of formworks of 
beams. Additionally, I have to thank Mr. Denis Tremblay and Mr. Martin Dupuis for their great help during preparation of the materials and the mixes for the beam specimens. I also need to thank Mr. Ted Anderson, and Mr. Ken Judge from NRCAN, who provided their expertise during the bond test setup. In addition, my thanks go out to Messrs Ken McMartin, Stanely Conley, and Pierre Trudel of Civil and Environmental Engineering Laboratories at Carleton University for their great help during beam testing program. It should also be mentioned that the RCA used in this study were donated by RichVan Holdings Ltd of Richmond, BC and Groupe Beauval, Montréal, PQ. The virgin aggregates from BC were provided by Lafarge Canada Inc. and Lehigh Northwest Materials. The cement was provided by Lafarge North America. Without the in-kind contributions of these companies, this research would be very difficult to complete. I would like to thank Prof. Mohsen Ali Shayanfar of Iran University of Science and Technology (IUST), and Prof. Saeed Pourzaynali and Prof. Javad Razzaghi from University of Guilan, who inspired me to pursue my $\mathrm{PhD}$ studies.

Finally, this undertaking could never have been achieved without the patience, encouragement, and understanding of my dear wife Leila and my wonderful son Arya, who have been the inspiration that has always driven me forward. They have kept me focused. They let me be quiet when I wanted to be quiet, and be noisy when I wanted to be noisy. Well, that is another story. 


\section{TABLE OF CONTENTS}

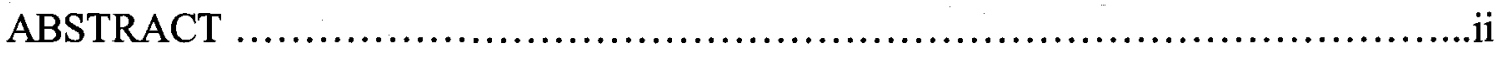

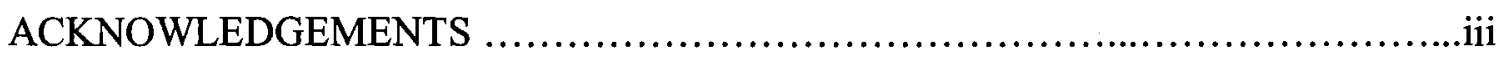

TABLE OF CONTENTS.................................................................

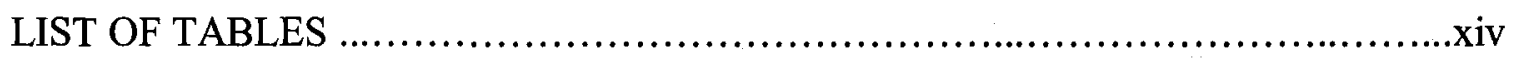

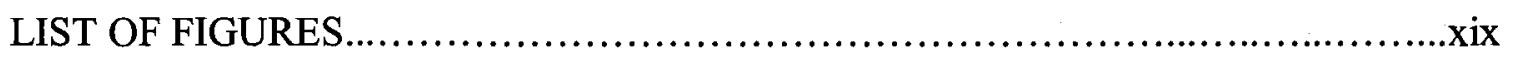

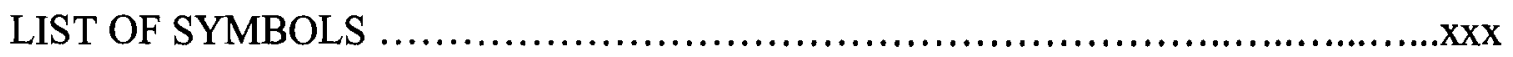

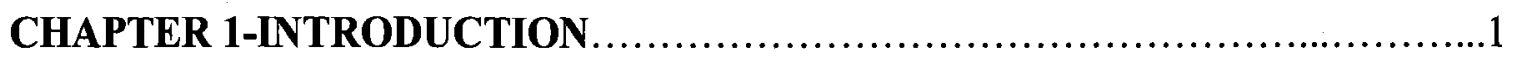

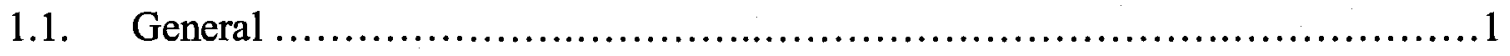

1.2. A Note on Important Definitions and Acronyms..............................5

1.3. Economic and Environmental Benefits of RAC ................................

1.4. Problem Definition, Objectives and Scope ................................. 10

1.5. Thesis Organization.......................................................... 15

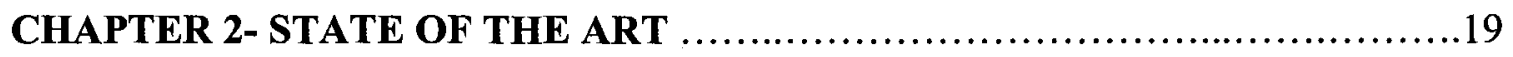

2.1. General ...........................................................19

2.2. Relevant Mechanical Properties of Concrete...................................19

2.3. Effect of RCA and Other Constituents of Concrete on its Properties.............26

2.3.1. Coarse RCA Content............................................27

2.3.2. Fine RCA Content .............................................. 30

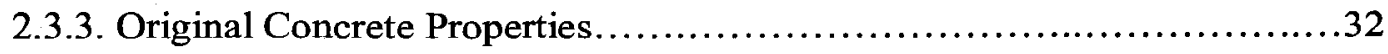

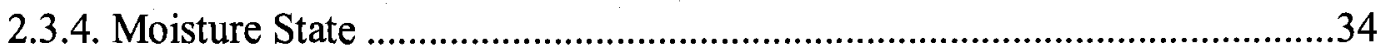

2.3.5. Mineral and Chemical Admixtures.................................... 34

2.3.6. Processing Level.................................................. 37 
2.4. Methods for Improvement of the Properties of RAC ...........................38

2.5. Structural Behaviour of Steel Reinforced Recycled Concrete Members...........41

2.5.1. Flexural and Shear Behaviour of RC Members.........................41

2.5.1.1. Flexural Behaviour ......................................41

2.5.1.2. Shear Behaviour..........................................43

2.5.2. Flexural and Shear Behaviour of RRC Beams...........................49

2.5.3. Stress-Strain Relationship........................................53

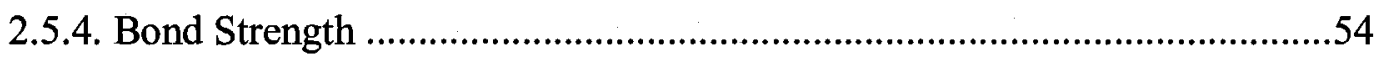

2.6. Selection of RAC Mixture Proportions...................................55

2.7. Existing Guidelines and Standards.......................................57

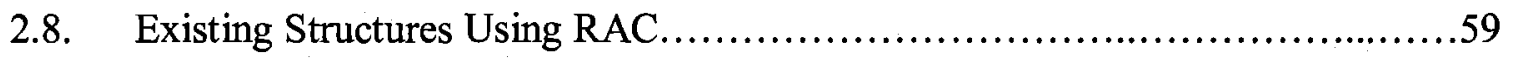

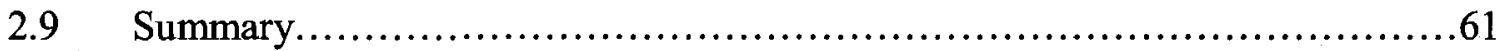

CHAPTER 3- PRELIMINARY INVESTIGATION OF CONCRETE MIXES

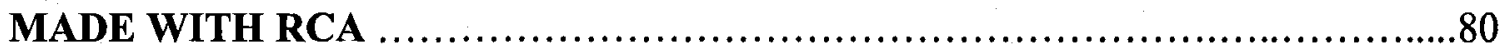

3.1. General................................................................. 80

3.2. Material Characterization of RCA ...................................... 83

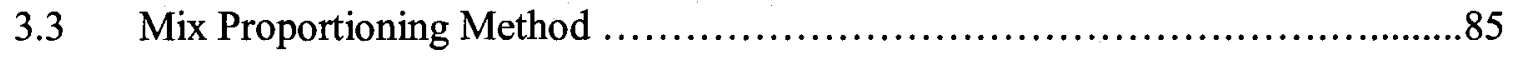

Preparation of Specimens............................................. 88

3.4.1 Preparation of Materials.........................................88

3.4.2 Water Content Adjustment........................................88

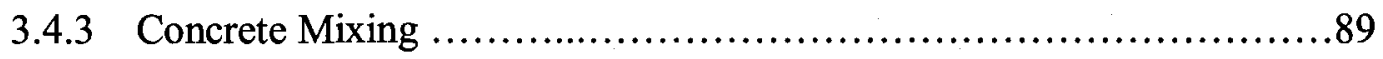

3.5 Effect of RCA on Fresh and Hardened Properties of RAC.....................90

3.5.1 Fresh Properties............................................91 
3.5.1.1 Slump..................................................91

3.5.1.2 Fresh Density............................................94

3.5.2 Hardened Properties.................................................97

3.5.2.1 Hardened Density... ........................................97

3.5.2.2 Compressive Strength........................................100

3.5.2.3 Splitting Tensile Strength... ....................................104

3.5.2.4 Elastic Modulus.............................................106

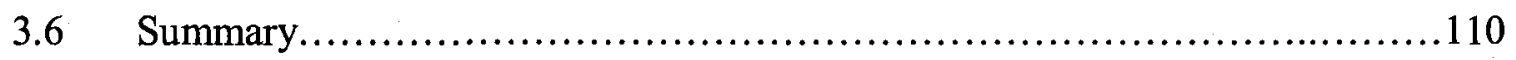

\section{CHAPTER 4- PROPOSED MIX PROPORTIONING APPROACH FOR}

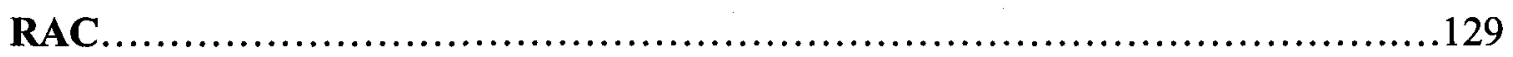

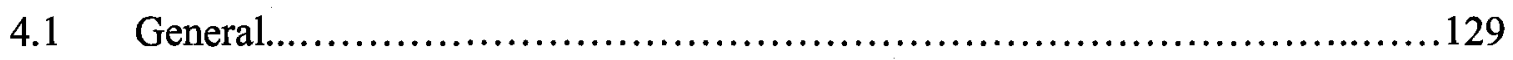

$4.2 \quad$ Hypothesis.........................................................................

4.3 Determination of Residual Mortar Specific Gravity.............................131

4.3.1 Direct Method.....................................................131

4.3.2 Indirect Method...................................................133

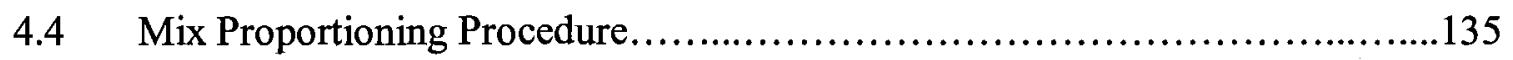

4.5 Maximum Permissible Coarse RCA and RM Content..........................138

4.5.1 Determining the Upper Limit of RCA Content in RAC ..................139

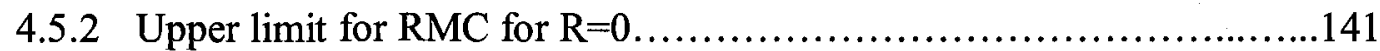

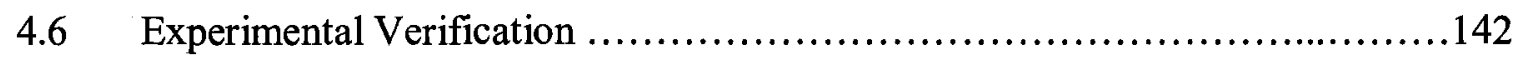

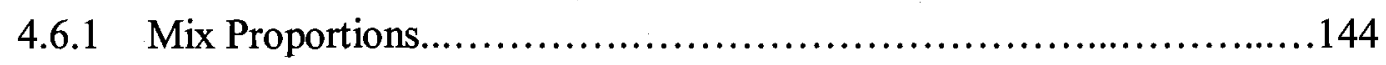

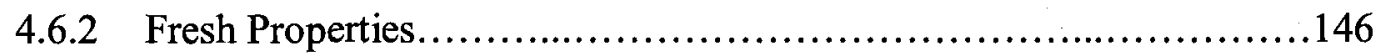

4.6.2.1 Slump..................................................... 146 
4.6.2.2 Fresh Density..........................................147

4.6.3 Hardened Properties.............................................. 148

4.6.3.1 Hardened Density........................................148

4.6.3.2 Compressive Strength......................................149

4.6.3.3 Elastic Modulus...........................................150

4.6.3.4 Stress-Strain Relationship................................152

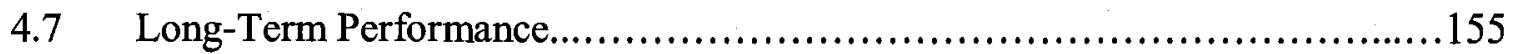

4.7 .1 Creep......................................................... 156

4.7.2 Drying Shrinkage................................................... 163

4.8 Summary of the Test Results.............................................. 167

CHAPTER 5- ANALYSIS OF THE EXPERIMENTAL DATA: SHORT AND

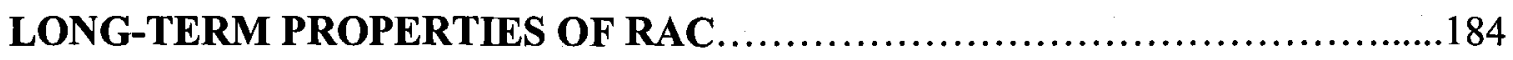

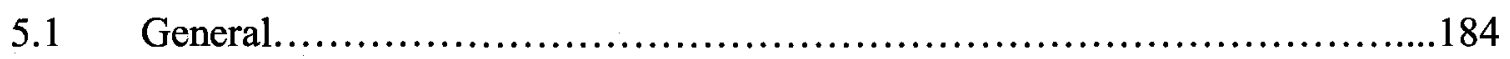

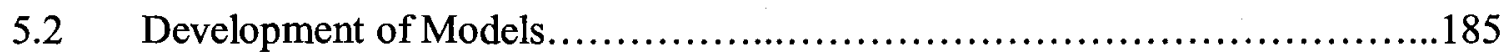

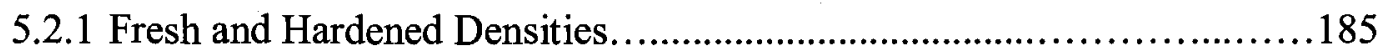

5.2.1.1 Phase-Based Models........................................186

5.2.1.2 Mixture-Based Models......................................188

5.2.1.3 Experimental Verification.....................................190

5.2.2 Elastic Modulus.........................................................191

5.2.2.1 Phase-Based Models........................................192

5.2.2.2 Mixture-Based Models.........................................195

5.2.2.3 Experimental Verification.................................197

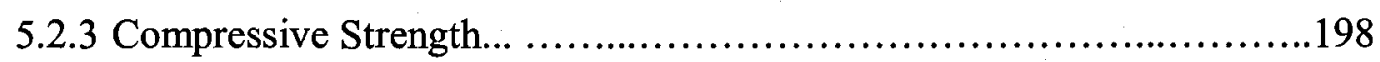


5.2.3.1 Equivalent-Mortar-Volume (EMV) Based Models

5.2.3.2 Light-Weight-Aggregate (LWA) Based Model. 203

5.2.3.3 Experimental Verification .204

5.2.4 Creep .206

5.2.5 Drying shrinkage .210

5.3 Evaluation of Existing Empirical Equations.............................211

5.3.1 Relationship between Compressive and Tensile Strengths.............212

5.3.2 Relationship between Compressive Strength and Elastic Modulus........213

5.3.3 Compressive Stress-Strain Relationship...........................216

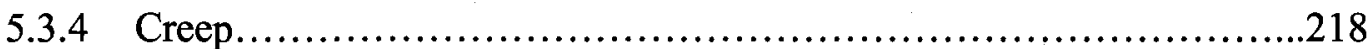

5.3.5 Drying Shrinkage..............................................221

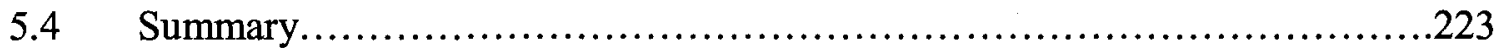

CHAPTER 6- STRUCTURAL PERFORMANCE OF RECYCLED REINFORCED CONCRETE BEAMS: FLEXURE.............................................. 258

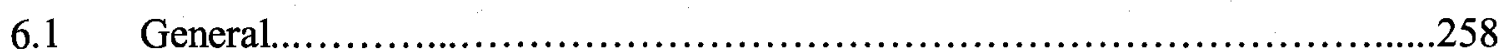

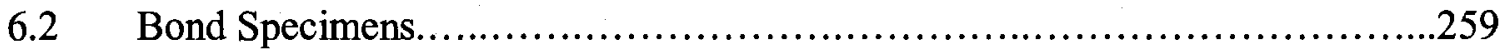

6.2.1 Mix Proportions................................................260

6.2.2 Test Setup.................................................261

6.2.3 Details of Bond Specimens......................................262

6.2.4 Experimental Observations........................................263

6.2.4.1 Effect of Mix Proportioning Method........................264

6.2.4.2 Effect of Test Bar Size...................................265

6.2.4.3 Effect of Aggregate Type....................................266 
6.2.4.4 Failure Modes............................................267

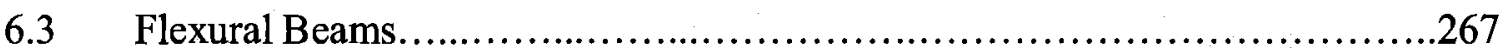

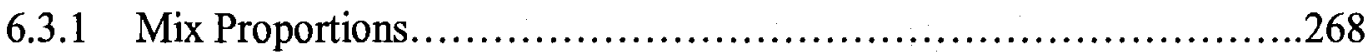

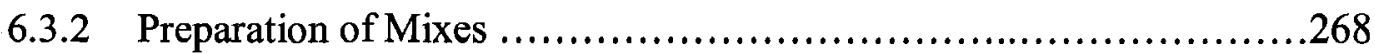

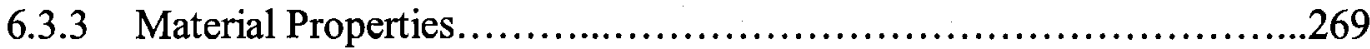

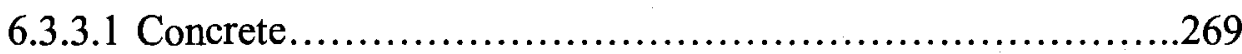

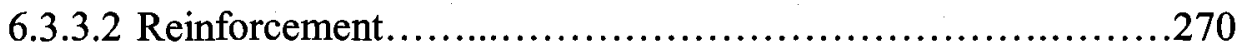

6.3.4 Details of RRC Beams.............................................270

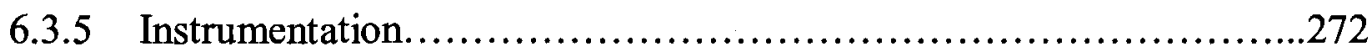

6.3.5.1 Strain Gauges ...............................................272

6.3.5.2 Linear Potentiometers.......................................273

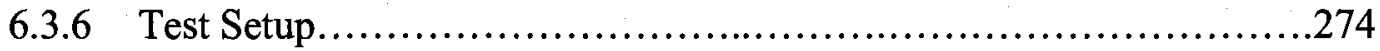

6.3.7 Flexural Behaviour of RRC Beams.................................275

6.3.7.1 Failure Modes.............................................277

6.3.7.2 Effect of Tension Reinforcement.............................278

6.3.7.3 Effect of Compression Steel...............................281

6.3.7.4 Effect of Concrete Type..................................283

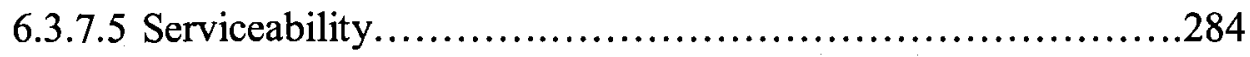

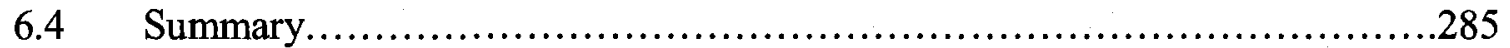

CHAPTER 7- STRUCTURAL PERFORMANCE OF RECYCLED REINFORCED

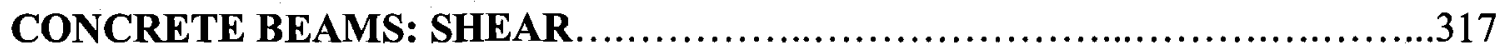

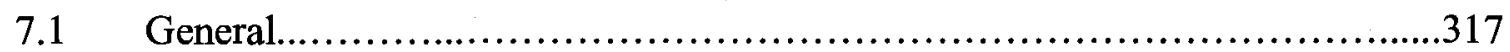

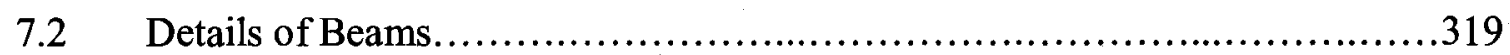


7.2.1 Beams without Shear Reinforcement...................................319

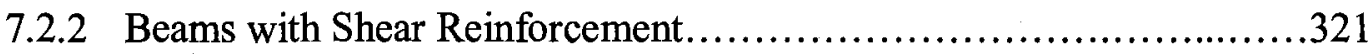

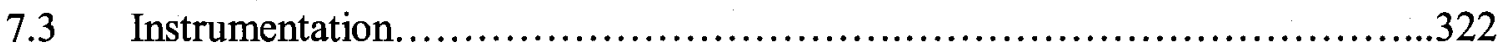

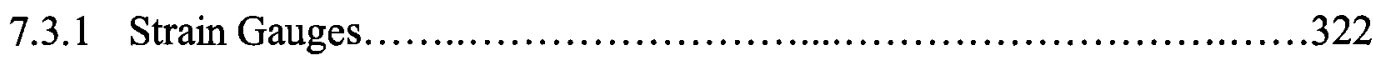

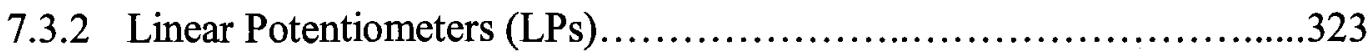

7.3.3 Linear Variable Displacement Transducers (LVDTs) ....................324

7.4 Shear Performance of RRC Beams without Shear Reinforcement.....................324

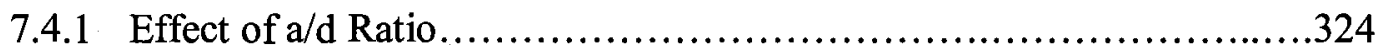

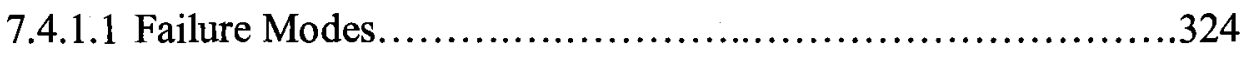

7.4.1.2 Ultimate Shear Strength....................................328

7.4.1.3 Shear Performance.............................................330

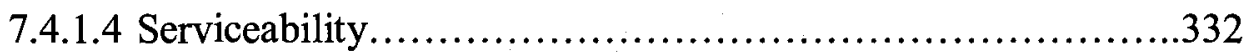

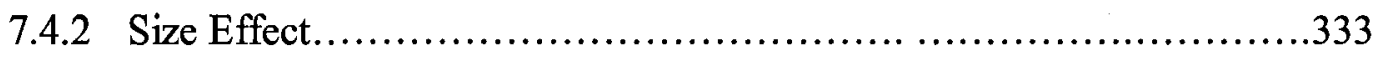

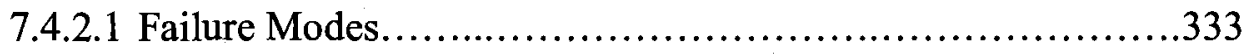

7.4.2.2 Ultimate Shear Strength......................................336

7.4.2.3 Shear Performance........................................337

7.4.2.4 Serviceability.............................................. 340

7.5 Shear Performance of RRC Beams with Transverse Reinforcement.............340

7.5.1.1 Failure Modes...........................................340

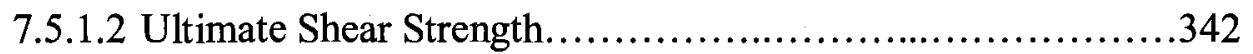

7.5.1.3 Shear Performance..............................................343

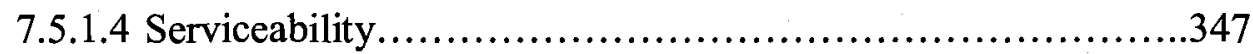

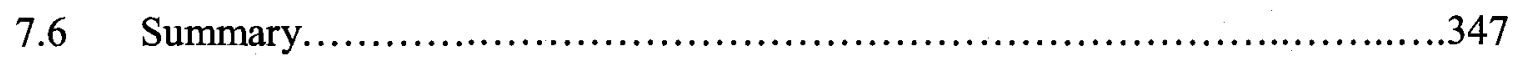




\section{CHAPTER 8- ANALYSIS OF THE EXPERIMENTAL DATA: ULTIMATE} FLEXURAL AND SHEAR STRENGTHS OF RRC BEAMS.

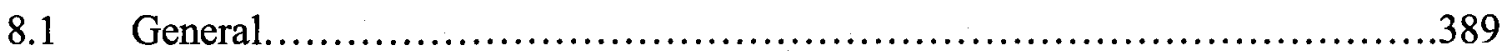

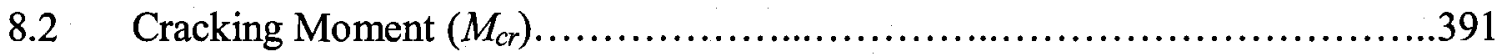

8.3 Equivalent Rectangular Stress Block Parameters $\left(\alpha_{1}\right.$ and $\left.\beta_{1}\right) \ldots \ldots \ldots \ldots \ldots \ldots . \ldots 394$

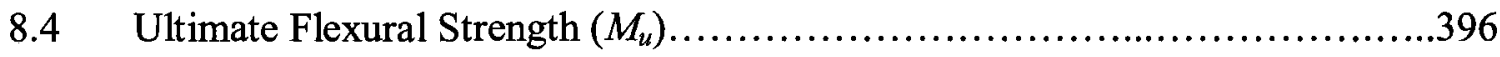

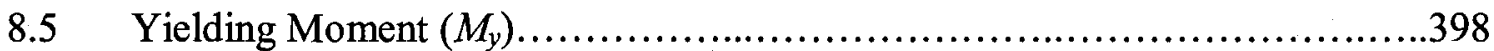

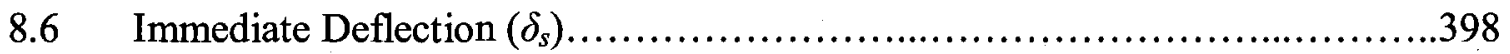

$8.7 \quad$ Ultimate Shear Strength...................................................401

8.7.1 Shear Strength without Shear Reinforcement $\left(\mathrm{v}_{\mathrm{c}}\right) \ldots \ldots \ldots \ldots \ldots \ldots \ldots \ldots \ldots \ldots . . . \ldots 1$

8.7.1.1 Shear Strength Variation with a/d..............................401

8.7.1.2 Shear Strength for Different Beam Depths (Size Effect)..........404

8.7.2 Shear Strength of Beams with Shear Reinforcement $\left(v_{u}\right) \ldots \ldots \ldots \ldots \ldots \ldots . . . .406$

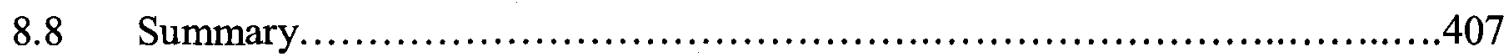

CHAPTER 9- CONCLUSIONS AND FUTURE WORK.............................425

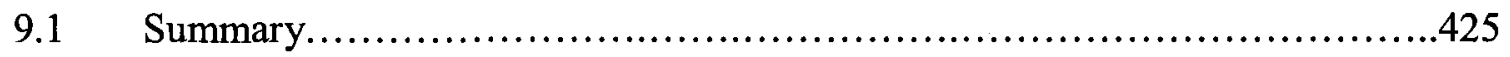

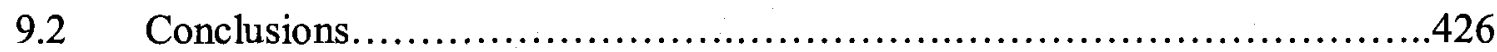

9.2.1 Evaluation of the Proposed Mix proportioning Method....................428

9.2.1.1 Short-Term properties..................................428

9.2.1.2 Stress-Strain Relationship...................................428

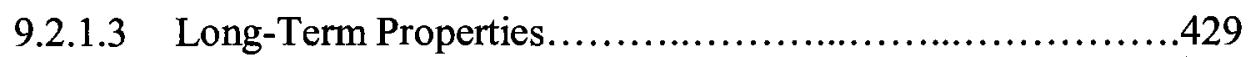

9.2.1.4 Bond Performance.........................................429

9.2.1.5 Flexural Performance..........................................430 
9.2.1.6 Shear Performance.......................................431

9.2.2 Developed Models for Short and Long Term Properties of RAC........432

9.2.3 Evaluation of Existing Empirical Equations.......................433

9.2.3.1 Short and Long term Properties of RAC...................434

9.2.3.2 Ultimate Flexural Strength................................434

9.2.3.3 Ultimate Shear Strength.................................435

9.3 Recommendations for Future Work..................................436

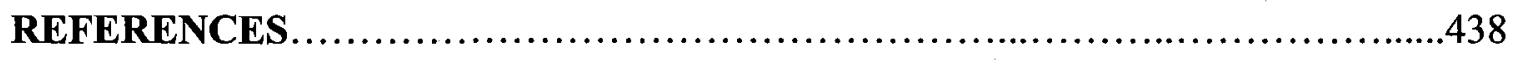

APPENDIX A: DETERMINATION OF RESIDUAL MORTAR SPECIFIC GRAVITY: DIRECT METHOD ...........................................457

APPENDIX B: DETAILS OF EXPERIMENTAL INVESTIGATION CARRIED

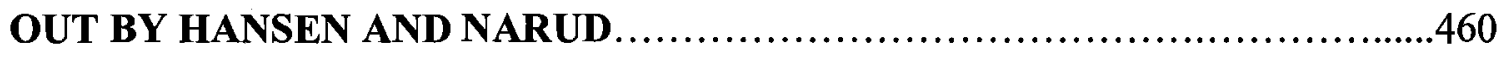
APPENDIX C: THE PRINCIPAL STEPS IN FULL RESPONSE ANALYSIS OF

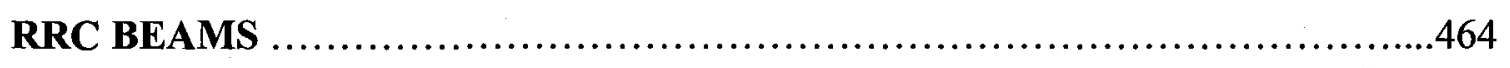




\section{LIST OF TABLES}

Table 1.1: Construction and demolition (C\&D) waste and percentage of concrete

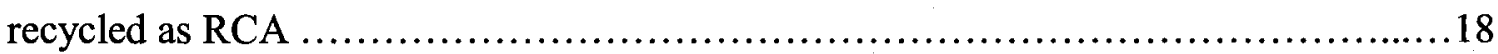

Table 2.1: Existing empirical models for elastic modulus of concrete .................64

Table 2.2: Summary of the creep empirical equations proposed by ACI Committee 209

Table 2.3: Summary of the creep empirical equations proposed by CEB-FIP .66

Table 2.4: Summary of the shrinkage empirical equations proposed by ACI Committee 209 .67

Table 2.5: Summary of the shrinkage empirical equations proposed by CEB-FIP ......68

Table 2.6: Summary of the studies performed by previous researchers on compressive, tensile, and flexural strength of RAC

Table 2.7: Summary of findings by previous researchers about elastic modulus of RAC

Table 2.8: Summary of the findings of previous researchers about shrinkage and creep of

RAC .72

Table 2.9: The improvements achieved by previous researchers on compressive, tensile, and flexural strength of RAC . .73

Table 2.10: The improvements achieved by previous researchers with respect to elastic modulus of RAC .74

Table 2.11: The improvements achieved by previous researchers with respect to shrinkage and creep of RAC. .74 
Table 2.12: Summary of the findings of previous researchers about structural behaviour

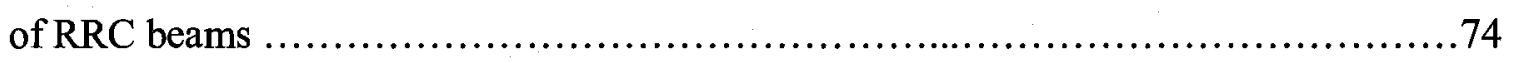

Table 2.13: Specified limitations on RCA properties by different countries ..............75

Table 2.14: Summary of simplified and general shear design methods of CSA A23.3-04

Table 2.15: Summary of existing expressions for the concrete contribution to shear resistance. .77

Table 2.16: Summary of existing expressions for the stress-strain relationship of normal

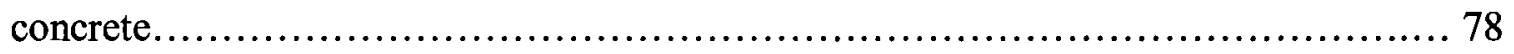

Table 3.1: Average physical properties for coarse and fine aggregates.................112

Table 3.2: Mix proportions of PM mixes.....................................113

Table 3.3: Mix proportions of PV mixes........................................113

Table 3.4: Fresh properties of PM mixes.......................................114

Table 3.5: Fresh properties of PV mixes........................................... 115

Table 3.6: Hardened properties of PM mixes...................................116

Table 3.7: Hardened properties of PV mixes.....................................117

Table 4.1: Measured properties for the residual mortar of RCA-MO...................169

Table 4.2: Measured properties for the residual mortar of RCA-VA ..................169

Table 4.3: Measured properties for the original virgin aggregate of RCA-MO.........169

Table 4.4: Measured properties for the original virgin aggregate of RCA-VA..........170

Table 4.5: Mix proportions of mixes............................................171

Table 4.6: Fresh properties of mixes ..........................................172

Table 4.7: Hardened properties of mixes ....................................... 172 
Table 4.8: Fresh and hardened properties of stress-strain test specimens

Table 4.9: Fresh and hardened properties of creep and shrinkage specimens. 173

Tables 5.1: The experimental/predicted density values using phase-based models for RAC mixes made with RCA-MO. 227

Tables 5.2: The experimental/predicted density values using phase-based models for RAC mixes made with RCA-VA .228

Table 5.3: The experimental/predicted hardened and fresh density values using mixturebased models for RAC mixes.

Table 5.4: The experimental/predicted elastic modulus values using phase-based models for RAC mixes .230

Table 5.5: The experimental/predicted elastic modulus values using mixture-based models for RAC mixes .231

Table 5.6: Comparison of experimental and predicted compressive strength values of RAC mixes using EMV based models. 232

Table 5.7: The experimental and predicted compressive strength value of RAC mixes using lightweight-aggregate-based models .233

Table 6.1: Fresh and hardened properties of bond specimens 287

Table 6.2: Details of bond specimens. 287

Table 6.3: Mechanical properties of steel bars used for bond test 287

Table 6.4: Summarized bond of test results .288

Table 6.5: Mix proportions of flexural RRC and control beams 288

Table 6.6: Fresh and hardened properties of flexural RRC and control beams .288

Table 6.7: Structural details of RRC beams tested in flexure .289 
Table 6.8: Summary test results for RRC beams under flexure.......................289

Table 7.1: Fresh and hardened properties of shear RRC and control beams..............349

Table 7.2: Structural details of shear RRC beams without shear reinforcement..........350

Table 7.3: Structural details of shear RRC beams with shear reinforcement.............351

Table 7.4: The measured strains in longitudinal and shear reinforcements and concrete at

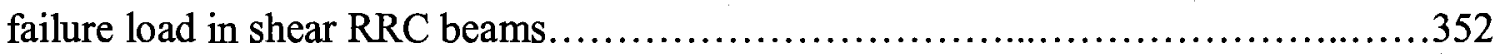

Table 8.1a: Predicted and observed cracking moments of RRC beams made of RCA-MO

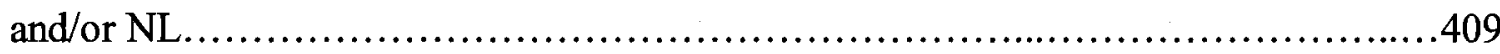

Table 8.1b: Predicted and observed cracking moments of RRC beams made of RCA-VA and/or NG.

Table 8.2: Predicted over observed equivalent stress block parameters for CM-C, EM-C and CL-C mixes 410

Table 8.3a: Predicted and observed yielding moment and ultimate flexural strength of FEM and FCL beams. .411

Table 8.3b: Predicted and observed yielding moment and ultimate flexural strength of FEV and FCG beams.

Table 8.4: Mid span deflections of flexural RRC beams

Table 8.5: Ratio of observed to predicted ultimate shear resistance of SEM and SCL beams with different $\mathrm{a} / \mathrm{d}$ ratios.

Table 8.6: Ratio of observed to predicted ultimate shear resistance of SEV and SCG beams with different $\mathrm{a} / \mathrm{d}$ ratios 414

Table 8.7: Ratio of observed to predicted ultimate shear resistance of SEM and SCL beams with different depths 
Table 8.8: Ratio of observed to predicted ultimate shear resistance of SEV and SCG beams with different depths........................................................ Table 8.9: Comparison of observed shear strength of RRC beams with their predicted

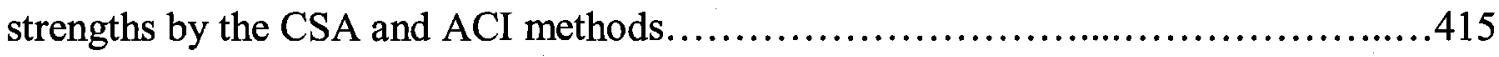

Table B.1: The Mix Proportions of Original Concretes..............................462

Table B.2: Physical Properties of Natural Gravel and Recycled Coarse Aggregates....462

Table B.3: The Mix Proportions of RAC and NAC mixes...........................462

Table B.4: The Compressive Strength of RAC and NAC mixes......................463 


\section{LIST OF FIGURES}

Figure 1.1: Schematic representation of recycled aggregate concrete (RAC).............18

Figure 2.1: Shear resistance components in beams..............................79

Figure 2.2: Definition of $s_{z}$ accounting for effect of well distributed longitudinal reinforcement on size effect in shear ............................................ 79

Figure 3.1: Slump versus RCA content for different $w / c$ ratios.....................118

Figure 3.2: Slump versus w/c ratio for different RCA content levels..................118

Figure 3.3: Fresh density versus RCA content for different w/c ratios..................119

Figure 3.4: Variation of fresh density with w/c ratio for different RCA content levels

Figure 3.5: Variation of fresh density with RCA content at different w/c ratios.........120

Figure 3.6: Variation of fresh density with TMV for different w/c ratios..............120

Figure 3.7: Hardened density versus RCA content for different w/c ratios...............121

Figure 3.8: Variation of hardened density with w/c ratio at RCA content levels........121

Figure 3.9: Variation of hardened density with RCA content for different w/c ratios. .122

Figure 3.10: Variation of hardened density with TMV at different $w / c$ ratios.

Figure 3.11: Compressive strength versus RCA content for different w/c ratios.... 123

Figure 3.12: Compressive strength versus $\mathrm{c} / \mathrm{w}$ ratio for different $\mathrm{RCA}$ content levels. 123

Figure 3.13: Variation of compressive strength with RCA content for different w/c ratios. 
Figure 3.14: Variation of compressive strength with TMV for different w/c ratios. 124

Figure 3.15: Splitting tensile strength versus RCA content for different w/c ratios. 125

Figure 3.16: Variation of tensile strength with w/c ratio for different RCA content levels.

Figure 3.17: Variation of tensile strength with RCA content for different w/c ratios 126

Figure 3.18: Variation of tensile strength with TMV for different $w / c$ ratios 126

Figure 3.19: Elastic modulus versus RCA content for different $w / c$ ratios .127

Figure 3.20: Variation of elastic modulus with w/c ratio for different RCA content levels.

Figure 3.21: Variation of elastic modulus with RCA content for different w/c ratios. 128

Figure 3.22: Variation of elastic modulus with TMV for different $w / c$ ratios 128

Figure 4.1: Schematic illustration of the composition of RAC proportioned by the conventional method

Figure 4.2: Schematic illustration of the composition of RAC proportioned by the proposed method 174

Figure 4.3: Variation of RCA volume with RMC

Figure 4.4: Schematic illustration of the maximum feasible RMC in a mix proportioned by the proposed method. 175

Figure 4.5: Effect of mix proportioning method on the slump 175 
Figure 4.6: Effect of mix proportioning method on the fresh density 176

Figure 4.7: Effect of mix proportioning method on the hardened density .............176

Figure 4.8: Effect of mix proportioning method on the compressive strength ............176

Figure 4.9: Effect of mix proportioning method on the elastic modulus ...............177

Figure 4.10: Schematic plan of stress-strain test setup.............................177

Figure 4.11: Observed stress-strain relationship of different mixes...................178

Figure 4.12: Effect of mix proportioning method on the compressive stress-strain relationship

Figure 4.13: Schematic view of creep test setup..................................179

Figure 4.14: Effect of mix proportioning method on the creep coefficient .............180

Figure 4.15: Effect of mix proportioning method on the (total strain/initial strain)......180

Figure 4.16: Effect of mix proportioning method on the specific creep.................180

Figure 4.17: Effect of mix proportioning method on the creep strain.................181

Figure 4.18: Effect of mix proportioning method on the (creep + shrinkage) strain...181

Figure 4.19: Effect of aggregate type on creep coefficient...........................182

Figure 4.20: Effect of mix proportioning method on the drying shrinkage strain.......182

Figure 4.21: Effect of aggregate type on drying shrinkage..........................183

Figure 5.1: Predicted versus experimental fresh density values of RAC mixes based on different models. .234

Figure 5.2: Predicted versus experimental hardened density values of RAC mixes based on different models. .235

Figure 5.3: Predicted versus experimental elastic modulus values of RAC mixes using parallel models .236 
Figure 5.4: Predicted versus experimental elastic modulus values of RAC mixes using series models.

Figure 5.5: Predicted versus experimental elastic modulus values of RAC mixes using Hirsch models. .238

Figure 5.6: Predicted Vs Experimental elastic modulus values of RAC mixes using Counto models. .239

Figure 5.7: Predicted Vs Experimental elastic modulus values of RAC mixes using Mindess models. 240

Figure 5.8: Stress-Strain variation of TM, RM, and NM in RAC .241

Figure 5.9: Predicted versus experimental compressive strength values of RAC mixes based on different models. .242

Figure 5.10: Experimental versus predicted splitting tensile strength values based on ACI-318 .243

Figure 5.11: Elastic modulus versus hardened density for all RAC and NAC mixes

Figure 5.12: Experimental versus predicted elastic modulus of mixes made with RCAMO\&NL based on CSA and ACI equations.

Figure 5.13: Experimental versus predicted elastic modulus of mixes made with RCAVA\&NG based on CSA and ACI equations .245

Figure 5.14: Experimental versus predicted elastic modulus of mixes made with RCAMO\&NL based on different equations. .246

Figure 5.15: Experimental versus predicted elastic modulus of mixes made with RCAVA\&NG based on different equations .247 
Figure 5.16: Experimental versus predicted elastic modulus based on Eurocode2 (2002)

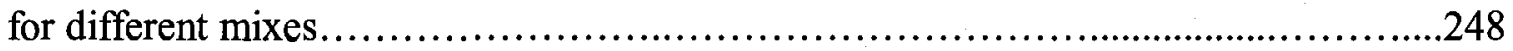
Figure 5.17: Normalized compressive stress-strain relationship based on experimental

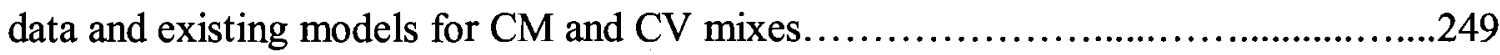
Figure 5.18: Normalized compressive stress-strain relationship based on experimental data and existing models for EM and EV mixes.

Figure 5.19: Normalized compressive stress-strain relationship based on experimental data and existing models for $\mathrm{CL}$ and $\mathrm{CG}$ mixes. .251

Figure 5.20: Normalized creep coefficient versus time relationship based on experimental data and $\mathrm{CEB}$ and $\mathrm{ACI}$ equations for $\mathrm{CM}$ and $\mathrm{CV}$ mixes. .252 Figure 5.21: Normalized creep coefficient versus time relationship based on experimental data and $\mathrm{CEB}$ and $\mathrm{ACI}$ equations for $\mathrm{EM}$ and $\mathrm{EV}$ mixes. .253

Figure 5.22: Normalized creep coefficient versus time relationship based on experimental data and $\mathrm{CEB}$ and $\mathrm{ACI}$ equations for $\mathrm{CL}$ and $\mathrm{CG}$ mixes. .254 Figure 5.23: Normalized shrinkage strain versus time relationship based on experimental data and $\mathrm{CEB}$ and $\mathrm{ACI}$ equations for $\mathrm{CM}$ and $\mathrm{CV}$ mixes .255 Figure 5.24: Normalized shrinkage strain versus time relationship based on experimental data and $\mathrm{CEB}$ and $\mathrm{ACI}$ equations for EM and $\mathrm{EV}$ mixes 256

Figure 5.25: Normalized shrinkage strain versus time relationship based on experimental data and $\mathrm{CEB}$ and $\mathrm{ACI}$ equations for $\mathrm{CL}$ and $\mathrm{CG}$ mixes. 257

Figure 6.1: Schematic of beam-end bond test setup. 290 Figure 6.2: Details of bond specimen. 291 
Figure 6.3: Effect of mix proportioning method on the bond stress loaded-end response.

Figure 6.4: Effect of mix proportioning method on the bond stress-free end slip response.

Figure 6.5: Effect of bar size on the bond stress variation with loaded and free-end displacements

Figure 6.6: Effect of aggregate type on the bond stress variation with loaded and freeend displacements. 294

Figure 6.7: Cracking patterns of bond specimens using bar No.30: Front view. 294

Figure 6.8: Cracking patterns of bond specimens using bar No.30: Top view .296

Figure 6.9: Preparation of materials prior to mixing. .297

Figure 6.10: Concrete mixing procedure. .298

Figure 6.11: Casting and curing procedures of beams. .299

Figure 6.12: Typical stress-strain relationship of steel reinforcement bars. .300

Figure 6.13: Typical dimensions and reinforcement details of the beams tested in flexure .300

Figure 6.14: Schematic instrumentation employed to test the beams under flexure.....301

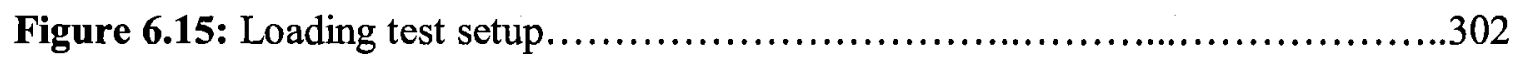

Figure 6.16: Typical crack patterns of FEM and control FCL beams..................303

Figure 6.17: Typical crack patterns of FEV and control FCG beams.................304

Figure 6.18: Effect of longitudinal tension reinforcement ratio on: a) cracking moment, b) yielding moment, and c) ultimate moment strengths. .305 
Figure 6.19: Effect of longitudinal tension reinforcement ratio on the moment- deflection

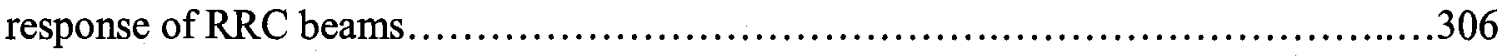
Figure 6.20: Deflection curve of singly reinforced beams at different load levels.....307 Figure 6.21: Variation of concrete compression strain along singly reinforced beams at different load levels. .308

Figure 6.22: Variation of longitudinal steel strain along singly reinforced beams at different load levels. .309

Figure 6.23: Effect of compression steel on flexural behaviour of RRC beams..........310 Figure 6.24: Deflection curve of doubly reinforced beams at different load levels. 311

Figure 6.25: Variation of concrete compression strain along doubly reinforced beams at different load levels

Figure 6.26: Variation of longitudinal steel strain along doubly reinforced beams at different load levels 312

Figure 6.27: Effect of concrete type on cracking, yield and ultimate moments of beams. .312

Figure 6.28: Effect of concrete type on flexural behaviour of RRC beams .313 Figure 6.29: Typical crack spacing of FEM and control FCL beams at service load. 315

Figure 6.30: Typical crack spacing of FEV and control FCG beams at service load....316

Figure 7.1: Details of shear beams .352

Figure 7.2: Schematic arrangement of strain gauges in shear beams. .353

Figure 7.3: Schematic arrangement of LPs for deflection measurements. 353 
Figure 7.4: Schematic arrangement of side LVDTs for shear deformation measurements

Figure 7.5: Typical crack patterns of RRC shear beams. .354

Figure 7.6: Experimental ultimate shear stress values for different a/d ratios. .355

Figure 7.7: The effect of shear span ratio on longitudinal steel strain near support (LS1) .356

Figure 7.8: Effect of a/d ratio on shear behaviour of RRC beams .357

Figure 7.9: Variation of concrete compression strain along the beams for different a/d ratios. .359

Figure7.10: Variation of longitudinal steel strain along beam for different a/d ratios 360

Figure 7.11: Effect of aggregate type on normalized shear stress resistance-midspan deflection response of $\mathrm{RRC}$ beams for different $\mathrm{a} / \mathrm{d}$ ratios. .362

Figure 7.12: Effect of aggregate type on normalized shear stress resistance-diagonal deformation response of RRC beams at different a/d ratios. 364

Figure 7.13: Typical crack patterns of shear RRC beams .366 Figure 7.14: Experimental nominal shear strength of RRC beams for different size beams. .367

Figure 7.15: Size effect on shear behaviour of RRC members 368 Figure 7.16: Variation of concrete compression strain along beam for different size beams. 370 Figure 7.17: Variation of longitudinal steel strain along beam for different size beams 371 
Figure 7.18: Effect of aggregate type on normalized shear stress -midspan deflection response of RRC beams with different depths.

Figure 7.19: Effect of aggregate type on normalized shear stress -diagonal deformation response of RRC beams with different depths.

Figure 7.20: Crack patterns of SEM and SCL beams with different shear reinforcement ratios.

Figure 7.21: Crack patterns of SEV and SCG beams with different shear reinforcement ratios.

Figure 7.22: The effect of shear reinforcement ratio on shear reinforcement strain in the west shear span (TS2) .379

Figure 7.23: Experimental ultimate shear stress resistance values. .380

Figure 7.24: Effect of shear reinforcement ratio on shear behaviour of RRC members.

Figure 7.25: Variation of shear reinforcement and concrete contribution to shear resistance along beams. 383

Figure 7.26: Effect of aggregate type on shear-midspan deflection response of RRC beams with different shear reinforcement ratios.

Figure 7.27: Effect of aggregate type on shear-diagonal deformation response of RRC beams with different shear reinforcement ratios. 385

Figure 7.28: Variation of concrete compression strain along beam with different shear reinforcement ratios. 387

Figure 7.29: Variation of longitudinal steel strain along beam with different shear reinforcement ratios. 388 
Figure 8.1a: Observed versus calculated cracking moments of SEM and FEM beams

Figure 8.1b: Observed versus calculated cracking moments of SEV and FEV beams .416

Figure 8.2: Calculated and observed equivalent stress block parameters.

Figure 8.3: Experimental and predicted ultimate flexural strength of RRC beams .418

Figure 8.4a: Experimental and predicted ultimate shear strength of RRC beams with $\mathrm{a} / \mathrm{d}=1.5$

Figure 8.4b: Experimental and predicted ultimate shear strength of RRC beams with $\mathrm{a} / \mathrm{d}=2.0$.

Figure 8.4c: Experimental and predicted ultimate shear strength of SEM and SCL beams with $\mathrm{a} / \mathrm{d}=2.7$.

Figure 8.4d: Experimental and predicted ultimate shear strength of SEV and SCG beams with $\mathrm{a} / \mathrm{d}=2.7$. .420

Figure 8.4e: Experimental and predicted ultimate shear strength of RRC beams with $\mathrm{a} / \mathrm{d}=4.0$

Figure 8.5a: Experimental and predicted ultimate shear strength of RRC beams with $\mathrm{d}=200 \mathrm{~mm}$.

Figure 8.5b: Experimental and predicted ultimate shear strength of SEM and SCL beams with $\mathrm{d}=300 \mathrm{~mm}$. 422

Figure 8.5c: Experimental and Predicted ultimate shear strength of SCG and SEV beams with $\mathrm{d}=300 \mathrm{~mm}$ .422 
Figure 8.5d: Experimental and predicted ultimate shear strength of RRC beams with $\mathrm{d}=400 \mathrm{~mm}$.

Figure 8.5e: Experimental and predicted ultimate shear strength of RRC beams with

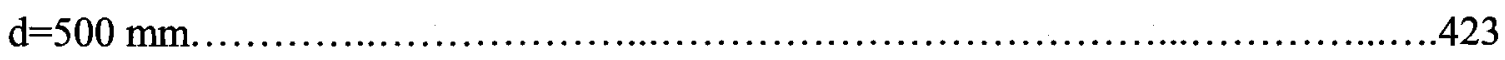

Figure 8.6: Experimental and predicted shear resistance of beams with stirrups........424 


\section{LIST OF SYMBOLS}

$$
\begin{array}{ll}
\alpha_{1} & =\text { equivalent rectangular stress block width parameter } \\
\beta_{1} & =\text { equivalent rectangular stress block height parameter } \\
\gamma_{c} & =\text { hardened density of concrete, } \mathrm{kg} / \mathrm{m}^{3} \\
\gamma_{D R}^{C A} & =\text { dry-rodded unit weight of coarse aggregate } \\
\gamma_{M} & =\text { density of mortar } \\
\gamma_{N A} & =\text { density of NA } \\
\gamma_{N A C} & =\text { density of NAC mix } \\
\gamma_{N M} & =\text { density of NM } \\
\gamma_{O V A} & =\text { density of OVA } \\
\gamma_{R A C} & =\text { density of RAC mix }
\end{array}
$$$$
\gamma_{R A C}^{100 \% R C A}=\text { density of RAC mix at } 100 \% \text { RCA content proportioned by the conventional }
$$$$
\text { method }
$$$$
\gamma_{R C A}=\text { density of RCA }
$$$$
\gamma_{R M}=\text { density of RM }
$$$$
\gamma_{T M}=\text { equivalent density of total mortar in RAC mix }
$$$$
\gamma_{T N A}=\text { equivalent density of TNA in RAC mix }
$$$$
\varepsilon \quad=\text { compressive strain in the specimen corresponding to the stress }
$$$$
\varepsilon_{0} \quad=\text { instantaneous strain of concrete immediately after loading }
$$$$
\varepsilon_{c}^{N A C}=\text { creep strain of NAC }
$$$$
\varepsilon_{c}^{R A C}=\text { creep strain of RAC }
$$ 
$\varepsilon_{c}(t)=$ creep strain of concrete at any age

$\varepsilon_{s h} \quad=$ drying shrinkage strain of concrete

$\varepsilon_{s p}(t)=$ unit or specific creep of concrete

$\varepsilon_{\text {total }}(t)=$ total creep strain of concrete at any age

$\lambda=$ factor to account for low density concrete

$\mu \quad=$ Poisson's ratio of concrete

$\mu_{a} \quad=$ Poisson's ratio of aggregate

$\mu_{N A} \quad=$ Poisson's ratio of NA

$\mu_{N A C}=$ Poisson's ratio of NAC

$\mu_{R A C}=$ Poisson's ratio of RAC

$\mu_{R C A}=$ Poisson's ratio of RCA

$v \quad=$ pulse velocity, $\mathrm{m} / \mathrm{s}$

$\rho_{s} \quad=$ percentage of shear reinforcement

$\sigma \quad=$ compressive stress due to applied load on the specimen

$\sigma_{c} \quad=$ sustained stress acting on concrete specimen

$\sigma_{N M}=$ corresponding stress in NM

$\sigma_{R M} \quad=$ corresponding stress in RM

$\sigma_{T M} \quad=$ corresponding stress in TM

$\tau_{b} \quad=$ bond strength

a = shear span

$A \quad=$ air content 
$A_{c} \quad=$ cross section area of the specimen

$A C^{C A}=$ absorption capacity of coarse aggregate

$A C^{F A}=$ absorption capacity of fine aggregate

$b \quad=$ section width

$c \quad=$ creep of concrete

$c_{p} \quad=$ creep of neat cement paste of the same quality as used in concrete

$c_{N A C}=$ creep coefficient of NAC

$c_{R A C}=$ creep coefficient of RAC

$C \quad=$ compressions force acting on the section at ultimate state

$C_{t} \quad=$ creep coefficient of concrete at any age

$C_{t}^{\text {Total }}=$ total creep coefficient of concrete at any age

$d_{b} \quad=$ nominal diameter of the test bar

$d_{v} \quad=$ effective shear depth, taken as the greater of $0.9 d$ and $0.72 h$

$d \quad=$ beam effective depth

$E_{a} \quad=$ Elastic modulus of aggregate

$E_{c} \quad=$ secant modulus of elasticity of concrete, GPa

$E_{d} \quad=$ dynamic modulus of elasticity of concrete, GPa

$E_{M} \quad=$ elastic modulus of mortar

$E_{N A} \quad=$ elastic modulus of NA

$E_{N A C}=$ elastic modulus of NAC

$E_{N M}=$ elastic modulus of NM 
$E_{\text {OVA }}=$ elastic modulus of OVA

$E_{p} \quad=$ elastic modulus of neat cement paste of the same quality as used in concrete

$E_{\text {RAC }}=$ elastic modulus of RAC

$E_{R A C}^{100 \% R C A}=$ elastic modulus of RAC mix at $100 \%$ RCA content proportioned by the conventional method

$E_{R C A}=$ elastic modulus of RCA

$E_{R M} \quad=$ elastic modulus of RM

$E_{t} \quad=$ tangent modulus of elasticity of concrete, $\mathrm{GPa}$

$E_{T M} \quad=$ equivalent elastic modulus of total mortar in RAC

$E_{T N A}=$ equivalent elastic modulus of TNA in RAC

$f_{c} \quad=$ compressive strength of concrete

$f_{c}^{\prime} \quad=$ specified compressive strength of concrete, $\mathrm{MPa}$

$f_{c}^{\prime N A C}=$ compressive strength of companion NAC

$f_{c}^{\prime N M}=$ compressive strength of NM

$f_{c}^{\prime O C}=$ compressive strength of original concrete

$f_{c}^{\prime R A C}=$ compressive strength of RAC

$f_{c}^{\prime R M}=$ corresponding stress in RM

$f_{c}^{\prime T M}=$ corresponding stress in TM

$f_{r} \quad=$ modulus of rupture of concrete, MPa

$f_{t} \quad=$ splitting tensile strength of concrete 


$$
\begin{array}{ll}
g & =\text { volume fraction of aggregate } \\
\mathrm{h} & =\text { section height } \\
I_{e} \quad & =\text { effective moment of inertia } \\
I_{g} \quad=\text { gross moment of inertia } \\
\mathrm{jd} \quad=\text { distance between equivalent compressive and tensile forces in a section } \\
K_{R M} \quad=\text { residual mortar creep coefficient } \\
l_{1} \quad=\text { initial comparator reading } \\
l_{2} \quad=\text { comparator reading at time } \mathrm{t} \\
l_{g} \quad=\text { effective gauge length between the inner most ends of the gauge studs }
\end{array}
$$

embedded in the specimens

$L_{b} \quad=$ embedded length of the test bar in concrete

$L_{i} \quad=$ initial length of LVDT

$L_{n} \quad=$ net beam span, measured from centre to centre of the supports

$m \quad=$ LVDT reading

$m_{c}^{C A}=$ moisture content of coarse aggregate

$\mathrm{M} \quad=$ Moment

$M_{a} \quad=$ applied maximum moment corresponding to the load for which deflection is calculated

$M_{c r} \quad=$ cracking moment

$M_{\text {crush }}=$ crushing moment

$M_{r} \quad=$ ultimate flexural resistance 


$$
\begin{array}{ll}
M_{y} & =\text { yielding moment } \\
M_{u} & =\text { ultimate moment } \\
\mathrm{n} & =\text { porosity of coarse aggregate } \\
P & =\text { applied load on the specimen } \\
P_{c r} \quad & =\text { cracking load } \\
P_{\text {crush }} & =\text { crushing load } \\
P_{y} \quad=\text { yielding load } \\
P_{u} \quad=\text { ultimate load } \\
R_{\min } \quad=\text { minimum replacement ratio of RCA by NA in RAC mix proportioned by the }
\end{array}
$$

\section{EMV method}

$R_{v} \quad=$ volume-based replacement ratio of RCA with NA

$R_{w} \quad=$ weighted-based replacement ratio of RCA with NA

$R M_{v}=$ residual mortar volume in RCA

$\mathrm{RMC}=$ residual mortar content in RCA

$R M C_{\max }=$ maximum permissible $\mathrm{RMC}$ in case of desired $100 \% \mathrm{RCA}$ content in RAC mix proportioned by the EMV method

$S_{c} \quad=$ shrinkage of concrete

$S_{p} \quad=$ shrinkage of neat cement paste of the same quality as used in concrete

$S_{N A C}=$ shrinkage of NAC

$S_{R A C}=$ shrinkage of RAC

$S_{R M}=$ residual mortar shrinkage coefficient 
$S G^{C}=$ specific gravity of cement

$S G_{b}^{N A}=$ bulk specific gravity of NA

$S G_{b}^{\text {OVA }}=$ bulk specific gravity of OVA

$S G_{b}^{R C A}=$ bulk specific gravity of RCA

$S G_{D R}^{N A}=$ dry-rodded specific gravity of NA

$S G_{S S D}^{C A}=$ specific gravity of the SSD coarse aggregates

$S G_{D R}^{R C A}=$ dry-rodded specific gravity of RCA

$S G_{S S D}^{F A}=$ specific gravity of the SSD fine aggregates

$S G_{S S D}^{N A}=$ specific gravity of SSD NA

$S G_{S S D}^{O V A}=$ specific gravity of SSD OVA

$S G_{S S D}^{R C A}=$ specific gravity of SSD RCA

$u \quad=$ volume fraction of unhydrated cement

$U \quad=$ fresh density of concrete

$\mathrm{v} \quad=$ shear stress

$v_{c} \quad=$ shear stress resistance provided by concrete

$v_{s} \quad=$ shear stress resistance provided by shear reinforcement

$v_{u} \quad=$ ultimate shear stress resistance

$\mathrm{V} \quad=$ Shear force

$V_{a}^{C A}=$ absolute volume of coarse aggregate

$V_{c} \quad=$ concrete contribution to shear resistance

$V_{u} \quad=$ ultimate shear force acting on the beam 


$$
\begin{aligned}
& V_{D R-R C A}^{R A C}=\text { dry-rodded volume of RCA in RAC mix } \\
& V_{D R-N A}^{N A C}=\text { dry-rodded volume of NA in NAC mix } \\
& V_{M}^{N A C}=\text { volume of mortar in NAC mix } \\
& V_{\max R C A}^{R A C}=\text { the maximum RCA volume that can be added to a unit volume of RAC } \\
& V_{N A}^{N A C}=\text { volume of NA in NAC mix } \\
& V_{N A}^{R A C}=\text { volume of NA in RAC mix } \\
& V_{N M}^{R A C}=\text { volume of NM in RAC mix } \\
& V_{\text {OVA }}=\text { volume of OVA } \\
& V_{R C A}=\text { volume of } \mathrm{RCA} \\
& V_{R M} \quad=\text { volume of RM } \\
& V_{R C A}^{R A C}=\text { volume of RCA in RAC mix } \\
& V_{s} \quad=\text { shear reinforcement contribution to shear resistance } \\
& V_{T M}^{R A C}=\text { volume of TM in RAC mix } \\
& V_{T M}^{R A C-100 R C A}=\text { total mortar volume of RAC at } 100 \% \text { RCA content proportioned by the } \\
& \text { conventional method } \\
& V_{T N A}^{R A C}=\text { volume of TNA in RAC mix } \\
& W^{C} \quad \text { = weight of cement } \\
& W^{W} \quad=\text { weight of water } \\
& W_{A D}^{C A} \quad=\text { weight of coarse aggregate in air-dry condition } \\
& W_{c}^{R A C}=\text { required weight of cement in RAC mix }
\end{aligned}
$$


$W_{c}^{N A C}=$ required weight of cement in NAC mix

$W_{N A}^{N A C}=$ weight of coarse NA in the companion NAC mix

$W_{O D}^{C A}=$ required oven-dry weight of coarse aggregate

$W_{S S D}^{C A}=$ weight of the SSD coarse aggregate

$W_{S S D}^{F A}=$ weight of SSD fine aggregate

$W_{S S D, a v}=$ weighted average specific gravity of fine and coarse aggregates

$W_{O D}^{F A} \quad=$ oven-dry weight of fine aggregate

$W_{O D}^{N A} \quad=$ oven-dry weight of NA

$W_{O D}^{O V A}=$ oven-dry weight of OVA

$W_{O D}^{R C A}=$ oven-dry weight of RCA

$W_{O D}^{R M}=$ oven-dry weight of RM

$W_{O D-F A}^{R A C}=$ required oven-dry weight of fine aggregates in RAC mix

$W_{O D-N A}^{R A C}=$ required oven-dry weight of NA in RAC mix

$W_{O D-R C A}^{R A C}=$ required oven-dry weight of RCA in RAC mix

$W_{w}^{R A C}=$ required weight of water in RAC mix

$W_{O D-F A}^{N A C}=$ required oven-dry weight of fine aggregates in NAC mix

$W_{R C A}^{R A C}=$ weight of coarse RCA in RAC mix

$W_{w}^{N A C}=$ required weight of water in NAC mix

$\mathrm{y} \quad=$ distance from neutral axis 


\section{CHAPTER 1: INTRODUCTION}

\subsection{General}

Concrete is the most prevalent modern construction material and is universally used for all types of structures. In the fresh state, concrete typically contains about $12 \%$ cement and $80 \%$ aggregate by mass (Neville, 1995). Global construction industry uses approximately 1.6 billion tons of cement and 10 billion tons of sand, gravel, and crushed rock annually (Mehta, 2001) while in Canada the total annual aggregate supply in 2003 was around 350.5 million tons (Panagapko, 2003). The world's annual cement production of 1.6 billion tons accounts for about $7 \%$ of the global carbon dioxide $\left(\mathrm{CO}_{2}\right)$ emission into the atmosphere (Mehta, 2001). Producing one ton of portland cement requires about four giga-joules of energy. Mining large quantities of raw materials for the production of cement such as limestone and clay, and fuel such as coal, often results in extensive deforestation or denudation and top-soil loss (Mehta, 2001). Besides, the extraction of natural aggregates also has several negative environmental consequences such as destruction of the natural habitat and loss of the water storage capacity of the ground (Winfield and Taylor, 2005), as well as increased greenhouse gas (GHG) emissions due to the energy consumption required for mining, processing, and transportation of natural aggregates (Abbas et al., 2006a).

Although concrete structures may have service lives of several decades to more than a century, for several reasons, including the following, in reality they may not last that long:

i) Structures becoming obsolete and not serving their basic functions. 
ii) Extensive deterioration.

iii) Changes in zoning and market demand for new buildings/structures.

iv) Severe damage due to natural disasters such as earthquakes.

v) War-inflicted damage.

The demolished materials are considered as construction and demolition (C\&D) waste. Up until very recently, in most countries, this demolition concrete was treated as another type of waste and no regulations existed concerning its production and disposal. This was mostly due to the fact that other types of waste, primarily toxic wastes, required more immediate attention. In the last couple of decades, however, there has emerged greater emphasis on sustainability and so-called green practices. The worldwide production of concrete and masonry rubble has been estimated roughly as one billion tons per year (Mehta, 2001), but only a small fraction of this waste is used in the construction of new structures. Therefore, the construction industry produces a large amount of C\&D waste, of which concrete, at approximate consumption of 4.5 billion tons per year (Salem and Burdette, 1998), constitutes a large portion of this waste (Nisbet et al., 2001).

The amount of C\&D waste and the percentage of concrete recycled are shown in Table 1.1 for different countries. In Canada, a large portion of the building stock and infrastructure are made of concrete. Many of these buildings were built in the 50's or 60's, and are near the end of their design life. It is expected that during the next two decades, a large amount of concrete, resulting from the demolition of these buildings and structures, will be available for either disposal or for being recycled for reuse as concrete aggregates. Nisbet et al. (2001) have reported the annual C\&D waste in Canada as 11 million tons, with concrete being $52 \%$ of this amount. It is estimated that approximately $73 \%$, or more 
than 4 million tons, of demolition concrete, is reused as backfill or road sub-base (George and Michael, 2001), which is about $3 \%$ and $1.2 \%$ of the total Ontario and Canadian annual aggregate supply of about 142 and 350 million tons, respectively (Panagapko, 2003; OARC, 2002).

The use of demolished concrete as aggregate for new concrete began in Europe after World War II (Wainwright et al., 1994). However, as soon as the demand for aggregate could be met by available natural aggregate, recycling was basically abandoned (Buck, 1976).

Some countries have had some kind of standards and guideline for RCA, albeit not for RAC. Since 1982, the ASTM "Standard specification for concrete aggregates", C33-82 (ASTM C33-82) defines coarse aggregate such that it includes crushed hydraulic cement concrete, and ASTM 125-79a (ASTM 125-79a) "Standard definitions of terms relating to concrete and concrete aggregates" defines manufactured sand to include hydrauliccement concrete, and there are no longer technical barriers to the use of recycled concrete as aggregates in the United States. In Japan, the Building Contractors' Society of Japan (BCSJ) issued a "Proposed standard for the use of recycled aggregate and recycled aggregate concrete" in 1977 (Hansen, 1986). However, the recycling law was established in 1991 to control the increase of waste and to promote recycling of useful resources (Noguchi and Tamura, 2001). In the Netherlands, a proposal for a Dutch standard for recycled concrete as aggregate for production of new concrete was developed by CUR (Commissie voor Uitvoering van Research ingesteli de Betonvereniging). This standard allows using up to $20 \%$ by weight of the total coarse or fine aggregates to be RCA (Hansen, 1986). In the United Kingdom, the New British Standard Guide 6543, "Use of 
industrial by-products and waste materials in buildings and civil engineering", covers the use of demolition waste and other waste materials in both road construction and buildings. In Russia, since 1984 NIIZBH of the former USSR Research Institute for Concrete and Reinforced Concrete has allowed the use of coarse RCA in production of up to $20 \mathrm{MPa}$ concrete, including reinforced concrete, but not in pre-stressed concrete (Hansen, 1986). Denmark issued a modification to the regular concrete code in 1990, which allows the use of RAC for certain structural purposes under mild exposure conditions (Hansen, 1992).

Currently, in North America recycled aggregates are primarily used in non-structural applications, such as many types of backfill, base or fill for drainage systems, pavement base and sub-base, lean-concrete bases, concrete blocks, sound barrier walls along highways, partition walls, embankments, etc. Although the use of RCA in structural grade concrete is limited, RCA has been used successfully for road construction in Canada, the United States, France, Netherlands, Denmark, Germany, Japan, Belgium, and United Kingdom.

However, there are a number of fundamental barriers in the construction industry to the use of recycled aggregates in structural grade concrete. These include (1) designers' and owners' reluctance to use RCA due to lack of clear guidelines and technical specifications that would ensure the quality of concrete made with RCA and would alleviate their preconceived concerns about the inferiority of RAC, (2) lack of quality control and quality assurance standards for recycling plants and RCA suppliers, (3) insufficient demand and lack of economic incentives to use RCA, (4) unsteady supply of structural grade RCA and the consequent difficulty of competing with a well-established 
material such as traditional natural aggregate. However, these obstacles can be overcome by developing technical specifications and quality control and quality assurance methodologies for producing structural grade RAC and by implementing policies that would encourage its use.

In Canada, the C-2000 Green Building Standards aims to make recyclable materials up to $75 \%$ of the existing structure, including all building materials (Mishulovich, 2003), but this program does not require any processing of concrete other than its separation from other demolition waste (Taschereau, 2001).

In 1993, according to the Cement Association of Canada, the use of recycled concrete was included in the Ontario Provincial Standard Specification (OPSS), which specifies that recycled aggregates can be used as road or parking lot base and sub-base as well as sewer bedding and backfills (Wilson, 2003). However, currently, in Canada, there are no established guidelines for producing structural-grade concrete by using RCA.

It is evident from the preceding discussion that the extent and type of usage of RCA varies from one country to another. In Canada, standards for the consistent production, quality control and design of RAC and its use as structural material are lacking.

\subsection{A Note on Important Definitions and Acronyms}

The terminology used in the literature on aggregate recycling can be confusing. In order to facilitate the discussion in the current study, the following terminology and acronyms will be consistently used throughout this thesis:

- NA (Natural Aggregate) = aggregate obtained from crushed rock or from natural sand and gravel deposits. 
- RA (Recycled Aggregate) = aggregate obtained from the re-processing of mineral waste materials, with the largest source being construction, renovation and demolition waste. However, RA is not the same as "recovered" aggregates, which are obtained by washing the cement paste out of fresh concrete and returning the aggregates to the aggregate stockpile.

- RCA (Recycled Concrete Aggregate) $=$ aggregate obtained from crushing demolition concrete, normally comprising original virgin aggregate (OVA) coated with cement paste residue and/or lumps of paste residue, called residual mortar (RM), as illustrated in Figure 1.1.

- OC (Original Concrete $)=$ concrete from demolished concrete structures which can be used as raw material for production of recycled aggregates. Original concrete is occasionally referred to as old concrete, demolished concrete, or conventional concrete.

- OVA (Original Virgin Aggregate) $=$ aggregate used in the production of the original concrete (see Figure 1.1).

- $\quad$ NAC (Natural Aggregate Concrete $)=$ concrete produced with gravel or crushed rock as coarse aggregate, natural sand as fine aggregate, and cement paste as binder material.

- $\quad$ RAC (Recycled Aggregate Concrete $)=$ concrete produced with combination of RCA and NA as coarse aggregate and natural sand as fine aggregate, and cement paste as binder material. Recycled fine aggregate is not used as replacement for sand due to its proven inferior properties. RAC in this study refers to recycled aggregate concrete in a generic sense. 
- $\mathrm{RC}($ Reinforced Concrete $)=$ conventional concrete reinforced with steel rebars

- $\quad$ RRC (Reinforced Recycled Concrete $)=$ recycled aggregate concrete reinforced with steel rebars.

- $\quad$ RM (Residual Mortar) $=$ the mortar fraction attached to original virgin aggregate in RCA (see Figure 1.1).

- $\mathrm{NM}($ New Mortar $)=$ the new mortar used to make RAC (see Figure 1.1).

- $\quad$ TMV (Total Mortar Volume) $=$ the sum of RM and NM volumes.

- ITZ (Interfacial Transition Zone) $=$ the interface between the natural aggregate particles and the mortar in conventional concrete.

- OITZ (Original Interfacial Transition Zone) $=$ the interface between the original virgin aggregate particles and the adhered residual mortar in RCA (see Figure 1.1).

- NITZ (New Interfacial Transition Zone) $=$ the interface between the RCA particles and the new mortar in RAC (see Figure 1.1).

\subsection{Economic and Environmental Benefits of RAC}

The global demand for regulating, recycling and reusing C\&D waste has increased in the last few decades due to both economic and environmental considerations. From the economical point of view, due to several reasons the cost of conventional aggregates will continue to increase while that of recycled aggregates will likely decrease. The reasons for this economic scenario include (1) exhaustion of quality natural aggregate resources used for concrete has modified the aggregate type from river gravel to crushed stone for coarse aggregate, and from river sand to sea sand and crushed sand for fine aggregate, which require high processing fees, resulting in higher overall cost. For example, in the United States, the unit cost of producing natural sand and crushed stone in the processing 
plants range from $\$ 6.5-\$ 8 /$ ton (MOTH, 1999). (2) Quality aggregates are in short supply in many countries, making it necessary to haul aggregates from distant locations, which increases their overall cost. As stated in the Environmental Council of Concrete Organization (ECCO, 1999) information sheet, it is quite common for contractors to haul NA 80 to $112 \mathrm{~km}$ on many projects; haul distances greater than $320 \mathrm{~km}$ are not uncommon in the United States. With RCA, there is potential for cost savings in hauling; it is estimated that the total transportation distance for hauling the aggregates can be reduced as much as $80 \%$ (Abbas et al., 2006a). (3) While the quantity of construction waste is increasing due to increased demolition work, the number of available landfills is decreasing; hence, the dumping fees are expected to rise (ECCO, 1999). For instance, the dumping fee in Holland has increased from approximately $\$ 20$ to $\$ 112-120$ per ton within only a few years (De Vries, 1993). Also, the 1985 tipping fees in Hartland landfill located in Hartland city in New Brunswick for general waste has increased from $\$ 8.50$ to $\$ 75$ per ton between 1985 and 1993 (DECPBC, 2001). (4) Using mobile recycling equipments will avert distant transportation of C\&D waste to dumping facilities. (5) Due to growing global demand for RCA, and increase in the number of recycling plants, the relative cost of RCA production is expected to decrease.

From an environmental point of view, the depletion of aggregate resources may lead to possible destruction of the pristine environment; therefore, the use of RCA can definitely contribute to a greener environment. Some of the environmental impacts of the extraction and the use of natural aggregates are presented as follows:

- Destruction of the natural habitats of many organisms (Winfield and Taylor, 2005). 
- Impact of the natural aggregate production on the quality of the surface and groundwater resources due to changes in ground slope, and hence changes in water drainage patterns (Winfield and Taylor, 2005).

- Loss of the water storage capacity of the ground (Winfield and Taylor, 2005).

- Increased green house gas (GHG) emissions due to the energy consumption required for mining, processing, and transportation of natural aggregates. As the hauling distances between the quarries to the processing plants and processing plants to construction sites increase, GHG emissions associated with the transportation of aggregates also increase.

The increased use of recycled aggregates in the construction industry will slow down the demand for fresh natural resources and the associated energy and GHG emissions required to produce fresh aggregates. Sustainable development was defined at the Earth Summit in Rio de Janeiro as an economic activity that is in harmony with the earth's ecosystem (United Nations, 1992). It envisages a philosophy of fulfilling the present needs without depriving future generations from meeting their needs. The concept of sustainable development consists of, first, the careful use of natural resources, which are rapidly being exhausted, by using industrial by-products and therefore reducing waste. Secondly, it is necessary to reduce energy consumption that is linked to carbon dioxide gas emissions, which are the major cause for the "greenhouse effect". Although Canada accounts for only $2.5 \%$ percent of global emissions, it is the world's third largest per capita emitter of these gases, after the United States and Australia (IPCC-GC, 2005 and EC-GC, 2006). According to the Kyoto Protocol, 38 industrialized countries, including Canada, committed to cut their emissions of GHG by 5.2\% below 1990 levels by 2008 to 
2012. It is envisaged that using the results of the current research could contribute to Canada's GHG emission reduction objective.

Recognizing the aforementioned economical and environmental issues, it would be prudent for governments that are concerned about insufficient environmental protection and sustainable development to encourage the use of recycled aggregates. The construction industry may be compelled to practice recycling through such policy instruments as higher landfill and rock excavation fees, and subsidies for using recycled aggregates. This policy is already adopted by Netherlands and Denmark (Collins, 1994), which have the highest concrete recycling rates in the world (see Table 1.1).

\subsection{Problem Definition, Objectives, and Scope}

In order to be used in structural members, concrete, including RAC, must possess appropriate mechanical, durability and physical properties. As it will be illustrated in the state of the art (Chapter 2), a number of studies have been performed to investigate the use of RCA in structural-grade concrete. Although these studies cover a relatively wide range, they do not follow a comprehensive and methodical approach that can direct to the standardization of the practice. In general, five major gaps in the existing literature on RAC have been identified, which are listed as follows:

\section{- Lack of research on the effect of RCA source on the properties of RAC}

In the majority of the previous studies, laboratory-produced RCA has been used. In a scant number of cases in which commercially-produced RCA has been used, no attempt has been made to investigate the effect of the RCA source. But this is an important issue because it is difficult to produce RCA of uniform quality in large quantities even when produced by the same processing plant because demolition concrete from different 
sources need to be used. On the other hand, it is not clear what effect the variability in the properties of RCA will have on the fresh and hardened properties of RAC.

\section{- No specific mix proportioning procedure for RAC}

Currently, to the writer's best knowledge, there is no specific mix proportioning method available for structural-grade RAC. Thus far, the conventional mix proportioning method for normal concrete has been basically used to produce RAC, with some adjustments, such as increase in cement content, without any special consideration to the existing residual mortar quantity in RCA. In fact, it is the quantity and quality of residual mortar (RM) that distinguishes RCA from natural aggregate. As described later, the RM has been identified as the key factor responsible for the inferior properties of RAC. Although some investigators have successfully achieved equal or higher compressive strength in RAC than $\mathrm{OC}$ by adjusting the proportions of the concrete mix ingredients, nevertheless, these adjustments have always resulted in higher creep and shrinkage and lower elastic modulus for RAC compared to OC. The extent of the difference varies over a wide range. From the point of view of users' of concrete, this kind of variability constitutes a drawback while from the designers' perspective it creates uncertainty with respect to the serviceability and long-term performance of RAC structures. Therefore, there is a need to develop a specific mix proportioning method for RAC to overcome these inferiorities. To achieve this objective, firstly, a comprehensive investigation of the fresh and hardened properties of concrete produced with RCA obtained from different commercial sources need to be carried out. Such a study can provide an extensive database which can contribute to the understanding of the effect of residual mortar quantity and quality on 
different properties of concrete produced with RCA and to the development of a specific mix proportioning procedure for RAC.

- Applicability of existing models for predicting different properties of RAC

Although several models have been developed to predict different properties of NAC, such as its elastic modulus and stress-strain relationship, many questions arise regarding the applicability of the existing models to RAC. To the best of the writer's knowledge, to date no specific research has been performed to assess the appropriateness of the existing models for RAC.

\section{- Applicability of existing design provisions to RAC}

Short-term properties of $R A C$ : Relating the other mechanical properties of concrete to its compressive strength is a common practice. For normal concrete, these interrelations have been well established and described in various design codes and standards. However, some previous studies have indicated that the existing empirical equations for normal concrete may or may not be valid for RAC. Therefore, questions regarding the applicability of the existing equations, such as those recommended by CSA A23.3-04 (CSA, 2004) or ACI 318-05 (ACI, 2005) to RAC, are understandable, and further work is needed to answer them.

Long-term properties of RAC: Although some researches have revealed higher creep and shrinkage values for RAC, no significant attempt has been made to overcome these inferiorities and to evaluate the applicability of existing creep and shrinkage prediction methods for normal concrete, such as those presented by CEB (CEB, 1990) or ACI 31805 (ACI, 2005), to RAC.

- Applicability of existing design provisions developed for RC to RRC 
Due to the paucity of information about the structural performance of RRC members, many questions arise regarding the applicability of the design provisions in standards such as the CSA Standard A23.3-04 and ACI 318-05 to these members. Many of the design parameters and equations in CSA A23.3-04, such as those related to flexural and shear capacities, concrete stress-strain relation, and bond and development strength of reinforcing bars are derived based on the results of tests carried out on conventional concrete members.

The following gaps have been identified in the existing literature on RAC:

Stress-strain relationship of RAC: The effect of RCA content on the stress-strain relationship has been investigated by limited researchers. However, the applicability of existing models for normal concrete to RAC has been inadequately studied. In addition, no research has been performed to evaluate the applicability of the existing equations for calculating the equivalent rectangular stress block distribution parameters of normal concrete $\left(\alpha_{1}\right.$ and $\left.\beta_{1}\right)$ - parameters used for flexural analysis of concrete members at ultimate state- to RAC. This is an important topic because knowledge of these parameters is indispensable for an accurate and expedient design of flexural members.

Bond strength of steel reinforcement embedded in RAC: Although limited studies have been carried out on the bond performance of steel rebars embedded in RAC, these studies have only utilized conventional pull-out tests. Beam end bond test is an alternative test method to direct pullout test; it provides a more realistic assessment of the bond performance of steel reinforcement in flexural members because the bond specimen more accurately simulates the behaviour of a beam in flexure by ensuring that the concrete around the bar is in tension. Therefore, there is a need to generate information about the 
bond strength of steel reinforcement embedded in RAC by using the so-called beam-end bond test.

Flexural behaviour of RRC beams: A substantial amount of research work has been done in the past on the flexural behaviour of $\mathrm{RC}$ beams made with $\mathrm{NAC}$, but there is not adequate information available about the flexural behaviour of RRC members. According to literature, slightly lower ultimate flexural strength, lower cracking moment, wider cracks, and higher deflections have been reported in RRC beams compared to conventional RC members. However, there is inadequate available information to fundamentally explain these differences. Therefore, there is a need to more systematically study the flexural behaviour of RRC beams and to evaluate the applicability of the existing models and standards to their design.

Shear behaviour of RRC beams: A substantial amount of research on shear in conventional reinforced concrete has been carried out over the last 50 years. Today, the shear resistance mechanisms of reinforced concrete are well known and the effects of the various material and geometric parameters on the shear strength of reinforced concrete members are well understood. On the other hand, due to lack of adequate research results, it is not evident whether RRC members have the same shear behaviour as conventional RC members. While intuitively one expects the same behaviour, the contribution of the various resistance mechanisms to the total shear resistance may not be the same. In particular, the contributions of the aggregate interlock mechanism and the uncracked concrete to the shear resistance of RAC may not be the same as in conventional concrete because these mechanisms are strongly dependent on concrete properties. 
The general objective of this research is to address these issues through a study following a systematic approach with the ultimate goal being the standardization of the design of reinforced or unreinforced concrete structures made with RCA. The research methodology that is used in this study consists of analytical and experimental investigation of each issue identified above as "gaps in research".

It should be pointed out that standardization of design and construction involving RCA would be a lengthy and time-consuming process, which would demand extensive evidence to check the veracity of any adopted procedure, and it would involve issues other than structural design, including aspects of durability, practicality as well as QC/QA guidelines. The research in this thesis is part of a wider collaborative effort with two main thrusts; namely, the structural performance and the durability of RAC. Matters related to durability of RAC were investigated by Abbas (Abbas, 2007), who examined in detail its chloride diffusion, carbonation and freeze-thaw resistance. A summary of the key findings of that study will be presented in Chapter 5. The current study deals primarily with the short-term and long-term structural performance of steel reinforced concrete members made with RAC as well as with the development of a new mix designed method for concrete made with RCA. The scope of the study is limited to the flexural and shear behaviour of reinforced RAC beams and to the creep and shrinkage properties of RCA.

\subsection{Thesis Organization}

This thesis is organized in nine chapters. In order to facilitate the readability of the thesis, the tables and figures are presented at the end of each chapter. 
In Chapter 1, a general introduction on the use of the RAC as structural material is presented, and the environmental and economic benefits of RAC are addressed. The problem definition, the objectives and the scope of the study are also outlined.

Chapter 2 presents the state of the art on the use of RCA in the production of RAC. In the first part of the literature review, the research on the effects of RCA on the different properties of RAC are discussed; followed by the review of studies carried out on different methods for improvement of its corresponding properties. The fine and coarse RCA content, original concrete properties, moisture state, supplementary cementitious materials (SCM's) and chemical admixtures, and processing level are the key factors known to be affecting the short- and long-term properties of RAC. The section on structural behaviour of steel recycled reinforced concrete members discusses the researches performed on flexural and shear behaviour of RRC members, stress-strain relationship of RAC, and the bond strength of steel rebars embedded in RAC. Thereafter, different available concrete mix proportioning methods are presented. Subsequently, existing guidelines and standards as well as existing structures using RAC are reviewed at the end of the chapter.

Chapter 3 deals with the experimental investigation of the effects of RCA content and source on the fresh and hardened properties of RAC.

Chapter 4 presents the proposed mix proportioning method for RAC and the performance of the corresponding mixes proportioned by the method. Performance is measured in terms of the fresh and hardened properties of the mixes, including their fresh and hardened density, tensile and compressive strength, elastic modulus, and stress-strain 
relationship. In addition, the long-term properties of RAC, i.e. its creep and shrinkage behaviour, are investigated.

In Chapter 5, the experimental data on the short- and long-term properties of RAC mixes are analyzed. Several models are presented for the fresh and hardened density, elastic modulus, and compressive strength of RAC. In addition, existing equations for interrelating different mechanical properties of RAC, and existing models for prediction of its stress-strain relation as well as its creep and shrinkage properties are investigated. Chapters 6 and 7 present the flexural and shear performance of RRC beams in terms of their flexural and shear behaviour and the bond performance of beams made with RAC. The effect of longitudinal reinforcement ratio, RCA source, compression steel, and mix type are studied in Chapter 6. In Chapter 7, the effect of beam shear span/depth ratio, size effect, transverse reinforcement ratio, RCA source, and mix type are investigated. In Chapter 8, the experimental data on the flexural and shear performance of RRC beams are analyzed. The cracking and yielding moments, as well as the ultimate flexural and shear strengths of RRC beams are calculated using different analytical approaches and are compared with the experimental data. In addition, the serviceability of RRC beams is evaluated based on existing design provisions. The conclusions and the recommendations for future work are presented in Chapter 9. 
Table 1.1: Construction and demolition(C\&D) waste and percentage of concrete recycled as RCA

(Source: Nisbet et al., 2001)

\begin{tabular}{|c|c|c|}
\hline Country & $\begin{array}{c}\text { C\&D waste } \\
\text { (million tons/year) }\end{array}$ & $\begin{array}{c}\text { Concrete recycled } \\
\%\end{array}$ \\
\hline Canada & 11 & 42 \\
\hline US & 136 & $50-57$ \\
\hline UK & 30 & 45 \\
\hline France & 24 & 15 \\
\hline EU-15 & 180 & 28 \\
\hline Australia & 5 & 41 \\
\hline Sweden & 2 & 21 \\
\hline Spain & 13 & $<5$ \\
\hline Belgium & 7 & 87 \\
\hline Germany & 59 & 17 \\
\hline Italy & 20 & 9 \\
\hline Netherlands & 11 & 90 \\
\hline Portugal & 3 & $>5$ \\
\hline Denmark & 8 & 81 \\
\hline Greece & 2 & $<5$ \\
\hline Finland & 1 & 41 \\
\hline Irland & 1 & $<5$ \\
\hline
\end{tabular}

* Sandler (2003).

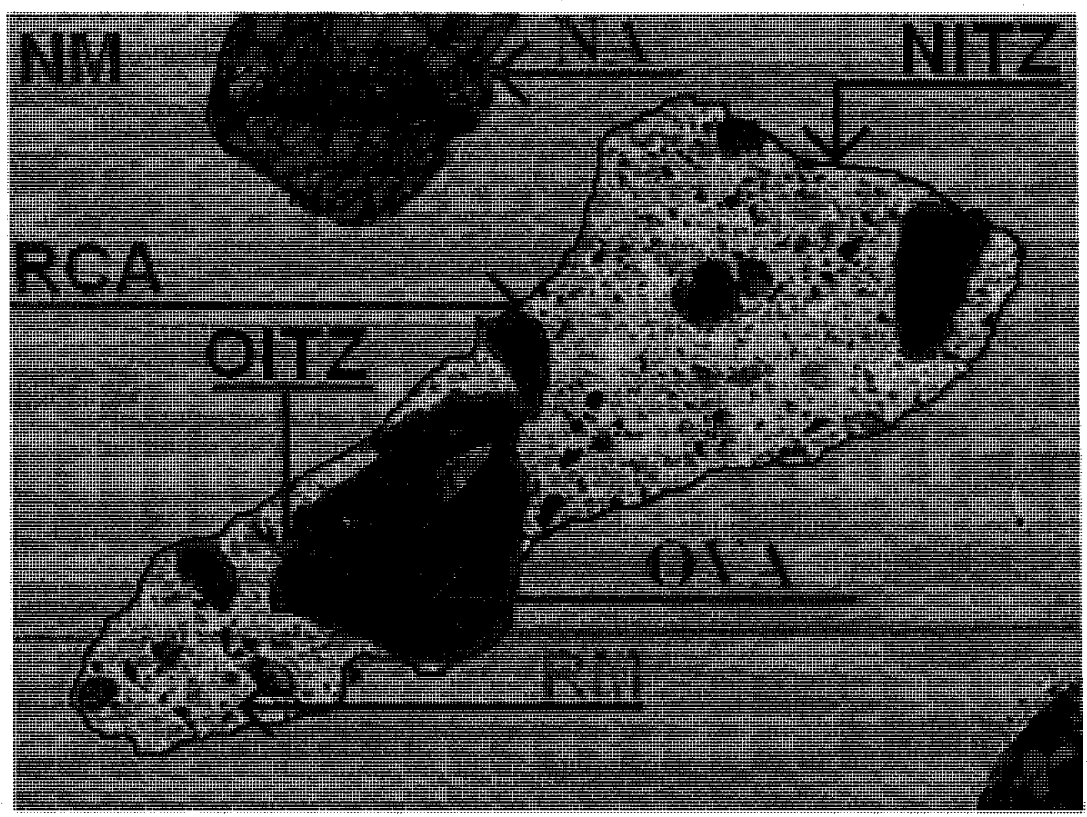

Figure 1.1: Schematic representation of recycled aggregate concrete (RAC) 


\section{CHAPTER 2: STATE OF THE ART}

\subsection{General}

This chapter presents a review of the existing literature on the use of RCA in the production of RAC. First, the research performed on the short-term as well as long-term properties of RAC, including its compressive, tensile and flexural strength, elastic modulus, creep, shrinkage, and bearing strength, are discussed, followed by the review of the studies that were carried out on the methods to improve some of these properties. The section on structural behaviour of reinforced concrete members made of RAC discusses the studies that have been carried out on the flexural and shear behaviour, and on the flexural and shear fatigue strength of RRC beams, on the bond strength of steel rebars embedded in RAC, and finally on the stress-strain relationship of RAC. In Section 2.6, the methods used in the literature for selecting mix proportions for structural-grade RAC are reviewed. Finally, some important existing guidelines and applications are summarized.

\subsection{Relevant Mechanical Properties of Concrete}

In order to evaluate the structural behaviour of a member, first one should have a good knowledge of the compressive and tensile strengths, elastic modulus, creep, and shrinkage characteristics of the materials from which it will be made.

\section{Compressive Strength}

The compressive strength of concrete is usually considered as its most important property because: 
- Compressive strength is usually a good indicator of the quality of concrete since it is directly related to the properties of the hydrated cement paste.

- Compressive strength is an essential element in structural design and is usually required for compliance purposes.

- Other concrete properties, such as its tensile and shear strengths and its elastic modulus are functions of its compressive strength.

\section{Tensile Strength}

The tensile strength of concrete is of interest when evaluating its shear strength and resistance to cracking. For conventional concrete, the tensile strength is found to be a function of its compressive strength, but it has not been established whether the same functional relationship applies to RAC. Although concrete is not normally designed to resist direct tension, the knowledge of tensile strength is of value in estimating the load under which cracking will develop. The absence of cracking is of considerable importance in maintaining the continuity of a concrete structure and in many cases in the prevention of corrosion of reinforcement. Cracking problems occur when due to applied loads tension stresses develop, but the most frequent cause of cracking is restrained shrinkage and temperature gradients. Tensile strength is of particular interest in unreinforced concrete structures, such as concrete dams, and in highways and airfield pavements as well as in prestressed concrete design.

There is a general acceptance that tensile strength increases with increase in the compressive strength of NAC, but there is no agreement about the exact form of the functional relationship. As indicated below, a number of empirical formulas relating 
tensile strength $\left(f_{t}\right)$ to compressive strength $\left(f_{c}\right)$ have been suggested in the literature. They are generally of the form:

$$
f_{t}=k\left(f_{c}\right)^{n}
$$

where $k$ and $n$ are empirical coefficients.

According to ACI 318-99 (ACI, 2005), the splitting tensile strength of NAC is proportional to the square root of its compressive strength,

$$
f_{t}=0.54 \sqrt{f_{c}^{\prime}}
$$

Based on the CSA Standard A23.3-04: Clause 8.6.4 (CSA, 2004), the modulus of rupture of NAC is proportional to the square root of its specified compressive strength,

$$
f_{r}=0.6 \lambda \sqrt{f_{c}^{\prime}}
$$

where $f_{r}$ is modulus of rupture of concrete in $\mathrm{MPa} ; f_{c}^{\prime}$ is specified compressive strength of concrete in MPa; and $\lambda$ is 1.00 for normal density concrete, 0.85 for structural semi-density concrete in which all the fine aggregate is natural sand, and 0.75 for structural low-density concrete in which none of the fine aggregate is natural sand.

\section{Elastic Modulus}

The elastic modulus is normally represented by the slope of the straight part of the stressstrain curve. However, in case of nonlinear stress-strain relationship, it is taken as the tangent to the curve at the origin. This is the initial tangent modulus, but it is of limited practical importance. For a material with a non-linear stress-strain curve it is possible to define the elastic modulus in different ways. For concrete, some definitions are:

- The secant elastic modulus $\left(E_{c}\right)$ : the chord connecting the point on the ascending part of the stress-strain curve with a stress value of $1.0 \mathrm{MPa}$ to another point with a stress value equal to approximately one-third of the compressive strength of the concrete. 
- The tangent elastic modulus $\left(E_{t}\right)$ : slope of the tangent to the stress-strain curve at the point of interest.

- The dynamic elastic modulus $\left(E_{d}\right)$ : determined by ultrasonic pulse velocity measurements,

$$
\begin{gathered}
E_{d}=\frac{V^{2} \times \rho}{K^{2}} \\
K=\sqrt{\frac{(1-v)}{(1+v) \cdot(1-2 v)}}
\end{gathered}
$$

where $V$ is the pulse velocity (Corinaldesi and Moriconi, 2001).

For normal concrete, it is quite well-known that the elastic modulus increases with increase in the compressive strength of concrete, but there is no agreement on the accurate form of the relationship. This is not surprising, as the elastic modulus of concrete is mainly a function of the elastic modulus of its components (aggregate and mortar) and their volume fractions. Since the former moduli are rarely known, in some advanced standards and in some research works, the effect of the elastic modulus and volume fractions of aggregate and mortar have been accounted for by a coefficient as a function of the density of the concrete, usually density raised to power 1.5. Most of the aforementioned empirical equations have the following format:

$$
E_{c}=\left(A \sqrt{f_{c}^{\prime}}+B\right) \times\left(\frac{\gamma_{c}}{C}\right)^{1.5}
$$

where $f_{c}^{\prime}$ and $\gamma_{c}$ are the compressive strength and hardened density of concrete, and $\mathrm{A}$, $\mathrm{B}$, and $\mathrm{C}$ are empirical coefficients. Some of the existing equations for predicting the secant elastic modulus of normal concrete are presented in Table 2.1. It is important to find out whether these expressions are applicable to RAC. 


\section{Creep}

Creep is the increase in strain under a sustained stress, which in the case of concrete can be several times as large as the initial strain. It should be noted that it is the hydrated cement paste which undergoes creep, while the aggregate in concrete restrains creep. Thus, creep is a function of the cement paste volume in concrete, but there are certain physical properties of aggregate which also influence the creep of concrete (Neville, 1996). Aggregates elastic modulus is an important factor because stiffer aggregates restrain creep more effectively. The following model has been presented to correlate the creep of concrete to the properties and volume fractions of aggregate and cement paste (Neville, 1996):

$$
\begin{gathered}
\log \frac{c_{p}}{c}=\alpha \log \frac{1}{1-g-u} \\
\alpha=\frac{3(1-\mu)}{1+\mu+2\left(1-2 \mu_{a}\right) \frac{E_{c}}{E_{a}}}
\end{gathered}
$$

where $c_{p}$ is creep of neat cement paste of the same quality as used in concrete, $c$ is the creep of concrete, $g$ is the volume fraction of aggregate, $u$ is the volume fraction of unhydrated cement, $\mu_{a}$ is Poisson's ratio of aggregate, $\mu$ is Poisson's ratio of concrete, $E_{a}$ is the modulus of aggregate, and $E_{c}$ is the elastic modulus of concrete.

Porosity is another factor which affects the creep of concrete because porous aggregates usually have a lower elastic modulus. Nevertheless, it is worth mentioning that the porosity of the aggregate is a significant parameter in the transfer of moisture (absorption capacity) within concrete. Therefore, it may be linked to creep in that it produces conditions conducive to the development of drying creep. 
Creep is directly proportional to the applied stress. There is no lower limit of proportionality since concrete creeps even at very low stress levels. However, the upper limit of proportionality corresponds to the advent of severe micro-cracking in concrete, usually between 0.4 and 0.6 of its compressive strength (Neville, 1996). Within the service stresses range, the proportionality assumption between creep and stress is reasonable. Creep is also inversely proportional to the strength of concrete at the time of application of the load. For the same stress/strength ratio, creep is essentially independent of the w/c ratio (Neville, 1996). Since both the creep and the elastic modulus of concrete are related to its compressive strength, it may be concluded that creep and elastic modulus are also related.

Another important external factor influencing creep is the relative humidity of the air surrounding the concrete, i.e. the lower the $\mathrm{RH}$, the higher the creep. Other factors which affect concrete creep are alternate wetting and drying, volume/surface ratio of member, temperature, and cyclic loading (Neville, 1996).

For normal concrete, the available methods for practical purposes are based on empirical relationships proposed by ACI Committee 209 (ACI, 1982) and CEB-FIP (CEB, 1993), which are summarized in Tables 2.2 and 2.3. In this study we will focus on the effect of aggregates on creep because RCA will be used to partially replace natural aggregate in the concretes mixes to be used in the creep tests.

\section{Shrinkage}

Concrete will contract on drying irrespective of the applied stress, and the magnitude of shrinkage is of the same order as the elastic strain under the usual range of stress (Neville, 1996). Water/cement ratio is one of the most important factors which contribute 
to cracking in concrete because its increase tends to increase shrinkage and simultaneously reduces the strength of concrete. For a given w/c ratio, higher cement content increases shrinkage; therefore, the cracking probability increases. On the other hand, for a given workability (or a fixed water content), shrinkage will not increase by increasing the cement content; it may even decreases because for a fixed water content the $w / c$ ratio will decrease. Alternatively, higher water content will increase shrinkage because it is generally accompanied by a reduction in the volume of restraining aggregates.

The size and grading of aggregate do not influence the magnitude of shrinkage; however, using higher nominal maximum size of aggregate leads to higher volume of aggregate, or lower amount of cement paste, and therefore less shrinkage. The following model has been presented to correlate the shrinkage of concrete to the corresponding properties and volume fractions of aggregate and cement paste (Neville, 1996):

$$
S_{c}=S_{p}(1-g)^{n}
$$

where $S_{p}$ is the shrinkage of neat cement paste of the same quality as used in concrete, $S_{c}$ is the shrinkage of concrete, $g$ is the volume fraction of aggregate, and $n$ is an empirical coefficient between 1.2 and 1.7.

Consideration of shrinkage in structures is important because it often leads to cracking. In the case of restrained structures, once shrinkage cracking occurs, it makes the exposed structural member more vulnerable to intrusion of external agents and to durability problems. However, even in unrestrained structures, shrinkage is harmful. For example, contiguous concrete elements will move away from one another thus creating gaps between them. 
If the stress induced by the free shrinkage (reduced by creep) is less than the tensile strength of concrete, no cracking will occur. Thus, time has a two fold effect: increase of strength with time leads to reduction of cracking, but the increase in elastic modulus will increase tensile stresses due to a given amount of shrinkage. In addition, with time the creep relief decreases so that the cracking tendency becomes greater. For normal concrete, the available methods for practical purposes are based on empirical relationships proposed by ACI Committee 209 (ACI, 1982) and CEB-FIP (CEB, 1993), which are summarized in Table 2.4 and 2.5.

We can see from the preceding discussion that the various mechanical and physical properties of concrete are interrelated because changes in the proportions or the properties of the constituents of concrete affect nearly all its properties. Since the introduction of RCA in RAC alters the constitution of the concrete mix, it is expected that it will affect the concrete mechanical and physical properties.

\subsection{Effect of RCA and Other Constituents of Concrete on its Properties}

In this section, previous research work conducted on the mechanical properties of RAC will be reviewed. According to different investigators (Hansen, 1986 \& 1992; De Oliveira and Vazquez, 1996; Yamasaki and Tatematsu, 1998; Limbachia et al., 2000; Kiuchi, 2001; Kiuchi and Horiuchi, 2003; Gomez-Soberon, 2003; Maruyama et al., 2004a \& 2004b; Sakata and Ayano, 2000), the mechanical properties of RAC could be inferior, identical, or superior to NAC depending on prevailing conditions. Therefore, in order to properly compare the findings of the different studies, the research results about the mechanical properties of RAC will be reviewed in accordance with the specific test parameters and conditions in each study. Some of these test parameters include coarse 
RCA content, fine RCA content, w/c ratio, binder content, original concrete properties, moisture state, processing level, and chemical admixtures.

\subsubsection{Coarse RCA Content}

According to available literature, for a given w/c ratio and identical mix proportions, the mechanical and long-term properties of concrete become inferior compared to NAC as the coarse RCA content is increased (Ray and Venkateswarlu, 1991; Limbachia et al., 2000; Topcu and Sengel, 2004). Similarly, the compressive strength of RAC using only coarse RCA has been found to be generally lower compared to the strength of similar control mixes of conventional concrete (Nixon, 1977; Hansen, 1986 \& 1992; De Vries, 1993; Topcu and Guncan, 1995; Kiuchi and Horiuchi, 2003; Maruyama et al., 2004a \& 2004b; Topcu and Sengel, 2004). However, partial replacement of 20-30\% coarse NA by coarse RCA does not significantly affect the compressive strength, creep, and shrinkage of RAC (De Vries, 1993; Limbachia et al., 2000; Dhir et al., 1999; Gomez-Soberon, 2002a, 2002b, \& 2002c). On the other hand, identical or even higher compressive strength in RAC, compared to a similar concrete made with NA (NAC), has been achieved by adjusting the $\mathrm{w} / \mathrm{c}$ ratio (either by water content, cement content, or water and cement content ratio) as a function of the RCA content (Limbachia et al., 2000; Dhir et al., 1999).

It has been shown that due to the large amount of attached residual mortar, the elastic modulus of RAC is always lower than that of companion NAC, while its drying shrinkage and creep are always higher than those of NAC (Hansen, 1986 \& 1992; Oliveira and Vazquez, 1996; Yamasaki and Tatematsu, 1998; Limbachia et al., 2000; Kiuchi, 2001; Kiuchi and Horiuchi, 2003; Gomez-Soberon, 2003; Maruyama et al., 
2004a \& 2004b; Sakata and Ayano, 2000). However, currently there are no guidelines for proportioning mixes of RAC which would yield the specified strength, slump and longterm properties.

Using different $w / c$ ratios $(0.45,0.55,0.65$, and 0.70$)$, Ray and Venkateswarlu (1991) carried out an experimental study to determine the compressive, tensile, and bearing strengths of RAC made with either coarse RCA alone or with mixed coarse RCA and NA ( $60 \%$ RCA and $40 \% \mathrm{NA})$. They reported that the compressive strength of RAC is markedly lower than that of NAC, but they emphasized that this does not imply that RAC is unsuitable in normal plain or reinforced concrete. Topcu (1997) evaluated the effect of coarse RCA content on the compressive strength of RAC by non-destructive techniques, such as Schmidt hammer test, impact test, and ultrasound velocity test. According to the results, the Schmidt hardness values decreased as the RCA percentage increased while the ultrasound velocity in RAC was more than in NAC due to the higher air voids in RAC. The impact test caused more damages in RAC due to its higher mortar volume. Mukherjee et al. (2003) presented the results of an experimental study and used statistical analysis to determine the strength related properties of RAC, made with coarse RCA and natural sand. Based on their results, with identical basic quantities such as mixing proportions and w/c ratio, they found that with $95 \%$ confidence level the strength satisfies both the normal and lognormal distribution. The statistical distribution and scatter generally did not differ significantly from that of normal concrete.

Katz (2003) reported the effect of partially hydrated waste concrete on the properties of RCA and RAC (made with only coarse RCA). He found a reduction of $25 \%$ in the compressive strength of RAC compared to NAC, regardless of the crushing age of the old 
concrete. Other properties, such as flexural and splitting strengths and drying shrinkage exhibited similar trends. He also pointed out that the effect of RCA on the new concrete properties (strength, elastic modulus, etc.) is similar to the effect of lightweight aggregate on concrete properties.

Kiuchi and Horiuchi (2003) and Maruyama et al. (2004a \& 2004b) reported a linear relationship between $\mathrm{c} / \mathrm{w}$ and the compressive strength of RAC similar to that in NAC. Due to the high water absorption of RCA, Fumoto and Yamada (2003) suggested that the quantity of water absorbed by RCA be added to the total water (tw) required in concrete. According to their results, the relationship between $\mathrm{c} / \mathrm{tw}$ and compressive and splitting tensile strengths for RAC is linear as for normal-strength (30-70MPa) concrete. Topcu and Sengel (2004) reported reduction in workability as the RCA content increased. The decrease was $15-20 \%$ for concrete with $100 \%$ of RCA compared to concrete without RCA due to the higher water absorption ratio of the mortar in RCA. However, this problem can be overcome by soaking RCA for 24 hours prior to mixing.

Otsuki et al. (2003a and 2003b) conducted a study to evaluate the flexural strength and the elastic modulus of the interfacial transition zones (ITZ) in concrete (using different w/c ratio, mixing time and curing conditions). They observed that the higher $w / c$ ratio led to the formation of a thick water film on the aggregate surface and caused bleeding, which resulted in a porous and weak interfacial transition zone (ITZ). On the other hand, higher mixing time increased the strength of the ITZ and the packing of cement particles near the aggregate produced a less porous ITZ. Similarly, curing under high RH (up to $90 \%$ ) led to better mechanical properties at the ITZ which may be attributed to the reduction in the porosity of the ITZ. 


\subsubsection{Fine RCA Content}

As stated earlier, for a given $\mathrm{w} / \mathrm{c}$ ratio and mix proportions, the mechanical and long-term properties of RAC made with coarse RCA have generally been found to be inferior to those of a control mix made with all natural aggregates. The situation becomes worse when natural fine aggregate is concurrently replaced by fine RCA. On the other hand, the rate of strength development has been found to be similar for all concretes made with the same types of cement regardless of aggregate type (Wainwright et al., 1994). Partial fine RCA replacement of less than $20 \%$ has been reported to have negligible effect on the strength of RAC (Wainwright et al., 1994; Merlet and Pimienta, 1994; Kikuchi, et al. 1994; Dhir et al., 1999; Ajdukiewicz and Kliszczewicz, 2002; Shayan and Xu, 2003). However, it has been recommended (Wainwright et al., 1994) to not replace more than $50 \%$ of fine natural aggregate by fine RCA.

Dhir et al. (1999) studied the effects of RCA content and the properties of the original concrete on the properties of RAC obtained from six different sources. In the first part of the study, RAC mixes were proportioned using NA and RCA blends with up to $100 \%$ coarse and 50\% fine RCA. For RAC mixes, the cement and free water contents were kept the same as in the corresponding NAC mixes. In the second part of the study, the RCA proportions of the RAC mixes which exhibited lower strength were adjusted by changing their w/c ratio. The modified w/c ratio was intended to recoup the loss of strength. Strength reduction was noted for the concrete mixes with more than $30 \%$ coarse or $20 \%$ fine RCA. On average, the strength of concrete with $100 \%$ coarse and $50 \%$ fine RCA was found to be $20-30 \%$ lower than that of the corresponding NAC. The difference in strength between NA and $100 \%$ coarse RCA mixes was between $6-18 \%$. The strength of $100 \%$ 
coarse-50\% fine RCA mixes was found to be $15-20 \%$ lower than for the corresponding reference concrete with $100 \%$ coarse RCA and natural sand. They also reported a gradual reduction in flexural strength and elastic modulus, particularly for the concrete made with $100 \%$ coarse and $50 \%$ fine RCA. They showed that the adjustment to $\mathrm{w} / \mathrm{c}$ ratio to achieve equal strength can be made by way of the water content, the cement content or both. By adjusting w/c ratio from 0.65 to 0.60 , it was possible to match the strength of concrete containing $100 \%$ coarse RCA with that of the corresponding NA concrete.

Sakata and Ayano (2000) studied the effect of size of RCA on the strength, slump, air content, and durability of RAC. Based on their results, the drying shrinkage strain was not different in the concrete mixes with the various types of RCA, except for the mix made with both fine and coarse RCA. The creep coefficient of concrete with fine RCA was higher while its compressive strength was lower than the other concrete mixes.

Fine RCA serves as a water reservoir for hydration under air-cured conditions. BuyleBodin and Hadjieva-Zaharieva (2002) and Nagataki et al. (2000) reported that due to the change from water to air curing, the compressive strength of RAC, using both fine and coarse RCA, decreased by no more than $3-6 \%$ while the strength of NAC and RAC, using only coarse RCA, decreased by three times more. Under air-curing conditions, compared to NAC, the presence of free water in RAC increased drying shrinkage by $80 \%$ in RAC made with coarse RCA, and $300 \%$ in RAC made with fine and coarse RCA. Ajdukiewicz and Kliszczewicz (2002) found that the joint influence of shrinkage and creep might be only slightly greater for recycled concrete in comparison with concrete from new aggregate. 
It is clear that the combination of coarse and fine RCA has significantly greater effect on both the short-term and long-term properties of RAC. Some of the findings are also contradictory. A case in point is the aforementioned results of Ajdukiewicz and Kliszczewicz (2002). It is difficult to justify their finding because many factors which increase creep also increase shrinkage; consequently, it is not clear how their combined effect can be less than their individual effects. In the present, study we will investigate the creep and shrinkage properties of RAC to supplement existing data and to possibly resolve some of these contradictory findings.

Tables 2.6, 2.7, and 2.8 show the effect of both coarse and fine RCA on the mechanical properties (compressive strength, tensile strength, flexural strength and elastic modulus), and on the long-term properties of concrete as reported by different researchers. It should be mentioned that some of the values in these tables are not given directly by the cited investigators, but are calculated by the writer based on the information and data provided by them.

\subsubsection{Original Concrete Properties}

There are three constituent elements in NAC that collectively determine its strength: aggregate, mortar, and aggregate-mortar bond or the interfacial transition zone (ITZ). In RAC, on the other hand, there are five elements that influence its strength: original virgin aggregate (OVA), residual mortar (RM), OVA-RM interface (OITZ), new mortar (NM), and RCA-NM interface (NITZ). The first three of the five elements are contained within the concrete from which the RCA is extracted. Therefore, RCA and its original concrete properties directly affect the mechanical properties of RAC (Ajdukiewicz and 
Kliszczewicz, 2002). The salient properties of the original concrete that are expected to affect the RCA properties are its w/c ratio, strength, aggregate type, etc.

According to the literature, in order to achieve the maximum strength in RAC, the w/c ratio in NM should be equal to or smaller than the w/c ratio in RM. In other words, the strength of NM should be higher than that of RM (Hansen, 1986 \& 1992; Yamasaki and Tatematsu, 1998). Ryu (2002a) used different coarse RCA (with different strengths and residual mortar content) and different w/c ratios to investigate this issue. His results showed that the strength of the RAC depends on the quality of the NITZ, provided the quality of the OITZ is better than that of the NITZ, but when the quality of NITZ is better than that of OITZ, it depends on the quality of OITZ. This has been confirmed by other researchers (Poon et al., 2004b; Tavakoli and Soroushian, 1996a). Note that the quality of ITZ in RCA can be assessed if the compressive strength of the recycled concrete is determined before crushing it into aggregate.

According to Nagataki et al. (2000), RAC produced with air-entrained high and medium quality recycled aggregates can have 3 to 16 percent higher strength than reference concrete mixes. They noted that even mixes with both coarse and fine fractions of the lowest quality RCA in the same mixture had only $6 \%$ lower compressive strength. These results may be attributed to the better mechanical compatibility of the recycled products with the new cement paste, and to the presence of an internal water reservoir in the form of pre-saturated porous RCA which contributes to improved cement hydration.

ITZ serves the link between the mortar and the coarse aggregate particles and as a strength-limiting phase in concrete, i.e. it may cause concrete failure at considerably lower stress level than the strength of either the aggregate or the mortar. Even when each 
component has high stiffness, the stiffness of the concrete may be lower because of the broken links (i.e. voids and micro-cracks in ITZ) because the latter does not transfer any stress (Poon et al., 2004b). Furthermore, at the crack tips high stresses may develop due to stress concentrations.

\subsubsection{Moisture State}

Due to the porous nature of its adhered mortar, RCA has a high water absorption capacity compared to NA; therefore, its moisture state influences its properties. The study by Poon et al. (2004a) has shown that using RCA in air-dried (AD) condition causes negligible change in the workability of the fresh concrete and the compressive strength of RAC compared to NAC. However, when used in the oven-dried (OD) or saturated surface dry (SSD) states, both the workability and the compressive strength of the concrete are significantly affected by the high water absorption capacity of the recycled aggregate. In the SSD state, the high water content inside the aggregate particles might result in "bleeding", leading to a relatively high local w/c in the vicinity of these particles, and consequently a lower compressive strength compared to NAC. Merlet and Pimienta (1994) also demonstrated that the pre-wetting of RCA with $30 \%$ of mixing water improved the mechanical properties of concrete, but it did not have any influence on its drying shrinkage.

\subsubsection{Supplementary Cementitious and Chemical Admixtures}

Generally speaking, supplementary cementitious materials and chemical admixtures have been used effectively to enhance the different fresh and hardened properties of conventional concrete. However, limited research has been performed about their effect on the properties of RAC. 
The effects of superplasticizers and partial replacement of fine RCA with pulverized fuel ash (PFA) and natural sand on the compressive strength of concrete made with fine and coarse RCA were investigated by Wainwright et al. (1994). Based on their results, using superplasticizers to reduce water demand resulted in increased strength and in reduced porosity and permeability in mixes containing all recycled aggregates. The improvement was more noticeable when both PFA and natural sand were incorporated in the mix. Salem and Burdette (1998) investigated the effect of fly ash and air entraining agents on the mechanical properties and freeze-thaw resistance of RAC made with only coarse RCA. By increasing the fly ash content from 14 to $28 \%$ by weight of cement, the compressive strength and modulus of rupture at 28 days decreased by $9 \%$ and $14 \%$ in RAC and $11 \%$ and $10 \%$ in NAC, respectively. These investigators and Salem et al. (2003) also investigated the effects of air entrainment on the physical properties of both RAC and NAC. They reported that $5 \%$ air entrainment resulted in 25 and $30 \%$ reduction in the compressive strengths of NAC and RAC, respectively.

The effect of blast furnace slag (bfs) on the mechanical properties and drying shrinkage of RAC was investigated by Sagoe-Crentsil et al. (2001) using 100\% commercially graded unwashed coarse RCA and natural fines. They reported no significant difference between the strength of portland cement concretes as a function of aggregate type for the grade of concrete investigated. The slag cement concrete with similar w/c ratio achieved much higher later-age strength owing to the pozzolanic properties of the slag. The splitting tensile-to-compressive strength ratio values ranged from 0.089 to 0.12 for RAC, which was close to the 0.08 to 0.14 ratio for equivalent NAC. While both NAC and RAC displayed similar trends with regard to the rate of shrinkage, one year shrinkage strain 
values of RAC made with and without bfs were $35 \%$ and $15 \%$ higher than those of the corresponding NAC.

Corinaldesi and Moriconi (2001) attempted to reduce the strength loss due to the use of RCA by partially replacing the fine RCA with SCM (fly ash and silica fume). After 14 days of curing, the compressive strength development of RAC without any SCM's addition slowed down compared to NAC. This effect was not evident in the case of mixes with silica fume and fly ash. On the other hand, the static elastic modulus was found to be substantially dependant on the compressive strength rather than the presence of SCM's whereas the dynamic elastic modulus was mainly influenced by aggregate type and to lesser extent by the kind of SCM's.

In another study, Corinaldesi et al. (2001) evaluated the structural properties of RAC containing fly ash or silica fume, using both coarse and fine RCA. They demonstrated $10 \%$ lower tensile strength for equal compressive strength in RAC and NAC, with or without SCM addition. The RAC had $20 \%$ or $30 \%$ lower elastic modulus than NAC depending on the absence or presence of fly ash- because of the lower or higher paste content, respectively. According to the results, the greatest drying shrinkage strain occurred in the concrete made with the recycled aggregate and fly ash and this was attributed to the largest volume of micro pores $(<50 \mathrm{~nm})$. Also, RAC had higher bond strength than NAC (with both smooth and ribbed bars), the increment being 15\% and 6\% with and without fly ash, respectively. This was probably due to the presence of fine RCA which tends to reduce bleeding in concrete.

Mandal et al. (2002) studied the durability of RAC made with $100 \%$ coarse RCA using fly ash and water-reducing agents. In general, RAC showed an average of $15 \%$ reduction 
in compressive strength at all ages compared to the companion NAC. The 28-day modulus of rupture for RAC was also reduced by $6 \%$ compared to NAC. It was observed that $10 \%$ fly ash addition substantially improved the cube strength of RAC at all ages, but higher shrinkage was reported for RAC compared to NAC.

Ajdukiewicz and Kliszczewicz (2002) produced recycled high-performance concrete (RHPC) using moderate or high-strength (35-70MPa) concrete as RCA, silica fume and superplasticizers. They succeeded to produce $80 \mathrm{MPa}$ RAC using recycled aggregate from $60 \mathrm{MPa}$ concrete. Tensile strengths for companion NAC were always higher than RAC, but the differences were not greater than $10 \%$ at 28 days. The influence of admixtures on tensile strength was much greater than the presence of RCA.

Gonzalez and Martinez (2004) investigated the effect of silica fume on the mechanical properties of RAC made of $50 \%$ coarse RCA and natural fine aggregate. According to their results, the compressive strength of RAC with $50 \%$ coarse RCA was close to that of NAC while its static elastic modulus was smaller. From the age of 7 days onward, concretes with silica fume showed more strength than that without it. However, the effects of aggregate type and silica fume were negligible on tensile strength. This would imply that the tensile strength is a function of the properties of the ITZ and not of the type of coarse aggregate.

\subsubsection{Processing level}

As stated earlier, RCA consists of two parts: the natural aggregate and the attached residual mortar. Due to the inferior physical properties of mortar compared to natural aggregate, higher mortar volume generally leads to lower quality of RCA and consequently the concrete made with it. Therefore, any attempt to reduce the mortar 
content will contribute to improvement of quality of RCA. This can be achieved in the processing plant by different methods.

Nagataki et al. (2004) studied the micro-structural changes introduced by the recycling process to each constituent of the RCA. The recycling process consisted of crushing the source concrete with combination of jaw crusher and impact crusher and processing the crushed material twice with a mechanical grinding equipment to minimize the residual mortar (RM). As a consequence of this two-stage crushing process, one of the interesting observations was a remarkable decrease in the extent of cracking within the natural aggregate particles in the coarse RCA. After double crushing, the damage to the residual mortar and to the ITZ was negligible. When the recycling process was extended to grinding, the natural aggregate particles experienced more cracking. At the end of the grinding process, in terms of the crack density, no micro-structural change was observed in the residual mortar. The splitting tensile and compressive strengths of the concretes made with the coarse RCA obtained from high and medium quality concrete were significantly higher than those of companion NAC. However, extending the recycling process to grinding level did not result in a significant change in compressive strength.

\subsection{Methods for the Improvement of the Properties of RAC}

One of the main reasons for the observed inferior properties of RAC compared to NAC is the presence of the residual mortar in RCA. Some research works have been performed to improve the RAC properties by improving the RCA quality, and/or adding some SCM's and/or chemical admixtures to RAC.

Polypropylene and Fibraflex (metallic) fibres were added to RAC by Mesbah and BuyleBodin (1999) to improve its shrinkage characteristics. Six hundred days after the end of 
curing, Fibraflex fibres decreased the value of free shrinkage in the order of $15 \%$ compared to a plain mortar, but polypropylene fibres were less effective than Fibraflex fibres. Fibraflex fibres were also more effective in reducing restrained shrinkage cracking because of their higher stiffness which endowed them with superior ability to transfer loads across cracks. With a fibre volume of $1 \%$, the crack width after 50 days was only $0.14 \mathrm{~mm}$ compared to $1.1 \mathrm{~mm}$ for plain recycled aggregate mortar.

The effects of brick powder and fibres (polypropylene and stainless steel) on the mechanical properties of RAC were studied by Corinaldesi et al. (2002). The recycledaggregate mortars showed excellent bond strength with bricks due to the high quality of the interfacial zone. The introduction of polypropylene fibres in recycled aggregate mortar to restrain its drying shrinkage did not improve its flexural strength but reduced its compressive strength significantly. On the other hand, it slightly lowered its bond strength with both the brick and with galvanized steel bars.

Decompression and Rapid Release (DRR) is another method for improving RAC quality (Tamura et al., 2001). In this method, during mixing initially the pressure in the mixer is lowered to a specified level below atmospheric pressure (decompression), then in order to bond the aggregate and the paste, it is returned rapidly to atmospheric level (rapid release). Tamura et al. (2001) used a modified DDR (MDDR) by not mixing the concrete during the decompression and rapid release stages. Using normal mixing method, the compressive strength of RAC was found to be about $10 \%$ lower than that of NAC at 28 days, while employing MDDR improved the strength of RAC by about $20 \%$, and rendered it almost equal to that of NAC. The MDDR was not effective in reducing the shrinkage of RAC, but reduced its creep strain by about $20 \%$, which would result in creep 
deformations similar to those of NAC. The carbonation depth of both RAC and NAC decreased about $30 \%$ by the MDDR procedure.

Double mixing method (DM) has been investigated as another method for improving RAC properties (Ryu, 2002b; Poon et al., 2004b). In this method, the necessary water is added at two different times during the mixing process. Using this method Ryu reported an increase of $17 \%$ and $26 \%$ in the compressive and tensile strengths of RAC, respectively, compared to NAC. This can be attributed to the improvement of the quality of ITZ due to double mixing.

Shayan and $\mathrm{Xu}(2003)$ attempted to improve the strength and drying shrinkage of 50MPa RAC by treating the surface of RCA with different combinations of sodium silicate, lime, and silica fume. The results showed that even though the sodium silicate treatments improved the surface characteristics, no strength gain was achieved. However, silica fume increased the strength. Similarly, Katz (2004) attempted to improve the quality of RCA by: (1) impregnation with a solution of silica fume (SF) for the purpose of densifying the surface of RCA, and (2) ultrasonic cleaning of RCA in order to remove the loose surface particles and improve the bond between the new mortar and RCA. The SF treatment improved the compressive strength of RCA by $13-16 \%$ at 28 days. However, a negative effect was observed in NAC due to the formation of a separating layer on the relatively impermeable surface of NA, which impaired the bond between the mortar and the aggregate, leading to a decrease in the strength properties of the concrete. The improvement achieved by using ultrasonic method was less effective compared to SF method. Finally, the dry mixing of RCA prior to the addition of other mix ingredients is 
another possible way to detach the mortar from recycled aggregate particles and to improve the properties of RAC (Tavakoli and Soroushian, 1996b).

Tables $2.9,2.10$, and 2.11 present a summary of the improved compressive, tensile, and flexural strengths, elastic modulus, and shrinkage and creep of RAC, achieved by different investigators through the application of different techniques. It should be mentioned that some of the values in these tables are not given directly by the original investigators, but are deduced by the writer based on the information provided in their published work.

\subsection{Structural Behaviour of Steel Reinforced Recycled Concrete}

\section{Members}

In order to evaluate the structural behaviour of steel reinforced concrete members made with RAC, first one should have a good knowledge of the corresponding behaviour of similar members made with normal concrete. Towards this goal, before reviewing the existing literature on the structural behaviour of RRC members, a brief review of the structural behaviour of RC members made with NAC will be presented.

\subsubsection{Flexural and Shear Behaviour of RC Members}

\subsubsection{Flexural Behaviour}

Extensive investigations of the flexural response of $\mathrm{RC}$ members have conclusively established their behaviour and failure mechanisms and results are reflected by the general theory of flexure which is contained in various design codes. Four basic assumptions are usually made when deriving a general theory for the flexural strength of reinforced concrete members:

1. Plane sections before bending remain plane after bending 
This assumption is based on Euler-Bernouli's principle and implies that the longitudinal strain in concrete and steel at various points across the section is proportional to their distance from the neutral axis. For normal concrete, an extensive number of tests have shown that this assumption is very nearly accurate up to flexural failure if good bond exists between the concrete and the steel reinforcement. For recycled aggregate concrete, the appropriateness of this assumption is highly dependant on the bond between the RAC and the steel, which will be discussed later in this chapter.

\section{The stress-strain curve for steel is known}

\section{The tensile strength of concrete may be neglected}

\section{The stress-strain curve for concrete is known}

The last assumption is required to accurately evaluate the full behaviour of the section. This stress-strain curve provides the information required for calculating the properties of the rectangular stress block parameters at failure, which is the basis of flexural design provisions in different codes. Therefore, the flexural response of RRC would be dependent on the stress-strain relationship of RAC, which will be discussed later in this chapter.

There are basically two failure modes in flexural members:

1. Tension Failure: If the steel content of the section is below the so-called balance reinforcement ratio, the steel will reach its yield strength before concrete reaches its maximum capacity. This type of failure is a ductile one.

2. Compression Failure: If the steel content of the section is large, the concrete may reach its maximum capacity before the steel yields. This type of failure is brittle. The failure modes of RRC members are expected to be the same. 


\subsubsection{Shear Behaviour}

Shear transfer in RC members is strongly dependent on the tensile and compressive strengths of concrete. Therefore, shear failure is generally nonductile and brittle. Therefore, like compression failure in flexure, it must be avoided.

\section{Shear Failure Mechanisms in Beams without Stirrups}

According to linear analysis, shear stress in a beam can be expressed as following:

$$
\begin{gathered}
v=\frac{1}{b \cdot j d} \frac{d M}{d X}\left[1-\left(\frac{2 y}{h}\right)^{2}\right] \\
v_{\max }=\frac{V}{b \cdot j d}
\end{gathered}
$$

where $V$ is the shear force, $v$ is the shear stress, $b$ is the section width, $j d$ is the distance between equivalent compressive and tensile forces in a section, $M$ is the moment, $y$ is the distance from neutral axis, and $h$ is the section height.

In regions of pure shear or where bending stresses are low, the only significant stress is the shear stress. In such cases, the pure shear results in principal tensile stresses acting at $45^{\circ}$ to the beam axis. In the post-cracking stages, particularly near failure, the distribution of the longitudinal normal stresses is not linear and hence the aforementioned equations do not directly apply. However, the relative magnitude obtained from these equations is still indicative of the potential for inclined cracking.

\section{Shear Resistance Components:}

Figure 2.1 illustrates part of a simply supported beam diagonally cracked in a pure shear region. With reference to this figure, it may be seen that the total shear force $(V)$ is resisted by three major components (ASCE-ACI, 1973):

- Shear stress in uncracked concrete $\left(V_{c}\right)$ 
Depending upon the strength of concrete under combined compressive and shear stresses and the depth of the uncracked zone, the contribution of this mechanism can be determined.

\section{- Interface shear transfer $\left(V_{a}\right)$}

The faces of a crack are generally rough, and as a result of relative motion along the crack surfaces, the rough asperities interlock, endowing the crack to transfer the shear. This form of shear transfer has invariably been referred to as interface shear, shear friction, and aggregate interlock.

\section{- Dowel action $\left(V_{d}\right)$}

Dowel action can be developed by three mechanisms: the flexure of the reinforcing bars, the shear strength across the bars, and the kinking of the reinforcement. Dowel action can only occur at the expense of large displacements. Therefore, at acceptable crack displacements, dowel action is not significant.

The contribution of each of these mechanisms varies depending upon the type of member and the relative magnitude of the stresses and the level of loading. For rectangular beams without shear reinforcement, it is reported (ASCE-ACI, 1973) that after an inclined crack has formed; the proportion of the shear transferred by the various mechanisms is as follows: 15 to $25 \%$ by dowel action, 20 to $40 \%$ by the uncracked concrete compression zone, and 33 to $50 \%$ by aggregate interlock or interface shear transfer. More recently, ASCE-ACI Committee 445 Report highlighted a new mechanism, called the residual tensile stresses, which are transmitted directly across the crack (ASCE-ACI, 1998). Among the abovementioned shear transfer mechanisms, the interface shear transfer in RRC members is of high interest due to the existence of the residual mortar and 
consequently lower maximum original virgin aggregate size in RAC, which might lead to less rough, and smoother diagonal crack interface, and consequently smaller shear strength.

\section{Principle Mechanisms of Shear Resistance}

The principal mechanisms of shear resistance are beam and arch actions (Park and Paulay, 1975). From first principles:

$$
V=\frac{d M}{d x}=\frac{d}{d x}(T \cdot j d)=j d \cdot \frac{d T}{d x}+T \cdot \frac{d(j d)}{d x}
$$

Based on elastic analysis, $\frac{d(j d)}{d x} \approx 0$ hence, the entire external shear is exclusively resisted by beam action. This is clearly based on the assumption of perfect bond. If the bond force is destroyed, then $\frac{d T}{d x}=0$. In this case, the external shear is resisted by arch action. However, generally, each mechanism may contribute a certain amount to the total resistance.

\section{Size Effect}

The shear strength of beams without web reinforcement generally decreases as the effective depth increases (Kani, 1967). In particular, the dowel and aggregate interlock components may decrease significantly as the crack width above the main reinforcement tends to increase. However, well distributed longitudinal reinforcements can contribute to size effect reduction (Collins and Kuchma, 1999). Therefore, it can be noticed that the size effect is also of significant interest in RRC members due to the role of aggregate interlock action in deeper beams. 


\section{Shear Failure Mechanisms}

The shear span to depth ratio a/d is a highly influential factor in establishing the shear strength of concrete members. Therefore, three general categories of failure may be established (Leonhardt, 1965):

1. For $3<\mathrm{a} / \mathrm{d}<7$ : Failure of the beam mechanism at or shortly after diagonal cracking load. This is usually a beam action failure as the subsequent arch action is not capable of supporting the cracking load.

2. For $2<\mathrm{a} / \mathrm{d}<3$ : Shear compression or flexural tension failure of the compression zone above diagonal cracking load. This is usually an arch action failure.

3. For $\mathrm{a} / \mathrm{d}<2.5$ : Failure by crushing or splitting of the concrete (failure of arch action).

\section{Shear Failure Mechanism in Beams with Stirrups}

When the diagonal cracks form, the ability of the concrete to transmit principal tensile stresses is severely reduced and unless appropriate reinforcement is present, failure may result soon after cracking. In the presence of shear reinforcement, a new system of internal stresses can develop after cracking which would enable the member to carry additional load (Figure 2.1b). The presence of transverse reinforcement does not prevent the formation of inclined cracks, but it becomes effective after the formation of diagonal cracks. Below some existing models for the shear failure mechanisms of beams with stirrups will be presented.

\section{- The $45^{\circ}$ Truss Model}

Both Ritter (1899) and Mörsch (1909) explained independently the flow of forces in a cracked reinforced concrete beam in terms of a truss model. The diagonal compressive stresses in the concrete act as the diagonal members of the truss while the stirrups act as 
vertical tension members. The bottom chord of the truss consists of the longitudinal tension reinforcement while the flexural compression zone of the beam acts as the top chord. They neglected the tensile stresses in the cracked concrete and assumed that after cracking the diagonal compression stresses would remain at $45^{\circ}$. While Mörsch recognized that the choice of $45^{\circ}$ was conservative, he could not conceive a practical way to determine the actual inclination. The conservatism of this model is due to the neglect of the tensile stresses in the concrete and the choice of $45^{\circ}$ for the compressive strut inclination.

\section{- The Variable-Angle Truss Model}

To reduce the conservatism of the $45^{\circ}$ Truss Model, the ACI Code modified it by adding a concrete contribution to shear resistance. Alternatively, this model can be made more accurate by considering the fact that $\theta$ is variable and is typically less than $45^{\circ}$. In predicting the shear strength of beams using the Variable-Angle Truss Model, it is necessary to use an effective concrete compressive strength less than the cylinder crushing strength.

\section{- Compression Field Theory (CFT)}

Before the equilibrium equations of the Variable-Angle Truss Model can be used to design a member for shear, the inclination, $\theta$, of the diagonal compressive struts must be known. In 1929, Wagner treated an analogous problem in studying the postbuckling shear resistance of thin-webbed metal girders. He assumed that after buckling, the thin webs would not resist compression and that the shear would be carried by a field of diagonal tension. To determine the angle of inclination of the diagonal tension, Wagner considered the deformations of the system. He assumed that the angle of inclination of the diagonal 
tensile stresses would coincide with the angle of inclination of the principal tensile strains. This approach became known as the tension field theory.

Mitchell and Collins (1974) developed the Compression Field Theory by applying Wagner approach to reinforced concrete and assuming that after cracking, the concrete carries no tension and that the shear is carried by a field of diagonal compression. However, because the compression field theory neglects the contribution of tensile stresses in the cracked concrete, it overestimates deformations and gives conservative estimates of strength. Later, Vecchio and Collins (1986) developed the Modified Compression Field Theory, which accounts for the influence of tension in the concrete.

\section{- Modified Compression Field Theory (MCFT)}

Prior to cracking, the shear is carried equally by diagonally tensile and diagonal compressive stresses acting at $45^{\circ}$. After diagonal cracks form, the tensile stresses in the concrete are substantially reduced. In the CFT Model, it is assumed that the principal tensile stress equals zero after the concrete has cracked. On the other hand, the Modified Compression Field Theory accounts for the contribution of the tensile stresses in the concrete between the cracks. This model is the basis of the General Method in CSA A23.3-04 Standard (CSA, 2004).

As it can be seen, although researchers have expended considerable effort to understand the shear behaviour of concrete, due to the lack of an universally accepted model for shear behaviour, shear design provisions still generally consist of empirical relationships that differ from code-to-code to such an extent that the shear strength of a particular member as calculated by one code-of-practice may be quite different from the strength predicted by another design code. Tables 2.14 and 2.15 summarize the empirical 
equations for shear strength of RC beams proposed by CSA A23.3-04 and other codes, and/or researchers, respectively.

\subsubsection{Flexural and Shear Behaviour of RRC Beams}

The flexural and shear static/fatigue behaviour of steel reinforced recycled concrete (RRC) beams was investigated by Yagishita et al. (1994) using three types of RCA with different qualities. The difference in quality was due to different crushing processes. His results indicated that the ultimate strength of the RRC beams was always about $12 \%$ less than that of conventional RC beams. Based on the load-displacement curves of beams subjected to cyclic flexure, there was no noticeable difference between the responses of the two sets of beams. However, the moment-curvature relations of the beams made of low-grade RCA showed lower rotational stiffness. The reduction in stiffness due to cyclic loading in conventional RC beams and in medium-grade RCA was similar. Similarly, there was little difference between the crack patterns and maximum crack widths of the two sets of beams after 300,000 cycles of loading and unloading, but the shear deformations caused wider cracks in RRC beams.

In the case of the beams made of low-grade RCA, cracks were indicative of bond deterioration along the longitudinal reinforcement in the tensile region, most probably due to the effects of the destruction of the residual mortar, as well as the drying shrinkage of the concrete at the bottom of the reinforcement. The stiffness of the RC beams showed little reduction after several cycles of loadings, while the stiffness of RRC beams gradually decreased with increased cycles of loading. In all beams, cracks due to diagonal tension cracks occurred at several places before 300,000 cycles. Additionally, in the 
beams made of low and medium-grade RCA, small cracks were observed in the tension zone, which were indicative of bond deterioration along the tensile reinforcement.

Nishiura et al. $(2000,2001)$ placed coarse RAC in-situ inside pre-cast U-shaped beams with or without lap joints. The U-shaped section was made of normal concrete and reinforced with flexural steel at the bottom and with stirrups for shear. The hollow part of the U was subsequently filled with in-situ concrete (either RAC or NAC). Based on their results, they concluded that fracture processes and cracking behaviour, maximum load, shapes of the hysteresis loops, and viscous damping coefficient for the composite Ushaped beams filled with RAC were nearly identical to those filled with NAC.

The applicability of the current ACI recommendations for conventional concrete contribution to shear resistance of members, $V_{c}$, to RRC members made with coarse RCA was studied by Han et al. (2001). The experimental variables were shearspan/depth ratio: $\frac{a}{d}(1.5,2,3$, and 4$)$, aggregate types (natural, washed recycled and nonwashed recycled aggregate), and percentage of shear reinforcement: $\rho_{s}(0,0.089,0.244$, 0.507 , and 0.823 percent). The test results indicated that the aggregate type had negligible effect on the failure mode of the beams with $a / d=2$. However, differences in the roughness of the cracks were observed, with smoother cracks in RAC beams. For the beams with $a / d=2.0$, but different aggregate types, based on the load-deflection response, the conventional concrete was more ductile due to the greater effective aggregate interlock mechanism. Based on their results, even the simplified ACI equation for $V_{c}$ overestimates the shear capacity of RAC beams with $a / d$ of 3.0 and 4.0 and no shear reinforcement. The effects of the $a / d$ on the diagonal tension parameter $\left(\frac{V_{c r}}{b d \sqrt{f_{c}^{\prime}}}\right)$ were 
correctly predicted by the pertinent relations given in the ACI Code (ACI, 1999). For increasing $a / d$, the degree of conservatism of the $\mathrm{ACI}$ equation decreased, but the code predictions became unconservative for beams with a/d of 3.0 and 4.0. The reserve capacity of beams after cracking was more for beams with a/d less than 2.50. For all the RAC beams with web reinforcement, their observed cracking shear was larger than that predicted by the ACI Code, but the scatter in the data was very large and the test trend was not obvious. Since all the beams with web reinforcement failed in flexure, the results could not be used to investigate their shear failure modes.

The flexural behaviour of reinforced recycled concrete beams was examined by Maruyama et al. (2004a). According to their results, crack width in wet cured reinforced recycled concrete $(\mathrm{RRC})$ beams was wider than in conventional reinforced concrete (RC), while crack spacing was smaller. Expansive additive, however, reduced crack width by $20-30 \%$. The deflection of RRC beams was larger than those of conventional RC beams, even in the case of beams with expansive additive, and this was due to the lower elastic modulus of RAC than NAC. Experimental ultimate moments were $10-20 \%$ larger than their calculated values based on measured yield stress of reinforcing bars. Finally, they concluded RAC would not decrease the flexural capacity of a beam, provided the reinforcing steel is sufficiently anchored to reach its yield stress before the advent of compression failure in concrete.

In another study, Maruyama et al. (2004b) studied the shear behaviour of reinforced recycled concrete (RRC) beams. Ten reinforced concrete beams were prepared using recycled fine and coarse aggregates obtained from concrete made in the laboratory and from demolition sites. The major factors were combination of aggregates, water to 
cement ratios, effective depth and usage of expansive additive. According to their results, RRC beams using RCA showed the same cracking patterns and failure mode as companion RC beams. The shear strength of beams without stirrups decreased by 10$20 \%$ when coarse recycled and virgin fine aggregates were used and by $10-30 \%$ when both coarse and fine recycled aggregates were used. Reducing the water-cement ratio increased the shear strength by $25 \%$ in case of $w / c=0.3$ and by $10 \%$ in case of $w / c=0.45$ compared with beams made of concrete with $w / c=0.6$. Expansive additive increased the strength by $10 \%$ regardless of aggregate type.

The shear behaviour of reinforced concrete beams made with RAC was also investigated by Gonzalez and Martinez (2004). According to their study, the cracking to ultimate load ratio in RRC beams was smaller than in RC beams. The tests revealed the premature cracking suffered by beams that included recycled aggregates. Considering this, and the relationship that exists between the phenomenon of cracking and the stirrups spacing, they suggested modification of the provisions of the standards with stricter limitations on the maximum stirrup spacing.

Santos et al. (2004) studied the flexural behaviour of RRC beams. The crack pattern in all beams during bending was reported to be similar to those of conventional beams. Failure of all beams was initiated by yielding of steel followed by compression failure in concrete. There were no pre-failure qualitative differences in the behaviour of the two types of beams. The stiffness of the RAC beams was lower than the NAC beams, but this was expected due to the lower elastic modulus of RAC.

The seismic performance of frame structures made of RCA was investigated by Xiao et al. (2006). Based on four $1 / 2$ scale frame specimens $(100 \%, 50 \%, 30 \%$ and $0 \%$ RCA 
content levels) tested under low-frequency cyclic lateral load and constant gravity load, they reported similar failure pattern for all the frames regardless of their RCA content. However, the yield, maximum and ultimate load capacities of frames made with RCA was smaller. From the analysis of characteristic displacements and hysteresis loops of the frames, the authors concluded that the frames made with properly mixed RAC behaved well enough to stand an earthquake. Table 2.12 presents a summary of the findings of several researchers with regards to the structural behaviour of RRC beams.

\subsubsection{Stress-Strain Relationship}

The relationship between the stress and strain of materials over their full range is of vital interest to structural engineers. The stress-strain relationship of conventional concrete in compression is well known. There are several empirical models for prediction of stressstrain response of normal concrete, which have been comprehensively reviewed by Popovics (1998). Some of the existing equations are presented in Table 2.16.

For RAC, the compressive stress-strain relationship has been reported to be similar to that of NAC (Ajdukiewicz and Kliszczewicz, 2002). Xiao et al. (2004) also investigated the compressive strength and the stress-strain relationship of concrete made of coarse RCA $(0 \%, 30 \%, 50 \%, 70 \%$, and $100 \%$ replacement $)$. The shape of the stress-strain curve for all the RAC specimens was found to be similar to that of NAC specimens, irrespective of the RCA replacement level, which leads to the conclusion that, in principle, there should be no objection in the structural design process to the plastic distribution of stresses in RRC members. The curvature of the non-linear portion of the ascending branch of the strain-stress curve improved with increase in the RCA content, while the slope of the descending branch decreased as the RCA content increased. This 
change was generally characterized by an increase in the strain at peak stress and a significant decrease in the ductility of the concrete. The value of the strain corresponding to peak stress increased by $20 \%$ at $100 \%$ RCA replacement.

\subsubsection{Bond Strength}

Since a basic requirement in reinforced concrete construction is that there should be adequate means for transfer of the force in the reinforcement to the surrounding concrete, investigating the bond strength in concrete made of RCA is obviously an important issue. Ajdukiewicz and Kliszczewicz (2002) reported that there was no significant difference between the bond strength of rebars embedded in NAC and RAC. It was shown that the greatest difference was between the bond behaviour of smooth and deformed bars. The next important factor was the presence of admixtures, followed by the influence of RCA. The value of bond stress at failure for recycled aggregate concrete was lower than that of NAC, particularly for plain round bars. For concrete with fully recycled aggregate, the average drop was up to $20 \%$ while in the case of concrete made with recycled fine aggregate instead of natural sand, it was up to $8 \%$ only.

Yagishita et al. (1994) performed the bond test by studying several parameters including the RCA type, concrete strength, the direction of concrete casting, and the concrete cover thickness. The highest bond strength was observed in NAC with low and medium w/c ratio and the lowest strength in RAC made with low quality RCA. On the contrary, the maximum bond strength for concretes with high w/c ratio was not affected by the presence of RCA, possibly due to the better quality of residual mortar compared to the new mortar with its high w/c ratio. In the case of concrete cast transversely to the length of the bars and with $80 \mathrm{~mm}$ cover over them, the aggregate type and concrete strength 
caused little variation in bond strength. This may be due to the effect of the water membrane formed by bleeding and by the settlement of concrete under the bars. It was indicated that an increase in the concrete cover was very effective in increasing the bond strength. For each aggregate type, the test specimens with $40 \mathrm{~mm}$ cover had $30-50 \%$ less bond strength than those with $80 \mathrm{~mm}$ cover.

\subsection{Selection of RAC Mixture proportions}

When using RCA instead of NA, the main factors which necessitate some modifications in conventional mix design are the RCA higher water absorption, lower specific gravity, and amount of residual mortar. In most studies in the past, mixes with the same proportion of RAC and NAC have been used to evaluate the properties of RAC. Therefore, no special mix design has been employed in order to achieve certain specified properties of fresh or hardened RAC. In fact, research on the mix design of RAC is practically non-existent. Currently, the same procedures that are used in the design of conventional concrete mixes are also used to design RAC mixtures (Dhir et al., 1999; Limbachiya et al., 2000; Mandal et al., 2002; and Gomez-Soberon, 2002a, 2002b, 2002c, and 2003). Generally, pre-soaking of RCA is recommended in order to offset its high water absorption and/or some other adjustments are made to the water content to offset the effects of the high moisture content and higher water absorption of RCA (Ray and Venkateswarlu,1991; Merlet and Pimienta, 1994; Yanagi et al., 1994; Ramamurthy and Gumaste, 1998; Nagataki et al., 2000; Sagoe-Crentsil, 2001; Buyle-Bodin and HadjievaZaharieva, 2002; Gomez-Soberon, 2002a, 2002b, 2002c, and 2003; Topcu and Sengel, 2004; and Xiao et al. 2004). In order to take into account the lower specific gravity of RCA, most investigators have employed the absolute volume method of conventional 
mix design (Salem and Burdette, 1998; Yamasaki and Tatematsu, 1998; Buyle-Bodin and Hadjieva-Zaharieva, 2002; Salem et al., 2003; Gomez-Soberon, 2002a, 2002b, 2002c, and 2003; Poon et al. 2004a; and Santos et al. 2004). Any anticipated reduction in the strength of RAC is averted by adjusting its w/c ratio (Dhir et al., 1999; Limbachiya et al., 2000; and Sagoe-Crentsil et al., 2000).

Lin et al. (2004) made an attempt to reduce the number of tedious and costly mix trials by optimizing the mix design process via controllable inputs and measurable outputs using a technique based on the design of experiments. In this study, based on the results of some preliminary tests, five control factors were identified, viz. (1) water/ cement ratio, (2) volume ratio of recycled coarse aggregate, (3) replacement ratio of river sand, (4) content of crushed brick, and (5) cleanness of aggregate. Overall assessment of both the slump and the compressive strength of RAC indicated that the optimal mix will have water/cement ratio of 0.5 , coarse aggregate volume ratio of $42.0 \%, 100 \%$ natural river sand, $0 \%$ crushed brick, and as-is recycled aggregate with no washing.

Lamond et al. (2002) specified some guidelines for mixture proportions using RCA. A higher standard deviation (4.83MPa) was specified for a concrete with RCA of variable quality than for concrete made with uniform quality RCA or with NA. They also stated that at the design stage, the w/c for RAC made with coarse RCA and natural sand can be assumed to be the same as in NAC. If trial mixtures showed that the compressive strength was lower than assumed, the w/c should be lowered. For the same slump, $5 \%$ increase in the free water requirement of coarse RAC than NAC was suggested. Pre-soaking of $\mathrm{RCA}$, and removing materials smaller than $2 \mathrm{~mm}$ from aggregates prior to production was recommended as well. 


\subsection{Existing Guidelines and Standards}

Currently, there is no standard in North America for the use of RCA in structural-grade concrete. The RILEM specifications are the most commonly accepted international standard for recycled aggregates (RILEM, 1994). ACI 555R-01 report on "Removal and Reuse of Hardened Concrete" provides some guidelines about hardened concrete removal and its reuse as new aggregate in RAC production (Lamond et al., 2002). In the United Kingdom, the use of recycled aggregates is permitted since the publication of BS EN 12620:2002 "Aggregates for Concrete"; however, this document does not give specific compositional limits. It is anticipated that some limitations will be imposed on the RA composition in the 2007 revision of the document. BS EN 8500-2:2002, Concrete Complementary British Standard to BS EN 206-1: Specification for Constituent Materials and Concrete permits the use of RCA in designated concrete types, subject to certain restrictions on concrete strength and exposure environment. In the UK, the combination of RCA and NA must also meet a default value of $0.075 \%$ for concrete drying shrinkage. According to BS $8500-2$, RCA can be used up to strength class C40/50 and in a range of exposure conditions. RCA is not generally permitted in concrete exposed to sea water, de-icing salts or severe freezing and thawing. It is suggested that concrete containing RCA be restricted to use in non-aggressive soils. Also BS 8500 restricts the amount of RCA to $20 \%$ by mass of the total coarse aggregate in concretes designated as RC25RC50 (Research Information Digest 1, 2005).

In Spain, the Ministry of the Environment published a National C\&D Waste Plan (PNRCD) in 2001 (Alaejos et al., 2004). This five year plan calls for a 10\% reduction of C\&D Waste and $25 \%$ recycling by 2006 . In order to attain these goals, specifically, in 
the area of structural concrete, in November 2002 a task force was created to draft the technical regulations governing structural concrete. The draft regulation will specify certain limitations on the use of RA for structural concrete, including:

- Allowing RCA in mass concrete and reinforced concrete but not in prestressed concrete.

- Allowing RCA obtained from the recycling of conventional concrete, but excluding lightweight and fiber-reinforced concrete and concrete made with aluminous cement.

- Depending on the effect of OC quality on RAC, specification of minimum strength for OC and identification of demolition reasons. The latter is intended to avoid using concrete affected and damaged by causes such as alkali-aggregate reaction, sulphate attack, etc.

- Limiting to maximum $20 \%$ the replacement of larger NA aggregates $(>4 \mathrm{~mm})$ by similar grade RA,

- Allowing the use of RA in concrete exposed to aggressive environments only where special precautions have been taken (i.e. use of single-source verified RA, additional testing, higher cement content or a lower w/c ratio in mixing, etc.).

In Germany, the national standard 'DIN 4226-1000 Aggregates for Concrete and Mortar, 2002" sets the guideline for recycled concrete content in concrete aggregates. In the Netherlands, the Dutch standard NEN 6720:1995 "Construction Requirements and Calculating Methods", allows the use of recycled concrete aggregate, provided the replacement percentage of natural aggregate is not higher than $20 \%$. Only a replacement of up to $10 \%$ is allowed for masonry aggregates (Corinaldesi et al., 2002). Similarly, in Japan, the Building Contractors' Society of Japan (BCSJ) issued a "proposed standard for 
the use of recycled aggregate and recycled aggregate concrete" in 1977. However, the recycling law was established in 1991 to control the increase of waste and promote recycling of useful resources (Noguchi and Tamura, 2001). Table 2.12 presents some specified limitations on use of RCA established by different countries (Alaejos et al., 2004).

In Canada, the C-2000 Green Building Standards aim at making recyclable materials up to $75 \%$ of existing structure. However, this program does not require any processing of concrete other than its separation from other demolition debris (Taschereau, 2001). According to Cement Association of Canada, in 1993 the use of recycled concrete was included in Ontario Provincial Standard Specification (OPSS) and RCA is to be used as road or parking lot base and sub-base aggregate as well as sewer bedding and backfills. The fact that there is no standard or specifications in Canada for the use of RCA in the production of the RAC provides the motivation for the current study.

\subsection{Existing Structures using RAC}

Some countries have successfully employed crushed concrete as a substitute for natural aggregate even in the construction of major motorways and other large structures during the past few decades. Hansen (1986) has extensively reviewed case histories around the world and some of the examples cited by him are briefly recapped as follows. In the United States, since 1976, RCA has been used in sub-base, cement treated sub-base, lean concrete base, and in concrete pavement of highways and airports. In Belgium, concrete from an old lock wall was crushed and used as new aggregate for construction of a new and larger lock at the same location. In the Netherlands, the first application of RCA was for partition walls in an apartment building, but since then it has been extended to some 
other projects in highway and airport construction. In Russia, coarse RCA has been used for foundations and fine RCA as mineral filler in asphalt. In Japan, RCA has been used in real structures since 1984 . Two small test structures were built by the Building Research Institute of the Ministry of Construction. In Germany, an office building using RCA was built in Darmstadt in 1998 (Xiao et al., 2006).

Excellent performance was reported for a concrete pavement made with $100 \% \mathrm{RCA}$ in Dundee, Scotland (Digest 1 Research Information, 2005). Also, RCA was used to build the Building Research Establishment (BRE) Cardington Laboratory in the UK, in which over 100 tons of coarse RCA was used in a ready-mix RAC (Digest 433, 1998). According to De Vries (1993), coarse NA was replaced by coarse RCA in the foundations and walls of the Caland canal, near Rotterdam-Holland, in order to protect the entrance to the lock on the canal. Collins (1994) reported the construction of a multistorey house in Copenhagen in which RCA from demolished houses was used.

From this literature survey we can see that extensive research has been conducted on many aspects of RAC, but most of the research has focussed on its basic mechanical and physical properties, rather than its structural performance. The research on its structural performance is rather limited and is not sufficient for developing detailed design guidelines. Furthermore, as stated earlier, a specific mix design method for RAC is presently lacking. For these reasons, the following comprehensive study is undertaken to develop a mix design method for RAC and to extensively investigate the structural performance of reinforced RAC members under flexure and shear. 


\subsection{Summary}

As presented in this state of the art review, a number of studies have been performed to investigate the use of RCA in structural-grade concrete. Although these studies cover a relatively wide range, they do not follow a comprehensive and methodical approach that can lead to the standardization of the practice. In general, the following major gaps in the existing literature on RAC have been identified:

- Lack of research on the RCA source effect on $R A C$. In the majority of the previous studies, laboratory-produced RCA has been used rather than commercially-produced one. Since Canada is a vast country, there is an evident need to investigate the RCA source effect by choosing materials from diverse geographical locations.

- No specific mix proportioning method for RAC. To the writer's best knowledge, currently there is no specific mix proportioning method available for structural-grade RAC which considers the influence of the residual mortar quantity in RCA. This is anticipated to be the key factor responsible for the inferior properties of RAC reported in the literature. To develop such a mix design procedure, firstly, a comprehensive investigation of the fresh and hardened properties of concrete produced with RCA obtained from different commercial sources need to be carried out.

- Applicability of existing models for predicting different properties of RAC. Although several models have been developed to predict different properties of NAC such as its elastic modulus and stress-strain relationship, many questions arise regarding the applicability of these models to RAC. To the writer's knowledge, no specific research has been performed to assess the appropriateness of the existing models for predicting 
different properties of RAC such as its elastic modulus and stress-strain relationship, and accordingly development of new models or modification of the existing ones.

- Applicability of existing design provisions to RAC. Previous studies have indicated that the existing empirical equations for normal concrete may or may not be valid for RAC. Therefore, questions regarding the applicability of the existing relations such as those recommended by CSA A23.3-04 or ACI 318-05 to RAC are understandable and further work is needed to answer them.

- Applicability of existing design provisions developed for RC to RRC:

Stress-strain relationship of $\boldsymbol{R A C}$. The effect of RCA content on the stress-strain relationship has been investigated by a limited number of researchers. However, the applicability of existing models for normal concrete, as well as existing equations for calculating the equivalent rectangular block stress distribution parameters of normal concrete $\left(\alpha_{1}\right.$ and $\left.\beta_{I}\right)$, to RAC is not evident. The latter is rather important for predicting the ultimate flexural strength of RC beams.

Bond strength of steel reinforcement embedded in RAC. Although limited studies have been carried out on the bond performance of steel rebars embedded in RAC, these are all based on conventional pull-out tests rather than the so-called beam-end bond test, which provides a better indication of the bond performance of RAC.

Flexural behaviour of RRC beams. A substantial amount of research work has been done in the past on the flexural behaviour of RC beams made with NAC, but there is not adequate information available about the flexural behaviour of RRC members. There is a need to develop information about the flexural behaviour of RRC beams and evaluate the 
applicability of the existing models and standards to ensure proper use of this material in structural applications.

Shear behaviour of RRC beams: A substantial amount of research on shear in conventional reinforced concrete has been carried out. On the other hand, due to lack of adequate research results, it is not evident whether RRC members have the same shear behaviour as conventional $\mathrm{RC}$ members. In particular, the contribution of the aggregate interlock mechanism and the uncracked concrete to the shear resistance of RAC may not be the same as in conventional concrete. 
Table 2.1: Existing empirical equations for elastic modulus of concrete

\begin{tabular}{|c|c|}
\hline Author/Source & Equation \\
\hline \multirow{2}{*}{ ACI 318 (ACI, 2005) } & $\begin{array}{c}\text { If } f_{c}^{\prime} \leq 41 \mathrm{MPa} \text { and } 1440 \leq \gamma_{c} \leq 2480 \mathrm{~kg} / \mathrm{m}^{3}: \\
E_{c}=43 \times 10^{-3} \gamma_{c}^{1.5} \sqrt{f_{c}^{\prime}}\end{array}$ \\
\hline \multirow{2}{*}{ ACI 363 (ACI, 1997) } & $\begin{array}{c}\text { If } f_{c}^{\prime} \leq 81 \mathrm{MPa}: \\
E_{c}=\left(3320 \sqrt{f_{c}^{\prime}}+6900\right) \times\left(\frac{\gamma_{c}}{2320}\right)^{1.5}\end{array}$ \\
\hline \multirow{2}{*}{ CSA A23.3-04 (CSA, 2004) } & $\begin{array}{c}\text { For normal density concrete, if } 20 \leq f_{c}^{\prime} \leq 40 \mathrm{MPa}: \\
E_{c}=4500 \sqrt{f_{c}^{\prime}}\end{array}$ \\
\cline { 2 - 3 } & For $1500 \leq \gamma_{c} \leq 2500 \mathrm{~kg} / \mathrm{m}^{3}:$ \\
\hline Gardner (2000) & $E_{c}=\left(3300 \sqrt{f^{\prime}{ }_{c}}+6900\right)\left[\frac{\gamma_{c}}{2300}\right]^{1.5}$ \\
\hline Eurocode 2 ( CEN, 2005) & $E_{c}=3500+4300 \sqrt{f^{\prime}}$ \\
\hline Nassif et al. (2005) & $E_{c}=21500\left(\frac{f_{c}^{\prime}}{10}\right)^{1 / 3}$ \\
\hline \multirow{2}{*}{ AIJ (Tomosawa and Noguchi, 1993) } & If $f_{c}^{\prime} \leq 36 \mathrm{MPa}:$ \\
\hline Tomasawa and Noguchi (1993) & $E_{c}=2.1 \times 10^{5}\left(\sqrt{\frac{f^{\prime}{ }_{c}}{200}}\right)\left[\frac{\gamma_{c}}{2.3}\right]^{1.5}$ \\
\hline
\end{tabular}


Table 2.2: Summary of the creep empirical equations proposed by ACI Committee 209

\begin{tabular}{|c|c|c|}
\hline \multicolumn{3}{|c|}{$\varepsilon_{c}(t)=\left(1+C_{t}\right) \times \varepsilon_{0}$} \\
\hline \multicolumn{3}{|c|}{$\begin{aligned} \text { where } \varepsilon_{0} & =\text { the initial strain at loading } \\
C_{t} & =\text { creep coefficient }\end{aligned}$} \\
\hline \multicolumn{3}{|c|}{$C_{t}=C_{u} \cdot K_{t} \cdot K_{a} \cdot K_{h} \cdot K_{t h} \cdot K_{s} \cdot K_{f} \cdot K_{e}$} \\
\hline Ultimate Creep Coefficient & For $\mathrm{H}=40 \%$ : & $1.30 \leq C_{u} \leq 4.15$ \\
\hline \multicolumn{3}{|c|}{ Where $\mathrm{H}=$ relative humidity of the ambient atmosphere } \\
\hline Time under load coefficient & \multicolumn{2}{|c|}{$K_{t}=\frac{t^{0.6}}{10+t^{0.6}}$} \\
\hline \multicolumn{3}{|c|}{ where $\mathrm{t}=$ time in days after application of load } \\
\hline \multirow{3}{*}{ Age when loaded coefficient } & curing method & $K_{a}$ \\
\hline & moist-cured & $1.25 t_{i}^{-0.118}$ \\
\hline & steam-cured & $1.13 t_{i}^{-0.095}$ \\
\hline \multicolumn{3}{|c|}{ where $t_{i}=$ age of concrete in days when load is first applied } \\
\hline Relative humidity coefficient & For $\mathrm{H}>40 \%$ : & $K_{h}=1.27-0.0067 H$ \\
\hline \multirow{3}{*}{$\begin{array}{l}\text { Minimum thickness of member } \\
\text { coefficient }\end{array}$} & Thickness (mm) & $K_{t h}$ \\
\hline & $\leq 150$ & 1.00 \\
\hline & 300 & 0.82 \\
\hline \multirow{6}{*}{ Slump of concrete Coefficient } & Slump (mm) & $K_{s}$ \\
\hline & 51 & 0.95 \\
\hline & 68.6 & 1.00 \\
\hline & 76.2 & 1.02 \\
\hline & 101.6 & 1.09 \\
\hline & 127 & 1.16 \\
\hline \multirow{4}{*}{ Fines coefficient } & Fine Content (\%) & $K_{f}$ \\
\hline & $\leq 30$ & 0.95 \\
\hline & 50 & 1.00 \\
\hline & 70 & 1.05 \\
\hline \multirow{4}{*}{ Air content coefficient } & Air Content (\%) & $K_{e}$ \\
\hline & $\leq 6$ & 1.00 \\
\hline & 7 & 1.09 \\
\hline & 8 & 1.17 \\
\hline
\end{tabular}


Table 2.3: Summary of the creep empirical equations proposed by CEB-FIP

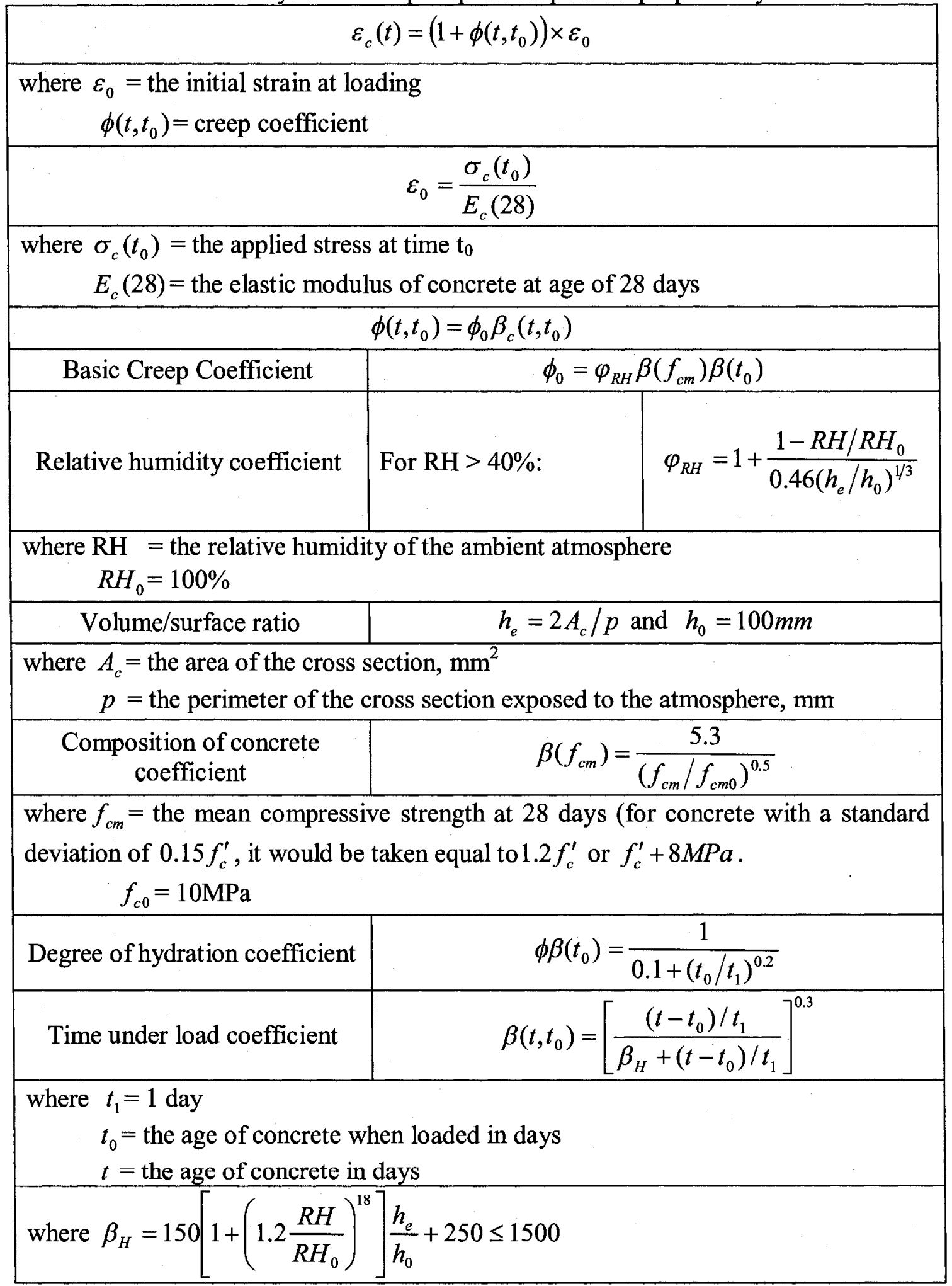


Table 2.4: Summary of the shrinkage empirical equations proposed by ACI Committee 209

\begin{tabular}{|c|c|c|c|}
\hline \multicolumn{4}{|c|}{$\varepsilon_{s h}=\varepsilon_{s h t} \cdot S_{t} \cdot S_{h} \cdot S_{t h} \cdot S_{s} \cdot S_{f} \cdot S_{e} \cdot S_{c}$} \\
\hline \multirow{3}{*}{ Ultimate Shrinkage Strain } & \multicolumn{2}{|c|}{ Curing Method } & $\varepsilon_{\text {shu }}$ \\
\hline & \multicolumn{2}{|c|}{ Moist-cured } & $0.000415 \leq \varepsilon_{s h u} \leq 0.00107$ \\
\hline & \multicolumn{2}{|c|}{ Steam-cured } & 0.00075 \\
\hline \multirow{3}{*}{$\begin{array}{l}\text { Time under exposure } \\
\text { coefficient }\end{array}$} & $\begin{array}{l}\text { Curing } \\
\text { Method }\end{array}$ & $\mathrm{t}$ (days) & $S_{t}$ \\
\hline & $\begin{array}{l}\text { Moist- } \\
\text { cured }\end{array}$ & $\begin{array}{c}\text { age from } 7 \\
\text { days }\end{array}$ & $\frac{t}{35+t}$ \\
\hline & $\begin{array}{c}\text { Steam- } \\
\text { cured }\end{array}$ & $\begin{array}{l}\text { age from 1- } \\
3 \text { days }\end{array}$ & $\frac{t}{55+t}$ \\
\hline \multirow{2}{*}{$\begin{array}{l}\text { Relative humidity } \\
\text { coefficient }\end{array}$} & \multicolumn{2}{|c|}{ For $40 \leq H \leq 80 \%$} & $S_{h}=1.4-0.01 \mathrm{H}$ \\
\hline & \multicolumn{2}{|c|}{ For $80 \leq H \leq 100 \%$} & $S_{h}=3.0-0.03 H$ \\
\hline \multirow{6}{*}{$\begin{array}{l}\text { Slump of concrete } \\
\text { Coefficient }\end{array}$} & \multicolumn{2}{|c|}{ Slump (mm) } & $S_{s}$ \\
\hline & \multicolumn{2}{|c|}{51} & 0.95 \\
\hline & \multicolumn{2}{|c|}{68.6} & 1.00 \\
\hline & \multicolumn{2}{|c|}{76.2} & 1.02 \\
\hline & \multirow{2}{*}{\multicolumn{2}{|c|}{$\begin{array}{c}\frac{101.6}{127} \\
\end{array}$}} & 1.09 \\
\hline & & & 1.16 \\
\hline \multirow{4}{*}{ Fines coefficient } & \multicolumn{2}{|c|}{ Fine Content (\%) } & $S_{f}$ \\
\hline & \multicolumn{2}{|c|}{$\leq 40$} & 0.86 \\
\hline & \multicolumn{2}{|c|}{50} & 1.00 \\
\hline & \multicolumn{2}{|c|}{70} & 1.04 \\
\hline \multirow{4}{*}{ Air content coefficient } & \multicolumn{2}{|c|}{ Air Content (\%) } & $S_{e}$ \\
\hline & \multicolumn{2}{|c|}{$\leq 4$} & 0.98 \\
\hline & \multirow{2}{*}{\multicolumn{2}{|c|}{$\begin{array}{c}6 \\
10 \\
\end{array}$}} & 1.00 \\
\hline & & & 1.03 \\
\hline \multirow{5}{*}{ Cement content coefficient } & \multicolumn{2}{|c|}{ Cement $\left(\mathrm{kg} / \mathrm{m}^{3}\right)$} & $S_{c}$ \\
\hline & \multicolumn{2}{|c|}{$\leq 223$} & 0.87 \\
\hline & \multicolumn{2}{|c|}{335} & 0.95 \\
\hline & \multirow{2}{*}{\multicolumn{2}{|c|}{$\begin{array}{l}418 \\
557\end{array}$}} & 1.00 \\
\hline & & & 1.09 \\
\hline \multirow{3}{*}{$\begin{array}{l}\text { Minimum thickness of } \\
\text { member coefficient }\end{array}$} & \multicolumn{2}{|c|}{ Thickness (mm) } & $S_{t h}$ \\
\hline & \multicolumn{2}{|c|}{$\leq 150$} & 1.00 \\
\hline & & 30 & 0.84 \\
\hline
\end{tabular}


Table 2.5: Summary of the shrinkage empirical equations proposed by CEB-FIP

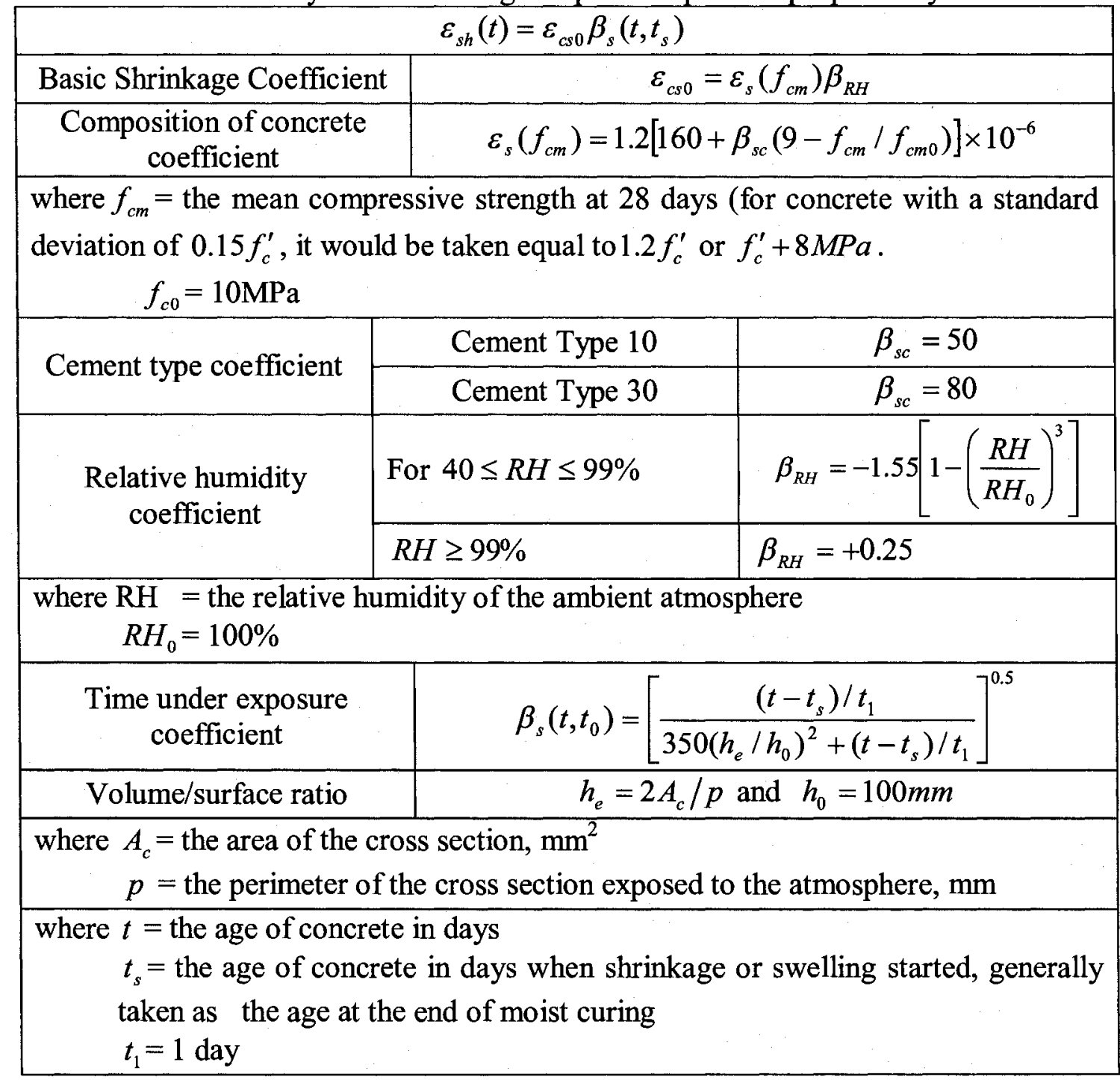


Table 2.6: Summary of the studies performed by previous researchers on compressive, tensile, and flexural strength of RAC

\begin{tabular}{|c|c|c|c|c|c|c|}
\hline \multirow[t]{2}{*}{ Investigator } & \multicolumn{3}{|c|}{$\begin{array}{c}\text { Strength Reduction Using } \\
\text { Coarse RCA } \\
\end{array}$} & \multicolumn{3}{|c|}{$\begin{array}{c}\text { Strength Reduction } \\
\text { Using Coarse / Fine RCA }\end{array}$} \\
\hline & $\mathbf{f}_{\mathbf{c}}$ & $\mathbf{f}_{\mathrm{t}}$ & $\mathbf{f}_{\mathbf{f}}$ & $\mathbf{f}_{\mathbf{c}}$ & $\mathbf{f}_{\mathbf{t}}$ & $\mathbf{f}_{\mathbf{f}}$ \\
\hline Malhotra (1976) & $1-19 \% \downarrow$ & - & $3-19 \% \downarrow$ & $\begin{array}{c}12- \\
16 \% \downarrow\end{array}$ & - & $8-20 \% \downarrow$ \\
\hline Frondistou (1977) & $4-14 \% \downarrow$ & - & - & - & - & - \\
\hline Buck (1976) & $0-8 \mathrm{MPa} \downarrow$ & - & - & $\downarrow$ & - & - \\
\hline De Pauw (1983) & $10-20 \% \downarrow$ & - & - & $\begin{array}{c}25- \\
50 \% \downarrow\end{array}$ & - & - \\
\hline Tomozo (1983) & $14 \% \downarrow$ & - & - & $32 \% \downarrow$ & - & - \\
\hline $\begin{array}{c}\text { Hansen-Narud } \\
(1983)\end{array}$ & $0-39 \% \downarrow$ & - & - & $\downarrow$ & - & - \\
\hline $\begin{array}{c}\text { Nishibayashi et al. } \\
\text { (1985) }\end{array}$ & $15-30 \% \downarrow$ & - & - & - & - & - \\
\hline $\begin{array}{c}\text { Sri Ravindrarajah } \\
\text { (1987) }\end{array}$ & - & - & - & $\begin{array}{c}13- \\
17 \% \downarrow\end{array}$ & $\begin{array}{c}11- \\
20 \% \downarrow\end{array}$ & $10-15 \% \downarrow$ \\
\hline $\begin{array}{c}\text { Ong-Sri } \\
\text { Ravindrarajah } \\
(1987)\end{array}$ & $1-16 \% \downarrow$ & $\begin{array}{c}7-21 \% \downarrow^{a} \\
12- \\
17 \% \uparrow^{b} \\
\end{array}$ & $\begin{array}{l}1-13 \% \downarrow^{a} \\
7-12 \% \uparrow^{b}\end{array}$ & $9-19 \% \downarrow$ & $4-7 \% \downarrow$ & $4-15 \% \downarrow$ \\
\hline Loo et al. (1987) & $15-21 \% \downarrow^{c}$ & $8-12 \% \downarrow$ & $1-10 \% \downarrow^{c}$ & $\begin{array}{c}17- \\
27 \% \downarrow^{c}\end{array}$ & $\begin{array}{c}19- \\
20 \% \downarrow^{c}\end{array}$ & $15-25 \% \downarrow^{c}$ \\
\hline \multirow{2}{*}{$\begin{array}{c}\text { Ray-Venkateswarlu } \\
\text { (1991) }\end{array}$} & \multirow[t]{2}{*}{$0-31 \% \downarrow^{d}$} & \multirow[t]{2}{*}{$5-20 \% \downarrow$} & $\begin{array}{l}\text { Bearing } \\
\text { Strength }\end{array}$ & \multirow[t]{2}{*}{ - } & \multirow[t]{2}{*}{. } & \multirow[t]{2}{*}{ - } \\
\hline & & & $23-45 \% \downarrow^{e}$ & & & \\
\hline De Vries (1993) & $10-20 \% \downarrow$ & $=$ & - & - & - & - \\
\hline $\begin{array}{l}\text { Merlet-Pimienta } \\
(1994)\end{array}$ & - & - & - & $\begin{array}{c}15- \\
25 \% \downarrow\end{array}$ & - & - \\
\hline Yanagi et al.(1994) & - & - & - & $\begin{array}{c}10- \\
20 \% \downarrow\end{array}$ & - & - \\
\hline $\begin{array}{c}\text { Wainwright et al. } \\
\text { (1994) }\end{array}$ & $11-20 \% \downarrow$ & - & - & $\begin{array}{c}21- \\
38 \% \downarrow\end{array}$ & - & - \\
\hline $\begin{array}{c}\text { Topcu-Gunkan } \\
(1995)\end{array}$ & $\downarrow$ & - & - & - & - & - \\
\hline Kiuchi (2001) & $2-3 \% \downarrow$ & - & - & - & - & - \\
\hline Tamura et al. (2001) & $10 \% \downarrow$ & - & - & - & - & - \\
\hline $\begin{array}{c}\text { Corinaldesi et al. } \\
(2001) \\
\end{array}$ & - & - & - & $8 \% \downarrow$ & $8 \% \downarrow$ & - \\
\hline Mandal et al. (2002) & $15 \% \downarrow$ & $6 \% \downarrow$ & - & - & - & - \\
\hline $\begin{array}{c}\text { Buyle-Zaharieva } \\
(2002)\end{array}$ & $3-6 \% \downarrow$ & & & $9-18 \% \downarrow$ & & 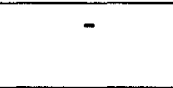 \\
\hline $\begin{array}{c}\text { Lamond et al. } \\
(2002)\end{array}$ & $5-24 \% \downarrow$ & $\begin{array}{c}\text { At most } \\
10 \% \downarrow\end{array}$ & - & $\begin{array}{c}15- \\
40 \% \downarrow\end{array}$ & $\begin{array}{c}10- \\
20 \% \downarrow\end{array}$ & - \\
\hline $\begin{array}{c}\text { Gomez-Soberon } \\
(2002 \mathrm{a}, \mathrm{b}, \mathrm{c})\end{array}$ & $11 \% \downarrow$ & $11 \% \downarrow$ & - & - & - & - \\
\hline $\begin{array}{l}\text { Ajdukiewicz- } \\
\text { Kliszew.(2002) }\end{array}$ & $5-8 \% \downarrow$ & - & - & $7-20 \% \downarrow$ & - & - \\
\hline
\end{tabular}


Table 2.6 continued: Summary of the studies performed by previous researchers on compressive, tensile, and flexural strength of RAC

\begin{tabular}{|c|c|c|c|c|c|c|}
\hline \multirow[t]{2}{*}{ Investigator } & \multicolumn{3}{|c|}{$\begin{array}{l}\text { Strength Reduction } \\
\text { Using } \\
\text { Coarse RCA }\end{array}$} & \multicolumn{3}{|c|}{$\begin{array}{c}\text { Strength Reduction } \\
\text { Using } \\
\text { Coarse / Fine RCA }\end{array}$} \\
\hline & $\mathbf{f}_{\mathbf{c}}$ & $\mathbf{f}_{\mathbf{t}}$ & $\mathbf{f}_{\mathrm{f}}$ & $\mathbf{f}_{\mathbf{c}}$ & $\mathbf{f}_{\mathbf{t}}$ & $\mathbf{f}_{\mathbf{f}}$ \\
\hline Poon et al. (2004a) & $\begin{array}{c}20 \% \downarrow \text { and } \\
7-9 \% \downarrow^{n}\end{array}$ & - & - & - & - & - \\
\hline Poon et al. (2004b) & - & - & - & - & - & - \\
\hline Santos et al. (2004) & $0-15 \% \downarrow$ & $\downarrow$ & - & - & - & - \\
\hline Xiao et al. (2004) & $11 \% \downarrow$ & - & - & - & - & - \\
\hline Katz (2004) & - & - & - & - & - & - \\
\hline Mukherjee et al. (2003) & $27 \% \downarrow$ & - & - & - & - & - \\
\hline $\begin{array}{c}\text { De Oliveira- Vazquez } \\
\text { (1996) }\end{array}$ & $10 \% \downarrow$ & - & $10 \% \downarrow$ & - & - & - \\
\hline $\begin{array}{c}\text { Salem-Burdette } \\
(1998)\end{array}$ & $2 \% \uparrow-9 \% \downarrow^{f}$ & $9-14 \% \downarrow^{g}$ & - & - & - & - \\
\hline $\begin{array}{l}\text { Ramamurthy- } \\
\text { Gumaste(1998) } \\
\end{array}$ & $15-42 \% \downarrow$ & - & - & - & - & - \\
\hline $\begin{array}{c}\text { Yamasaki-Tatematsu } \\
(1998 \\
\end{array}$ & $\downarrow^{h}$ & - & - & $\downarrow^{1}$ & - & - \\
\hline Dhir et al. (1999) & $6-18 \% \downarrow$ & - & $\downarrow$ & $20-30 \downarrow$ & - & $\downarrow$ \\
\hline $\begin{array}{c}\text { Sakata-Ayano } \\
(2000)\end{array}$ & - & - & - & $20 \% \downarrow$ & - & - \\
\hline $\begin{array}{c}\text { Nagataki et al. } \\
(2000)\end{array}$ & $\begin{array}{c}14 \% \uparrow, 12 \% \\
\uparrow, 9 \% \downarrow^{j}\end{array}$ & - & - & $\begin{array}{c}5 \% \uparrow, 9 \% \uparrow \\
.4 \% \downarrow^{j} \\
\end{array}$ & - & - \\
\hline Limbachiya et al. (2000) & - & - & $\begin{array}{l}4 \% \downarrow- \\
3 \% \uparrow^{k}\end{array}$ & - & - & - \\
\hline $\begin{array}{l}\text { Sagoe-Crentsil, et } \\
\text { al.(2001) }\end{array}$ & $\approx 0 \%$ & - & - & - & - & - \\
\hline Ryu (2002a and 2002b) & $2-27 \% \downarrow$ & - & - & - & - & - \\
\hline Katz (2003) & -- & - & - & $25-40 \% \downarrow$ & to $46 \% \downarrow$ & to $30 \% \downarrow$ \\
\hline Chen et al. (2003) & $25-40 \% \downarrow 1$ & - & - & - & - & - \\
\hline Kiuchi-Horiuchi (2003) & $8-22 \% \downarrow$ & - & - & - & - & - \\
\hline Shayan-Xu (2003) & $28 \% \downarrow$ & - & - & - & - & - \\
\hline Topcu-Sengel (2004) & $23.5-33 \% \downarrow$ & - & - & - & - & - \\
\hline & & & & & & \\
\hline
\end{tabular}

${ }^{a}$ For high-strength RAC

${ }^{c}$ bFor low-strength RAC

${ }^{c}$ Different for water or air curing conditions

${ }^{d}$ Higher w/c ratio, lower reduction

${ }^{e}$ Higher w/c, lower difference, Always bearing strength $>$ compressive strength

$\sigma_{b r}=\lambda \sigma_{c} \sqrt{R}$ where $\lambda=0.45,0.3$, and 0.40 for conventional concrete, RAC with $100 \%$ coarse RCA, and concrete made with combined coarse aggregate containing of $60 \%$ of recycled aggregate and $40 \%$ of natural aggregate, $\mathrm{R}$ is the ratio of bearing area to the 
punching area during bearing strength test and loading trough steel plates, and $\sigma_{c}$ is the compressive strength of concrete.

$f_{2 \%} \uparrow$ and $9 \% \downarrow$ by using $14 \%$ and $28 \%$ fly ash by weight of cement, respectively

${ }^{9} 9-14 \% \downarrow$ by using $14 \%$ and $28 \%$ fly ash by weight of cement, respectively

${ }^{h} \mathrm{~F}_{c}=19.6 \mathrm{C} / \mathrm{W}-1.36 \mathrm{MPa}$

${ }^{i} \mathrm{~F}_{c}=18.8 \mathrm{C} / \mathrm{W}+3.73 \mathrm{MPa}$

${ }^{j}$ Change in compressive strength for $\mathrm{RCA}$ with $\mathrm{w} / \mathrm{c}=0.55$ made of air-entrained concrete with low, medium, and high w/c ratio, respectively

$k_{4 \% \downarrow}$ for $\mathrm{C} 50$ and $3 \% \uparrow$ for C70 (Using super plasticizers)

${ }^{l} \mathrm{RCA}$ containing brick and tile

${ }^{m}$ Tensilestrength $=\frac{1}{10}$ to $\frac{1}{14}$ Compressivestrength (The same as normal concrete)

${ }^{n} 20 \% \downarrow$ for normal-strength concrete and $6.8-9 \% \downarrow$ for high-strength concrete

Table 2.7: Summary of findings by previous researchers about elastic modulus of RAC

\begin{tabular}{|c|c|c|c|c|}
\hline \multirow[t]{2}{*}{ Investigator } & \multicolumn{2}{|c|}{$\begin{array}{c}\text { Elastic Modulus } \\
\text { Reduction Using Coarse } \\
\text { RCA\% }\end{array}$} & \multicolumn{2}{|c|}{$\begin{array}{c}\text { Elastic Modulus } \\
\text { Reduction Using } \\
\text { Fine/Coarse RCA\% }\end{array}$} \\
\hline & Static & Dynamic & Static & Dynamic \\
\hline Frondistou (1977) & $40 \% \downarrow$ & - & - & - \\
\hline Nishibayashi et al. (1985) & $15 \% \downarrow$ & - & - & - \\
\hline Hansen-Boegh (1985) & $14-28 \% \downarrow$ & $14-28 \% \downarrow$ & - & - \\
\hline Sri Ravindrarajah (1987) & - & - & $28-32 \% \downarrow$ & - \\
\hline Ong-Ravindrarajah (1987) & $6-40 \% \downarrow$ & up to $30 \% \downarrow$ & $16-45 \downarrow$ & up to $30 \% \downarrow$ \\
\hline Loo et al. (1987) & $25-35 \% \downarrow$ & $13-18 \% \downarrow$ & - & - \\
\hline Merlet-Pimienta (1994) & - & - & $23-28 \% \downarrow$ & - \\
\hline Topcu-Gunkan (1995) & $20 \% \downarrow$ & - & - & - \\
\hline De Oliveira-Vazquez (1996) & $25 \%$ & - & - & - \\
\hline Salem-Burdette (1998) & $16 \%$ & - & - & - \\
\hline Nishiura et al. (2000) & $\begin{array}{c}2 \% \downarrow, 4 \% \downarrow \\
\text { and } 2 \% \uparrow\end{array}$ & - & - & - \\
\hline Kiuchi (2001) & $15-20 \% \downarrow$ & - & - & - \\
\hline Corinaldesi et al. (2001) & - & - & - & $21 \% \downarrow$ \\
\hline Lamond et al. (2002) & $10-33 \% \downarrow$ & - & $25-40 \% \downarrow$ & - \\
\hline Gomez-Soberon $(2002 a, b, c)$ & $10 \% \downarrow$ & - & - & - \\
\hline Ajdukiewicz -Kliszewicz (2002) & $3-11 \% \downarrow$ & - & $20-31 \% \downarrow$ & - \\
\hline Gomez-Soberon (2003) & $10 \% \downarrow$ & - & - & - \\
\hline Katz (2003) & - & - & $37-50 \% \downarrow$ & - \\
\hline Chen et al.(2003) & $30 \% \downarrow^{a}$ & - & - & - \\
\hline Santos et al.(2004) & $14-24 \% \downarrow$ & - & - & - \\
\hline Xiao et al. (2004) & $45 \% \downarrow$ & - & - & - \\
\hline Summary & $2-45 \% \downarrow$ & $13-30 \% \downarrow$ & $20-50 \% \downarrow$ & $\begin{array}{l}\text { Up to } \\
50 \% \downarrow\end{array}$ \\
\hline
\end{tabular}

${ }^{a}$ RCA containing brick and tiles 
Table 2.8: Summary of the findings of previous researchers about shrinkage and creep of RAC

\begin{tabular}{|c|c|c|c|c|}
\hline \multirow[t]{2}{*}{ Investigator } & \multicolumn{2}{|c|}{$\begin{array}{c}\text { Reduction Using Coarse } \\
\text { RCA, \% }\end{array}$} & \multicolumn{2}{|c|}{$\begin{array}{c}\text { Reduction Using } \\
\text { Fine/Coarse RCA,\% }\end{array}$} \\
\hline & Shrinkage & Creep & Shrinkage & Creep \\
\hline Tomozo (1983) & $30 \% \uparrow$ & - & - & - \\
\hline Nishibayashi,et al. (1985) & $37-110 \% \uparrow$ & $\begin{array}{c}28- \\
43 \% \uparrow\end{array}$ & - & - \\
\hline Hansen-Boegh (1985) & $50 \% \uparrow$ & - & - & - \\
\hline Sri Ravindrarajah (1987) & - & - & $63-98 \% \uparrow$ & - \\
\hline Loo et al. (1987) & $59-111 \% \uparrow^{a}$ & - & $49-82 \% \uparrow^{a}$ & - \\
\hline Merlet-Pimienta (1994) & - & - & $20-46 \% \uparrow$ & - \\
\hline Yanagi et al. (1994) & - & - & $17 \% \uparrow$ & - \\
\hline Mesbah-Buyle (1999) & - & - & - & - \\
\hline Limbachiya et al. (2000) & $6-9 \% \uparrow$ & $\begin{array}{c}33- \\
65 \% \uparrow\end{array}$ & - & - \\
\hline Han et al. (2001) & $20 \% \uparrow$ & $20 \% \uparrow$ & - & - \\
\hline Buyle-Zaharieva (2002) & $80 \% \uparrow$ & - & $200 \% \uparrow$ & - \\
\hline Lamond et al. (2002) & $20-50 \% \uparrow$ & $\begin{array}{c}30- \\
60 \% \uparrow \\
\end{array}$ & $70-100 \uparrow$ & - \\
\hline Gomez-Soberon (2002a) & $\begin{array}{c}20-70 \% \uparrow \text { and } \\
263 \% \uparrow \\
\end{array}$ & - & - & - \\
\hline Gomez-Soberon (2002b) & - & $\begin{array}{c}30- \\
47 \% \uparrow\end{array}$ & - & - \\
\hline Gomez-Soberon (2003) & $\begin{array}{c}20-70 \% \uparrow \text { and } \\
263 \% \uparrow b\end{array}$ & $\begin{array}{c}30- \\
47 \% \uparrow\end{array}$ & - & - \\
\hline Summary & $6-111 \% \uparrow$ & $\begin{array}{c}20- \\
65 \% \uparrow \\
\end{array}$ & $17-200 \% \uparrow$ & - \\
\hline
\end{tabular}

${ }^{a}$ Different for water or air curing conditions after 90 days ${ }^{b} 20-70 \% \uparrow$ in shrinkage for up to $60 \%$ RCA replacement and $263 \% \uparrow$ for $100 \%$ RCA replacement 
Table 2.9: The improvements achieved by previous researchers on compressive, tensile, and flexural strength of RAC

\begin{tabular}{|c|c|c|c|c|c|}
\hline \multirow[t]{2}{*}{ Investigator } & \multicolumn{3}{|c|}{$\begin{array}{c}\text { Strength } \\
\text { Enhancement } \\
\text { Using Coarse RCA }\end{array}$} & \multicolumn{2}{|c|}{$\begin{array}{c}\text { Strength } \\
\text { Enhancement } \\
\text { Using } \\
\text { Coarse/Fine RCA }\end{array}$} \\
\hline & $\mathbf{f}_{\mathbf{c}}$ & $\mathbf{f}_{t}$ & $\mathbf{f}_{\mathbf{f}}$ & $\mathbf{f}_{\mathbf{c}}$ & $\mathbf{f}_{\mathbf{t}}$ \\
\hline Buck (1976) & $50 \% \uparrow$ & - & - & - & - \\
\hline Merlet-Pimienta(1994) & - & - & - & $\begin{array}{c}22- \\
27 \% \uparrow \\
\end{array}$ & $\begin{array}{c}15- \\
21 \% \uparrow\end{array}$ \\
\hline Wainwright et al. (1994) & - & - & - & $\begin{array}{c}44- \\
81 \% \downarrow^{a} \\
21- \\
24 \% \uparrow^{b}\end{array}$ & - \\
\hline Dhir et al. (1999) & $\begin{array}{c}6- \\
18 \% \uparrow \\
\end{array}$ & - & - & - & - \\
\hline Sagoe-Crentsil, et al.(2001) & $22 \% \uparrow$ & - & - & - & - \\
\hline Tamura et al. (2001) & $20 \% \uparrow$ & - & - & - & - \\
\hline Corinaldesi et al. (2001) & - & - & $\begin{array}{c}10 \% \\
\uparrow\end{array}$ & $2 \% \downarrow$ & - \\
\hline Mandal et al. (2002) & $18 \% \uparrow$ & $18 \% \uparrow$ & - & - & - \\
\hline Ajdukiewicz - Kliszew.(2002) & $\begin{array}{c}42- \\
70 \% \uparrow\end{array}$ & & - & $\begin{array}{c}46- \\
70 \% \uparrow\end{array}$ & - \\
\hline Ryu (2002a and 2002b) & $8-17 \uparrow$ & $\begin{array}{c}17- \\
26 \% \uparrow\end{array}$ & - & - & - \\
\hline Shayan-Xu(2003) & $\begin{array}{c}3- \\
22 \% \uparrow\end{array}$ & - & - & - & - \\
\hline Poon et al.(2004b) & $13 \% \uparrow$ & $\begin{array}{c}13- \\
22 \% \uparrow\end{array}$ & - & - & - \\
\hline Katz(2004) & $\begin{array}{c}7- \\
16 \% \uparrow \\
\end{array}$ & - & - & - & - \\
\hline Summary & $\begin{array}{c}3- \\
70 \% \uparrow \\
\end{array}$ & $\begin{array}{c}13- \\
26 \% \uparrow\end{array}$ & $\begin{array}{c}10 \% \\
\uparrow \\
\end{array}$ & $\begin{array}{c}44- \\
81 \% \uparrow\end{array}$ & $\begin{array}{c}15- \\
21 \% \uparrow \\
\end{array}$ \\
\hline
\end{tabular}

${ }^{a}$ Using Pulverized Fuel Ash substituting fine RCA

${ }^{b}$ Using Super plasticizers 
Table 2.10: The improvements achieved by previous researchers with respect to elastic modulus of RAC

\begin{tabular}{|c|c|c|c|c|}
\hline \multirow{2}{*}{ Investigator } & \multicolumn{2}{|c|}{$\begin{array}{c}\text { Elastic Modulus } \\
\text { Enhancement } \\
\text { Using Coarse RCA }\end{array}$} & \multicolumn{2}{c|}{$\begin{array}{c}\text { Elastic Modulus } \\
\text { Enhancement Using } \\
\text { Coarse/Fine RCA }\end{array}$} \\
\cline { 2 - 5 } & Static & Dynamic & Static & Dynamic \\
\hline $\begin{array}{c}\text { Merlet-Pimienta } \\
(1994)\end{array}$ & - & - & $9-17 \% \uparrow$ & - \\
\hline $\begin{array}{c}\text { Corinaldesi et al. } \\
(2001)\end{array}$ & - & - & $13 \% \downarrow$ & - \\
\hline $\begin{array}{c}\text { Ajdukiewicz- } \\
\text { Kliszczewicz (2002) }\end{array}$ & $8-25 \% \uparrow$ & - & $11-25 \% \uparrow$ & - \\
\hline Summary & $8-25 \% \uparrow$ & - & $9-25 \% \uparrow$ & - \\
\hline
\end{tabular}

Table 2.11: The improvements achieved by previous researchers with respect to shrinkage and creep of RAC

\begin{tabular}{|c|c|c|c|c|}
\hline \multirow{2}{*}{ Investigator } & \multicolumn{2}{|c|}{$\begin{array}{c}\text { Enhancement } \\
\text { Using Coarse RCA } \\
\%\end{array}$} & \multicolumn{2}{c|}{$\begin{array}{c}\text { Enhancement } \\
\text { Using Fine/Coarse RCA \% }\end{array}$} \\
\cline { 2 - 5 } & Shrinkage & Creep & Shrinkage & Creep \\
\hline Merlet-Pimienta(1994) & - & - & $\downarrow$ almost as NAC & \\
\hline Mesbah-Buyle(1999) & - & - & $6-15 \% \downarrow^{a}$ & - \\
\hline Han et al.(2001) & - & $20 \% \downarrow$ & - & - \\
\hline Summary & - & $20 \% \downarrow$ & $6-15 \% \downarrow$ & - \\
\hline
\end{tabular}

${ }^{a} 6 \%$ and $15 \%$ reduction in free shrinkage by using Polypropylene and Fibraflex fibres, respectively

Table 2.12: Summary of the findings of previous researchers about structural behaviour

\begin{tabular}{|c|c|c|c|c|c|c|c|}
\hline \multirow[t]{2}{*}{ Investigator } & \multicolumn{2}{|c|}{ RCA\% } & \multirow{2}{*}{$\begin{array}{c}\mathbf{b} \\
\mathrm{mm}\end{array}$} & \multirow{2}{*}{$\begin{array}{c}\mathbf{h} \\
\mathrm{mm}\end{array}$} & \multirow[t]{2}{*}{$\mathbf{a} / \mathbf{d}$} & \multirow{2}{*}{$\begin{array}{c}\mathbf{W} / \mathbf{C} \\
\%\end{array}$} & \multirow{2}{*}{$\begin{array}{l}\text { No. of } \\
\text { Beams }\end{array}$} \\
\hline & $\mathrm{C}$ & $\mathrm{F}$ & & & & & \\
\hline \multirow{2}{*}{$\begin{array}{c}\text { Yagishita et al. } \\
\text { (1994) }\end{array}$} & 100 & 0 & 120 & 180 & $2.29^{\text {Shear }}$ & 61 & $3 \mathrm{RAC}+1 \mathrm{NAC}$ \\
\hline & 100 & 0 & 120 & 180 & $3.17^{\text {flexure }}$ & 61 & $3 \mathrm{RAC}+1 \mathrm{NAC}$ \\
\hline \multirow{2}{*}{ Han et al. (2001) } & 100 & 0 & 170 & 300 & $1.5,2,3,4$ & 40 & $5 \mathrm{RAC}+1 \mathrm{NAC}$ \\
\hline & 100 & 0 & 170 & 300 & 2 & 40 & $5 \mathrm{RAC}+1 \mathrm{NAC}$ \\
\hline Santos et al. (2004) & 100 & 0 & 150 & 200 & 3.94 & 55 & $2 \mathrm{RAC}+1 \mathrm{NAC}$ \\
\hline \multirow{5}{*}{$\begin{array}{l}\text { Maruyama et al. } \\
\text { (2004a and 2004b) }\end{array}$} & 100 & 100 & 150 & 200 & 3.1 & $30,45,60$ & 4 \\
\hline & 100 & 0 & 150 & 200 & 3.1 & $30,45,60$ & 4 \\
\hline & 0 & 0 & 150 & 200 & 3.1 & $30,45,60$ & 4 \\
\hline & 100 & 100 & 150 & 400 & 3.1 & 55 & $2 \mathrm{RAC}+1 \mathrm{NAC}$ \\
\hline & 100 & 100 & 150 & 200 & 3.1 & 55 & $2 \mathrm{RAc}+1 \mathrm{NAC}$ \\
\hline $\begin{array}{c}\text { Gonzalez-Martinez } \\
\text { (2004) }\end{array}$ & 50 & 0 & 200 & 350 & 3.7 & 55 & $8 \mathrm{RAC}+1 \mathrm{NAC}$ \\
\hline
\end{tabular}


Table 2.13: Specified limitations on RCA properties by different countries

\begin{tabular}{|c|c|c|c|c|c|c|c|c|c|c|c|c|c|}
\hline \multirow{2}{*}{ Country } & \multicolumn{2}{|c|}{$\begin{array}{c}\text { Compressive } \\
\text { Strength } \\
(\mathrm{MPa})\end{array}$} & \multirow{2}{*}{ 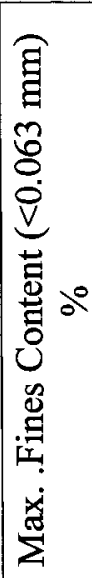 } & \multicolumn{2}{|c|}{ 莺莺 } & \multicolumn{2}{|c|}{ 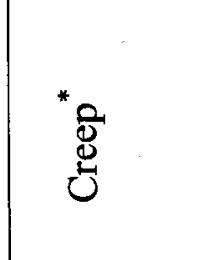 } & \multicolumn{2}{|c|}{ 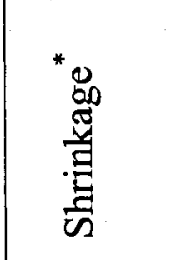 } & \multicolumn{4}{|c|}{$\begin{array}{l}\text { Maximum } \\
\text { Impurities } \\
\text { Content (\%) }\end{array}$} \\
\hline & 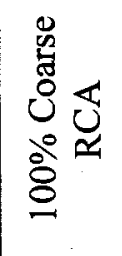 & 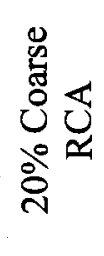 & & 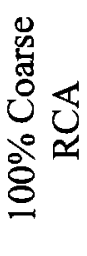 & 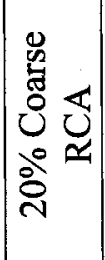 & 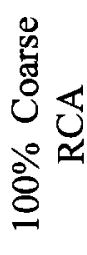 & 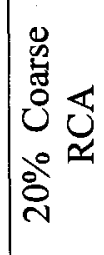 & 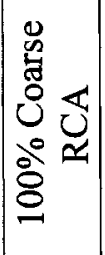 & 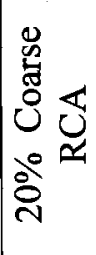 & 息 & 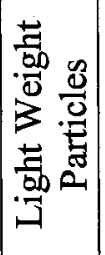 & 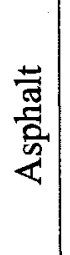 & 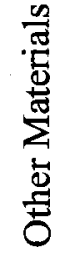 \\
\hline RILEM & 50 & $\begin{array}{l}\text { No } \\
\text { Limit }\end{array}$ & 2 & 0.8 & - & 1.25 & 1 & 1.5 & 1 & \multirow{3}{*}{20} & \multirow{3}{*}{\multicolumn{3}{|c|}{1}} \\
\hline Belgium & 30 & - & 3 & 0.8 & 1 & 1.25 & 1 & 1.5 & 1 & & & & \\
\hline $\begin{array}{l}\text { Hong } \\
\text { Kong }\end{array}$ & 20 & $25-30$ & 4 & - & - & - & - & - & - & & & & \\
\hline Holland & $45^{(4)}$ & $\begin{array}{l}\text { No } \\
\text { Limit }\end{array}$ & - & - & - & $\begin{array}{l}1.25 \\
-\overline{1.45}\end{array}$ & 1 & $\begin{array}{c}1.35 \\
- \\
1.55\end{array}$ & 1 & & & & - \\
\hline Germany & - & - & 4 & - & - & - & - & - & - & - & - & - & - \\
\hline $\mathrm{Japan}^{2}$ & 24 & - & - & - & - & - & - & - & - & - & - & - & - \\
\hline UK & 40 & $\begin{array}{c}\text { No } \\
\text { Limit }\end{array}$ & $\begin{array}{c}5(3 \\
)\end{array}$ & - & - & - & - & - & - & 5 & 0.5 & 5 & 1 \\
\hline Australia $^{1}$ & 40 & - & 6 & - & - & - & - & - & - & & Total $A$ & Amo & unt) \\
\hline
\end{tabular}

* The modification factors given in the table should be applied to the conventional equations for normal concrete

${ }^{1}$ Non Structural application

${ }^{2}$ Civil works

${ }^{3}$ BS-EN-206-1

${ }^{4}$ Cubic strength 
Table 2.14: Summary of simplified and general shear design methods of CSA A23.3-04

\begin{tabular}{|c|c|c|c|c|c|}
\hline $\begin{array}{c}\text { Code/ } \\
\text { Researcher }\end{array}$ & \multicolumn{5}{|c|}{ Equations } \\
\hline \multirow{9}{*}{ 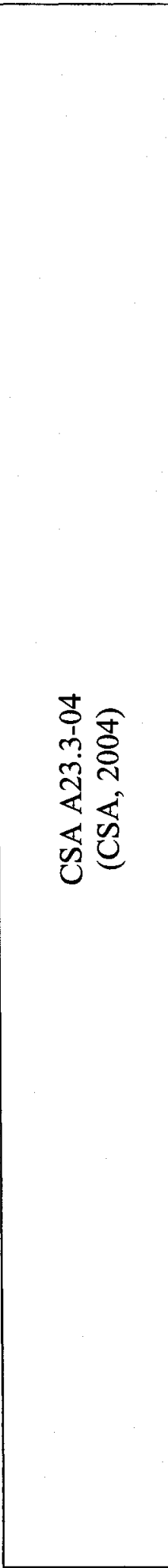 } & \multicolumn{4}{|c|}{$\begin{array}{c}V_{s}=\frac{A_{v} f_{y} d_{v}(\cot \theta+\cot \alpha) \sin \alpha}{s} \\
V_{r}+V_{s} \leq 0.25 f_{c}^{\prime} b_{w} d_{v} \\
\lambda=\text { as defined in Equation }(2-3) \\
d_{v}=\operatorname{Max}(0.92 d, 0.72 h)\end{array}$} & \\
\hline & \multirow{4}{*}{ 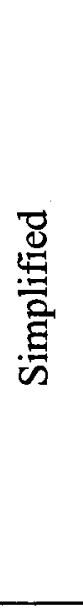 } & \multirow[b]{3}{*}{$\beta$} & if $A_{v} \pi 0.06 \sqrt{f_{c}^{\prime}} \frac{b_{w} s}{f_{y}}$ & \multicolumn{2}{|r|}{0.18} \\
\hline & & & \multirow[b]{2}{*}{ if $A_{v} \geq 0.06 \sqrt{f_{c}^{\prime}} \frac{b_{w} s}{f_{y}}$} & $a_{g} \geq 20 \mathrm{~mm}$ & $\beta=\frac{230}{1000+d_{v}}$ \\
\hline & & & & $a_{g} \pi 20 \mathrm{~mm}$ & $\begin{array}{c}\beta=\frac{230}{1000+s_{z e}} \\
s_{z e}=\frac{35 s_{z}}{15+a_{g}} \geq 0.85 s_{z} \\
s_{z}=\text { see Figure } 2.2\end{array}$ \\
\hline & & $\theta$ & \multicolumn{3}{|r|}{$z$} \\
\hline & \multirow{4}{*}{ 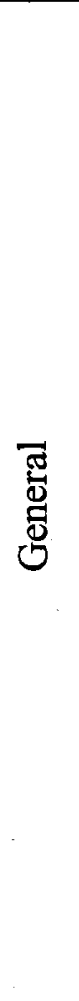 } & \multirow{3}{*}{$\beta$} & \multicolumn{3}{|c|}{$\begin{array}{l}\beta=\frac{0.40}{\left(1+1500 \varepsilon_{x}\right)} \cdot \frac{1300}{1000+s_{z e}} \\
\varepsilon_{x}=\frac{M_{f} / d_{v}+V_{f}+0.5 N_{f}}{2 E_{s} A_{s}}\end{array}$} \\
\hline & & & $\begin{array}{c}\text { If } \\
A_{v} \pi 0.06 \sqrt{f_{c}^{\prime}} \frac{b_{w} s}{f_{y}}\end{array}$ & \multicolumn{2}{|c|}{$\begin{array}{c}s_{z e}=\frac{35 s_{z}}{15+a_{g e}} \geq 0.85 s_{z} \\
s_{z}=\text { see Figure } 2.2 \\
\text { if } f_{c}^{\prime} \leq 60 M P a: a_{g e}=a_{g} \\
\text { if } \\
60 \pi f_{c}^{\prime} \leq 70 M P a: a_{g e}=a_{g}\left(1-\frac{f_{c}^{\prime}-60}{10}\right) \\
\text { if } f_{c}^{\prime} \phi 70 M P a: a_{g e}=0\end{array}$} \\
\hline & & & $\begin{array}{c}\text { If } \\
A_{v} \geq 0.06 \sqrt{f_{c}^{\prime}} \frac{b_{w} s}{f_{y}} \\
\end{array}$ & \multicolumn{2}{|r|}{$s_{z e}=300 \mathrm{~mm}$} \\
\hline & & $\theta$ & \multicolumn{3}{|c|}{$29+7000 \varepsilon_{x}$} \\
\hline
\end{tabular}


Table 2.15: Summary of existing expressions for the concrete contribution to shear resistance

\begin{tabular}{|c|c|}
\hline $\begin{array}{c}\text { Code/ } \\
\text { Researcher }\end{array}$ & Equations \\
\hline $\begin{array}{l}\text { Spanish } \\
\text { EHE-99 } \\
(1999)\end{array}$ & $\begin{array}{l}V_{c}=\left[0.15 \xi\left(100 \rho_{s} f_{c k}\right)^{1 / 3}-0.15 \sigma_{c d}^{\prime}\right] b_{o} d \\
f_{c}^{\prime} \leq 60 M P a \\
\xi=1+\sqrt{\frac{200}{d}} \quad \rho_{s}=\frac{A_{s l}}{b_{0} d} \leq 0.02 \quad \sigma_{c d}^{\prime}=\frac{N_{d}}{A_{c}}\end{array}$ \\
\hline $\begin{array}{l}\text { Eurocode-2 } \\
\quad(2002)\end{array}$ & $\begin{array}{l}V_{c}=\left[\frac{0.18}{\gamma_{c}} k\left(100 \rho_{l} f_{c k}\right)^{1 / 3}+0.15 \sigma_{c p}^{\prime}\right] b_{w} d \geq\left(0.035 k^{3 / 2} f_{c k}{ }^{1 / 2}\right) b_{w} d \\
f_{c}^{\prime} \leq 100 M P a \\
k=1+\sqrt{\frac{200}{d}} \leq 2.0 \quad \rho_{l}=\frac{A_{s}}{b_{w} d} \leq 0.02 \quad \sigma_{c p}^{\prime}=\frac{N_{E d}}{A_{c}} \leq 0.2 f_{c d} N_{E d}\end{array}$ \\
\hline ACI-318- & \begin{tabular}{|l|l|} 
Simplified & $V_{c}=\frac{\sqrt{f_{c}^{\prime}}}{6} b_{w} d$ \\
\end{tabular} \\
\hline 05 & \begin{tabular}{l|l} 
General & $V_{c}=\left(\sqrt{f_{c}^{\prime}}+120 \rho_{w} \frac{d}{a}\right) \frac{b_{w} d}{7} \leq 0.3 \sqrt{f_{c}^{\prime}} b_{w} d \quad f_{c}^{\prime} \leq 70 M P a$ \\
\end{tabular} \\
\hline $\begin{array}{l}\text { Niwa et al. } \\
\text { (1986) }\end{array}$ & $\begin{array}{l}V_{c}=0.2\left(1+\frac{M_{0}}{M_{d}}\right) f_{c}^{1 / 3}\left(100 \rho_{w}\right)^{1 / 3}(1 / d)^{1 / 4}(0.75+1.4(a / d)) \\
M_{d}: \text { design moment } \\
M_{o}: \text { Moment to cancel the stress produced by axial force in tension fiber }\end{array}$ \\
\hline $\begin{array}{l}\text { Zsutty } \\
(1968)\end{array}$ & For $\mathrm{a} / \mathrm{d}>2.5: V_{c}=2.21\left(f_{c}^{\prime} \rho_{w} d / a\right)^{1 / 3} b_{w} d$ \\
\hline $\begin{array}{l}\text { Zsutty } \\
(1971)\end{array}$ & For $\mathrm{a} / \mathrm{d} \leq 2.5: V_{c}=\left[2.21\left(f_{c}^{\prime} \rho_{w} d / a\right)^{1 / 3}\right] \cdot\left(\frac{2.5 d}{a}\right) b_{w} d$ \\
\hline $\begin{array}{l}\text { Gastebled } \\
\text { and May } \\
(2001)\end{array}$ & $V_{c}=0.15 \frac{37.41}{\sqrt{d}}\left(\frac{3 d}{a_{s}}\right)^{1 / 3}\left(100 \rho_{s}\right)^{1 / 6}\left(1-\sqrt{\rho_{s}}\right)^{2 / 3} f_{c}^{0.35} b_{w} d$ \\
\hline $\begin{array}{l}\text { CEB-FIP- } \\
1990\end{array}$ & $V_{c}=0.15\left(\left(1+\sqrt{\frac{200}{d}}\right) \cdot\left(\frac{3 d}{a}\right)^{1 / 3} \cdot\left(100 \rho_{s} \cdot f_{c}^{\prime}\right)^{1 / 3}-0.15 \sigma_{c d}^{\prime}\right)$ \\
\hline \multirow{3}{*}{$\begin{array}{l}\text { Bažant and } \\
\mathrm{Yu}(2005)\end{array}$} & \begin{tabular}{l|l} 
Simplified & $V_{c}=\left(3.5 \sqrt{f_{c}^{\prime} / d}\right) b_{w} d \leq 3.5 \sqrt{f_{c}^{\prime}} b_{w} d$
\end{tabular} \\
\hline & $V_{c}=\frac{5 \sqrt{f_{c}^{\prime}}}{\sqrt{1+d / d_{0}}} \quad d_{0}=71120\left(\frac{\rho}{f_{c}^{\prime}}\right)^{2 / 3}$ \\
\hline & $V_{c}=\frac{5.5 \sqrt{f_{c}^{\prime}}}{\sqrt{1+d / d_{0}}} d_{0}=1350\left(\frac{\rho}{f_{c}^{\prime}}\right)^{2 / 3}\left(\frac{d}{a}\right)^{1 / 3}$ \\
\hline
\end{tabular}


Table 2.16: Summary of existing expressions for the stress-strain relationship of normal concrete

\begin{tabular}{|c|c|c|}
\hline Research & & Equations \\
\hline \multirow[t]{2}{*}{$\begin{array}{l}\text { Thorenfeldt } \\
\text { et al. } \\
\text { (1987) }\end{array}$} & 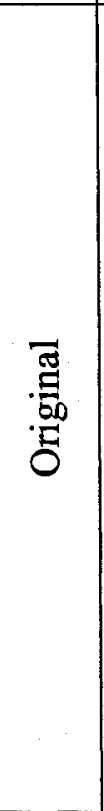 & $\begin{array}{l}\frac{f_{c}}{f_{c}^{\prime}}=\frac{n\left(\varepsilon_{c} / \varepsilon_{c}^{\prime}\right)}{n-1+\left(\varepsilon_{c} / \varepsilon_{c}^{\prime}\right)^{n k}} \\
f_{c}^{\prime}=\text { Peak stress obtained from a cylinder test, MPa } \\
\varepsilon_{c}^{\prime}=\text { strain when } \mathrm{f}_{\mathrm{c}} \text { reaches } \mathrm{f}_{\mathrm{c}} \\
\varepsilon_{c}=\text { given strain at any point } \\
n=E_{c} /\left(E_{c}-E_{c}^{\prime}\right) \\
E_{c}=\text { Initial tangent modulus, } \mathrm{MPa} \\
E_{c}^{\prime}=\frac{f_{c}^{\prime}}{\varepsilon_{c}^{\prime}} \\
\text { For } \frac{\varepsilon_{c}}{\varepsilon_{c}^{\prime}} \leq 1.0: k=1 \\
\text { For } \frac{\varepsilon_{c}}{\varepsilon_{c}^{\prime}} \phi 1.0: k \geq 1.0\end{array}$ \\
\hline & 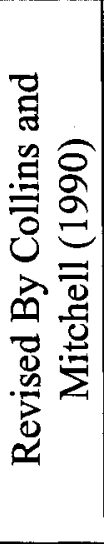 & $\begin{array}{l}\frac{f_{c}}{f_{c}^{\prime}}=\frac{n\left(\varepsilon_{c} / \varepsilon_{c}^{\prime}\right)}{n-1+\left(\varepsilon_{c} / \varepsilon_{c}^{\prime}\right)^{n k}} \\
n=0.8+\frac{f_{c}^{\prime}}{17} \\
E_{c}=\text { Secant tangent modulus } \\
\text { For } \frac{\varepsilon_{c}}{\varepsilon_{c}^{\prime}} \leq 1.0: k=1 \\
\text { For } \frac{\varepsilon_{c}}{\varepsilon_{c}^{\prime}} \phi 1.0: k=0.67+\frac{f_{c}^{\prime}}{62} \geq 1.0\end{array}$ \\
\hline $\begin{array}{l}\text { Hognestad } \\
\text { (1951) }\end{array}$ & $\begin{array}{l}\text { For } \frac{\varepsilon}{\varepsilon} \\
\text { For } \frac{\varepsilon}{\varepsilon}\end{array}$ & $\begin{array}{ll}\frac{\varepsilon_{c}}{\varepsilon_{0}} \leq 1.0: & \frac{f_{c}}{f_{c}^{\prime \prime}}=\frac{2 \varepsilon_{c}}{\varepsilon_{0}}-\left(\frac{\varepsilon_{c}}{\varepsilon_{0}}\right)^{2} \\
\varepsilon_{c} & \frac{f_{c}}{f_{c}^{\prime \prime}}=1-\frac{0.15\left(\varepsilon_{c}-\varepsilon_{0}\right)}{0.0038-\varepsilon_{0}}\end{array}$ \\
\hline $\begin{array}{l}\text { Desayi and } \\
\text { Krishnan }\end{array}$ & $f_{c}=$ & $\frac{E \varepsilon_{c}}{1+\left(\varepsilon_{c} / \varepsilon_{0}\right)^{2}}$ \\
\hline Model 1 & $\frac{f_{c}}{f_{c}^{\prime}}=$ & $=\frac{\varepsilon_{c}}{\varepsilon_{0}} \cdot e^{1-\varepsilon_{c} / \varepsilon_{0}}$ \\
\hline
\end{tabular}




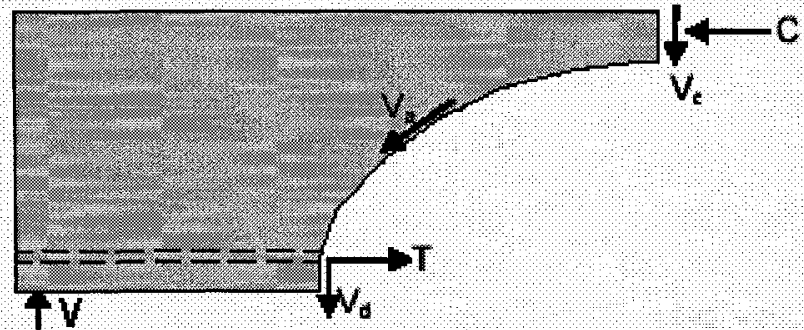

a) without stirrups

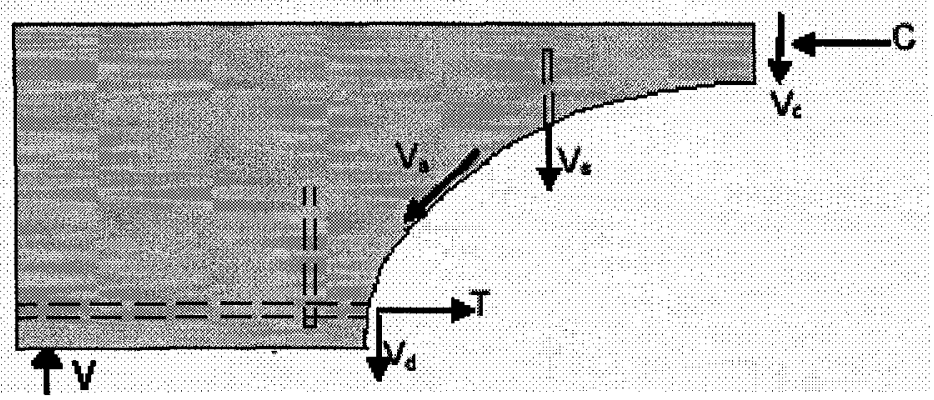

b) with stirrups

Figure 2.1: Shear resistance components in beams

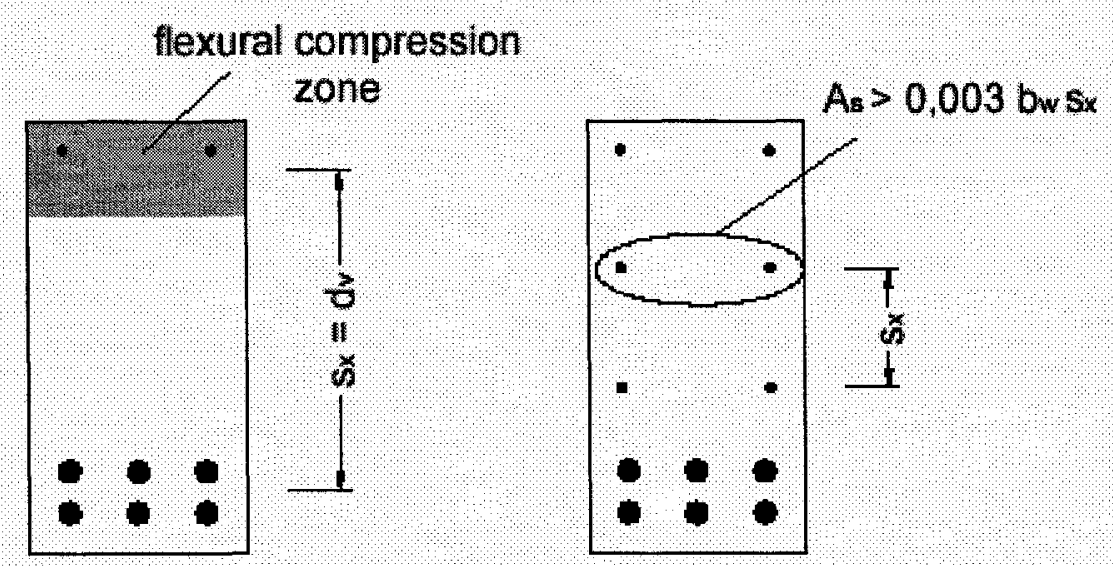

Figure 2.2: Definition of $s_{z}$ accounting for effect of well distributed longitudinal reinforcement on size effect in shear 


\section{CHAPTER 3: PRELIMINARY INVESTIGATION OF CONCRETE MIXES MADE WITH RCA}

\subsection{General}

As discussed in Chapter 2, the residual mortar in RCA is the key factor which differentiates RCA from NA in terms of its lower specific gravity, higher absorption capacity, etc. In practice RCA is pre-soaked and/or some other adjustments are made to the water content of concrete to offset the effects of the higher or lower moisture content and higher water absorption of RCA on RAC. RCA may also have different surface roughness and angularity due to the type of crushing process employed in its production. Thus, in order to produce a structural-grade concrete, it is essential to carry out a preliminary study to investigate the effect of residual mortar on the fresh and hardened properties of RAC. Since no specific mix proportioning method exists for structuralgrade RAC, such an investigation will provide valuable information for the structural phase.

Two different sources of RCA were used in this study: RCA-MO and RCA-VA, obtained from recycled aggregate processing plants in Montreal (Québec) and Vancouver (British Columbia). The original virgin aggregates in the above RCA were Navan limestone (NL) from Ottawa (Ontario) and natural river gravel (NG) from Vancouver (British Columbia), respectively. The coarse natural aggregates used to supplement the RCA in the mixes prepared for the purposes of the present investigation were selected based on the similarity of their physical and mechanical properties to the corresponding OVA's in 
RCA-MO and RCA-VA. For simplicity, RAC mixes made with RCA-MO and RCA-VA will be designated as PM and PV, respectively, in which P stands for preliminary and M and V stand for Montreal and Vancouver, respectively. The fine aggregate used in this study was natural sand (derived from granite) obtained from Cantley (Quebec).

The physical properties of RCA-MO, RCA-VA, NL, and NG will be discussed later in Section 3.2.

In order to investigate the effect of the attached residual mortar on the fresh and hardened concrete properties, several RAC specimens with different combinations of RCA and coarse natural aggregate, and w/c ratio were produced. Initially, the RAC was produced using $100 \%$ RCA as coarse aggregate. Subsequently, the RCA was partially replaced by NA (50\% and $70 \%$ replacement by weight). The replacement of the RCA with NA allows producing concrete with different total mortar volumes (i.e., residual mortar in RCA plus the new mortar in the fresh concrete). Control specimens made with $100 \%$ NA were also prepared. The TMV in RAC can be calculated based on the RCA and NA volumes, the RM content, the RCA specific gravity, and OVA specific gravity as follows

$$
V_{T M}^{R A C}=1-\left((1-R M C) \cdot \frac{S G_{b}^{R C A}}{S G_{b}^{O V A}}\right) \cdot V_{R C A}^{R A C}-V_{N A}^{R A C}
$$

where $V_{T M}^{R A C}, V_{R C A}^{R A C}$ and $V_{N A}^{R A C}$ are the volume of TM, RCA and NA (partial replacement of RCA) in RAC mix; and $S G_{b}^{R C A}$ and $S G_{b}^{O V A}$ are the bulk specific gravity of RCA and OVA. Note that the second term on the right-hand side of Eq.3.1 represents the volume of OVA. In Eq.3.1 it is assumed that the specific gravity of NA and OVA are the same albeit this is not essential for applying the equivalent mortar volume (EMV) mix proportioning method in next Chapter. 
As the compressive strength of concrete is mostly dependant on the strength of its two principal constituents, aggregate and mortar, and on the quality of their interfacial transition zone (ITZ), the residual mortar quality and the RCA surface roughness are expected to be important factors affecting the compressive strength of RAC. However, other concrete properties, such as elastic modulus, fresh and hardened density, creep and shrinkage, are mainly a function of the volume fractions and the corresponding properties of the aggregate and the mortar. Both the quantity and quality of RM and NM play an important role in the production of high quality RAC. Therefore, it is reasonable to focus on the effect of the TMV rather than on the other parameters, such as RCA content, RM volume of RCA, or the total RM content of RAC. The combined effect of the latter parameters will be reflected by the TMV effect. In the opinion of the writer, the reason for the wide range of reported inferior physical and mechanical properties of RAC may be lack of consideration of the role of TMV. In other words, low quality or varying quantity of residual mortar in RCA can lead to different inferiority levels in RAC compared to NAC. However, by taking advantage of TMV concept, it is possible to correlate the properties of RAC mixes to their TMV, which automatically takes the residual mortar quality (RCA source effect) and residual mortar quantity (RM content) into account. Note that residual mortar of low quality normally has a lower specific gravity which results in higher residual mortar, or total mortar volume, in a concrete mix. To recap, in the present study, four types of RAC mixes with RCA content equal to $100 \%, 50 \%, 30 \%$ and $0 \%$ of the total coarse aggregate content were made. In order to study the effect of w/c ratio on the physical and mechanical properties of both airentrained (AE) and non-air-entrained (NAE) RAC, for each mix type, three w/c ratios 
$(0.35,0.45$ and 0.55$)$ were used. Since there is no standard mix design method for proportioning structural-grade RAC, the preliminary mixes were proportioned using a slightly modified version of the ACI standard mix design procedure (ACI committee 211, 1997) for conventional concrete.

\subsection{Material Characterization of RCA}

Specified mechanical and durability related properties of concrete are achieved through proper mix proportioning, which involves the proportioning of the main ingredients of concrete; i.e., cementitious materials, fine and coarse aggregates, water and chemical admixtures. This requires precise characterization of the relevant properties of these ingredients. The material properties of coarse aggregates are particularly important in the design of mixes since they occupy approximately $75 \%$ of the total volume of concrete. The material characterization of RCA, when used as coarse aggregates in new concrete, requires special attention since its properties may vary depending on the source, the production method and the quantity and quality of its attached residual mortar.

In this chapter, the material characterization of the two types of RCA used in the current study is presented. The recycling plants that provided the RCA receive concrete from various demolition projects, involving a blend of different types and sources of virgin aggregates. The first batch of RCA was obtained from a recycled aggregate processing plant located in Montreal, Québec, and the second was from a plant in Vancouver, British Columbia. The RCA from Montreal was obtained from concrete in which the coarse natural aggregate was crushed limestone, the most widely used aggregate in the St. Lawrence Lowlands of the Province of Québec, where the main urban areas are located. The RCA from British Columbia was obtained from demolition concrete made 
predominantly with well-rounded river-bed gravel. Although the RCA from both locations likely included concrete from various demolition projects, and they were probably a blend of several different concrete mixtures, possibly fly-ash concrete in the case of the RCA from Vancouver, no attempt was made to separate or to quantify the RCA originating from the different demolition concrete sources. In terms of their shape and surface characteristics, the two types of RCA showed differences as well. The RCA particles from Montreal showed more uniformity of size and angularity and they had rougher surface than the RCA-VA particles. The RCA aggregate particles generally followed the shape of their corresponding OVA particles. Both RCA types were observed to have rougher surfaces than their corresponding OVA due to the presence of the attached residual mortar. For brevity, henceforth, the RCA from Montreal and Vancouver will be referred to as RCA-MO and RCA-VA, respectively.

As stated earlier, the present research is part of a broader research program with two main thrusts; namely, the durability of RAC and the structural performance of RAC reinforced RAC members. The former aspect is not covered in this study, but it was investigated by Abbas (2007) in a parallel study within the framework of the wider research program. Abbas et al. (2006c, 2006b, and 2007a) performed a comprehensive investigation to determine the mechanical and physical properties of the RCA used in the present study. The material characterization of RCA involved the determination of specific gravity, absorption capacity, moisture content, and porosity. The specific gravity and absorption capacity tests were performed per ASTM C 127-01 (ASTM, 2003). Since the aggregates from both sources were received unscreened and ungraded, before starting the material characterization testing, they were screened from impurities and fines, and were 
separated according to their size. The following section discusses this preparation process of the aggregates before material characterization testing. The residual mortar content of each RCA was determined based on a new method developed by Abbas et al. (2006d and 2007b) which involved immersion of RCA in sodium sulphate solution and its subjection to several freeze-and-thaw cycles. Table 3.1 shows the weighted average properties for RCA-MO, RCA-VA, NL, NG, and river sand.

\subsection{Mix Proportioning Method}

In order to investigate the effect of RCA content on the fresh and hardened properties of the mixes in this preliminary study, first RAC mixes with coarse aggregate comprising only RCA were proportioned using the conventional mix design method of ACI 211.1-97 (ACI committee 211,1997 ), but the lower specific gravity and higher absorption capacity of RCA, due to the presence of $\mathrm{RM}$, were taken into account. In the preliminary study, no water reducing admixtures (WRA) or supplementary cementitious materials were used in any mix. Three w/c ratios, $0.35,0.45$ and 0.55 , were used to produce both air-entrained and non-air-entrained mixes. Next mixes with combined NA and RCA were made in which the RCA was replaced by NA at 50\%, 70\%, and $100 \%$ replacement levels based on weight. The selection of the mix ingredients (water, air, cement, and coarse/fine aggregate contents) are described next.

\section{- Estimation of water and air contents}

Slump in the range of 75 to $100 \mathrm{~mm}$ is suitable for most types of structural applications such as reinforced foundation, beams, slabs and columns (ACI Committee 211, 1997); therefore, this slump range was selected for the RAC specimens investigated in this preliminary study. Using Table A1.5.3.3 of ACI 211.1-97 (ACI Committee 211, 1997), 
water contents of $205 \mathrm{~kg} / \mathrm{m}^{3}$ and $184 \mathrm{~kg} / \mathrm{m}^{3}$, respectively, were determined for non-airentrained and air-entrained concretes assuming $19 \mathrm{~mm}$ nominal maximum aggregate size and $75-100 \mathrm{~mm}$ slump. Similar to conventional concrete, RAC with $6 \%$ air content is expected to withstand freeze-and-thaw action due to severe exposure conditions.

\section{- Calculation of cement content}

For each w/c ratio, the required amount of cement was determined by dividing the estimated water content by the $\mathrm{w} / \mathrm{c}$ ratio.

\section{- Calculation of coarse and fine aggregate contents}

An analytical approach was used for calculating the coarse and fine aggregate contents. According to ACI-211.1-97 (ACI Committee 211, 1997), the required weight of coarse aggregate can be found from the volume of the dry-rodded coarse aggregate per unit volume of concrete, $V_{D R}^{C A}$, as suggested in Table A.1.5.3.6 of ACI 211.1-97 (ACI Committee 211, 1997),

$$
W_{O D}^{C A}=V_{D R}^{C A} \cdot \gamma_{D R}^{C A}
$$

where $W_{O D}^{C A}$ and $\gamma_{D R}^{C A}$ are the required oven-dry weight and the dry-rodded unit weight of coarse aggregate, respectively. Another approach for calculating the required coarse aggregate amount is to use the absolute volume of coarse aggregate, $V_{a}^{\mathrm{CA}}$, as:

$$
\begin{aligned}
& W_{O D}^{C A}=V_{a}^{C A} \cdot 1000 S G_{b}^{C A} \\
& V_{a}^{C A}=\frac{\frac{W_{S S D}^{C A}}{S G_{S S D}^{C A}}}{\frac{W^{C}}{S G^{C}}+\frac{W_{S S D}^{F A}}{S G_{S S D}^{F A}}+\frac{W_{S S D}^{C A}}{S G_{S S D}^{C A}}+W^{W}+A}
\end{aligned}
$$

where $W_{S S D}^{C A}$ is the weight of the SSD coarse aggregate, $W_{S S D}^{F A}$ is the weight of SSD fine aggregate, $W^{C}$ is the weight of cement, $W^{W}$ is the weight of water, $A$ is the air content, 
and $S G_{S S D}^{C A}, S G_{S S D}^{F A}$ and $S G^{C}$, respectively, are the specific gravity of the SSD coarse and fine aggregates, and of cement.

The fine aggregate content can be obtained using either of the two recommended methods in ACI 211.1-97 (ACI Committee 211, 1997): the weight method, or absolute volume method. In this preliminary study, the former method was used. Therefore, the required fine aggregate weight, $W_{S S D}^{F A}$, can be calculated from:

$$
W_{S S D}^{F A}=U-W_{S S D}^{C A}-W^{C}-W^{W}
$$

where $U$ is the fresh density of concrete and can be calculated by the equation provided by ACI 211.1-97 (ACI Committee 211, 1997):

$$
U=10 S G_{S S D, a v}(100-A)+W^{C}\left(1-\frac{S G_{S S D, a v}}{S G^{C}}\right)-W^{W}\left(S G_{S S D, a v}-1\right)
$$

where $S G_{S S D, a v}$ is the weighted average specific gravity of fine and coarse aggregates.

By combining Eq.3.2 to 3.6, the coarse aggregate weight can be obtained from

$$
W_{S S D}^{C A}=\frac{V_{D R}^{C A}\left[W^{C}\left(\frac{1}{S G^{C}}-\frac{1}{S G_{S S D}^{F A}}\right)+\frac{U-W^{W}}{S G_{S S D}^{F A}}+W^{W}\right]}{\left[\frac{1}{S G_{S S D}^{C A}}-\left(\frac{1}{S G_{S S D}^{C A}}-\frac{1}{S G_{S S D}^{F A}}\right) V_{D R}^{C A}\right]}
$$

Knowing the coarse aggregate weight, and using Eq.3.5 and Eq.3.6, the fine aggregate weight can be obtained. Note that the specific gravity of the cement was measured to be 3.15. A summary of the proportions for the preliminary mixes is presented in Table 3.2 and 3.3. In these table, PM and PV represent the preliminary mixes made with RCA-MO and RCA-VA; and CL and CG stand for control mixes made with natural limestone and gravel, respectively. In addition, 100,50 and 30 represent the percentage weight contribution of RCA to the total coarse aggregate weight in the various mixes; L, M and $\mathrm{H}$ represent the low (0.35), medium (0.45) and high (0.55) w/c ratios; and $\mathrm{A}$ and $\mathrm{N}$ stand 
for air-entrained and non-entrained mixes, respectively. For instance, PM-100LA stands for preliminary air-entrained RAC made with $100 \% \mathrm{RCA}-\mathrm{MO}$ and low w/c=0.35.

\subsection{Preparation of Mixes}

The PM and PV mixes were made following three main steps:

1) Preparation of materials

2) Adjustment of mixing water

3) Concrete mixing

These steps are discussed in more detail as follows.

\subsubsection{Preparation of Materials}

The materials required for concrete mixes were prepared 24 hours prior to mixing time as described below:

- Weighing the air-dried fine aggregate and the air-dried coarse aggregates in individual size fractions $(35 \%, 25 \%$ and $40 \%$ for $4.75 \mathrm{~mm}, 9.5 \mathrm{~mm}$ and $12.5 \mathrm{~mm}$ fraction sizes, respectively) and mixing these quantities to produce the desired grading.

- Soaking the recombined coarse aggregates in water for 24 hours prior to using them in the concrete mix.

- Spraying the fine aggregate with water and keeping it in a damp state (4\% moisture content), by covering it with a plastic sheet, for 24 hours prior to its use in the concrete mix.

- Weighing and storing the cementitious materials in dry plastic pails.

\subsubsection{Water Content Adjustment}

Due to the pre-soaking of coarse aggregate and the damp state of the fine aggregate, the estimated mixing water quantity needed adjustment prior to the concrete mixing. The 
quantity of the adsorbed water on coarse and fine aggregate surfaces can be obtained by subtracting the SSD weight of the coarse aggregate from the weight of the coarse aggregate in wet condition. The revised weight of required water is calculated by subtracting the weight of the adsorbed surface water from the previously estimated mixing water weight.

The weight of coarse and fine aggregates in SSD condition can be calculated as

$$
\begin{gathered}
W_{S S D}^{C A}=\frac{W_{A D}^{C A}}{1+m_{c}^{C A}} \cdot\left(1+A C^{C A}\right) \\
W_{S S D}^{F A}=W_{O D}^{F A} \cdot\left(1+A C^{F A}\right)
\end{gathered}
$$

where $W_{O D}^{F A}$ is the weight of fine aggregate in oven-dry condition, $W_{A D}^{C A}$ is the weight of coarse aggregate in air-dry condition, $A C^{C A}$ is the absorption capacity of coarse aggregate, $A C^{F A}$ is the absorption capacity of fine aggregate, and $m_{c}^{C A}$ is the moisture content of coarse aggregate.

The water adjustment for the coarse and fine aggregates was performed as follows:

- Removing the pre-soaked coarse aggregate from water, draining the excess water, and weighing the aggregate in its wet state. The adsorbed water weight was subtracted from the estimated water weight.

- The damp fine aggregate was weighed and, from this weight, its SSD weight was subtracted. The difference between the two weights was subtracted from the estimated water content to arrive at a revised weight of water.

\subsubsection{Concrete Mixing}

A pan mixer was used for mixing the concrete. The mixing sequence followed ASTM C 192/C 192M-02 (ASTM, 2003) procedure as detailed below:

- Buttering the mixer with a mortar proportioned closely to the test mix proportions. 
- Adding the coarse aggregate and the air entraining admixture (if applicable).

- Starting the mixer and adding the fine aggregate.

- Adding the cement and water while the mixer is running.

- Mixing the concrete for 3 minutes.

- Stopping the mixer for 3 minutes; while at rest, keeping the mixer covered.

- Remixing the concrete for another 2 minutes.

- Measure the slump, temperature, air content, and weight (for the fresh density of concrete).

After the mixing process, concrete specimens were cast and cured following the procedures described in ASTM C 192/C 192M-02 (ASTM, 2003). For each mix, six cylindrical specimens $(100 \times 200 \mathrm{~mm})$ were prepared for the 28 -day compressive strength (3 specimens per test) and the splitting tensile strength tests $(3$ specimens). Three cylinders $(150 \times 300 \mathrm{~mm})$ were also prepared for the determination of elastic modulus. Specimens were stored for 28 days in a moist room in accordance with the requirements of ASTM C 511-98 (ASTM, 2003). The fresh and hardened properties of the mixes prepared in this preliminary study are presented in the following section.

\subsection{Effect of RCA on Fresh and Hardened Properties of RAC}

In this chapter, the results of the fresh and hardened properties of the concrete mixes described in Section 3.4 are presented. It is worth mentioning that the main purpose of this section is to identify the major parameters affecting the fresh and hardened properties of both air-entrained and non-air-entrained RAC mixes at different w/c ratios and with different RCA contents. Therefore, during the preliminary study, the effect of three main parameters on fresh and hardened properties of RAC were investigated: (1) water-cement 
ratio, (2) RCA content and (3) total mortar volume (TMV). Since the w/c ratio is a key factor which is known to affect various fresh and hardened properties of conventional concrete, its effect on the present RAC properties will be investigated. However, the main focus of this section will be on RCA content, especially on the TMV. As discussed in Section 3.1, it is more pertinent to study the effect of TMV rather than the effect of quantities such as RM volume and RCA content. The outcome of this section will be a basis for the development of a new mix design for RAC (Chapter 4) and the development of rational and/or empirical models for density, compressive strength, and elastic modulus of RAC.

The mix proportions of each mix, whose test results are reported here, can be found in Tables 3.2 and 3.3 .

\subsubsection{Fresh Properties}

The slump, air content and fresh density were measured once for each mix. Tables 3.4 and 3.5 present the summary of the fresh properties for the mixes tested. In the following sections, these results are presented in detail.

\subsubsection{Slump}

The conventional slump test as per ASTM C143/C 143M-00 (ASTM, 2003) was used to measure the slump of the mixes. Tables 3.4 and 3.5 present the slump results for PM and PV mixes, respectively. It can be observed from Table 3.4 that the slump of air-entrained PM mixes with different RCA replacement levels ranges from $60 \mathrm{~mm}$ (PM-30LA) to 200 mm (PM-50HA). The slump of the conventional concrete produced with limestone is in the range of $40 \mathrm{~mm}$ (CL-LA) to $180 \mathrm{~mm}$ (CL-HA). From Table 3.4, it can be observed that the slump of non-air-entrained PM mixes with different RCA replacement levels 
range from $55 \mathrm{~mm}(\mathrm{PM}-50 \mathrm{HN})$ to $205 \mathrm{~mm}(\mathrm{PM}-100 \mathrm{HN})$. The slump of the conventional concrete produced with limestone was found to be in the range of $90 \mathrm{~mm}$ (CL-LN) to 200 $\mathrm{mm}(\mathrm{CL}-\mathrm{HN})$.

Similarly, it can be observed from Table 3.5 that the slump of air-entrained PV mixes with different RCA replacement levels range from $50 \mathrm{~mm}$ (PV-100LA) to $215 \mathrm{~mm}$ (PV50HA).The slump of the accompanying conventional concrete produced with river gravel is in the range of $130 \mathrm{~mm}$ (CG-LA) to $230 \mathrm{~mm}$ (CG-HA). From Table 3.5, it can be observed that the slump of non-air-entrained PV mixes with different RCA replacement levels range from $100 \mathrm{~mm}(\mathrm{PV}-100 \mathrm{LN})$ to $220 \mathrm{~mm}(\mathrm{PV}-30 \mathrm{MN})$. The slump of the conventional concrete produced with river gravel is in the range of $160 \mathrm{~mm}(\mathrm{CG}-\mathrm{LN})$ to $230 \mathrm{~mm}(\mathrm{CG}-\mathrm{HN})$. All of the minimum observed slump values are practically within 75 $\pm 25 \mathrm{~mm}$, which is the specified range for minimum target slump.

\section{- Effect of water-cement ratio}

Water-cement ratio is known to be the most important factor affecting the slump of RAC. The experimental data in Fig. 3.1 and 3.2 illustrate the general effect of this parameter on the slump of both air-entrained and non-air entrained RAC mixes. It is evident from Figures 3.1 and 3.2 that the slump is linearly related to the $w / c$ ratio, with increase in w/c ratio resulting in higher slump. Since the water content was fixed for these mixes, an increase in $\mathrm{w} / \mathrm{c}$ ratio led to lower cement content, and consequently less total surface area of cement in the mix, which is expected to result in lower water demand and consequently higher slump. 


\section{- Effect of RCA content}

For constant w/c ratio, aggregate type and RCA content were found to be the other two main factors affecting the slump of RAC mixes. The crushing of recycled concrete generally results in a type of aggregate which is geometrically similar to crushed aggregate. Although the outer surface of RCA, covered by adhered mortar, partially follows the shape and angularity of OVA, the angular corners of RCA tend to be smoother due to subsequent handling and mixing. Depending on the level of angularity of NA, RCA may be less or more angular than NA. For instance, RCA-MO was found to be relatively less angular compared to crushed natural limestone, while RCA-VA was noticed to be more angular than the round natural gravel. In addition, both RCA-MO and RCA-VA had rougher surface texture (due to the porous nature of the attached RM) compared to natural limestone aggregate and gravel, respectively. Less angular and smoother aggregate surface normally results in higher slump values.

Figure 3.1 illustrates the variation of slump values with RCA content for different w/c ratios for both air-entrained and non-air-entrained mixes. In air-entrained batches, increase in the RCA content resulted in higher slump for PM mixes while the converse happened in the PV mixes. For PM mixes this trend can be mainly attributed to the difference between the angularity of the constituent RCA and the NA. It appears that airentraining can better mitigate the effect of the rougher surface texture of RCA-MO than that of the relatively smoother surface of NL. In PV mixes, at higher RCA content levels, higher angularity and rougher surface texture both resulted in lower slump values. At higher w/c ratios, the rate of slump change decreased; presumably the effect of the higher 
water content in the mix became predominant rather than the effects of the angularity and/or surface roughness of aggregates.

For non-air-entrained PM and PV mixes, the general trend was a reduction in slump (with a slight improvement at 100\% RCA content in PM mixes) with increased RCA content. In the absence of any air-entraining admixture, in PM mixes, the rougher surface texture of RCA-MO compared to NL was the dominant effect and led to lower slump values. In PV mixes, at higher RCA content levels the greater angularity and surface roughness both resulted in lower slump. At higher w/c ratios, the rate of slump change decreased, presumably because the effect of higher water content in the mix became predominant rather than the effects of angularity and/or surface roughness of aggregates.

\subsubsection{Fresh Density}

The density of fresh concrete mixes was measured according to ASTM C 138/C 138M01a (ASTM, 2003). The fresh concrete density and air content were determined using the same container. The fresh density was obtained by dividing the net weight of the fresh concrete by the volume of the container.

For air-entrained PM mixes, fresh density ranged from $2181 \mathrm{~kg} / \mathrm{m}^{3}$ (PM-100MA) to 2366 $\mathrm{kg} / \mathrm{m}^{3}$ (CL-LA). For non-air-entrained PM mixes, it ranged from $2324 \mathrm{~kg} / \mathrm{m}^{3}$ (PM$100 \mathrm{HN})$ to $2409 \mathrm{~kg} / \mathrm{m}^{3}$ (CL-LN). The range for air-entrained PV specimens was found to be between $2224 \mathrm{~kg} / \mathrm{m}^{3}$ (PV-100MA) and $2349 \mathrm{~kg} / \mathrm{m}^{3}$ (CG-LA). For non-air entrained PV specimens, the range was from $2366 \mathrm{~kg} / \mathrm{m}^{3}$ (PV-100MN) to $2446 \mathrm{~kg} / \mathrm{m}^{3}$ (CG-MN). Based on the preceding ranges of fresh density in air-entrained and non-air-entrained PM and PV mixes, it is observed, and as expected, that the fresh density of air-entrained 
mixes is generally lower than that of non-air-entrained mixes due to the higher air void content of air-entrained mixes.

\section{- Effect of water-cement ratio}

The charts in Fig. 3.3 and 3.4 illustrate the general effect of w/c ratio on the fresh density of both air-entrained and non-air entrained RAC mixes tested in this study. From these figures, it is evident that the fresh density of RAC is not linearly and solely correlated to its $w / c$ ratio. This can be attributed to the fact that the fresh density of RAC is a function of the volume fraction and density of its constituents (aggregate, cement, water, sand, and air content). Since the water and aggregate contents were kept constant in these mixes, increasing w/c ratio resulted in lower cement content for the same water content. Consequently, more sand had to be added to the mix to compensate for the reduction in RAC yield caused by its lower cement content. The addition of sand partially recoups the fresh density of the mix at higher w/c ratios.

\section{- Effect of RCA content}

It has been reported in the literature (Topcu and Sengel, 2004) that an increase in the RCA content of a mix would lead to a decrease in its fresh density. With reference to the present test results, as plotted in Fig. 3.3 and 3.5 , the preceding trend can be easily observed for both the PM and PV mixes. Generally, higher RCA content resulted in lower fresh density for all w/c ratios in both air-entrained and non-air-entrained PM and PV mixes. This is largely due to the fact that the fresh density of concrete is mainly dominated by the volume fractions and density of its mortar and aggregate. Due to the presence of RM in RCA, the overall mortar volume (TMV) in PM and PV mixes 
increased as the RCA content increased, which in turn led to the lower overall fresh density of these mixes.

The rate of reduction in the fresh density of PM mixes was found more noticeable compared to that of PV mixes. This can be mainly attributed to the higher residual mortar content in RCA-MO (41\%) compared to that in RCA-VA (23\%), which resulted in higher overall RM and TM volumes in PM mixes compared to PV mixes, and consequently higher changes in the fresh density of the corresponding mixes.

Such correlation has traditionally been used by other investigators to conclude that an increase in RCA content of a mix results in a decrease in its fresh density compared to NAC. However, this argument may not be entirely valid because in none of the previous studies the effect of the total mortar volume on fresh density has been investigated. Based on conventional mix design methods for concrete, an increase in RCA content automatically leads to an increase in the total mortar volume, consequently, the conclusion that the increase in RCA content leads to a decrease in the fresh density value seems plausible. In fact, this conclusion may not be generally valid because, based on the rule of mixtures, it would be the increase in the total mortar volume (TMV) of RAC, rather than the increase in its RCA content alone, that would reduce its fresh density. Since the TMV includes both the residual mortar and the new mortar, it reflects the combined effect of the quality and quantity of both mortars on the mix properties. The residual mortar of low quality usually has a lower specific gravity which results in higher residual or total mortar volume. To verify the above statement with respect to the effect of TMV on the fresh density of RAC, in the next section, the relationship between the fresh density and the total mortar volumes of the current mixes is analyzed. 


\section{- Effect of TMV}

Since the fresh density of RAC is a function of the proportion and density of its components, if these proportions are maintained essentially constant, the fresh density of RAC could be related to its TMV only. Figure 3.6 illustrates the variation of the fresh density with TMV for both air-entrained and non-air-entrained PM and PV mixes with different $w / c$ ratios. A practically linear relation is observed between the TMV and the fresh density of all the mixes. In Chapter 6 some rational models will be proposed for estimating the fresh density of RAC based on its mix proportions and the data presented in this section will be used to validate them.

\subsubsection{Hardened Properties}

In this section, the results of the hardened density $\left(\gamma_{c}\right)$, compressive strength $\left(f_{c}^{\prime}\right)$, elastic modulus $\left(E_{c}\right)$, and splitting tensile strength $\left(f_{t}\right)$ measurements of the hardened concrete mixes described earlier are presented. The proportions of each mix can be found in Tables 3.2 and 3.3 while Tables 3.6 and 3.7 present the hardened density values for PM and PV mixes, respectively. All the reported values in these tables are based on the average of three replicate test samples.

\subsubsection{Hardened Density}

The hardened density of RAC was obtained by weighing the $100 \times 200 \mathrm{~mm}$ cylinders in air and in water 24 hours after their casting. The density was calculated by dividing the weight of the specimen in the air by the volume of the specimen.

The hardened density values ranged from $2138 \mathrm{~kg} / \mathrm{m}^{3}$ (PM-100MA) to $2322 \mathrm{~kg} / \mathrm{m}^{3}$ (CLLA) for air-entrained PM mixes and $2298 \mathrm{~kg} / \mathrm{m}^{3}$ (PM-100HN) to $2388 \mathrm{~kg} / \mathrm{m}^{3}$ (CL-LN), for non-air-entrained PM mixes. For air-entrained PV specimens, it was between 2196 
$\mathrm{kg} / \mathrm{m}^{3}$ (PV-100HA) and $2329 \mathrm{~kg} / \mathrm{m}^{3}$ (PV-30LA) while for non-air entrained PV mixes, it was from $2275 \mathrm{~kg} / \mathrm{m}^{3}$ (PV-100HN) to $2462 \mathrm{~kg} / \mathrm{m}^{3}$ (CG-HN).

According to the range of hardened density values in air-entrained and non-air-entrained PM and PV mixes, as expected, the hardened densities of air-entrained mixes are generally lower than those of non-air-entrained mixes due to the higher void content of air-entrained mixes.

\section{- Effect of water-cement ratio}

The charts in Fig. 3.7 and 3.8 illustrate the general effect of w/c ratio on the hardened density of the current RAC mixes. It appears that the densities of both the air-entrained and the non-air entrained PM and PV mixes are related to their w/c ratios. From these figures, it is evident that the fresh density of RAC is linearly correlated to its w/c ratio. However, upon closer scrutiny, these relationships are not reflecting the effect of the w/c ratio only because, in these mixes, the water and aggregate contents were kept constant, thus increasing the w/c ratio resulted in lower cement content for the same water content. Consequently, as the w/c ratio was lowered, concomitantly more sand was added to the mix to compensate for the reduction in RAC yield caused by its low cement content. It is this inverse relationship between the sand content and the w/c ratio that is responsible for the apparent decrease in density with increased w/c ratio as observed in Figs. 3.7 and 3.8.

\section{- Effect of RCA Content}

It has been reported in the literature (Topcu and Sengel, 2004) that an increase in the RCA content of a mix leads to a decrease in its hardened density. With reference to the 
current test results plotted in Fig. 3.7 and 3.9, the preceding trend can be easily observed for both the PM and PV mixes. Generally, higher RCA content resulted in lower hardened density in both air-entrained and non-air-entrained PM and PV mixes for all w/c ratios. This is largely due to the fact that the hardened density of concrete is dominated by the volume fractions and densities of its mortar and aggregate; generally, the higher the natural aggregate content (assuming normal density aggregates) of a mix, the greater its density. In the present tests, due to presence of RM in RCA, the overall mortar volume (TMV) in PM and PV mixes increased as the RCA content increased, which in turn led to the lower overall hardened density of these mixes.

The rate of reduction in the hardened density of PM mixes was found more perceptible compared to that of PV mixes. This can be ascribed mainly to the higher residual mortar content in RCA-MO (41\%) compared to that in RCA-VA (23\%), which results in higher overall RM and TM volumes in PM mixes compared to PV mixes, and consequently higher changes in the hardened density of the former mixes.

Such correlation has traditionally been used by previous researchers to indicate that an increase in RCA content of a mix leads to a decrease in its hardened density. However, this argument may not be entirely valid because in none of the previous studies the effect of the total mortar volume on hardened density was investigated. Based on conventional concrete mix design methods, an increase in RCA content automatically leads to an increase in the total mortar volume, consequently, the conclusion that the increase in RCA content leads to a reduction hardened density seems plausible. In fact, this conclusion may not be generally valid because based on the rule of mixtures it would be the increase in the total mortar volume (TMV) of RAC, rather than the increase in its 
RCA content alone, that would reduce its hardened density. Since the TMV includes the residual mortar and the new mortar, the TMV effect subsumes the effect of RCA on density of RAC because it reflects the combined effects of both the quality and the quantity of the residual and the new mortar. To verify the above statement with respect to the effect of TMV on the hardened density of RAC, in the next section, the relationship between the hardened density and the total mortar volumes of the current mixes is presented.

\section{- Effect of TMV}

Figure 3.10 illustrates the variation of the hardened density with TMV in both airentrained and non-air-entrained PM and PV mixes with different w/c ratios. A linear relationship is observed between the TMV and the hardened density of all air-entrained and non-air-entrained PM and PV mixes. The results of this section will be used in Chapter 6 to develop and validate some proposed rational models for estimating the hardened density of RAC.

\subsubsection{Compressive Strength}

The conventional compressive strength testing method per ASTM C 39/C 39M-01 (ASTM, 2003) was used to determine the 28-day compressive strength, $f_{c}^{\prime}$, of the present mixes. In general, as it can be observed in Tables 3.6 and 3.7, the compressive strength was within the expected range for structural-grade concrete. For air-entrained PM mixes, it was from 19.8 MPa (PM-100HA) to 47.8 MPa (PM-30LA); for non-air-entrained PM mixes; it ranged from $31.5 \mathrm{MPa}(\mathrm{PM}-100 \mathrm{HN})$ to $57.5 \mathrm{MPa}(\mathrm{PM}-30 \mathrm{LN})$. Air-entrained PV specimens performed well with their strength ranging from 26.5 MPa (PV-50HA) to 44.9 MPa (PV-100LA) while for non-air-entrained PV specimens, it ranged from 36.1 
$\mathrm{MPa}(\mathrm{PV}-50 \mathrm{HN})$ to $54.7 \mathrm{MPa}(\mathrm{PV}-100 \mathrm{LN})$. Note that practically all of the above mixes have 28-day compressive strength of at least $25 \mathrm{MPa}$ (except PM-100HA), which is adequate for normal structural concrete applications.

According to the range of compressive strengths in air-entrained and non-air-entrained PM and PV mixes, it is evident, and as expected, that the compressive strengths of airentrained mixes are generally lower than those of non-air-entrained mixes due to the higher air void content of the former than the latter.

\section{- Effect of water-cement ratio}

One of the main factors affecting the compressive strength of RAC is the w/c ratio of the fresh mix. The experimental charts in Fig. 3.11and 3.12 illustrate the general effect of w/c ratio on the compressive strength of the current RAC mixes. As expected, the compressive strengths of both air-entrained and non-air-entrained PM and PV mixes were found to be related to the respective w/c ratio of the mixes, irrespective of their RCA content. Similar to conventional concrete, one can see that an increase in the w/c ratio of RAC resulted in a decrease in its compressive strength.

\section{- Effect of RCA content}

The correlation between the compressive strength and the RCA content are demonstrated in Fig. 3.11 and 3.13. From these figures, it is evident that there is not a correlation between the compressive strength and the RCA content. This is a key and important finding because it is often believed by many practitioners that RCA generally results in lower strength concrete. The lack of correlation between the RCA content and compressive strength is due to the fact that the compressive strength of RAC is mainly dependant on the relative strength of: (1) RM, (2) NM, (3) the quality of ITZ at interface 
of aggregate (RCA or NA) and NM, (4) the quality of ITZ at interface of OVA and RM, (5) the mechanical bond between aggregate (RCA or NA) and NM, and (6) the mechanical bond between OVA and RM. In an aggregate such as RCA-MO and NL, in which OVA and NL are crushed limestone with irregular and angular corners, good mechanical bond is expected between OVA and RM or NL and NM. However in RCAVA and NG, in which OVA and NG are round shaped gravel, the bond between OVA and RM or NG and NM is expected to be relatively weaker than that between NL and mortar. Therefore, the weaker bond most likely led to early initiation of failure in PV mixes.

For both air-entrained and non-air-entrained PM mixes, generally the compressive strength of mixes with $100 \%$ RCA content was slightly less than those with $0 \%$ RCA, which was more evident at higher w/c ratios. This is partially due to the lower air content at $0 \%$ RCA content compared to $100 \%$ RCA content. However, it may be mainly attributed to the greater angularity of the crushed NL compared to RCA-MO, and consequently the quality of the mechanical bond between the aggregate particles and the mortar. In other words, the low compressive strength of RAC at high RCA contents (e.g. PM-100HA) is not necessarily due to the RCA content, but due to other effects such as the RCA angularity level, mix air content, etc.

For both air-entrained and non-air-entrained PV mixes, generally the compressive strength at $100 \%$ RCA content was slightly higher than that at $0 \%$ RCA, which was less noticeable at higher $w / c$ ratios. Once again, this can be mainly ascribed to the smaller angularity of the round NG compared to RCA-VA; which may result in weaker mechanical bond between the aggregate and the mortar. The higher compressive strength 
of CG-HA and CG-HN compared to PV-100HA and PV-100HN was mainly due to lower void content of the former mixes.

\section{- Effect of TMV}

Figure 3.14 illustrates the variation of the compressive strength of both air-entrained and non-air-entrained PM and PV mixes with their TMV and w/c ratios. As expected, the compressive strength of RAC was found not to be correlated to its TMV value. This is not unexpected because the strength of RAC is mainly dependant on the relevant properties of (1) OVA, RM, and OITZ; and (2) NA, new mortar (NM), and the mechanical bond between the aggregates (RCA or NA) and the NM, i.e. the quality of the new ITZ (NITZ). If the RM and the OITZ have comparable strength to the NM and NITZ, respectively, the overall strength of RAC is expected to be comparable to that of the companion NAC; otherwise, its strength characteristics will be affected by the properties of $\mathrm{OC}$, and it might be inferior compared to the companion NAC.

Since there was no information available for the strength characteristics of RCA-MO and RCA-VA (and accordingly RM) in the present study, it was not feasible to compare directly the strength of RM with that of the NM. However, according to the observed linear relationship between the $\mathrm{c} / \mathrm{w}$ ratio and the compressive strength, and also due to comparable compressive strengths of RAC and NAC mixes with w/c ratio of 0.35 , it may be concluded that the quality of RM in RCA-MO and RCA-VA (i.e., the compressive strength of OC) was higher than the corresponding quality of NM in the PM and PV mixes. To further investigate this point, i.e. the effect of RCA quality on the strength of RAC, in Chapter 6, the experimental results of Hansen and Narud (1983) will also be examined because they used RCA with relatively low strength compared to the specified 
strength of the new mix made with it. In Chapter 6, some empirical models will be presented which attempt to relate the strength of RAC to the strength of its constituent RCA.

\subsubsection{Splitting Tensile Strength}

The splitting tensile tests were carried out following the method described in ASTM 49696 (ASTM, 2003). In general, as it can be observed in Tables 3.6 and 3.7, the splitting tensile strength, $f_{t}$, for the all mixes is basically within a narrow range. For air-entrained PM mixes, it varies from 3.3 MPa (PM-50HA) to 4.5 MPa (PM-100LA) while for nonair-entrained PM mixes it varies from 3.4 MPa (PM-30HN) to $4.9 \mathrm{MPa}(\mathrm{PM}-50 \mathrm{HN})$. Similarly, for air-entrained PV mixes, it varies from 2.5 MPa (PV-100HA) to 5.3 MPa (PV-30LA) while for non-air entrained PV mixes, it ranges between 3.0 MPa (PV$100 \mathrm{HN})$ and 5.6 MPa (PV-30MN).

Based on the above data, it is evident that the splitting tensile strength of air-entrained mixes is generally lower than that of non-air-entrained mixes and this is due to the higher air void content created by air entrainment.

\section{- Effect of water-cement ratio}

The experimental charts in Fig. 3.15 and 3.16 illustrate the general effect of w/c ratio on the splitting tensile strength of the current RAC mixes. As expected, the tensile strength of both air-entrained and non-air-entrained PM and PV mixes was found to be linearly related to the respective w/c ratio of the mix, irrespective of its RCA content. According to the theses figures, it can be observed that the splitting tensile strength values of both air-entrained and non-air entrained PM specimens vary practically linearly with the w/c ratio; an increase in the $\mathrm{w} / \mathrm{c}$ ratio results in a decrease in the splitting tensile strength. The 
non-air-entrained PM specimens at $100 \%$ RCA seem to be an exception to the above trend, for in the latter case the w/c ratio seems to have negligible effect on the splitting tensile strength. In contrast, as it may be observed in Figure 3.16, the PV mixes do not exhibit any systematic relationship between the w/c ratio and the tensile strength. This may be attributed principally to the lower mechanical bond between NG and NM and/or between the OVA and the RM in PV mixes compared to those in PM, which would result in initiation of failure at mortar-aggregate interfaces rather than within the RM or the NM. Visual observation of the failure planes of the test specimens confirmed this hypothesis.

\section{- Effect of RCA content}

The correlation between the splitting tensile strength and the RCA content are demonstrated in Fig. 3.15 and 3.17. From these figures, it is concluded that there is not a correlation between the splitting tensile strength of RAC and its RCA content. Although the latter conclusion is contrary to the popular perception, it is not unexpected because the splitting tensile strength of RAC is mainly dependant on the strength of (1) RM, (2) NM, (3) the mechanical bond between aggregate (RCA or NA) and the NM, and (4) the mechanical bond between the OVA and the RM. In an aggregate such as RCA-MO and NL, in which the OVA and the NL are crushed limestone with irregular and angular corners, good bond between the OVA and the RM on the one hand and the NL and the NM on the other hand is expected. On the contrary, in the case of RCA-VA and NG, in which the OVA and the NG are round shaped gravel, the bond between OVA the RM or between the NG and the NM is expected to be relatively weaker. Therefore, these interfacial regions with low strength are susceptible to failure under lower stresses. 
Note, however, that generally in both air-entrained and non-air-entrained PM mixes, the splitting tensile strength was found to be lower at $0 \% \mathrm{RCA}$ content than at higher RCA contents. This may be attributed to the higher quality of RM compared to the NM. It should be stated that in both air-entrained and non-air-entrained PV mixes with at $\mathrm{w} / \mathrm{c}=0.35$ or 0.45 , the splitting tensile strength generally increased with increased RCA content. However, for $w / c=0.55$, the tensile strength dropped dramatically for mixes with $100 \%$ RCA content compared to the companion mix with no RCA. This reduction in strength was $33 \%$ and $39 \%$ for air-entrained and non-air-entrained mixes, respectively.

\section{- Effect of TMV}

Figure 3.18 illustrates the variation of the splitting tensile strength of both air-entrained and non-air-entrained PM and PV mixes with their TMV and w/c ratios. As expected, the TMV has negligible effect on the tensile strength of RAC because it is well known that the tensile strength of RAC, or of concrete and other similar composites in general, is a function of the strength of its constituent mortar (matrix) and aggregate (solid), as well as the strength of the mortar-aggregate interface, but it is not a function of the proportions of these quantities in the mix.

\subsubsection{Elastic Modulus}

The elastic modulus test for concrete was carried out following the method described in ASTM C469-02 (ASTM, 2003). The elastic modulus described here is a secant modulus and is calculated based on the procedure described in ASTM (2003). Briefly, two points on the compressive stress-strain curve of concrete are chosen and are connected by a straight line, i.e. the secant. The slope of this secant line is denoted as the elastic 
modulus. The first point corresponds to a strain of 0.00005 and the second to a stress of $0.4 f_{c}^{\prime}$.

As it can be observed in Tables 3.6 and 3.7, and Fig. 3.19 the elastic moduli of airentrained PM mixes range from 20.8 GPa (PM-100MA) to 34.4 GPa (CL-LA) while those of the non-air-entrained PM mixes range from 27.1 GPa (PM-100HN) to $36.7 \mathrm{GPa}$ (CL-LN). The air-entrained PV specimens elastic moduli range from $23.0 \mathrm{GPa}$ (PV100HA) to $32.9 \mathrm{GPa}$ (PV-30LA) while those of the companion non-air entrained PV specimens range from 29.3 GPa (PV-100HN) to $36.4 \mathrm{GPa}(\mathrm{PV}-30 \mathrm{LN})$. Again, it may be noticed, that air-entrainment reduces the elastic modulus because it creates more voids in the concrete.

The above values are generally within the expected range of the elastic modulus values for normal concrete; however, in Chapter 6, a detailed analysis of these results will be performed and some semi-empirical models will be presented for predicting the elastic modulus of RAC based on the properties of its constituents.

\section{- Effect of water-cement ratio}

The w/c ratio is a key parameter which is known to affect many hardened properties of concrete, including its strength and elastic modulus. The experimental bar charts in Fig.3.19 and 3.20 illustrate the general effect of this parameter on the elastic moduli of the current RAC mixes. One can easily discern the effect of this parameter on the elastic moduli of both air-entrained and non-air entrained mixes. Observe that generally the elastic modulus of RAC is linearly related to its w/c ratio, wherein an increase in the w/c ratio of the mix results in a decrease in its elastic modulus. 
It should be stated that for a composite material such as concrete, its elastic modulus is mainly a function of the void ratio of the mix and the volume fractions and elastic moduli of its aggregate and the mortar. At constant water and aggregate content, an increase in w/c ratio results in a decrease in the elastic modulus of RAC because of the cement content reduction and sand content increase of the resulting concrete, both of which result in a decrease of its elastic modulus. The current results generally conform to this hypothesis.

\section{- Effect of RCA content}

It has been reported in the literature (Ray and Venkateswarlu, 1991; Limbachia et al., 2000; Topcu and Sengel, 2004) that an increase in the RCA content of a mix leads to a decrease in its elastic modulus. With reference to the current test results as plotted in Fig.3.19 and 3.21, the preceding trend can be easily observed for both the PM and PV mixes. Generally, higher RCA content resulted in lower elastic modulus in both airentrained and non-air-entrained PM and PV mixes for all w/c ratios. Due to presence of RM in RCA, the overall mortar volume (TMV) in PM and PV mixes increased as the RCA content increased, which in turn led to the lower overall elastic modulus of these mixes.

Such correlation has traditionally been used by previous researchers to indicate that an increase in RCA content of a mix leads to a decrease in its elastic modulus and consequently to an overall inferiority of RAC compared to NAC. However, this argument may not be entirely valid because in none of the previous studies the effect of the total mortar volume on elastic modulus was investigated. Based on conventional mix design methods for concrete, an increase in RCA content of RAC automatically leads to an 
increase in its total mortar volume; consequently, the conclusion that the increase in RCA content leads to a decrease in the elastic modulus value seems plausible. In fact, this conclusion may not be generally valid because based on the rule of mixtures it would be the increase in the total mortar volume (TMV) of RAC, rather than the increase in its RCA content alone, that would reduce its elastic modulus. Since the TMV comprises the residual mortar and the new mortar, and since the residual mortar is part of the RCA, the TMV automatically reflects the combined effects of both their quality and quantity. Of course, the latter supposition is based on the premise that the residual and the new mortars are qualitatively similar. On the other hand, significant discrepancy between their properties, such as their strength and stiffness, would require that the contribution of each mortar to the total stiffness be separately determined. This contribution would be function of both the quantity and the stiffness of each type of mortar within the mix.

To verify the above statement with respect to the effect of TMV on the elastic modulus of $\mathrm{RAC}$, in the next section, the relationship between the elastic moduli and the total mortar volumes of the current mixes is analyzed.

\section{- Effect of TMV}

The variation of the elastic modulus values of the tested specimens with their TMV is illustrated in Fig.3.22. The figure illustrates the variation of the elastic modulus with TMV for both air-entrained and non-air-entrained PM and PV mixes with different w/c ratios. There is a linear relation between the TMV and the elastic modulus of all the airentrained and the non-air-entrained PM and PV mixes. Hence for the current mixes both the TMV and the RCA content correlate linearly with the elastic modulus, but later in this thesis it will be demonstrated that the RCA content is not the critical parameter. It is the 
total mortar volume -- assuming the new and residual mortar to be qualitatively similar -that determines the value of the elastic modulus of RAC.

\subsection{Summary}

- The $w / \mathrm{c}$ ratio is the main factor affecting the slump of the RAC mixes. For identical w/c ratios, the angularity and surface roughness of the RCA are the other two factors affecting the slump of both air-entrained and non-air-entrained RAC mixes.

- For air-entrained PM mixes with lower RCA content, air-entraining effect tends to dominate the slump and the other effects, such as the rougher texture of RCA-MO compared to NL, plays a secondary role. However, at higher RCA content, it would appear that the lesser angularity of RCA-MO compared to NL becomes the dominant factor. On the other hand, for non-air-entrained PM mixes, the rougher surface texture of RCA-MO appears to be the dominating factor.

- In both air-entrained and non-entrained PV mixes, the combined rougher surface texture and greater angularity of RCA-VA compared to NG resulted in lower slump values at higher RCA contents.

- The main factors affecting the compressive and splitting tensile strength of RAC are the w/c ratio and air content. In fact, RCA content is not a major factor affecting the compressive strength of RAC, provided the RM quality is better than the NM. The degree of angularity of RCA rather than the RCA content has greater effect on the compressive strength of RAC.

- It has been observed that the main factors affecting the fresh and hardened density and elastic modulus of RAC are its w/c ratio, air content, and RCA content. In all PM and PV mixes, increasing the $\mathrm{w} / \mathrm{c}$ ratio, air content and RCA content, led to a corresponding 
decrease in the values of the preceding properties. The reduction was more dramatic for the PM mixes compared to the PV mixes due to the higher RMC in RCA-MO compared to RCA-VA.

- The TMV of RAC, rather than its RCA content, is the major factor governing its overall fresh and hardened density and elastic modulus. It can be asserted that the TMV captures the combined effect of w/c ratio, air content and RCA content on the above properties. 
Table 3.1: Average physical properties for coarse and fine aggregates (Abbas, 2007)

\begin{tabular}{|c|c|c|c|c|c|c|}
\hline & \multirow{2}{*}{ Aggregate } & \multirow{2}{*}{$\begin{array}{c}\text { Moisture } \\
\text { Content } \\
\text { (\%) }\end{array}$} & $\begin{array}{c}\text { Absorption } \\
\text { Content } \\
\text { (\%) }\end{array}$ & & \multicolumn{3}{|c|}{ Specific Gravity } & \\
\cline { 4 - 7 } & & & Bulk & SSD & Apparent & $\begin{array}{c}\text { RMC } \\
\text { (\%) }\end{array}$ \\
\hline RCA-MO & 1.1 & 5.4 & 2.31 & 2.42 & 2.64 & 41 \\
\hline RCA-VA & 1.3 & 3.3 & 2.42 & 2.50 & 2.64 & 23 \\
\hline Limestone (NL) & 0.2 & 0.34 & 2.70 & 2.71 & 2.73 & - \\
\hline River Gravel & 0.2 & 0.89 & 2.72 & 2.74 & 2.79 & - \\
\hline River Sand $^{*}$ & 4 & 0.54 & 2.70 & 2.72 & 2.76 & - \\
\hline
\end{tabular}

*Fineness modulus (F.M) of 2.60 .

Table 3.2: Mix proportions of PM mixes

\begin{tabular}{|c|c|c|c|c|c|c|c|c|}
\hline \multirow{3}{*}{ Mix ID } & \multirow{3}{*}{$\begin{array}{c}\text { RCA } \\
\text { Content } \\
\%\end{array}$} & \multirow{3}{*}{$\mathbf{w} / \mathbf{c}$} & \multicolumn{5}{|c|}{ Mix Proportions $\left(\mathrm{kg} / \mathrm{m}^{3}\right)$} & \multirow{3}{*}{$\begin{array}{l}\mathbf{A E}^{1} \\
\text { (ml) }\end{array}$} \\
\hline & & & \multirow{2}{*}{ Water } & \multirow{2}{*}{ Cement } & \multirow{2}{*}{ Sand } & \multicolumn{2}{|c|}{$\begin{array}{c}\text { Coarse } \\
\text { Aggregate } \\
\end{array}$} & \\
\hline & & & & & & RCA & NA & \\
\hline PM-100LA & 100 & 0.35 & 185 & 528 & 643 & 874 & 0 & 159 \\
\hline PM-100MA & 100 & 0.45 & 183 & 406 & 732 & 864 & 0 & 122 \\
\hline PM-100HA & 100 & 0.55 & 184 & 335 & 799 & 868 & 0 & 100 \\
\hline PM-100LA & 100 & 0,35 & 204 & 583 & 631 & 923. & 0 & 0 \\
\hline PMT-100MN & 100 & 0.45 & 205. & $=456$ & 743 & 925 & 0. & 0 \\
\hline PMe100HN & 100 & 0.55 & 206 & $\sqrt{375}$ & 816 & 9930 & 0 & 0 \\
\hline PM-50LA & 50 & 0.35 & 189 & 541 & 658 & 447 & 447 & 163 \\
\hline PM-50MA & 50 & 0.45 & 188 & 417 & 751 & 443 & 443 & 125 \\
\hline PM-50HA & 50 & 0.55 & 186 & 339 & 809 & 440 & 440 & 101 \\
\hline PM-50LN & 50 & 0.35 & 209 & 597 & 646 & 473 & 473. & 0 \\
\hline PM-50MN & 50 & 0.45 & 209 & 464 & 756 & 471 & 471 & 0 \\
\hline PM-50HN & 50 & 0.55 & 209 & 380 & 826 & 472 & 470 & 0 \\
\hline PM-30LA & 30 & 0.35 & 194 & 555 & 675 & 275 & 642 & 167 \\
\hline PM-30MA & 30 & 0.45 & 188 & 417 & 751 & 266 & 621 & 125 \\
\hline PM-30HA & 30 & 0.55 & 186 & 339 & 809 & 263 & 616 & 101 \\
\hline PM-30LN & 30 & 0.35 & 208 & 594 & 642 & 282 & 659. & 0 \\
\hline PM-30NAN & 30 & 0.45 & 210 & -467 & 761 & 285 & 663 & 0 \\
\hline PM-30109: & $3-30$ & 0.55 & 210 & $3822^{25}$ & 83.1 & 284 & 663 & 0. \\
\hline CL-LA & 0 & 0.35 & 199 & 570 & 693 & 0 & 942 & 171 \\
\hline CL-MA & 0 & 0.45 & 195 & 434 & 782 & 0 & 923 & 130 \\
\hline CL-HA & 0 & 0.55 & 194 & 353 & 842 & 0 & 915 & 103 \\
\hline CL-LN & 0 & 0.35 & 213 & 609 & 659 & 0. & 965 & 0 \\
\hline CL-MN & 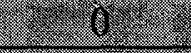 & 0,45 & 215 & -479 & 781 & 0 & 973 & 0 \\
\hline CLHIN & $x=$ & 0.55 & Six & 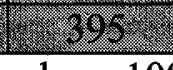 & 15ren & 0 & 977 & 0 \\
\hline
\end{tabular}

${ }^{1} \mathrm{AEA}$ : air entraining agent (Micro Air 940) $30 \mathrm{ml}$ per $100 \mathrm{~kg} / \mathrm{m}^{3}$ cement was used 
Table 3.3: Mix proportions of PV mixes

\begin{tabular}{|c|c|c|c|c|c|c|c|c|}
\hline \multirow{3}{*}{ Mix ID } & \multirow{3}{*}{$\begin{array}{c}\text { RCA } \\
\text { Content } \\
\%\end{array}$} & \multirow{3}{*}{$\mathbf{w} / \mathbf{c}$} & \multicolumn{5}{|c|}{ Mix Proportions $\left(\mathrm{kg} / \mathrm{m}^{3}\right)$} & \multirow{3}{*}{$\begin{array}{l}\mathbf{A E}^{1} \\
(\mathrm{ml})\end{array}$} \\
\hline & & & \multirow[t]{2}{*}{ Water } & \multirow[t]{2}{*}{ Cement } & \multirow[t]{2}{*}{ Sand } & \multicolumn{2}{|c|}{$\begin{array}{c}\text { Coarse } \\
\text { Aggregate }\end{array}$} & \\
\hline & & & & & & RCA & NA & \\
\hline PV-100LA & 100 & 0.35 & 190 & 543 & 661 & 899 & 0 & 163 \\
\hline PV-100MA & 100 & 0.45 & 184 & 410 & 738 & 871 & 0 & 123 \\
\hline PV-100HA & 100 & 0.55 & 186 & 340 & 810 & 880 & 0 & 101 \\
\hline PV-100EN & -100 & 0.35 & 206 & 588 & 636 & 931 & 0 & 0 \\
\hline PV-100MN & 100 & 0,45 & 206 & 459 & 748 & 932 & 0 & 0 \\
\hline PV-100HN & 100 & 0.55 & 209 & 380 & 826 & 941 & 0. & 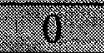 \\
\hline PV-50LA & 50 & 0.35 & 192 & 548 & 667 & 453 & 453 & 165 \\
\hline PV-50MA & 50 & 0.45 & 188 & 418 & 754 & 445 & 445 & 126 \\
\hline PV-50HA & 50 & 0.55 & 190 & 346 & 824 & 448 & 448 & 102 \\
\hline PV-50IN & 50 & 0,35 & 208 & 593. & 642 & 470 & 470 & 0. \\
\hline PV-50MA & 50 & 0.45 & 209 & 464 & 756 & 471 & 471 & 0. \\
\hline PV-50HN & 50 & 0.55 & 210 & 382 & 831 & 473 & 473 & 0 \\
\hline PV-30LA & 30 & 0.35 & 192 & 549 & 668 & 272 & 636 & 165 \\
\hline PV-30MA & 30 & 0.45 & 189 & 421 & 758 & 268 & 626 & 126 \\
\hline PV-30HA & 30 & 0.55 & 190 & 345 & 824 & 268 & 627 & 102 \\
\hline PV-30LN & 30 & 0.35 & 209 & 598 & 647 & 284 & 663. & 0 \\
\hline PV-30MN & 30 & 0.45 & 212 & 471 & 767 & 287 & 669 & 0. \\
\hline DV-30HN & 30 & 0.55 & 211 & 384 & 836 & 286 & 667. & 0 \\
\hline CG-LA & 0 & 0.35 & 194 & 555 & 675 & 0 & 918 & 167 \\
\hline CG-MA & 0 & 0.45 & 191 & 424 & 765 & 0 & 903 & 128 \\
\hline CG-HA & 0 & 0.55 & 196 & 356 & 849 & 0 & 923 & 103 \\
\hline CG-EN & 0 & 0.35 & 212 & 606 & 655 & 0 & 959 & 0 \\
\hline CG-MN & 0 & 0.45 & 215 & 477. & 778 & 0 & 969 & 0 \\
\hline CG-HN & 0 & 0.55 & 215 & 390 & 849 & 0 & 967 & 0 \\
\hline
\end{tabular}

${ }^{1}$ AEA: air entraining agent (Micro Air 940) $30 \mathrm{ml}$ per $100 \mathrm{~kg} / \mathrm{m}^{3}$ cement was used. 
Table 3.4: Fresh properties of PM mixes

\begin{tabular}{|c|c|c|c|}
\hline Mix ID & Slump (mm) & Air Content (\%) & Fresh Density $\left(\mathrm{kg} / \mathrm{m}^{3}\right)$ \\
\hline PM-100LA & 85 & 5.8 & 2227 \\
\hline PM-100MA & 150 & 7.4 & 2181 \\
\hline PM-100HA & 195 & 6.6 & 2181 \\
\hline PM-100LIN & 105 & $2=2.2$ & 2338 \\
\hline PM-100MN & -6185 & 2.0 & -2324 \\
\hline PM-10014N & 205 & $3-1.4 .2$ & $2=2324$ \\
\hline PM-50LA & 95 & 6.0 & 2281 \\
\hline PM-50MA & 145 & 8.0 & 2238 \\
\hline PM-50HA & 200 & 7.0 & 2210 \\
\hline PM-50LN & 55 & 2.0 & 2395 \\
\hline PM-50MN & 140 & 1.9 & 2366 \\
\hline PM-50MN & $180=$ & $x=1.6$ & 2352 \\
\hline PM-30LA & 60 & 5.0 & 2338 \\
\hline PM-30MA & 150 & 7.2 & 2238 \\
\hline PM-30HA & 165 & 7.9 & 2210 \\
\hline PM-30LN & 90 & $x \sqrt{2.1}$ & 2381 \\
\hline PM-30MN & $=165$ & $2=-1.7$ & $(13 x-2381$ \\
\hline PM-30HN & 200 & 2.0 & 2366 \\
\hline CL-LA & 40 & 4.5 & 2366 \\
\hline CL-MA & 110 & 6.4 & 2295 \\
\hline CL-HA & 180 & 6.4 & 2267 \\
\hline CL-LN & 90 & $3 \quad 1.8$ & 2409. \\
\hline Cl-MN & 155 & 1201.3 & 2409 \\
\hline $\mathrm{CL}-\mathrm{HN}$ & 200 & $20.9-1.0$ & -2409 \\
\hline
\end{tabular}


Table 3.5: Fresh properties of PV mixes

\begin{tabular}{|c|c|c|c|}
\hline Mix ID & Slump (mm) & Air Content (\%) & Fresh Density $\left(\mathrm{kg} / \mathrm{m}^{3}\right)$ \\
\hline PV-100LA & 50 & 5.0 & 2315 \\
\hline PV-100MA & 150 & 6.9 & 2224 \\
\hline PV-100HA & 190 & 6.6 & 2238 \\
\hline PV-100LN: & $100: 3$ & 1.8 & 2381 \\
\hline PV-100MN & 160 & $5=1.8$ & 2366 \\
\hline PV-100HN & 25200 & $2 x=-1.25=9$ & 2381 \\
\hline PV-50LA & 90 & 5.2 & 2335 \\
\hline PV-50MA & 165 & 6.0 & 2272 \\
\hline PV-50HA & 215 & 5.6 & 2278 \\
\hline PV-50LN & 120 & 1.8 & 2404 \\
\hline PV-50MN & 215 & 1.0 & 2393 \\
\hline PV-5.0AN & 220 & 0.6 & 2394 \\
\hline PV-30LA & 95 & 5.0 & 2339 \\
\hline PV-30MA & 170 & 6.2 & 2284 \\
\hline PV-30HA & 215 & 6.0 & 2277 \\
\hline PV-30LN & 9105 & $1.8=2-x-2$ & 2422 \\
\hline PV-30MN & -200 & $1.1=2$ & 2427 \\
\hline PV-30HiN & 225 & 0.6 & $2409=$ \\
\hline CG-LA & 130 & 5.4 & 2349 \\
\hline CG-MA & 175 & 6.4 & 2290 \\
\hline CG-HA & 230 & 4.8 & 2331 \\
\hline CGalen & 160 & 1.7 & 2438 \\
\hline CG-MN & 220 & -1.0 & 2446 \\
\hline CG-HN = & 230 & 0.4 & 2429 \\
\hline
\end{tabular}


Table 3.6: Hardened properties of PM mixes

\begin{tabular}{|c|c|c|c|c|}
\hline Mix ID & $\begin{array}{c}\gamma_{\mathbf{c}} \\
\left(\mathbf{k g} / \mathbf{m}^{3}\right)\end{array}$ & $\begin{array}{c}\mathrm{f}_{\mathrm{t}} \\
(\mathrm{MPa})\end{array}$ & $\begin{array}{c}\mathbf{f}_{\mathrm{c}}(28 \text { days }) \\
(\mathrm{MPa})\end{array}$ & $\begin{array}{c}\mathrm{E}_{\mathrm{c}} \\
(\mathrm{GPa})\end{array}$ \\
\hline PM-100LA & 2233 & 4.5 & 44.8 & 27.4 \\
\hline PM-100MA & 2138 & 3.8 & 30.9 & 20.8 \\
\hline PM-100HA & 2144 & 3.6 & 19.8 & 24.5 \\
\hline PM-100LN & 2299 & 4.2 & 51.5 & 31.0 \\
\hline PM-100MN & 2302 & 4 & 43.0 & 28.5 \\
\hline PM-100HN & 2298 & 4.2 & 31.5 & 27.1 \\
\hline PM-50LA & 2274 & 4.2 & 41.3 & 28.4 \\
\hline PM-50MA & 2179 & 3.8 & 32.5 & 25.8 \\
\hline PM-50HA & 2167 & 3.3 & 25.2 & 24.3 \\
\hline PM-50LN & 2325 & 4.9 & 54.2 & 33.8 \\
\hline PM-50MN & 2333 & 4.3 & 49.6 & 32 \\
\hline PM-50HN & 2352 & 4.1 & 42.4 & 28.8 \\
\hline PM-30LA & 2293 & 4.2 & 47.8 & 31.7 \\
\hline PM-30MA & 2234 & 3.9 & 33.1 & 26.8 \\
\hline PM-30HA & 2208 & 3.4 & 28.4 & 25.2 \\
\hline PM-30MN & 2374 & 4.5 & 57.5 & 34.1 \\
\hline PM-30MN & 2372 & 4.4 & 48.1 & 32.4 \\
\hline PM-30HN & 2359 & 3.4 & 38.7 & 30.6 \\
\hline CL-LA & 2322 & 4.2 & 46.6 & 34.4 \\
\hline CL-MA & 2257 & 3.5 & 36.2 & 31 \\
\hline CL-HA & 2236 & 3.4 & 28.8 & 26 \\
\hline CL-LN & 2388 & 4.5 & 54.4 & 36.7 \\
\hline CL-MN & 2377 & 4 & 45.3 & 34.5 \\
\hline CL-11N & 2377 & 3.5 & 37.5 & 32.1 \\
\hline & & & & \\
\hline \hline
\end{tabular}


Table 3.7: Hardened properties of PV mixes

\begin{tabular}{|c|c|c|c|c|}
\hline Mix ID & $\begin{array}{c}\gamma_{\mathbf{c}} \\
\left(\mathbf{k g} / \mathbf{m}^{3}\right)\end{array}$ & $\begin{array}{c}\mathbf{f}_{\mathbf{f}} \\
(\mathrm{MPa})\end{array}$ & $\begin{array}{c}\mathbf{f}_{\mathbf{c}}(28 \text { days }) \\
(\mathrm{MPa})\end{array}$ & $\begin{array}{c}\mathrm{E}_{\mathrm{c}} \\
(\mathrm{GPa})\end{array}$ \\
\hline PV-100LA & 2307 & 4.2 & 44.9 & 30.9 \\
\hline PV-100MA & 2233 & 3.4 & 34.3 & 25.9 \\
\hline PV-100HA & 2196 & 2.5 & 26.8 & 23.0 \\
\hline PV-100LN & 2356 & 4.0 & 54.7 & 34.2 \\
\hline PV-100MN & 2289 & 4.4 & 43.9 & 31.4 \\
\hline PV-100HN & 2275 & 3.0 & 37.9 & 29.3 \\
\hline PV-50LA & 2325 & 3.9 & 36.6 & 32.9 \\
\hline PV-50MA & 2273 & 4.0 & 36.6 & 29.4 \\
\hline PV-50HA & 2255 & 3.5 & 26.5 & 26.8 \\
\hline PV-50LN & 2388 & 4.1 & 48.6 & 35.3 \\
\hline PY-50MN & 2392 & 4.6 & 42.6 & 32.3 \\
\hline PV-50HN & 2393 & 4.3 & 36.1 & 30.2 \\
\hline PV-30LA & 2329 & 5.3 & 40.9 & 32.9 \\
\hline PV-30MA & 2277 & 3.7 & 34.7 & 28.7 \\
\hline PV-30HA & 2252 & 3.5 & 27.0 & 27.0 \\
\hline PV-30LN & 2396 & 4.9 & 53.5 & 36.4 \\
\hline PY-30MN & 2393 & 5.6 & 46.4 & 32.8 \\
\hline PY-30HN & 2392 & 4.8 & 39.1 & 32.1 \\
\hline CG-LA & 2308 & 3.9 & 40.8 & 29.9 \\
\hline CG-MA & 2281 & 3.8 & 33.7 & 28.8 \\
\hline CG-HA & 2270 & 4.1 & 30.0 & 29.1 \\
\hline CG-16N & 2416 & 3.7 & 53.1 & 35.1 \\
\hline CG-MN & 2417 & 4.4 & 40.7 & 35.4 \\
\hline CG-HN & 2462 & 4.5 & 40.5 & 33.5 \\
\hline
\end{tabular}




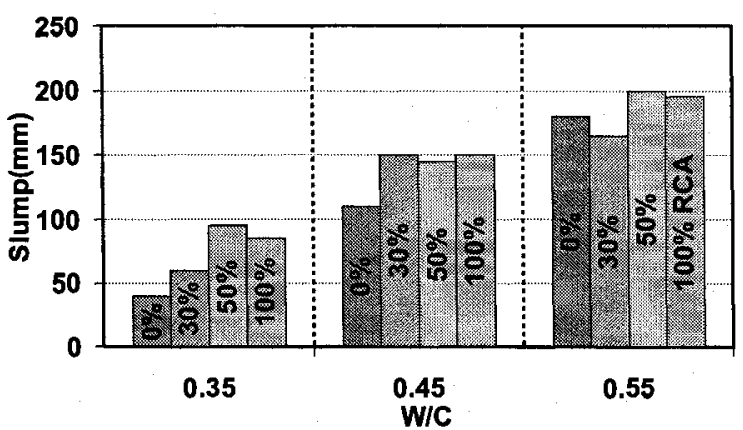

(a) PM - AE

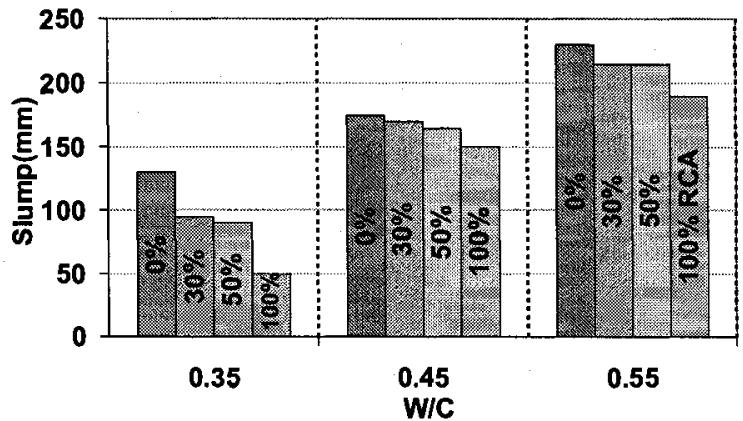

(c) PV - AE

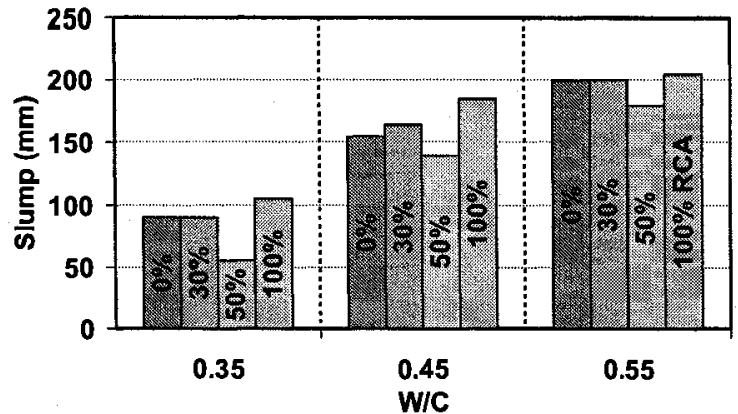

(b) PM - NAE

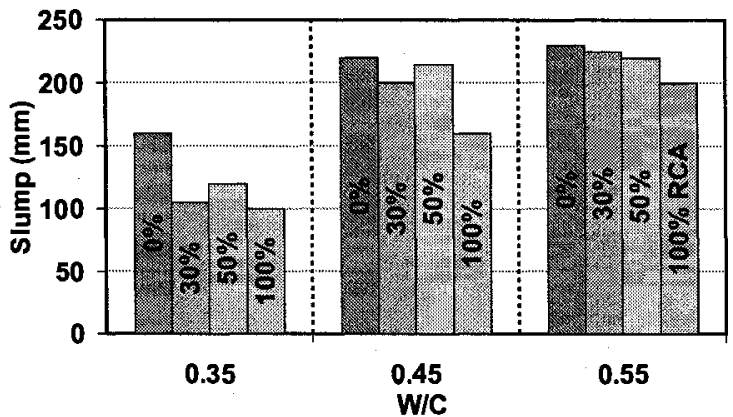

(d) PV - NAE

Figure 3.1: Slump versus RCA content for different $w / c$ ratios

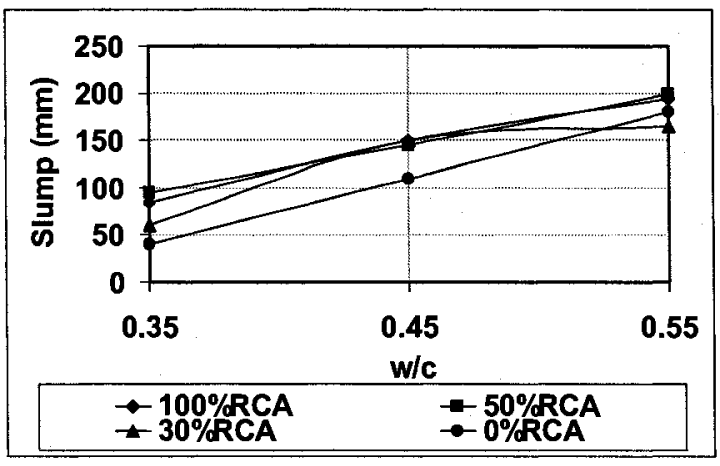

(a) $\mathrm{PM}-\mathrm{AE}$

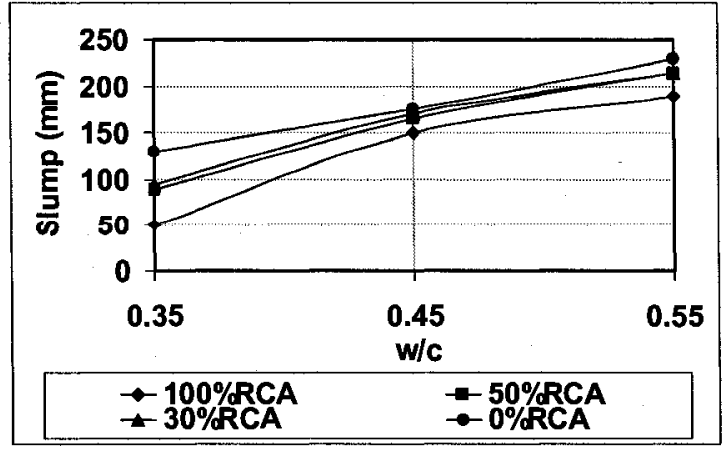

(c) PV - AE

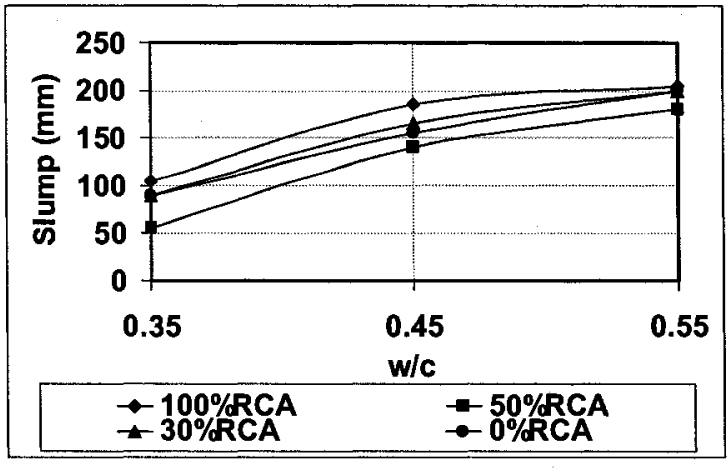

(b) PM - NAE

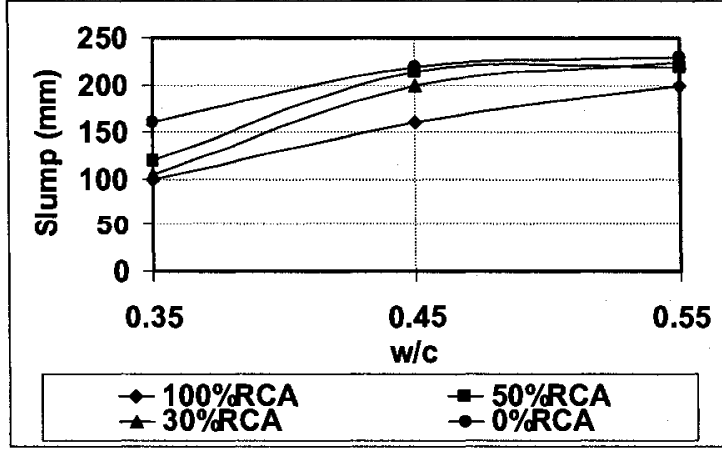

(d) PV - NAE

Figure 3.2: Slump versus $w / c$ ratio for different RCA content levels 


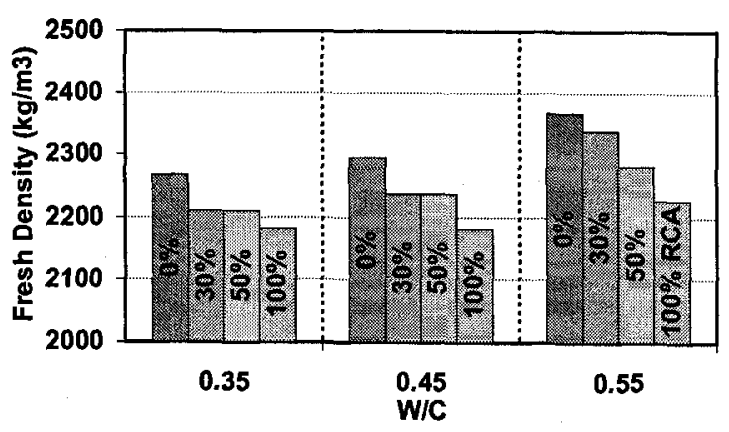

(a) $\mathrm{PM}-\mathrm{AE}$

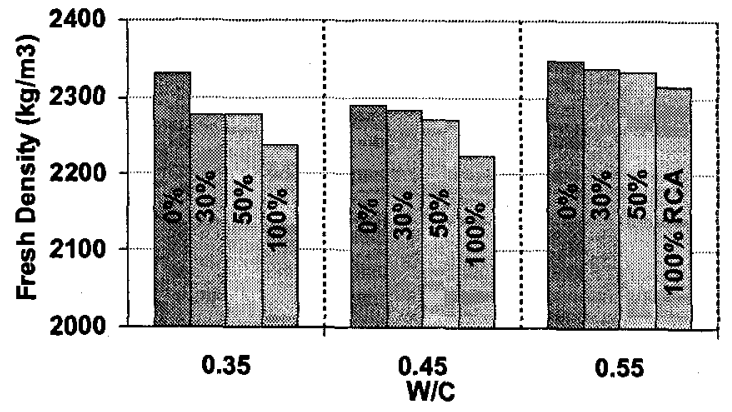

(c) $\mathrm{PV}-\mathrm{AE}$

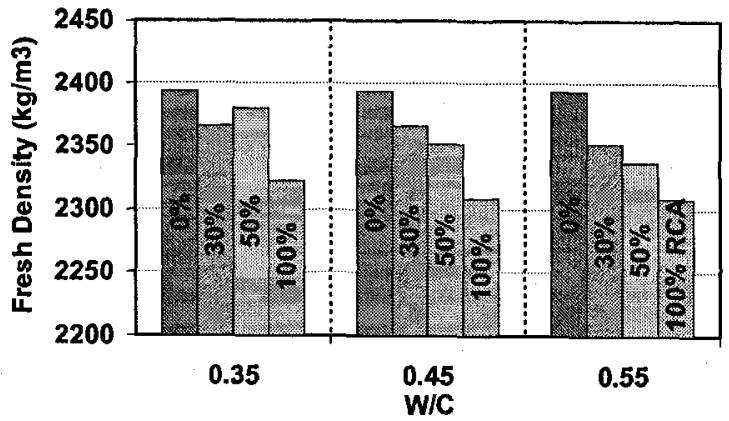

(b) PM - NAE

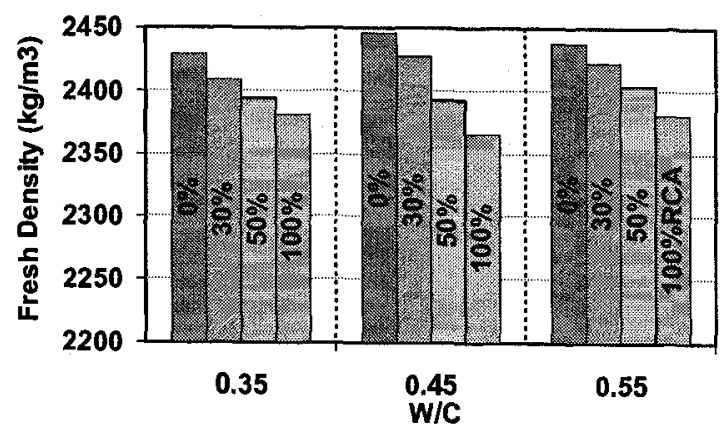

(d) PV - NAE

Figure 3.3: Fresh density versus RCA content for different $w / c$ ratios

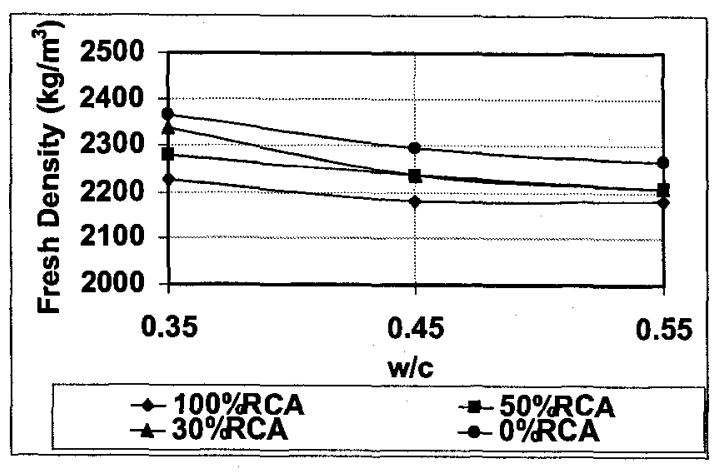

(a) PM - AE

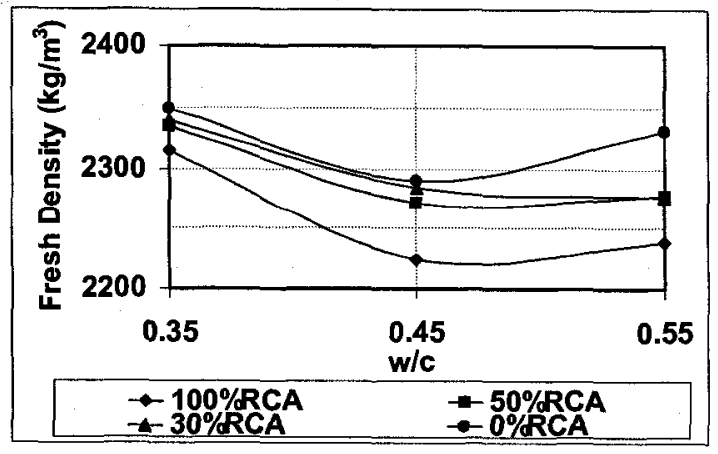

(c) PV - AE

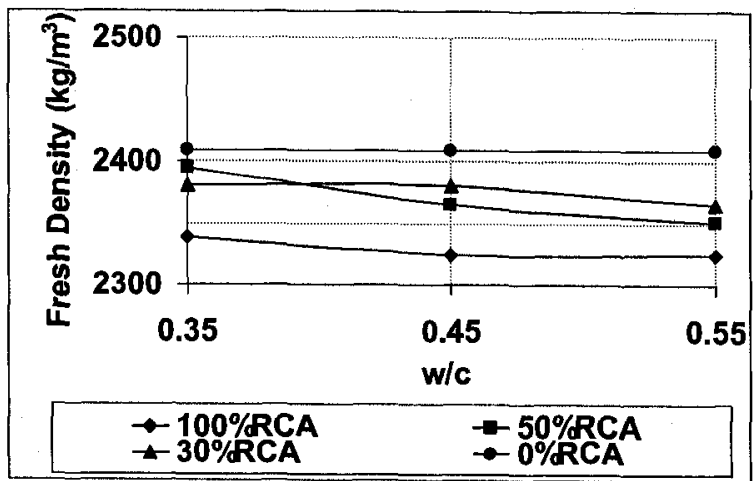

(b) PM - NAE

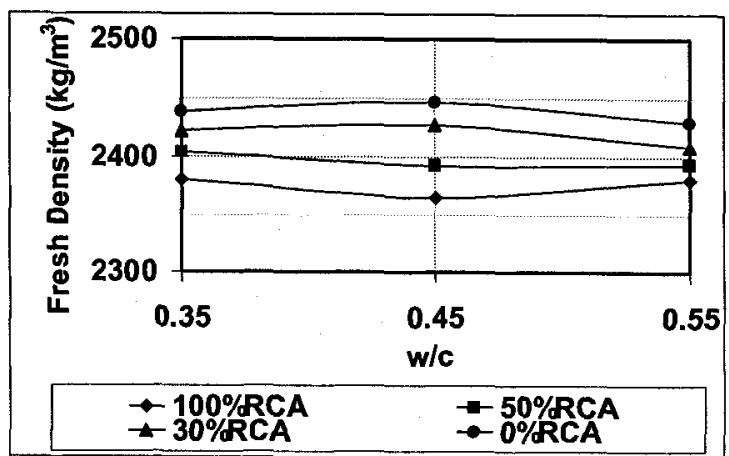

(d) PV - NAE

Figure 3.4: Variation of fresh density with $w / c$ ratio for different RCA content levels 


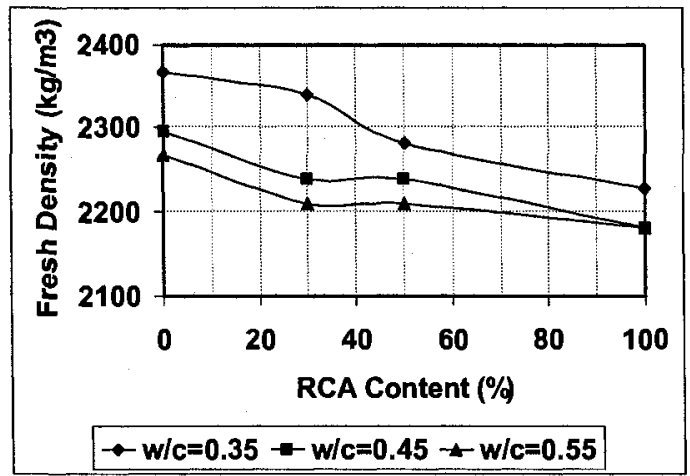

(a) PM (AE)

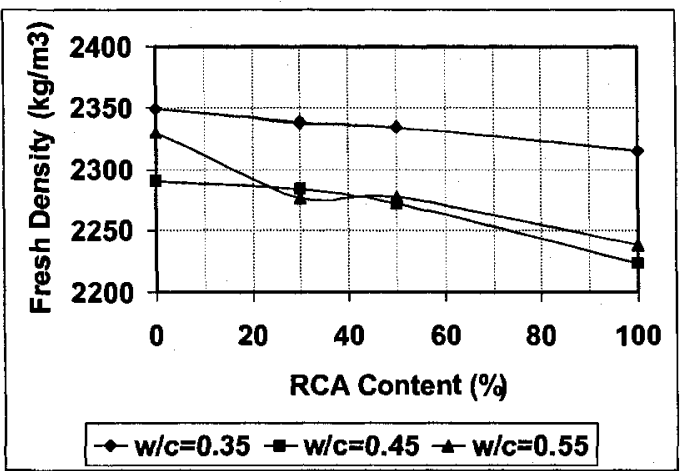

(c) PV (AE)

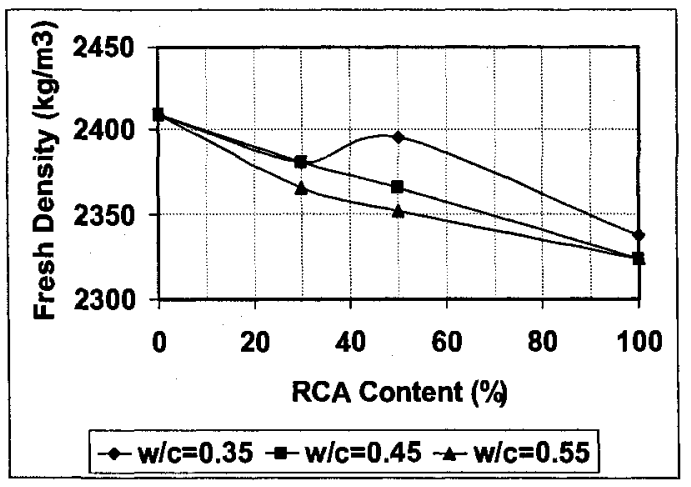

(b) PM (NAE)

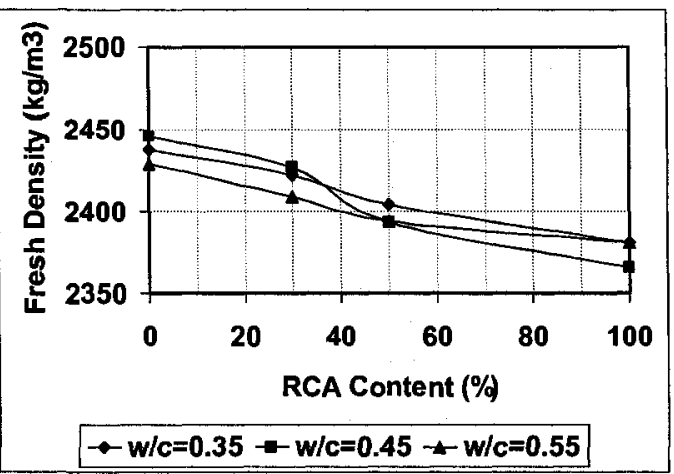

(d) PV (NAE)

Figure 3.5: Variation of fresh density with RCA content at different $w / c$ ratios

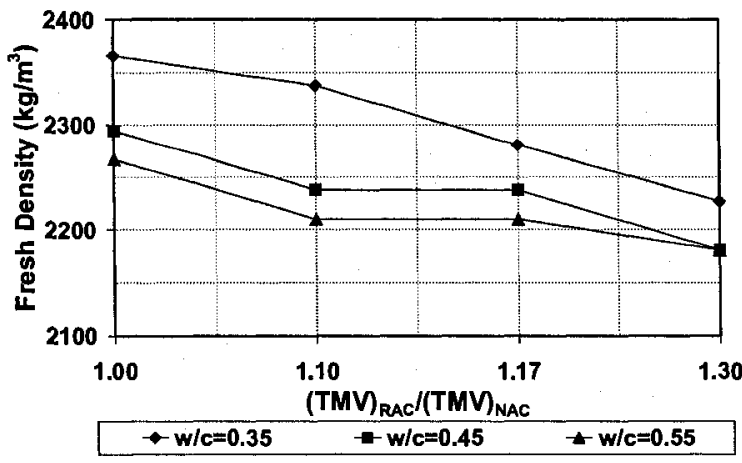

(a) PM (AE)

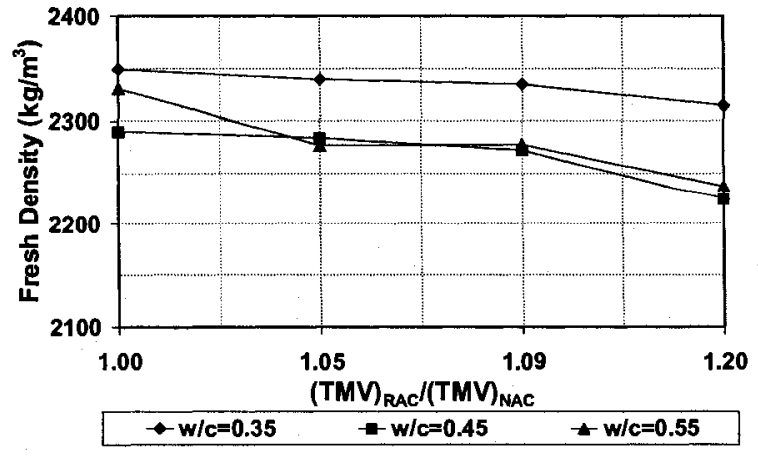

(c) PV (AE)

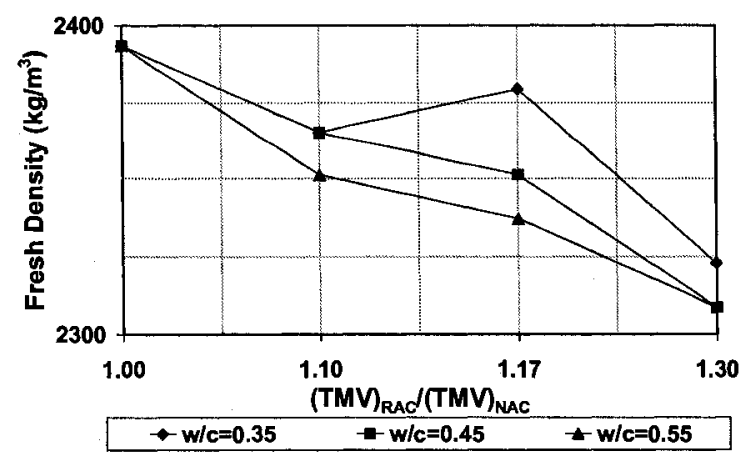

(b) PM (NAE)

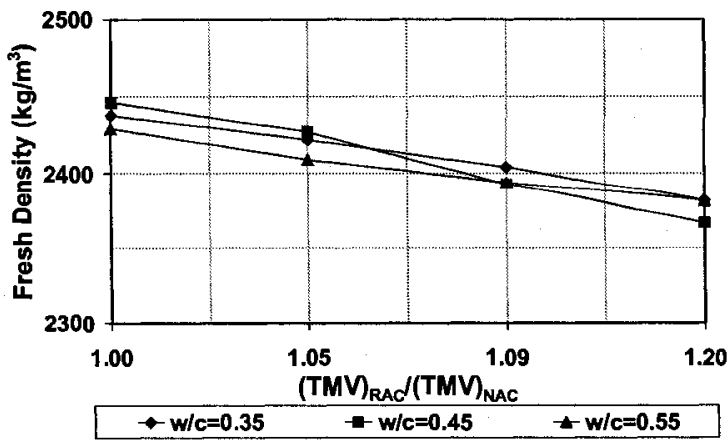

(d) PV (NAE)

Figure 3.6: Variation of fresh density with TMV for different $w / c$ ratios 


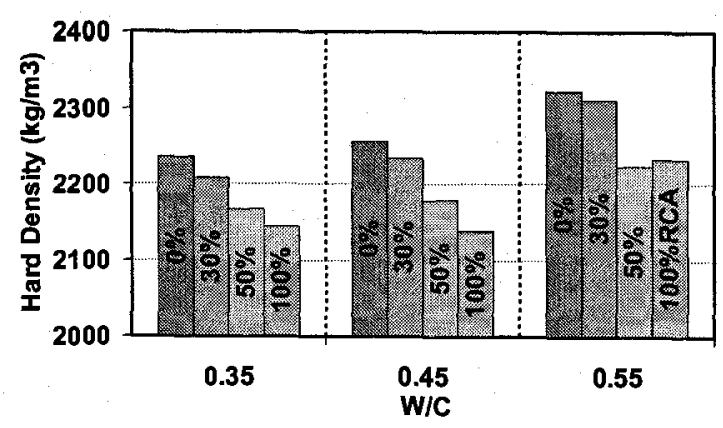

(a) $\mathrm{PM}-\mathrm{AE}$

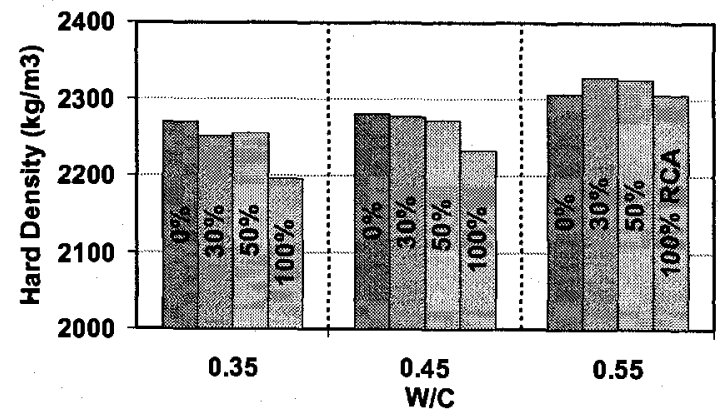

(c) $\mathrm{PV}-\mathrm{AE}$

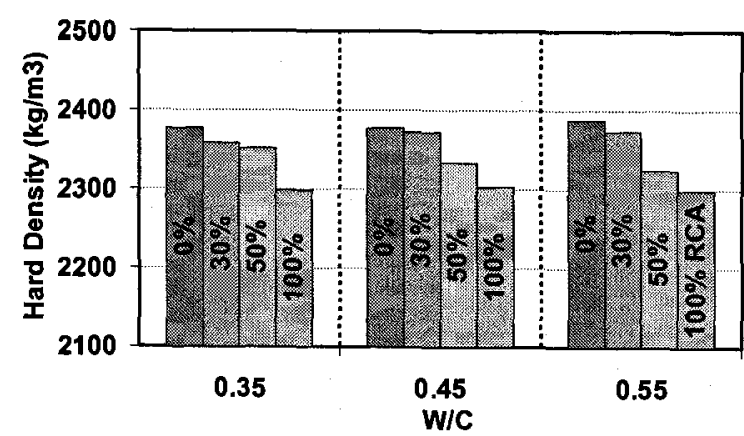

(b) PM - NAE

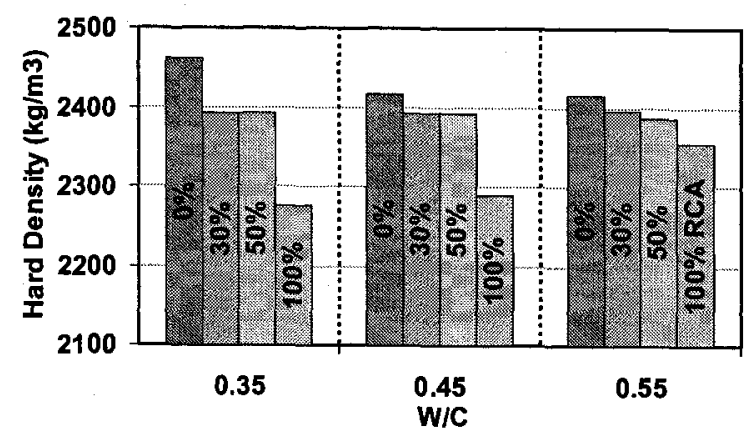

(d) PV - NAE

Figure 3.7: Hardened density versus RCA content for different w/c ratios

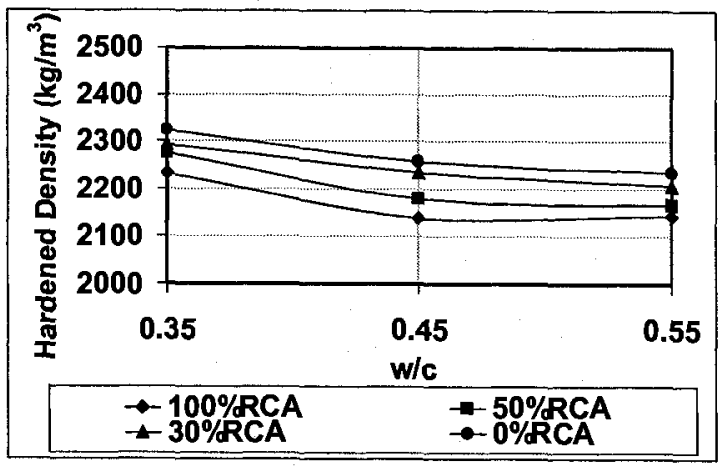

(a) $\mathrm{PM}-\mathrm{AE}$

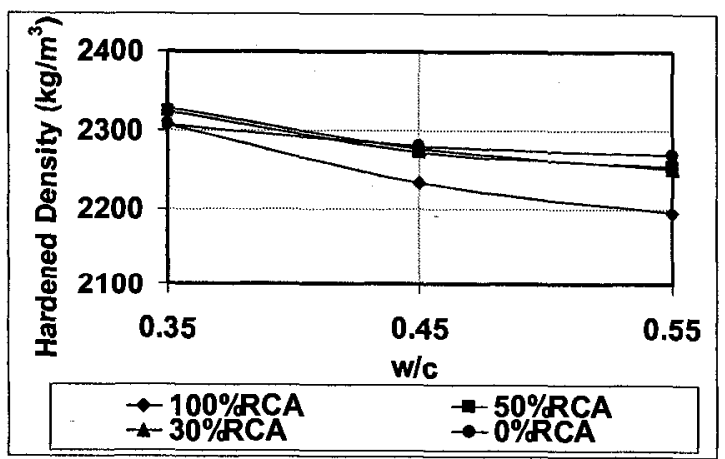

(c) $\mathrm{PV}-\mathrm{AE}$

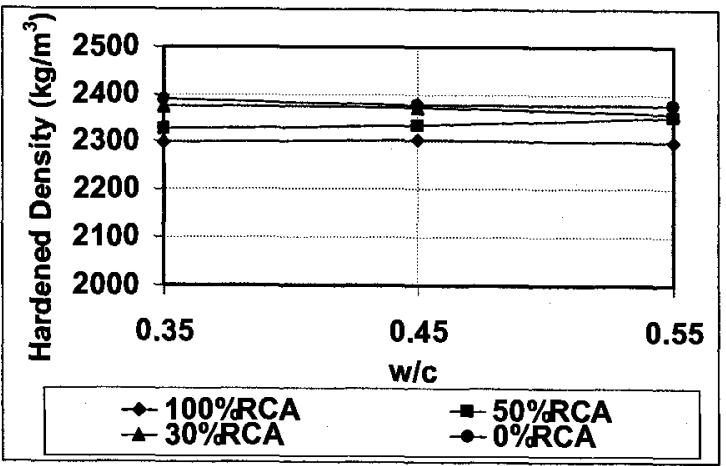

(b) PM - NAE

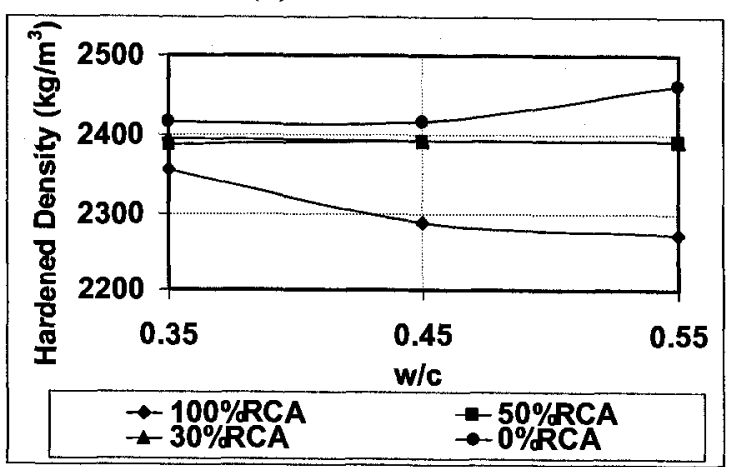

(d) PV - NAE

Figure 3.8: Variation of hardened density with $w / c$ ratio at RCA content levels 


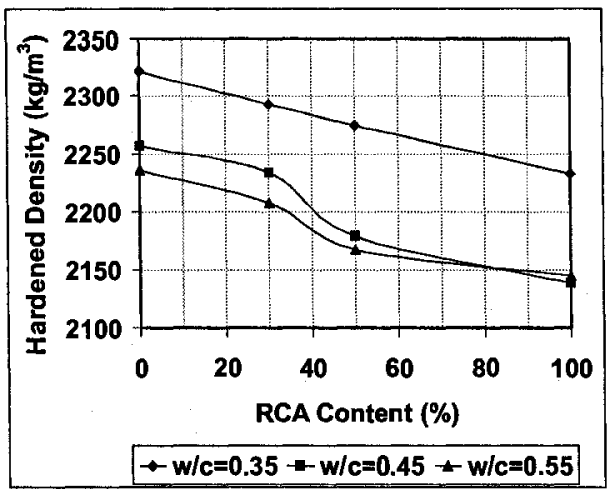

(a) PM - AE

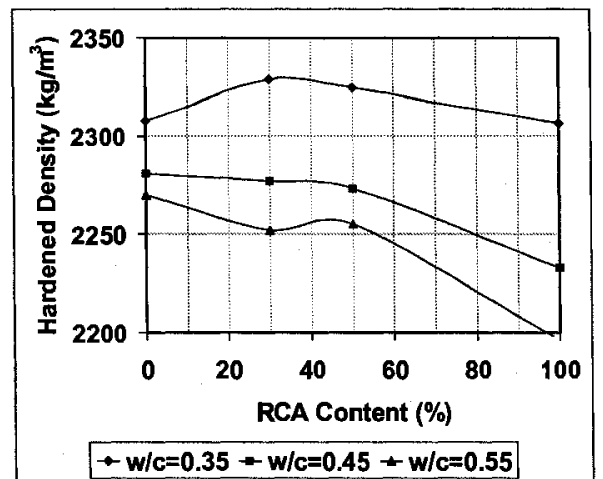

(c) PV - AE

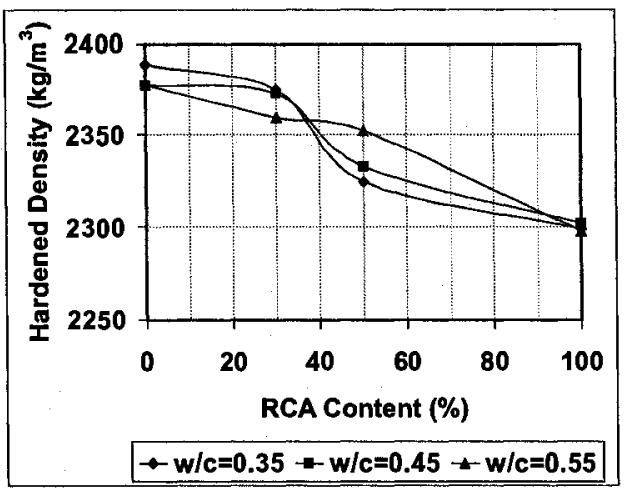

(b) PM - NAE

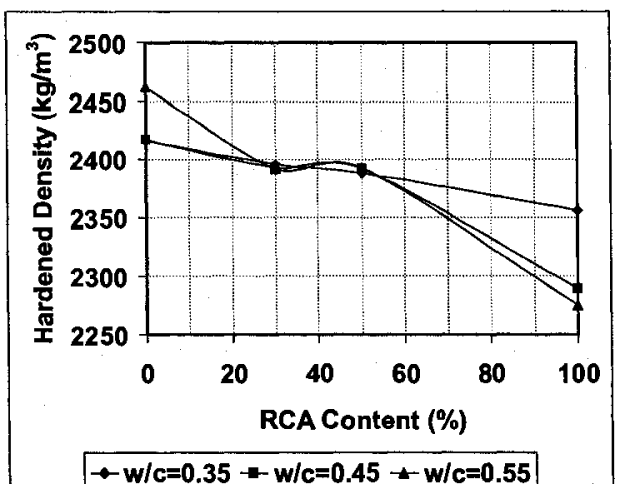

(d) PV - NAE

Figure 3.9: Variation of hardened density with RCA content for different w/c ratios

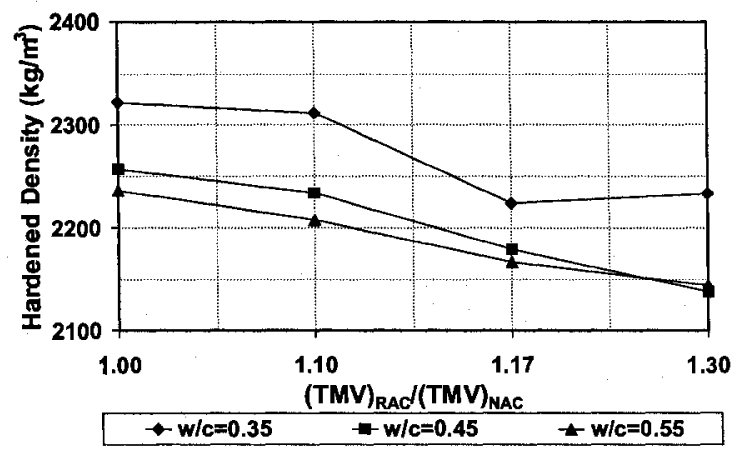

(a) PM - AE

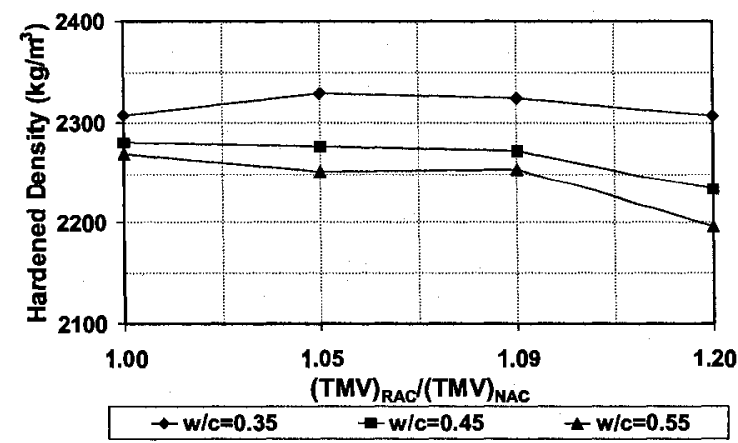

(c) PV - AE

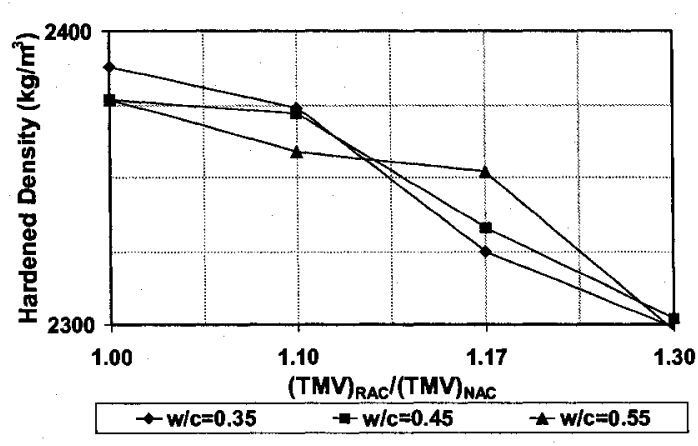

(b) PM - NAE

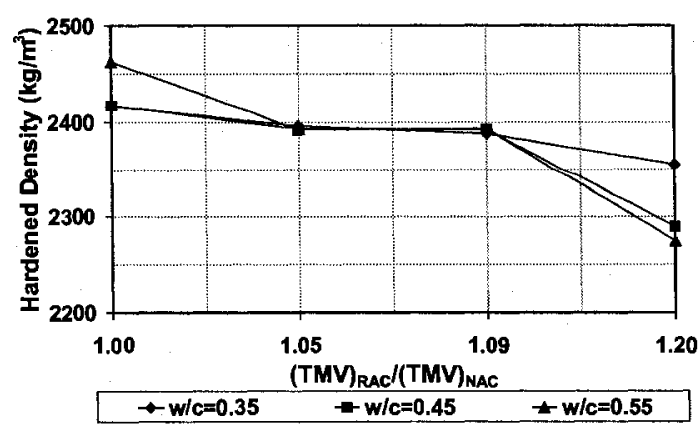

(d) PV - NAE

Figure 3.10: Variation of hardened density with TMV at different w/c ratios 


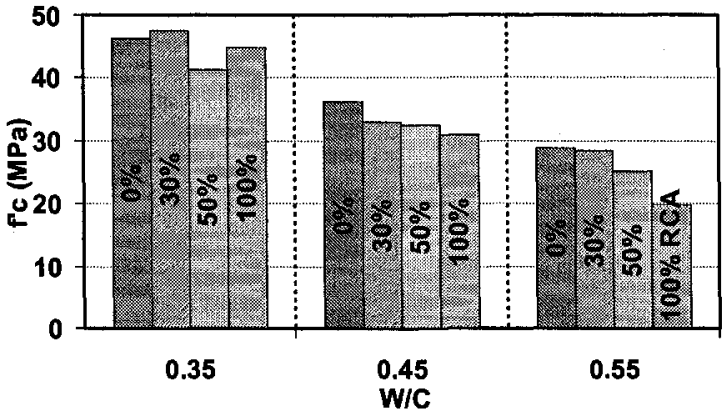

(a) $\mathrm{PM}-\mathrm{AE}$

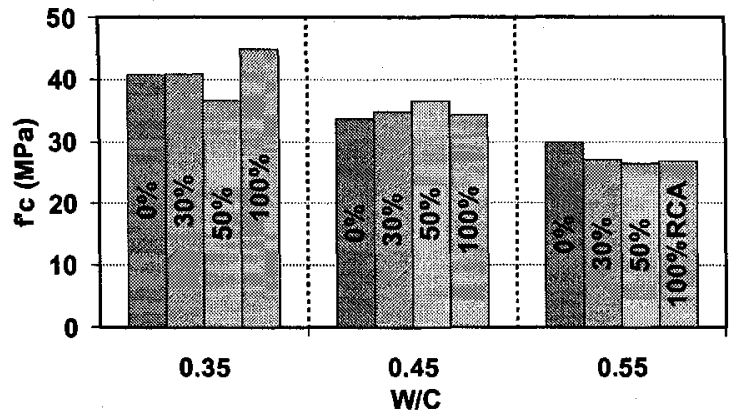

(c) PV - AE

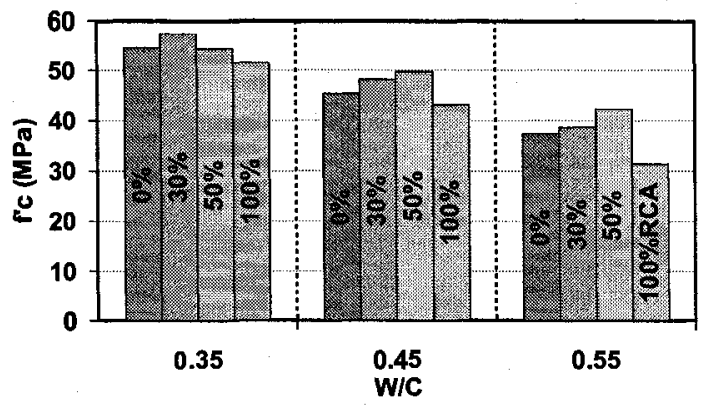

(b) PM - NAE

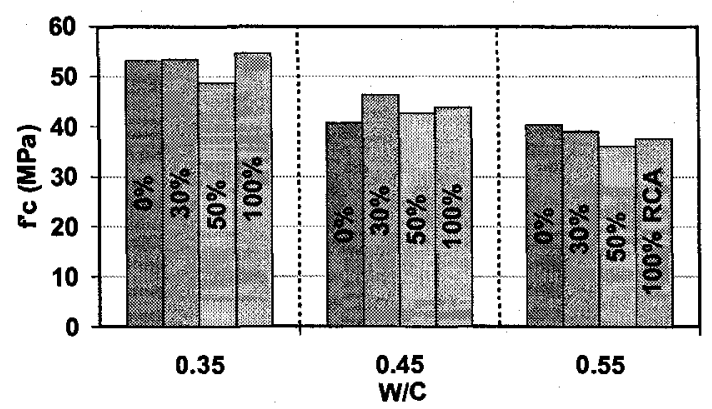

(d) PV - NAE

Figure 3.11: Compressive strength versus RCA content for different $w / c$ ratios

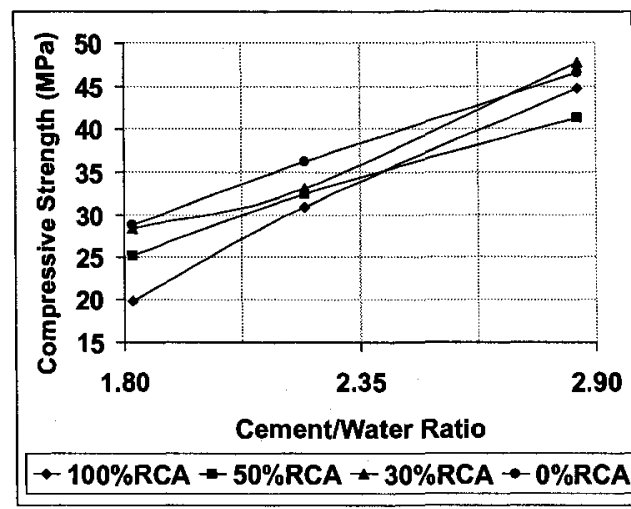

(a) $\mathrm{PM}-\mathrm{AE}$

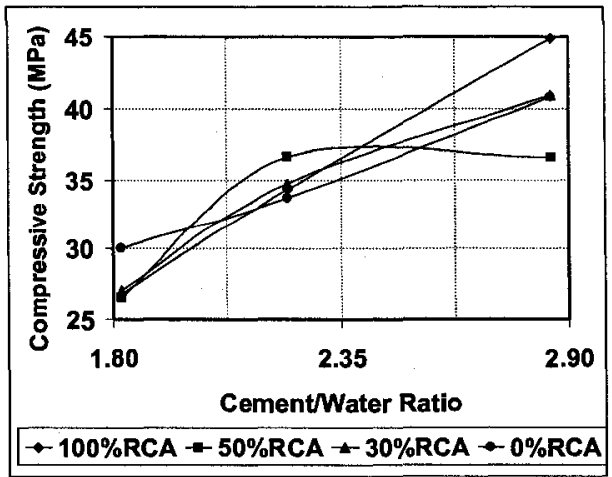

(c) PV - AE

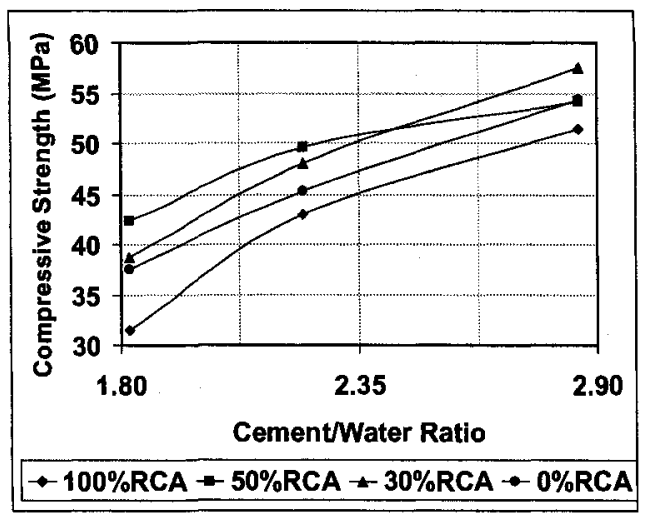

(b) PM - NAE

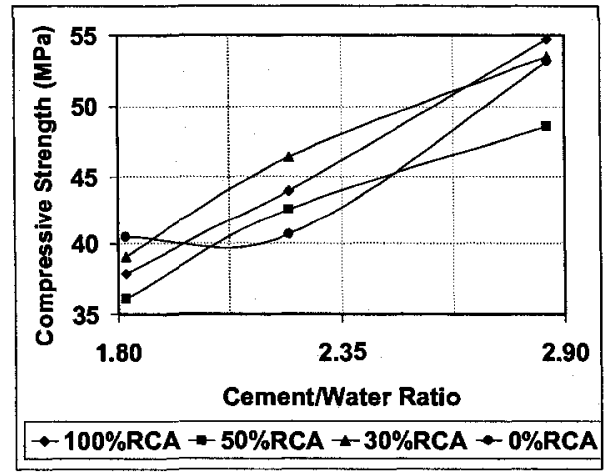

(d) PV - NAE

Figure 3.12: Compressive strength versus $\mathrm{c} / \mathrm{w}$ ratio for different RCA content levels 


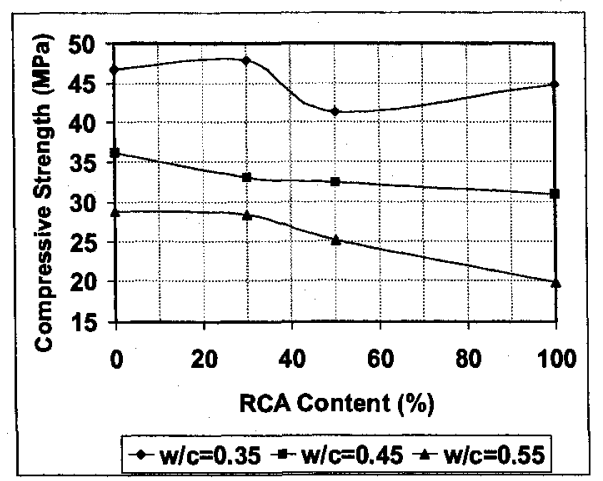

(a) $\mathrm{PM}-\mathrm{AE}$

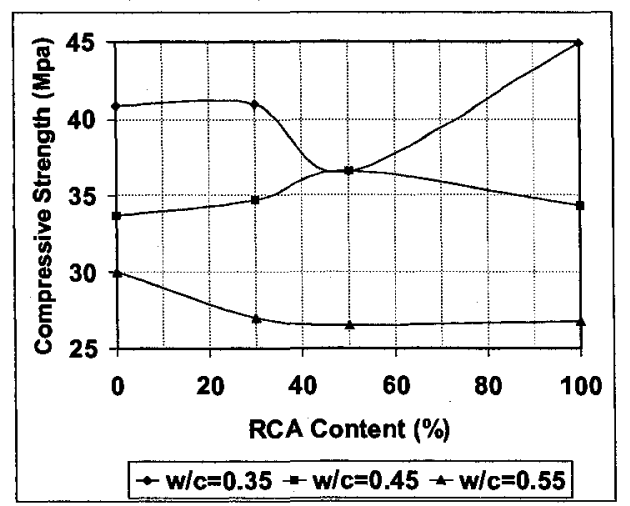

(c) PV - AE

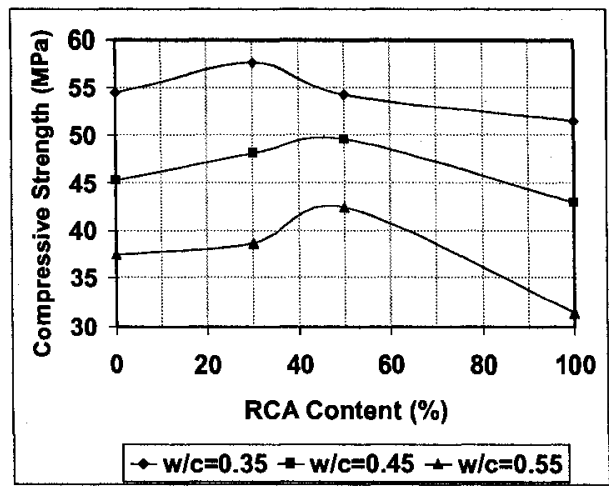

(b) PM - NAE

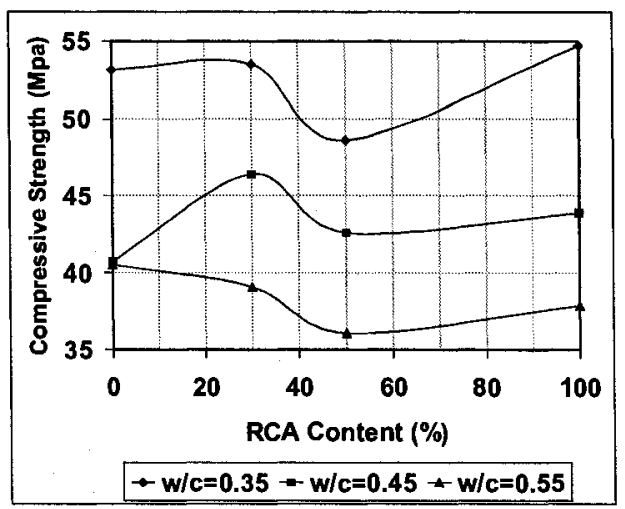

(d) PV - NAE

Figure 3.13: Variation of compressive strength with RCA content for different $w / c$ ratios

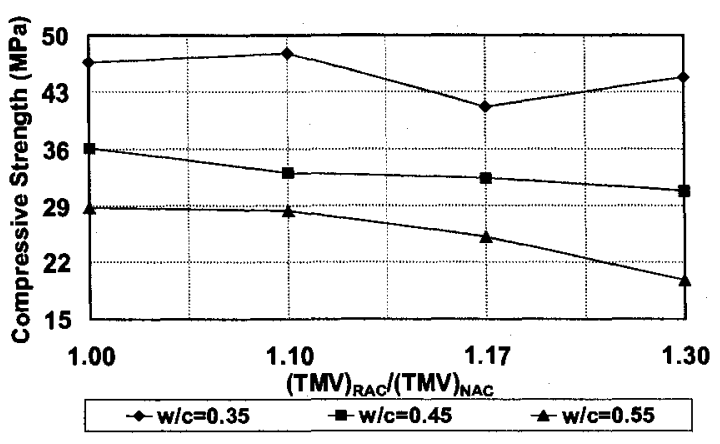

(a) $\mathrm{PM}-\mathrm{AE}$

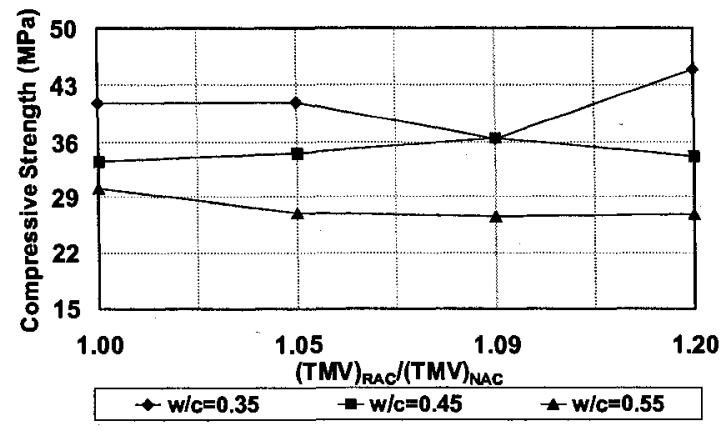

(c) PV - AE

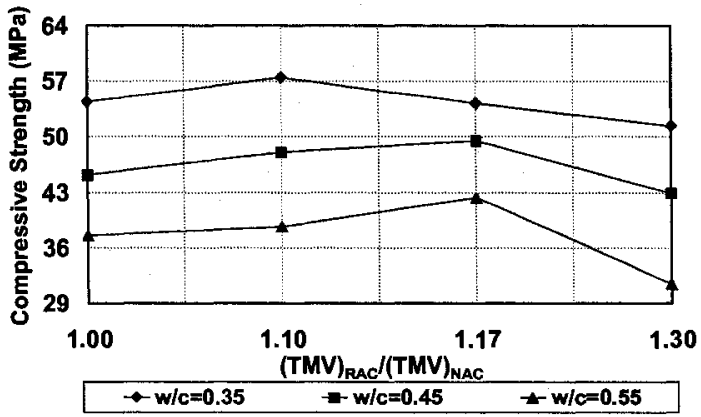

(b) PM - NAE

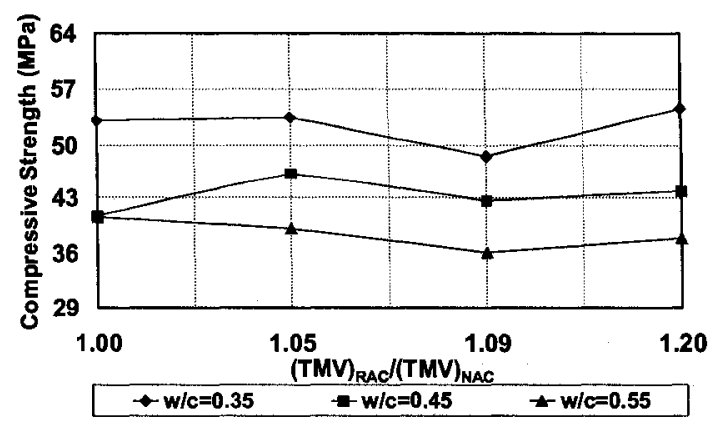

(d) PV - NAE

Figure 3.14: Variation of compressive strength with TMV for different $w / c$ ratios 


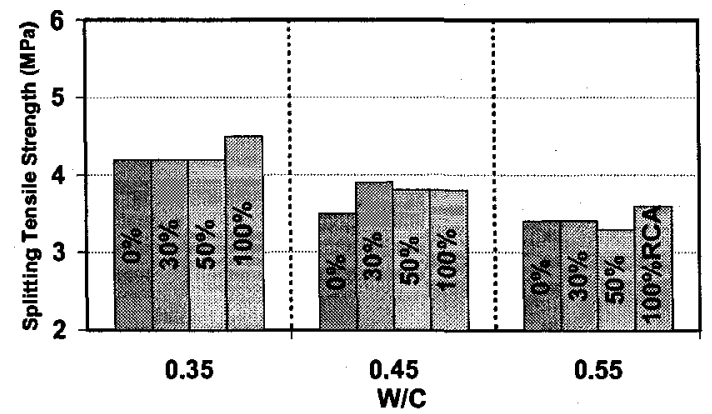

(a) PM - AE

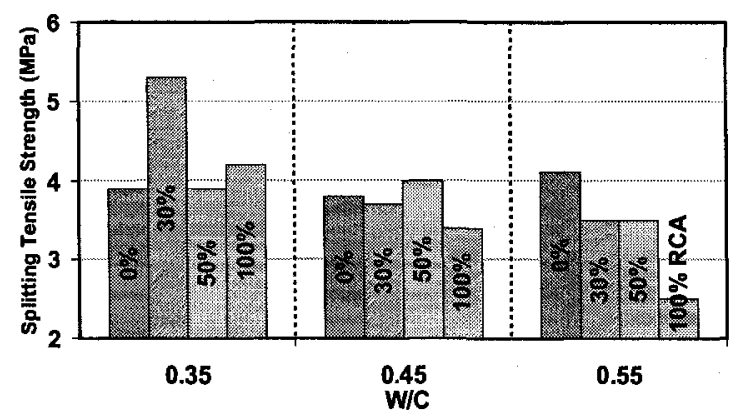

(c) PV - AE

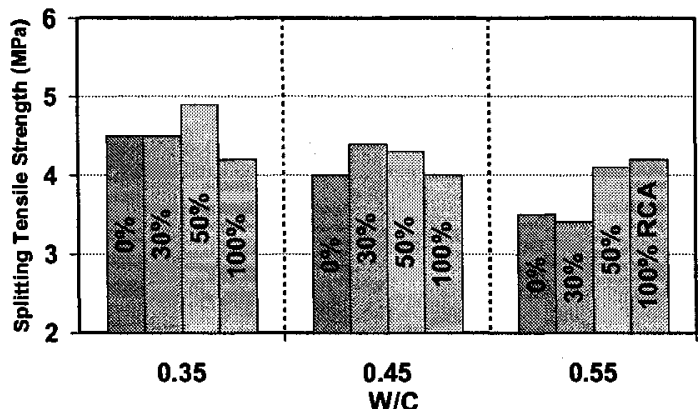

(b) PM - NAE

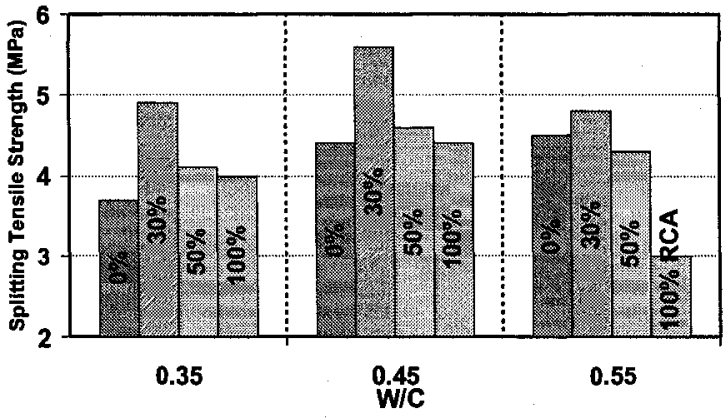

(d) PV - NAE

Figure 3.15: Splitting tensile strength versus RCA content for different $w / c$ ratios

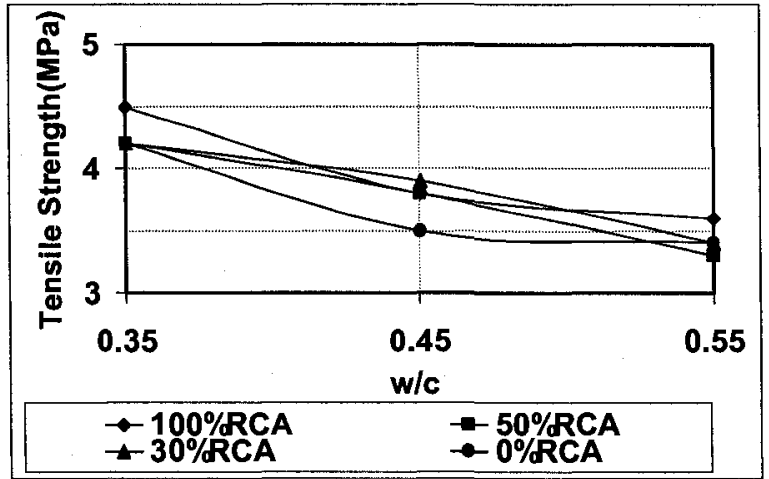

(a) $\mathrm{PM}-\mathrm{AE}$

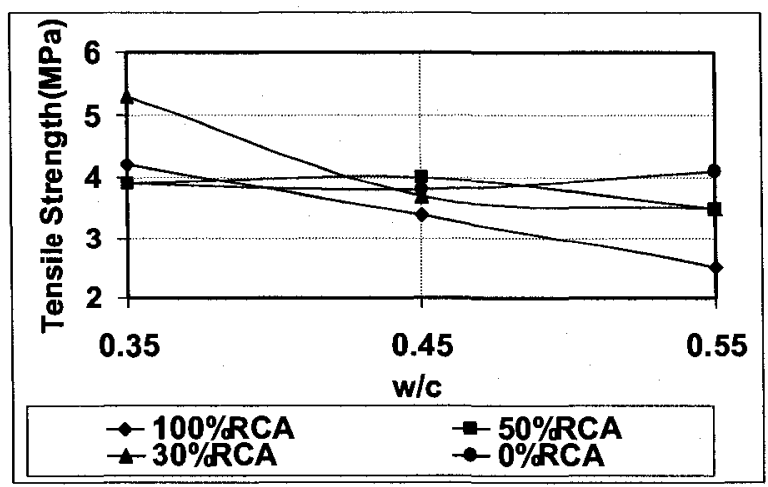

(c) $\mathrm{PV}-\mathrm{AE}$

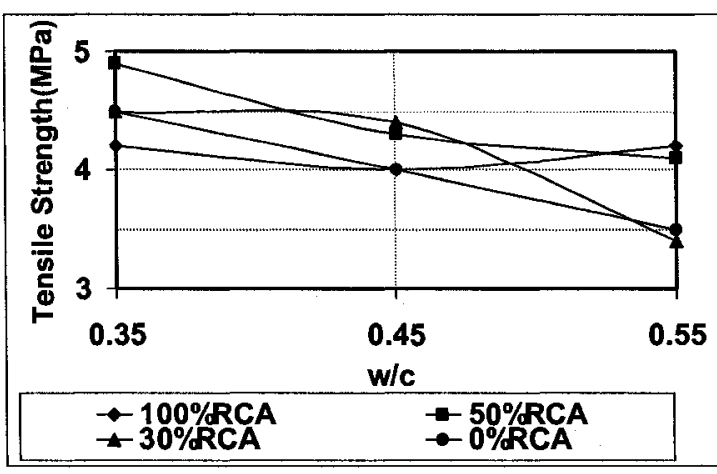

(b) PM - NAE

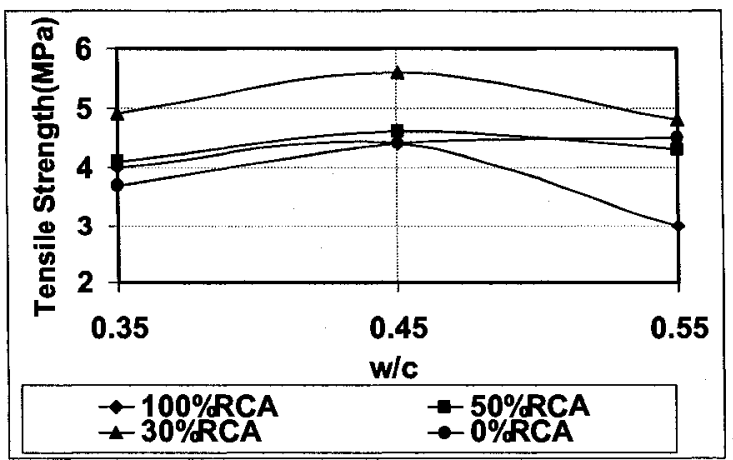

(d) PV - NAE

Figure 3.16: Variation of tensile strength with $w / c$ ratio for different RCA content levels 


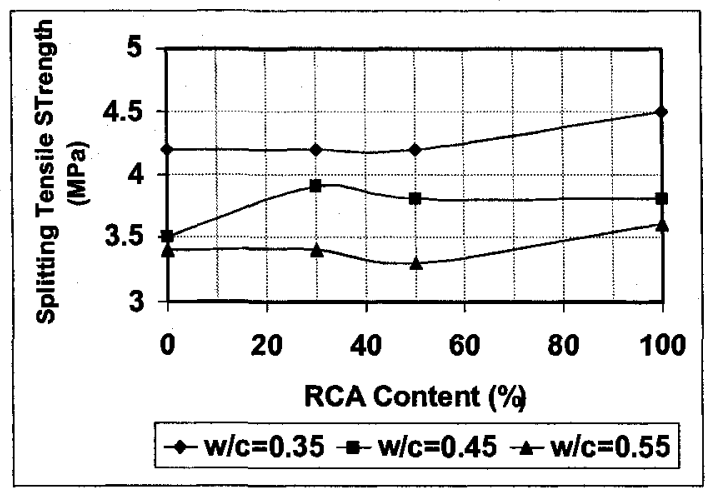

(a) PM - AE

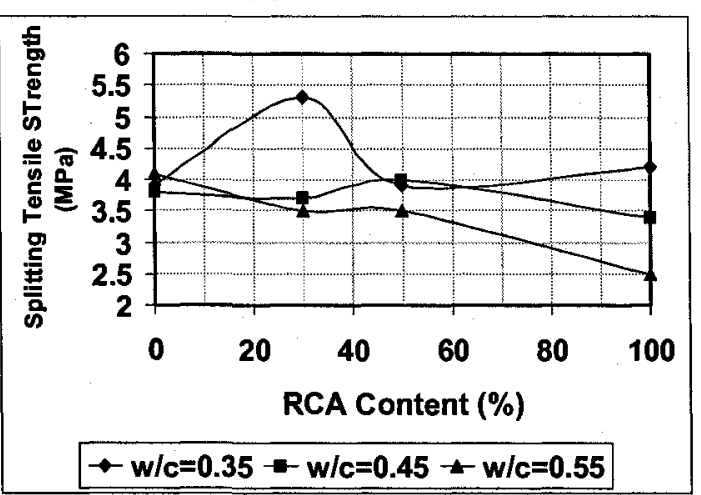

(c) PV - AE

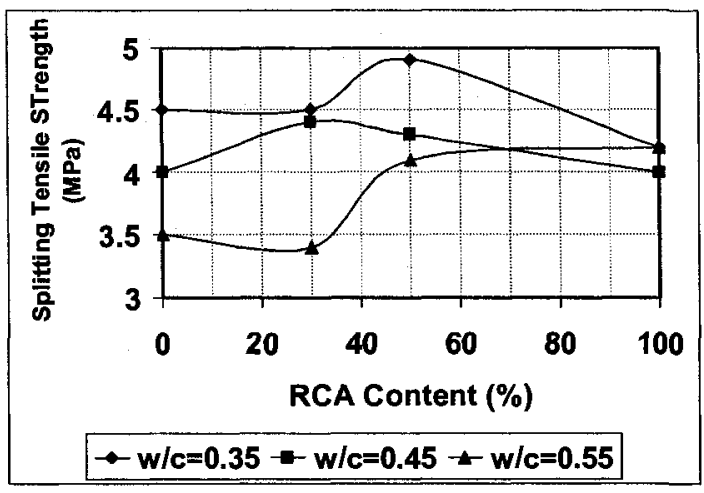

(b) PM - NAE

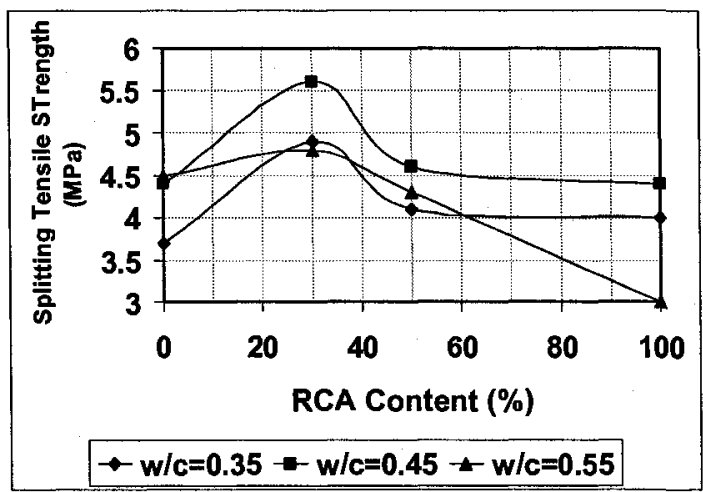

(d) PV - NAE

Figure 3.17: Variation of tensile strength with RCA content for different w/c ratios

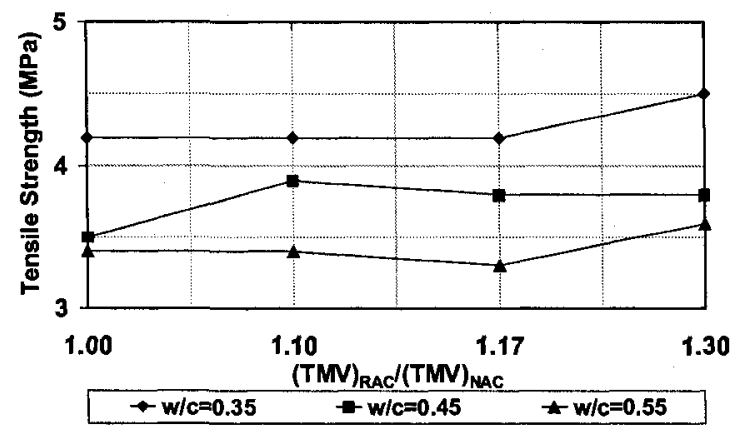

(a) PM - AE

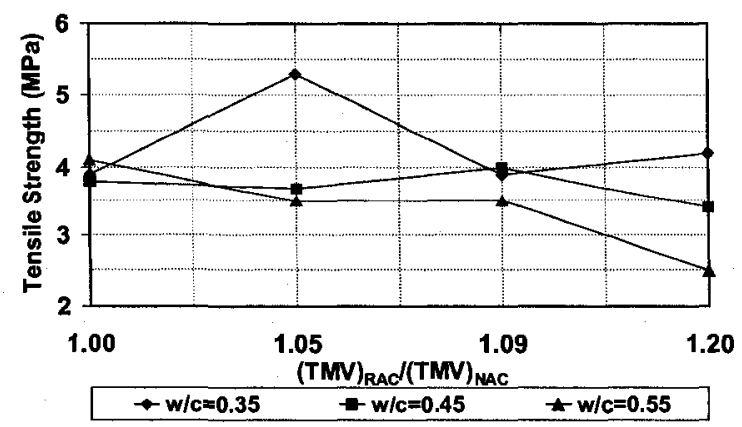

(c) PV - AE

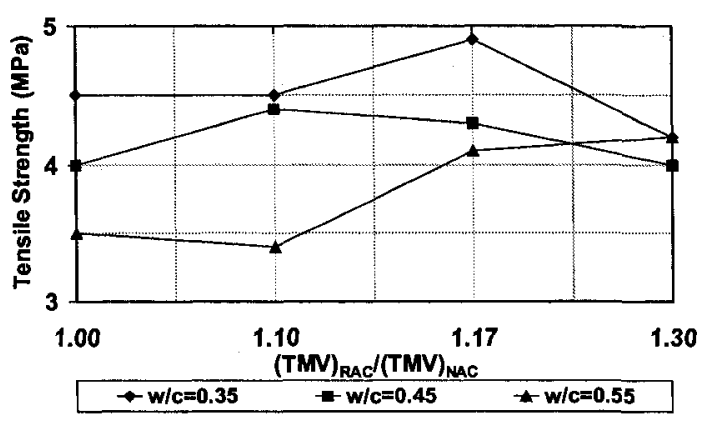

(b) PM - NAE

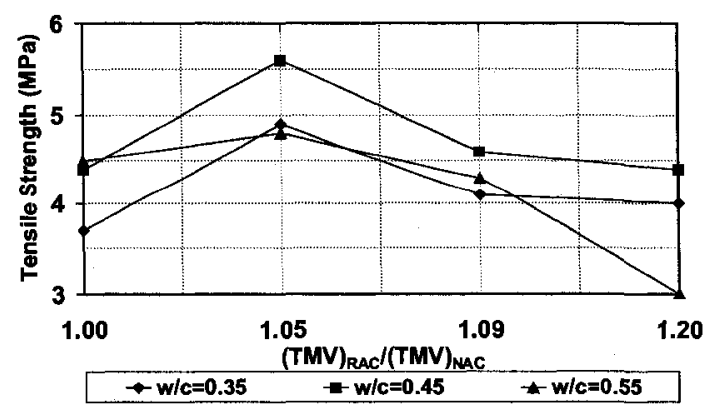

(d) PV - NAE

Figure 3.18: Variation of tensile strength with TMV for different $w / c$ ratios 


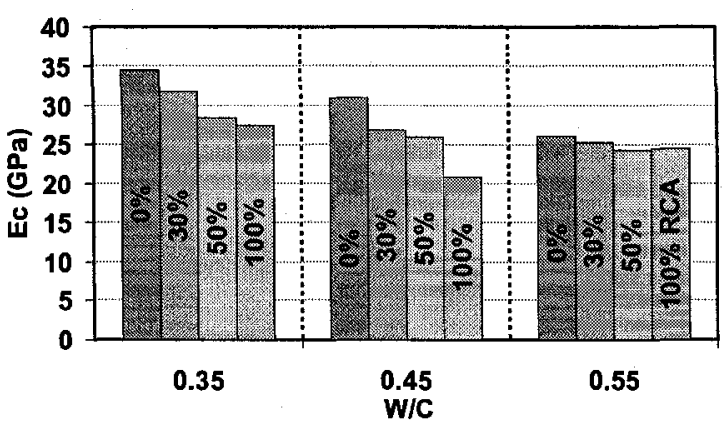

(a) $\mathrm{PM}-\mathrm{AE}$

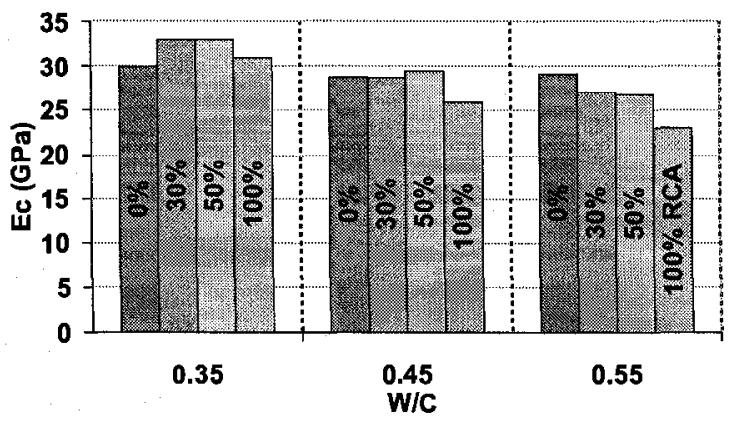

(c) PV - AE

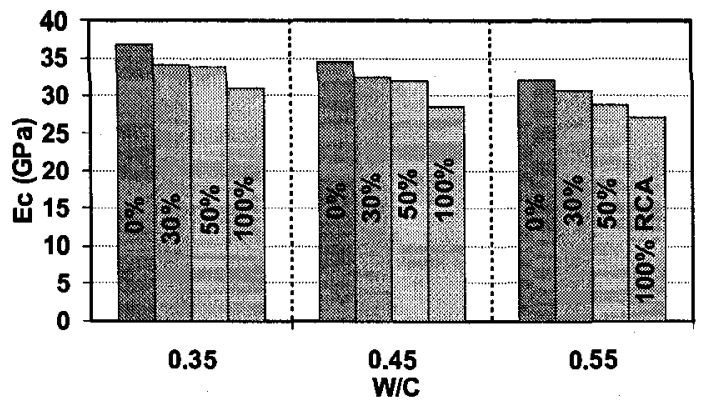

(b) PM - NAE

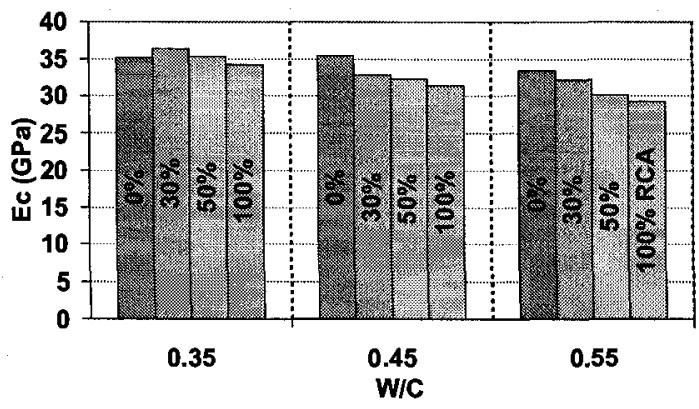

(d) PV - NAE

Figure 3.19: Elastic modulus versus RCA content for different $w / c$ ratios

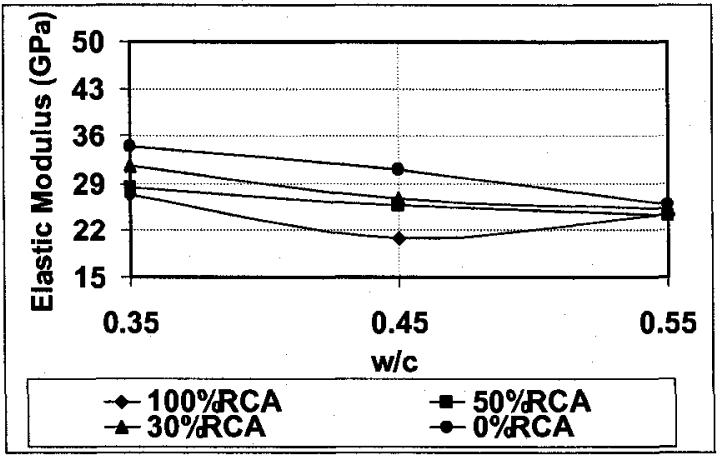

(a) PM - AE

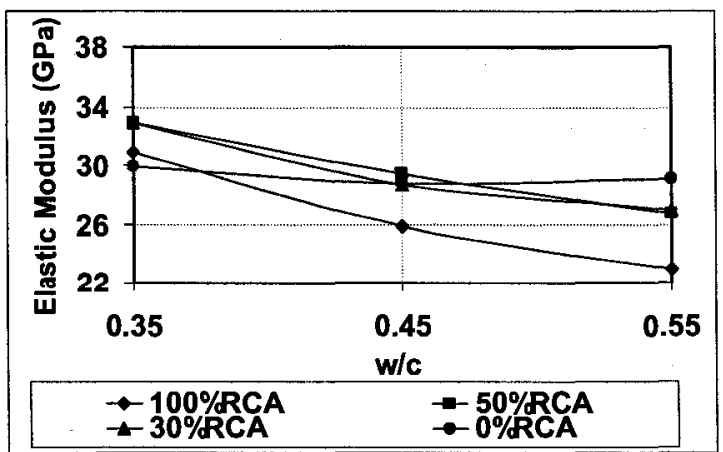

(c) PV - AE

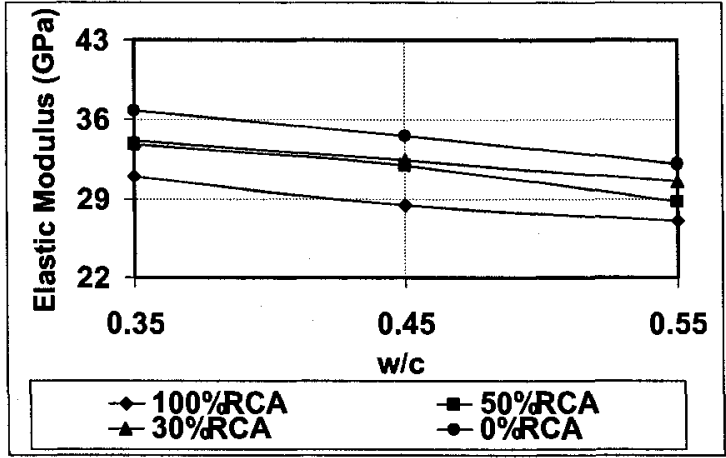

(b) PM - NAE

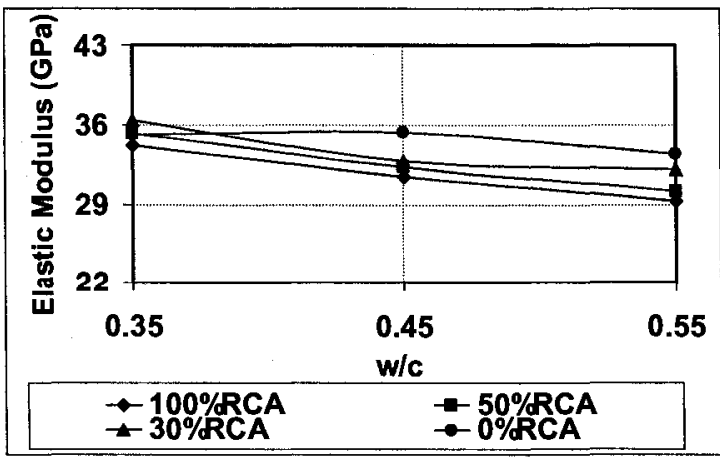

(d) PV - NAE

Figure 3.20: Variation of elastic modulus with $w / c$ ratio for different RCA content levels 


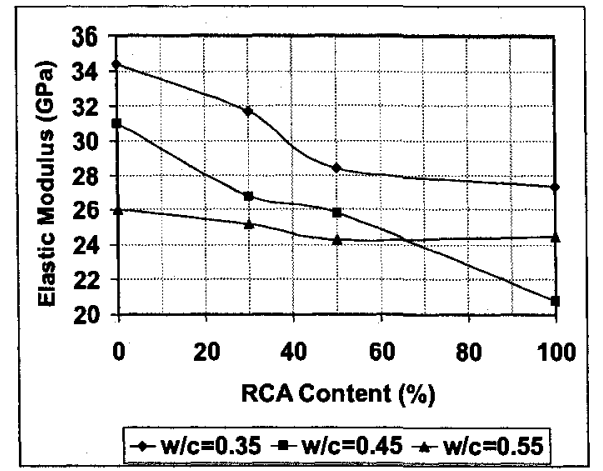

(a) PM - AE

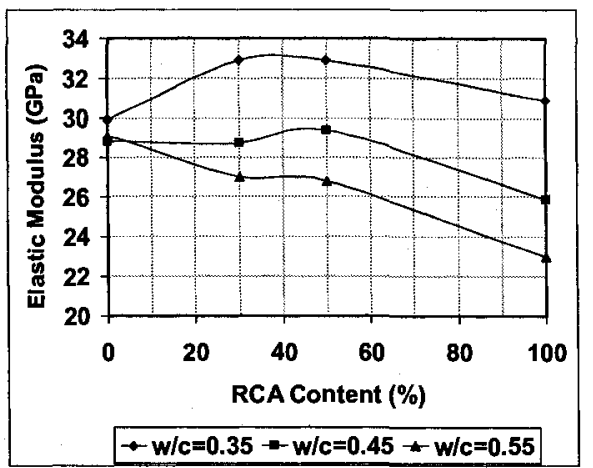

(c) PV - AE

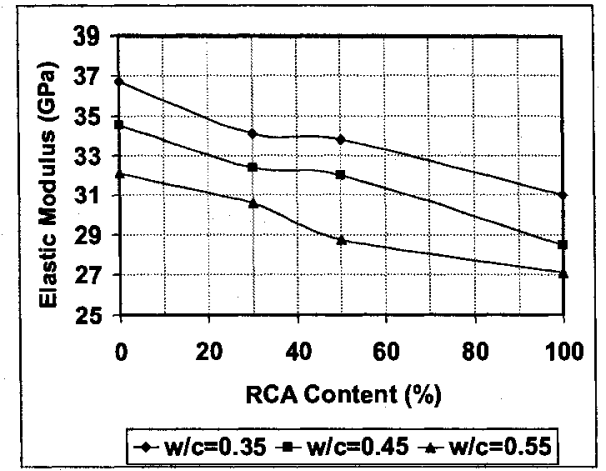

(b) PM - NAE

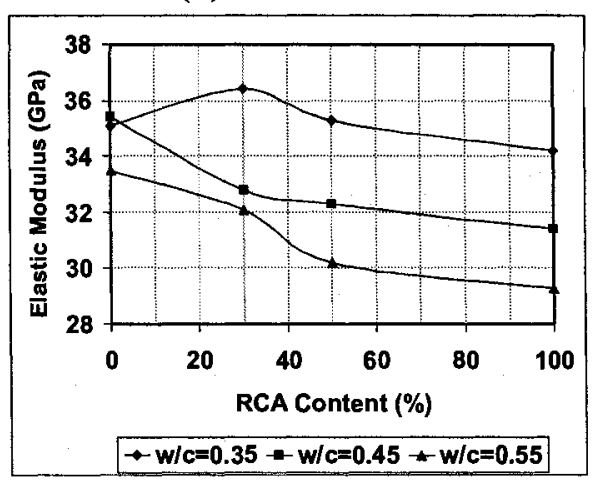

(d) PV - NAE

Figure 3.21: Variation of elastic modulus with RCA content for different $w / c$ ratios

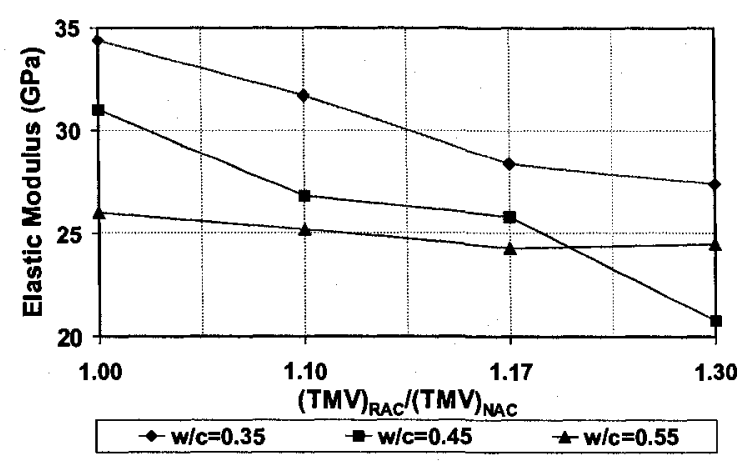

(a) PM - AE

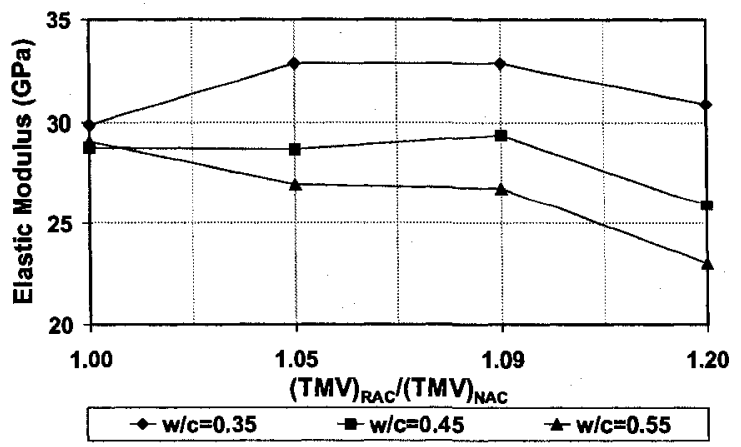

(c) PV - AE

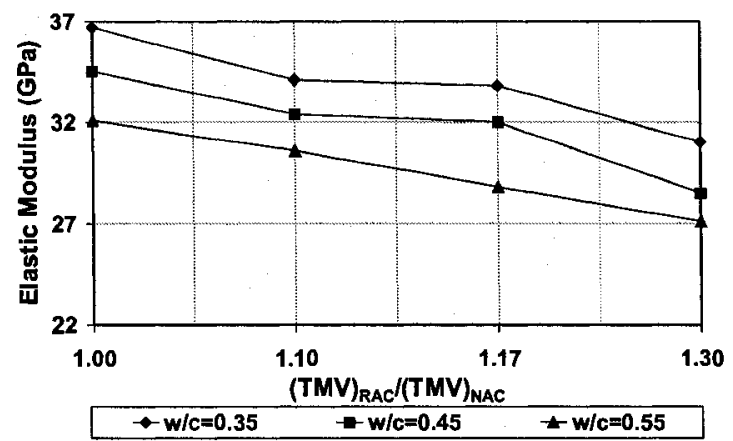

(b) PM - NAE

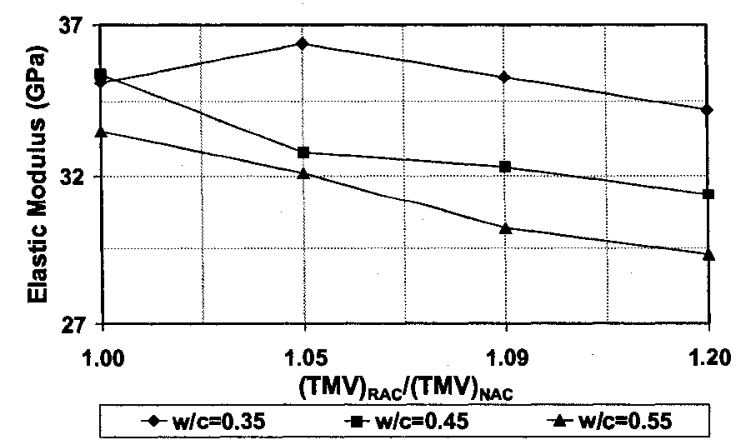

(d) PV - NAE

Figure 3.22: Variation of elastic modulus with TMV for different $w / c$ ratios 


\section{CHAPTER 4: PROPOSED MIX PROPORTIONING METHOD FOR RECYCLED AGGREGATE CONCRETE}

\subsection{General}

As stated earlier in Chapter 2, it has always been reported that RCA has inferior shortterm and long-term properties compared to NAC. Notwithstanding the fact that RAC with equal compressive strength to NAC has been produced by means of adjusting the w/c ratio of RAC; the RAC has always been reported to have lower fresh and hardened densities, lower elastic modulus, lower freeze-and-thaw resistance, higher permeability, and higher creep and shrinkage (Hansen, 1992; Kiuchi and Horiuchi, 2003; Maruyama et al., 2004a and 2004b; De Oliveira and Vazquez, 1996). The extent of the inferiority differs from one investigation to another and from one property to another. Whereas the compressive strength of concrete is mostly dependant on: (1) the strength of its mortar, (2) the quality of its interfacial transition zone (ITZ), and (3) the quality of the mechanical bond between its aggregates and mortar, its other properties, such as density, elastic modulus, creep, and shrinkage, are mainly a function of the volume fractions and the corresponding properties of its aggregate and mortar. Therefore, the characteristics of RCA, i.e. the quantity and quality will influence the latter properties of RAC.

When conventional mix design procedures, such as the ACI 211.1-97 (ACI Committee $211,1997)$ are used, the RCA is simply treated as another single-phase aggregate with no consideration given to the quantity of its residual mortar. However, RCA is a two-phase material comprising residual mortar and the original virgin or natural aggregate; 
consequently, using conventional methods of mixture proportioning, the concrete made with RCA would have larger total mortar volume (i.e., residual mortar plus new mortar) and less NA compared to a companion mix made with equal volume of NA. Figure 4.1 schematically illustrates the concept of conventional mix proportioning for RAC according to which RCA is a one-phase material which could be used as a simple substitute for NA. From this figure, it is quite evident that the TMV in RAC is higher than that in NAC.

It is contended in the present investigation that the higher mortar content of RAC is the key factor that is responsible for its reported inferior properties. It is also contended that the effects of this factor can be practically eliminated through a proper mix design method. Yet, to the author's knowledge, no systematic and comprehensive study has been performed to date with the objective of eliminating these inferiorities through the development of a specific mix design or proportioning method for concrete produced with RCA. Therefore, to address this issue, in this chapter a new method of concrete mix design will be developed and verified experimentally.

\subsection{Hypothesis}

The fundamental premise of the proposed equivalent mortar volume (EMV) mix design method is that RCA is a two-phase material comprising residual mortar and original virgin aggregate. Therefore, when designing a concrete mix with RCA, one must account for the relative amount and property of each phase. Furthermore, in a new mix involving RCA, the residual mortar phase must be treated as part of the total mortar rather than the aggregates in the new mix. In other words, it cannot be assumed, as is currently customary, that RCA is a simple substitute for natural aggregates because in fact it partly 
replaces the aggregate and partly the mortar. In the EMV method, the amount of coarse RCA in a mix is determined based on the amount of coarse NA that has been used in a companion NAC with the same specified properties. This approach guarantees that the volume of total mortar in RAC is equivalent to that in NAC. Figure 4.2 schematically illustrates the concept behind the EMV method in which RCA is treated as a two-phase material. In this figure, for the RAC mix its RCA content is adjusted until its total mortar and total natural aggregate volumes are equal to the corresponding volumes in the companion NAC mix. The details of this method are presented as follows.

\subsection{Determination of Residual Mortar Specific Gravity}

In order to satisfy the requirement of identical total mortar volumes in RAC and the companion NAC mixes, firstly the volume of the residual mortar in RCA need be determined, which requires knowledge of the specific gravity of residual mortar. There are two methods for determining this quantity: direct experimental method and indirect method.

\subsubsection{Direct Method}

Currently, there is no standard method available to measure the specific gravity and absorption capacity of residual mortar. Hence, it is suggested to determine these quantities following ASTM C 128-01 (ASTM 2003), the standard test method for determining the absorption capacity and specific gravity of fine aggregate. Since the properties of residual mortar are different from those of fine aggregate, the application of this method is not expected to give accurate results; however, the method will furnish a general idea about these properties of residual mortar. 
Using the latter method, the residual mortar samples were prepared by initially crushing the RCA and sieving it. The particle sizes passing the $1 / 2$ in. $(12.5 \mathrm{~mm})$ sieve, but retained on $3 / 8$ in. $(9.5 \mathrm{~mm})$ sieve were collected and crushed again using a jaw crusher machine. The crushed mortar was sieved again and particle sizes passing sieve No. $4(4.75 \mathrm{~mm})$ but retained on sieve No. $8(2.36 \mathrm{~mm})$ were collected. After this sieving process, by visual inspection and using tweezers and a magnifying glass, the RM particles were manually separated from the OVA. The residual mortar thus collected was dried in the oven (at $105^{\circ} \mathrm{C}$ ) to constant weight and its oven dry weight was determined. Thereafter, it was immersed in water for 24 hours to achieve full absorption, and was subsequently surface dried to achieve the SSD condition.

To determine the specific gravity of the mortar, the volumetric procedure of ASTM C128 test was followed. The Le Chatelier flask was used to measure and calculate the specific gravity and absorption capacity of the residual mortar and original virgin aggregates. All the test results reported here are based on the average of three repeat test samples.

Tables 4.1 to 4.4 show some of the measured physical properties, including the specific gravity of the residual mortar and its associated original virgin aggregate for RCA-MO and RCA-VA, respectively. In general, no significant difference was observed between the measured properties of the residual mortar and the original virgin aggregate of the RCA from the two sources. The absorption capacity of the residual mortar was $9.97 \%$ for RCA-MO and $10.08 \%$ for RCA-VA, while their SSD specific gravities were 2.31 and 2.32, respectively. Their respective bulk specific gravities were 2.10 and 2.11, respectively. Similarly, for original virgin aggregate of RCA-MO and RCA-VA, respectively, their absorption capacities were $0.95 \%$ and $0.97 \%$, their SSD specific 
gravities were 2.75 and 2.81 , and their bulk specific gravities were 2.72 and 2.78 . It is clear that the residual mortar and natural aggregate components of the two types of RCA have practically the same physical properties despite the fact that the natural aggregate in one case is limestone and in the other case it is river gravel.

Due to the high porosity of RM compared to natural fine aggregate, as soon as the ovendry RM was placed in the flask, the water immediately started to fill the micro pores of its surface. This surface absorption made it difficult to distinguish the air bubbles due to entrapped air in the water from those due to the air within the pores of the mortar. Given that the ASTM method requires that measurement be taken once air bubble formation due to entrapped air in the water has ceased, it was not possible to accurately measure the volume of the residual mortar by this method. Although the method was slightly modified, as described in Appendix A, nevertheless, it was concluded that the indirect method described below would yield better estimate of the specific gravity of the residual mortar.

\subsubsection{Indirect Method}

Considering recycled concrete aggregate as a two-phase composite material, it is possible to calculate its physical properties as a function of the corresponding properties and volume fractions of its residual mortar (RM) and original virgin aggregate (OVA).

Basically, the volume and weight of each RCA particle can be presented as the sum of the volumes and weights of its two components:

$$
\begin{gathered}
V_{R C A}=V_{R M}+V_{O V A} \\
W_{O D}^{R C A}=W_{O D}^{R M}+W_{O D}^{O V A}
\end{gathered}
$$

The volume of RCA, RM or OVA can be calculated by dividing its oven-dry weight by its bulk specific gravity 


$$
\begin{gathered}
V_{R C A}=\frac{W_{O D}^{R C A}}{S G_{b}^{R C A}} \\
V_{R M}=\frac{W_{O D}^{R M}}{S G_{b}^{R M}} \\
V_{O V A}=\frac{W_{O D}^{O V A}}{S G_{b}^{O V A}}
\end{gathered}
$$

where $V, W_{O D}$ and $S G_{b}$ denote volume, oven-dry weight and bulk specific gravity, respectively, while superscripts $R M, O V A$ and $R C A$ stand for residual mortar, original virgin aggregate and recycled concrete aggregate, respectively. Let the residual mortar content, $R M C$, be defined as

$$
R M C=\frac{W_{O D}^{R M}}{W_{O D}^{R C A}}
$$

then using Eqs.4.1 to 4.6 the bulk specific gravity of RM can be expressed as

$$
S G_{b}^{R M}=\frac{R M C}{\frac{1}{S G_{b}^{R C A}}-\frac{1-R M C}{S G_{b}^{\text {OVA }}}}
$$

Since in this study the NA used to supplement the OVA in RAC was chosen to be similar to the companion OVA, instead of finding the specific gravity of the OVA in RCA-MO and RCA-VA, the bulk specific gravities of Navan limestone and natural river gravel were determined and were assumed to be equal to the specific gravities of the OVA in the corresponding RCA.

Using Eq.4.7, the bulk specific gravity of the residual mortar for RCA-MO and RCA-VA were calculated to be 1.91 and 1.79 , respectively, which are lower than the corresponding values obtained by the direct method described earlier (i.e., 2.10 and 2.11). This can be firstly attributed to the breaking of the air void system in the residual mortar during sample preparation, i.e. while separating them from the OVA particles, which would lead to the decrease of the volume of the residual mortar and would consequently increase its 
bulk specific gravity. Secondly, it was observed during the test that the residual mortar contained some small OVA particles, thus increasing its specific gravity and decreasing its porosity. The latter is also responsible for the apparently higher bulk specific gravity of the residual mortar determined by the direct method. As a result of these observations, it was decided to consider the calculated residual mortar properties as being more accurate.

Knowing the bulk specific gravity of residual mortar, the volume of the residual mortar in RCA can be determined, and knowledge of the latter would enable one to satisfy the requirement of equal total mortar volume in the $\mathrm{RAC}$ and the companion NAC mixes.

\subsection{Mix Proportioning Procedure}

As stated earlier, the basic premise of the proposed mix proportioning procedure is maintenance of equal total mortar volume in the RAC and the companion NAC mixes. The procedure commences by the assumption that the mix proportions of the companion NAC are known. This assumption does not pose any difficulty because the NAC is designed using traditional mix ingredients and conventional design method. When proportioning RAC mixes, the ideal case may be using only RCA and no fresh coarse natural aggregate (NA). However, to generalize the proposed method, it is assumed that the coarse aggregate in RAC may comprise NA and RCA. Let the NA content of RAC, $V_{N A}^{R A C}$, be a fraction $R$ of the coarse NA volume in the companion NAC, $V_{N A}^{N A C}$, i.e.

$$
R=\frac{V_{N A}^{R A C}}{V_{N A}^{N A C}}
$$

The main requirement of the proposed method is equal total mortar volume (TMV) in RAC and the companion NAC, i.e.

$$
V_{T M}^{R A C}=V_{M}^{N A C}
$$


where $V_{T M}^{R A C}$ is the total mortar volume in RAC, and $V_{M}^{N A C}$ is the volume of mortar in the companion NAC. The equal total mortar volume requirement can be expressed alternatively as equal total natural aggregate (TNA) in RAC and the companion NAC, i.e.

$$
V_{T N A}^{R A C}=V_{N A}^{N A C}
$$

where $V_{T N A}^{R A C}$ is the total coarse natural aggregate volume in RAC, and $V_{N A}^{N A C}$ is the volume of coarse natural aggregate in the companion NAC. However, the total mortar volume in RAC consists of the residual mortar in RCA and the new or fresh mortar, which can be expressed as

$$
V_{T M}^{R A C}=V_{R M}^{R A C}+V_{N M}^{R A C}
$$

where $V_{R M}^{R A C}$ is the residual mortar volume in RAC, and $V_{N M}^{R A C}$ is the volume of the new mortar in RAC. Similarly,

$$
V_{T N A}^{R A C}=V_{O V A}^{R A C}+V_{N A}^{R A C}
$$

where $V_{O V A}^{R A C}$ is the original coarse virgin aggregate volume in RAC, and $V_{N A}^{R A C}$ is the volume of the fresh coarse natural aggregate in RAC.

Knowing the bulk specific gravities of residual mortar (Section 4.3) and the RCA, and the residual mortar content (RMC), the volume of residual mortar can be calculated using Eqs.4.3, 4.4, 4.6 and 4.7 as follows:

$$
V_{R M}^{R A C}=V_{R C A} \times R M C \times \frac{S G_{b}^{R C A}}{S G_{b}^{R M}}
$$

In addition, the volume of original coarse virgin aggregate in the RCA can be calculated by subtracting the volume of residual mortar from the RCA volume

$$
V_{O V A}^{R A C}=V_{R C A} \times(1-R M C) \times \frac{S G_{b}^{R C A}}{S G_{b}^{O V A}}
$$

Using Eqs.4.8, 4.9a, 4.10b, and 4.11b, the required volume of coarse RCA $\left(V_{R C A}^{R A C}\right)$ and $\mathrm{NA}\left(V_{N A}^{R A C}\right)$ in RAC can be found as follows: 


$$
\begin{gathered}
V_{R C A}^{R A C}=\frac{V_{N A}^{N A C} \times(1-R)}{(1-R M C) \times \frac{S G_{b}^{R C A}}{S G_{b}^{O V A}}} \\
V_{N A}^{R A C}=V_{N A}^{N A C} \times R
\end{gathered}
$$

Knowing the bulk specific gravities of NA and RCA and having calculated the RCA and NA volumes, the required oven-dry weights of RCA $\left(W_{O D-R C A}^{R A C}\right)$ and NA $\left(W_{O D-N A}^{R A C}\right)$ can be obtained as

$$
\begin{aligned}
W_{O D-R C A}^{R A C} & =V_{R C A}^{R A C} \times S G_{b}^{R C A} \times 1000 \\
W_{O D-N A}^{R A C} & =V_{N A}^{R A C} \times S G_{b}^{N A} \times 1000
\end{aligned}
$$

Having determined the volumes of the residual mortar in RAC (Eq.4.11a) and the mortar in the companion NAC, one can use Eqs.4.9a and 4.10a to determine the required volume of new mortar in $\operatorname{RAC}\left(V_{N M}^{R A C}\right)$

$$
V_{N M}^{R A C}=V_{M}^{N A C}-V_{R M}^{R A C}
$$

By multiplying the quantities of the ingredients of the mortar in the companion NAC by the ratio of $\frac{V_{N M}^{R A C}}{V_{M}^{N A C}}$, the required weights of oven-dry fine aggregate $\left(W_{O D-F A}^{R A C}\right)$, water $\left(W_{w}^{R A C}\right)$ and cement $\left(W_{c}^{R A C}\right)$ can be obtained as

$$
\begin{gathered}
W_{w}^{R A C}=W_{w}^{N A C} \times \frac{V_{N M}^{R A C}}{V_{M}^{N A C}} \\
W_{c}^{R A C}=W_{c}^{N A C} \times \frac{V_{N M}^{R A C}}{V_{M}^{N A C}} \\
W_{O D-F A}^{R A C}=W_{O D-F A}^{N A C} \times \frac{V_{N M}^{R A C}}{V_{M}^{N A C}}
\end{gathered}
$$

where $W_{w}^{N A C}, W_{c}^{N A C}$ and $W_{O D-F A}^{N A C}$ are the weights of water, cement and oven-dry fine aggregate, respectively, in the companion NAC mix.

It is noteworthy that in the proposed method, by assuming different $R$ values, many alternatives RAC mix proportions with identical TM and TNA volumes can be designed. 
In other words, in contrary to the conventional mix proportioning methods, adoption of different $\mathrm{R}$ values in the $E M V$ method does not result in overall higher mortar volume in RAC compared to the companion NAC. However, depending on the quantity of its residual mortar, based on the proposed method, and as explained next, there is an upper limit to the RCA content of a RAC mix.

\subsection{Maximum Permissible Coarse RCA and RM Content}

As stated earlier, it may be ideal to have $100 \% \mathrm{RCA}$ as the coarse aggregate in $\mathrm{RAC}$, which is equivalent to $\mathrm{R}=0$ in Eq.4.8. Figure 4.3 illustrates the schematic variation of $V_{R C A}^{R A C}$ (Eq.4.12) with $\mathrm{RMC}$, assuming $\mathrm{R}=0$. In order to investigate the effect of $\mathrm{RMC}$ in Eq.4.12, let us examine the lower and upper limits of RMC ( $0 \%$ and $100 \%)$.

- For $\mathrm{RMC}=0$, there would be no residual mortar attached to original virgin aggregate, and therefore, the bulk specific gravity of RCA would be the same as that of original virgin aggregate $\left(\frac{S G_{b}^{R C A}}{S G_{b}^{O V A}}=1\right)$ and the required $\frac{V_{R C A}^{R A C}}{V_{N A}^{N A C}}=1$.

- As the RMC is increased from zero to $100 \%, V_{R C A}^{R A C}$ increases hyperbolically and approaches infinity $\left(\frac{V_{R C A}^{R A C}}{V_{N A}^{N A C}} \rightarrow \infty\right)$. Of course, this is not physically feasible or meaningful. However, the maximum amount of any aggregate, including RCA, that can be accommodated within a unit volume of concrete is equal to the dry-rodded unit volume of that aggregate (ACI Committee 211,1997$)$. The maximum amount of RMC $\left(R M C_{\max }\right)$ in a RAC mix is determined by the upper limit of its companion RCA. In the following sections, firstly, the upper limit for RCA content in the proposed method will be 
quantitatively established, followed by the upper limit for RMC, assuming all the coarse aggregates to be RCA, i.e. $100 \%$ RCA.

\subsubsection{Determining the Upper Limit of RCA Content in RAC}

Assuming the dry-rodded volume of RCA in RAC, $V_{D R-R C A}^{R A C}$, to be equal to one, the volume of the maximum amount of RCA that can be added to a unit volume of RAC $\left(V_{\max R C A}^{R A C}\right)$ can be calculated as follows:

$$
V_{\max R C A}^{R A C}=\frac{S G_{D R}^{R C A}}{S G_{b}^{R C A}}
$$

where $S G_{D R}^{R C A}$ is the dry-rodded specific gravity of RCA.

For RCA with high RMC, it may not be possible to place the required $V_{R C A}^{R A C}$ in a unit volume of concrete. In other words, it may not be possible to use $100 \%$ RCA in RAC and some NA may need be added to the mix $(R>0)$ to compensate for the TNA of RAC.

The absolute volume of NA in NAC ( $\left.V_{N A}^{N A C}\right)$ in Eq.4.12 can be related to its dry-rodded volume $\left(V_{D R-N A}^{N A C}\right)$ as:

$$
V_{N A}^{N A C}=V_{D R-N A}^{N A C} \times \frac{S G_{D R}^{N A}}{S G_{b}^{N A}}
$$

where $S G_{D R}^{N A}$ is the dry-rodded specific gravity of NA.

By substituting Eqs.4.20 and 4.21 in Eq.4.12, the minimum replacement ratio $\left(R_{\min }\right)$ can be calculated as:

$$
R_{\min }=1-\frac{(1-R M C)}{V_{D R-N A}^{N A C}} \times \frac{S G_{D R}^{R C A}}{S G_{D R}^{O V A}} \times \frac{S G_{b}^{N A}}{S G_{b}^{O V A}} \geq 0
$$

By assuming identical shape and size grading in RCA and NA, it can be written that: 


$$
\frac{S G_{D R}^{R C A}}{S G_{D R}^{N A}}=\frac{S G_{b}{ }^{R C A}}{S G_{b}{ }^{N A}}
$$

Assuming the fresh NA that is used as replacement of RCA to be of similar nature to the OVA in RCA, the ratio $\frac{S G_{b}^{N A}}{S G_{b}^{\text {OVA }}}$ in Eq.4.22 may be taken as 1. Therefore, by substituting Eq.4.23 in Eq.4.22 one obtains

$$
R_{\min }=1-\frac{(1-R M C)}{V_{D R-N A}^{N A C}} \times \frac{S G_{b}^{R C A}}{S G_{b}^{N A}} \geq 0
$$

Knowing the dry-rodded volume of NA in the companion NAC $\left(V_{D R-N A}^{N A C}\right)$, e.g. from Table A.1.5.3.6 of ACI 211.1-97 (ACI Committee 211, 1997), as well as the bulk specific gravity of RCA and NA, the minimum fresh NA content of RAC can be calculated using Eq.4.24. For instance, by using a nominal maximum aggregate size of $19 \mathrm{~mm}$ and fineness moduli of 2.6 for fine aggregate, Table A.1.5.3.6 of ACI 211.1-97 (ACI Committee 211 , 1997) gives $V_{D R-N A}^{N A C}=0.64$. For RCA-MO, by using $S G_{b}^{R C A}=2.31$, $\mathrm{RMC}=41 \%$, and $S G_{b}^{N A}=2.70, R_{\min }=21 \%$ for NA content will be obtained. For RCAVA, by using $S G_{b}^{R C A}=2.42$, RMC $=23 \%$, and $S G_{b}^{N A}=2.72$, the negative value of $-7 \%$ for $R_{\min }$ will be obtained. The latter negative value implies that RAC can be made with $100 \%$ RCA-VA without any fresh NA added to the mix.

As stated earlier, as the RMC increases and approaches $100 \%$, the required $V_{R C A}^{R A C}$ in Eq.4.12 hyperbolically increases and approaches infinity $\left(\frac{V_{R A A}^{R A C}}{V_{N A}^{N A C}} \rightarrow \infty\right)$. However, if the $(1-R)$ in the numerator of Eq.4.12 is set equal to its denominator, $(1-R M C) \times \frac{S G_{b}^{R C A}}{S G_{b}^{O V A}}$ 
the resulting equation would be valid for any RMC. The physical interpretation of the latter action is replacement of residual mortar volume in RCA with fresh natural aggregate $\left(V_{N A}^{R A C}=V_{R M}^{R A C}\right)$ to compensate for the deficiency in TNA of RAC compared to the companion NAC. Therefore:

$$
R=\frac{R M_{v}}{V_{N A}^{N A C}}
$$

where

$$
R M_{v}=\frac{R M C}{S G_{b}^{R M}}
$$

By inserting in Eq.4.26 the bulk specific gravity of RM of RCA-MO a value of 1.91 and $41 \%$ for its residual mortar content, the residual mortar volume of RCA-MO can be calculated $21.47 \%$. Similarly, for RCA-VA, inserting 1.79 for its RM specific gravity and $23 \%$ for its residual mortar content, its residual mortar volume is determined $12.85 \%$.

In this study, this approach has been employed to calculate the required NA and RCA contents in RAC mixes proportioned by the EMV method. By inserting Eq.4.25 in Eq.4.12 and using Eq.4.13 to 4.19, the required mix proportions (i.e., coarse RCA and NA, fine aggregate, cement and water) in RAC can be determined. The RCA content in RAC can be correspondingly calculated using

$$
\text { RCA Content }=\frac{W_{O D-R C A}^{R A C}}{W_{O D-R C A}^{R A C}+W_{O D-N A}^{R A C}}
$$

Accordingly, RCA-MO and RCA-VA contents values were found $63.5 \%$ and $74.3 \%$.

\subsubsection{Upper Limit for $R M C$ for $R=0$}

If RMC of a given coarse RCA is less than its upper limit, it is possible to make RAC mixes made entirely with RCA as coarse aggregate. If its RMC exceeds its upper limit, the same cannot be done. However, one could still make an RAC mix in the latter case 
but with a blend of RAC and fresh coarse natural aggregates. The foregoing upper limit for RMC can be obtained by using Eqs.4.12, 4.20, 4.21 and 4.23 as follows

$$
R M C_{\max }=1-V_{D R-N A}^{N A C} \times \frac{S G_{D R}^{N A}}{S G_{D R}^{R C A}} \times \frac{S G_{b}^{O V A}}{S G_{b}^{N A}}
$$

Using Eq.4.23 and assuming $\frac{S G_{b}^{O V A}}{S G_{b}^{N A}}=1$, Eq.4.28 can be rearranged as follows:

$$
R M C_{\max }=1-V_{D R-N A}^{N A C} \times \frac{S G_{b}^{N A}}{S G_{b}^{R C A}}
$$

Knowing the dry-rodded volume of NA in the companion NAC $\left(V_{D R-N A}^{N A C}\right)$ from Table A.1.5.3.6 of ACI 211.1-97 (ACI Committee 211, 1997), as well as bulk specific gravity of RCA and NA, the upper limit can be calculated using Eq.4.29. For instance, assuming a nominal maximum aggregate size of $19 \mathrm{~mm}$ and fineness moduli of 2.6 for fine aggregate, Table A.1.5.3.6 of ACI 211.1-97 (ACI Committee 211, 1997) gives $V_{D R-N A}^{N A C}=0.64$. For RCA-MO, letting $S G_{b}^{R C A}=2.31 \quad$ and $S G_{b}^{N A}=2.70$ leads to $R M C_{\max }=25.2 \%$. Since the actual RMC content of RCA-MO is $41 \%$, it can be seen that it is not feasible to make a concrete mix comprising only RCA-MO as coarse aggregate. On the other hand, for RCA-VA, $S G_{b}^{R C A}=2.42$ and $S G_{b}^{N A}=2.72$, which yields $R M C_{\max }=28.1 \%$. Since the actual RMC of RCA-VA is $23 \%$, it is feasible to make an RAC mix in which the coarse aggregate content is $100 \%$ RCA-VA.

\subsection{Experimental Verification}

To verify the validity of the assertion that RAC mixes proportioned by the proposed EMV method would not suffer from inferior physical or mechanical properties when 
compared to an equivalent mix made entirely with coarse natural aggregate, a number of concrete mixes were designed, made and tested. Three types of mixes were made:

- Type 1: RAC mixes made with $100 \%$ RCA coarse aggregate and proportioned by the conventional method.

- Type 2: NAC mixes made with $100 \%$ coarse NA and proportioned by the conventional method.

- Type 3: RAC mixes proportioned by the equivalent mortar volume (EMV) method. The mortar volume of these mixes was equivalent to that of Type 2 mixes.

The mix Type 1 was made for the following purposes: (1) to investigate the deterioration in the short-term and long-term properties of RAC mixes produced with $100 \% \mathrm{RCA}$; (2) to study the effect of TMV on the fresh and hardened properties of RAC; (3) to compare the relevant properties of RAC mixes proportioned by the conventional method to those of mixes proportioned by the EMV method. Mix Type 2 serves as the control or reference for Mix Type 1 and Type 3. By comparing the properties of mixes with and without RCA, it can be established whether the reported inferiorities of RAC are intrinsic or they are the result of improper mix proportioning methods. It will also be established whether the proposed method would indeed lead to RAC mixes free of any inferiority. To isolate the effect of RCA properties from the effect of other known factors on concrete properties, the w/c ratio and air content of all mixes were held at 0.45 and $6 \%$, respectively. Due to their prevalence in modern concrete mixes, fly ash and ground granulated blast furnace slag (bfs) were added to some mixes and their effects on the short- and long-term properties of RAC are investigated. The percent replacements of cement by fly ash and bfs were chosen to be $25 \%$ and $35 \%$ by weight of cement, which 
are common levels of replacement in current practice. The fly-ash was CSA CI from Sundance (Alberta) with average specific gravity of 2.01 , and the blast furnace slag was Grade 80 with average specific gravity of 2.99 .

\subsubsection{Mix Proportions}

Table 4.5 presents the mixtures proportions, where the mix designations are based on the following notation:

- First letter E or C: mix proportioned based on EMV (E) or conventional method (C).

- Second letter M, V, L or G: mix made with RCA-MO (M), RCA-VA (V), natural limestone (L) or natural gravel (G).

- Third letter C, F or B: mix made with ordinary Portland cement only (C), cement plus fly ash (F), or cement plus bfs (B).

For example, CM-B represents a mix proportioned based on conventional method, made with RCA-MO and incorporating 35\% blast furnace slag (as replacement by mass of the portland cement). The RCA contents in Table 4.5 are calculated by dividing the air-dried weight of the coarse RCA by the air-dried weight of total coarse aggregate.

The following points are important to note regarding the mixes:

1. NAC mixes were made with Portland cement without any fly ash or bfs.

2. Limestone was the coarse natural aggregate in some NAC mixes (Type 2) and also partially the coarse aggregate in Type 3 mixes made with RCA-MO.

3. River gravel was the coarse natural aggregate in other NAC mixes (Type 2) and also partially the coarse aggregate in Type 3 mixes made with RCA-VA.

4. A low-range water reducing admixture was used in all the mixes except for the NAC and RAC mixes made with fly-ash. 
5. The target cementitious material content of mix Type 1 was $350 \mathrm{~kg} / \mathrm{m}^{3}$.

6. The target cementitious material content of mix Type 3 was $352 \mathrm{~kg} / \mathrm{m}^{3}$ to $377 \mathrm{~kg} / \mathrm{m}^{3}$, depending on the RMC of the RCA.

In Table 4.5, the properties of PM-100MA and PV-100MA mixes from preliminary study (Tables 3.2 and 3.3) are also represented. As no water reducing admixtures (WRA) was employed in preliminary mixes, it may be possible to compare the properties of some preliminary mixes with those of the current mixes in order to investigate the effect of WRA on the fresh and hardened properties of RAC.

It is important to mention that the EMV method decreases the cement requirement of the mix because it requires less fresh mortar than required by the conventional method. The smaller cement requirement makes RAC more environmentally friendly. As it can be seen from Table 4.5, the cement content of the NAC (with $0 \% \mathrm{RCA}$ ) proportioned by the conventional method is above $400 \mathrm{~kg} / \mathrm{m}^{3}$. When a companion RAC mix, with a total mortar volume equal to the preceding NAC mix, is designed by the EMV method; the cement content drops to $377 \mathrm{~kg} / \mathrm{m}^{3}$ and $352 \mathrm{~kg} / \mathrm{m}^{3}$ for the mixes made with RCA-VA and RCA-MO.

Concrete specimens were cast and cured following the procedures described in ASTM C 192/C 192M-00 (ASTM, 2003). Six cylindrical specimens $(100 \times 200 \mathrm{~mm})$ were prepared for 28-day compressive strength ( 3 specimens) and splitting tensile strength tests (3 specimens). Three cylindrical specimens $(150 \times 300 \mathrm{~mm})$ were prepared for the determination of elastic modulus. Specimens were stored for 28 days in a moist room in accordance with the requirements of ASTM C 511-98 (ASTM, 2003). The fresh and hardened properties of the mixes are presented in the following section. 


\subsubsection{Fresh Properties}

The slump, air content and fresh density were measured once for each mix. Table 4.6 presents the summary of the measured fresh properties. In the following sections, these results are explained in further detail.

\subsubsection{Slump}

As it can be seen from Table 4.6, in general, all CM and EM mixes proportioned with either conventional or EMV mix proportioning method were found to be workable, with their slump ranging from $55 \mathrm{~mm}(\mathrm{CM}-\mathrm{B})$ to $130 \mathrm{~mm}(\mathrm{CM}-\mathrm{F})$. The slump of the companion CL-C produced with limestone was $175 \mathrm{~mm}$. Similarly, the slump values of all $\mathrm{CV}$ and $\mathrm{EV}$ mixes proportioned by either conventional or EMV mix proportioning method were found to be about $70 \mathrm{~mm}$ for the $\mathrm{CV}$ and about $150 \mathrm{~mm}$ for the $\mathrm{EV}$ mixes. The slump of the companion CG-C produced with river gravel was $210 \mathrm{~mm}$.

Figure 4.5 illustrates the effect of mix proportioning method on the slump of different mixes. Generally, for mixes with the same w/c ratio, the EMV method resulted in higher slump compared to conventional method. This can be seen by comparing the $50 \%$ and $45 \%$ higher slump of mixes EM-C and EM-B compared to those of CM-C and CM-B, respectively. Similarly, for EV-C, EV-F and EV-B mixes, the slump values were $100 \%$, $56 \%$ and $36 \%$ higher than those of CV-C, CV-F and CV-B mixes, respectively. However, the slump of CM-F was slightly higher than that of EM-F mix. Note that mainly due to their higher water content, the CL-C and CG-C mixes had higher slump compared to all the EM, EV, CM and CV mixes. 


\subsubsection{Fresh Density}

According to the results in Table 4.6, the fresh concrete density of EM and CM mixes proportioned by the conventional and the EMV mix proportioning methods ranged from $2226 \mathrm{~kg} / \mathrm{m}^{3}$ (CM-F) to $2306 \mathrm{~kg} / \mathrm{m} 3$ (EM-B). The fresh density of CL-C was determined as $2332 \mathrm{~kg} / \mathrm{m}^{3}$. Similarly, the fresh concrete density of CV and EV mixes proportioned by the conventional and the EMV mix proportioning methods ranged from $2286 \mathrm{~kg} / \mathrm{m}^{3}$ (CVB) to $2328 \mathrm{~kg} / \mathrm{m} 3$ (EV-C) while the fresh density of CG-C was $2315 \mathrm{~kg} / \mathrm{m}^{3}$.

Figure 4.6 illustrates the effect of mix proportioning method on the fresh density of mixes. Generally, the conventional mix design method resulted in lower fresh density in both the $\mathrm{CM}$ and the $\mathrm{CV}$ mixes (with or without fly ash or bfs) compared to the companion CL-C and CG-C mixes, respectively. This is once again due to the higher total mortar volume in $\mathrm{CM}$ and $\mathrm{CV}$ mixes compared to the companion CL-C and CG-C mixes. On the other hand, using the EMV method resulted in higher fresh density of both the EM and the EV mixes. In all the EM and EV mixes, the fresh density values were higher than those of the $\mathrm{CM}$ and the $\mathrm{CV}$ mixes, but slightly lower than the fresh density of the companion CL-C and CG-C mixes, respectively. The latter higher densities are attributed to the equal total mortar volume of EM and EV and the corresponding CL-C and CG-C mixes, respectively. The slightly higher fresh density of CL-C and CG-C mixes, compared to EM and EV mixes, is due to the lower specific gravity of residual mortar (as part of total mortar) in the EM and EV mixes compared to the specific gravity of fresh mortar in CL-C and CG-C mixes. According to the figures, it can also be observed that the fresh density of both the CM-C and the CV-C mixes are higher than the corresponding values of PM-100MA and PV-100MA mixes, respectively. This may be 
due to the use of WRA in the former mixes which resulted in a fresh mortar with higher fine aggregate content and consequently higher overall density.

\subsubsection{Hardened Properties}

In this section, the results for the hardened density $\left(\gamma_{c}\right)$, compressive strength $\left(f_{c}^{\prime}\right)$ and elastic modulus $\left(E_{c}\right)$ of the hardened concrete mixes described earlier in this chapter are presented. The proportions of each mix whose results are reported here can be found in Table 4.7.

\subsubsection{Hardened Density}

According to the results in Table 4.7, the hardened concrete density of the EM and CM mixes proportioned by the conventional and by the EMV mix proportioning method range from $2217 \mathrm{~kg} / \mathrm{m}^{3}$ (CM-F) to $2319 \mathrm{~kg} / \mathrm{m} 3$ (EM-B), while the hardened density of CL-C is $2300 \mathrm{~kg} / \mathrm{m}^{3}$. Similarly, the hardened density of CV and EV mixes proportioned by the conventional and by the EMV mix proportioning method ranged from $2281 \mathrm{~kg} / \mathrm{m}^{3}$ (CV-F) to $2325 \mathrm{~kg} / \mathrm{m} 3$ (EV-B), and the hardened density of CG-C is $2315 \mathrm{~kg} / \mathrm{m}^{3}$.

Figure 4.7 illustrates the effect of mix proportioning method on the hardened density of the different mixes. Generally, the conventional mix design method resulted in lower hardened density in both the CM and the CV mixes (with or without fly ash or bfs) compared to the companion CL-C and CG-C mixes, respectively. This is once again due to the higher total mortar volume in the $\mathrm{CM}$ and $\mathrm{CV}$ mixes compared to the companion CL-C and CG-C mixes, respectively. On the other hand, using the EMV resulted in higher hardened density for both the EM and EV mixes. In all the EM and EV mixes, the hardened density values are higher than those of the $\mathrm{CM}$ and $\mathrm{CV}$ mixes, and even slightly higher than the hardened density of the companion CL-C and CG-C mixes, respectively. 
The higher densities are attributed to the same total mortar volume of the EM and EV and the corresponding CL-C and CG-C mixes, respectively. According to the figures, it can also be observed that the hardened density of both CM-C and CV-C mixes are higher than the corresponding values of the PM-100MA and PV-100MA mixes, respectively. This may be due to the use of WRA in the former mixes which resulted in a mortar with higher fine aggregate content and consequently higher overall density.

\subsubsection{Compressive Strength}

In general, as it can be observed in Table 4.7, the 28-day compressive strengths of the tested specimens are in the range from $34 \mathrm{MPa}$ (CM-F) to $42 \mathrm{MPa}$ (CM-B). The compressive strength of CL-C is $36.9 \mathrm{MPa}$. Similarly, the compressive strength of the CV and EV mixes proportioned by the conventional and the EMV mix proportioning method range from $35 \mathrm{MPa}(\mathrm{CV}-\mathrm{F})$ to $40 \mathrm{MPa}$ (EV-C and EV-B), while the compressive strength of CG-C is $35.6 \mathrm{MPa}$.

Figure 4.8 illustrates the effect of mix proportioning method on the compressive strength of the different mixes. Except for the CM-F and CV-F mixes, the compressive strength of all other $\mathrm{CM}$ and the $\mathrm{CV}$ mixes are $12-14 \%$ and $10-12 \%$ higher than that of the companion CL-C and CG-C mixes, respectively. This is mainly due to the addition of WRA to the CM-C and CM-B mixes, and consequently the better quality of their mortars compared to those of the CL-C and CG-C mixes, respectively.

Using the EMV proportioning method resulted in $6 \%$ and $13 \%$ higher compressive strength for all the EM and the EV mixes compared to the companion CL-C and CG-C mixes, respectively. The higher compressive strength of EM-B compared to EM-C may be attributed to the densifying effect of bfs on the microstructure of hydrated cement 
paste which leads to higher density and strength. These higher strengths confirm the findings of the preliminary study which revealed that the compressive strength of RAC is primarily a function of the relative strength of its components rather than of its total mortar volume.

Note, however, that both the CM-F and EM-F mixes had 7-8\% lower strength than the companion CL-C mix, regardless of the mixture proportioning method. This is attributed to the slower hydration rate of fly ash compared to portland cement. However, for the CV-F and EV-F mixes, their compressive strengths were comparable to that of the companion CG-C mix, regardless of the mixture proportioning method. Once again, the latter behaviour is ascribed to the greater angularity and surface roughness of RCA-VA compared to NG.

In Figure 4.8, it can also be observed that the compressive strengths of both CM-C and CV-C mixes were higher than those of the corresponding PM-100MA and PV-100MA mixes, respectively. This may be attributed to the addition of WRA to the former mixes, which resulted in a fresh mortar with higher overall density and strength.

\subsubsection{Elastic Modulus}

As it can be observed in Table 4.7, the elastic modulus of CM and EM mixes range from 27.2 $\mathrm{GPa}(\mathrm{CM}-\mathrm{C})$ to $32.3 \mathrm{GPa}(\mathrm{EM}-\mathrm{B})$, while the elastic modulus of CL-C is $31.1 \mathrm{GPa}$. Similarly, the elastic modulus of the CV and EV mixes proportioned by the conventional and the EMV mix proportioning method range from $28.8 \mathrm{GPa}(\mathrm{CV}-\mathrm{C})$ to $34.2 \mathrm{GPa}$ (EVF), while the elastic modulus of CG-C is $30.7 \mathrm{GPa}$.

Figure 4.9 illustrates the effect of mix proportioning method on the elastic modulus of different mixes. Generally, using conventional mix proportioning method resulted in 
noticeably lower elastic modulus for the $\mathrm{CM}$ and the $\mathrm{CV}$ mixes compared to the companion CL-C and CG-C mixes, respectively. Conversely, using the EMV mix proportioning method resulted in higher elastic moduli for EM-C and EV-C mixes ( $11 \%$ and $14 \%$ higher) compared to the CM-C and CV-C mixes. The elastic modulus of EV-C was even higher than the elastic modulus of the companion CG-C mix. These results clearly show that the use of the EMV method would not result in inferior elastic modulus for RAC mixes. The observed higher moduli can be attributed to the equality of the total natural aggregate volumes of the EM and CL-C mixes on the one hand and the EV and the CG-C mixes on the other. Note that the addition of WRA to the EMV mixes did not alter the total mortar volume, w/c ratio or other proportions of these mixes compared to the corresponding NAC mixes. The WRA was simply used to improve their workability. Hence, the higher moduli cannot be ascribed to the presence of WRA in the EMV mixes. The $7 \%$ higher elastic modulus of EM-B compared to EM-C can be attributed to the densifying effect of bfs on the microstructure of hydrated cement paste and consequently its higher elastic modulus. Due to the lower RMC of RCA-VA, the difference between the elastic moduli of EV-C and EV-B mixes was negligible. Using the EMV method, the elastic moduli of the EM-F and the EV-F mixes were found to be comparable to or higher than those of the companion CL-C and CG-C mixes, but were $10 \%$ and $12 \%$ higher than those of CM-F and CV-F mixes, respectively. With reference to Figure 4.9, it can be observed that using WRA resulted in 31\% and $11 \%$ higher elastic moduli for CM-C and CV-C mixes compared to the moduli of the corresponding PM-100MA and PV-100MA mixes, but the elastic moduli of the former mixes are $13 \%$ and $6 \%$ lower than those of the companion CL-C and CG-C mixes, respectively. 


\subsubsection{Stress-Strain Relationship}

In this section, only the mixes made with ordinary Portland cement, without the addition of any fly ash or ground granulated blast furnace slag (bfs), will be used to study the effect of the proposed mix proportioning method on the stress-strain relationship of RAC. The mixes considered are:

1. RAC produced with $100 \%$ RCA content using the conventional mix proportioning method (CM-C and CV-C mixes).

2. NAC produced with $100 \%$ NA content proportioned by the conventional method (CL-C and CG-C mixes).

3. RAC proportioned by the equivalent mortar volume (EMV) method (EM-C and EVC mixes).

Three cylindrical specimens $(100 \times 200 \mathrm{~mm})$ were prepared and cast for each mix type. After moist curing for 24 hours and demolding the specimens, they were kept moist for another 7 days by covering them with a plastic sheet and wet burlap, and stored in an airdry condition waiting until the test date. The mix proportions of each mix, whose test results are presented in Table 4.8, can be found in Table 4.5.

For investigating the stress-strain behaviour of RAC, an LVDT was used for measuring the average deformation of the specimens. The test jig, Fig.4.10, consisted of two rings: one at the top and one at the bottom. The top ring was rigidly attached to the specimen by means of two diametrically opposite pins so that it was free to rotate (see Figure 4.10). The bottom ring was rigidly attached to the specimen at three points by using three pins uniformly distributed around the perimeter of the specimen. At one point on the circumference of the rotating ring, midway between the two support points, a pivot rod 
was used to maintain a constant distance between the rings. At the opposite point on the circumference of the rotating ring, the change in distance between the rings recorded by the LVDT is equal to the sum of the specimen deformation of the specimen and the displacement due to the rotation of the ring about the pivot rod. Since the distances of the pivot rod and the LVDT from the vertical plane passing through the support pins of the rotating ring were chosen equal, the deformation of the specimen is equal to one-half the LVDT reading. Therefore, the stress and strain values at any load point can be calculated as follows:

$$
\begin{gathered}
\sigma=\frac{P}{A_{c}} \\
\varepsilon=\frac{m}{2 L_{i}}
\end{gathered}
$$

where $P$ is the applied load on the specimen, $A_{c}$ is the cross section area of the specimen, $\sigma$ is the compressive stress due to $P, m$ is the LVDT reading, $L_{i}$ is the initial length of LVDT (5 inches), and $\varepsilon$ is the compressive strain in the specimen corresponding to the stress $\sigma$.

Figure 4.11 presents the observed stress-strain curves of the different mixes. Three samples for each mix type were tested to investigate its stress-strain relationship, except for CV and CG mixes, for which two samples were tested. Generally, the stress-strain curves of the repeated samples for each mix type followed by the same ascending branch. However, there were some minor differences observed among the descending branches. It should be acknowledged that the descending branch of the curve is greatly dependent on the rigidity of the testing machine. In flexible testing machines, the testing sample may not be able to absorb the release in strain energy from the testing machine when the 
load drops after the peak load. Therefore, the minor differences among the descending branches may be attributed to the difference in the concrete characteristics within different samples and consequently different responses to the strain release after the peak load.

In order to compare the stress-strain curves of the mixes proportioned by the different mix proportioning methods, the corresponding curves have been normalized to the stress corresponding to their peak strain values. Since there were minor differences among the three repeat samples for each mix type, one sample was taken as the reference for each mix type. Figure 4.12 illustrates the effect of mix proportioning method on the stressstrain curves of the different mixes. For concrete mixes produced with RCA-MO and/or NL, generally, the ascending branch of stress-strain curve of EM was essentially coinciding with that of $\mathrm{CL}$, while for $\mathrm{CM}$; it was found to be different from those of $\mathrm{CL}$ and EM. This can be mainly attributed to the lower elastic modulus of the RAC mixes proportioned by the conventional method compared to RAC proportioned by the EMV method. Furthermore, the descending branch of EM was found to have a larger tail than that of mix CL. The larger tail is indicative of greater ductility. The descending branch of the CM mix could not be fully traced due to the sudden drop of the curve during the test. For concrete mixes produced with RCA-VA and/or NG, generally the ascending part of the curves for the $\mathrm{CV}$ and $\mathrm{EV}$ mixes were found to be rather similar, while for the CG; it was found to be only slightly stiffer. This may be attributed to (1) the different composition of RM compared to NM due to the observed fly ash particles in the composition of RM in RCA-VA (Abbas et al., 2007c), and (2) the presence of more interfaces between coarse aggregate and mortar. As stated earlier, there are two types of 
interfaces in RAC: (1) the interface between OVA with RM, and (2) the interface between the coarse RCA and/or NA with NM. Since the secant elastic modulus is usually calculated based on the stress and strain values at $40 \%$ of the peak stress, the stress-strain curve generally does not deviate from linearity dramatically within this range of loading. After this point, usually the cracks at the interface of the coarse aggregate and matrix propagate, and this is the start of the descent of concrete toward failure. In EM and EV mixes, although the TM volume concept increased their elastic moduli, nevertheless due to the high number of OVA particles in the RAC mixes, the overall interface area in an RAC mix is larger than that in the companion NAC, which is expected to increase the number of cracks at the interface of the coarse OVA and RM and accelerates the degradation of strength.

The mean values of the strains corresponding to the peak stress, $\varepsilon_{0}$, in CM, EM and CL mixes were $0.00270,0.00230$, and 0.00203 , respectively, while in $\mathrm{CV}, \mathrm{EV}$ and CG mixes, they were $0.00243,0.00240$, and 0.00190 , respectively. The higher $\varepsilon_{0}$ in $C M$ and CV mixes compared to CL and CG mixes can be attributed to the lower elastic modulus of the former.

\subsection{Long-Term Performance}

To investigate the long-term performance of RAC, including its creep and shrinkage characteristics, three mix types were prepared for each RCA source using only Type 10 Portland cement without any fly ash or bfs:

1. RAC using $100 \%$ RCA content proportioned by the conventional method (CM and CV mixes). 
2. NAC using $100 \%$ NA content proportioned by the conventional method (CL and CG mixes).

3. RAC proportioned by the EMV method (EM and EV mixes).

The mix proportions of each mix, whose fresh and hardened properties are presented in Table 4.9, can be found in Table 4.5.

In the following, effort is made to study the effects of mix proportioning method and aggregate type on the creep and shrinkage of RAC.

\subsubsection{Creep}

The creep tests were carried out following the procedure described in ASTM C 512-02 (ASTM, 2003). The creep investigation was conducted on $150 \times 300 \mathrm{~mm}$ cylindrical specimens. The specimens were demolded 24 hours after casting, and cured until age of 7 days in a moist curing room. After the completion of moist curing, they were stored at a temperature of $23.0 \pm 1.1^{\circ} \mathrm{C}$ and at a relative humidity of $50 \pm 4 \%$ until completion of the test.

The loading procedure and measurement techniques used in these tests are based on standard methods, and their details can be found in the above reference, therefore only a brief description of the test is provided below.

\section{Loading Frame (Creep jig)}

The loading frame, i.e. creep jig, consists of the header plates bearing on the ends of the loaded specimens, a load-maintaining element that is either a hydraulic capsule or ram, and threaded rods to take the reaction of the loaded system (Figure 4.13a). In each creep jig, the three test specimens for one mix type were stack and loaded simultaneously. Between the test specimen and the steel bearing plate at each end of the stack, a 
supplementary non-instrumented cylinder whose diameter was equal to that of the test cylinders and whose length was $75 \mathrm{~mm}$ was placed.

In order to measure the load at the nearest $2 \%$ of the total applied load, a hydraulic pressure gauge was permanently installed on each creep jig. The hydraulic jack was separately inserted into each loading frame whenever the load was being applied or adjusted.

\section{Strain-Measuring Device}

In order to measure the longitudinal strain in each specimen to the nearest 10 millionth, a Demec mechanical strain gauge was used (Figure 4.13b). The gauge length was $200 \mathrm{~mm}$ and each division on the dial gauge represented 10 microstrains. Strains were measured along three gauge lines spaced uniformly around the periphery of the specimen. Two Demec points were attached on each gauge line (6 Demec points per specimen) spaced $200 \mathrm{~mm}$ from each other. The Demec strain gauge consisted of an invar main beam with two conical locating points, one fixed and the other pivoting on a special knife edge. Movement of the pivoting point was measured by the strain gauge attached to a base plate on the invar beam. An invar reference bar was also used for control measurements, as well as a setting out bar to enable accurate positioning of Demec points (Figure 4.13c). Nine specimens were made for each mix; three were tested for compressive strength, three were subjected to sustained load and the remaining three were kept unloaded and used as controls to measure deformations due to causes other than load such as thermal and shrinkage deformations. Each strength and control specimen was kept under the same curing and storage treatment as the loaded specimen. 
As the purpose of the test was to compare the creep behaviour of different RAC mixes proportioned by the conventional and EMV methods with the companion NAC, the specimens loading was commenced at the age of 28 days.

Immediately before loading the creep specimens, the compressive strength of each mix was determined by testing the pertinent cylinders. Then, the companion creep specimens were loaded by a sustained load equal to $40 \%$ of their compressive strength at the age of loading. The strain readings were recorded immediately before and after loading, 2 to 6 hours later, then daily for 1 week, weekly until the end of 1 month, and monthly until the end of 1 year. Before taking each reading, the load was monitored and adjusted whenever deviated more than $2 \%$ from its set value using a hand operated hydraulic pump. The strain readings on the control specimens were taken following the same schedule as the loaded specimens.

The total strain, $\varepsilon_{\text {total }}(t)$, at any time was calculated as the difference between the average strain readings of the loaded and control specimens. To determine the creep strain at any age, $\varepsilon_{c}(t)$, the instantaneous strain immediately after loading, $\varepsilon_{0}$, was subtracted from the total strain at the same age as follows:

$$
\varepsilon_{c}(t)=\varepsilon_{\text {total }}(t)-\varepsilon_{0}
$$

The unit creep or specific creep, $\varepsilon_{s p}(t)$, at any age was also determined as follows:

$$
\varepsilon_{s p}(t)=\frac{\varepsilon_{c}(t)}{\sigma_{c}}
$$

where $\sigma_{c}$ is the sustained stress acting on the specimen, i.e. $40 \%$ of its compressive strength. 
Since the initial or instantaneous strain is basically a function of the elastic modulus of concrete, for the sake of comparison, it is reasonable to remove the effect of this parameter and normalize the creep strain by the initial strain. Alternatively one could express the results in the form of creep coefficient, $C_{t}$, as follows:

$$
C_{t}=\frac{\varepsilon_{c}(t)}{\varepsilon_{0}}
$$

Another alternative is to simply divide the total strain by the initial strain as:

$$
C_{t}^{\text {Total }}=\frac{\varepsilon_{\text {total }}(t)}{\varepsilon_{0}}
$$

At $\mathrm{t}=0$, the value of $C_{t}$ would be zero while the value of $C_{t}^{\text {Total }}$ would be equal to one. However, the creep coefficient is commonly defined as given by Eq.4.34.

Figure 4.14 illustrates the effect of mix proportioning method on the creep coefficient of the different mixes. Generally, the mixes made with RCA-MO and/or NL proportioned by the proposed method (EM mix) had the lowest creep coefficient values compared to the RAC mixes proportioned by the conventional method and even compared to the companion CL mix. At the age of 305 days, the creep coefficient of EM was $30 \%$ lower than that of CL, while that of CM was $31 \%$ higher. Since all of the factors affecting the creep properties of concrete (w/c ratio, applied stress, curing condition, temperature, and moisture) in this study were kept identical for the different mix types, the total coarse aggregate content (or total mortar volume) can be identified as the main reason for the higher creep coefficient of the RAC mixes proportioned by the conventional method compared to those proportioned by the EMV method. These results validate the fundamental hypothesis of the EMV method. The better performance of EM compared to 
the companion CL with identical TM volume in the two mixes may be ascribed to the better performance of RM in RCA-MO compared to the NM.

In the mixes produced with RCA-VA and/or NG, the RAC mixes proportioned by the proposed method (EV mix) had 3\% lower creep values than the companion NAC (i.e., $\mathrm{CG}$ ), while for the RAC proportioned by the conventional method (i.e., CV), it was found to be $11 \%$ higher at the age of 305 days. This can be mainly attributed to the higher total mortar in RAC mixes proportioned by the conventional method.

Figure 4.15 illustrates the effect of mix proportioning method on the $C_{t}^{\text {Total }}$ value of the different mixes. Generally, the $C_{t}^{\text {Total }}$ follows the same basic trend as the creep coefficient. For the mixes produced with RCA-MO and/or NL, the RAC mixes proportioned by the proposed method (EM mix) had the lowest $C_{t}^{\text {Total }}$ values compared to the companion RAC mixes proportioned by the conventional method and even compared to the companion CL mix. At the age of 305 days, the $C_{t}^{\text {Total }}$ value of EM was $23 \%$ lower than that of CL, while the $C_{t}^{\text {Total }}$ of CM was $24 \%$ higher than that of CL. In the mixes produced with RCA-VA and/or NG, the RAC proportioned by the proposed method (EV mix) had about $2 \%$ lower $C_{t}^{\text {Total }}$ values than that of the companion NAC, while for the RAC mixes proportioned by the conventional method; it was higher than the companion NAC ( $8 \%$ higher at the age of 305 days). This can be again attributed to the higher total mortar volume in RAC mixes proportioned by the conventional method.

Figure 4.16 illustrates the effect of mix proportioning method on the specific creep of the different mixes. The specific creep values follow the same trend as the creep coefficient. 
Figure 4.17 illustrates the effect of mix proportioning method on the creep strain of the different mixes. Similar to the creep coefficient values, in the mixes made with RCA-MO and/or NL, the RAC mixes proportioned by the proposed method (EM mix) had the lowest creep strain values compared to the RAC mixes proportioned by the conventional method and even compared to the companion CL mix. At the age of 305 days, the creep strain of EM was $22 \%$ lower than that of CL, while in CM it was $49 \%$ higher. Once more, the higher creep strain of CM compared to that of the companion CL can be ascribed to the higher TM volume of CM.

In the mixes produced with RCA-VA and/or NG, both the RAC mixes proportioned by the proposed method (EV mix) and by the conventional method (CV) had higher creep strain compared to the companion NAC, i.e. CG (13\% and $19 \%$ higher, respectively). The higher creep strain in CV can once again be attributed to its higher total mortar content of this mix compared to that in the CG. Since the TM volume in EV and CG were identical, the higher creep strain in the former is mainly due to its higher initial or elastic strain (0.007020 and 0.006016 initial strain in EV and CG mixes, respectively).

Figure 4.18 illustrates the effect of mix proportioning method on the total creep plus shrinkage strain of the different mixes.

Similar to the creep strain values, in the mixes made with RCA-MO and/or NL, the RAC mixes proportioned by the proposed method (EM mix) had the lowest total creep plus shrinkage values compared to the RAC mixes proportioned by the conventional method and even compared to the companion CL mix. At the age of 305 days, the total creep plus shrinkage strain of EM was $21 \%$ lower than that of CL, while in CM it was $48 \%$ higher 
than that of CL. Once more, the higher total creep plus shrinkage strain in CM compared to the companion CL can be attributed to the higher TM volume of the former.

For the mixes made with RCA-VA and/or NG, the RAC mixes proportioned by the proposed method (EV mix) and those proportioned by the conventional method (CV) had higher total creep plus shrinkage strain compared to the companion NAC, i.e. CG ( $16 \%$ and $23 \%$ higher, respectively). The higher total creep plus shrinkage strain in CV can once again be ascribed to its higher total mortar content compared to that in CG. Since the TM volume in EV and CG were identical, the higher total creep plus shrinkage strain in the former mix may be mainly due to its higher elastic strain at initial loading (0.007020 and 0.006016 initial strain in EV and CG mixes, respectively).

Figure 4.19 illustrates the effect of aggregate type on the creep coefficient of different mixes. According to Figure 4.19c, the creep coefficient variation over time was found quite similar for the mixes made with the two different natural aggregates (CL and CG). However, for RAC mixes, the creep coefficient of the mixes made with RCA-MO and RCA-VA were found different, regardless of the mix proportioning method. For RAC mixes proportioned by the conventional method, this can be ascribed to the different RM volumes in RCA-MO and RCA-VA aggregates, and consequently different TM volumes in CM and CV mixes. For the RAC mixes proportioned by the EMV method, this can be attributed to the difference in the composition of RM in RCA-MO and RCA-VA aggregates. In fact, due to the presence of fly ash in the RM of RCA-VA (Abbas et al., 2007c), the composition of RM was different than that in NM, which could result in overall higher creep in TM in CV compared to that in CM (Mindess and Young, 1981). 


\subsubsection{Drying Shrinkage}

The shrinkage tests were carried out following the procedure described in ASTM C 157/C 157M-99 (ASTM, 2003). The shrinkage investigation was conducted on $100 \times 100 \times 285 \mathrm{~mm}$ prisms. The test specimens were cured in the molds in the moist room and covered with plastic sheets to protect them from dripping water. Twenty four hours after mixing, the specimens were demolded. After the initial comparator reading, the prisms were stored in lime-saturated water at $23 \pm 2^{\circ} \mathrm{C}$ until the age of 28 days. At the end of the curing period, a second comparator reading was made and the specimens were stored in a drying room.

Readings were taken after $4,7,14$, and 28 days, and $8,16,32$, and 64 weeks after the end of moist curing. During each measurement, the reference bar was placed in the comparator, and the comparator dial was set to zero. Then, the specimen was placed in the comparator and the comparator dial reading was recorded. Using these readings, the shrinkage of the specimens is calculated as:

$$
\varepsilon_{s h}=\left(\frac{l_{2}-l_{1}}{l_{g}}\right)
$$

where $\varepsilon_{s h}$ is the drying shrinkage of the specimen, $l_{1}$ is the initial comparator reading, $l_{2}$ is the comparator reading at time $\mathrm{t}$, and $l_{g}$ is the effective gauge length between the inner most ends of the gauge studs embedded in the specimens.

Figure 4.20 illustrates the effect of mix proportioning method on the drying shrinkage strain of the different mixes. Generally, at early ages, the EM mix proportioned by the EMV method experienced the highest shrinkage compared to the CM mix proportioned by the conventional method and the companion CL. However, after age of 75 days, the 
shrinkage strain in the EM started to decrease and became less than the shrinkage of CM, but still slightly higher than that of CL. At the age of 224 days, the drying shrinkage in EM and CM were $7 \%$ and $26 \%$ higher than that of the companion CL. Since well known factors affecting the shrinkage properties of concrete (w/c ratio, curing condition, temperature, moisture, etc.) were kept the same for the current test specimens, the total coarse aggregate content or total mortar volume can be considered as the key factor for the higher drying shrinkage in the RAC mix proportioned by the conventional method compared to that proportioned by the EMV method. This again confirms the fundamental hypothesis of the EMV method.

Similarly, at early ages, the EV mix proportioned by the proposed method experienced the highest shrinkage strain values compared to those $\mathrm{CV}$ mixes proportioned by the conventional method and the companion CG. However, after the age of 75 days, the corresponding drying shrinkage strain in EV started to decrease to values lower than those of CM and CG. At the age of 224 days, the drying shrinkage in EM was 15\% lower than that of the companion CG mix, while in CV it was $14 \%$ higher than in CG.

Figure 4.21 illustrates the effect of aggregate type on the drying shrinkage of different mixes. Generally, the drying shrinkage strain in CV, EV and CG mixes (produced with RCA-VA and/or NG) were found to be slightly less than those of CM, EM and CL (made of RCA-MO and/or NG), respectively. The difference was more noticeable in EM compared to EV.

As stated earlier, in a separate, but parallel collaborative study, matters related to durability of RAC were investigated by Abbas(2007d). To put the focus of the present study in context, a summary of the results of that investigation follows: 
1. From all durability performance indicators, RAC produced with either conventional mix design method or with the equivalent total volume of mortar method have strong resistance against freeze-and-thaw action, a resistance expected from structural-grade concrete. However, since lower total residual mortar content values can be achieved using the equivalent mortar volume method, this method would be preferable for RCA with very large RMC values and when freeze-and-thaw resistance is considered to be a serious problem for the structure under consideration.

2. The resistance to chloride penetration of RAC is improved by using SCM's (fly ash or bfs) as partial replacement for OPC. The use of bfs reduces the apparent diffusion coefficient of all mixes, designed by either mix design method, 1.2-2 times more than the reduction achieved by the addition of fly ash. Both conventional mix design method and the equivalent mortar volume method provide good quality concrete against chloride penetration when SCM's are used.

3. The investigation of the results of the RAC specimens produced from different sources of RCA showed that pre-contamination is one of the main factors affecting the apparent diffusion coefficient. If the RCA is not excessively pre-contaminated, increased amounts of residual mortar have the potential to increase the binding capacity of the RAC; hence decreasing the diffusion of chloride ions in concrete. Therefore, when RCA is clear from pre-contamination, the use of the conventional mix design method improves concrete resistance to chloride ion penetration.

4. In the case of pre-contaminated RCA, the increased amounts of residual mortar will not improve the binding capacity of RAC; therefore, the use of equivalent mortar volume method can be considered to be more appropriate to design RAC with these RCA. 
5. The carbonation depths of RAC with and without SCM's fall in the expected range for structural-grade conventional concrete. RAC specimens with no SCM addition showed the lowest level of carbonation; these specimens were followed by the specimens with bfs. On the other hand, mixes with fly ash had the largest amount of carbonation depth and coefficient (low resistance to carbonation).

6. The investigation of the results of the specimens designed with different mix design methods showed that the main factor affecting the carbonation of RAC is the total reserve alkalinity (alkalinity from hydration of new cement content and residual old mortar). Those specimens with the high total cement content (i.e., total reserve alkalinity) have been found to give higher resistance to carbonation. The effect of mix design method on the carbonation of GC is not significant when fly ash is used. Therefore, when fly ash is used, both general mix design and equivalent mortar volume methods can be used for designing the mixes.

7. When fly ash is not used, the carbonation of RAC produced with RCA with high RMC (e.g. $>40 \%$ as in the case of RCA-MO) was found to be higher in mixes designed with the equivalent mortar volume method. Therefore, for these types of RCA, the conventional mix design method may be preferred.

8. When fly ash is not used, the carbonation of RAC produced with RCA with low RMC (e.g. $<25 \%$ as in the case of RCA-VA) was found to be similar in all mixes designed with either mix design method. Therefore, for these types of RCA, both either method may be used. 


\subsection{Summary of Test Results}

- The slump of RAC mixes proportioned by the proposed EMV method was comparable to the slump of the mixes proportioned by the conventional method, given equal cement and water contents and with or without the addition of fly ash or bfs.

- All RAC mixes proportioned by the proposed EMV method had comparable or higher fresh and hardened density values compared to the corresponding densities of the companion NAC mixes, while they were lower for the RAC mixes proportioned by the conventional method. This was true for both RCA sources and with or without addition of fly ash or bfs.

- The mix proportioning method and the total mortar volume did not have appreciable effect on the compressive strength of RAC mixes. Generally, the compressive strength was found to be mostly dependant on the strength of the mortar and the quality of bond between the aggregate and the mortar rather than the TMV.

- The mix proportioning method and the TMV had a significant effect on the elastic modulus of concrete made with RCA. Increasing the TMV decreases elastic modulus. All concrete mixes produced with RCA proportioned by the EMV method had comparable or higher elastic modulus values compared to the corresponding values for the companion NAC mixes, while they were lower for those mixes proportioned by the conventional method. This was true for both RCA sources and with or without the addition of fly ash or bfs.

- In case of the stress-strain relationship of concrete made with RCA, both the mix proportioning method and the quality of the aggregate-mortar bond were observed to have significant effect on the ascending branch of the curve and on the strain values 
corresponding to the peak stress. In the case of RCA-MO, the mix proportioning method was the dominant effect, and TMV concept resulted in the similar stress-strain relation of RAC being practically the same as that of NAC. However, for RCA-VA, the quality of aggregate-mortar interface and bond was observed to dominate their response, which resulted in higher strain values corresponding to peak stress compared to NAC.

- As for the descending branch of the curve, the RAC mixes proportioned by the proposed EMV method were found to exhibit more ductile behaviour compared to those proportioned by the conventional method and the companion NAC.

- The effect of mix proportioning on the creep of concrete produced with RCA was quite noticeable. Generally, the RAC mixes proportioned by the proposed EMV method had lower or comparable creep coefficient values as those of the companion NAC mixes, while the RAC mixes proportioned by the conventional method always had higher creep values.

- The effect of aggregate on creep was also observed to be more noticeable in RAC mixes proportioned by either method compared to the companion NAC.

- Despite the slightly higher drying shrinkage values at early ages, generally the RAC mixes proportioned by the EMV method were found to have comparable or lower shrinkage values than the companion NAC, while the RAC mixes proportioned by the conventional method, had higher shrinkage strain. 
Table 4.1: Measured properties for the residual mortar of RCA-MO

\begin{tabular}{|c|c|c|c|c|c|c|c|c|c|}
\hline \multirow{3}{*}{$\begin{array}{c}\text { Sample } \\
\#\end{array}$} & \multirow{3}{*}{$\begin{array}{c}\text { WOD }_{\text {OD }} \\
\text { (g) }\end{array}$} & \multirow{3}{*}{$\begin{array}{c}W_{\text {SSD }} \\
\text { (g) }\end{array}$} & \multirow{2}{*}{\multicolumn{2}{|c|}{$\begin{array}{l}\text { Reading of water } \\
\text { Level in } \\
\text { Le Chatelier Flask }\end{array}$}} & \multicolumn{3}{|c|}{ Specific Gravity } & \multirow{3}{*}{$\begin{array}{l}\mathrm{AC} \\
(\%)\end{array}$} & \multirow{3}{*}{$\begin{array}{c}n \\
(\%)\end{array}$} \\
\hline & & & & & \multirow{2}{*}{ SSD } & \multirow{2}{*}{ Bulk } & \multirow{2}{*}{ App. } & & \\
\hline & & & initial & final & & & & & \\
\hline 1 & 48.78 & 54.00 & 0.10 & 23.90 & 2.27 & 2.05 & 2.63 & 10.7 & 21.9 \\
\hline 2 & 45.00 & 49.48 & 0.10 & 21.50 & 2.32 & 2.11 & 2.67 & 9.96 & 20.9 \\
\hline 3 & 45.00 & 49.35 & -0.10 & 21.30 & 2.31 & 2.11 & 2.65 & 9.67 & 20.3 \\
\hline 4 & 45.00 & 49.30 & -0.10 & 21.20 & 2.32 & 2.12 & 2.66 & 9.56 & 20.2 \\
\hline \multicolumn{5}{|c|}{ Average } & 2.31 & 2.10 & 2.65 & 9.97 & 20.9 \\
\hline
\end{tabular}

Table 4.2: Measured properties for the residual mortar of RCA-VA

\begin{tabular}{|c|c|c|c|c|c|c|c|c|c|}
\hline \multirow{3}{*}{$\underset{\#}{\text { Sample }}$} & \multirow{3}{*}{$\begin{array}{l}\text { Wod }_{\text {OD }} \\
\text { (g) }\end{array}$} & \multirow{3}{*}{$\begin{array}{c}W_{\text {SSD }} \\
\text { (g) }\end{array}$} & \multirow{2}{*}{\multicolumn{2}{|c|}{$\begin{array}{l}\text { Reading of water } \\
\text { Level in } \\
\text { Le Chatelier Flask }\end{array}$}} & \multicolumn{3}{|c|}{ Specific Gravity } & \multirow{3}{*}{$\begin{array}{l}\text { AC } \\
(\%)\end{array}$} & \multirow{3}{*}{$\begin{array}{c}n \\
(\%)\end{array}$} \\
\hline & & & & & \multirow{2}{*}{ SSD } & \multirow{2}{*}{ Bulk } & \multirow{2}{*}{ App. } & & \\
\hline & & & initial & final & & & & & \\
\hline 1 & 49.22 & 54.40 & 0.00 & 23.70 & 2.30 & 2.08 & 2.67 & 10.5 & 21.9 \\
\hline 2 & 45.00 & 49.55 & 0.50 & 21.90 & 2.32 & 2.11 & 2.68 & 10.1 & 21.3 \\
\hline 3 & 45.00 & 49.45 & 0.00 & 21.30 & 2.33 & 2.12 & 2.68 & 9.89 & 20.9 \\
\hline 4 & 45.00 & 49.41 & -0.10 & 21.10 & 2.34 & 2.13 & 2.69 & 9.80 & 20.8 \\
\hline \multicolumn{2}{|c|}{ Average } & & & & 2.32 & 2.11 & 2.68 & 10.0 & 21.2 \\
\hline
\end{tabular}

Table 4.3: Measured properties for the original virgin aggregate of RCA-MO

\begin{tabular}{|c|c|c|c|c|c|c|c|c|c|}
\hline \multirow{3}{*}{$\begin{array}{c}\text { Sample } \\
\#\end{array}$} & \multirow{3}{*}{$\begin{array}{c}\text { WoD }_{\text {od }} \\
(\mathrm{g})\end{array}$} & \multirow{3}{*}{$\begin{array}{c}\text { WSSD }_{\text {SD }} \\
(\mathrm{g})\end{array}$} & \multirow{2}{*}{\multicolumn{2}{|c|}{$\begin{array}{l}\text { Reading of water } \\
\text { Level in } \\
\text { Le Chatelier Flask }\end{array}$}} & \multicolumn{3}{|c|}{ Specific Gravity } & \multirow{3}{*}{$\begin{array}{l}\mathbf{A C} \\
(\%)\end{array}$} & \multirow{3}{*}{$\begin{array}{c}\mathrm{n} \\
(\%)\end{array}$} \\
\hline & & & & & \multirow{2}{*}{ SSD } & \multirow{2}{*}{ Bulk } & \multirow{2}{*}{ App. } & & \\
\hline & & & initial & final & & & & & \\
\hline 1 & 50.00 & 50.50 & -0.10 & 18.40 & 2.74 & 2.71 & 2.78 & 1.00 & 2.71 \\
\hline 2 & 50.00 & 50.45 & -0.10 & 18.30 & 2.75 & 2.72 & 2.79 & 0.90 & 2.45 \\
\hline 3 & 50.00 & 50.48 & 0.00 & 18.40 & 2.75 & 2.72 & 2.80 & 0.96 & 2.62 \\
\hline \multicolumn{2}{|c|}{ Average } & & & & 2.75 & 2.72 & 2.79 & 0.95 & 2.59 \\
\hline
\end{tabular}


Table 4.4: Measured properties for the original virgin aggregate of RCA-VA

\begin{tabular}{|c|c|c|c|c|c|c|c|c|c|}
\hline \multirow{3}{*}{$\begin{array}{c}\text { Sample } \\
\#\end{array}$} & \multirow{3}{*}{$\begin{array}{c}\text { Wod } \\
\text { (g) }\end{array}$} & \multirow{3}{*}{$\begin{array}{c}W_{\text {SSD }} \\
(\mathrm{g})\end{array}$} & \multirow{2}{*}{\multicolumn{2}{|c|}{$\begin{array}{l}\text { Reading of water } \\
\text { Level in } \\
\text { Le Chatelier Flask }\end{array}$}} & \multicolumn{3}{|c|}{ Specific Gravity } & \multirow{3}{*}{$\begin{array}{l}\text { AC } \\
(\%)\end{array}$} & \multirow{3}{*}{$\begin{array}{c}\mathrm{n} \\
(\%)\end{array}$} \\
\hline & & & & & \multirow{2}{*}{ SSD } & \multirow{2}{*}{ Bulk } & \multirow{2}{*}{ App. } & & \\
\hline & & & initial & final & & & & & \\
\hline 1 & 50.00 & 50.51 & 0.60 & 18.70 & 2.80 & 2.77 & 2.85 & 1.02 & 2.82 \\
\hline 2 & 50.00 & 50.48 & 0.20 & 18.30 & 2.80 & 2.77 & 2.84 & 0.96 & 2.66 \\
\hline 3 & 50.00 & 50.46 & 0.30 & 18.20 & 2.83 & 2.80 & 2.87 & 0.92 & 2.58 \\
\hline \multicolumn{2}{|c|}{ Average } & & & & 2.81 & 2.78 & 2.86 & 0.97 & 2.69 \\
\hline
\end{tabular}




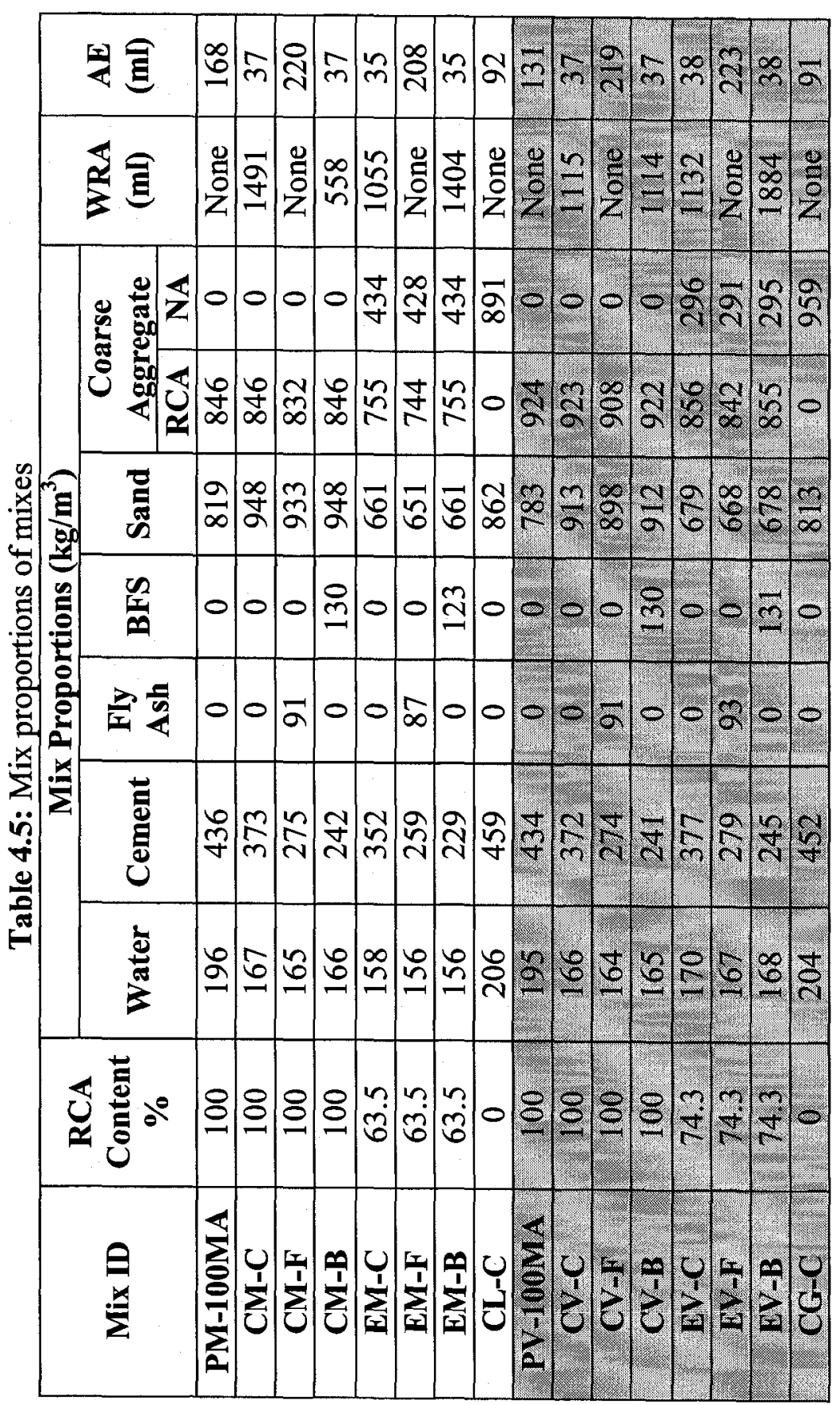


Table 4.6: Fresh properties of mixes

\begin{tabular}{|c|c|c|c|}
\hline Mix ID & $\begin{array}{c}\text { Slump } \\
(\mathrm{mm})\end{array}$ & $\begin{array}{c}\text { Air Content } \\
(\%)\end{array}$ & $\begin{array}{c}\text { Fresh Density } \\
\left(\mathrm{kg} / \mathrm{m}^{3}\right)\end{array}$ \\
\hline PM-100MA & 190 & 7.4 & 2181 \\
\hline CM-C & 70 & 6.9 & 2262 \\
\hline CM-F & 130 & 7.4 & 2226 \\
\hline CM-B & 55 & 6.0 & 2281 \\
\hline EM-C & 105 & 6.0 & 2305 \\
\hline EM-F & 120 & 5.7 & 2291 \\
\hline EM-B & 80 & 5.7 & 2306 \\
\hline CL-C & 175 & 6.3 & 2332 \\
\hline PV-100MA & 150 & 6.9 & 2224 \\
\hline CV-C & 70 & 7.4 & 2285 \\
\hline CV-F & 90 & 6.0 & 2281 \\
\hline CV-B & 110 & 7.1 & 2289 \\
\hline EV-C & 140 & 6.0 & 2321 \\
\hline EV-F & 140 & 5.5 & 2304 \\
\hline EV-B & 150 & 6.8 & 2325 \\
\hline CG-C & 210 & 6.3 & 2315 \\
\hline
\end{tabular}

Table 4.7: Hardened properties of mixes

\begin{tabular}{|c|c|c|c|}
\hline Mix ID & $\begin{array}{c}\gamma_{\mathbf{c}} \\
\left(\mathbf{k g} / \mathbf{m}^{3}\right)\end{array}$ & $\begin{array}{c}\mathbf{f}_{\mathbf{c}}(28 \text { days }) \\
(\mathrm{MPa})\end{array}$ & $\begin{array}{c}\mathrm{E}_{\mathrm{c}} \\
(\mathrm{GPa})\end{array}$ \\
\hline PM-100MA & 2138 & 30.9 & 20.8 \\
\hline CM-C & 2264 & 41.8 & 27.2 \\
\hline CM-F & 2217 & 34.0 & 28.1 \\
\hline CM-B & 2269 & 42.0 & - \\
\hline EM-C & 2295 & 39.2 & 30.3 \\
\hline EM-F & 2293 & 34.2 & 31 \\
\hline EM-B & 2319 & 41.8 & 32.3 \\
\hline CL-C & 2300 & 36.9 & 31.1 \\
\hline PV-100MA & 2233 & 34.3 & 25.9 \\
\hline CV-C & 2285 & 39.7 & 28.8 \\
\hline CV-F & 2281 & 35.0 & 30.5 \\
\hline CV-B & 2290 & 39.3 & 32.8 \\
\hline EV-C & 2321 & 40.0 & 32.8 \\
\hline EV-F & & 35.2 & 34.2 \\
\hline EV-B & 2325 & 40.0 & 32.8 \\
\hline CG-C & 2315 & 35.6 & 30.7 \\
\hline
\end{tabular}


Table 4.8: Fresh and hardened properties of stress-strain test specimens

\begin{tabular}{|c|c|c|c|c|c|c|c|c|}
\hline \multirow[b]{2}{*}{$\begin{array}{c}\text { Mix } \\
\text { ID }\end{array}$} & \multicolumn{3}{|c|}{ Fresh Properties } & \multicolumn{3}{|c|}{ Hardened properties } & \multirow[b]{2}{*}{$\begin{array}{l}\text { Peak } \\
\text { Strain } \\
\left(\mathrm{x} 10^{-3}\right)\end{array}$} & \multirow[b]{2}{*}{$\begin{array}{l}\text { Peak } \\
\text { Stress }\end{array}$} \\
\hline & $\begin{array}{l}\text { Slump } \\
\text { (mm) }\end{array}$ & $\begin{array}{c}\text { Air } \\
\text { Content } \\
\%\end{array}$ & $\begin{array}{c}\text { Fresh } \\
\gamma_{\mathrm{c}} \\
\left(\mathrm{kg} / \mathrm{m}^{3}\right)\end{array}$ & $\begin{array}{c}28 \text { days } \\
\mathrm{f}_{\mathrm{c}} \\
(\mathrm{MPa})\end{array}$ & $\begin{array}{c}28 \text { days } \\
E_{c} \\
(\mathrm{GPa})\end{array}$ & $\begin{array}{c}\text { Hardened } \\
\gamma_{\mathrm{c}} \\
\left(\mathrm{kg} / \mathrm{m}^{3}\right)\end{array}$ & & \\
\hline $\mathbf{C M}$ & 75 & 5.9 & 2298 & 43.9 & 27.2 & 2290 & 2.70 & 48.5 \\
\hline EM & 95 & 6.4 & 2341 & 41.6 & 29.8 & 2333 & 2.30 & 34.4 \\
\hline $\mathbf{C L}$ & 185 & 5.9 & 2333 & 37.1 & 30.3 & 2308 & 2.03 & 40.4 \\
\hline CV & 25 & 5 & 2378 & 45.9 & 28.8 & 2348. & 2.40 & 51.2 \\
\hline EV & $60=$ & 4.5 & 2398 & 49.1 & 31.8 & 2364 & 2.43. & 45.5 \\
\hline CG & 220 & 6.4 & 2347 & 33.8 & 30.5 & 2308 & 1.90 & 34.5 \\
\hline
\end{tabular}

Table 4.9: Fresh and hardened properties of creep and shrinkage specimens

\begin{tabular}{|c|c|c|c|c|c|}
\hline \multirow{2}{*}{$\begin{array}{c}\text { Mix } \\
\text { ID }\end{array}$} & \multicolumn{2}{|c|}{ Fresh Properties } & \multicolumn{2}{c|}{ Hardened properties } \\
\cline { 2 - 6 } & $\begin{array}{c}\text { Slump } \\
(\mathrm{mm})\end{array}$ & $\begin{array}{c}\text { Air } \\
\text { Content } \\
\%\end{array}$ & $\begin{array}{c}\text { Fresh } \\
\gamma_{\mathrm{c}} \\
\left(\mathrm{kg} / \mathrm{m}^{3}\right)\end{array}$ & $\begin{array}{c}\mathrm{f}_{\mathrm{c}}(\mathrm{MPa}) \\
28 \text { days }\end{array}$ & $\begin{array}{c}\text { Hardened } \\
\gamma_{\mathrm{c}} \\
\left(\mathrm{kg} / \mathrm{m}^{3}\right)\end{array}$ \\
\hline CM & 75 & 5.9 & 2298 & 43.9 & 2290 \\
\hline EM & 110 & 4.9 & 2338 & 41.4 & 2303 \\
\hline CL & 160 & 6.4 & 2330 & 35.2 & 2324 \\
\hline CV & 25 & 5 & 2378 & 45.9 & 2348 \\
\hline EY & 70 & 5.6 & 2398 & 4944.8 & 2364 \\
\hline CG & 200 & 6.2 & 2358 & 34.1 & 2322 \\
\hline
\end{tabular}
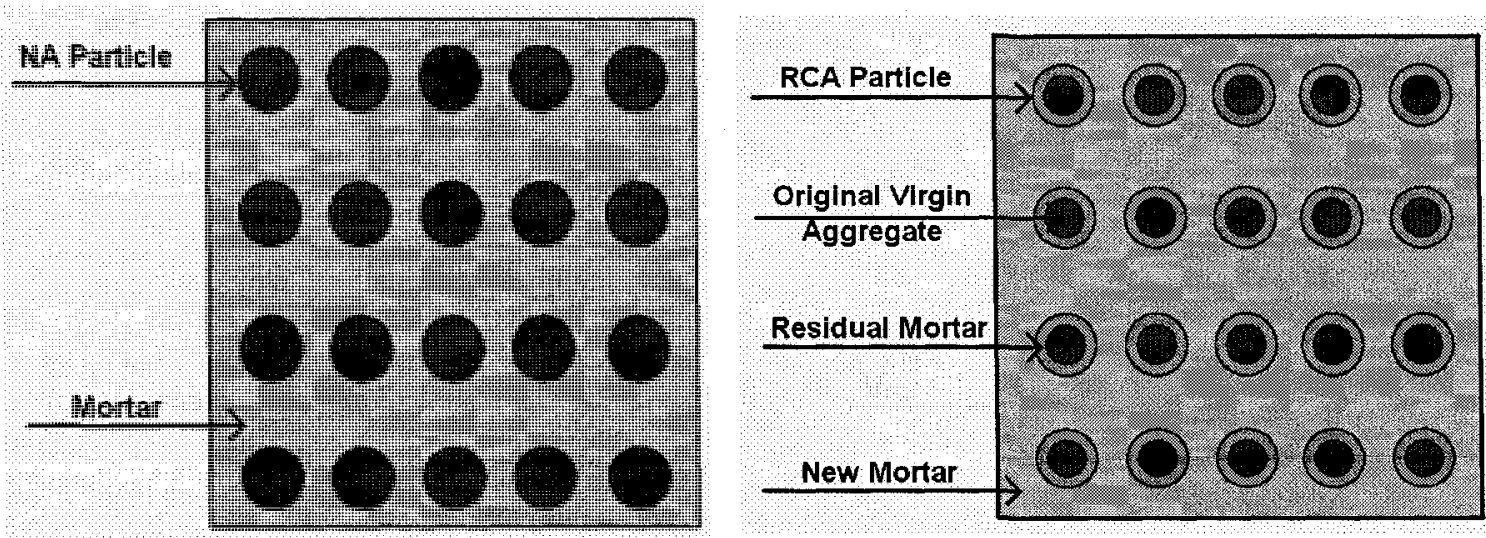

(New Mortar Volume) $\left.)_{\mathrm{RAC}}+(\text { Residual Mortar Volume })_{\mathrm{RAC}}\right\rangle_{(\text {Mortar Volume) }}$

a) Natural aggregate concrete (NAC) $\quad$ b) Recycled aggregate concrete (RAC)

Figure 4.1: Schematic illustration of the composition of RAC proportioned by the conventional method 


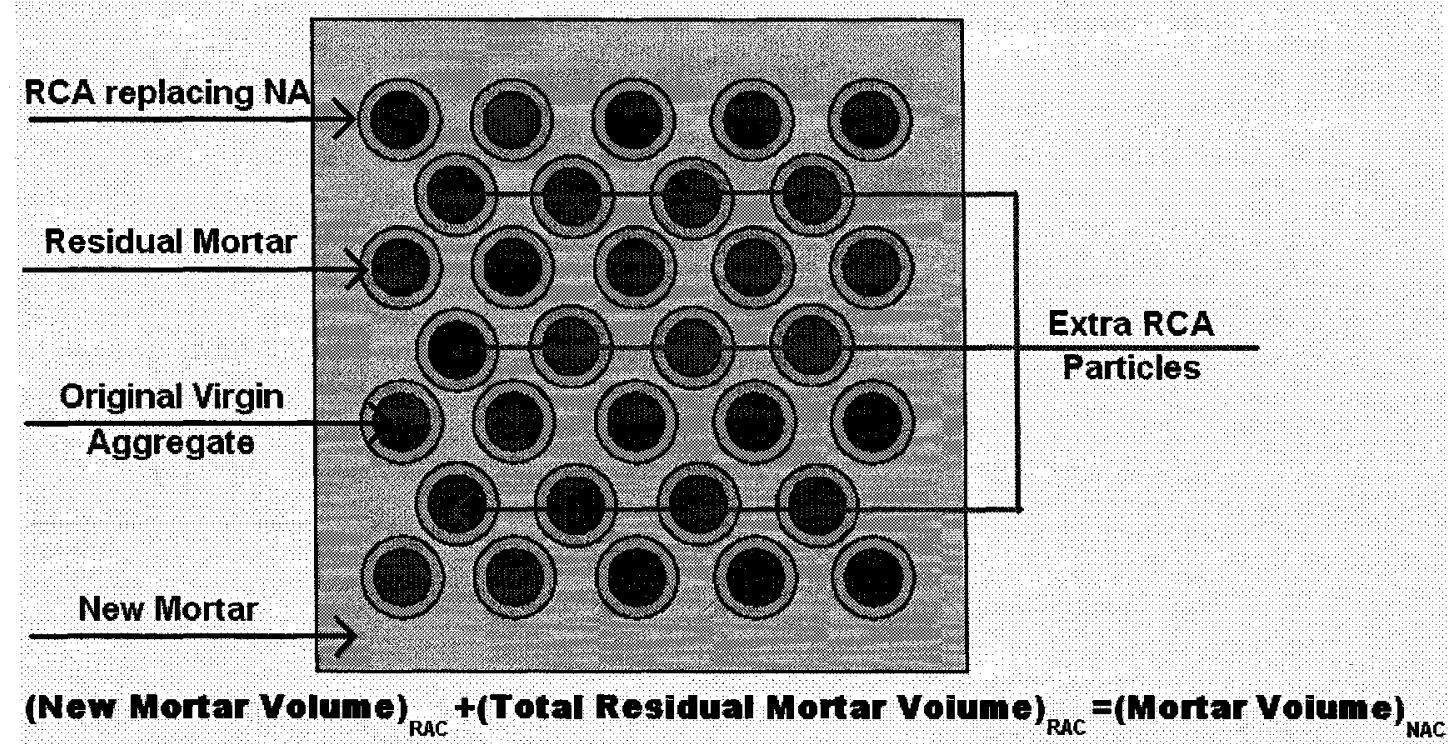

Figure 4.2: Schematic illustration of the composition of RAC proportioned by the proposed method

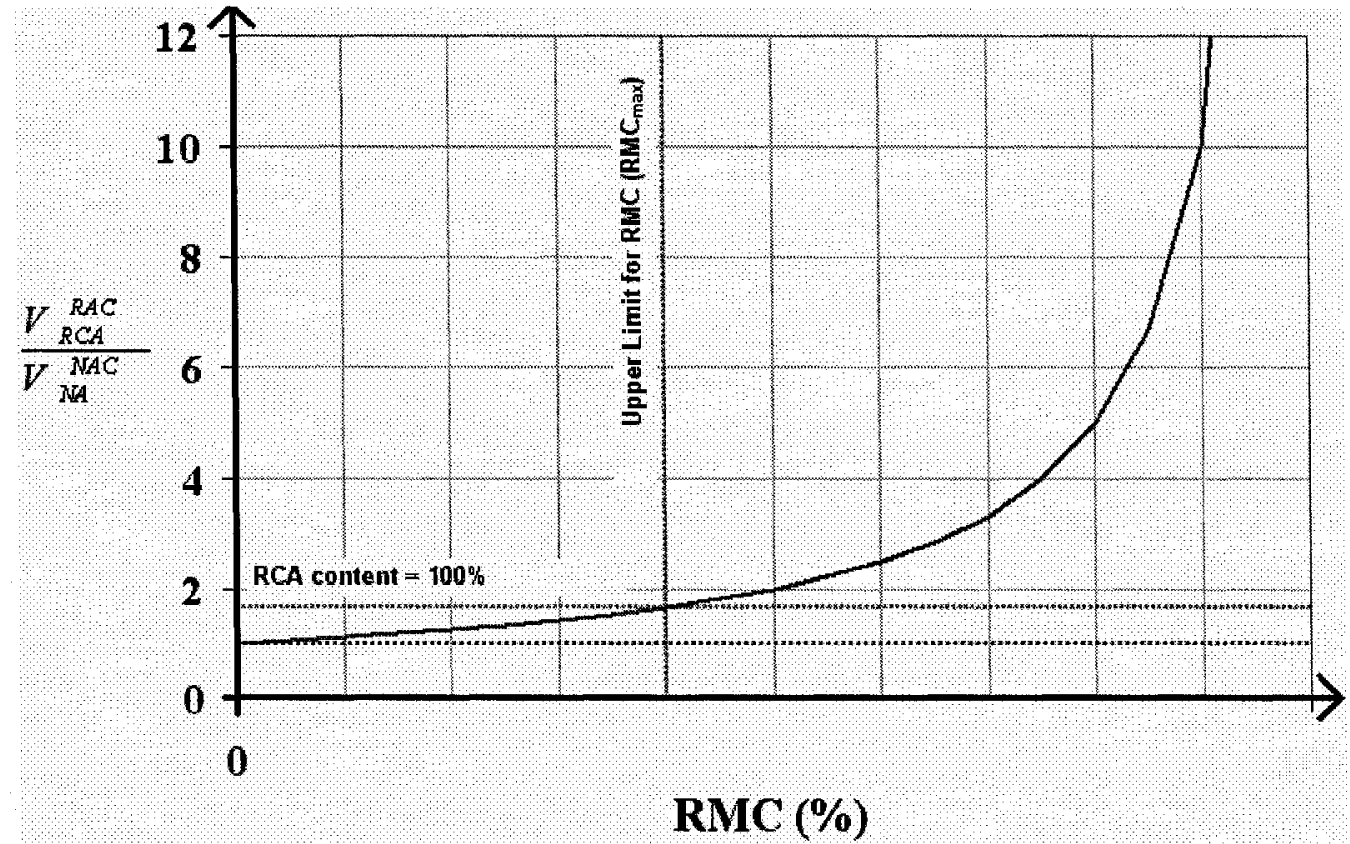

Figure 4.3: Variation of RCA volume with RMC 


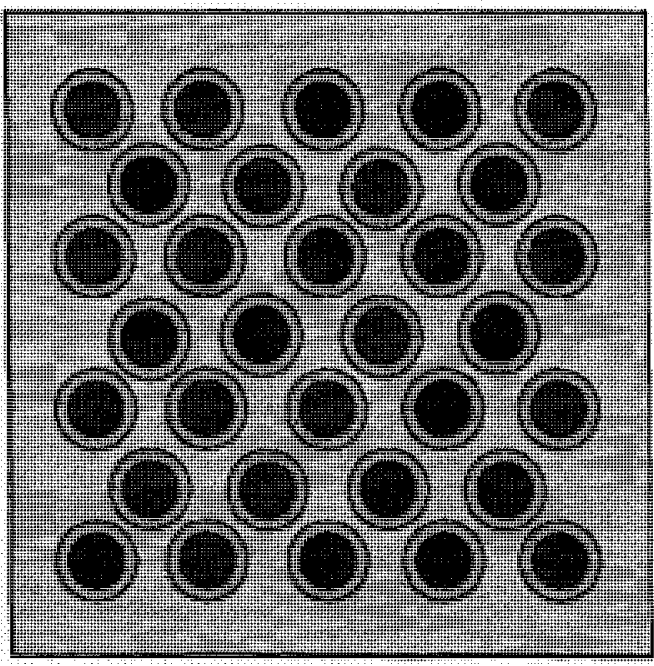

a) $\mathrm{RMC}<\mathrm{RMC}_{\max }$

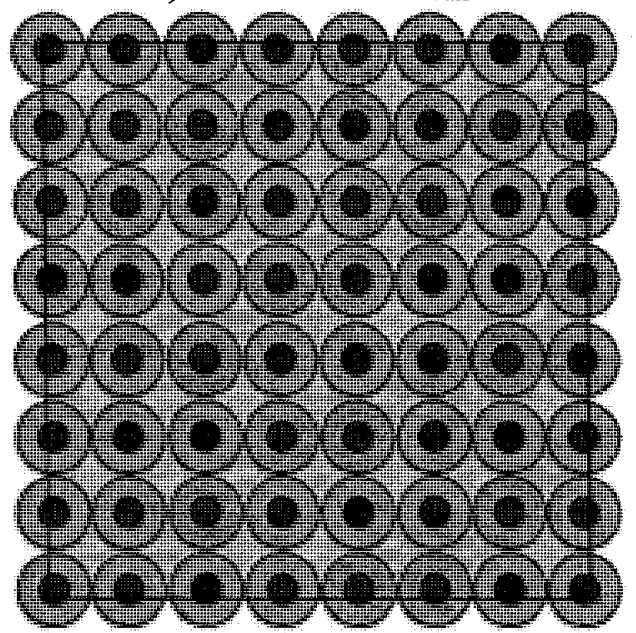

c) $\mathrm{RMC}>\mathrm{RMC}_{\max }$

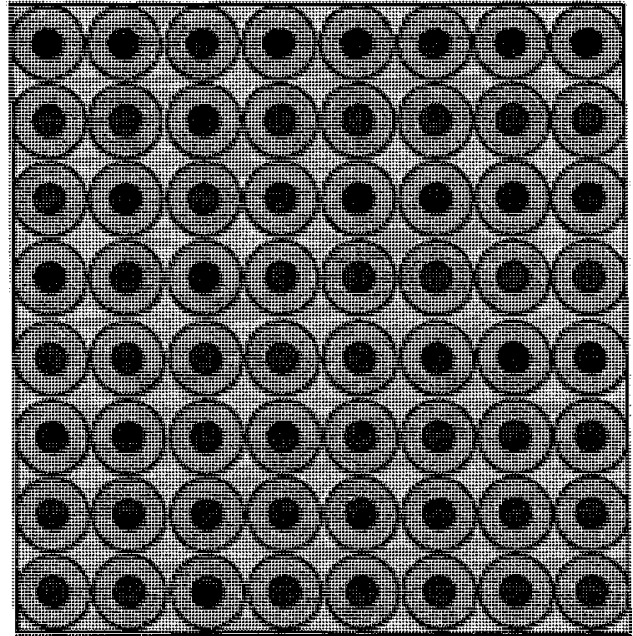

b) $\mathrm{RMC}=\mathrm{RMC}_{\max }$

Figure 4.4: Schematic illustration of the maximum feasible RMC in a mix proportioned by the proposed method

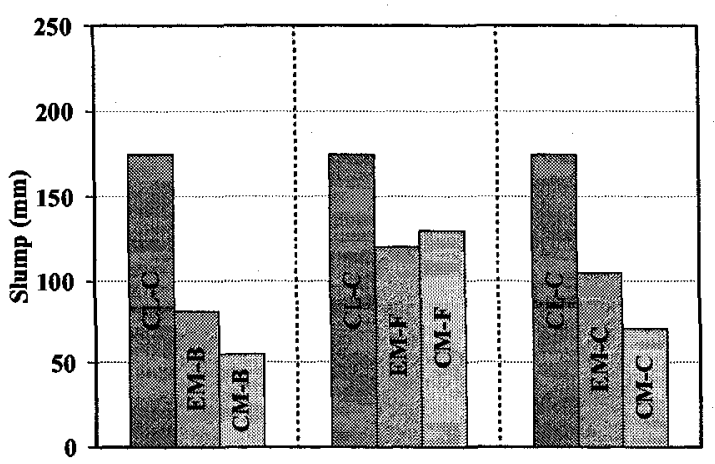

a) EM, CM and CL mixes

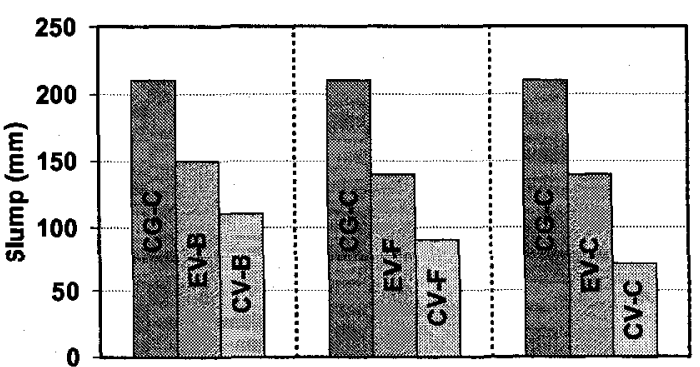

b) $\mathrm{EV}, \mathrm{CV}$, and $\mathrm{CG}$ mixes

Figure 4.5: Effect of mix proportioning method on the slump 


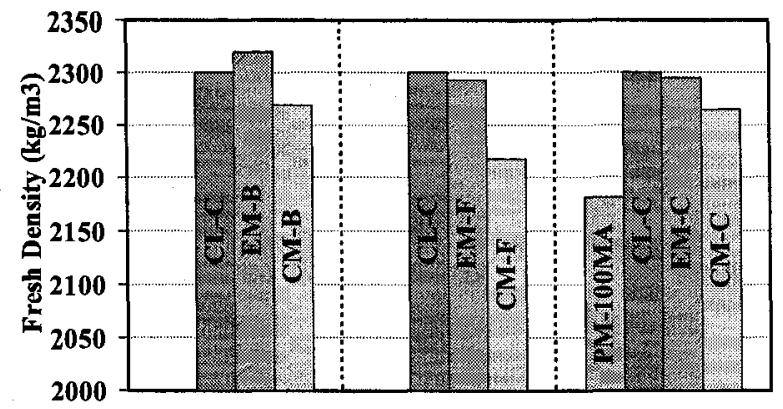

a) EM, CM and CL mixes

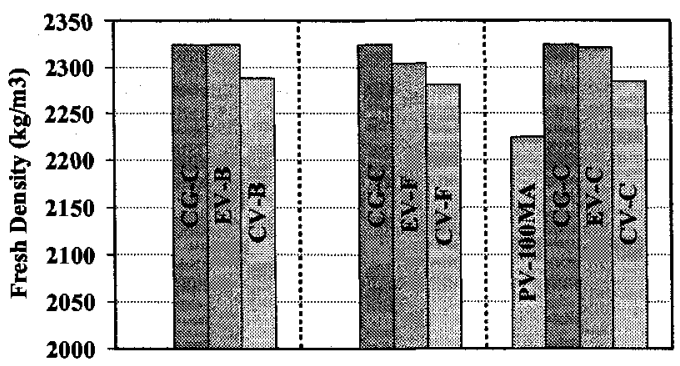

b) $\mathrm{EV}, \mathrm{CV}$, and CG mixes

Figure 4.6: Effect of mix proportioning method on the fresh density

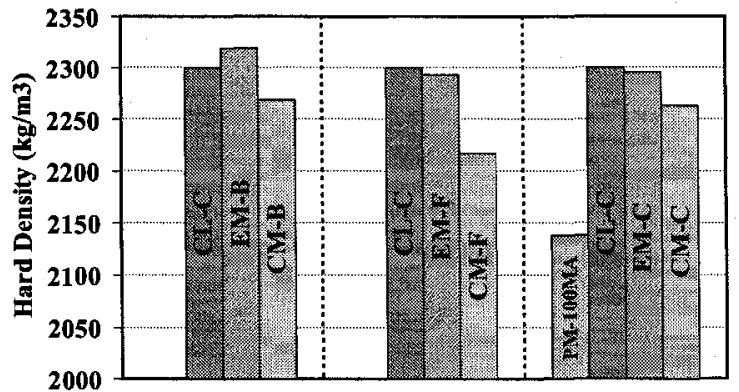

a) EM, CM and CL mixes

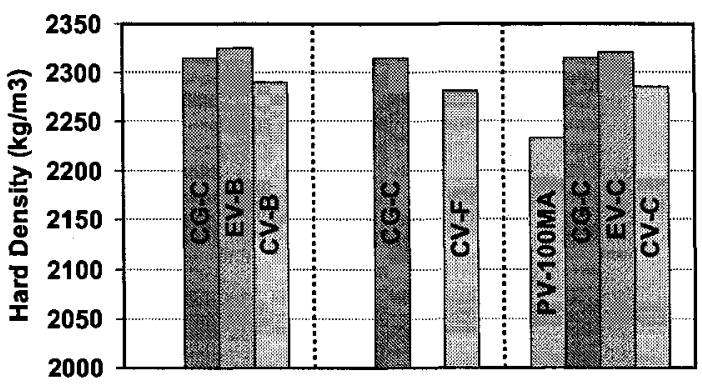

b) EV, CV, and CG mixes

Figure 4.7: Effect of mix proportioning method on the hardened density

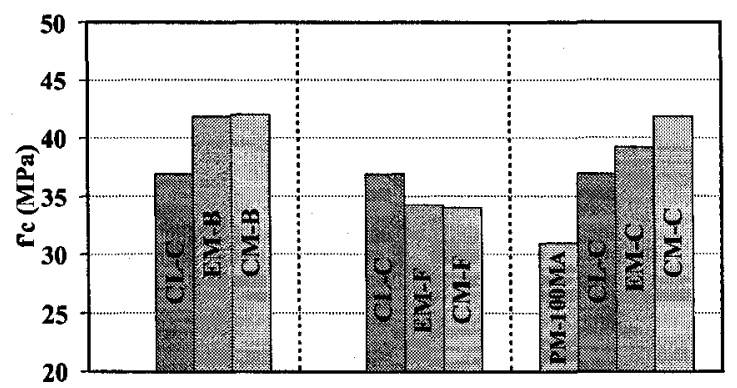

a) EM, CM and CL mixes

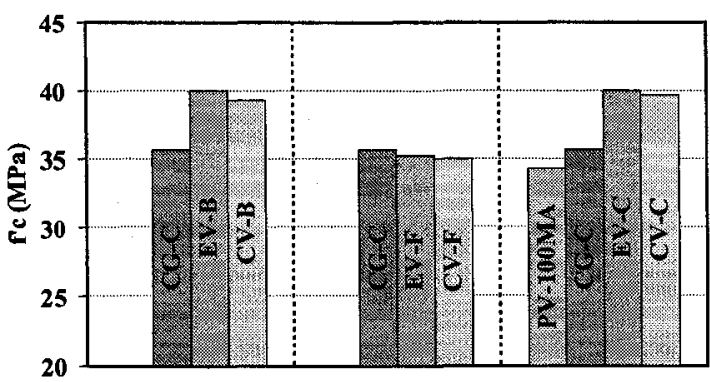

b) EV, CV, and CG mixes

Figure 4.8: Effect of mix proportioning method on the compressive strength 


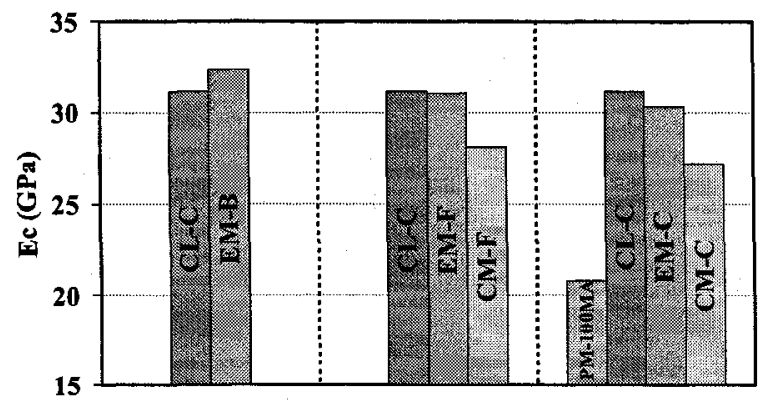

a) EM, CM and CL mixes

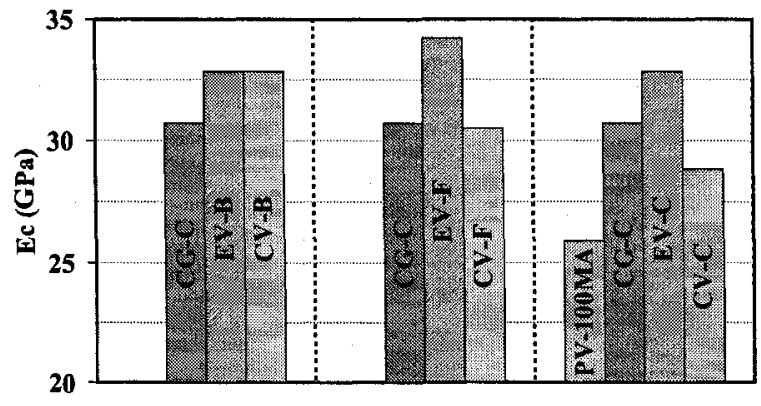

b) EV, CV, and CG mixes

Figure 4.9: Effect of mix proportioning method on the elastic modulus

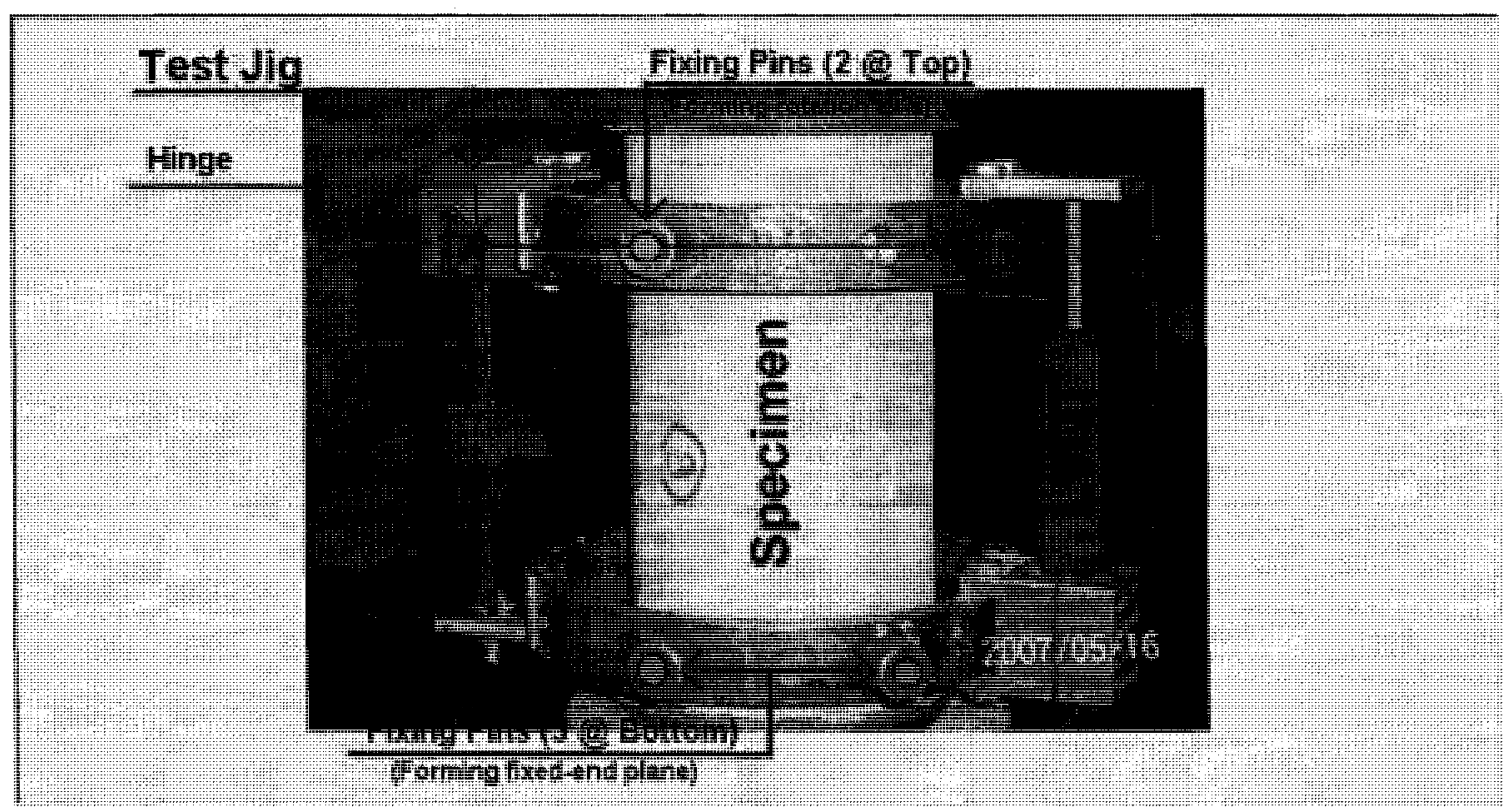

Figure 4.10: Schematic plan of stress-strain test setup 


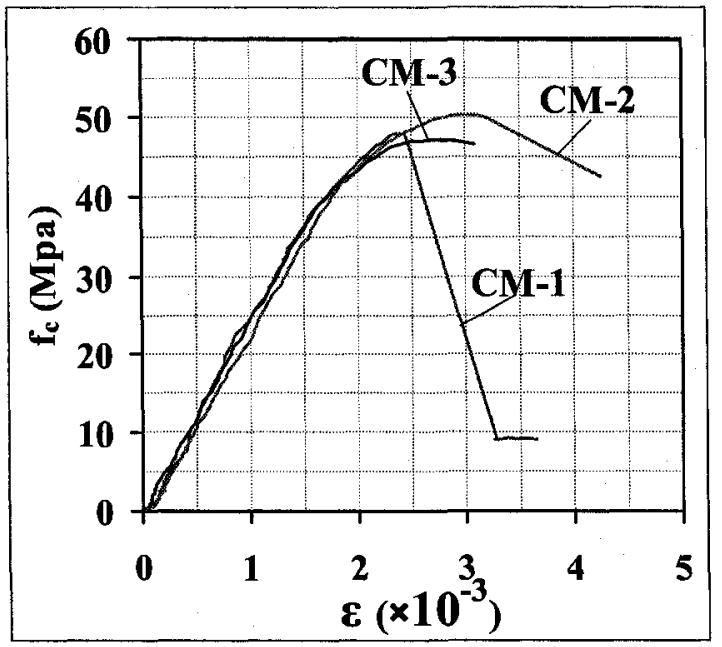

a) $\mathrm{CM}$

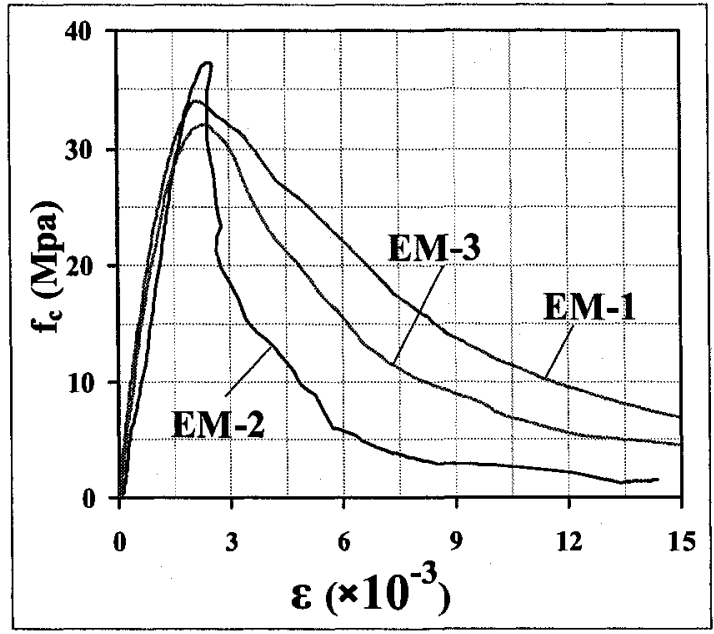

c) $\mathrm{EM}$

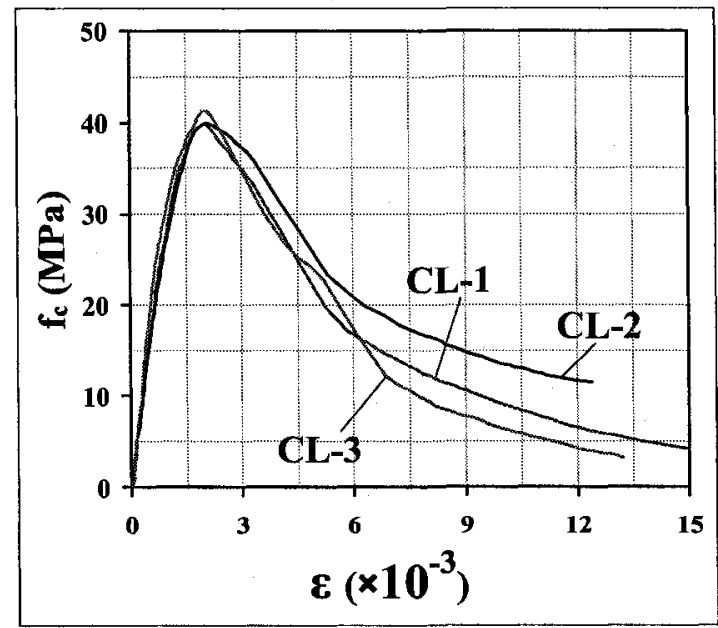

e) $\mathrm{CL}$

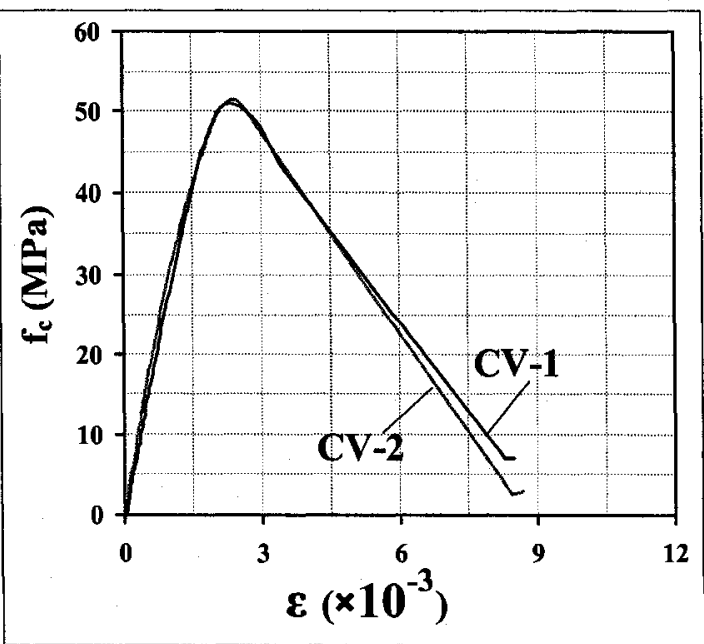

b) $\mathrm{CV}$

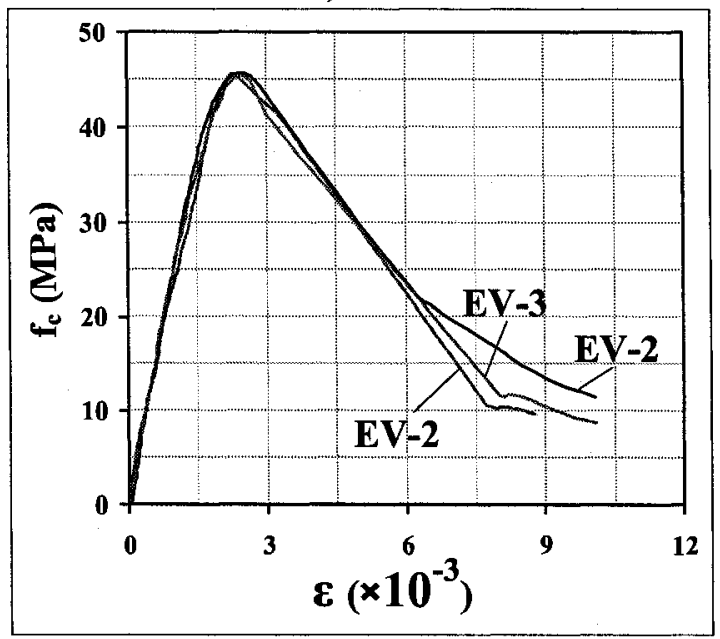

d) EV

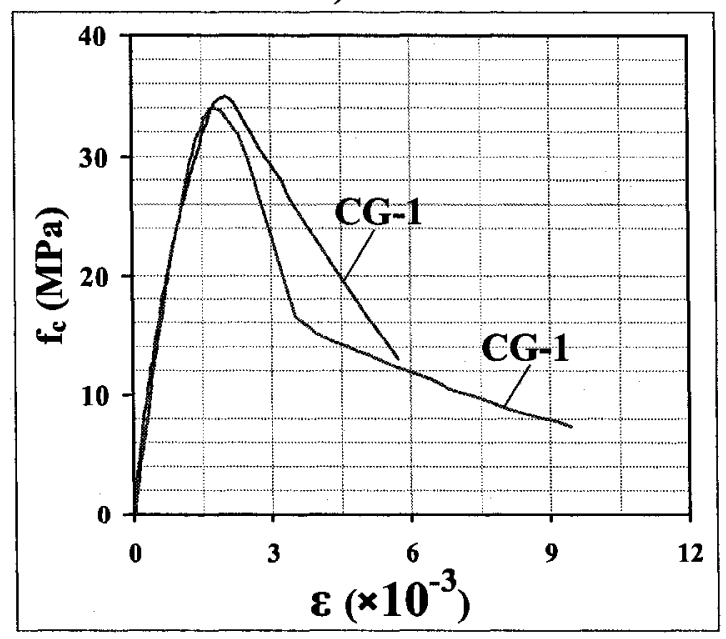

f) $\mathrm{CG}$

Figure 4.11: Observed stress-strain relationship of different mixes 


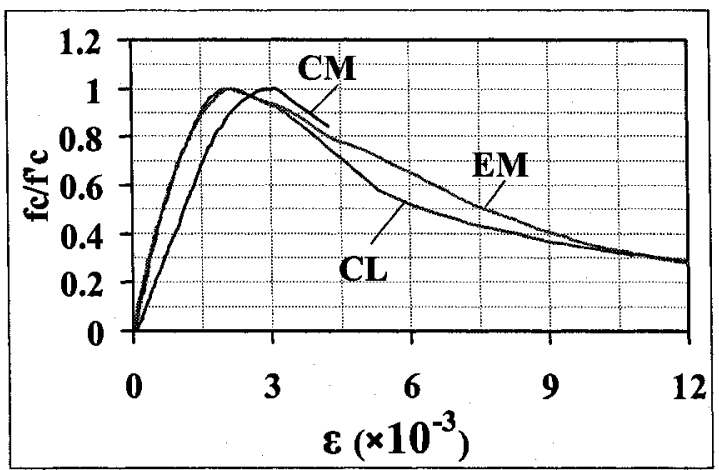

a) MO Specimens

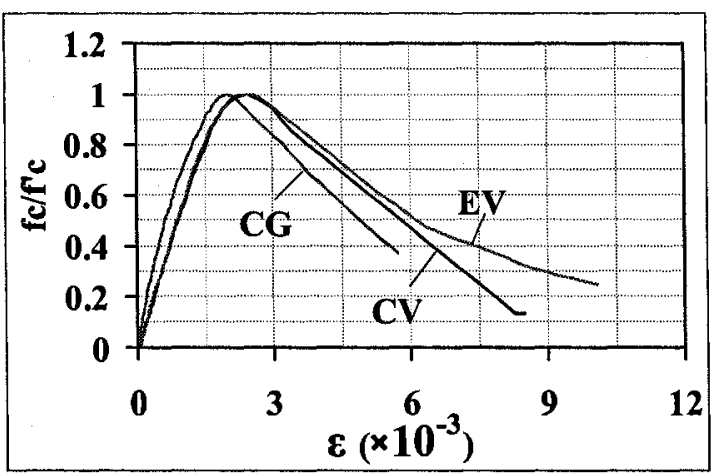

b) VA specimens

Figure 4.12: Effect of mix proportioning method on the compressive stress-strain relationship

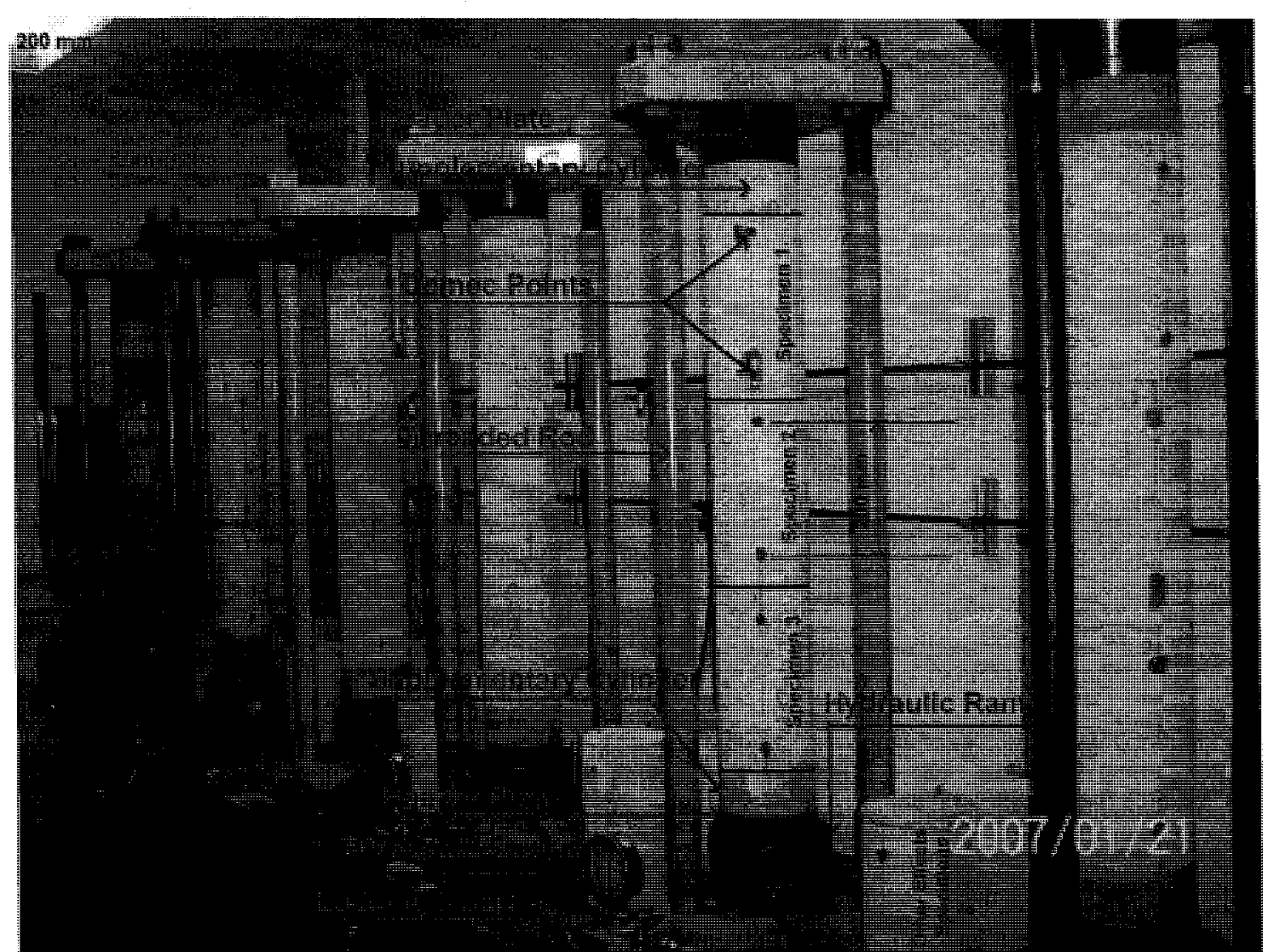

a) Loaditig frame (creep jig)

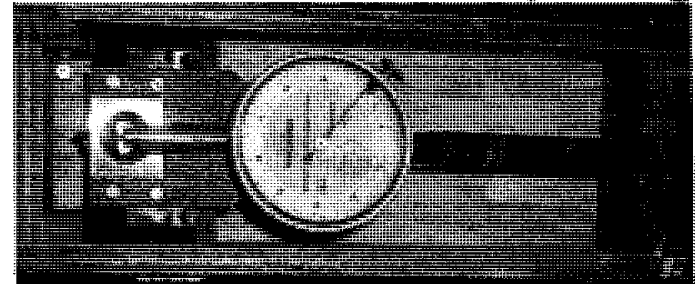

b) Demec mechanical strain gauge

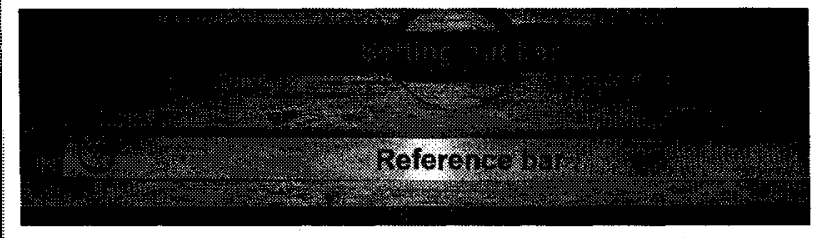

c) Setting-out and reference bars

Figure 4.13: Schematic view of creep test setup 


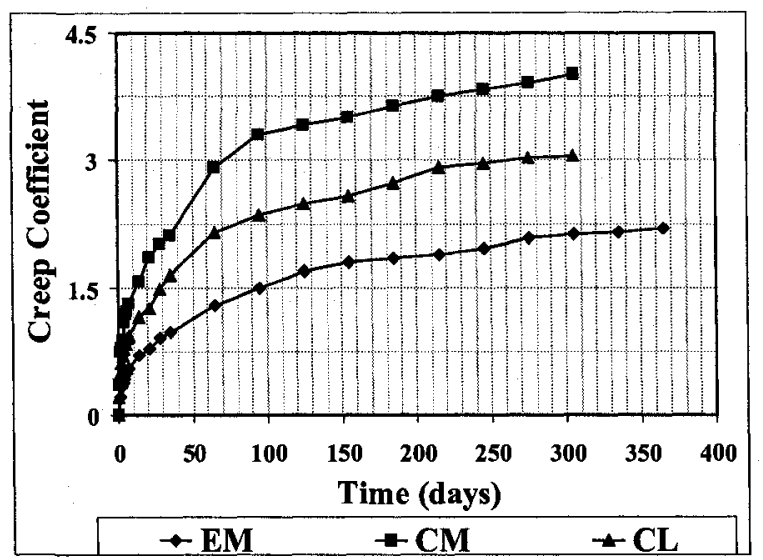

a) EM, CM and CL mixes

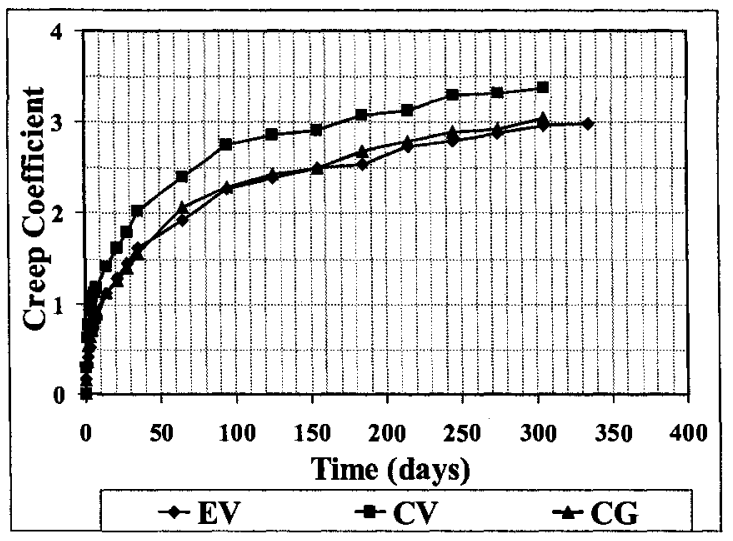

b) $\mathrm{EV}, \mathrm{CV}$, and $\mathrm{CG}$ mixes

Figure 4.14: Effect of mix proportioning method on the creep coefficient

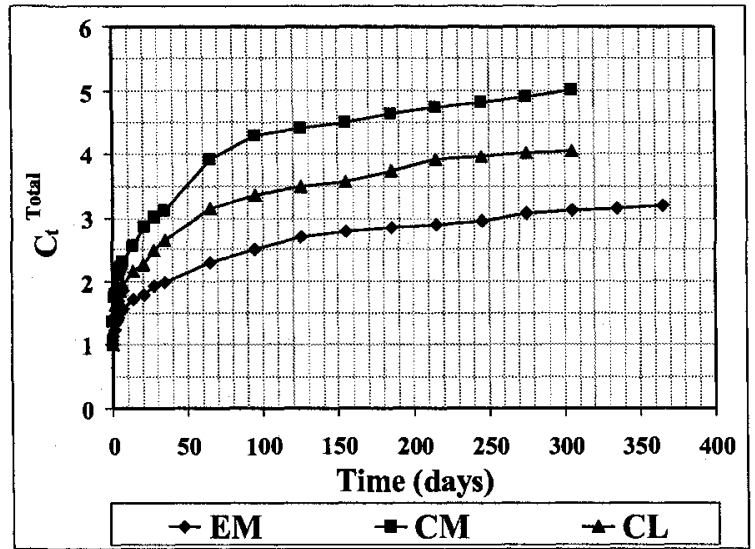

a) EM, CM and CL mixes

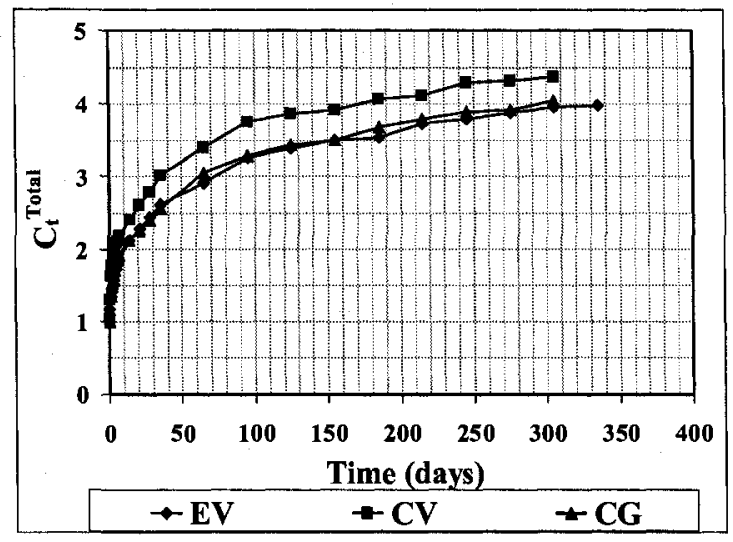

b) EV, CV, and CG mixes

Figure 4.15: Effect of mix proportioning method on the (total strain/initial strain)

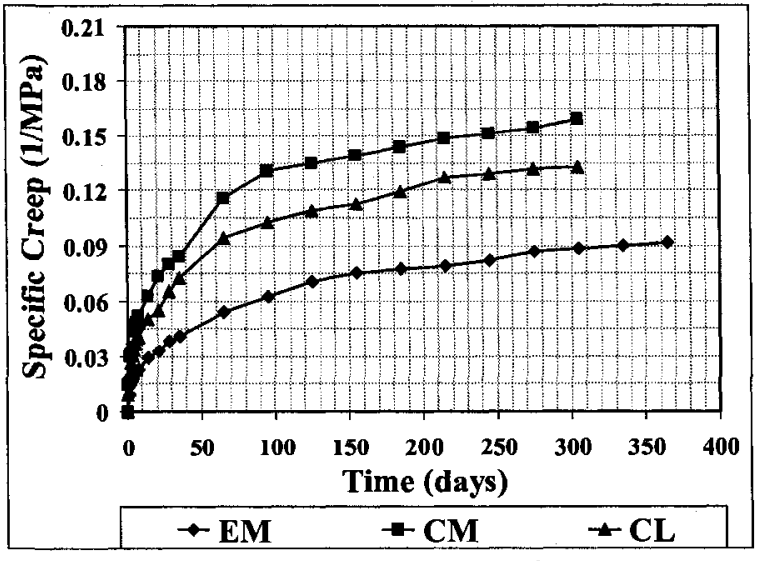

a) EM, CM and CL mixes

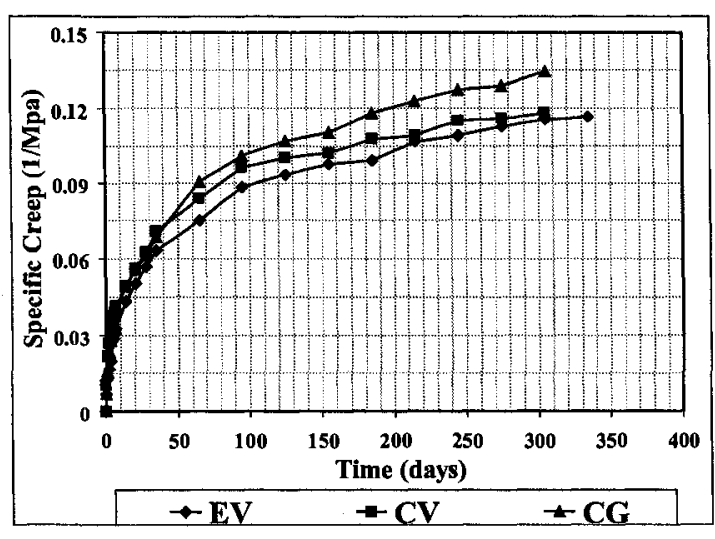

b) EV, CV, and CG mixes

Figure 4.16: Effect of mix proportioning method on the specific creep 


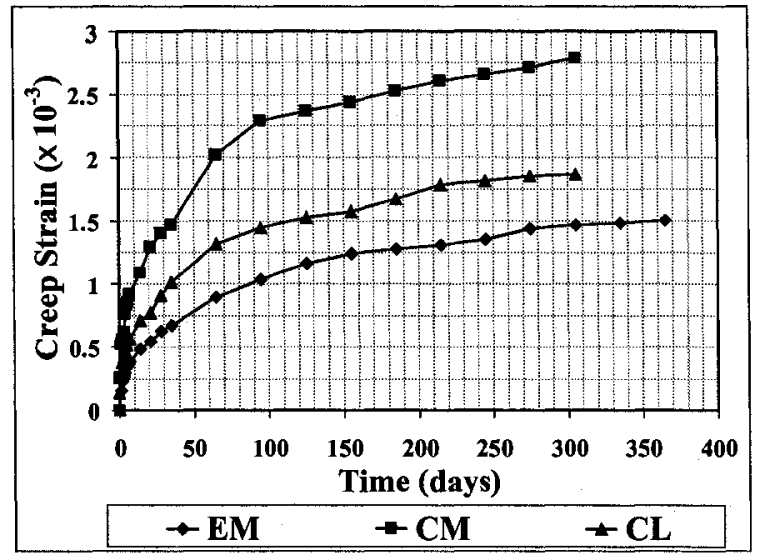

a) EM, CM and CL mixes

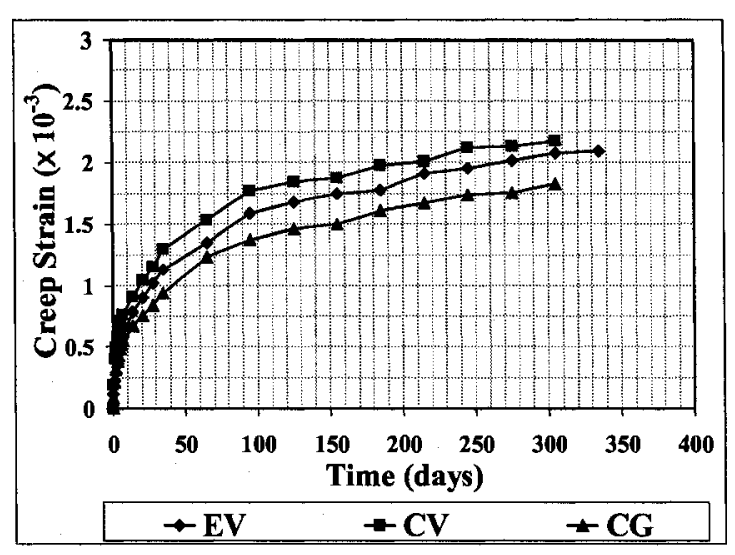

b) $\mathrm{EV}, \mathrm{CV}$, and CG mixes

Figure 4.17: Effect of mix proportioning method on the creep strain

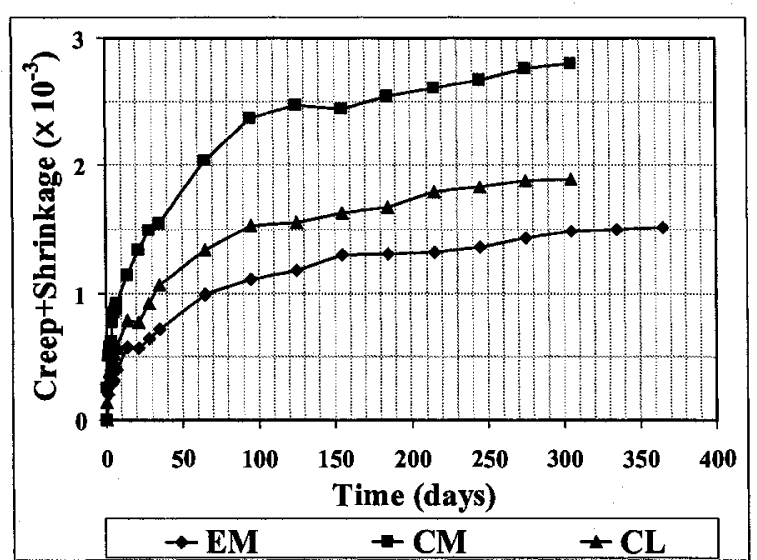

a) EM, CM and CL mixes

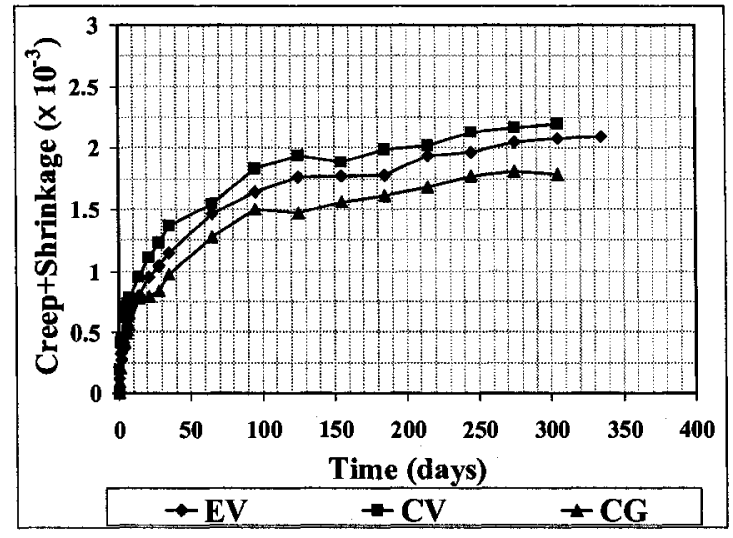

b) EV, CV, and CG mixes

Figure 4.18: Effect of mix proportioning method on the (creep + shrinkage) strain 


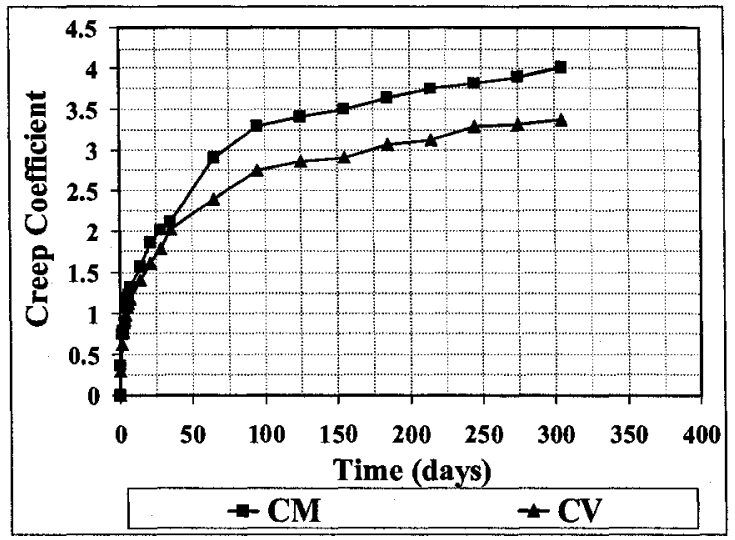

a) $\mathrm{CM}$ and $\mathrm{CV}$

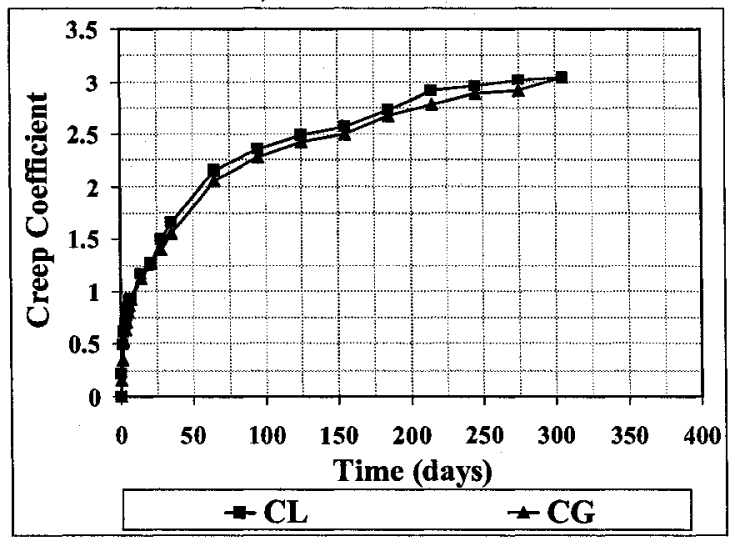

c) CL and CG

Figure 4.19: Effect of aggregate type on creep coefficient

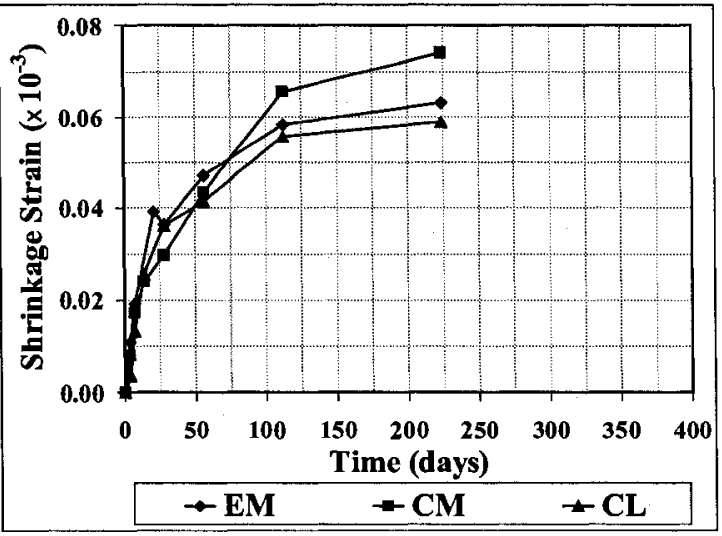

a) $\mathrm{EM}, \mathrm{CM}$ and $\mathrm{CL}$ mixes

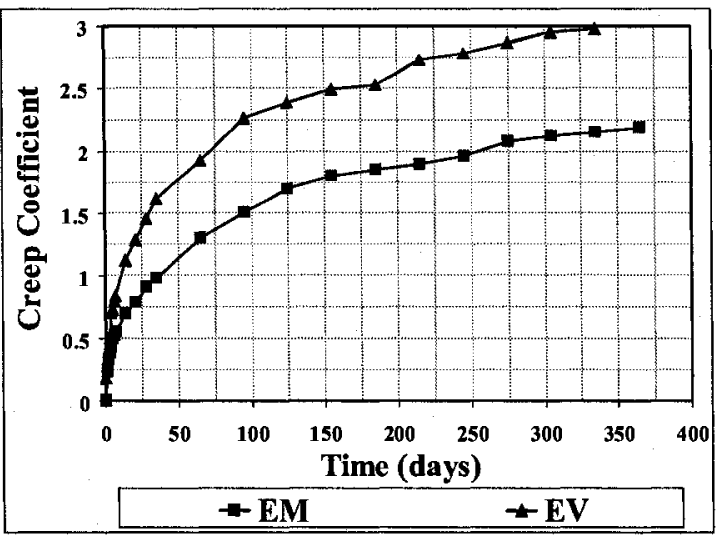

b) EM and EV

Figure 4.20: Effect of mix proportioning method on the drying shrinkage strain 


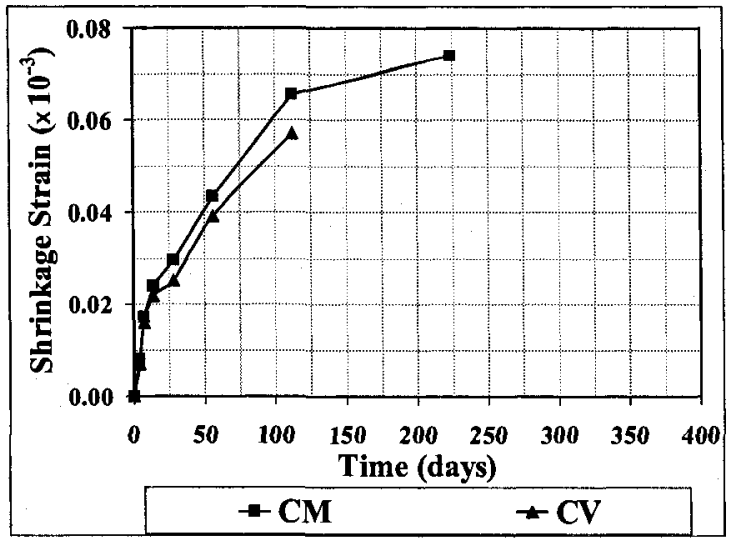

a) CM-C and CV-C

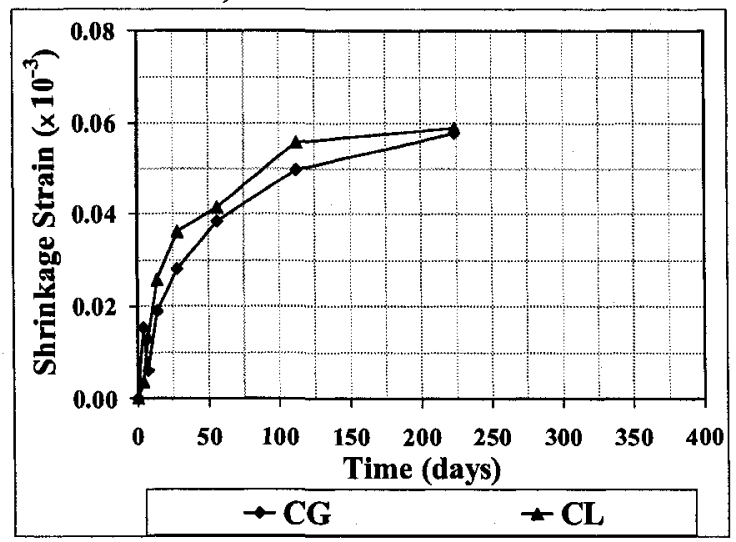

c) CL-C and CG-C

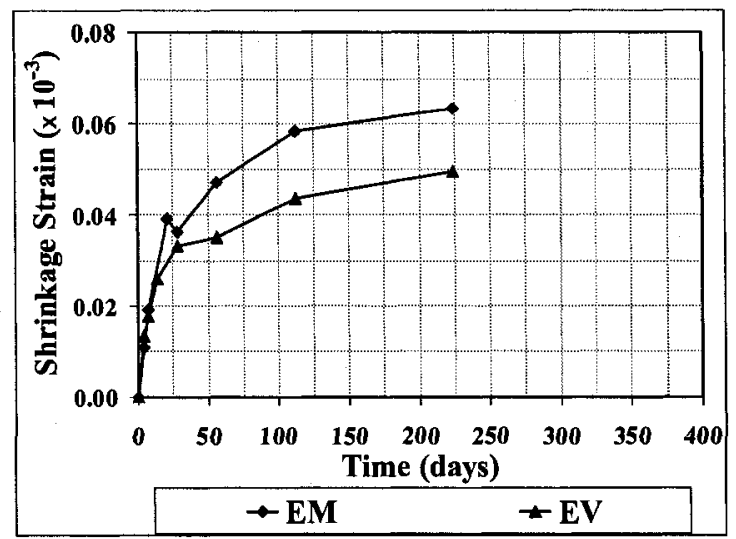

b) EM-C and EV-C

Figure 4.21: Effect of aggregate type on drying shrinkage 


\section{CHAPTER 5: ANALYSIS OF THE EXPERIMENTAL DATA: SHORT AND LONG-TERM PROPERTIES OF RAC}

\subsection{General}

As stated in Chapter 2, several models have been developed by different researchers in order to correlate a number of short-term and long-term properties of conventional concrete to various parameters. However, to the author's best knowledge, there has been no study performed to evaluate and/or revise the existing models to fit RAC, or develop some new models specified for RAC. It should be noted that these models have served as the foundation for various design codes, standards and handbooks and generally appear as some empirical equations interrelating different mechanical properties of conventional concrete. While these interrelations are well established for NAC, it is not the same in the case of RAC. Although some investigations have been carried to determine their applicability to RAC, the results have been contradictory. Therefore, it is essential to systematically and more broadly assess the applicability of these empirical equations to RAC.

The major objective of this chapter is to firstly determine the applicability to RAC of some available relations which attempt to interrelate some properties of NAC. Secondly, to develop new models for predicting certain short- and long-term properties of RAC from the basic properties of its constituents and their proportions in the mix. These properties include compressive and tensile strength, elastic modulus, fresh and hardened density, creep and shrinkage. 


\subsection{Development of Models}

\subsubsection{Fresh and Hardened Density}

The fresh density of concrete is generally required to design the formwork of concrete structures while its hardened density is used for estimating the self-weight of the structures. As demonstrated in Chapters 3 and 4, the paramount factor governing the fresh and hardened densities of RAC is its total mortar volume rather than its RMC or RCA content. Although others have presented some empirical equations for the fresh and hardened densities of RAC as a function of its RCA content [Topcu and Sengel, 2004], to the best knowledge of the author, no rational model exists which can be used to determine the fresh and hardened densities of RAC. In this section, a rational model is developed to correlate the fresh and hardened densities of RAC to the corresponding properties of its constituents. The model is based on the fact that RAC is a composite material comprising the new and residual mortars and the new and original virgin aggregates. Two sets of models are presented: (1) Phase-Based Models, predicting the density of RAC as a function of the corresponding properties and volume fractions of either its RCA, NA and NM; or of its TM and TNA; and (2) Mixture-Based Models, predicting the fresh and hardened densities of RAC as a function of either its RCA volume fraction or its TM volume content, and the same properties for concrete mixes with $0 \%$ and $100 \%$ RCA content levels. To validate these models, the results of the preliminary study in Chapter 3 as well as the proposed mix design results in Chapter 4 will be used. 


\subsubsection{Phase-Based Models}

The density of concrete as a composite material can be calculated by knowing the density and volume fractions of its aggregate and mortar as:

$$
\gamma_{N A C}=V_{N A}^{N A C} \times \gamma_{N A}+V_{M}^{N A C} \times \gamma_{M}
$$

where $\gamma_{N A C}$ is the density of NAC; $\gamma_{N A}$ is the density of NA, $\gamma_{M}$ is the density of mortar, $V_{N A}^{N A C}$ is the volume fraction $\mathrm{f}$ NA in a unit volume of NAC, and $V_{M}^{N A C}$ is the volume fraction of the mortar in a unit volume of NAC.

It should be mentioned that for calculating the fresh density of concrete, the aggregate and mortar densities are based on the SSD condition, while for hardened density, they are the bulk values.

For RAC, as a three-phase composite material including NA, RCA, and mortar. Eq.5.1 can be rewritten as:

$$
\gamma_{R A C}=V_{N A}^{R A C} \times \gamma_{N A}+V_{R C A}^{R A C} \times \gamma_{R C A}+V_{N M}^{R A C} \times \gamma_{N M}
$$

where $\gamma_{R A C}, \gamma_{N A}, \gamma_{R C A}$ and $\gamma_{N M}$ are the densities of RAC, NA, RCA and NM; and $V_{N A}^{R A C}$, $V_{R C A}^{R A C}$ and $V_{N M}^{R A C}$ are the volume fractions of NA, RCA, and NM in unit volume of RAC.

However, RCA itself is a two-phase composite material including OVA and RM, in which its density can be obtained as

$$
\begin{gathered}
V_{R C A}^{R A C} \times \gamma_{R C A}=V_{O V A}^{R C A} \times \gamma_{O V A}+V_{R M}^{R C A} \times \gamma_{R M} \\
V_{R C A}^{R A C}=V_{O V A}^{R C A}+V_{R M}^{R C A}
\end{gathered}
$$

where $\gamma_{R M}$ and $\gamma_{O V A}$ are the densities and $V_{R M}^{R C A}$ and $V_{O V A}^{R C A}$ are the volume fractions of RM and OVA, respectively, in the given volume of RCA in RAC ( $\left.V_{R C A}^{R A C}\right)$.

By treating the total mortar (TM) in RAC as a two-phase composite mortar comprising NM and RM; and the total natural aggregate (TNA) as a two-phase composite aggregate 
consisting of OVA and NA, an equivalent density can be defined for TNA and for TM as follows:

$$
\begin{gathered}
\gamma_{T M}=\frac{V_{N M}^{R A C} \times \gamma_{N M}+V_{R M}^{R C A} \times \gamma_{R M}}{V_{T M}^{R A C}} \\
\gamma_{T N A}=\frac{V_{N A}^{R A C} \times \gamma_{N A}+V_{O V A}^{R C A} \times \gamma_{O V A}}{V_{T N A}^{R A C}}
\end{gathered}
$$

where $\gamma_{T M}$ is the equivalent density of total mortar, and $\gamma_{T N A}$ is the equivalent density of TNA in RAC. The total volume of TM and TNA in RAC can be obtained as follows:

$$
\begin{aligned}
& V_{T M}^{R A C}=V_{R M}^{R C A}+V_{N M}^{R A C} \\
& V_{T N A}^{R A C}=V_{O V A}^{R C A}+V_{N A}^{R A C}
\end{aligned}
$$

where $V_{T M}^{R A C}$ and $V_{T N A}^{R A C}$ are the total mortar and total natural aggregate volumes in unit volume of RAC, respectively.

By using Eq.5.2 to Eq.5.8, the density of RAC can be written as:

$$
\gamma_{R A C}=V_{T M}^{R A C} \times \gamma_{T M}+V_{T N A}^{R A C} \times \gamma_{T N A}
$$

Although not essential to the development of the proposed models, for sake of simplicity, the density of TM and TNA in RAC may be taken equal to the density of mortar and aggregate in NAC, and Eq.5.9 can be rewritten as

$$
\gamma_{R A C}=V_{T M}^{R A C} \times \gamma_{M}+V_{T N A}^{R A C} \times \gamma_{N A}
$$

In this study, assuming equal density values for TNA in RAC and NA in NAC was reasonable since NA was chosen same as OVA in both RCA-VA and RCA-MO aggregates. Furthermore, as the volume fraction of RM in TM was not generally too large in RAC mixes (maximum $21.5 \%$ and $15.5 \%$ for PM and PV mixes at $100 \%$ RCA content), assuming equal density values for TM in RAC and mortar in NAC was also not very erroneous. 


\subsubsection{Mixture-Based Models}

RAC can also be assumed as a blended concrete composed of NAC ( $0 \%$ RCA content) and RAC (100\% RCA content) with different volume fractions. Since the fresh and hardened densities of RAC at different RCA contents vary between the corresponding properties of NAC and RAC with $100 \%$ RCA content, it is reasonable to correlate its fresh and hardened densities to the volume fractions and densities of the companion NAC and RAC with $100 \%$ RCA content.

In conventional mix proportioning method, it is customary to partially or fully replace NA by RCA based on volume or weight. The replacement ratio varies between zero and one and can be obtained as:

$$
\begin{aligned}
& R_{v}=\frac{V_{R C A}^{R A C}}{V_{N A}^{N A C}} \\
& R_{w}=\frac{W_{R C A}^{R A C}}{W_{N A}^{N A C}}
\end{aligned}
$$

where $V_{R C A}^{R A C}$ is the volume of coarse RCA in RAC, $V_{N A}^{N A C}$ is the volume of coarse NA in the companion NAC, $R_{v}$ is the volume-based replacement ratio, $W_{R C A}^{R C}$ is the weight of coarse RCA in RAC, $W_{N A}^{N A C}$ is the weight of coarse NA in the companion NAC, and $R_{w}$ is the weighted-based replacement ratio. Since in this method RAC simply replaces NA, the fresh mortar volume of RAC and companion NAC are equal

$$
V_{M}^{N A C}=V_{N M}^{R A C}
$$

where $V_{M}^{N A C}$ and $V_{N M}^{R A C}$ are the volume of fresh mortars in the companion NAC and RAC, respectively. Furthermore, the total coarse aggregate volume in RAC (RCA and NA) is identical to NA volume in companion NAC:

$$
V_{N A}^{N A C}=V_{R C A}^{R A C}+V_{N A}^{R A C}
$$


where $V_{N A}^{N A C}$ is the coarse NA volume in the companion NAC, $V_{N A}^{R A C}$ is the volume of coarse NA in RAC, and $V_{R C A}^{R A C}$ is the volume of coarse RCA in RAC.

Using Eqs.5.11 and 5.13, the volume of coarse NA and RCA in RAC can be obtained as follows:

$$
\begin{gathered}
V_{R C A}^{R A C}=R_{v} \times V_{N A}^{N A C} \\
V_{N A}^{R A C}=\left(1-R_{v}\right) \times V_{N A}^{N A C}
\end{gathered}
$$

By breaking $V_{M}^{N A C}$ to two parts as $V_{M 1}^{N A C}=R_{v} \times V_{M}^{N A C}$ and $V_{M 2}^{N A C}=\left(1-R_{v}\right) \times V_{M}^{N A C}$, and using Eqs.5.1, 5.2, and 5.11 to 5.15, the density of RAC can be calculated as a function of replacement ratio $\left(\mathrm{R}_{\mathrm{v}}\right)$ and the corresponding density values of companion NAC $\left(\gamma_{N A C}\right)$ and RAC at $100 \%$ RCA content $\left(\gamma_{R A C}^{100 \% R C A}\right)$ as follows

$$
\gamma_{R A C}=\gamma_{N A C}+\left(\gamma_{R A C}^{100 \% R C A}-\gamma_{N A C}\right) \times R_{v}
$$

In Eq.5.16, the effects of both NA and NM on the overall density of RAC are accumulated in $\gamma_{N A C}$, while the effects of OVA and RM are bulked in $\gamma_{R A C}^{100 \% R C A}$.

Equation 5.16 is only valid for the RAC mixes designed according to conventional mix proportioning method which simply involves the replacement of some NA by RCA. In order to have a general format of Eq.5.16, which is applicable to the RAC mixes proportioned by either the conventional method or the proposed EMV method, a new parameter, denoted as $\chi$, will be defined and utilized as follows

$$
\chi=\frac{V_{T M}^{R A C}-V_{M}^{N A C}}{V_{T M}^{R A C-100 \% R C A}-V_{M}^{N A C}}
$$

where $V_{T M}^{R A C}$ is the total mortar volume in RAC proportioned either by the conventional or the proposed EMV method, $V_{M}^{N A C}$ is the volume of the mortar in the companion NAC, and 
$V_{T M}^{R A C-100 \% R C A}$ is the total mortar volume of RAC at $100 \%$ RCA content proportioned by the conventional method.

By replacing $R_{v}$ with $\chi$, Eq.5.16 can be rewritten as

$$
\gamma_{R A C}=\gamma_{N A C}+\left(\gamma_{R A C}^{100 \% R C A}-\gamma_{N A C}\right) \times \chi
$$

Using Eq.5.18, it can be determined that RAC and NAC with equal TM volume would have comparable densities.

\subsubsection{Experimental Verification}

In order to verify phase-based models (Eqs.5.2 and 5.10), one first needs to know the density of mortar $\left(\gamma_{M}\right)$, which can be obtained using Eq.5.1 and knowing $\gamma_{N A C}$ and $\gamma_{N A}$ values. Knowing the preceding quantities $\gamma_{R A C}$ can be calculated at any RCA content by either using Eq.5.2 and having $\gamma_{R C A}$, or using Eq.5.10.

Tables 5.1 and 5.2 present the experimental/predicted fresh and hardened density values using phase-based models for RAC mixes made with RCA-MO and RCA-VA, respectively. The experimental over predicted values of hardened density were found between 1.00-1.12 and 0.98-1.06 using Eq.5.2 and 5.10, respectively. For the fresh density, the ratio varies between 0.98-1.13 and 0.98-1.04 using Eq.5.2 and 5.10, respectively. Based on these values, Eq.5.10 gives more accurate results for both the fresh and hardened densities of RAC. This is more evident from Figures 5.1a, 5.1b, 5.2a and $5.2 \mathrm{~b}$ which illustrate the predicted versus experimental fresh and hardened density values of all RAC mixes using phase-based models. The figure also shows the $95 \%$ confidence limits which correlate the accuracy of Eq.5.10.

Table 5.3 presents the ratio of experimental/predicted fresh and hardened density values using mixture-based model for all RAC mixes. The ratio varies between $0.98-1.02$ and 
0.99-1.01 using Eq.5.16 and 5.18, respectively. For the fresh density, the ratio ranges between 0.99-1.01 and 0.99-1.02 using Eq.5.16 and 5.18, respectively. According to the latter results, Eqs.5.16 and 5.18 have the same degree of accuracy. Since the $R_{w}$, $R_{v}$ and $\chi$ values in these tables are very close for RAC mixes proportioned by the conventional method, any one of them may be used in mixture-based models.

Generally the mixture-based models were found to more accurately predict the fresh density of RAC mixes proportioned by both the conventional and the proposed EMV method compared to phase-based models. This is quite evident in Figures 5.1 which illustrate the predicted versus experimental fresh density values of all RAC mixes using phase-based and mixture-based models. This conclusion was also found to be true for the hardened density of RAC mixes (Figure 5.2).

\subsubsection{Elastic Modulus}

The elastic modulus of concrete is the key parameter used to estimate the deformations of buildings and structures, as well as the modular ratio used for design of flexural members. Therefore, it is crucial to have a good understanding about different factors affecting the elastic modulus in RAC.

As established in Chapters 3 and 4, the paramount factor governing the elastic modulus of RAC is the TM volume rather than its RMC or RCA content. Although some researchers have tried to derive some empirical equations for the elastic modulus of RAC (De Juan and Gutiérrez, 2004, Topcu and Sengel, 2004, Kheder and Windawi, 2005), to the best knowledge of the author, no specific model exists for predicting the elastic modulus of RAC. Several models have been developed for NAC as a two-phase material (aggregate and mortar) correlating its elastic modulus to the volume fractions and the 
corresponding moduli of its components. However they do not apply to RAC as a fourphase composite material including OVA and RM in addition to NA and NM. In this section, two sets of models are presented: (1) Phase-Based Models, predicting the elastic modulus of RAC as a function of TM and TNA volumes and their corresponding moduli; and (2) Mixture-Based Models, predicting the elastic modulus of RAC as a function of either its RCA content or its TM volume and of the elastic moduli of RAC mixes with $0 \%$ and $100 \%$ RCA content levels. To validate these models, the results of the preliminary study in Chapter 3 as well as the proposed mix design results in Chapter 4 will be used.

\subsubsection{Phase-Based Models}

As stated in Chapter 2, several models have been developed for the elastic modulus of NAC as a two-phase composite material (i.e., parallel, series, Hirsch, Counto, and Mindess models). For instance, in the parallel model, the elastic moulus of conventional concrete as a composite material is correlated to the elastic modulus and volume fractions of its aggregate and mortar as:

$$
E_{N A C}=V_{N A}^{N A C} \times E_{N A}+V_{M}^{N A C} \times E_{M}
$$

where $E_{N A C}$ is the elastic modulus of NAC; $E_{N A}$ is the elastic modulus of NA, $E_{M}$ is the elastic modulus of mortar, $V_{N A}^{N A C}$ is the volume fraction of NA in a unit volume of NAC, and $V_{M}^{N A C}$ is the volume fraction of mortar in a unit volume of NAC.

For RAC, as a three-phase composite material including NA, RCA, and mortar, Eq.5.19 can be rewritten as:

$$
E_{R A C}=V_{N A}^{R A C} \times E_{N A}+V_{R C A}^{R A C} \times E_{R C A}+V_{N M}^{R A C} \times E_{N M}
$$


where $E_{R A C}, E_{N A}, E_{R C A}$ and $E_{N M}$ are the elastic modulus of RAC, NA, RCA and NM; and $V_{N A}^{R A C}, V_{R C A}^{R A C}$ and $V_{N M}^{R A C}$ are the volume fractions of NA, RCA, and NM in unit volume of RAC. However, RCA actually is a two-phase composite material including OVA and RM, in which its elastic modulus can be obtained as:

$$
\begin{gathered}
V_{R C A}^{R C C} \times E_{R C A}=V_{O V A}^{R C A} \times E_{O V A}+V_{R M}^{R C A} \times E_{R M} \\
V_{R C A}^{R A C}=V_{O V A}^{R C A}+V_{R M}^{R C A}
\end{gathered}
$$

where $E_{R M}$ and $E_{O V A}$ are the elastic moduli, and $V_{R M}^{R C A}$ and $V_{O V A}^{R C A}$ are the volume fractions of RM and OVA, respectively, in the RCA used. By assuming the total mortar (TM) in RAC as a two-phase composite mortar (i.e., NM and RM); and total natural aggregate (TNA) in RAC as a two-phase composite aggregate (i.e., OVA and NA), an equivalent elastic modulus can be defined for TNA and TM as follows:

$$
\begin{gathered}
E_{T M}=\frac{V_{N M}^{R A C} \times E_{N M}+V_{R M}^{R C A} \times E_{R M}}{V_{T M}^{R A C}} \\
E_{T N A}=\frac{V_{N A}^{R A C} \times E_{N A}+V_{O V A}^{R C A} \times E_{O V A}}{V_{T N A}^{R A C}}
\end{gathered}
$$

where $E_{T M}$ is the equivalent elastic modulus of total mortar, and $E_{T N A}$ is the equivalent elastic modulus of TNA in RAC. The total volume of TM and TNA in RAC can be obtained using Eq.5.7 and 5.8.

Using Eqs.5.20 to 5.24, the elastic modulus of RAC can be written as:

$$
E_{R A C}=V_{T M}^{R A C} \times E_{T M}+V_{T N A}^{R A C} \times E_{T N A}
$$

For sake of simplicity, the elastic modulus of TM and TNA in RAC may be set equal to those of mortar and aggregate in NAC; consequently Eq.5.25 can be rewritten as:

$$
E_{R A C}=V_{T M}^{R A C} \times E_{M}+V_{T N A}^{R A C} \times E_{N A}
$$


In this study, assuming equal elastic modulus values for TNA in RAC and NA in NAC was reasonable since the NA used was the same as the corresponding OVA in both the RCA-VA and RCA-MO aggregates. Furthermore, as the volume fraction of RM in TM was not generally too large in RAC mixes (maximum $21.5 \%$ and $15.5 \%$ for PM and PV mixes at $100 \%$ RCA content), assuming equal elastic modulus values for TM in RAC and mortar in NAC was a reasonable approximation.

By applying the same concept to other models, i.e. the series, the Hirsch, the Counto and the Mindess models (see Chapter 2: Table 2.1), the modified form of the corresponding models can be obtained as follows:

\section{Series Model}

$$
\frac{1}{E_{R A C}}=\frac{V_{T M}^{R A C}}{E_{M}}+\frac{V_{T N A}^{R A C}}{E_{N A}}
$$

Hirsch Model

$$
\frac{1}{E_{R A C}}=0.5 \times\left(\frac{1}{V_{T M}^{R A C} \times E_{M}+V_{T N A}^{R A C} \times E_{N A}}\right)+0.5 \times\left(\frac{V_{T M}^{R A C}}{E_{M}}+\frac{V_{T N A}^{R A C}}{E_{N A}}\right)
$$

\section{Counto Model}

$$
\frac{1}{E_{R A C}}=\frac{1-\sqrt{V_{T N A}^{R A C}}}{E_{M}}+\frac{1}{\left(\frac{1-\sqrt{V_{T N A}^{R A C}}}{\sqrt{V_{T N A}^{R A C}}}\right) \times E_{M}+E_{N A}}
$$

\section{Mindess Model}

$$
E_{R A C}=\frac{\left(1-V_{T N A}^{R A C}\right) \times E_{M}+\left(1+V_{T N A}^{R A C}\right) \times E_{N A}}{\left(1+V_{T N A}^{R A C}\right) \times E_{M}+\left(1-V_{T N A}^{R A C}\right) \times E_{N A}} \times E_{M}
$$

It should be mentioned that low quality of RM compared to NM in RAC might result in overall lower elastic modulus of TM in RAC compared to the companion NAC; however, it is possible, by adjusting the w/c ratio of NM in RAC, to achieve equal overall elastic modulus for TM in RAC as in the companion NAC. 


\subsubsection{Mixture-Based Models}

RAC can also be assumed as a blended concrete including NAC ( $0 \%$ RCA content) and RAC (100\% RCA content) with different volume fractions. Since the elastic modulus of RAC at different RCA contents varies between the corresponding properties of NAC and $\mathrm{RAC}$ at $100 \% \mathrm{RCA}$ content, it is possible to correlate its elastic modulus to the corresponding volume fractions and properties of NAC and RAC at 100\% RCA content.

By breaking $V_{M}^{N A C}$ to two parts as $V_{M 1}^{N A C}=R_{v} \times V_{M}^{N A C}$ and $V_{M 2}^{N A C}=\left(1-R_{v}\right) \times V_{M}^{N A C}$, and using Eqs.5.19, 5.20, and Eqs.5.11 to 5.15, the elastic modulus of RAC can be calculated as a function of replacement ratio $\left(R_{v}\right)$ and the corresponding elastic moduli of the companion NAC $\left(E_{N A C}\right)$ and RAC at $100 \%$ RCA content $\left(E_{R A C}^{100 \% R C A}\right)$ as follows:

$$
E_{R A C}=E_{N A C}+\left(E_{R A C}^{100 \% R C A}-E_{N A C}\right) \times R_{v}
$$

In Eq.5.31, the effects of both NA and NM on the overall elastic modulus of RAC are accumulated in $E_{N A C}$, while the effects of OVA and RM are reflected by $E_{R A C}^{100 \% R C A}$.

By applying the same concept to the series, the Hirsch, the Counto and the Mindess, the modified form of the corresponding models can be obtained as follows:

Series Model

$$
\frac{1}{E_{R A C}}=\frac{1}{E_{N A C}}+\left(\frac{1}{E_{R A C}^{100 \% R C A}}-\frac{1}{E_{N A C}}\right) \times R_{v}
$$

Hirsch Model

$$
\begin{gathered}
\frac{1}{E_{R A C}}=0.5 \times\left(\frac{1}{E_{N A C}+\left(E_{R A C}^{100 \% R C A}-E_{N A C}\right) \times R_{v}}\right) \\
+0.5 \times\left(\frac{1}{E_{N A C}}+\left(\frac{1}{E_{R A C}^{100 \% R C A}}-\frac{1}{E_{N A C}}\right) \times R_{v}\right)
\end{gathered}
$$




\section{Counto Model}

$$
\frac{1}{E_{R A C}}=\frac{1-\sqrt{1-R_{v}}}{E_{R A C}^{100 \% R C A}}+\frac{1}{\left(\frac{1-\sqrt{1-R_{v}}}{\sqrt{1-R_{v}}}\right) \times E_{R A C}^{100 \% R C A}+E_{N A C}}
$$

\section{Mindess Model}

$$
E_{R A C}=\frac{R_{v} \times E_{R A C}^{100 \% R C A}+\left(2-R_{v}\right) \times E_{N A C}}{\left(2-R_{v}\right) \times E_{R A C}^{100 \% R C A}+R_{v} \times E_{N A C}} \times E_{R A C}^{100 \% R C A}
$$

Eqs.5.31 to 5.35 are only valid for the RAC mixes in which NA is simply replaced by RCA using the conventional mix proportioning method. In order to have a general format of Eq.5.31 to Eq.5.35 which fit both the RAC mixes proportioned by the conventional and the proposed EMV method, the parameter $\chi$ (as defined in Eq.5.17) can be used instead of $R_{v}$ in Eqs.5.31 to 5.35. Therefore,

$$
E_{R A C}=E_{N A C}+\left(E_{R A C}^{100 \% R C A}-E_{N A C}\right) \times \chi
$$

\section{Modified Series Model}

$$
\frac{1}{E_{R A C}}=\frac{1}{E_{N A C}}+\left(\frac{1}{E_{R A C}^{100 \% R C A}}-\frac{1}{E_{N A C}}\right) \times \chi
$$

Modified Hirsch Model

$\frac{1}{E_{R A C}}=0.5 \times\left(\frac{1}{E_{N A C}+\left(E_{R A C}^{100 \% R C A}-E_{N A C}\right) \times \chi}\right)+0.5 \times\left(\frac{1}{E_{N A C}}+\left(\frac{1}{E_{R A C}^{100 \% R C A}}-\frac{1}{E_{N A C}}\right) \times \chi\right)$

Modified Counto Model

$$
\frac{1}{E_{R A C}}=\frac{1-\sqrt{1-\chi}}{E_{R A C}^{100 \% R C A}}+\frac{1}{\left(\frac{1-\sqrt{1-\chi}}{\sqrt{1-\chi}}\right) \times E_{R A C}^{100 \% R C A}+E_{N A C}}
$$

\section{Modified Mindess Model}

$$
E_{R A C}=\frac{\chi \times E_{R A C}^{100 \% R C A}+(2-\chi) \times E_{N A C}}{(2-\chi) \times E_{R A C}^{100 \% R C A}+\chi \times E_{N A C}} \times E_{R A C}^{100 \% R C A}
$$


By following this methodology, the RAC mixes having equal TM volume as the companion NAC would be expected to have comparable elastic moduli to the NAC.

\subsubsection{Experimental Verification}

In order to verify phase-based models (Eqs.5.26 and 5.30), one needs to know the elastic moduli of aggregate $\left(E_{N A}\right)$ and mortar $\left(E_{M}\right)$, which can be obtained by using any of equations Eqs.5.26 to 5.30. Of course the elastic moduli of the companion NAC ( $\left.E_{N A C}\right)$

and RAC at $100 \%\left(E_{R A C}^{100 \% R C A}\right)$ can be obtained experimentally which the volume fractions of TM and TNA would be known for the mixes under consideration.

Tables 5.4 present the experimental/predicted elastic modulus values using the phasebased models for all the RAC mixes tested. Generally, the difference between the parallel, the series, the Hirsch, the Counto and the Mindess models are negligible and the range of the experimental over predicted values of elastic modulus are between 0.931.12. This is more evident from Figures 5.3a to $5.7 \mathrm{a}$ which illustrate the predicted versus experimental elastic modulus values of all RAC mixes using different phase-based models. The figure also shows the $95 \%$ confidence limits, which clearly indicate the relatively high level of accuracy with which the elastic moduli of these mixes can be predicted.

Table 5.5 presents the ratio of experimental/predicted elastic modulus values using mixture-based models for all RAC mixes. The ratio is between $0.93-1.12$ using any of Eqs.5.31 to 5.35, or Eqs.5.36 to 5.40. Generally there is negligible difference observed between the phase-based and mixture-based models in predicting the elastic modulus of all RAC mixes. This is also evident in Figures 5.3 to 5.7, which illustrate the predicted versus experimental elastic modulus values of all RAC mixes using phase-based and 
mixture-based models. The figures also show the $95 \%$ confidence limits of the predicted values and it is evident that practically all the points fall within these limits. However, the mixture-based models employing $\chi$ instead of $R_{v}$ (Eq.5.35 to 5.40) are found to give more accurate results for RAC mixes proportioned by the proposed EMV method.

\subsubsection{Compressive Strength}

Amongst the different properties of concrete, the compressive strength is commonly considered as its most important property since other mechanical and long-term properties of concrete are directly or indirectly dependent on it. Therefore, it is crucial to have a good understanding of the various factors which affect the compressive strength of RAC.

As stated in Chapter 2, the compressive strength of RAC can be equal to or even higher than that of the companion NAC $\left(f_{c}^{\prime R A C}=f_{c}^{\prime N A C}\right)$ if the qualities of OITZ and RM is equal or better than those of NITZ and NM. Otherwise, its compressive strength may be lower and its value will depend on the strength of the recycled or original concrete (OC). In this case, although the cracking might initiate forming in RCA (either in OITZ or RM), they may not be interconnected to form a continuous crack system unless they extend to the NITZ and the NM. Therefore, the lower strength of RCA does not necessarily impose a limit on the overall strength of RAC, but the overall strength of RAC would be somewhere between those of the OC and the companion NAC, depending on the RCA content level of RAC.

In this section, two different approaches are presented to correlate the compressive strength of RAC to those of the OC and the companion NAC. 


\subsubsection{Equivalent-Mortar-Volume (EMV) Based Models}

It is well-known that the two components of normal concrete, i.e. aggregate and hydrated cement paste, show a practically linear stress-strain relation if individually loaded; however due to their substantially different elastic moduli and the advent of micro cracking, concrete exhibits inelastic behaviour, and consequently a high degree of nonlinearity in its stress-strain relation. This is mainly attributed to the composite action of the material, the presence of interfaces between the cement paste and the aggregates, and the advent and propagation of micro-cracks (Neville, 1996).

In RAC, as a four-phase material (including, OVA, NA, RM, and NM), it is reasonable to treat it as an equivalent two-phase concrete, comprising TM (RM plus NM) and TNA (NA and ONA) phases, akin to mortar and NA in normal concrete, but with the understanding that equivalent elastic moduli and compressive strength values must be used for TM and TNA. By treating TM as a two-phase mortar and using any of the existing models for the elastic modulus of normal concrete (Chapter 2: Table 2.1), the equivalent elastic modulus of TM can be obtained as a function of the elastic moduli and volume fractions of its RM and NM,

$$
E_{T M}=\frac{V_{N M}^{R A C} \times E_{N M}+V_{R M}^{R A C} \times E_{R M}}{V_{T M}^{R A C}} \text { (Parallel Model) }
$$

The stress-strain relation of TM is expected to be practically linear (Fig. 5.8) due to: (1) the small difference between the elastic moduli of its components (RM and NM), and (2) the presence of few early bond micro-cracks due to lower cracking tendency due to small difference between the elastic moduli and between the hydro-thermal properties of the two mortars. 
According to Figure 5.8, for a given strain $\varepsilon$, the corresponding stresses in TM, NM, and RM can be obtained as:

$$
\begin{aligned}
\sigma_{N M} & =E_{N M} \times \varepsilon \\
\sigma_{R M} & =E_{R M} \times \varepsilon \\
\sigma_{T M} & =E_{T M} \times \varepsilon
\end{aligned}
$$

By combining Eqs.5.41 to 5.44, the corresponding stress in TM can be obtained as

$$
\sigma_{T M}=\frac{V_{R M}^{R A C} \times \sigma_{R M}+V_{N M}^{R A C} \times \sigma_{N M}}{V_{T M}^{R A C}}
$$

Eq.5.45 is based on the so-called parallel model. Strictly speaking, it may not be completely valid but it does provide an upper-bound to the strength of the RAC. Note that the development of a model for RAC compressive strength is not one of the main objectives of this study, nevertheless, it is instructive to check the validity of this model for RAC. A lower bound to RAC compressive strength can be estimated by ignoring of the two terms in the nominator of Eq.5.45. Clearly, both of these are limiting conditions. An additional factor which could affect the compressive strength of RAC is the presence of voids and micro-cracks which are not accounted for by Eq.5.45. Although micromechanical models for predicting the compressive strength of concrete, involving the density, type and distribution of cracks in the mortar phase and concrete, have been proposed, such as the differential scheme and the Mori-Tanaka method (Mehta and Monteiro, 2006), it is beyond the scope of the present investigation to discuss them here. Assuming linear stress-strain relations for TM, RM, and NM up to failure, the following expression may be used to relate the compressive strength of TM to the corresponding strengths and volume fractions of RM and NM,

$$
V_{T M} \times f_{c}^{\prime T M}=f_{c}^{\prime R M} \times V_{R M}+f_{c}^{\prime N M} \times V_{N M}
$$


where $f_{c}^{\prime T M}, f_{c}^{\prime R M}$, and $f_{c}^{\prime N M}$ are the compressive strengths of TM, RM, and NM, respectively. Once again Eq.5.46 will furnish an upper bound to the compressive strength of TM. If the strengths of TNA, ONA, and NA are higher than the corresponding strengths of TM, RM, and NM, which will be normally the case, the failure is expected to occur in the mortars and/or the interfacial transition zones rather than the aggregates, and consequently the compressive strengths of TM, RM, and NM are expected to be equal to those of RAC, OC, and the companion NAC, respectively. Therefore:

$$
f_{c}^{\prime R A C}=\frac{f_{c}^{\prime O C} \times V_{R M}^{R A C}+f_{c}^{\prime N A C} \times V_{N M}^{R A C}}{V_{T M}^{R A C}}
$$

where $f_{c}^{\prime R A C}, f_{c}^{\prime O C}$, and $f_{c}^{\prime N A C}$ are the compressive strengths of RAC, OC, and NAC, respectively.

In the case of inferior OC compared to the companion NAC, Eq.5.46 may be used to determine the required level of improvement in the quality of NM in RAC compared to that in the companion NAC in order to achieve the target compressive strength of NAC $\left(f_{c}^{\prime N A C}=f_{c}^{\prime R A C}\right.$ ). According to Eq.5.46, the compressive strength of RAC may be improved by:

1. Increasing $V_{N M}^{R A C}$ or decreasing $V_{R M}^{R A C}$ at constant $V_{T M}^{R A C}$, which means lower RCA content, or

2. Increasing $f_{c}^{\prime N M}$ in RAC by adjusting the w/c ratio either by decreasing its water content or increasing its cement content. The required strength of NM may be obtained as:

$$
f_{c}^{\prime N M}=\frac{V_{T M}^{R A C} \times f_{c}^{\prime N A C}-f_{c}^{\prime O C} \times V_{R M}^{R A C}}{V_{N M}^{R A C}}
$$


In the case of equal compressive strength of NM (or NAC) and RM (or OC), no increase in $f_{c}^{\prime N M}$ for the new mortar in RAC would be necessary. However, for $f_{c}^{\prime R M}=0$, $f_{c}^{\prime N M}$ should be increased by $\frac{V_{T M}}{V_{N M}} \times f_{c}^{\prime N A C}$. Therefore, smaller RM content leads to lower required increase in the quality of NM in RAC. In the case of zero RM content $\left(V_{R M}=0\right)$, the ratio of $\frac{V_{T M}}{V_{N M}}=1.0$, and no increase in $f_{c}^{\prime N M}$ in RAC would be necessary.

By applying the same concept to series, Hirsch, Counto and Mindess models (Chapter 2: Table 2.1), the following models may be used instead of parallel model (Eq.5.46 and 5.41):

Series Model

$$
\begin{aligned}
& \frac{V_{T M}^{R A C}}{f_{c}^{\prime T M}}=\frac{V_{N M}^{R A C}}{f_{c}^{\prime N M}}+\frac{V_{R M}^{R A C}}{f_{c}^{\prime R M}} \\
& \frac{V_{T M}^{R A C}}{E_{T M}}=\frac{V_{N M}^{R A C}}{E_{N M}}+\frac{V_{R M}^{R A C}}{E_{R M}}
\end{aligned}
$$

\section{Hirsch Model}

$$
\begin{gathered}
\frac{1}{f_{c}^{\prime T M}}=0.5 \times\left(\frac{V_{N M}}{f_{c}^{\prime N M}}+\frac{V_{R M}}{f_{c}^{\prime R M}}\right)+0.5 \times\left(\frac{1}{V_{N M} \times f_{c}^{\prime N M}+V_{R M} \times f_{c}^{\prime R M}}\right) \\
\frac{1}{E_{T M}}=0.5 \times\left(\frac{1}{V_{N M} \times E_{N M}+V_{R M} \times E_{R M}}\right)+0.5 \times\left(\frac{V_{N M}}{E_{N M}}+\frac{V_{R M}}{E_{R M}}\right)
\end{gathered}
$$

\section{Counto Model}

$$
\begin{gathered}
\frac{1}{f_{c}^{\prime T M}}=\frac{(1-\sqrt{\eta})}{f_{c}^{\prime R M}}+\frac{1}{\left(\frac{(1-\sqrt{\eta})}{\sqrt{\eta}}\right) \times f_{c}^{\prime R M}+f_{c}^{\prime N M}} \\
\frac{1}{E_{T M}}=\frac{(1-\sqrt{\eta})}{E_{R M}}+\frac{1}{\left(\frac{(1-\sqrt{\eta})}{\sqrt{\eta}}\right) \times E_{R M}+E_{N M}} \\
\eta=\frac{V_{N M}^{R A C}}{V_{T M}^{R A C}}
\end{gathered}
$$


Mindess Model

$$
\begin{aligned}
f_{c}^{\prime T M} & =\frac{(1-\eta) \times f_{c}^{\prime R M}+(1+\eta) \times f_{c}^{\prime N M}}{(1+\eta) \times f_{c}^{\prime R M}+(1-\eta) \times f_{c}^{\prime N M}} \times f_{c}^{\prime R M} \\
E_{T M} & =\frac{(1-\eta) \times E_{R M}+(1+\eta) \times E_{N M}}{(1+\eta) \times E_{R M}+(1-\eta) \times E_{N M}} \times E_{R M}
\end{aligned}
$$

\subsubsection{Light-Weight-Aggregate (LWA) Based Model}

As mentioned earlier, the strength characteristics of the RAC would be affected by the properties of OC in the case of inferior quality of OITZ or RM compared to NITZ or NM. This situation is similar to the behaviour of lightweight aggregate concrete, in which the strength of lightweight aggregate is either close or lower compared to that of mortar. For this type of concrete made with normal-weight sand, Mindess (1981) has proposed the following empirical equation for the compressive strength of lightweight concrete:

$$
\sigma_{c}=\sigma_{a}{ }^{n} \cdot \sigma_{m}{ }^{(1-n)}
$$

where $\sigma_{c}, \sigma_{a}$, and $\sigma_{m}$ are the compressive strengths of the lightweight concrete, aggregate, and mortar, respectively; and $n$ is the volume fraction of the lightweight coarse aggregate.

By applying the same concept to RAC, the following empirical equation is proposed to correlate the compressive strength of RAC to the corresponding strength of RM and NM, as well as to the volume fraction of RM:

$$
\begin{array}{cc}
\text { If } f_{c}^{\prime R M} \leq f_{c}^{\prime N M}: & f_{c}^{\prime R A C}=\left(f_{c}^{\prime R M}\right)^{1-\eta} \cdot\left(f_{c}^{\prime N M}\right)^{\eta} \\
\text { If } f_{c}^{\prime R M} \geq f_{c}^{\prime N M}: & f_{c}^{\prime R A C}=f_{c}^{\prime N A C}
\end{array}
$$

where $\eta$ was defined in Eq.5.51b. 


\subsubsection{Experimental Verification}

To verify the proposed models, the experimental data from the comprehensive study performed by Hansen and Narud (1983) are used.

In their investigation, three types of RAC mixes were produced: high-strength $(\mathrm{H})$, medium strength (M), and low strength (L), using each of the three grades of RCA (H, M, and $\mathrm{L}$ ). The RAC mixes were designated as $\mathrm{H} / \mathrm{H}, \mathrm{H} / \mathrm{M}, \mathrm{H} / \mathrm{L}, \mathrm{M} / \mathrm{H}, \mathrm{M} / \mathrm{M}, \mathrm{M} / \mathrm{L}, \mathrm{L} / \mathrm{H}, \mathrm{L} / \mathrm{M}$, and $\mathrm{L} / \mathrm{L}$, where the first letter stands for the strength-grade of RAC, and the second letter indicates the strength-grade of RCA used. More details of their experimental investigation are presented in Appendix B.

Using Hansen and Narud's experimental data (Appendix B), both EMV models (Eq.5.47, and Eqs.5.49 to 5.52) and LWA model (Eq.5.54a and b) were used to predict the compressive strength of all RAC mixes made of RCA (H), (M), and (L). For those RAC mixes with lower target compressive strength than the compressive strength of $\mathrm{OC}(\mathrm{M} / \mathrm{H}$, $\mathrm{L} / \mathrm{H}$, and $\mathrm{L} / \mathrm{M}$ ), it will be assumed that the compressive strength of RAC is equal to that of the companion NAC. For the rest of RAC mixes with lower graded OC than the target compressive strength in RAC, the proposed models will be used to predict their compressive strength.

Table 5.6 presents the experimental over predicted values of the compressive strength of RAC mixes based on Hansen and Narud's experimental data using the different $E M V$ based models. The experimental over predicted compressive strength values after 14 days normal curing were found $0.88-1.10,1.03-1.49,1.03-1.20,1.03-1.18$, and 1.03-1.22 for parallel, series, Hirsch, Counto, and Mindess models, respectively, while for the 38-days- 
accelerated-cured specimens, they were $0.76-1.09,0.96-1.10,0.93-1.09,0.9-1.09$, and 0.95-1.09.

Table 5.7 presents the experimental over predicted values of the compressive strength for RAC mixes based on Hansen and Narud's experimental data and using LWA-based models. Generally, the LWA-based models are capable of predicting the compressive strength of RAC within a reasonable range: The experimental over predicted compressive strength values were found to be 1.03-1.18 and 0.89-1.09 for 14-days-normal-cured and 38-days-accelerated-cured specimens, respectively.

It is noteworthy that for all RAC mixes (regardless of RCA quality), the parallel and series models provided the upper and lower bound solutions, respectively. Higher difference between the strength-grade of RAC and RCA generally resulted in higher difference between the upper and the lower bound solutions. The EMV-based models tend to give less accurate prediction of the strength when the difference between the quality of RM and NM (or RAC and RCA strength-grades) is high. Since the main assumption in the EMV method is the close similarity between the mechanical properties of RM and NM, the observed lack of accuracy is explainable. However, the LWA-based model was found not to be as sensitive as the EMV-based models to the difference between the strength-grade of RAC and RCA. This is more evident from Figure 5.9, which illustrates the predicted versus experimental compressive strength values of RAC mixes using different EMV and LWA- based models.

Based on the results in Tables 5.6 and 5.7, it is apparent that designing a high-strength RAC made with low-strength RCA is possible. In $\mathrm{M} / \mathrm{L}$ and $\mathrm{H} / \mathrm{L}$ mixes, using even lowstrength RCA (L) (compressive strength of $9.1 \mathrm{MPa}$ ), RAC with the compressive 
strengths of 23.2 and $34.6 \mathrm{MPa}$ has been achieved by increasing the compressive strength of NM in RAC from 9.1 to 26.2 and $49.5 \mathrm{MPa}$, respectively. Also, in $\mathrm{H} / \mathrm{M}$ mix using medium-strength RCA (compressive strength of $26.2 \mathrm{Mpa}$ ), the RAC with compressive strengths of $46.3 \mathrm{MPa}$ was achieved by increasing the compressive strength of NM in RAC from 26.2 to $49.5 \mathrm{MPa}$.

\subsubsection{Creep}

Amongst the different properties of concrete, creep is a key factor which influences the long-term deformations in buildings and structures, as it may cause the deflections to increase several times over the initial deflection. Therefore, it is important to identify the important parameters which affect the creep of RAC.

As stated in Chapter 2, RAC has been commonly reported to have higher creep rate compared to NAC, which has been attributed to the RM in RCA. However, as shown in Chapter 4, the principal factor governing the creep of RAC is its total mortar volume rather than the RM content of RCA or the RCA content perse.

It is usually the hydrated cement paste which undergoes creep, and the role of the NA in concrete is primarily that of providing restraint to creep deformations. Creep, therefore is a function of the volumetric content of cement paste in concrete. The grading, maximum size and the shape of the aggregate have been suggested to affect creep, but, their global effect is normally reflected by the aggregate content.

Some models have been developed for NAC as a two-phase material (aggregate and mortar) correlating its creep to the volume fractions of its components (i.e., aggregate and mortar) and the corresponding property of cement paste. However they do not apply to 
$\mathrm{RAC}$ as a four-phase composite material, due to the two extra components: OVA and RM in RCA.

In Chapter 2, Neville's (1970) model for creep of conventional concrete was described. The model relates the creep of concrete $\left(c_{N A C}\right)$ and the volume fraction of aggregate $\left(V_{N A}^{N A C}\right)$ as

$$
\log \frac{c_{p}}{c_{N A C}}=\alpha_{N A C} \log \frac{1}{1-V_{N A}^{N A C}}
$$

where $c_{p}$ is the creep coefficient of neat cement paste of the same quality as used in concrete, $c_{N A C}$ is the creep coefficient of conventional concrete, $V_{N A}^{N A C}$ is the natural aggregate content in NAC, and $\alpha_{N A C}$ is a parameter defined as:

$$
\alpha_{N A C}=\frac{3\left(1-\mu_{N A C}\right)}{1+\mu_{N A C}+2\left(1-2 \mu_{N A}\right) \frac{E_{N A C}}{E_{N A}}}
$$

where $\mu_{N A}$ is the Poisson's ratio of aggregate, $\mu_{N A C}$ is the Poisson's ratio of concrete, $E_{N A}$ is the elastic modulus of aggregate, and $E_{N A C}$ is the elastic modulus of concrete.

For RAC, it is reasonable to assume that it is a two-phase concrete comprising the total mortar (TM) and the total natural aggregate (TNA). By assuming $\mu_{N A} \approx \mu_{R C A}$ and $\mu_{N A C} \approx \mu_{R A C}$, Eqs.5.55 and $5.56^{\circ}$ can be modified for RAC as follows

$$
\log \frac{c_{p}}{c_{R A C}}=\alpha_{R A C} \log \frac{1}{1-V_{T N A}^{R A C}}
$$

where $c_{R A C}$ is the creep of RAC, $V_{T N A}^{R A C}$ is the total natural aggregate content in RAC, and $\alpha_{R A C}$ is a parameter defined as:

$$
\alpha_{R A C}=\frac{3\left(1-\mu_{N A C}\right)}{1+\mu_{N A C}+2\left(1-2 \mu_{N A}\right) \frac{E_{R A C}}{E_{N A C}} \times \frac{E_{N A C}}{E_{N A}}}
$$


where $E_{R A C}$ is the elastic modulus of RAC.

Assuming identical creep for the cement pastes in RAC and NAC, and dividing Eq.5.56

by Eq.5.58, the creep of RAC can be correlated to the creep of NAC as follows:

$$
\frac{c_{R A C}}{c_{N A C}}=\frac{\left(1-V_{T N A}^{R A C}\right)^{\alpha_{R A C}}}{\left(1-V_{N A}^{N A C}\right)^{\alpha_{N A C}}}
$$

For the materials used, it is reasonable to assume $\mu_{N A}=\mu_{R C A}=0.10, \mu_{R A C}=\mu_{N A C}=0.12$ and $\frac{E_{N A C}}{E_{N A}}=0.5$. Therefore, Eq.5.59 can be written as

$$
\frac{c_{R A C}}{c_{N A C}}=\frac{\left(1-V_{T A A}^{R A C}\right)^{\frac{2.64}{1.12+0.8 E_{R A C} / E_{N A C}}}}{\left(1-V_{N A}^{N A C}\right)^{1.37}}
$$

By using the appropriate elastic modulus values and aggregate contents in the EM and EV mixes proportioned by the proposed EMV method in Chapter 4, Eq.5.60 indicates that RAC and the companion NAC have similar creep rates due to the same elastic moduli and aggregate contents in the corresponding mixes (see Chapter 4).

On the other hand, Eq.5.60 predicts the creep of $\mathrm{CM}$ and $\mathrm{CV}$ mixes proportioned by conventional mix proportioning method to be $27 \%$ and $20 \%$ higher than those of the companion CL and CG mixes, respectively. Based on the results in Chapter 4, the creep coefficient of $\mathrm{CM}$ and $\mathrm{CV}$ mixes at the age of 305 days are $31 \%$ and $11 \%$ higher than those of CL and CG mixes, respectively.

Another creep model proposed by Dantu (1958) for conventional concrete is as follows:

$$
\frac{\varepsilon_{p}}{\varepsilon_{c}^{N A C}}=\frac{1}{1-V_{N A}^{N A C}} \times \frac{E_{N A C}-E_{N A}}{E_{P}-E_{N A}}
$$

where $E_{p}$ and $\varepsilon_{p}$ are the elastic modulus and creep strain of neat cement paste of the same quality as used in concrete, and $\varepsilon_{c}^{N A C}$ is the creep strain of conventional concrete. 
If this model is applied to RAC, it would be reasonable to treat it as a two-phase concrete including total mortar (TM) and total natural aggregate (TNA) and rearrange Eq.5.61 as follows:

$$
\frac{\varepsilon_{p}}{\varepsilon_{c}^{R A C}}=\frac{1}{1-V_{T N A}^{R A C}} \times \frac{E_{R A C}-E_{N A}}{E_{P}-E_{N A}}
$$

where $\varepsilon_{c}^{R A C}$ is the creep strain of RAC.

Assuming the same creep rate for cement paste in RAC and NAC, and dividing Eq.5.61 by 5.62, the creep of RAC can be related to the creep of the companion NAC as

$$
\frac{\varepsilon_{c}^{R A C}}{\varepsilon_{c}^{N A C}}=\frac{E_{N A C}-E_{N A}}{E_{R A C}-E_{N A}} \times \frac{1-V_{T N A}^{R A C}}{1-V_{N A}^{N A C}}
$$

For the materials used here, it is reasonable to assume $\frac{E_{N A C}}{E_{N A}}=0.5$. Therefore, Eq.5.63 can be written as

$$
\frac{\varepsilon_{c}^{R A C}}{\varepsilon_{c}^{N A C}}=\frac{1}{2-E_{R A C} / E_{N A C}} \times \frac{1-V_{T N A}^{R A C}}{1-V_{N A}^{N A C}}
$$

By using the appropriate elastic modulus values and aggregate contents in the EM and EV mixes proportioned by the proposed method in Chapter 4, Eq.5.64 gives comparable creep rates for RAC and the companion NAC due to the same elastic moduli and aggregate contents in two mixes. The latter theoretical finding is confirmed by the current experimental results reported in Chapter 4 .

On the other hand, Eq.5.64 predicts the creep of $\mathrm{CM}$ and $\mathrm{CV}$ mixes proportioned by the conventional mix proportioning method to be $7 \%$ and $8 \%$ higher than those of the companion CL and CG mixes, respectively. According to the results in Chapter 4, the creep strains of CM and CV mixes are $49 \%$ and $19 \%$ higher than those of CL and CG 
mixes, respectively at age of 305 days. Generally, Eq.5.60 was found better in prediction of creep values of RAC compared to Eq.5.64.

\subsubsection{Drying Shrinkage}

Shrinkage is one of the key factors responsible for deformations in concrete structures, and it is one of the principal causes of cracking. It is also commonly believed by many investigators that RAC undergoes higher shrinkage than NAC. Of course these findings are based on data obtained from mixes designed by the conventional methods.

As stated in Chapter 2, RAC has been reported to have higher shrinkage rate demonstrated due to the presence of RM in RCA. However, as demonstrated in Chapter 4, the key factor governing the shrinkage of RAC is its total mortar volume rather than the RM content in RCA or the RCA content.

Some models have been developed for NAC as a two-phase material (aggregate and mortar) to correlate its shrinkage to the volume fractions of its aggregate and mortar, and to the shrinkage of cement paste. However, they do not directly apply to RAC as a fourphase composite material, including the two extra components; namely OVA and RM.

In Chapter 2 one of the models for predicting the shrinkage of conventional concrete was presented (Mindess and Young, 1981), which relates the shrinkage of concrete $\left(S_{N A C}\right)$ to the volume fraction of aggregate $\left(V_{N A}^{N A C}\right)$ as

$$
S_{N A C}=S_{p}\left(1-V_{N A}^{N A C}\right)^{n}
$$

where $n$ is a coefficient between 1.2 and 1.7 (average of 1.45).

For RAC, it would be reasonable that it is a two-phase concrete, including the total mortar (TM) and the total natural aggregate (TNA) and then write Eq.5.65 in the following modified form: 


$$
S_{R A C}=S_{p}\left(1-V_{T N A}^{R A C}\right)^{n}
$$

Assuming identical shrinkage for cement paste in RAC and NAC, and dividing Eq.5.65 by Eq.5.66, the shrinkage of RAC can be correlated to the shrinkage of the companion NAC as follows:

$$
\frac{S_{R A C}}{S_{N A C}}=\left(\frac{1-V_{T N A}^{R A C}}{1-V_{N A}^{N A C}}\right)^{n}
$$

By using the pertinent aggregate contents of the EM and EV mixes proportioned by the proposed method, Eq.5.67 leads to the conclusion RAC and NAC mixes with identical aggregate content proportions would have the same shrinkage rate, irrespective of the OVA and fresh NA. Clearly, implicit in this conclusion is the fact that the two types of aggregate are of similar quality, with similar absorption and shrinkage properties.

For $n=1.20$, Eq.5.67 predicts the shrinkage of $\mathrm{CM}$ and $\mathrm{CV}$ mixes proportioned by conventional mix proportioning method to be $24 \%$ and $16 \%$ higher than those of the companion CL and CG mixes, respectively, which compare favourably with the observed $26 \%$ and $14 \%$ higher shrinkage of $\mathrm{CM}$ and $\mathrm{CV}$ than those of CL and CG mixes, respectively at the age of 224 days.

\subsection{Evaluation of Existing Empirical Equations}

Generally, the compressive strength of concrete is considered as its most important property, while in fact its durability characteristics may be more important in some situations. However, the compressive strength is a fundamental element of structural design and is practically specified always since most other mechanical and long-term properties of concrete are assumed to be dependent on it directly or indirectly. Therefore, relating the other mechanical properties of concrete to its compressive strength is critical 
from design point of view. As stated in Chapter 2, for normal concrete, these interrelations have been well established and are described in various design codes, standards and handbooks. However, some previous studies have indicated that the existing empirical equations for normal concrete may not be valid for RAC. The major objective of this section is to examine the applicability of the existing equations in the literature for predicting some of the mechanical properties of normal concrete to estimating the short-term and long-term properties of RAC.

\subsubsection{Relationship between Compressive and Tensile strengths}

As stated in Chapter 2, some previous studies have indicated that the conventional relationships between the tensile and the compressive strengths of normal concrete may not be valid for RAC, while other studies have found no significant difference between the tensile strengths of RAC and NAC. In the following, this issue will be explored for the mixes tested in this study.

Figure 5.10 presents the splitting tensile strength values for all the RAC and NAC mixes whose mechanical properties were presented in Tables 3.6 and 3.7 as well as their corresponding predicted values based on ACI 318 (2005) ( $f_{t}=0.54 \sqrt{ } f_{c}$ for SI Unit). Generally, the ACI 318 predicted splitting tensile strength values are found to be $100 \%$ and $83 \%$ conservative for the PM and the PV mixes, respectively. In fact, for both airentrained and non-air-entrained RAC mixes made with RCA-MO at all RCA content levels and w/c ratios (PM mixes), the ACI-318 predicted values are conservative. Furthermore, all of the experimental splitting tensile strength values of the companion NAC mixes made with natural limestone (CL mixes) are also higher than the predicted values. 
Similarly, for air-entrained and non-air-entrained RAC mixes made with RCA-VA at all RCA content levels and w/c ratios (PV mixes), the predicted ACI-318 predicted values are conservative, except for PV-100HA and PV-100HN mixes, in which the experimental values are lower than the predicted values. Furthermore, all of the experimental splitting tensile strength values of the companion NAC made with natural river gravel (CG mixes) are higher than the predicted values except for CG-MN. Therefore, it may be concluded that the unconservatism of ACI-318 is not only due to the presence of RCA. Since the splitting tensile and compressive strengths of concrete are mainly dependant on the strength of its mortar, the mechanical bond between the aggregate and the mortar, and the interfacial transition zone quality, rather than just the RCA content or RM quantity, the relationship between the splitting tensile and compressive strengths of NAC can also be valid for RAC at any RCA content.

\subsubsection{Relationship between Compressive strength and Elastic Modulus}

For normal concrete, it is quite well-known that the elastic modulus of concrete increases with an increase in the compressive strength of concrete, but there is no agreement on the unique form of the relationship. This is not surprising, as the elastic modulus of concrete is mainly a function of the elastic modulus and volume fractions of its aggregate and mortar. As the former is rarely known, in some advanced standards and research works, the effect of the elastic modulus and volume fractions of aggregate and mortar have been reflected by the density of the concrete. Some of these empirical equations were presented in Chapter 2 (Table 2.1).

According to the results of the preliminary tests in Chapter 3, and the data in the existing literature, the hardened density and elastic modulus of RAC mixes proportioned by the 
conventional method are lower than those of the NAC due to the lower specific gravity and elastic modulus of RCA, while the proposed EMV mix proportioning method yields to comparable values for RAC and NAC mixes. While the elastic modulus of concrete is mainly a function of the volume fractions and elastic moduli of its components, the compressive strength is mainly dependant on the strength of the mortar and the aggregate and the interfacial transition zone. In other words, by adjusting the w/c ratio in NM in the RAC proportioned by the conventional method, the same or higher compressive strength as the companion NAC can be achieved, but its elastic modulus may be still lower than that of the companion NAC due to either lower TNA or higher TM content. Therefore, using the conventional expressions which simply correlate the elastic modulus of concrete to its compressive strength (like CSA A23.3-04: clause 8.6.2.3), one would overestimate the elastic modulus of RAC proportioned by the conventional method. However, by using the general form of the current relationships which correlate the elastic modulus of concrete to both its compressive strength and hardened density (like ACI-363 and CSA A23.3-04: clause 8.6.2.2), the effect of the elastic modulus and volume fractions of aggregate and mortar would be included since the concrete density is a function of its mortar and aggregate volume fractions as well as their densities. Figure 5.11 shows the variation of the elastic moduli of RAC mixes (proportioned by the conventional and EMV methods) versus their hardened density values. It is quite evident that the elastic moduli of these mixes tend to increase with increased hardened density. Figures 5.12 and 5.13 illustrate the experimental versus predicted elastic modulus values based on the ACI-318, ACI-363, and both the simple and general empirical equations of CSA A23.3-04 (clauses 8.6.2.3 and 8.6.2.2) for all mixes tested in this study. 
For the PM mixes proportioned by the conventional method, the experimental over predicted values based on simplified CSA A23.3-04 (clause 8.6.2.3) and ACI-318 equations, are 0.83-1.22 and 0.88-1.29 respectively; while for PV mixes, they are 0.981.21 and $0.94-1.13$. Generally, all the overestimated values by the simplified equations of both codes are for those mixes with compressive strength of $40 \mathrm{MPa}$ or higher. By using the general equations of ACI-363 and CSA A23.3-04 (clause 8.6.2.2), the experimental over predicted values of the PM mixes proportioned by conventional method are $0.92-$ 1.26 and $0.93-1.27$, while those of the PV mixes are 1.01-1.21 and 1.02-1.22. Therefore, the simplified equations recommended by both the ACI-318 and the CSA A23.3-04 (clause 8.6.2.3) standards are not applicable to RAC mixes proportioned by the conventional method, while the general forms of these code equations are applicable.

On the other hand, for the EM mixes proportioned by the proposed EMV method, the experimental over predicted values based on the simplified CSA and ACI equations are 1.08-1.18 and 1.02-1.12, while for the EV mixes, they are 1.15-1.28 and 1.08, respectively. By using the general equations of $\mathrm{ACI}$ and CSA, the experimental over predicted values of EM mixes proportioned by the EMV method are 1.10-1.19 and 1.111.20, while for EV mixes; they are 1.16-1.17 and 1.17. Therefore, both the simplified and general formats of the equations recommended in $\mathrm{ACI}$ and CSA standards are found to give reasonable results for the RAC mixes proportioned by the EMV method.

For the CL mixes made with natural limestone, the experimental over predicted values based on the simplified CSA and $\mathrm{ACI}$ equations are 1.08-1.16 and 0.99-1.12, while for the CG mixes made with river gravel, they are 1.04-1.23 and 0.94-1.14. By using the general equations of $\mathrm{ACI}$ and CSA, the experimental over predicted values of CL mixes 
are 1.10-1.19 and 1.11-1.20, while for CG mixes; they are 1.05-1.19 and 1.06-1.20, respectively. These numbers indicate that the level of accuracy for the mixes designed by the EMV method is comparable to that obtained for the NAC mixes designed by the conventional method. Consequently, the recommended equations of both ACI and CSA can be applied to RAC, provided that the mixes are designed by the EMV method.

Figures 5.14 and 5.15 also demonstrate the experimental versus predicted elastic modulus values using the empirical equations proposed by Gardner (2000), Nassif et al. (2005), Tomosawa et al. (1993) and Architectural Institute of Japan (AIJ) for all the mixes made with RCA-MO \& NL and RCA-VA \& NG, respectively. Generally, the recommended expressions by Gardner, Nassif et al., and Tomasawa et al. were all found applicable to RAC. However, the AIJ equation was valid mostly for those mixes with compressive strength of $36 \mathrm{MPa}$ or less, as recommended by AIJ.

Figure 5.16 also demonstrates the experimental versus predicted elastic modulus values using the Eurocode2 (2002) recommended expression for all the mixes made with RCAMO \& NL and RCA-VA \& NG, respectively. Generally, the elastic moduli for all RAC mixes (proportioned by the conventional and EMV methods) and NAC mixes are overestimated by this code.

\subsubsection{Compressive Stress-Strain Relationship}

Knowledge of the stress-strain relation of concrete is important when studying the behaviour and strength of RC beams. In flexural design, this data is needed to calculate the rectangular stress block parameters for concrete compression zone. Indeed, the structural performance of RC members, including their flexural and shear strength, is directly related to this relationship. Although the stress-strain relationship of normal 
concrete in compression is well known, there is limited research available for RAC. The majority of the research works have been focused on different mechanical properties of RAC, including its compressive and tensile strengths, elastic modulus, etc.

The present section specifically investigates the applicability of the existing stress-strain models for normal concrete (Table 2.16) to RAC.

Figures 5.17 to 5.19 illustrate the experimental and predicted stress-strain relationships of the different concrete mixes tested in this study using the empirical equations proposed by Thorenfeldt et al. (modified by Collins and Mitchell (1990)), Hognestad (1951), Desayi and Krishnan (1964) and Smith and Young (1955) for all the mixes made with RCA-MO \& NL and RCA-VA \& NG, respectively. For comparison purposes, all of the experimental curves have been normalized to the corresponding peak stress and corresponding strain values of the mixes. For the empirical relations, the strain was normalized by the strain corresponding to peak stress as recommended by the particular equation developer (Table 2.16).

Generally, the equation proposed by Thorenfeldt et al. (1987), and modified by Collins and Mitchell (1990), was found to fit best the ascending branch of the stress-strain curve of almost all the NAC and RAC mixes, regardless of the mix proportioning method. The Hognestad model (1951) was found to fit slightly better the ascending part of the stressstrain curves of CL-3.

On the other hand, although there was not a single empirical equation that fitted well the descending branch of the observed stress-strain relations of all RAC and NAC mixes, the Smith-Young model (1955) was found to fit relatively well most of the RAC and the 
NAC mixes. For CM mix, the descending part was not fully traced due to sudden drop of the load during the test.

It is noteworthy that the descending branch of the diagram is mainly important in the ultimate flexural strength calculations of members and in determining the ductility of reinforced concrete members. Normally the maximum design or useable strain in concrete under compression is set equal to 0.003 (ACI-318-05) or 0.0035 (CSA A23.304). Therefore, despite the minor differences among the descending shapes of the stressstrain curves based on existing models, this aspect of the relationship will not significantly influence the ultimate flexural strength of members nor the rectangular stress block parameters for the concrete compression zone in ultimate strength design. This will be discussed in more detail in Chapter 8 .

\subsubsection{Creep}

In this section the applicability of the conventional methods for predicting the creep coefficient of normal concrete to RAC is studied. The empirical equations proposed by ACI Committee 209 (ACI, 1982) and CEB-FIP (CEB, 1993), which are presented in Tables 2.2 and 2.3 in Chapter 2, will be examined.

Figures 5.20 to 5.22 show the experimental and predicted creep coefficient variation with time using the ACI Committee 209 and CEB-FIP equations for all the mixes made with RCA-MO \& NL and RCA-VA \& NG, respectively. For comparison purposes, all of the experimental creep coefficient-time curves have been normalized by the latest measured creep coefficient for each mix. For the empirical relations, the creep coefficients were normalized by the calculated creep coefficient at the same time as the corresponding experimental value measurements for each mix. 
Generally, the predicted normalized creep coefficient variation with time using both the $\mathrm{CEB}$ and the $\mathrm{ACI}$ equations agreed well with the observed variations for all the RAC and the NAC mixes, irrespective of aggregate type or mix proportioning method. At the early ages, the observed creep rate was found slightly lower compared to the predicted ones. This was more evident for the EM mix.

By reviewing the empirical equations proposed by $\mathrm{ACI}$ (Table 2.2), it can be seen that the fine aggregate and air contents are two factors which affect the creep properties of NAC. Increasing either quantity would lead to an increase in creep of concrete. By simply applying the conventional models to RAC, the amount of fine and air content existing in the residual mortar will not be taken into account. However, the latter may be difficult because there might not be enough information regarding the composition of the RM in RCA, therefore alternatively one could introduce a new coefficient which would indirectly takes these factors into account. Toward this end, a new factor, the so-called residual mortar coefficient $\left(K_{R M}\right)$ could be obtained by using Eq.5.59 the proposed empirical equation proposed for creep of RAC. For RAC mix proportioned by the EMV method, the creep of RAC is predicted to be similar to that of normal concrete due to its equivalent total mortar volume to that of the companion NAC. Therefore the residual mortar coefficient can be taken equal to $1\left(K_{R M}=1\right)$. However, the creep of RAC mix proportioned by conventional method is predicted to be higher than that of NAC due to its higher total mortar volume compared to that of the companion NAC. By assuming $\alpha_{N A C} \approx \alpha_{R A C}=1.37$, Eq.5.59 can be written as

$$
c_{R A C}=\left(\frac{1-V_{T N A}^{R A C}}{1-V_{N A}^{N A C}}\right)^{1.37} \times c_{N A C}
$$


Since $c_{N A C}$ can be found using either the ACI or the CEB method, the $\left(\frac{1-V_{T N A}^{R A C}}{1-V_{N A}^{N A C}}\right)^{1.37}$ term can be considered as a new coefficient which takes the effect of RM into account. Therefore:

$$
K_{R M}=\left(\frac{1-V_{N A}^{R A C}}{1-V_{N A}^{N A C}}\right)^{1.37}
$$

Since in the conventional mix proportioning method, RCA is replacing NA by weight or volume, the volume of coarse RCA in RAC can be taken equal to the coarse NA volume in NAC. Furthermore, the nominator in Eq.5.69 is equal to the total mortar volume in RAC. Therefore, Eq.5.69 can be rewritten as:

$$
K_{R M}=\left(\frac{V_{N M}^{R A C}+V_{R M}^{R A C}}{1-V_{R C A}^{R A C}}\right)^{1.37}
$$

By virtue of Eqs.4.7 and 4.11a in Chapter 4, Eq.5.70 can be rearranged as

$$
K_{R M}=\left[\frac{1-\left((1-R M C) \times \frac{S G_{b}^{R C A}}{S G_{b}^{O V A}}\right) \times V_{R C A}^{R A C}}{1-V_{R C A}^{R A C}}\right]^{1.37}
$$

The coefficient $K_{R M}$ based on Eq.5.71 can be multiplied by the creep coefficient obtained by the existing $\mathrm{ACI}$ or $\mathrm{CEB}$ equations for normal concrete to determine the creep coefficient. Knowing the volume of coarse RCA in a unit volume of RAC $\left(V_{R C A}^{R A C}\right)$, the residual mortar content (RMC), the bulk specific gravity of RCA $\left(S G_{b}^{R C A}\right)$ and OVA $\left(S G_{b}^{O V A}\right), K_{R M}$ values of 1.35 and 1.22 were calculated for $\mathrm{CM}$ and $\mathrm{CV}$ mixes, respectively, compared to the observed values of 1.29 and 1.14 at the age of 305 days. 
Since determining $S G_{b}^{\text {OVA }}$ might not be practical, Eq.5.71 can be simplified by assuming $\frac{S G_{b}^{R C A}}{S G_{b}^{O V A}} \approx 1$ as:

$$
K_{R M}=\left(\frac{1-(1-R M C) \times V_{R C A}}{1-V_{R C A}}\right)^{1.37}
$$

By using Eq.5.72 $K_{R M}$ values of 1.29 and 1.16 are calculated for $\mathrm{CM}$ and $\mathrm{CV}$ mixes, respectively, which are 5\% lower than the values calculated by Eq.5.71.

\subsubsection{Drying Shrinkage}

The focus of this section is on the applicability of the conventional methods for predicting the drying shrinkage of normal concrete to RAC. The empirical equations proposed by ACI Committee 209 (ACI, 1982) and CEB-FIP (CEB, 1993), which were presented in Tables 2.4 and 2.5 , will be examined.

Figure 5.23 to 5.25 show the experimental and predicted drying shrinkage strain variation with time using the empirical equations proposed by ACI and CEB-FIP for all mixes made with RCA-MO \& NL and RCA-VA \& NG, respectively. For comparison purposes, all of the experimental shrinkage strain-time curves have been normalized by the latest value of the measured shrinkage strain for each mix. For the empirical relations, the shrinkage strains were normalized by the relevant value of the calculated shrinkage strain corresponding to the latest measurements for each mix.

Generally, the predicted normalized drying shrinkage strain variation with time according to the $\mathrm{CEB}$ and $\mathrm{ACI}$ empirical equations were found to fit relatively well the observed variation for all the RAC and the NAC mixes, irrespective of aggregate type or mix proportioning method. 
By reviewing the empirical equations proposed by $\mathrm{ACI}$ (Tables 2.4 ), it can be seen that the fine aggregate, cement and air contents in conventional concrete are the three factors which affect the shrinkage properties of NAC. Increasing the fine, cement, or air content leads to an increase in shrinkage of concrete. By simply applying existing shrinkage models to RAC, the amount of cement, fine and air existing in the residual mortar will not be taken into consideration. Since there might not be enough information regarding the composition of the RM in RCA, one way of including the effects of latter quantities would be to introduce a new coefficient which would indirectly reflect their influence. Accordingly, a new factor, a so-called residual mortar coefficient $\left(S_{R M}\right)$ would be introduced based on the proposed empirical equation for drying shrinkage of RAC (see Eq.5.67 in Section 5.2.5). For RAC mixes proportioned by the proposed EMV method, Eq.5.67 predicts the shrinkage of RAC to be equal to that of normal concrete due to their equivalent total mortar volumes. Therefore, the residual mortar coefficient for such RAC mixes can be taken equal to $1\left(S_{R M}=1\right)$. However, the shrinkage of RAC mixes proportioned by the conventional method is predicted to be higher than that of NAC due to its higher total mortar volume compared to that of the companion NAC. By assuming an average value of 1.45 for $\mathrm{n}$, Eq.5.67 can be rewritten as:

$$
S_{R A C}=\left(\frac{1-V_{T N A}^{R A C}}{1-V_{N A}^{N A C}}\right)^{1.45} \times S_{N A C}
$$

By following the same procedure as that used to derive $K_{R M}$, Eq.5.73 can be rearranged as: 


$$
S_{R M}=\left[\frac{1-\left((1-R M C) \times \frac{S G_{b}^{R C A}}{S G_{b}^{O V A}}\right) \times V_{R C A}^{R A C}}{1-V_{R C A}^{R A C}}\right]^{1.45}
$$

The coefficient $S_{R M}$ given by Eq.5.74 can be used in conjunction with ACI and CEB methods in order to take the effect of RM in RCA into consideration. Using Eq.5.74, the residual mortar coefficient for $\mathrm{CM}$ and $\mathrm{CV}$ mixes were calculated to be 1.36 and 1.24 , respectively. The latter values compare favourably to the observed values of 1.26 and 1.14 at age of 224 days, respectively. Since determining $S G_{b}^{O V A}$ might not be practical, Eq.5.74 can be simplified by assuming $\frac{S G_{b}^{R C A}}{S G_{b}^{O V A}} \approx 1$,

$$
S_{R M}=\left(\frac{1-(1-R M C) \times V_{R C A}^{R A C}}{1-V_{R C A}^{R A C}}\right)^{1.45}
$$

Using Eq.5.75, the residual mortar coefficients of $\mathrm{CM}$ and $\mathrm{CV}$ were calculated as 1.31 and 1.17, respectively which are $6 \%$ lower than the values given by Eq.5.74.

\subsection{Summary}

- The predicted fresh and hardened densities of RAC using the proposed phase-based method agreed well with the experimental densities.

- The predicted fresh and hardened densities of RAC using either of the proposed mixture-based Eq.5.16 or Eq.5.18 were found more accurate compared to the predicted values using the phase-based models. Furthermore, Eq.5.16 was found to be mainly applicable to RAC mixes proportioned by the conventional method, while Eq.5.18 was found to be applicable to RAC mixes proportioned by both the conventional and the proposed EMV methods. 
- The predicted elastic modulus of RAC based on the phase-based models (Eq.5.26 to 5.30) agreed well with the corresponding experimental values for all RAC mixes proportioned by the conventional and the proposed EMV methods.

- The predicted elastic modulus of RAC based on either of the proposed mixture-based in Eq.5.31 to 5.35 or Eq.5.36 to 5.40 were found in good agreement with the experimental observations. Furthermore, Eqs.5.31 to 5.35 were mainly applicable to RAC mixes proportioned by the conventional method, while Eqs.5.36 to 5.40 were applicable to RAC mixes proportioned by both the conventional and the proposed EMV methods.

- The predicted compressive strength of RAC using the proposed EMV models in Eqs.5.47 to 5.52 were agreed well with the experimental values provided by Hansen and Narud (1983). However, in the case of high strength RAC made of low strength RCA, light-weight-aggregate based model (Eq.5.54a) was found more accurate.

- Both the EMV and the LWA models revealed that it is feasible to design a highstrength RAC by using even low-strength RCA by adjusting the quality of NM in RAC.

- The predicted creep and shrinkage of RAC using the proposed Eq.5.60 and Eq.5.67 were in relatively good agreement with the experimental values of all RAC mixes, irrespective of the mix proportioning method.

- The empirical equation of ACI 318-05 for the splitting tensile strength was found applicable to both air-entrained and non-air-entrained RAC mixes.

- The simplified empirical equations of ACI 318-05 and CSA A23.3-04 for elastic modulus of concrete were found to overestimate the elastic modulus of RAC mixes proportioned by the conventional method, while for RAC mixes proportioned by the proposed EMV method, they gave reasonable results. 
- The general empirical equations of ACI 363 and CSA A23.3-04 for elastic modulus of concrete were found to be applicable to all RAC mixes, irrespective of the mix proportioning method.

- Generally, the recommended expressions by Gardner, Nassif et al., and Tomasawa et al. for estimating the elastic modulus of conventional concrete were all found applicable to RAC. However, the AIJ equation was applicable mostly to those RAC mixes with compressive strength of $36 \mathrm{MPa}$ or less, as recommended by AIJ. The Euocode-2 equation overestimated the elastic modulus of all the RAC mixes appreciably.

- The empirical equation for the compressive stress-strain relationship of concrete proposed by Thorenfeldt et al. (1987) and modified by Collins and Mitchell (1990) was found to fit better the ascending branch of the stress-strain curve of all NAC and RAC mixes, regardless of the mix proportioning method, compared to other empirical equations. On the other hand, the Smith-Young model (1955) was found to fit relatively better the descending branch of the curves.

- The normalized creep coefficient and drying shrinkage strain variation with time predicted by both the $\mathrm{CEB}$ and the $\mathrm{ACI}$ empirical equations were found to agree well with the observed values for all the RAC and NAC mixes, irrespective of aggregate type or mix proportioning method.

- Neither the ACI nor the CEB empirical equations for creep and shrinkage of normal concrete consider the effects of RCA content or residual mortar volume on the creep and shrinkage of RAC. After applying the proposed residual mortar coefficients of $K_{R M}$ and $S_{R M}$ to the empirical equations of $\mathrm{ACI}$ and CEB, they were found applicable to 
the RAC mixes proportioned by both the conventional and the proposed EMV mix proportioning methods. 
Tables 5.1: The experimental/predicted density values using phase-based models for RAC mixes made with RCA-MO

\begin{tabular}{|c|c|c|c|c|c|c|}
\hline \multirow{2}{*}{$\begin{array}{c}\text { Mix } \\
\text { ID }\end{array}$} & \multicolumn{3}{|c|}{$\begin{array}{c}\text { Hardened density } \\
\left(\mathbf{k g} / \mathbf{m}^{\mathbf{3}} \mathbf{)}\right.\end{array}$} & \multicolumn{3}{c|}{$\begin{array}{c}\text { Fresh density } \\
\left(\mathbf{k g} / \mathbf{m}^{\mathbf{3}} \mathbf{)}\right.\end{array}$} \\
\cline { 2 - 7 } & \multirow{2}{*}{ Exp. } & \multicolumn{2}{|c|}{ Exp./Pred. } & \multirow{2}{*}{ Exp. } & \multicolumn{2}{|c|}{ Exp./Pred. } \\
\cline { 3 - 7 } & $\mathbf{E q . 5 . 2}$ & $\mathbf{E q . 5 . 1 0}$ & & $\mathbf{E q . 5 . 2}$ & Eq.5.10 \\
\hline PM-100LA & 2233 & 1.04 & 1.03 & 2227 & 1.00 & 1.00 \\
\hline PM-100MA & 2138 & 1.02 & 1.01 & 2181 & 1.01 & 1.01 \\
\hline PM-100HA & 2144 & 1.03 & 1.02 & 2181 & 1.02 & 1.03 \\
\hline PM-100LN & 2299 & 1.03 & 1.01 & 2338 & 1.03 & 1.02 \\
\hline PM-100MN & 2302 & 1.04 & 1.02 & 2324 & 1.02 & 1.01 \\
\hline PM-100HN & 2298 & 1.04 & 1.01 & 2324 & 1.02 & 1.01 \\
\hline PM-50LA & 2224 & 1.00 & 1.00 & 2281 & 1.00 & 1.00 \\
\hline PM-50MA & 2179 & 1.01 & 1.00 & 2238 & 1.01 & 1.01 \\
\hline PM-50HA & 2167 & 1.01 & 1.01 & 2210 & 1.01 & 1.01 \\
\hline PM-50LN & 2325 & 1.01 & 1.00 & 2395 & 1.03 & 1.02 \\
\hline PM-50MN & 2333 & 1.02 & 1.01 & 2366 & 1.02 & 1.01 \\
\hline PM-50HN & 2352 & 1.03 & 1.02 & 2352 & 1.01 & 1.00 \\
\hline PM-30LA & 2312 & 1.02 & 1.02 & 2338 & 1.01 & 1.01 \\
\hline PM-30MA & 2234 & 1.02 & 1.01 & 2238 & 1.00 & 1.00 \\
\hline PM-30HA & 2208 & 1.02 & 1.02 & 2210 & 1.00 & 1.00 \\
\hline PM-30LN & 2374 & 1.02 & 1.01 & 2381 & 1.01 & 1.01 \\
\hline PM-30MN & 2372 & 1.02 & 1.02 & 2381 & 0.98 & 1.01 \\
\hline PM-30HN & 2359 & 1.02 & 1.01 & 2366 & 1.00 & 1.00 \\
\hline CM-C & 2264 & 1.04 & 1.02 & 2262 & 1.01 & 1.00 \\
\hline CM-F & 2217 & 1.01 & 0.99 & 2226 & 0.99 & 0.98 \\
\hline CM-B & 2269 & 1.03 & 1.01 & 2281 & 1.00 & 1.00 \\
\hline EM-C & 2295 & 1.02 & 1.00 & 2305 & 1.00 & 0.99 \\
\hline EM-F & 2293 & 1.01 & 0.99 & 2291 & 0.98 & 0.98 \\
\hline EM-B & 2319 & 1.02 & 1.00 & 2306 & 0.99 & 0.98 \\
\hline Average & & 1.02 & 1.01 & & 1.01 & 1.00 \\
\hline St.Dev. & & 0.011 & 0.010 & & 0.013 & 0.012 \\
\hline
\end{tabular}


Tables 5.2: The experimental/predicted density values using phase-based models for RAC mixes made with RCA-VA

\begin{tabular}{|c|c|c|c|c|c|c|}
\hline \multirow{3}{*}{$\begin{array}{l}\text { Mix } \\
\text { ID }\end{array}$} & \multicolumn{3}{|c|}{$\begin{array}{c}\text { Hardened density } \\
\left(\mathbf{k g} / \mathbf{m}^{3}\right)\end{array}$} & \multicolumn{3}{|c|}{$\begin{array}{c}\text { Fresh density } \\
\left(\mathrm{kg} / \mathbf{m}^{\mathbf{3}}\right)\end{array}$} \\
\hline & \multirow{2}{*}{ Exp. } & \multicolumn{2}{|c|}{ Exp./Pred. } & \multirow{2}{*}{ Exp. } & \multicolumn{2}{|c|}{\begin{tabular}{|l} 
Exp./Pred. \\
\end{tabular}} \\
\hline & & Eq.5.2 & Eq.5.10 & & Eq.5.2 & Eq.5.10 \\
\hline PV-100LA & 2307 & 1.07 & 1.06 & 2315 & 1.05 & 1.04 \\
\hline PV-100MA & 2233 & 1.05 & 1.04 & 2224 & 1.04 & 1.03 \\
\hline PV-100HA & 2196 & 1.05 & 1.04 & 2238 & 1.03 & 1.03 \\
\hline PV-100LN & 2356 & 1.12 & 1.03 & 2381 & 1.11 & 1.03 \\
\hline PV-100MN & 2289 & 1.09 & 1.00 & 2366 & 1.11 & 1.02 \\
\hline PV-100HN & 2275 & 1.07 & 0.98 & 2381 & 1.13 & 1.04 \\
\hline PV-50LA & 2325 & 1.05 & 1.04 & 2335 & 1.03 & 1.02 \\
\hline PV-50MA & 2273 & 1.03 & 1.03 & 2272 & 1.02 & 1.02 \\
\hline PV-501 & 2255 & 1.04 & 1.03 & 2278 & 1.02 & 1.01 \\
\hline PV-50LN & 2388 & 1.06 & 1.02 & 2404 & 1.06 & 1.01 \\
\hline PV-50MN & 2392 & 1.07 & 1.02 & 2393 & 1.05 & 1.01 \\
\hline PV-50HN & 2393 & 1.05 & 1.00 & 2394 & 1.06 & 1.02 \\
\hline PV-30LA & 2329 & 1.03 & 1.03 & 2339 & 1.01 & 1.01 \\
\hline PV-30MA & 2277 & 1.02 & 1.02 & 2284 & 1.02 & 1.02 \\
\hline PV-30HA & 2252 & 1.02 & 1.02 & 2277 & 1.00 & 1.00 \\
\hline PV-30LN & 2396 & 1.04 & 1.01 & 2422 & 1.04 & 1.01 \\
\hline PV-30MN & 2393 & 1.03 & 1.01 & 2427 & 1.03 & 1.01 \\
\hline PV-30HN & 2392 & 1.02 & 1.00 & 2409 & 1.04 & 1.02 \\
\hline $\mathrm{CV}-\mathrm{C}$ & 2285 & 1.05 & 1.03 & 2306 & 1.04 & 1.03 \\
\hline $\mathrm{CV}-\mathrm{F}$ & 2281 & 1.03 & 1.02 & 2291 & 1.02 & 1.01 \\
\hline$C V-B$ & 2290 & 1.04 & 1.02 & 2286 & 1.02 & 1.01 \\
\hline $\mathrm{EV}-\mathrm{C}$ & 2321 & 1.04 & 1.02 & 2328 & 1.02 & 1.01 \\
\hline EV-F & $5-2$ & - & $2-$ & 2321 & 1.01 & 1.00 \\
\hline EV-B & 2325 & 1.03 & 1.01 & 2316 & 1.04 & 1.00 \\
\hline Averag & 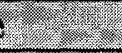 & 1.05 & 1.02 & $5=$ & 1.04 & 1.02 \\
\hline ot. Dev. & & 0.024 & 0.011 & & 0.033 & 0.012 \\
\hline
\end{tabular}


Table 5.3: The experimental/predicted hardened and fresh density values using mixturebased models for RAC mixes

\begin{tabular}{|c|c|c|c|c|c|c|c|c|c|}
\hline \multirow{3}{*}{$\begin{array}{l}\text { Mix } \\
\text { ID }\end{array}$} & \multirow{3}{*}{$\begin{array}{l}R_{w} \\
\%\end{array}$} & \multirow{3}{*}{$\begin{array}{l}R_{v} \\
\%\end{array}$} & \multirow{3}{*}{$\begin{array}{l}\chi \\
\%\end{array}$} & \multicolumn{3}{|c|}{$\begin{array}{c}\text { Hardened density } \\
\left(\mathrm{kg} / \mathrm{m}^{3}\right)\end{array}$} & \multicolumn{3}{|c|}{$\begin{array}{c}\text { Fresh density } \\
\left(\mathrm{kg} / \mathrm{m}^{3}\right)\end{array}$} \\
\hline & & & & \multirow{2}{*}{ Exp. } & \multicolumn{2}{|c|}{ Exp./Clc. } & \multirow{2}{*}{ Exp. } & \multicolumn{2}{|c|}{ Exp./Clc. } \\
\hline & & & & & Eq.5.16 & Eq.5.18 & & Eq.5.16 & Eq.5.18 \\
\hline PM-50LA & 50.0 & 53.4 & 54.2 & 2224 & 1.02 & 1.02 & 2281 & 1.00 & 1.00 \\
\hline PM-50MA & 50.0 & 53.7 & 54.4 & 2179 & 1.01 & 1.01 & 2238 & 1.00 & 1.00 \\
\hline PM-50HA & 50.0 & 53.5 & 53.0 & 2167 & 1.01 & 1.01 & 2210 & 1.00 & 1.01 \\
\hline PM-50LN & 50.0 & 53.7 & 52.0 & 2325 & 1.01 & 1.01 & 2395 & 0.99 & 0.99 \\
\hline PM-50MN & 50.0 & 53.7 & 52.7 & 2333 & 1.00 & 1.00 & 2366 & 1.00 & 1.00 \\
\hline PM-50HN & 50.0 & 53.8 & 52.9 & 2352 & 0.99 & 0.99 & 2352 & 1.00 & 1.01 \\
\hline PM-30LA & 30.0 & 32.8 & 32.4 & 2312 & 0.99 & 0.99 & 2338 & 0.99 & 0.99 \\
\hline PM-30MA & 30.0 & 33.0 & 33.1 & 2234 & 0.99 & 0.99 & 2238 & 1.01 & 1.01 \\
\hline PM-30HA & 30.0 & 33.1 & 35.2 & 2208 & 1.00 & 1.00 & 2210 & 1.01 & 1.01 \\
\hline PM-30LN & 30.0 & 33.1 & 32.2 & 2374 & 0.99 & 0.99 & 2381 & 1.00 & 1.00 \\
\hline PM-30MN & 30.0 & 33.0 & 32.4 & 2372 & 0.99 & 0.99 & 2381 & 1.00 & 1.00 \\
\hline PM-30HN & 30.0 & 32.9 & 34.0 & 2359 & 1.00 & 1.00 & 2366 & 1.01 & 1.01 \\
\hline EM-C & 63.5 & 67.0 & 0 & 2295 & 0.99 & 1.00 & 2305 & 0.99 & 1.01 \\
\hline $\mathrm{CM}-\mathrm{E}$ & 63.5 & 66.7 & 0 & 2293 & 0.98 & 1.00 & 2291 & 0.99 & 1,02 \\
\hline EM-B & 63.5 & 66.7 & 0 & 2319 & & & 2306 & & $x^{2}$ \\
\hline PV-50LA & 50.0 & 52.4 & 51.7 & 2307 & 0.99 & 0.99 & 2335 & 1.00 & 1.00 \\
\hline PV-50MA & 50.0 & 52.4 & 48.9 & 2233 & 0.99 & 0.99 & 2272 & 0.99 & 0.99 \\
\hline PV-50HA & 50.0 & 52.4 & 52.2 & 2196 & 0.99 & 0.99 & 2278 & 1.00 & 1.00 \\
\hline PV-50LN & 50.0 & 48.3 & 52.2 & 2356 & 1.00 & 1.00 & 2404 & 1.00 & 1.00 \\
\hline PV-50MN & 50.0 & 48.2 & 50.4 & 2289 & 0.98 & 0.98 & 2393 & 1.01 & 1.01 \\
\hline PV-50HN & 50.0 & 48.2 & 56.0 & 2275 & 0.99 & 0.99 & 2394 & 1.00 & 1.00 \\
\hline PV-30LA & 30.0 & 32.0 & 30.0 & 2325 & 0.99 & 0.99 & 2339 & 1.00 & 1.00 \\
\hline PV-30MA & 30.0 & 32.1 & 29.9 & 2273 & 0.99 & 1.00 & 2284 & 0.99 & 0.99 \\
\hline PV-30HA & 30.0 & 32.0 & 35.5 & 2255 & 1.00 & 1.00 & 2277 & 1.01 & 1.01 \\
\hline PV-30LN & 30.0 & 28.5 & 33.6 & 2388 & 1.00 & 1.00 & 2422 & 1.00 & 1.00 \\
\hline PV-30MN & 30.0 & 28.4 & 28.7 & 2392 & 0.99 & 0.99 & 2427 & 1.00 & 1.00 \\
\hline PV-30HN & 30.0 & 28.5 & 38.8 & 2393 & 1.01 & 1.00 & 2409 & 1.00 & 1.00 \\
\hline$=B V-C$ & 74.3 & 76.3 & 0 & 2321 & 0.99 & 1.00 & 2328 & 0.99 & 1.01 \\
\hline $\mathrm{E} \times-\mathrm{E}$ & 74.3 & 76.1 & 0 & & & & 2321 & 0.99 & 1.01 \\
\hline$E V-B$ & 94.3 & 76.1 & 0 & 2325 & 0.99 & 1200 & 2316 & 0.99 & 101 \\
\hline \multicolumn{5}{|c|}{ Average } & 1.00 & 1.00 & & 1.00 & 1.00 \\
\hline \multicolumn{5}{|c|}{ St. Dev. } & 0.010 & 0.009 & & 0.007 & 0.008 \\
\hline
\end{tabular}


Table 5.4: The experimental/predicted elastic modulus values using phase-based models for RAC mixes

\begin{tabular}{|c|c|c|c|c|c|c|}
\hline \multirow{2}{*}{ Mix ID } & \multirow{2}{*}{$\begin{array}{l}\mathbf{E}_{\mathbf{c}}^{\text {Exp }} \\
(\mathbf{G P a})\end{array}$} & \multicolumn{5}{|c|}{$\mathbf{E}_{\mathbf{c}}^{\text {Exp. } / \mathbf{E}_{\mathbf{c}}^{\text {Pred. }}}$} \\
\hline & & Parallel & Series & Hirsch & Counto & Mindess \\
\hline PM-50LA & 28.4 & 0.94 & 0.94 & 0.93 & 0.94 & 0.94 \\
\hline PM-50MA & 25.8 & 1.03 & 1.06 & 0.95 & 1.00 & 1.01 \\
\hline PM-50HA & 24.3 & 0.97 & 0.96 & 0.96 & 0.96 & 0.97 \\
\hline PM-50LN & 33.8 & 1.01 & 1.01 & 1.00 & 1.00 & 1.01 \\
\hline PM-50MN & 32 & 1.03 & 1.03 & 1.02 & 1.03 & 1.03 \\
\hline PM-50HN & 28.8 & 0.98 & 0.98 & 0.98 & 0.98 & 0.98 \\
\hline PM-30LA & 31.7 & 0.99 & 1.00 & 0.99 & 0.99 & 1.00 \\
\hline PM-30MA & 26.8 & 0.98 & 1.01 & 0.94 & 0.97 & 0.98 \\
\hline PM-30HA & 25.2 & 1.00 & 0.99 & 0.99 & 0.99 & 0.99 \\
\hline PM-30LN & 34.1 & 0.98 & 0.98 & 0.98 & 0.98 & 0.98 \\
\hline PM-30MN & 32.4 & 1.00 & 1.00 & 1.00 & 1.00 & 1.00 \\
\hline PM-30HN & 30.6 & 1.01 & 1.01 & 1.01 & 1.01 & 1.01 \\
\hline EM-C & 30,3 & 0.98 & 0,98 & 0.98 & 0.98 & 0.98 \\
\hline EM-E & 318 & 0.99 & 1.00 & 1.00 & 1.00 & 1.00 \\
\hline LM-B & 32.3 & $x=$ & $x-4$ & 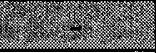 & 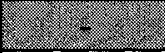 & $5-2$ \\
\hline PV-50LA & 32.9 & 1.08 & 1.08 & 1.08 & 1.06 & 1.08 \\
\hline PV-50MA & 29.4 & 1.07 & 1.08 & 1.07 & 1.07 & 1.08 \\
\hline PV-50HA & 26.8 & 1.04 & 1.05 & 1.00 & 1.04 & 1.05 \\
\hline PV-50LN & 35.3 & 1.02 & 1.02 & 1.02 & 1.02 & 1.02 \\
\hline PV-50MN & 32.3 & 0.97 & 0.97 & 0.97 & 0.97 & 0.97 \\
\hline PV-50HN & 30.2 & 0.97 & 0.97 & 0.97 & 0.97 & 0.97 \\
\hline PV-30LA & 32.9 & 1.09 & 1.09 & 1.09 & 1.06 & 1.09 \\
\hline PV-30MA & 28.7 & 1.03 & 1.03 & 1.03 & 1.03 & 1.03 \\
\hline PV-30HA & 27 & 1.01 & 1.02 & 0.98 & 1.01 & 1.01 \\
\hline PV-30LN & 36.4 & 1.05 & 1.05 & 1.05 & 1.05 & 1.05 \\
\hline PV-30MN & 32.8 & 0.96 & 0.96 & 0.96 & 0.96 & 0.96 \\
\hline PV-30HN & 32.1 & 1.01 & 1.01 & 1.01 & 1.01 & 1.01 \\
\hline EV-C & 32.8 & 1.09 & 1.05 & 1.07 & 1.07 & 1,08 \\
\hline EV-T & 34.2 & 1.12 & 1.41 & 1.11 & 1.11 & 1.11 \\
\hline$E V-B$ & 32.8 & 8 & 1.06 & 1.07 & 1.07 & 1.07 \\
\hline Avera & e & $\begin{array}{l}.02 \\
\end{array}$ & 1.02 & 1.01 & 24.01 & 1.02 \\
\hline $2.51 . \mathrm{Be}$ & 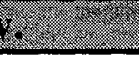 & 0.045 & 0.043 & 0.047 & 0.041. & 0.044 \\
\hline
\end{tabular}


Table 5.5: The experimental/predicted elastic modulus values using mixture-based models for RAC mixes

\begin{tabular}{|c|c|c|c|c|c|c|c|c|c|c|}
\hline \multirow{3}{*}{ Mix ID } & \multicolumn{10}{|c|}{ 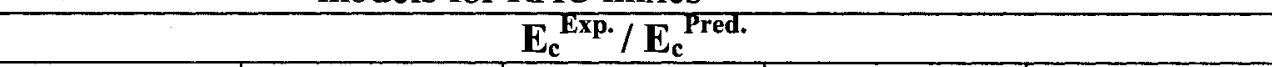 } \\
\hline & \multicolumn{2}{|c|}{$\begin{array}{l}\text { Parallel } \\
\text { Based on }\end{array}$} & \multicolumn{2}{|c|}{$\begin{array}{c}\text { Series } \\
\text { Based on } \\
\end{array}$} & \multicolumn{2}{|c|}{$\begin{array}{c}\text { Hirsch } \\
\text { Based on } \\
\end{array}$} & \multicolumn{2}{|c|}{$\begin{array}{c}\text { Counto } \\
\text { Based on } \\
\end{array}$} & \multicolumn{2}{|c|}{$\begin{array}{l}\text { Mindess } \\
\text { Based on }\end{array}$} \\
\hline & $\begin{array}{l}\mathbf{E q} . \\
5.31 \\
\end{array}$ & $\begin{array}{l}\text { Eq. } \\
5.36\end{array}$ & \begin{tabular}{l|}
$\mathbf{E q}$. \\
5.32
\end{tabular} & $\begin{array}{c}\mathbf{E q} \cdot \\
5.37\end{array}$ & \begin{tabular}{l|} 
Eq. \\
5.33
\end{tabular} & $\begin{array}{l}\text { Eq. } \\
5.38\end{array}$ & $\begin{array}{l}\mathbf{E q} . \\
5.34\end{array}$ & $\begin{array}{c}\mathbf{E q} \cdot \\
5.39\end{array}$ & $\begin{array}{l}\text { Eq. } \\
5.35\end{array}$ & $\begin{array}{l}\text { Eq. } \\
5.40\end{array}$ \\
\hline PM-50LA & 0.93 & 0.93 & 0.94 & 0.94 & 0.93 & 0.93 & 0.93 & 0.93 & 0.93 & 0.93 \\
\hline PM-50MA & 1.01 & 1.01 & 1.05 & 1.05 & 1.03 & 1.03 & 1.03 & 1.03 & 1.03 & 1.04 \\
\hline PM-50HA & 0.96 & 0.96 & 0.97 & 0.96 & 0.96 & 0.96 & 0.96 & 0.96 & 0.96 & 0.96 \\
\hline PM-50LN & 1.00 & 1.00 & 1.01 & 1.01 & 1.01 & 1.01 & 1.01 & 1.01 & 1.01 & 1.01 \\
\hline PM-50MN & 1.02 & 1.02 & 1.03 & 1.03 & 1.03 & 1.03 & 1.03 & 1.03 & 1.03 & 1.03 \\
\hline PM-50HN & 0.98 & 0.98 & 0.99 & 0.98 & 0.98 & 0.98 & 0.98 & 0.98 & 0.98 & 0.98 \\
\hline PM-30LA & 0.99 & 0.99 & 1.00 & 1.00 & 0.99 & 0.99 & 0.99 & 0.99 & 0.99 & 0.99 \\
\hline PM-30MA & 0.97 & 0.97 & 1.00 & 1.00 & 0.99 & 0.99 & 0.99 & 0.99 & 0.99 & 0.99 \\
\hline РМ-30HA & 0.99 & 0.99 & 0.99 & 0.99 & 0.99 & 0.99 & 0.99 & 0.99 & 0.99 & 0.99 \\
\hline PM-30LN & 0.98 & 0.98 & 0.99 & 0.98 & 0.98 & 0.98 & 0.98 & 0.98 & 0.98 & 0.98 \\
\hline PM-30MN & 1.00 & 1.00 & 1.00 & 1.00 & 1.00 & 1.00 & 1.00 & 1.00 & 1.00 & 1.00 \\
\hline PM-30HN & 1.00 & 1.01 & 1.01 & 1.01 & 1.01 & 1.01 & 1.01 & 1.01 & 1.01 & 1.01 \\
\hline & 106 & 0.98 & 0.98 & 008 & 1,02 & 0.98 & 1.07 & 6088 & 1007 & 0.98 \\
\hline $\mathrm{AM}-\mathrm{P}$ & $10 \%$ & 100 & 107 & 100 & 1.07 & 1.00 & 407 & 1.00 & 1107 & 1600 \\
\hline DM-B & 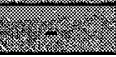 & 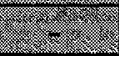 & $\sqrt{2+28}$ & 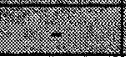 & 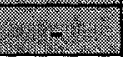 & 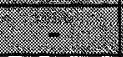 & 2.28 & & $\sqrt{430}$ & $18-2$ \\
\hline PV-50LA & 1.08 & 1.08 & 1.08 & 1.08 & 1.08 & 1.08 & 1.08 & 1.08 & 1.08 & 1.08 \\
\hline PV-50MA & 1.08 & 1.07 & 1.08 & 1.08 & 1.08 & 1.08 & 1.08 & 1.08 & 1.08 & 1.08 \\
\hline PV-5 & 1.03 & 1.03 & 1.05 & 1.05 & 1.04 & 1.04 & 1.04 & 1.04 & 1.04 & 1.04 \\
\hline PV-50LN & 1.02 & 1.02 & 1.02 & 1.02 & 1.02 & 1.02 & 1.02 & 1.02 & 1.02 & 1.02 \\
\hline PV-50MN & 0.96 & 0.97 & 0.97 & 0.97 & 0.97 & 0.97 & 0.97 & 0.97 & 0.97 & 0.97 \\
\hline PV-5 & 0.96 & 0.97 & 0.96 & 0.97 & 0.96 & 0.97 & 0.96 & 0.97 & 0.96 & 0.97 \\
\hline PV-30LA & 1.09 & 1.09 & 1.09 & 1.09 & 1.09 & 1.09 & 1.09 & 1.09 & 1.09 & 1.09 \\
\hline PV-30MA & 1.03 & 1.03 & 1.03 & 1.03 & 1.03 & 1.03 & 1.03 & 1.03 & 1.03 & 1.03 \\
\hline PV-30HA & 0.99 & 1.00 & 1.01 & 1.02 & 1.00 & 1.01 & 1.00 & 1.01 & 1.00 & 1.01 \\
\hline PV-30LN & 1.04 & 1.05 & 1.04 & 1.05 & 1.04 & 1.05 & 1.04 & 1.05 & 1.04 & 1.05 \\
\hline PV-30MN & 0.96 & 0.96 & 0.96 & 0.96 & 0.96 & 0.96 & 0.96 & 0.96 & 0.96 & 0.96 \\
\hline PV-30HN & 0.99 & 1.01 & 1.00 & 1.01 & 1.00 & 1.01 & 1.00 & 1.01 & 1.00 & 1.01 \\
\hline $2 \mathrm{EV}-\mathrm{C}$ & 1.12 & 1.06 & 1.12 & 106 & 1.12 & 1100 & 1.12 & 106 & 1.12 & 1.06 \\
\hline DV T & $\sqrt{1412}$ & 1.11 & 1.12 & 11112 & 1412 & 1.118 & 112 & 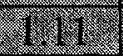 & 1712 & 9.118 \\
\hline 9048 & 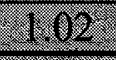 & 21088 & 2102 & 2008 & 1002 & 1098 & 1.02 & 108 & 1.02 & 1.08 \\
\hline trenge & 1102 & 1.012 & 1.02 & 10.02 & 21.02 & 1,62 & 1.102 & 87028 & 802 & 1102 \\
\hline atciser. & 0.050 & 00044 & 0.047 & 0.044 & 0.048 & 0.044 & 0.049 & 0044 & 0.049 & 0.045 \\
\hline
\end{tabular}




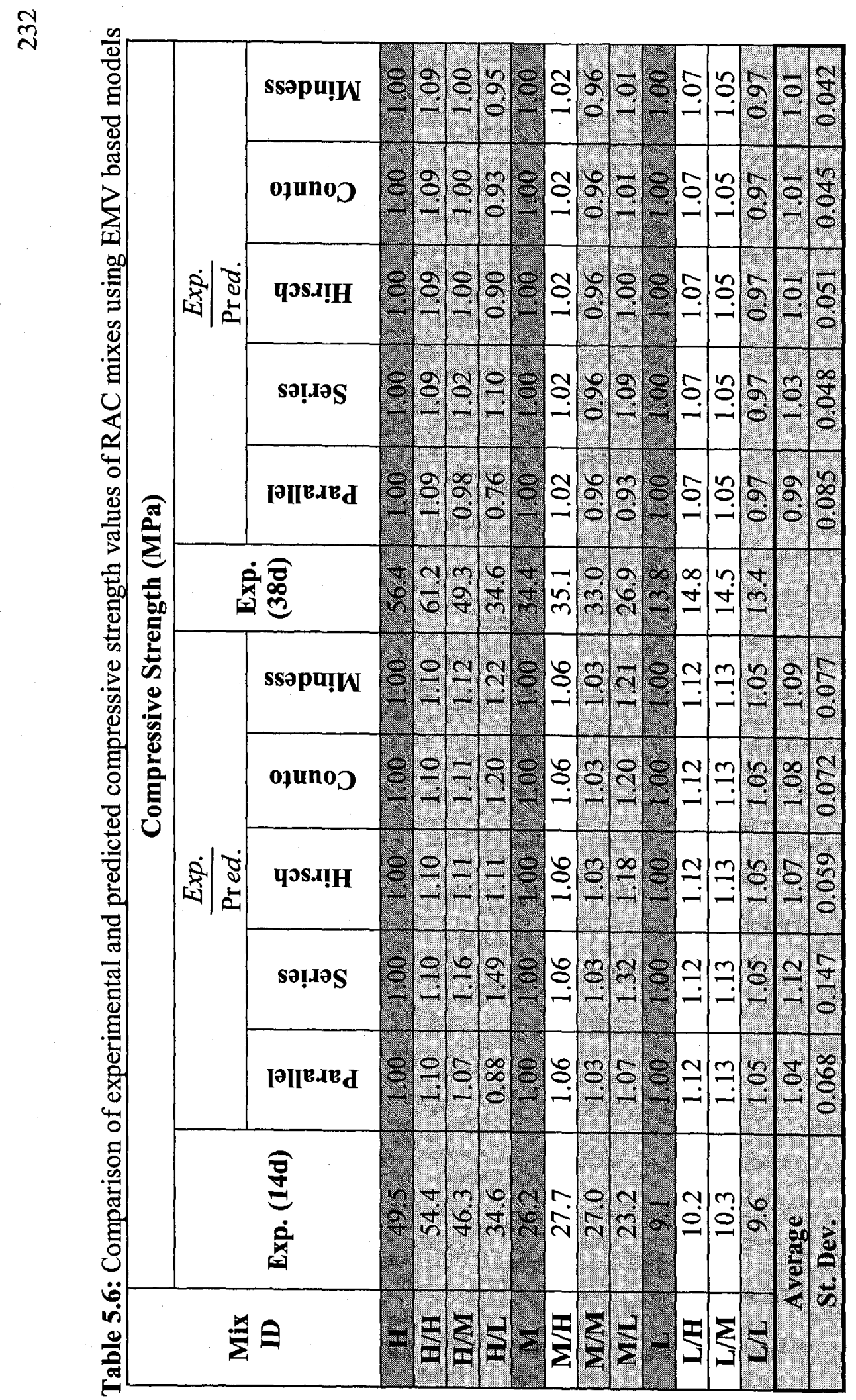


Table 5.7: The experimental and predicted compressive strength value of RAC mixes using lightweight-aggregate-based models

\begin{tabular}{|c|c|c|c|c|}
\hline \multirow{3}{*}{ Mix ID } & \multicolumn{4}{|c|}{ Compressive Strength (MPa) } \\
\hline & \multirow{2}{*}{ Exp. (14d) } & Exp. & \multirow{2}{*}{ Exp. (38d) } & \multirow{2}{*}{$\frac{\text { Exp. }}{\operatorname{Pr} e d .}$} \\
\hline & & Pred. & & \\
\hline Hi & 49.5 & 1.00 & 56.4 & 1.00 \\
\hline Н/H & 54.4 & 1.10 & 61.2 & 1.09 \\
\hline H/M & 46.3 & 1.12 & 49.3 & 1.00 \\
\hline H/L & 34.6 & 1.10 & 34.6 & 0.89 \\
\hline M & 26.2 & 1.00 & 34.4 & 1.00 \\
\hline M/H & 27.7 & 1.06 & 35.1 & 1.02 \\
\hline $\mathbf{M} / \mathbf{M}$ & 27.0 & 1.03 & 33.0 & 0.96 \\
\hline M/L & 23.2 & 1.18 & 26.9 & 1.00 \\
\hline L & 9.1 & 1.00 & 13.8 & 99.00 \\
\hline $\mathrm{L} / \mathrm{H}$ & 10.2 & 1.12 & 14.8 & 1.07 \\
\hline $\mathbf{L} / \mathbf{M}$ & 10.3 & 1.13 & 14.5 & 1.05 \\
\hline L/L & 9.6 & 1.05 & 13.4 & 0.97 \\
\hline \multicolumn{2}{|c|}{ Average } & 1.07 & 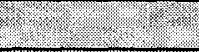 & $=1.00$ \\
\hline \multicolumn{2}{|c|}{ St. Dev. } & 0.060 & 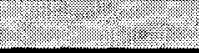 & 0.052 \\
\hline
\end{tabular}




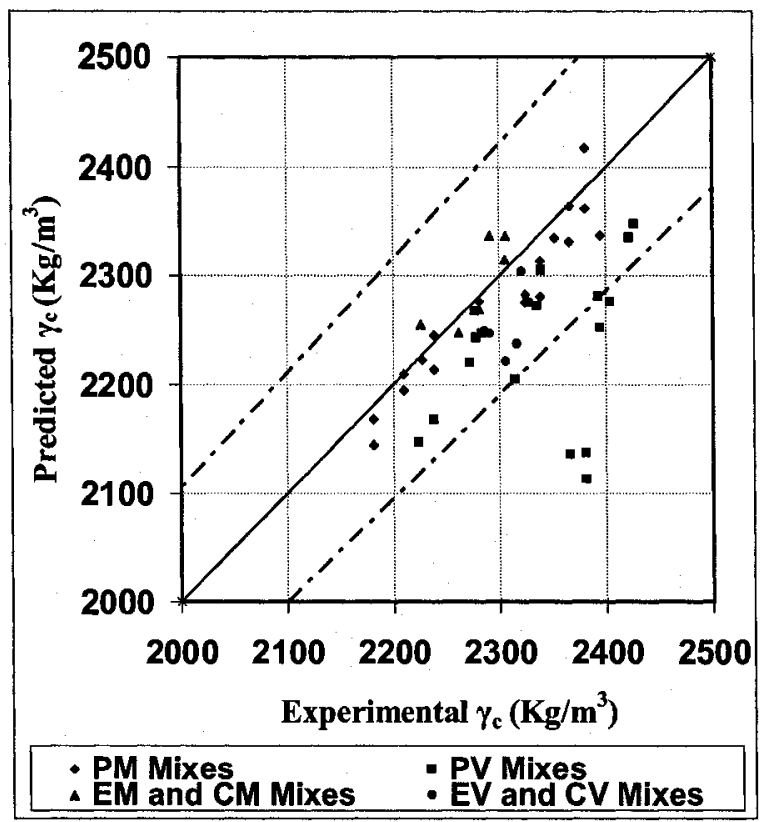

(a) Phase-Based Model (Eq.5.2)

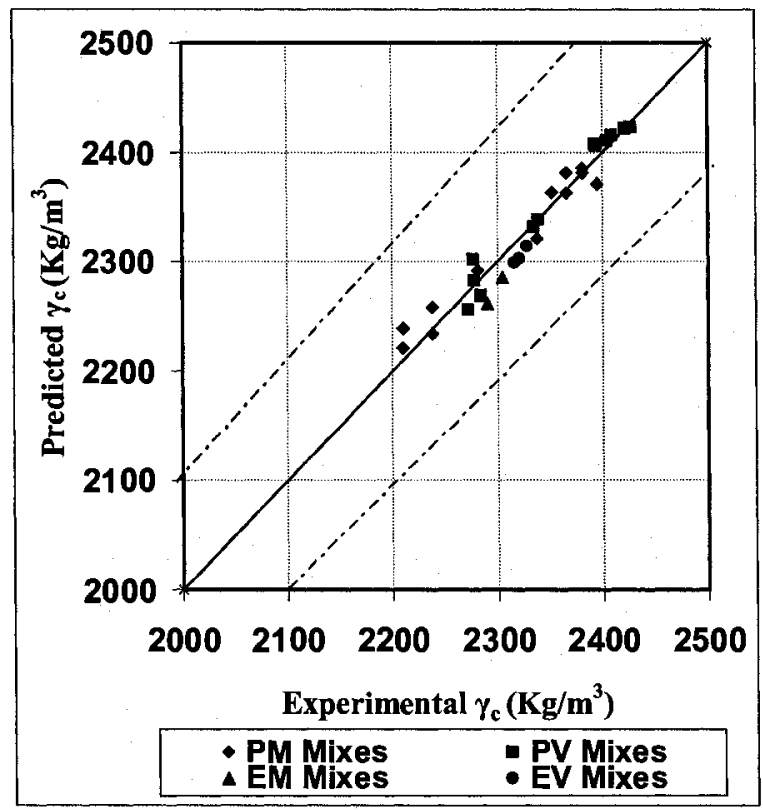

(a) Mixture-Based Model (Eq.5.16)

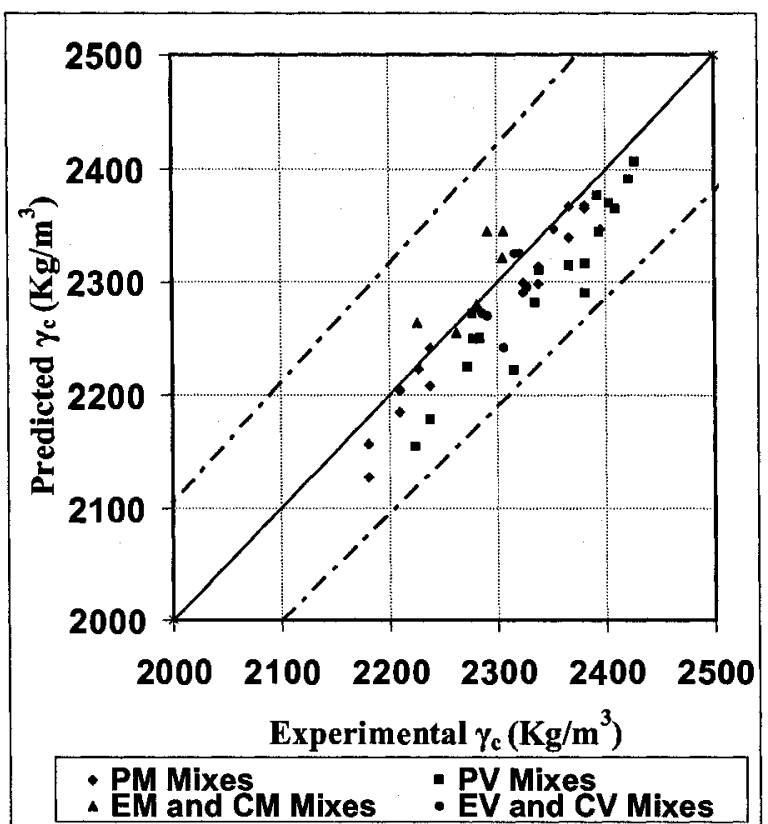

(b) Phase-Based Model (Eq.5.10)

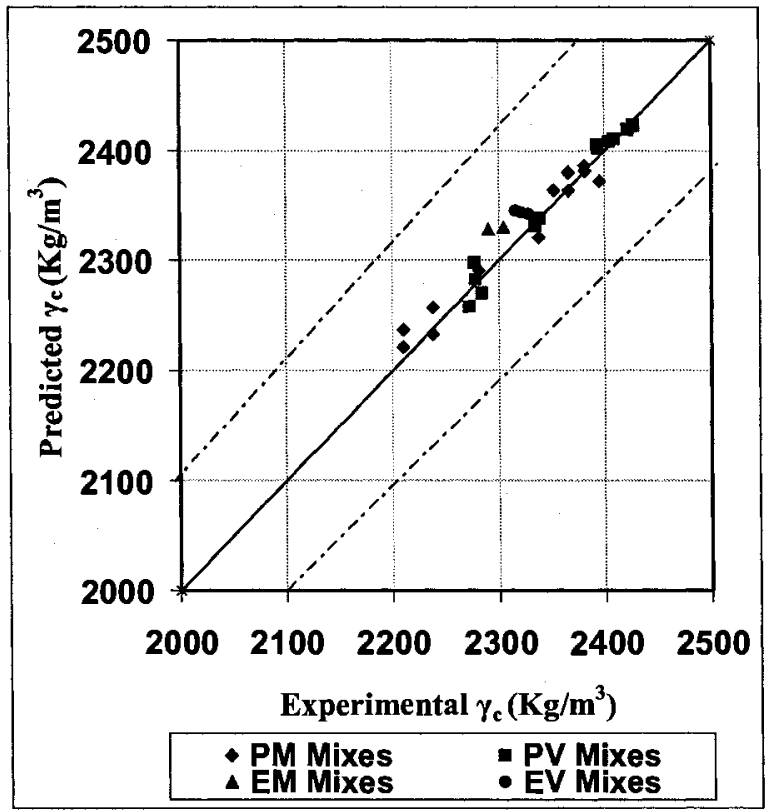

(b) Mixture-Based Model (Eq.5.18)

Figure 5.1: Predicted versus experimental fresh density values of RAC mixes based on different models 


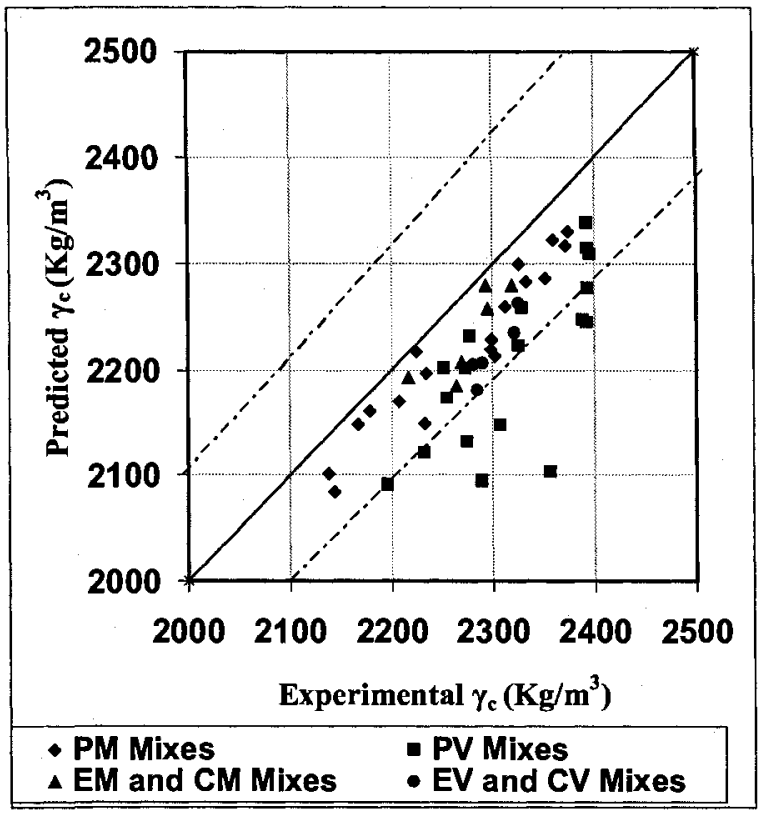

(a) Phase-Based Model-1

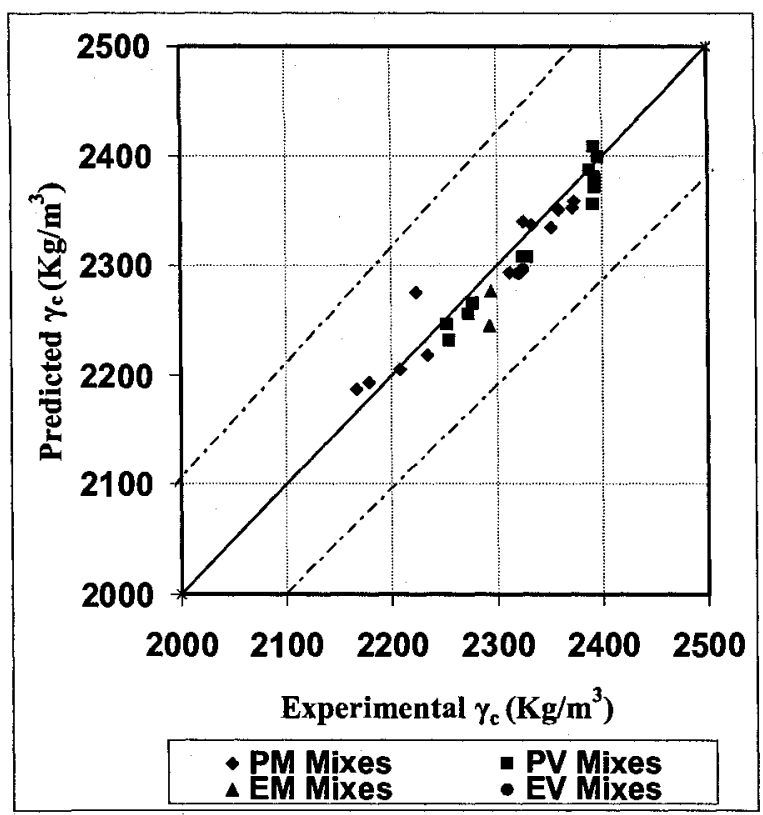

(a) Mixture-Based Model-1

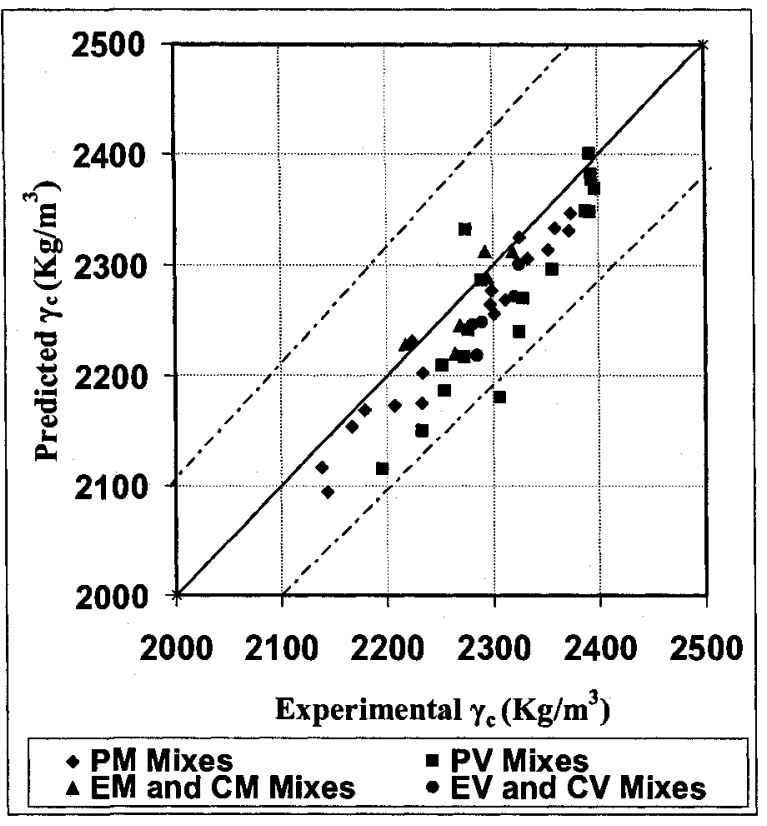

(b) Phase-Based Model-2

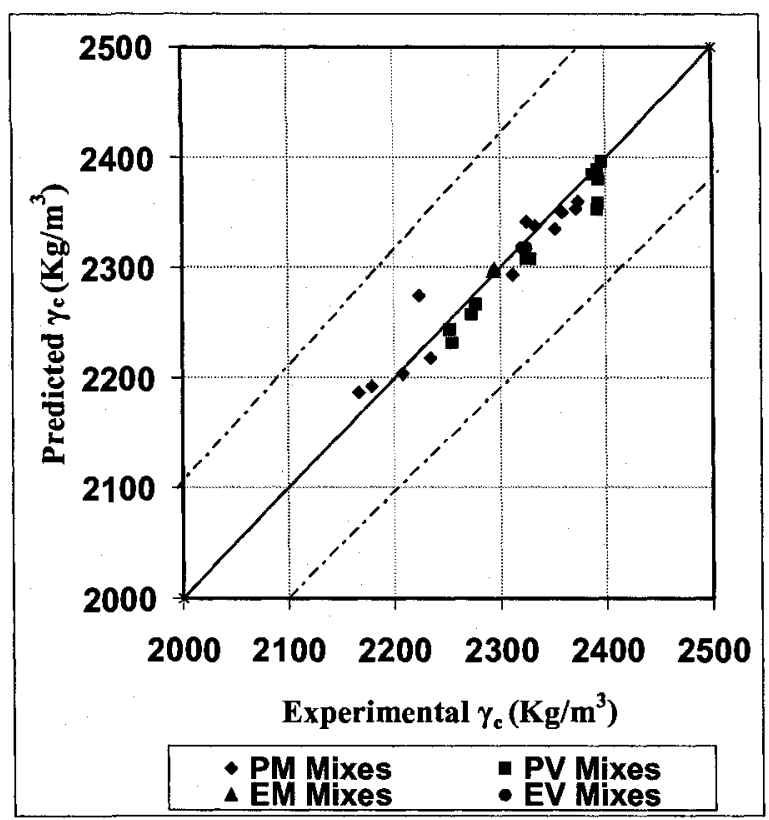

(a) Mixture-Based Model-2

Figure 5.2: Predicted versus experimental hardened density values of RAC mixes based on different models 


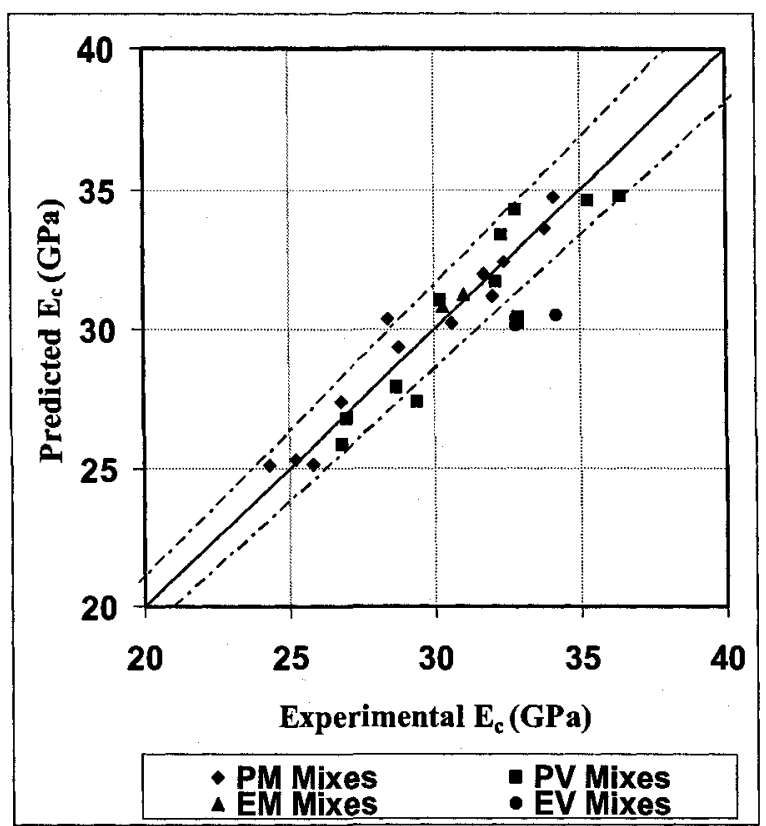

(a) Phase-based model (Eq.5.26)

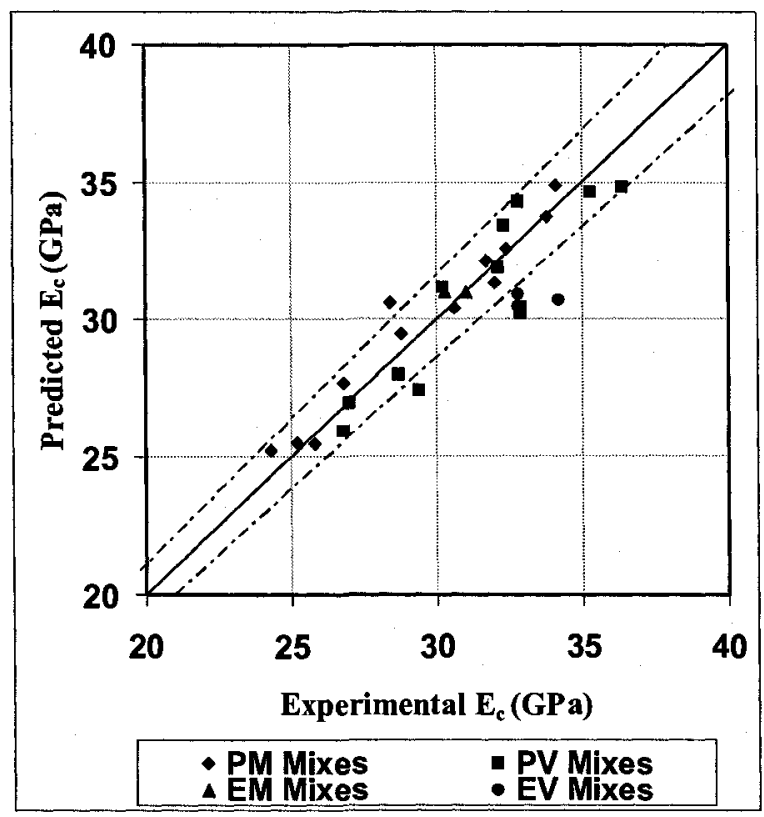

(b) Mixture-based model (Eq.5.36)

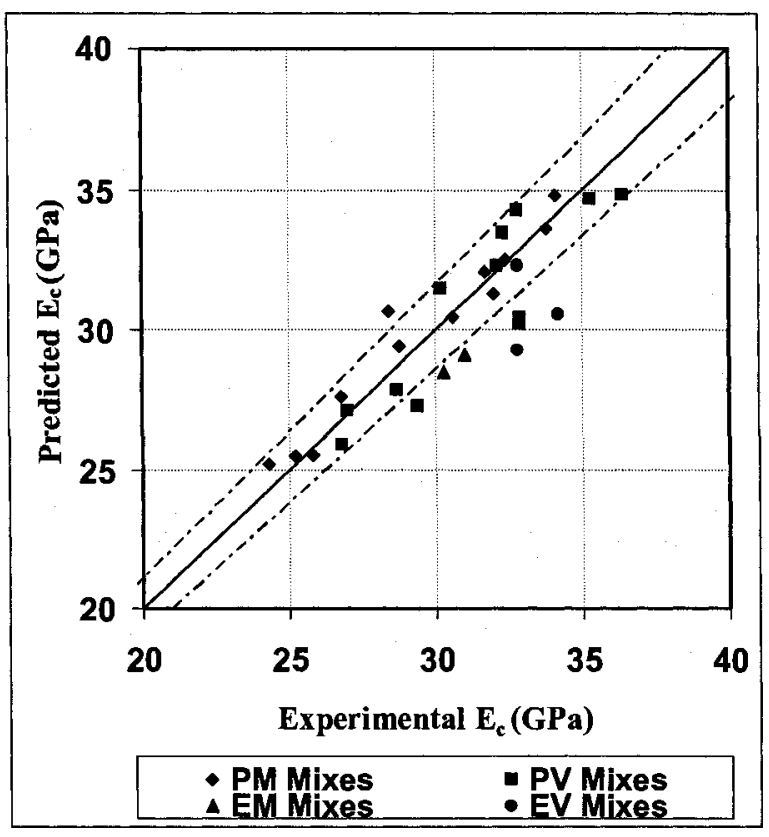

(b) Mixture-based model (Eq.5.31)

Figure 5.3: Predicted versus experimental elastic modulus values of RAC mixes using parallel models 


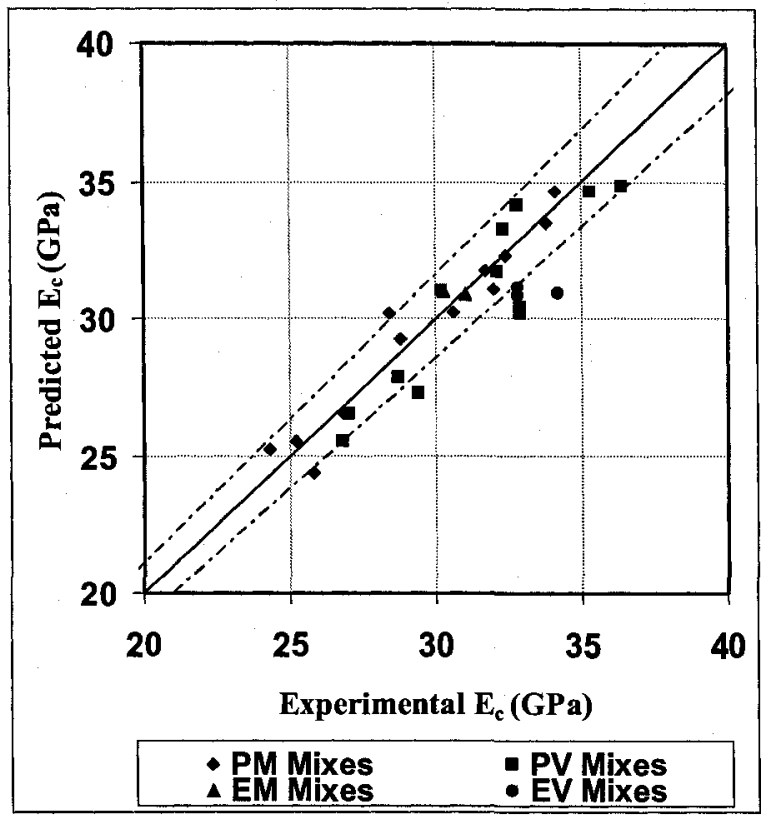

(a) Phase-based model (Eq.5.27)

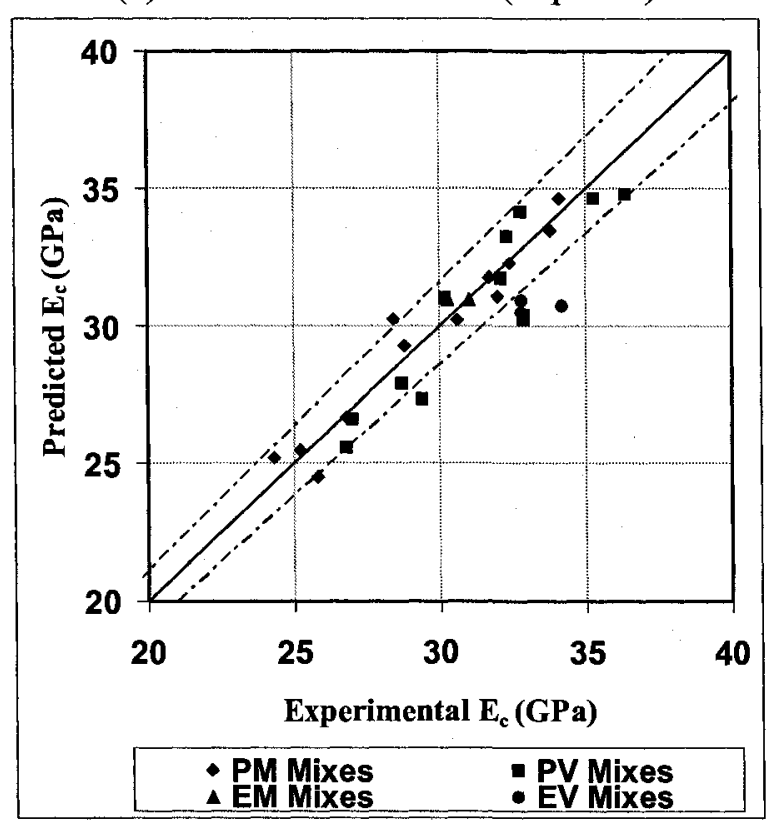

(b) Mixture-based model (Eq.5.37)

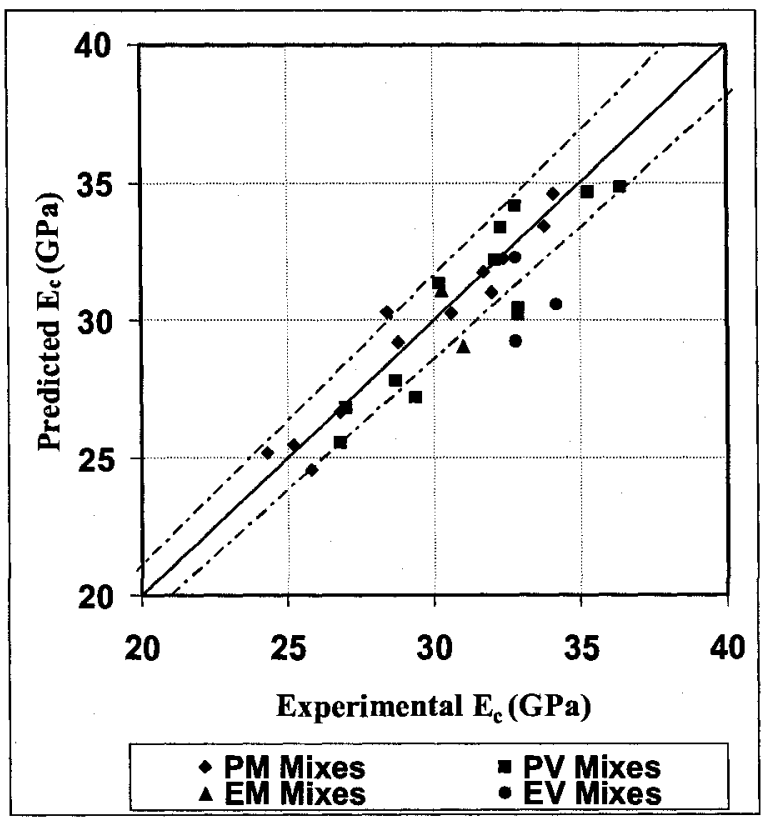

(b) Mixture-based model (Eq.5.32)

Figure 5.4: Predicted versus experimental elastic modulus values of RAC mixes using series models 


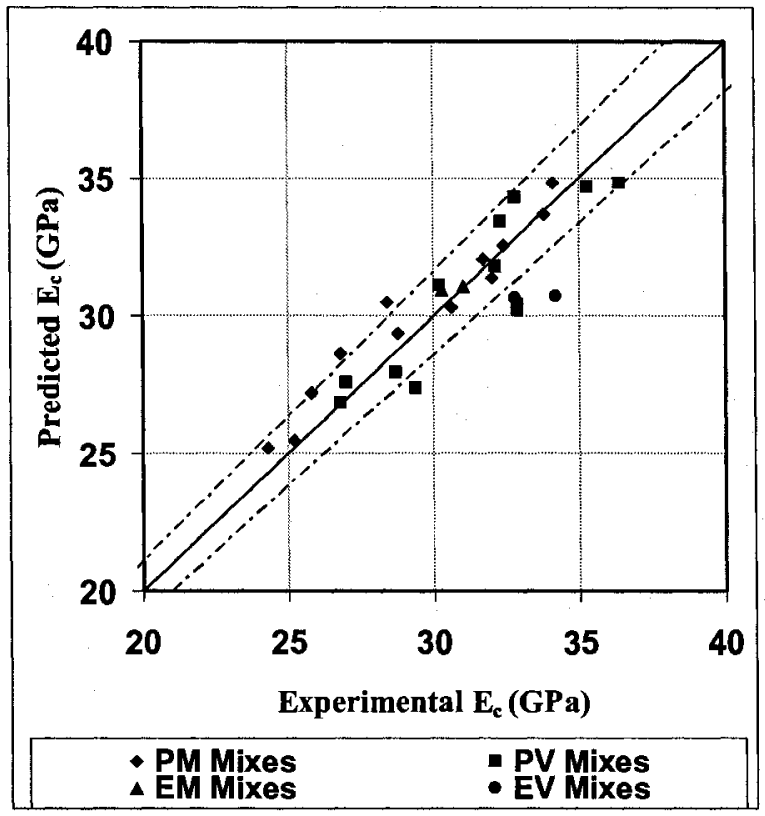

(a) Phase-based model (Eq.5.28)

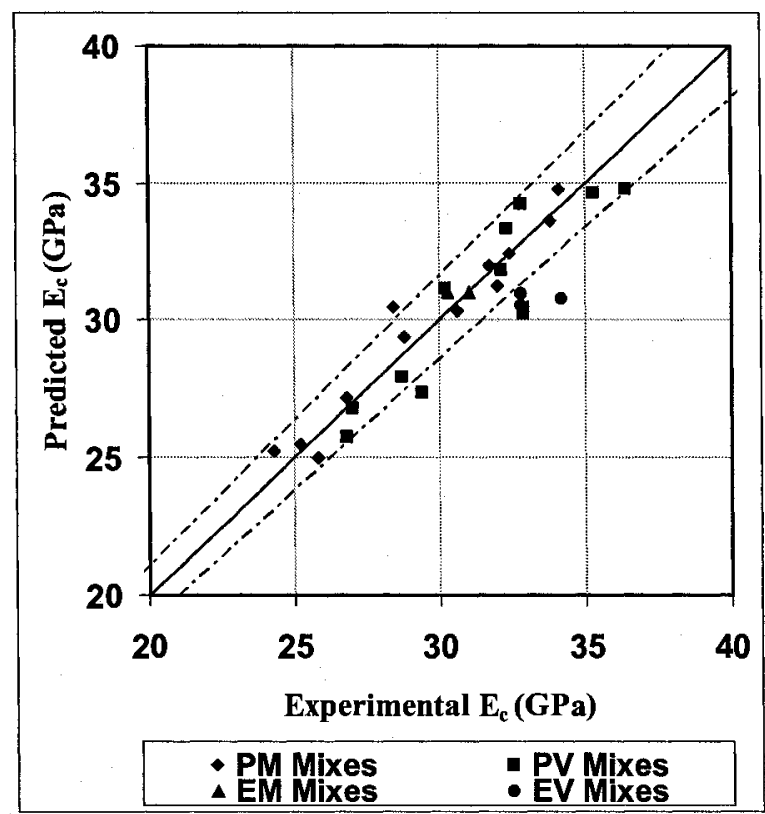

(b) Mixture-based model (Eq.5.38)

Figure 5.5: Predicted versus experimental elastic modulus values of RAC mixes using Hirsch models 


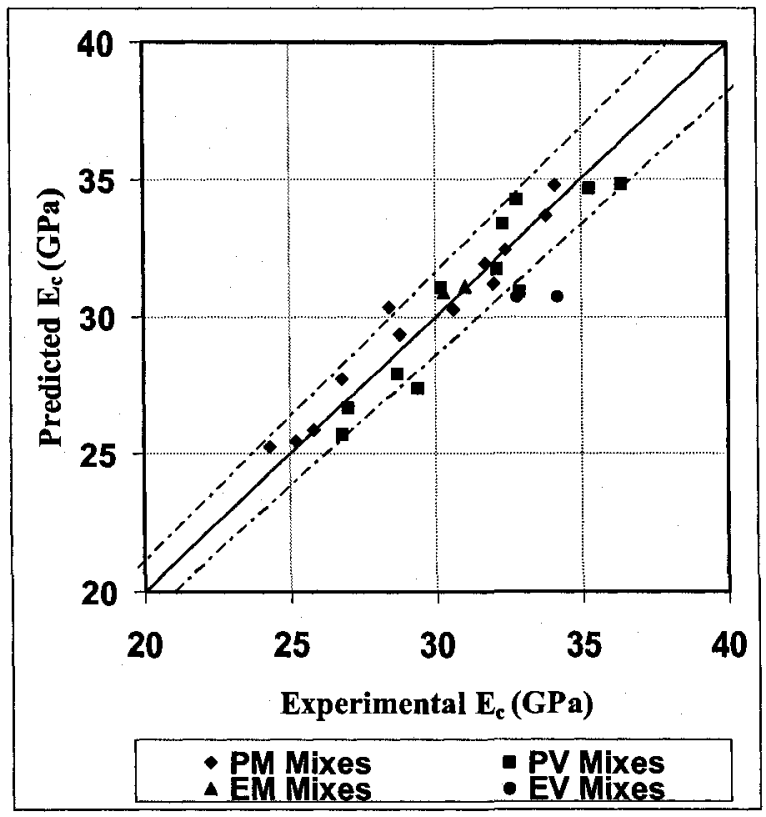

(a) Phase-based model (Eq.5.29)

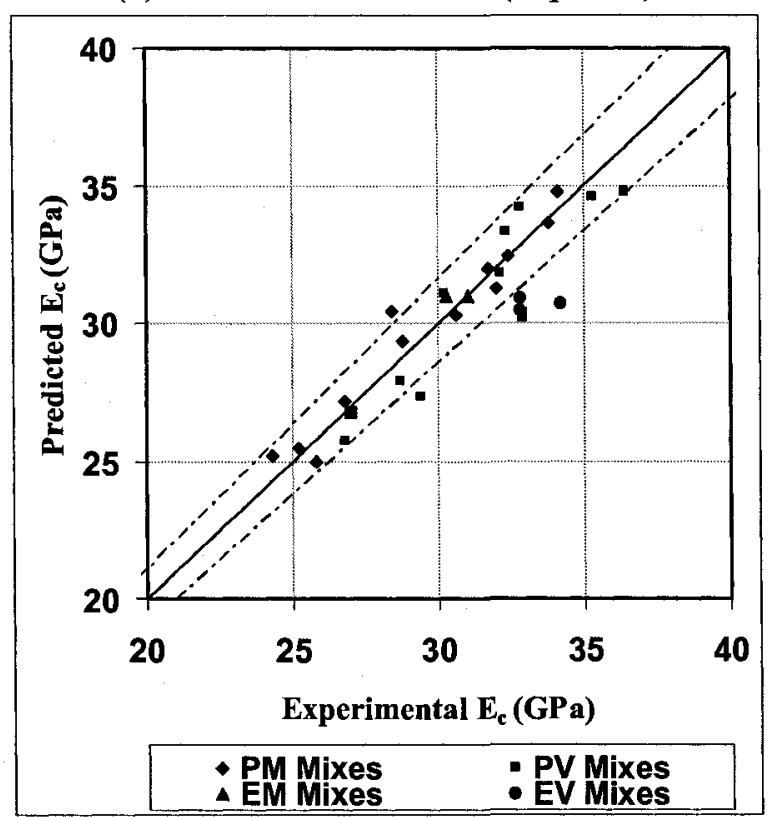

(b) Concrete-based model (Eq.5.39)

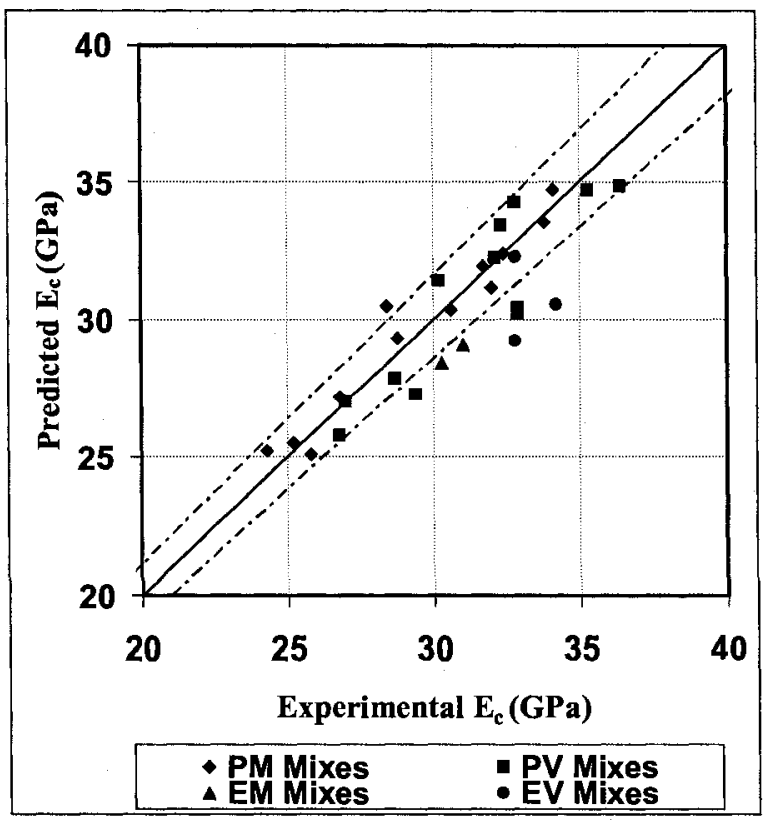

(b) Mixture-based model (Eq.5.34)

Figure 5.6: Mixture versus experimental elastic modulus values of RAC mixes using Counto models 


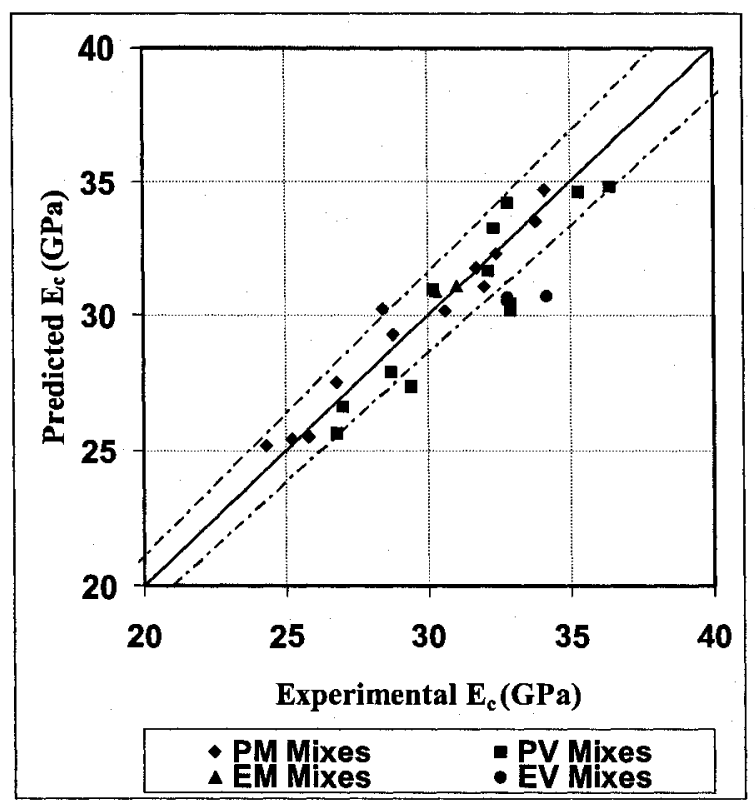

(a) Phase-based model (Eq.5.30)

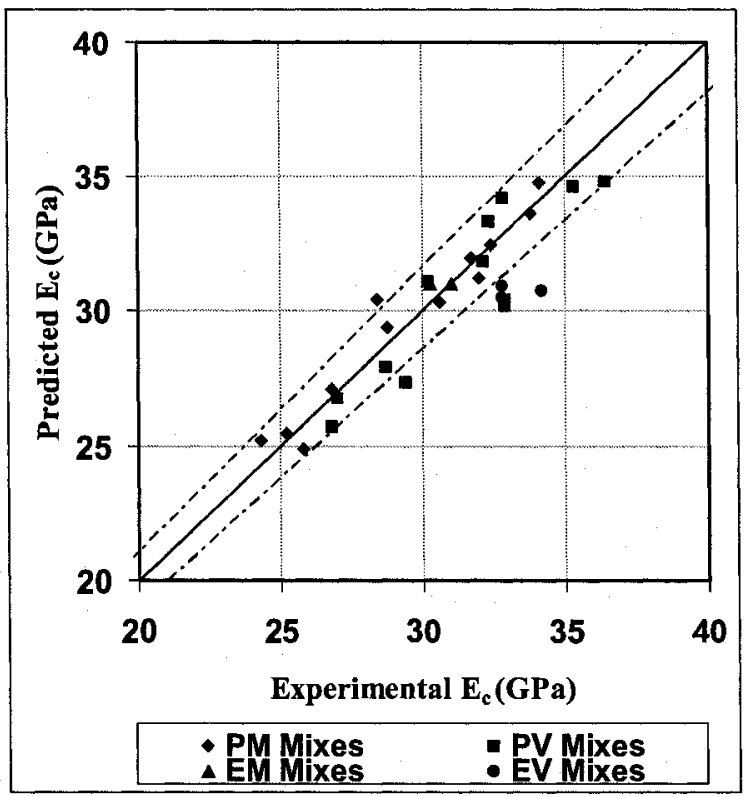

(b) Mixture-based model (Eq.5.40)

Figure 5.7: Predicted versus experimental elastic modulus values of RAC mixes using Mindess models 


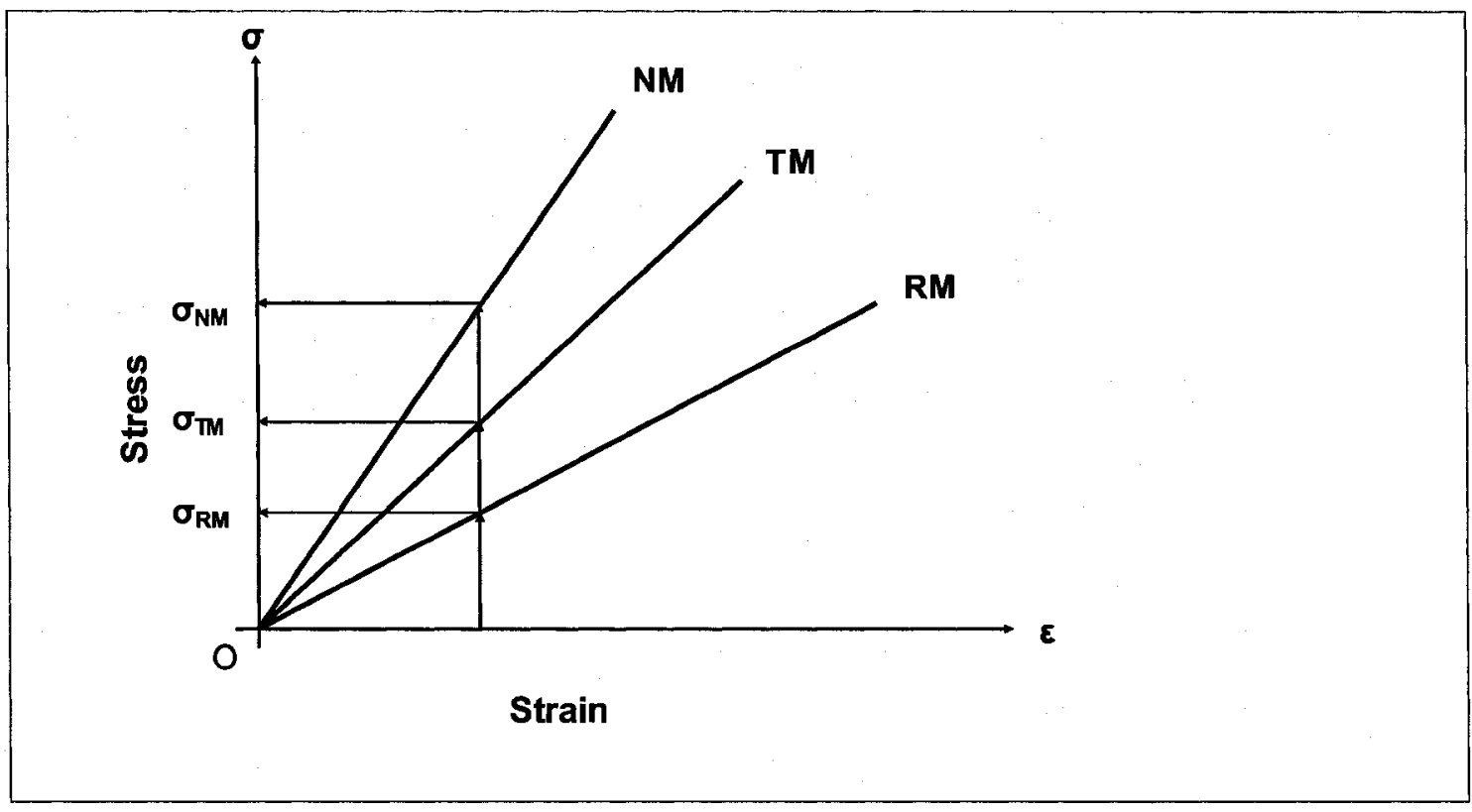

Figure 5.8: Stress-strain variation of TM, RM, and NM in RAC 


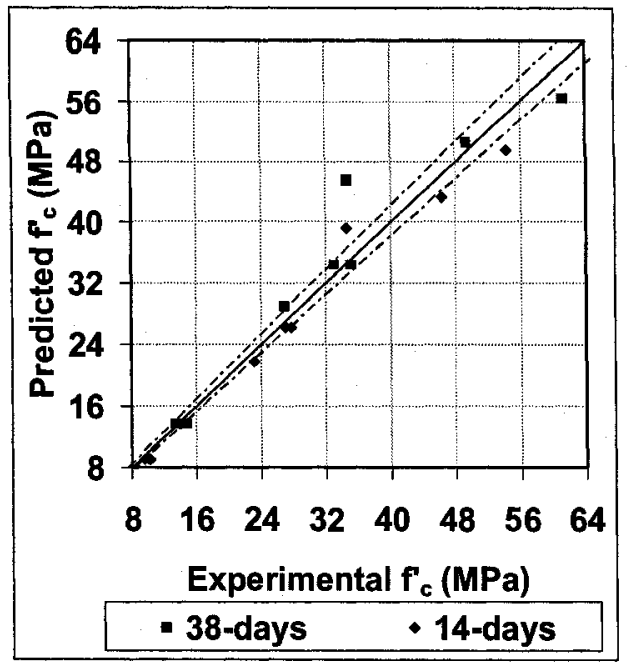

a) Parallel model

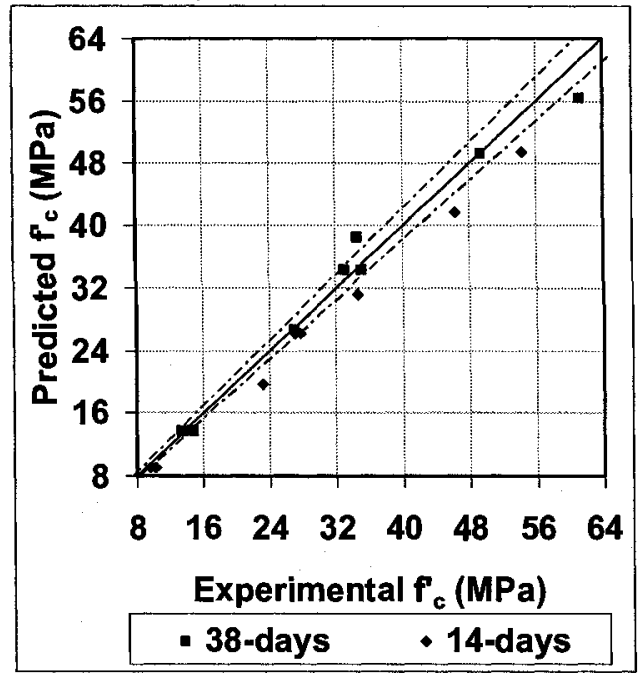

c) Hirsch's model

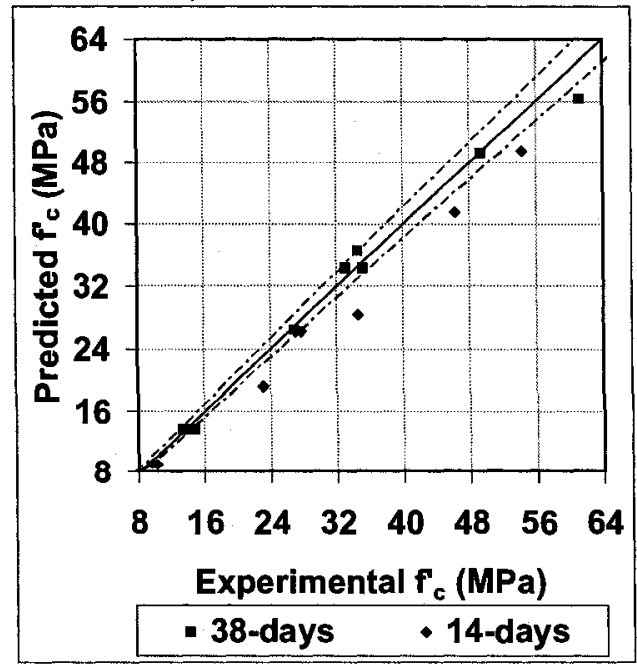

e) Mindess model

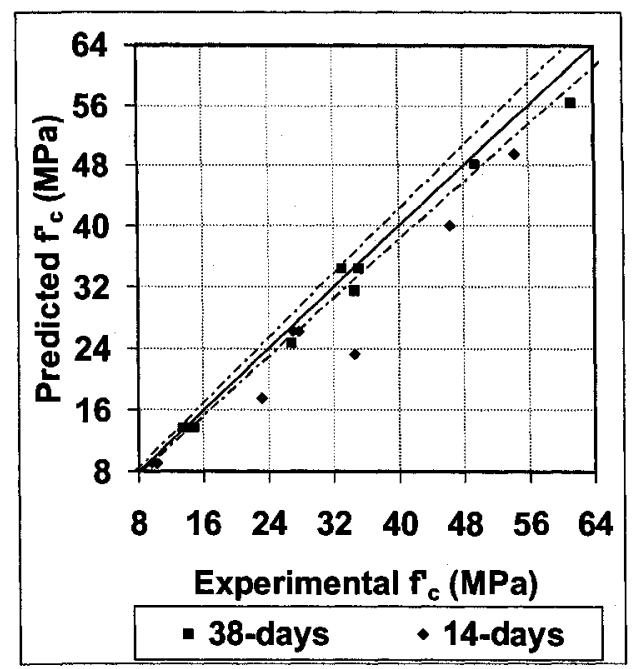

b) Series model

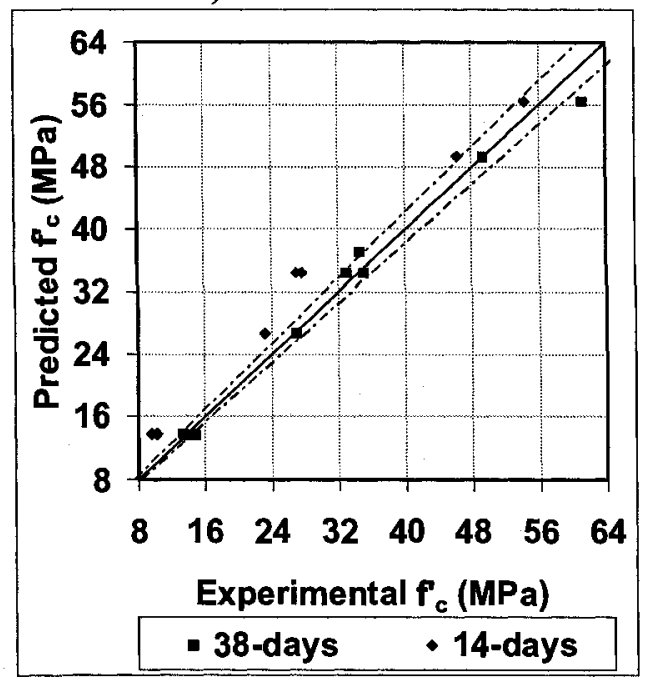

d) Counto's model

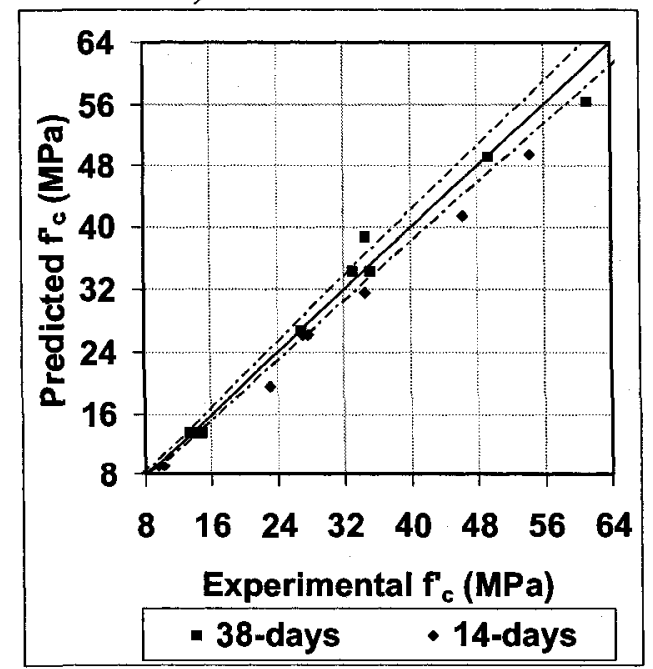

f) LWA-based model

Figure 5.9: Predicted versus experimental compressive strength values of RAC mixes based on different models 


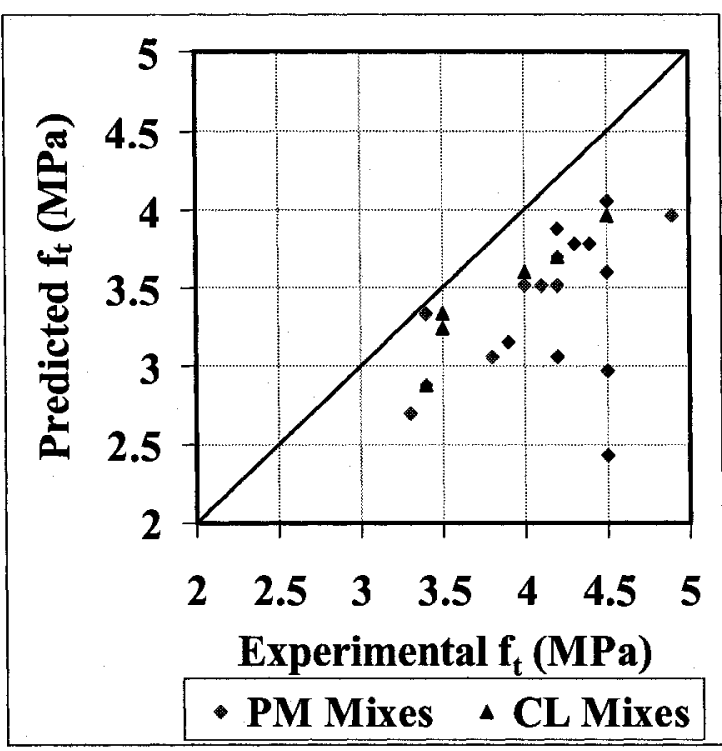

(a) PM mixes

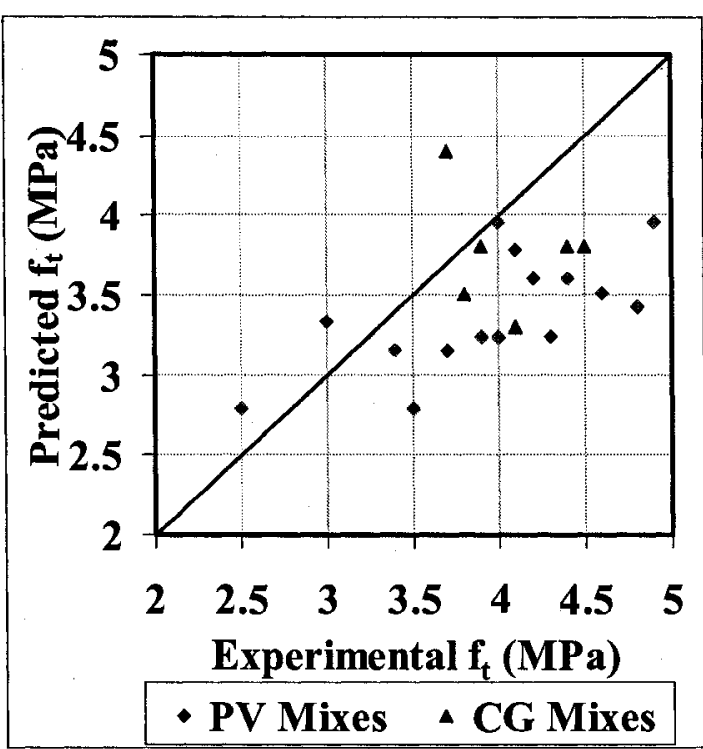

(b) PV mixes

Figure 5.10: Experimental versus predicted splitting tensile strength values based on ACI-318

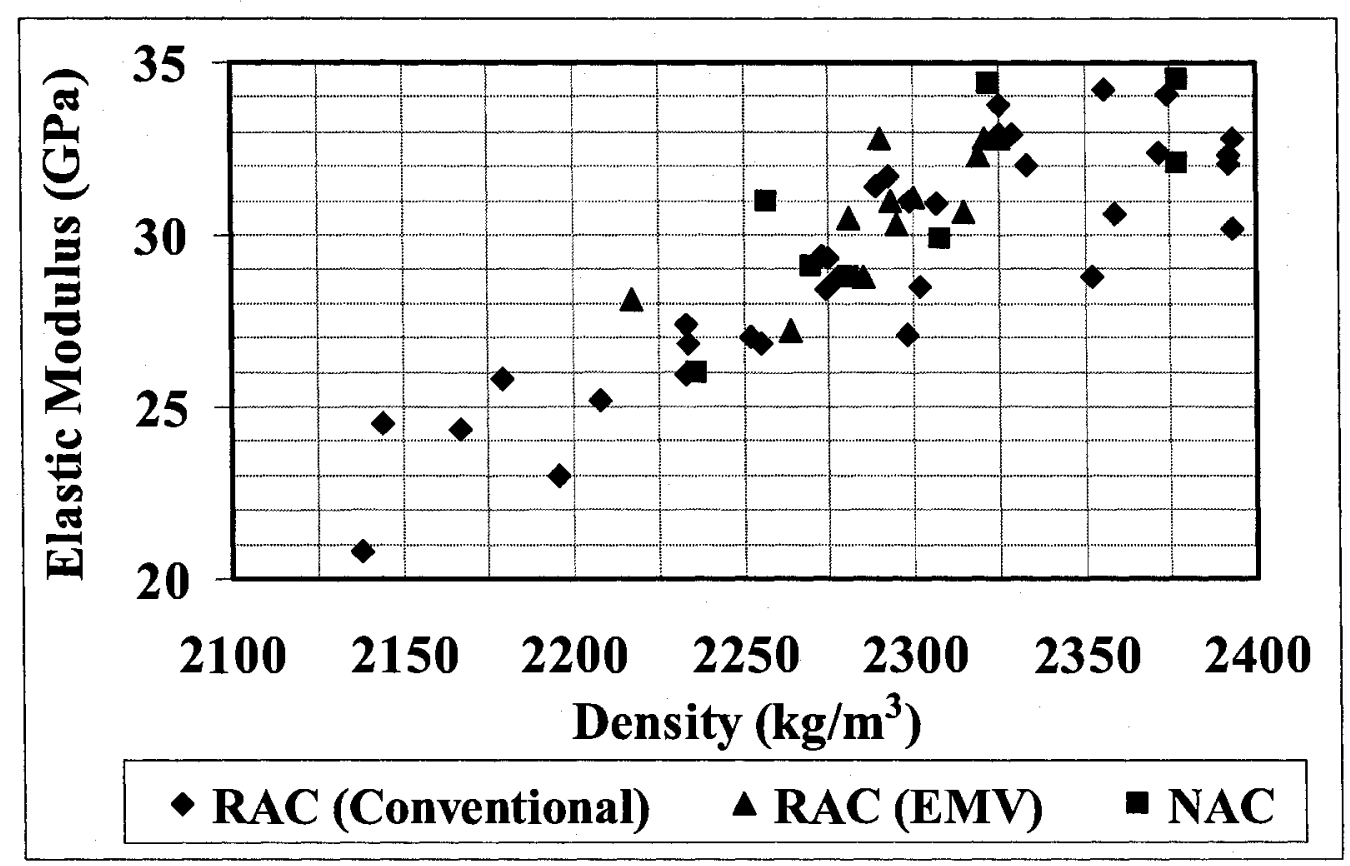

Figure 5.11: Elastic modulus versus hardened density for all RAC and NAC mixes 


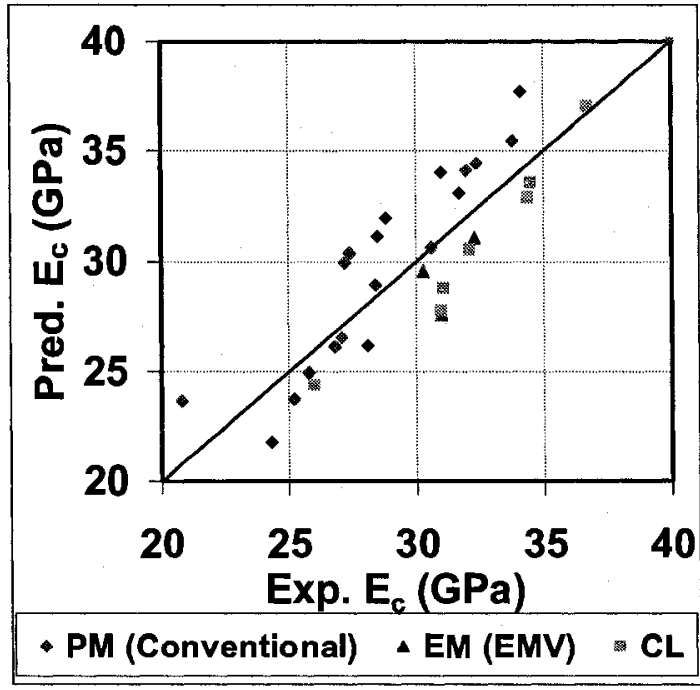

(a) ACI-318

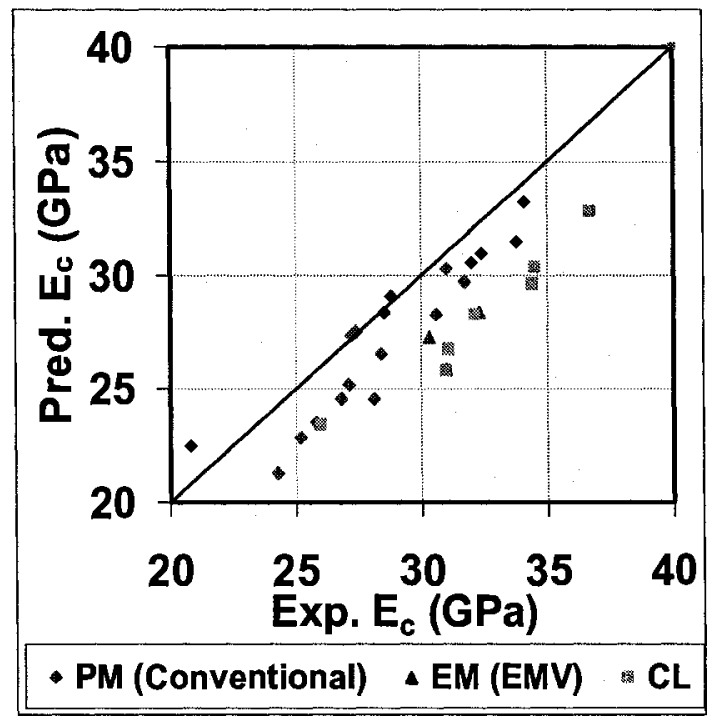

(c) ACI-363

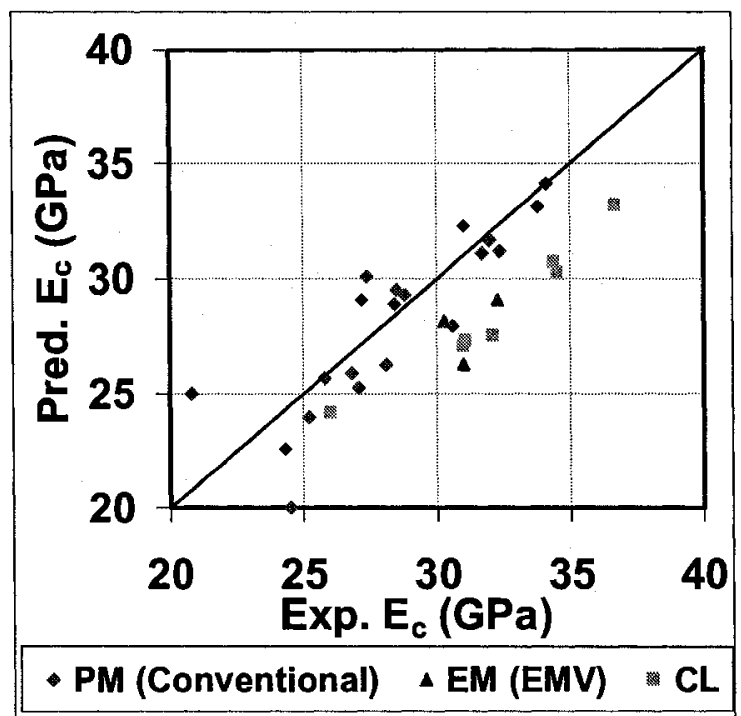

(b) CSA A23.3-04 (Clause 8.6.2.3)

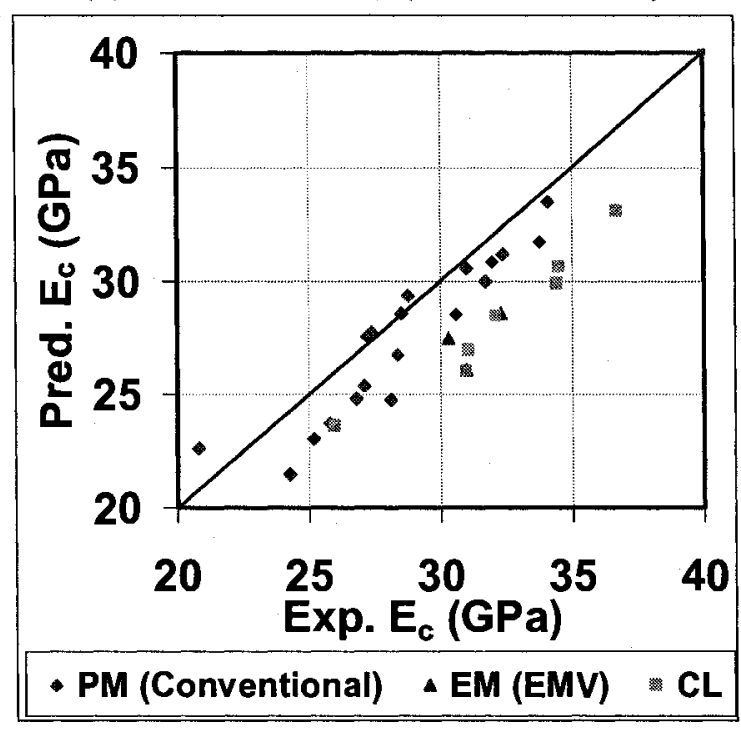

(d) CSA A23.3-04 (Clause 8.6.2.2)

Figure 5.12: Experimental versus predicted elastic modulus of mixes made with RCAMO\&NL based on CSA and ACI equations 


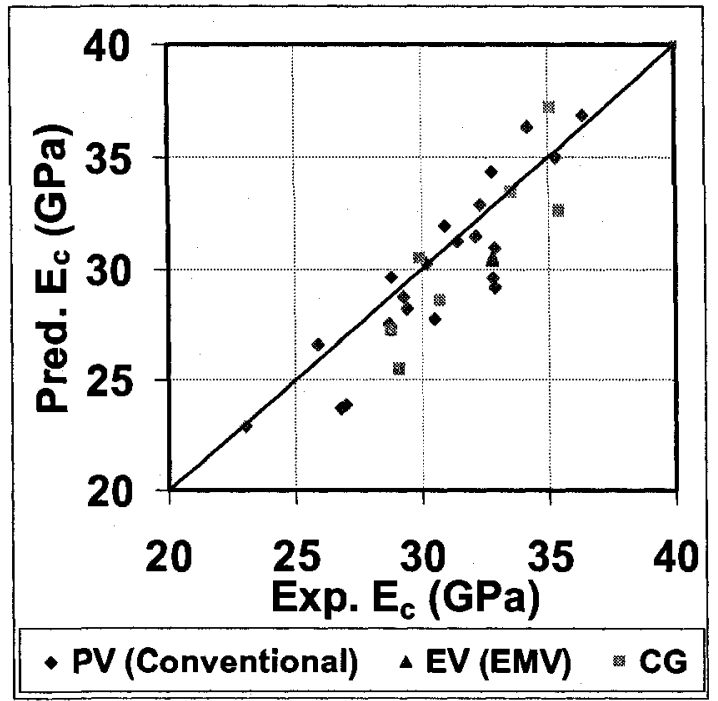

(a) ACI-318

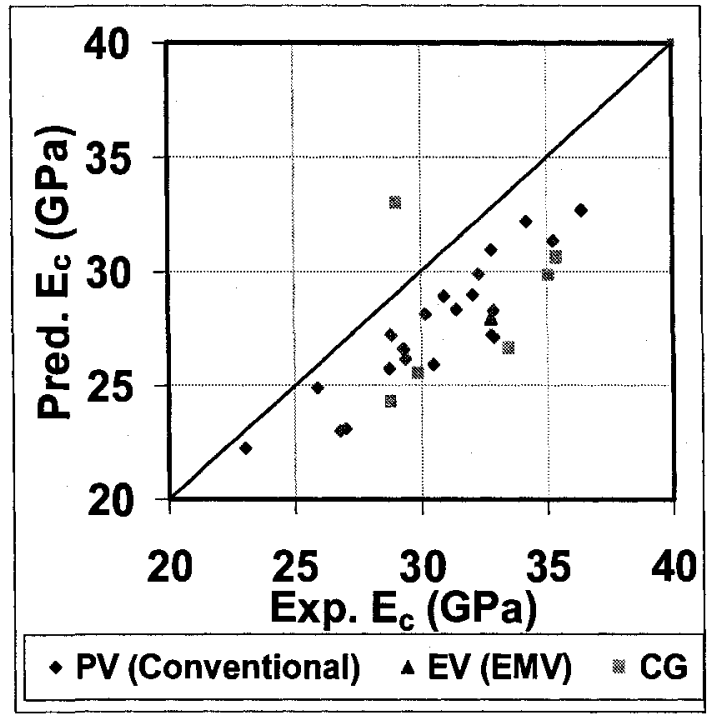

(c) ACI-363

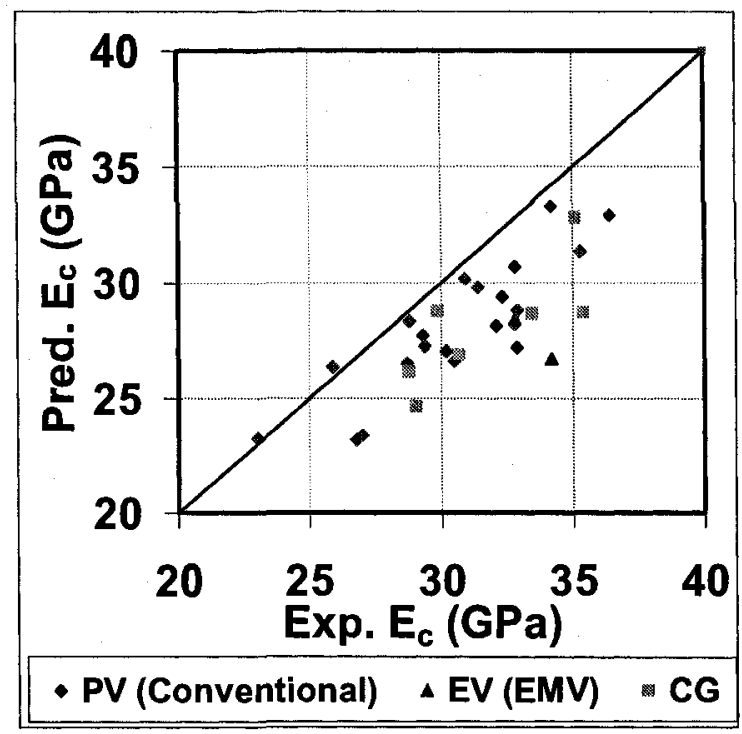

(b) CSA A23.3-04 (Clause 8.6.2.3)

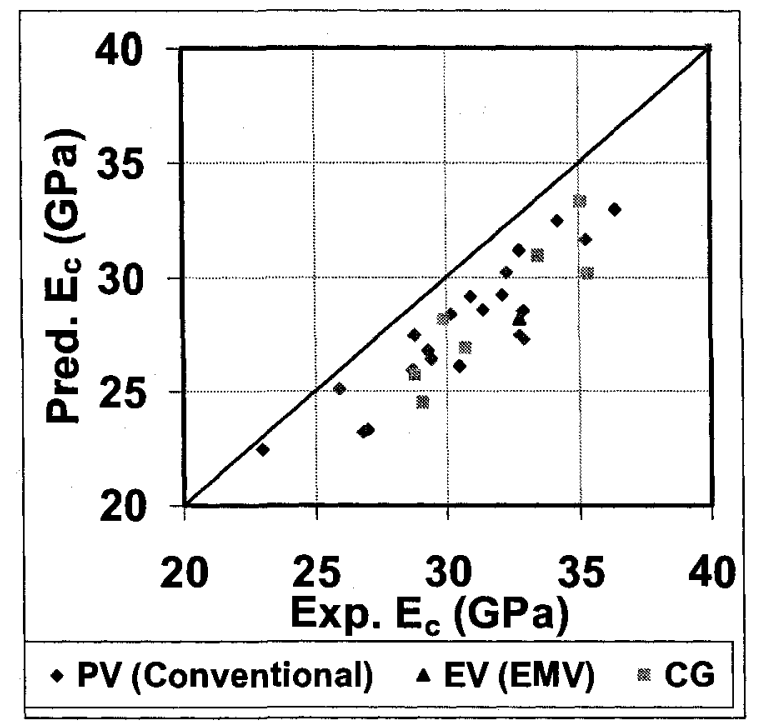

(d) CSA A23.3-04 (Clause 8.6.2.2)

Figure 5.13: Experimental versus predicted elastic modulus of mixes made with RCAVA\&NG based on CSA and $\mathrm{ACI}$ equations 


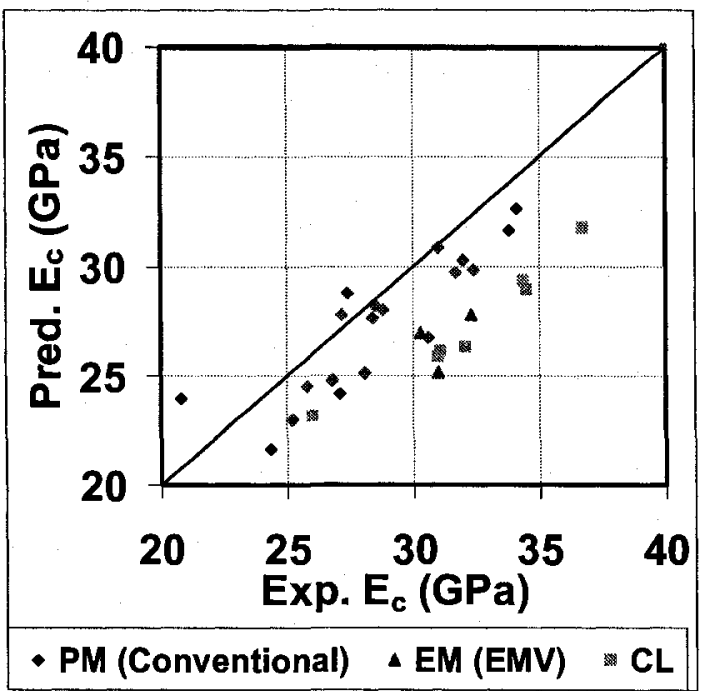

(a) Gardner (2000)

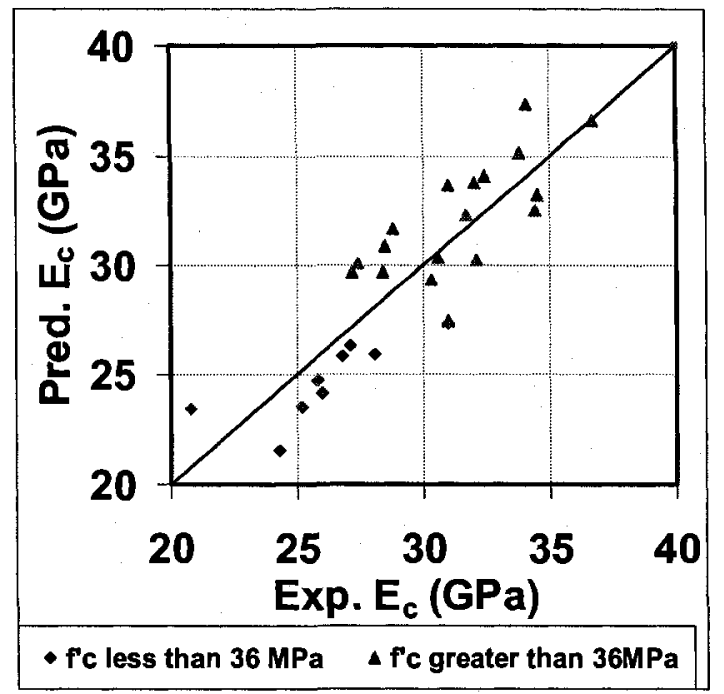

(c) AIJ (Japan)

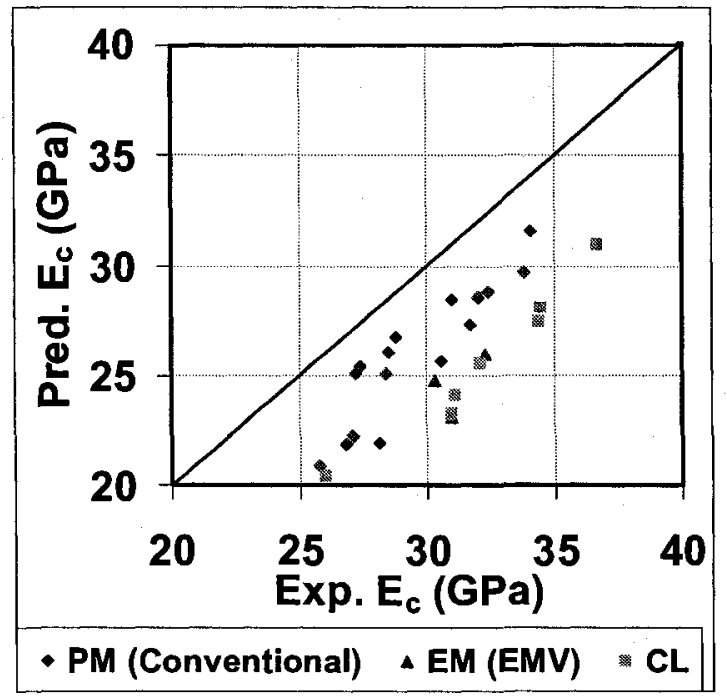

(b) Nassif etal. (2005)

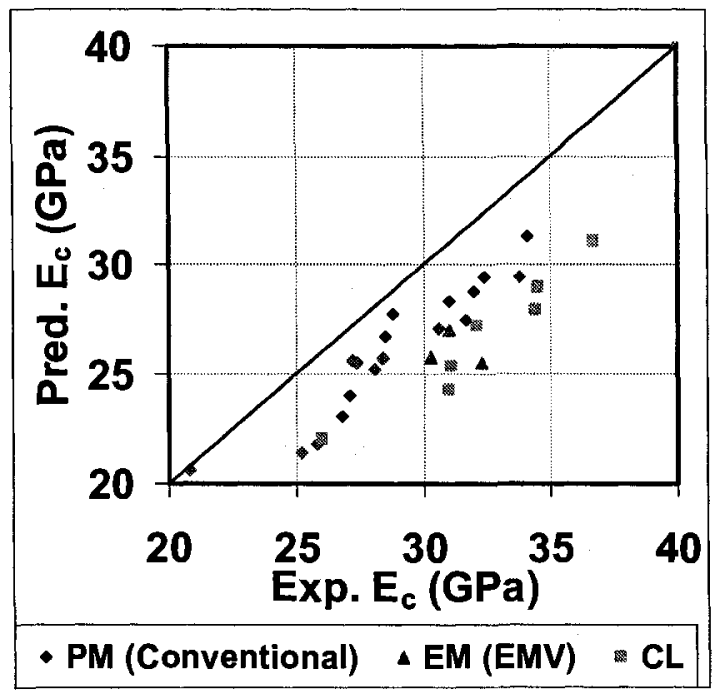

(d) Tomosawa et al. (1993)

Figure 5.14: Experimental versus predicted elastic modulus of mixes made with RCAMO\&NL based on different equations 


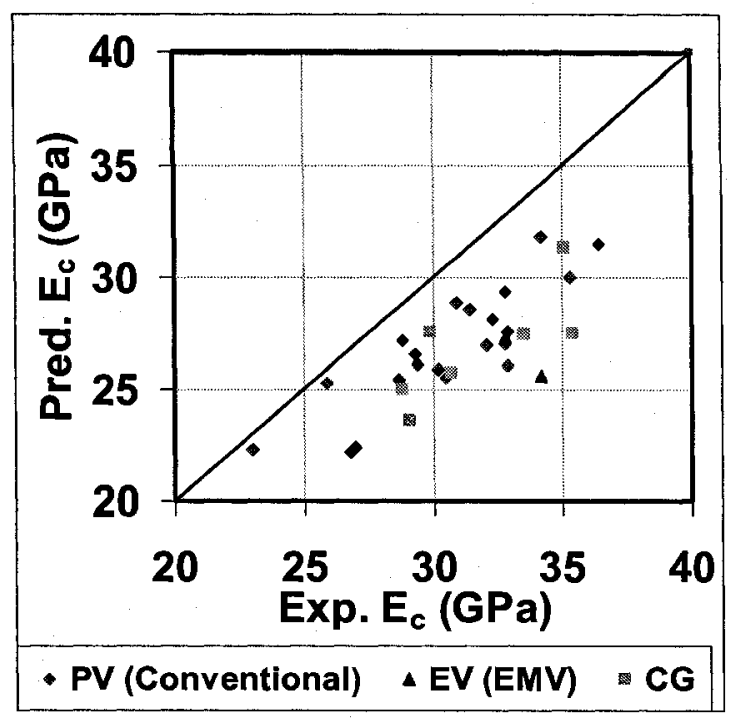

(a) Gardner (2000)

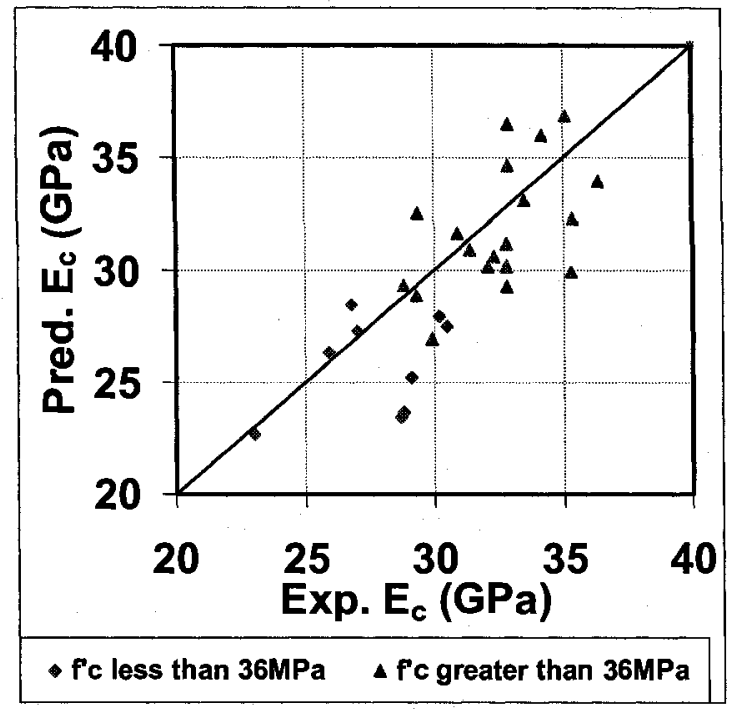

(c) AIJ (Japan)

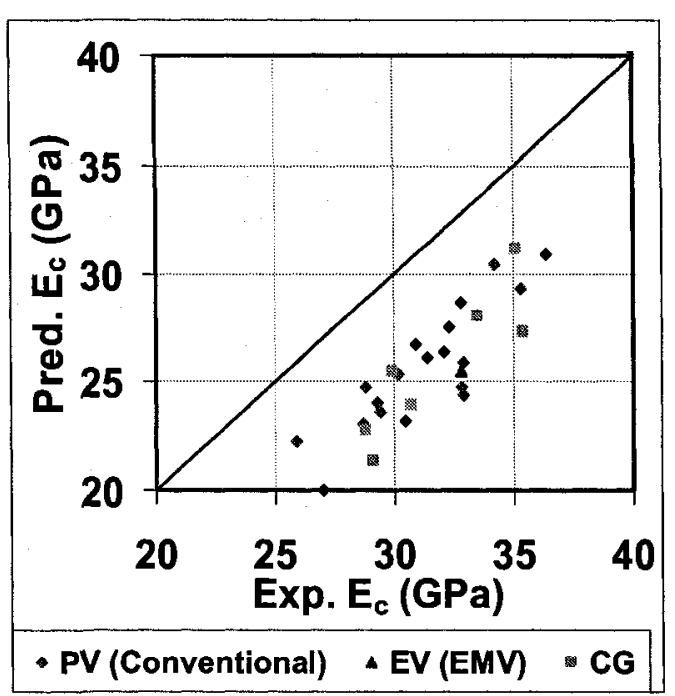

(b) Nassif et al. (2005)

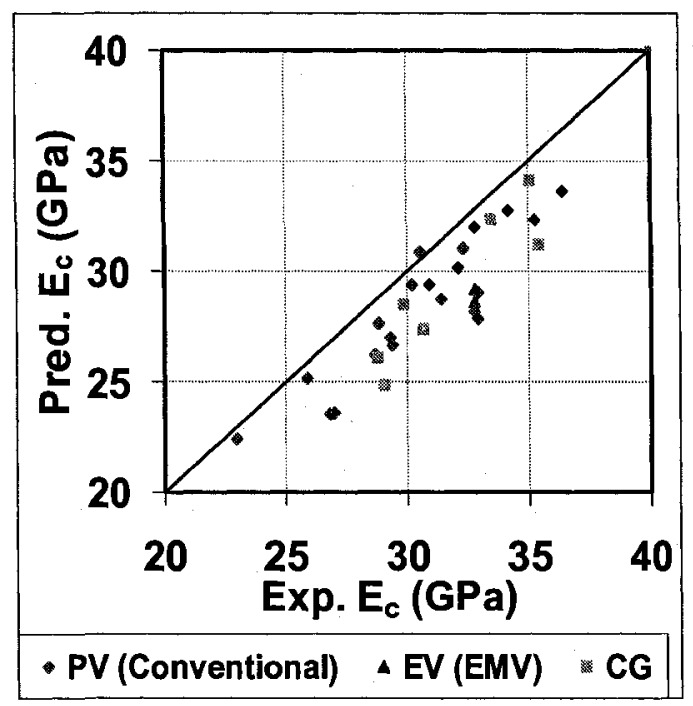

(d) Tomosawa et al. (1993)

Figure 5.15: Experimental versus predicted elastic modulus of mixes made with RCA-VA\&NG based on different equations 


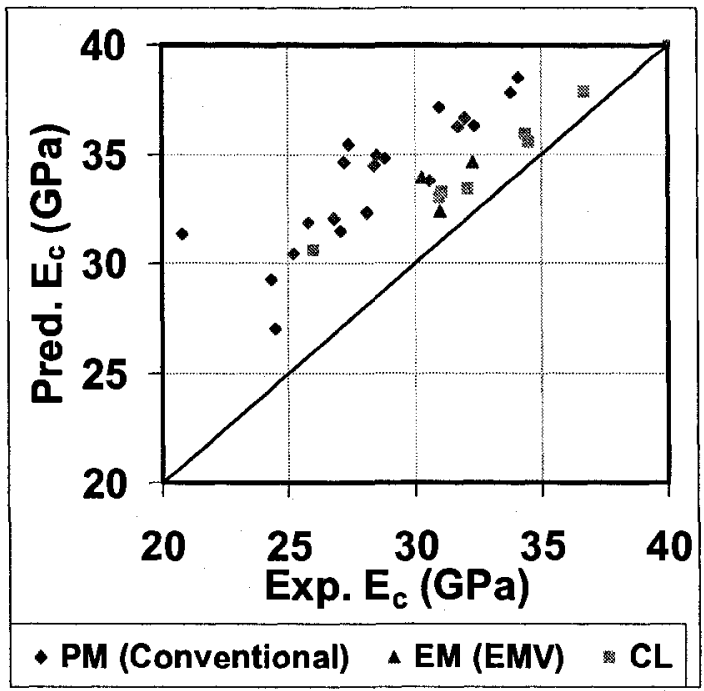

(a) RCA-MO\&NL

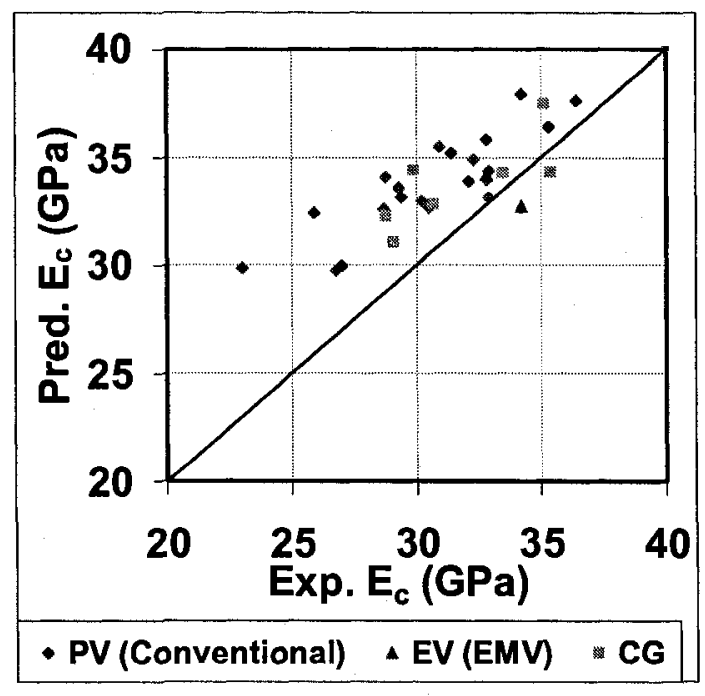

(b) RCA-VA\&NG

Figure 5.16: Experimental versus predicted elastic modulus based on Eurocode2 (2002)for different mixes 


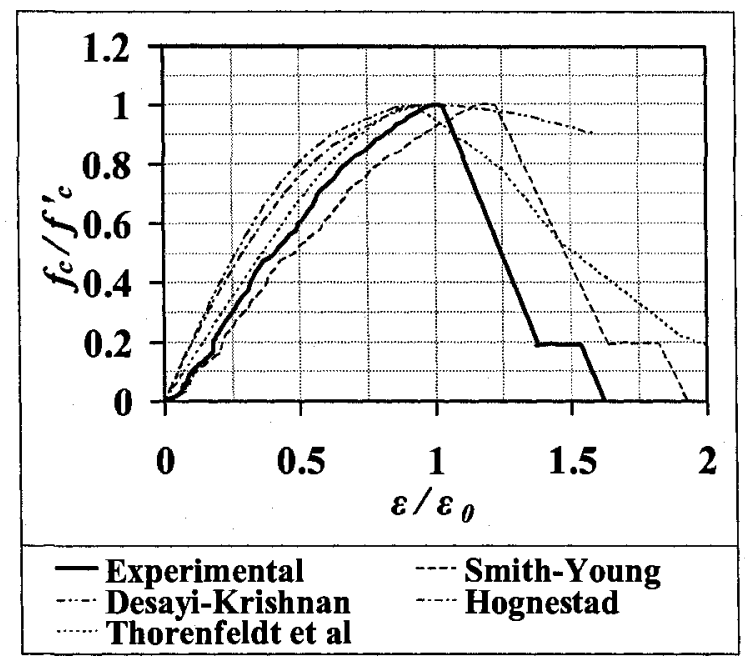

a) $\mathrm{CM}-1$

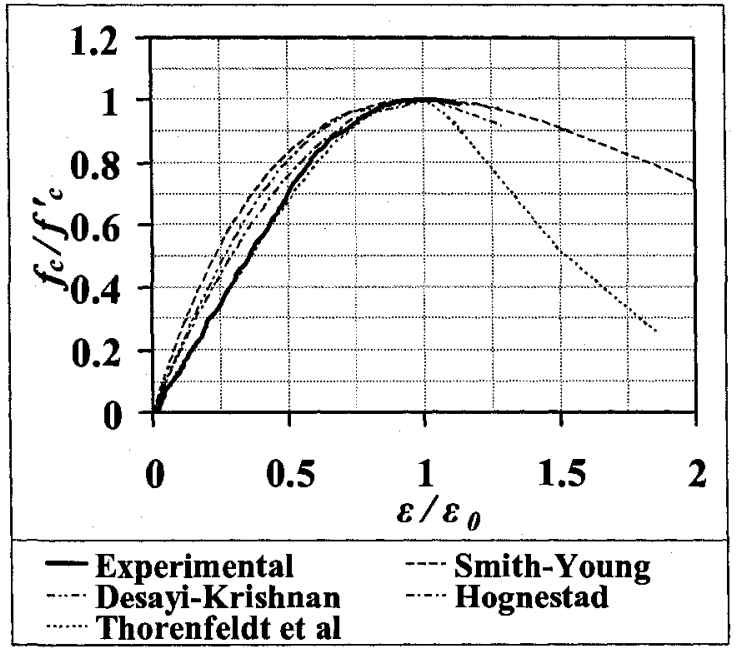

c) $\mathrm{CM}-2$

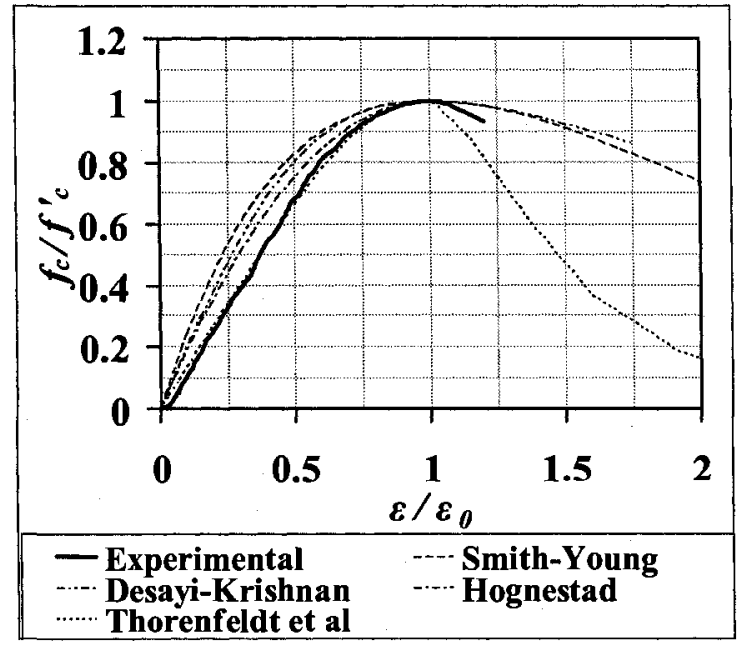

e) CM-3

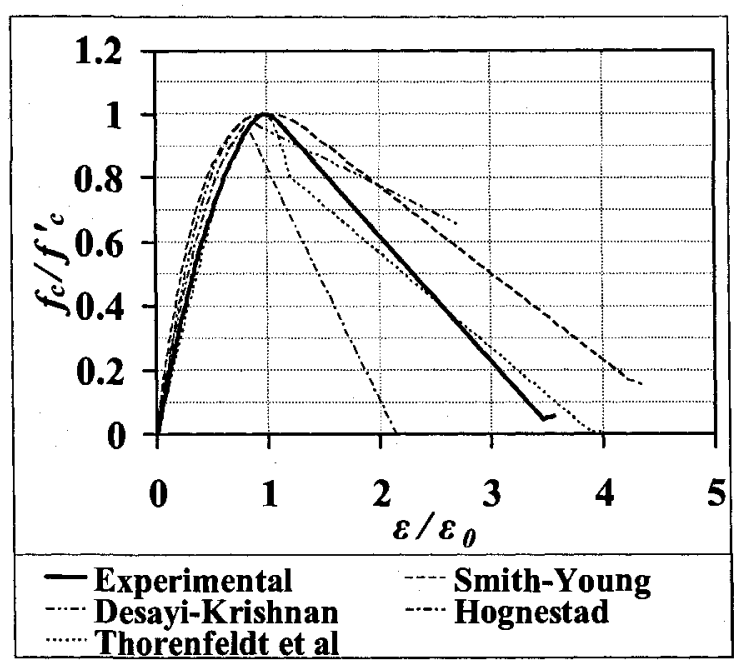

b) $\mathrm{CV}-1$

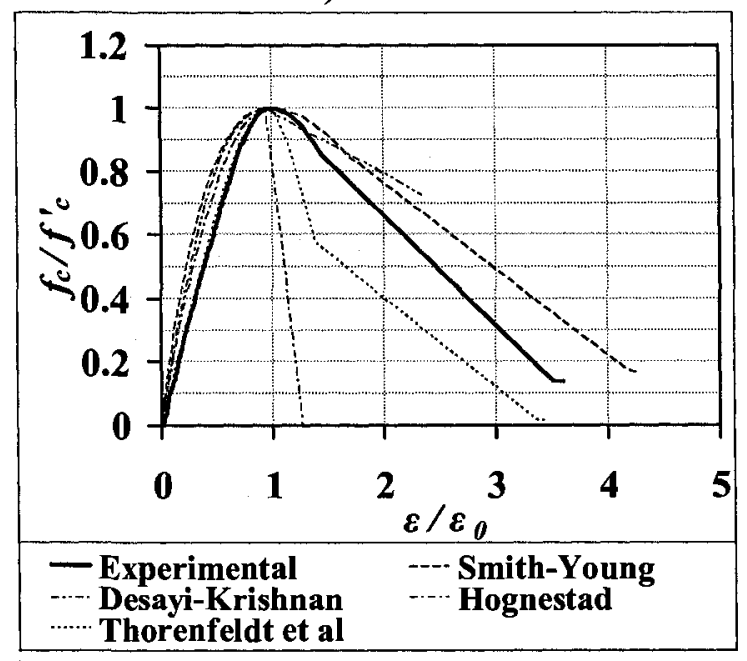

d) CV-2

Figure 5.17: Normalized compressive stress-strain relationship based on experimental data and existing models for $\mathrm{CM}$ and $\mathrm{CV}$ mixes 


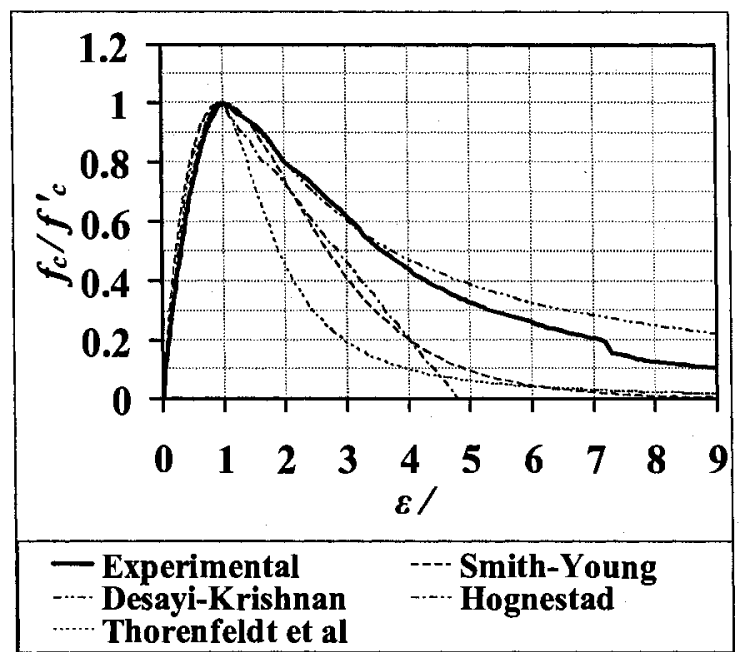

a) EM-1

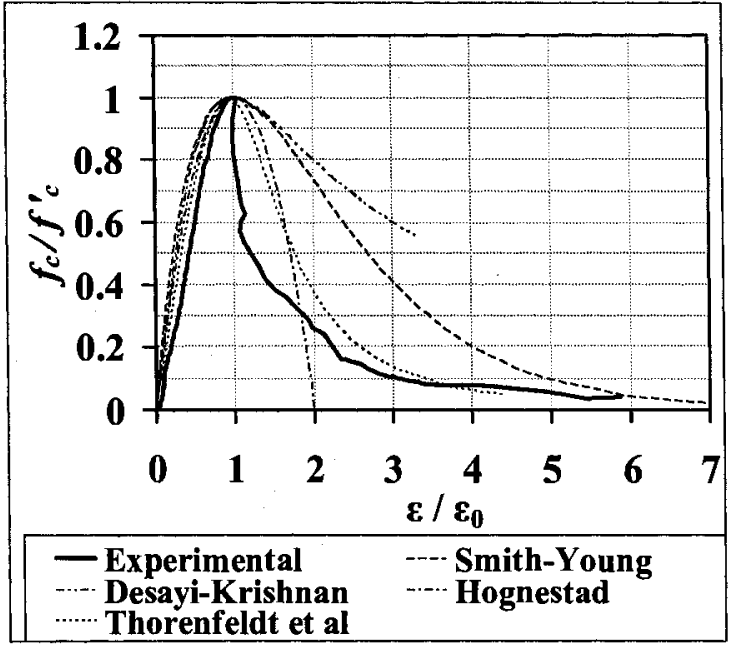

c) EM-2

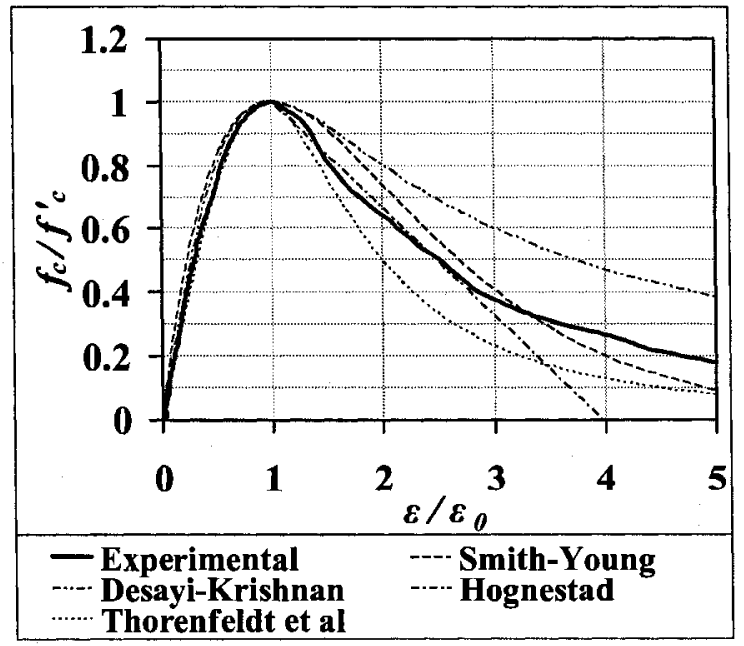

e) EM-3

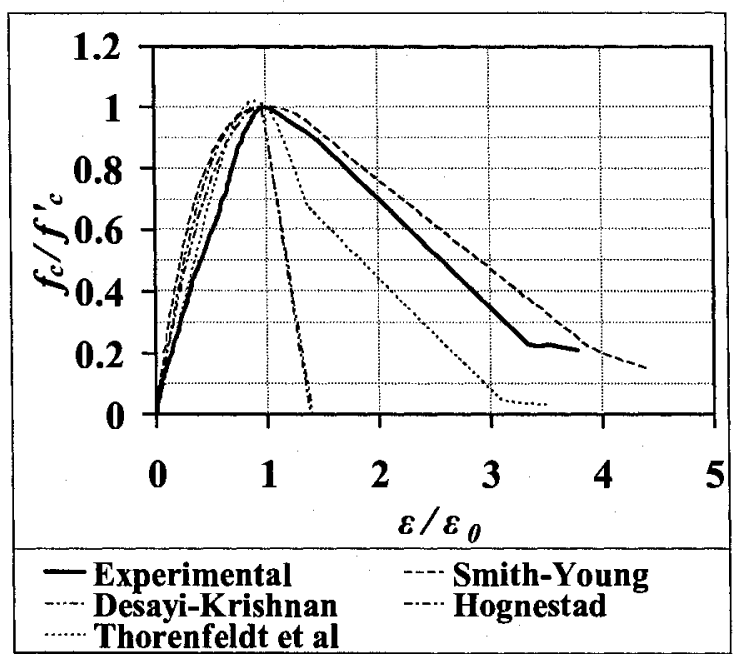

b) EV-1

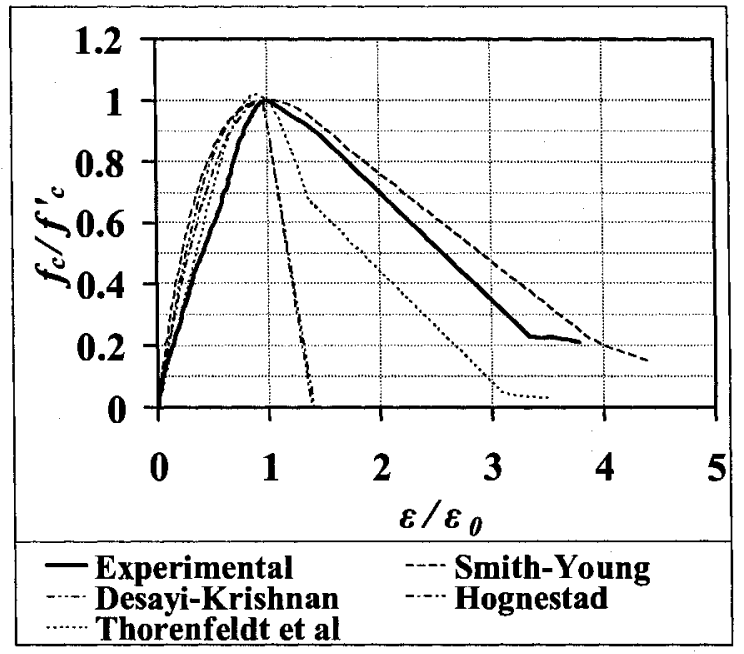

d) EV-2

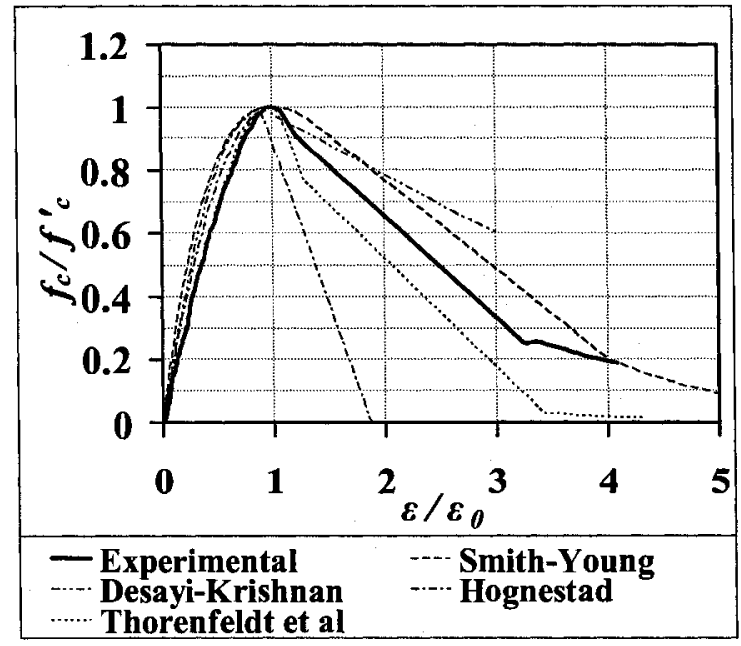

f) EV-3

Figure 5.18: Normalized compressive stress-strain relationship based on experimental data and existing models for EM and EV mixes 


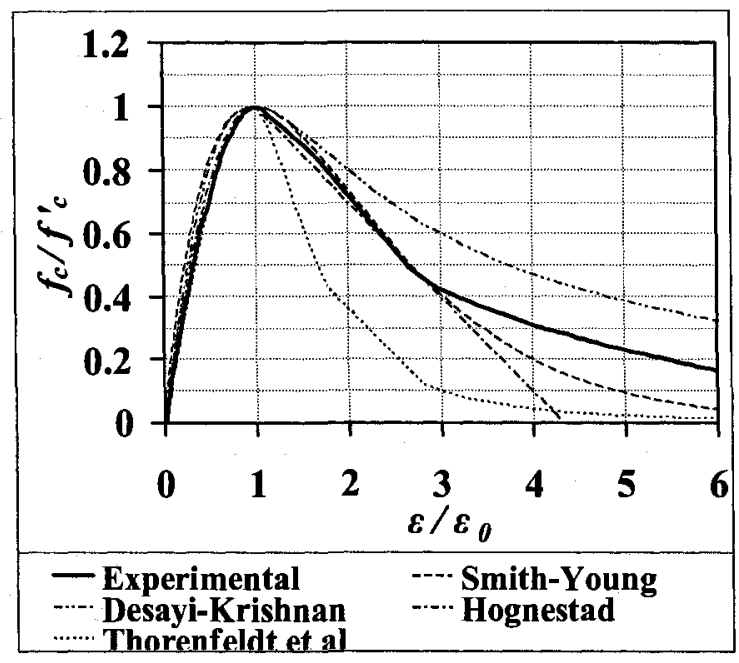

a) CL-1

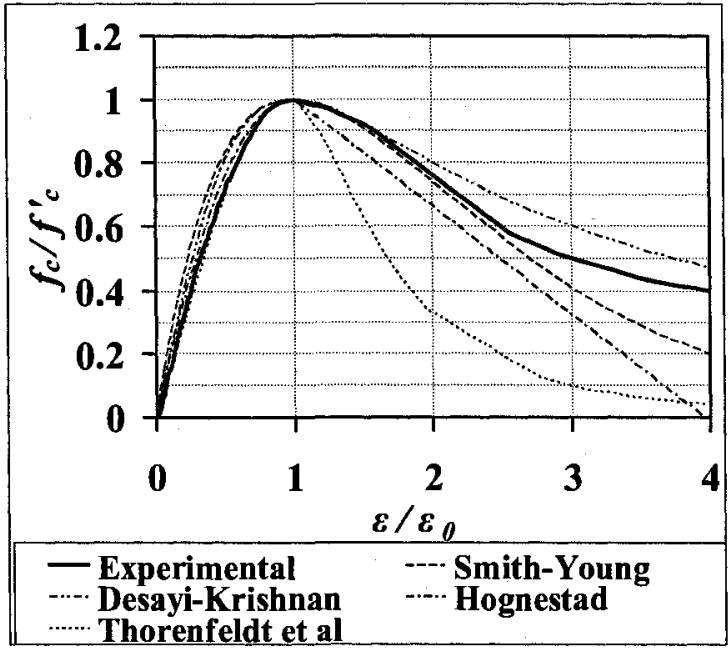

c) CL-2

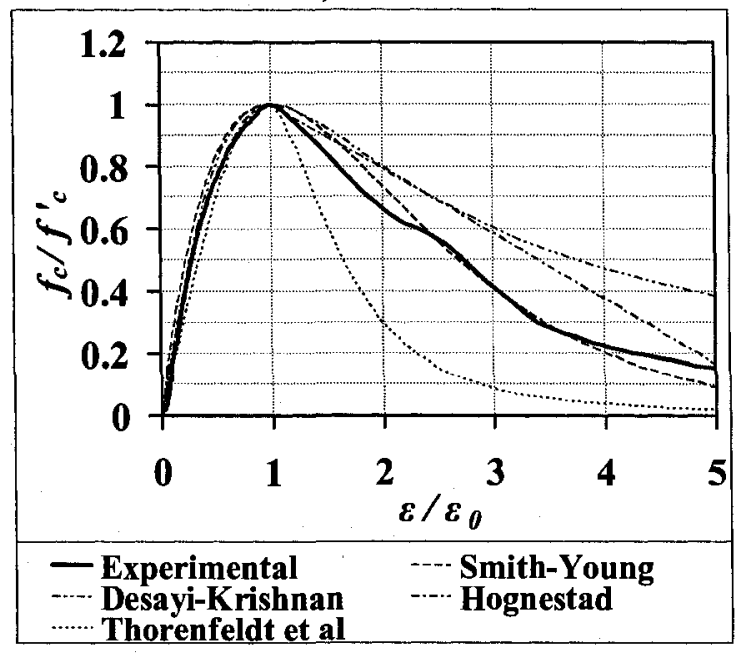

e) CL-3

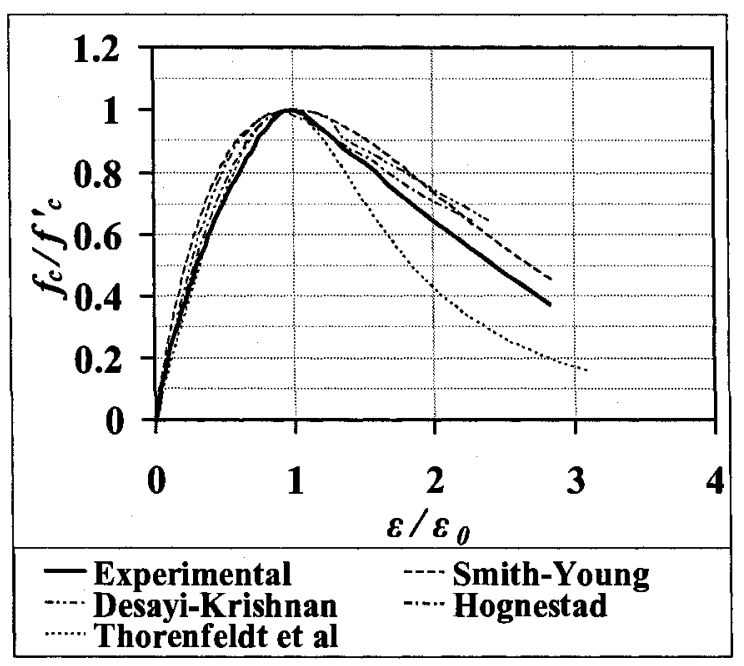

b) CG-1

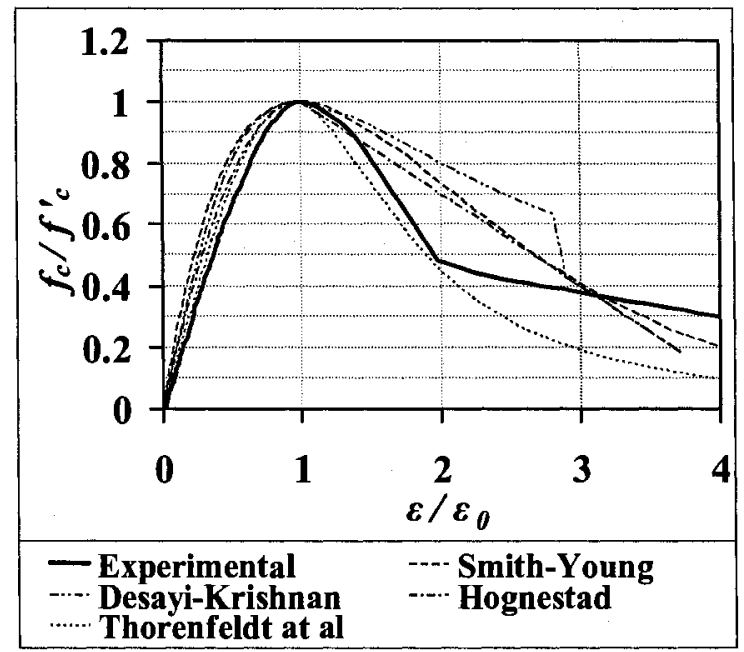

d) CG-2

Figure 5.19: Normalized compressive stress-strain relationship based on experimental data and existing models for CL and CG mixes 


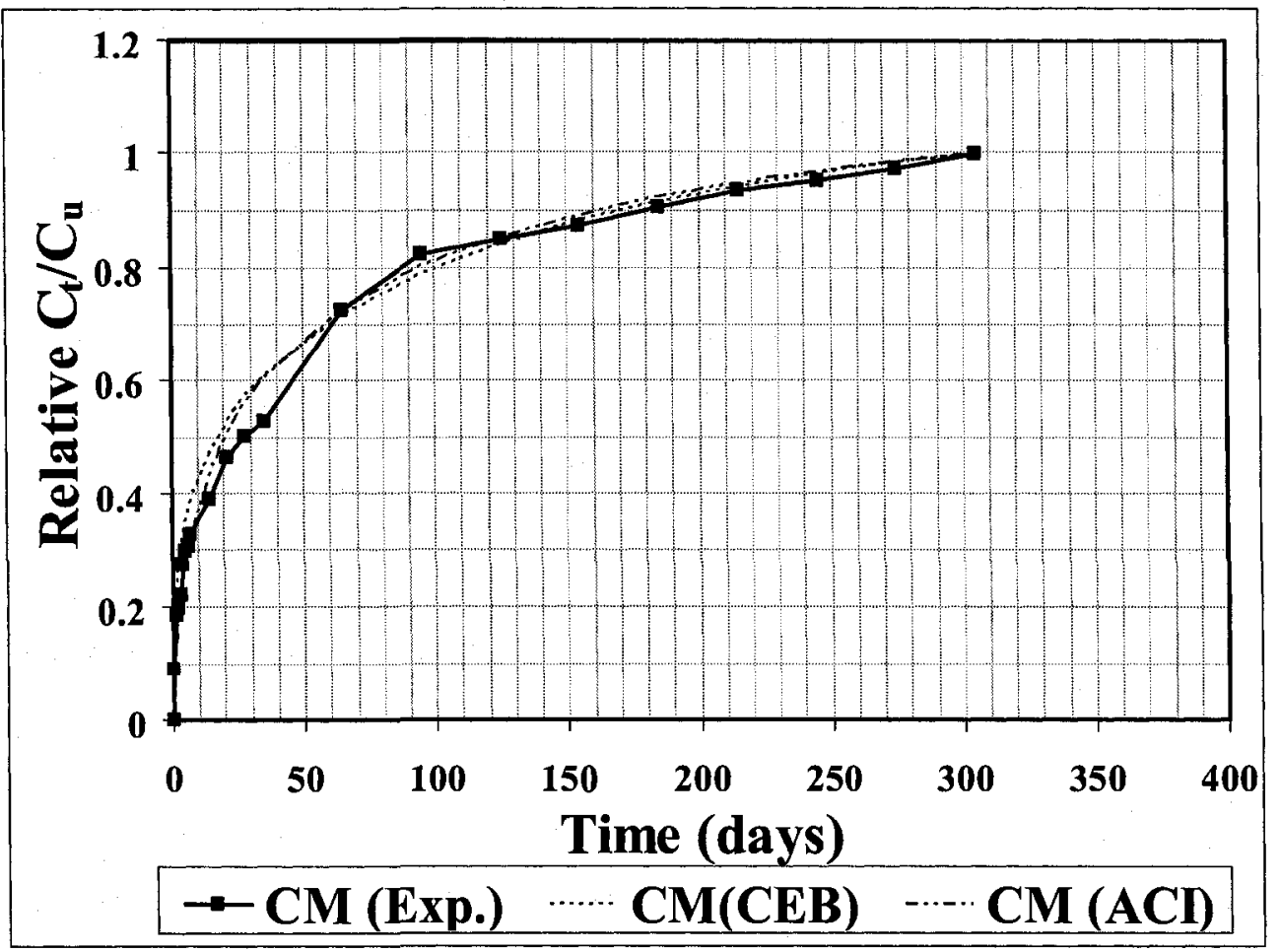

a) $\mathrm{CM}$

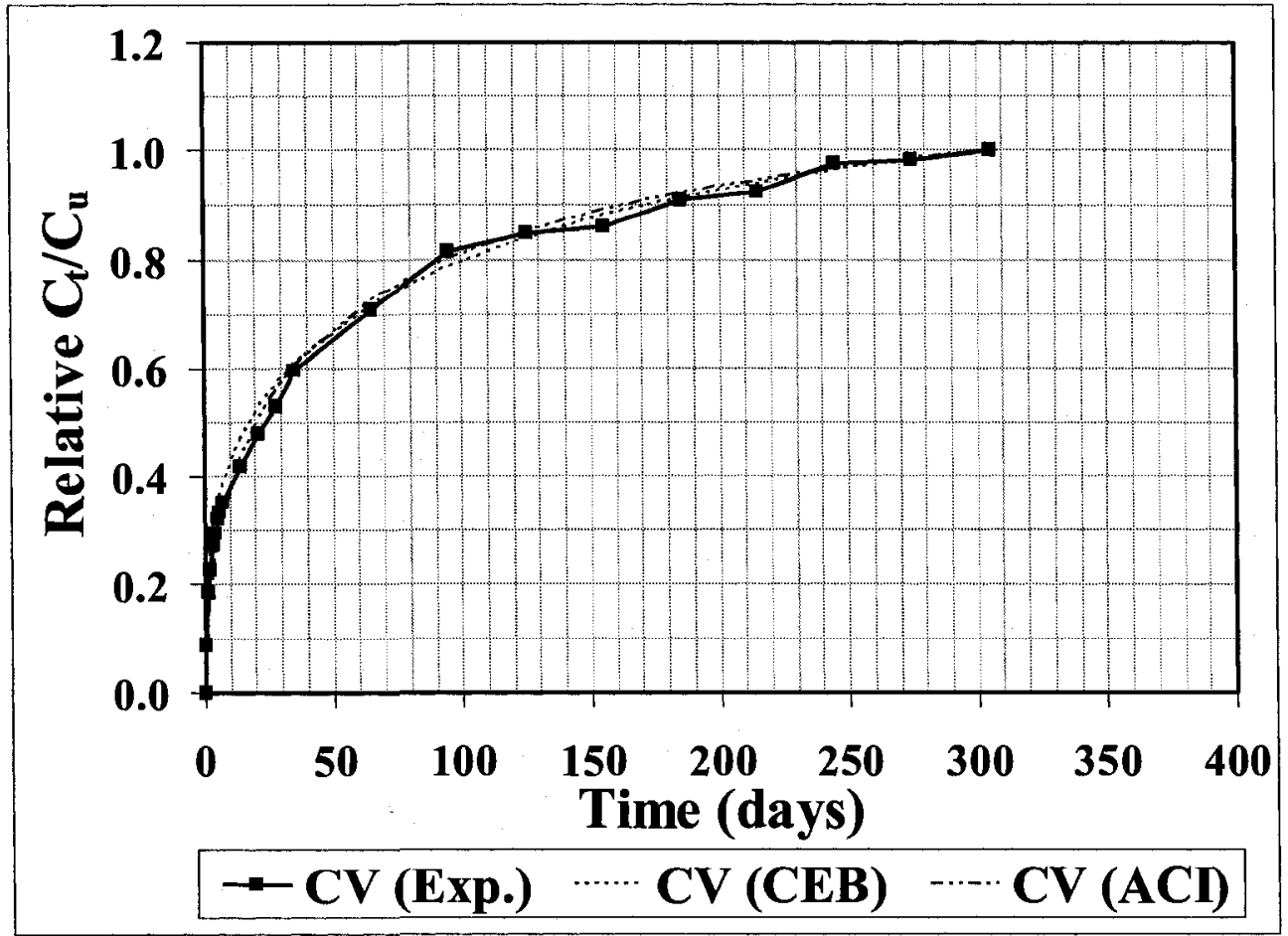

b) $\mathrm{CV}$

Figure 5.20: Normalized creep coefficient versus time relationship based on experimental data and $\mathrm{CEB}$ and $\mathrm{ACI}$ equations for $\mathrm{CM}$ and $\mathrm{CV}$ mixes 


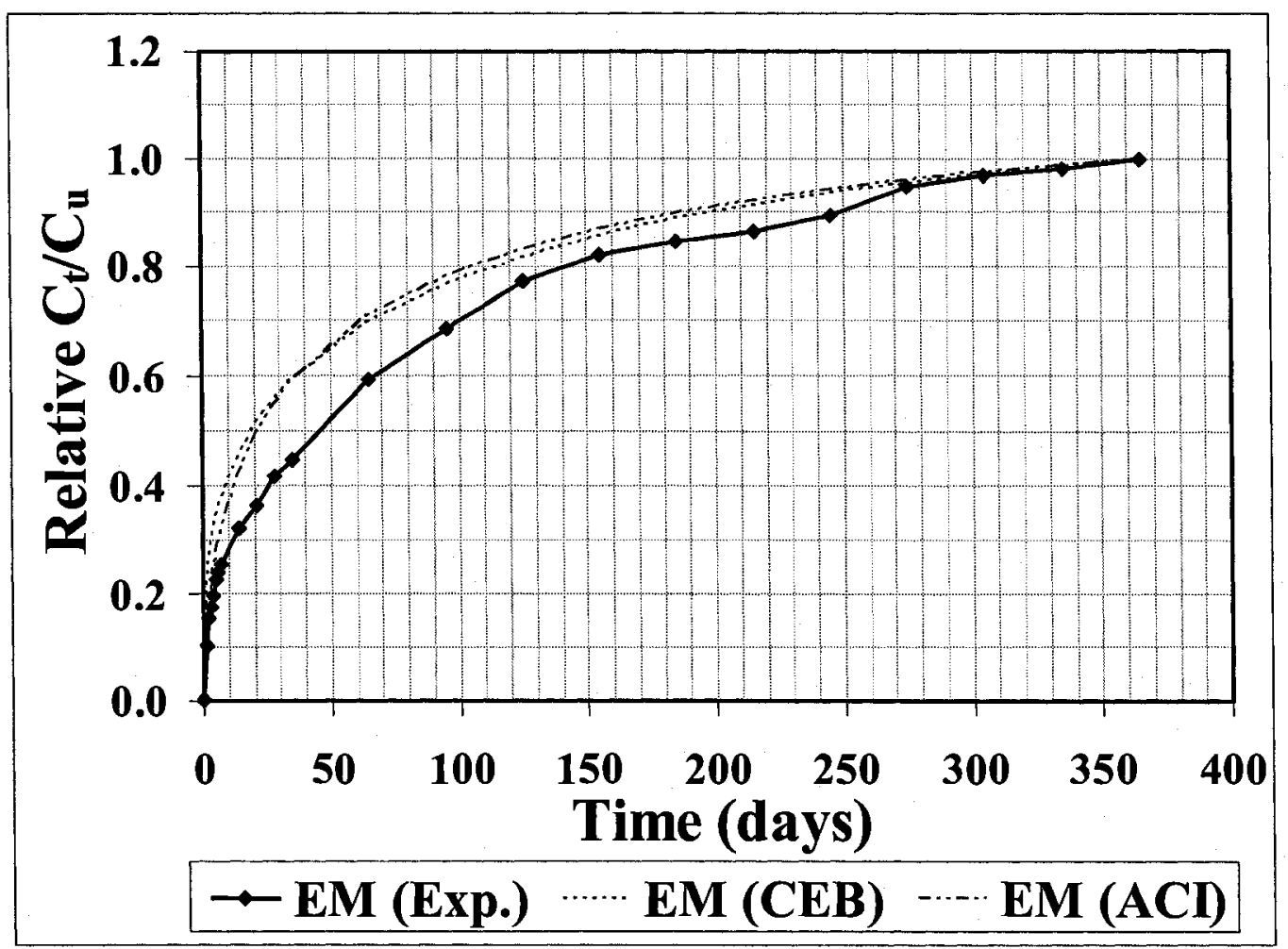

a) EM

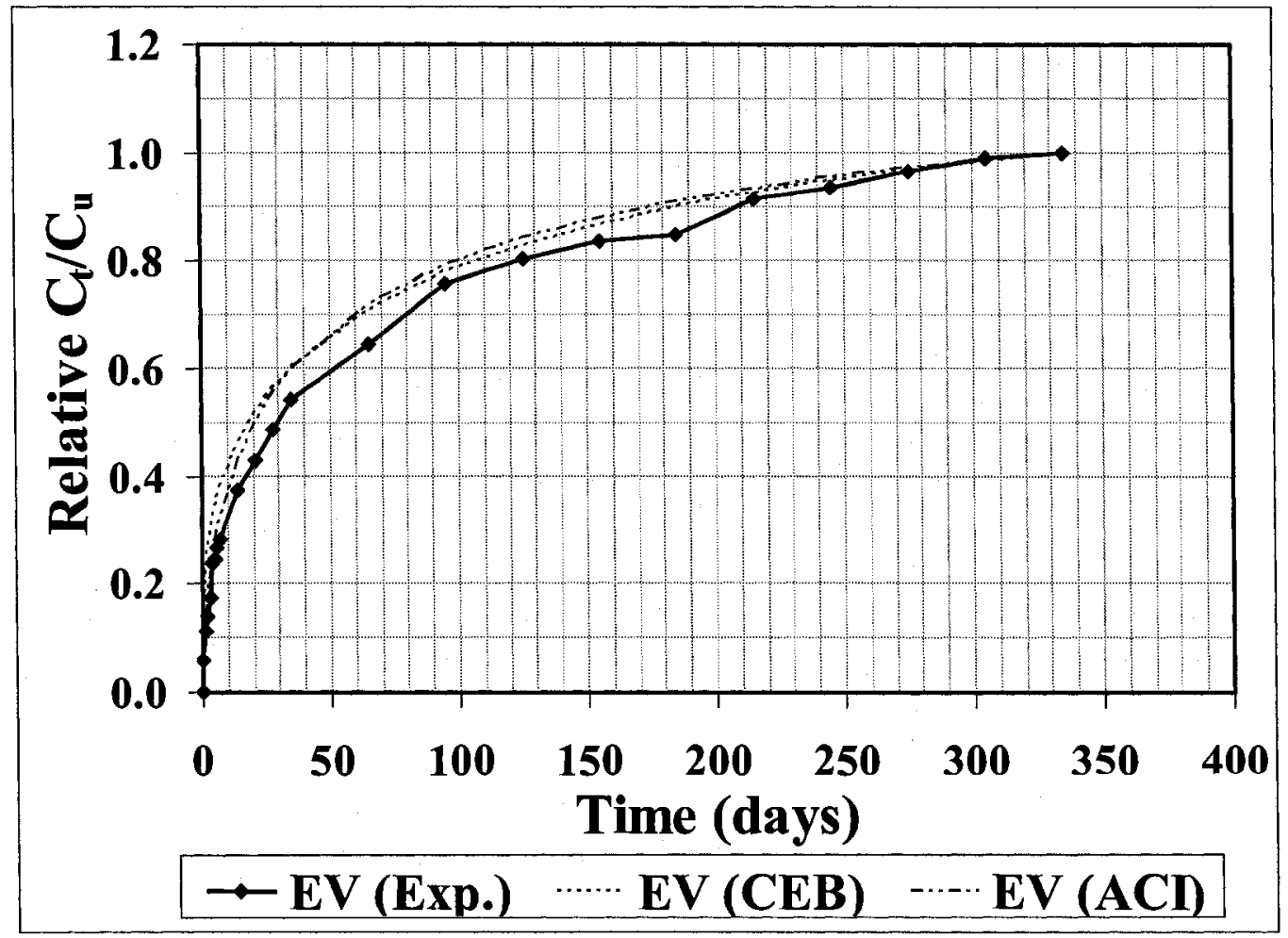

b) EV

Figure 5.21: Normalized creep coefficient versus time relationship based on experimental data and $\mathrm{CEB}$ and $\mathrm{ACI}$ equations for $\mathrm{EM}$ and $\mathrm{EV}$ mixes 


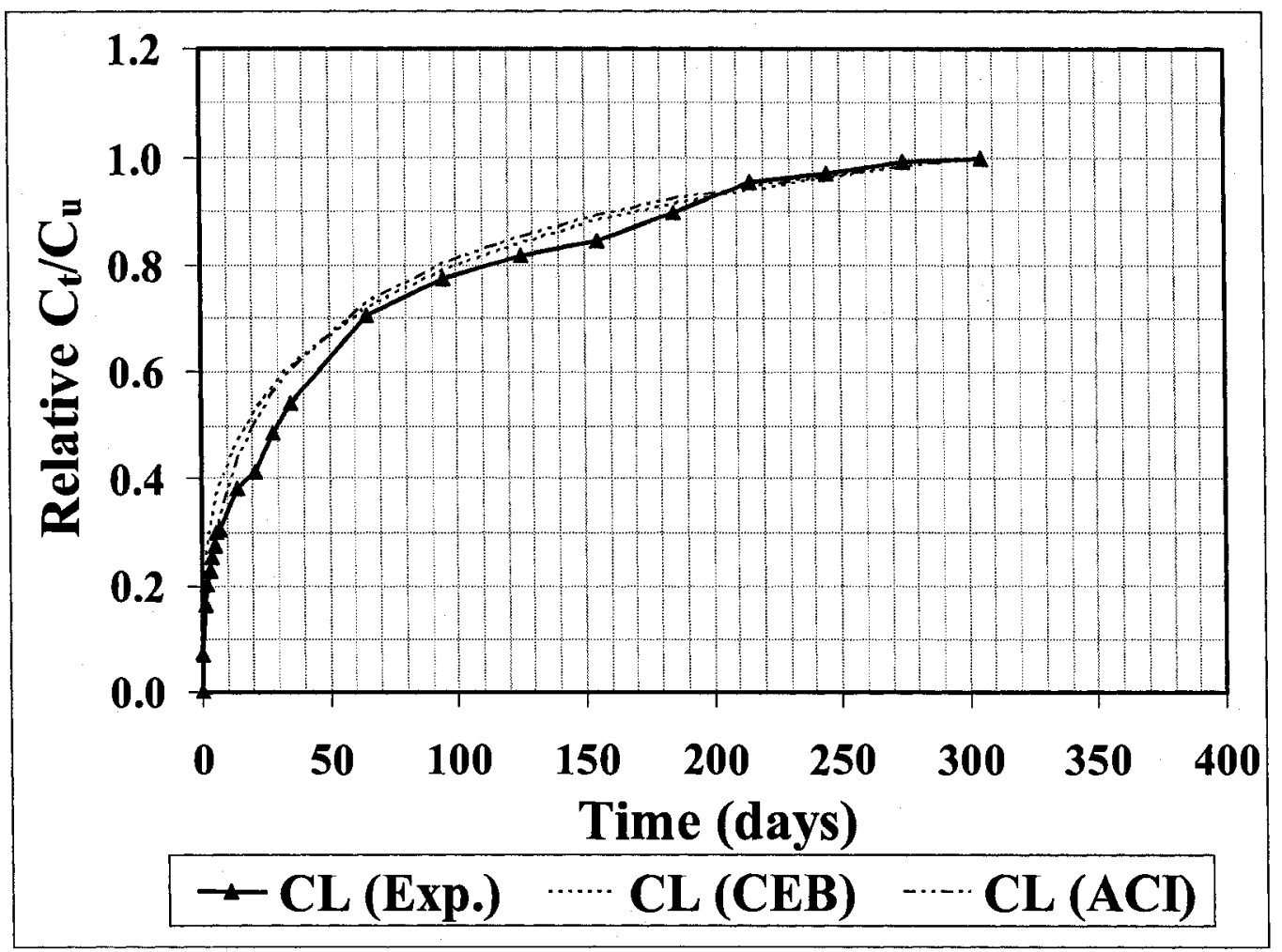

a) $\mathrm{CL}$

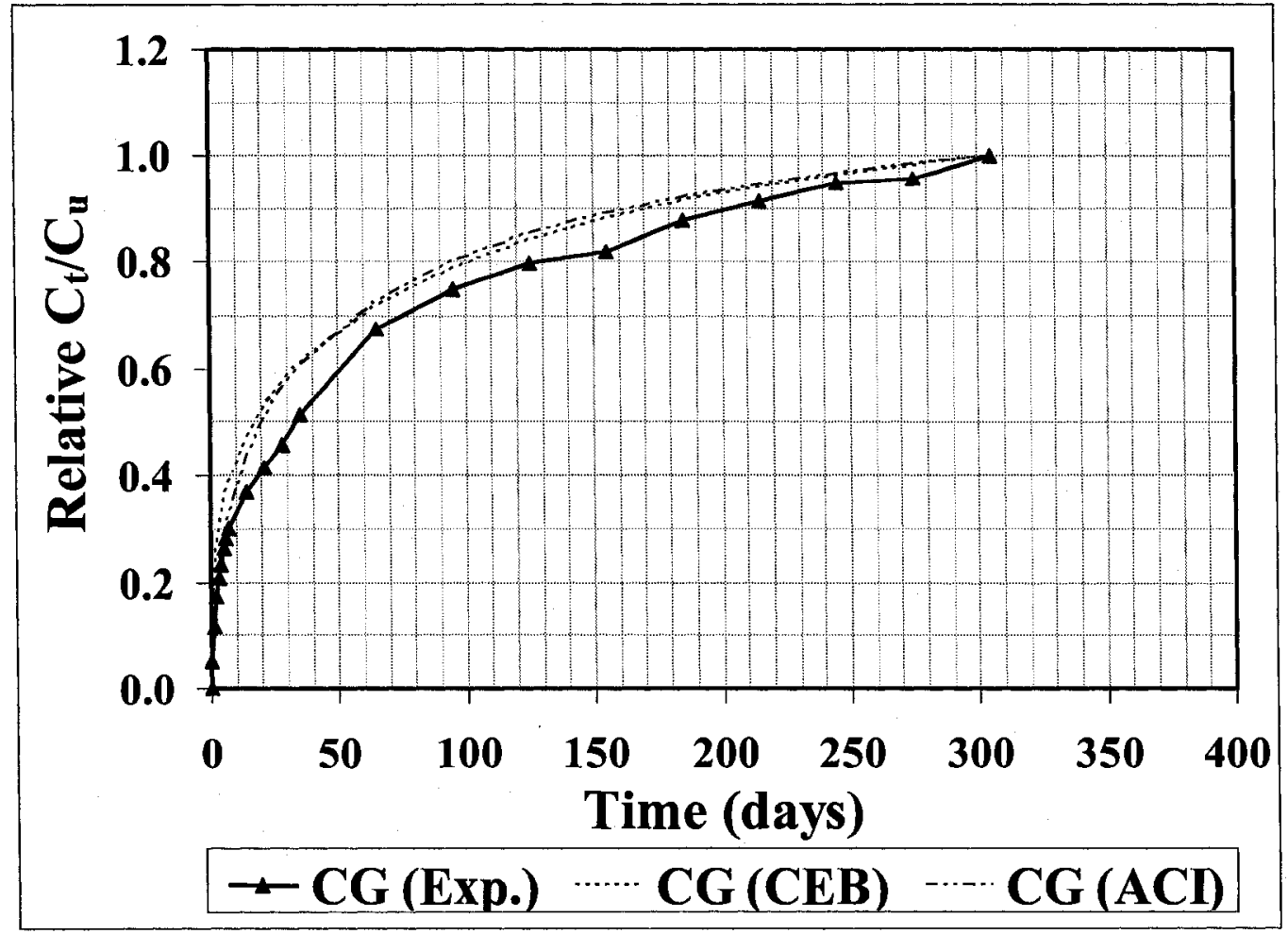

b) $\mathrm{CG}$

Figure 5.22: Normalized creep coefficient versus time relationship based on experimental data and $\mathrm{CEB}$ and $\mathrm{ACI}$ equations for $\mathrm{CL}$ and $\mathrm{CG}$ mixes 


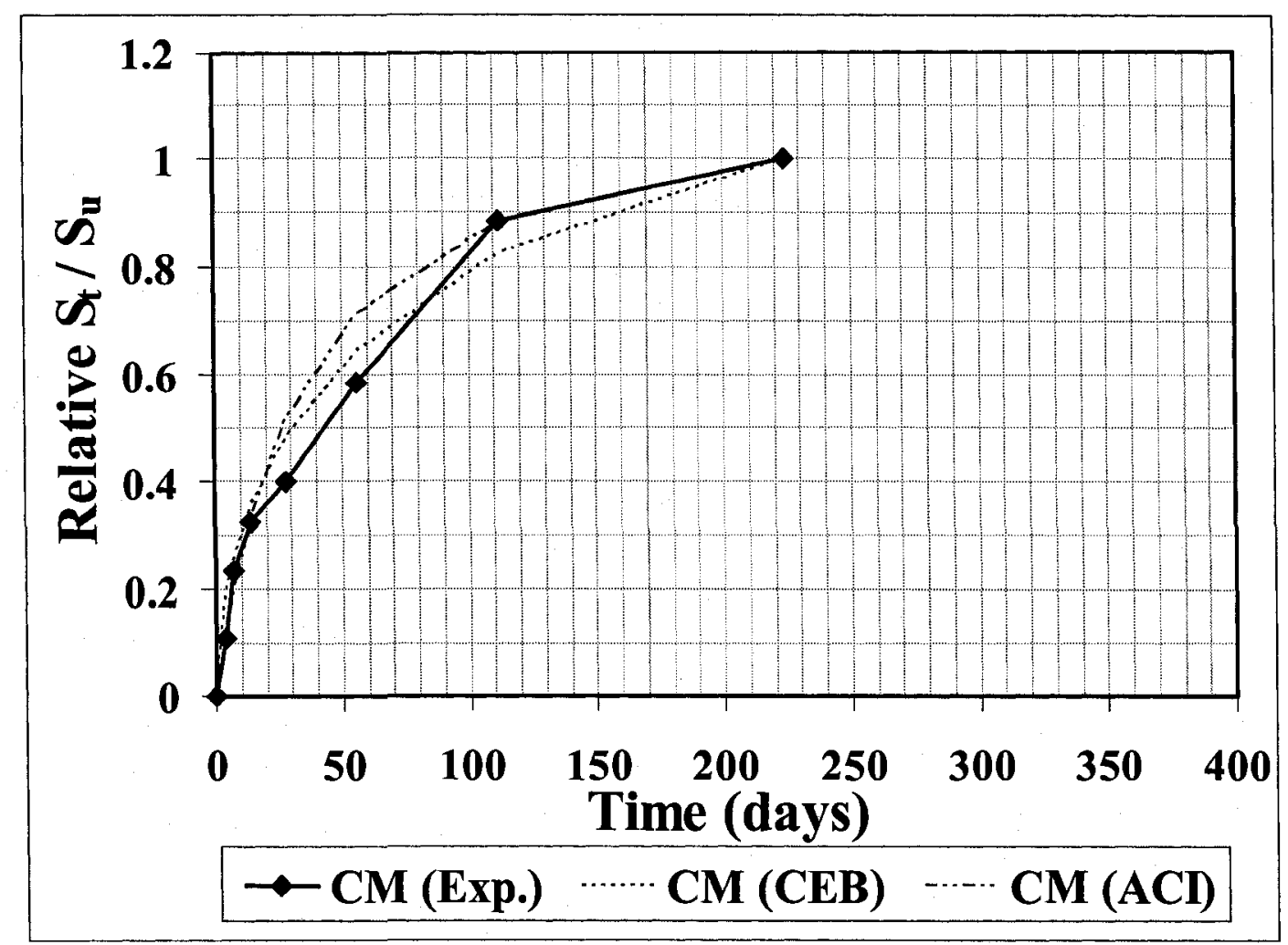

a) $\mathrm{CM}$

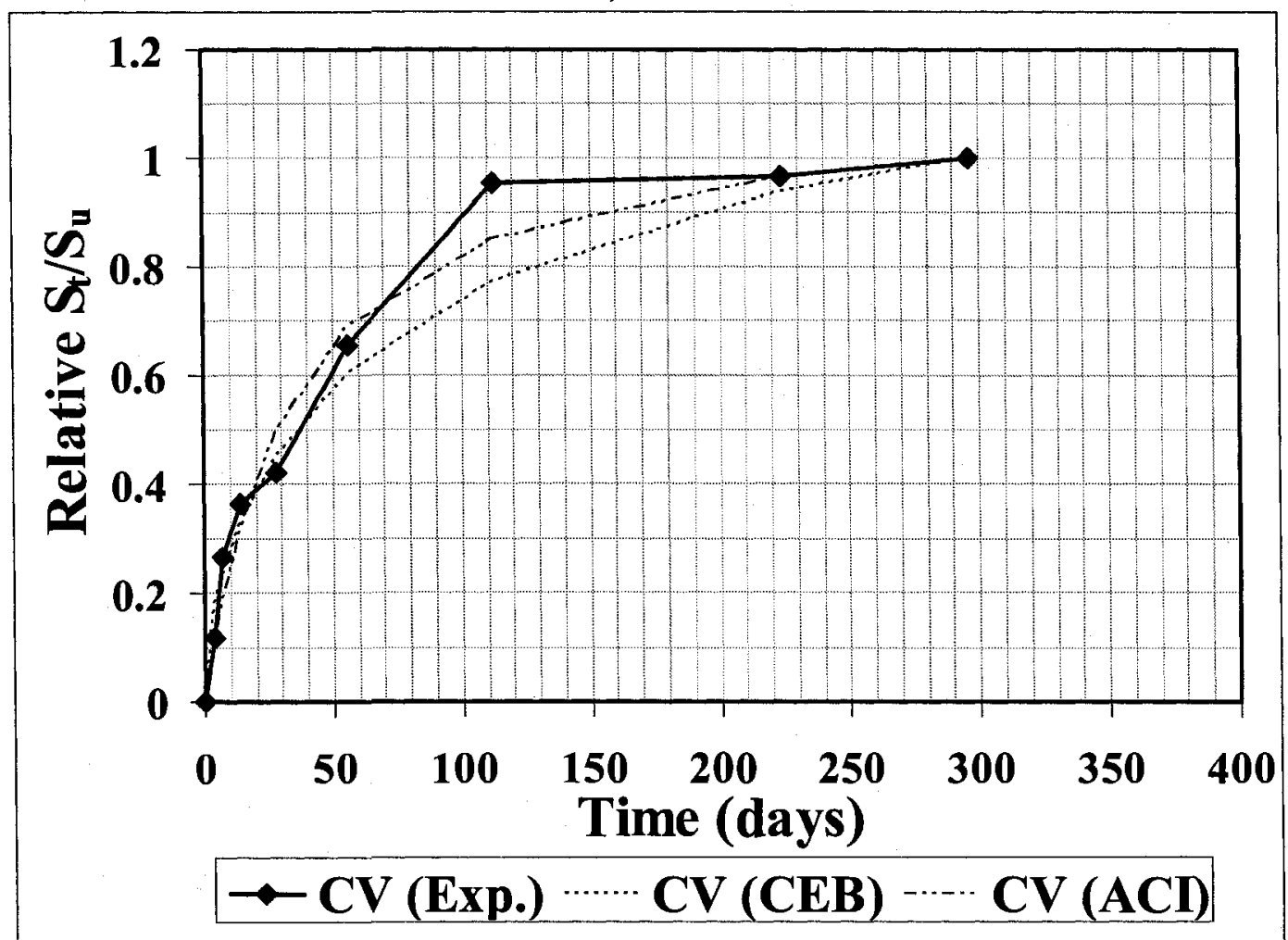

b) $\mathrm{CV}$

Figure 5.23: Normalized shrinkage strain versus time relationship based on experimental data and $\mathrm{CEB}$ and $\mathrm{ACl}$ equations for $\mathrm{CM}$ and $\mathrm{CV}$ mixes 


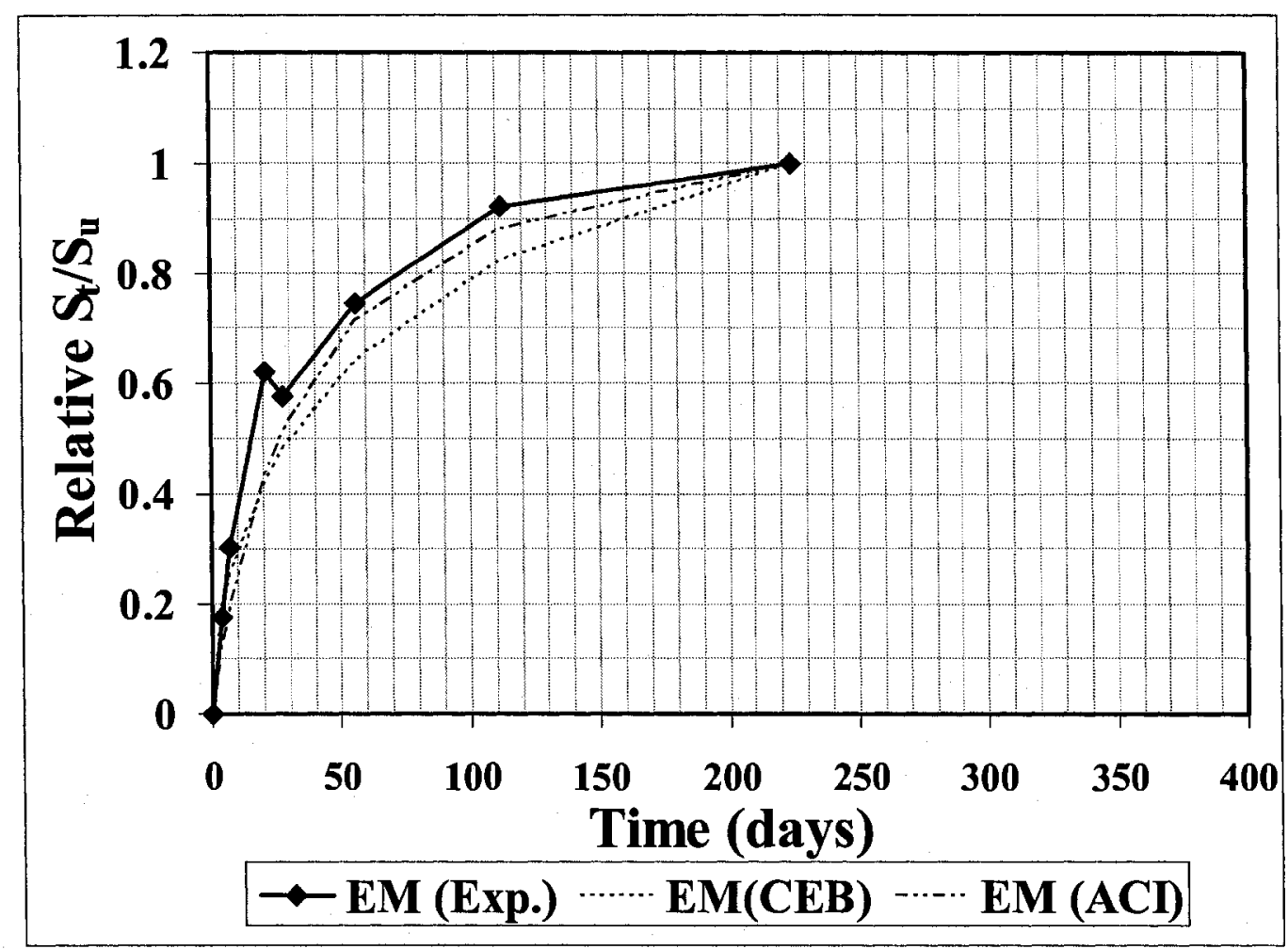

a) EM

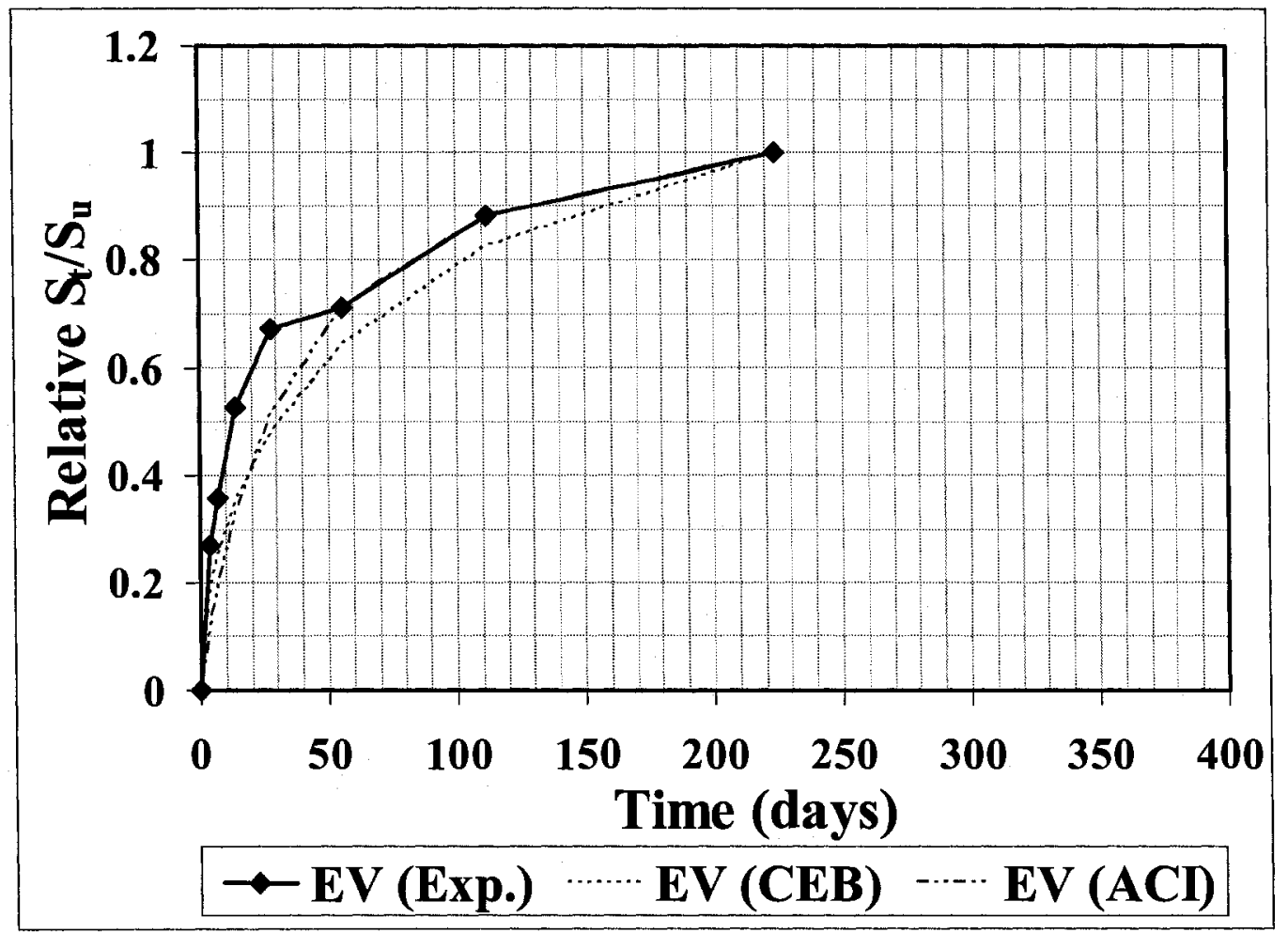

b) EV

Figure 5.24: Normalized shrinkage strain versus time relationship based on experimental data and $\mathrm{CEB}$ and $\mathrm{ACI}$ equations for $\mathrm{EM}$ and $\mathrm{EV}$ mixes 


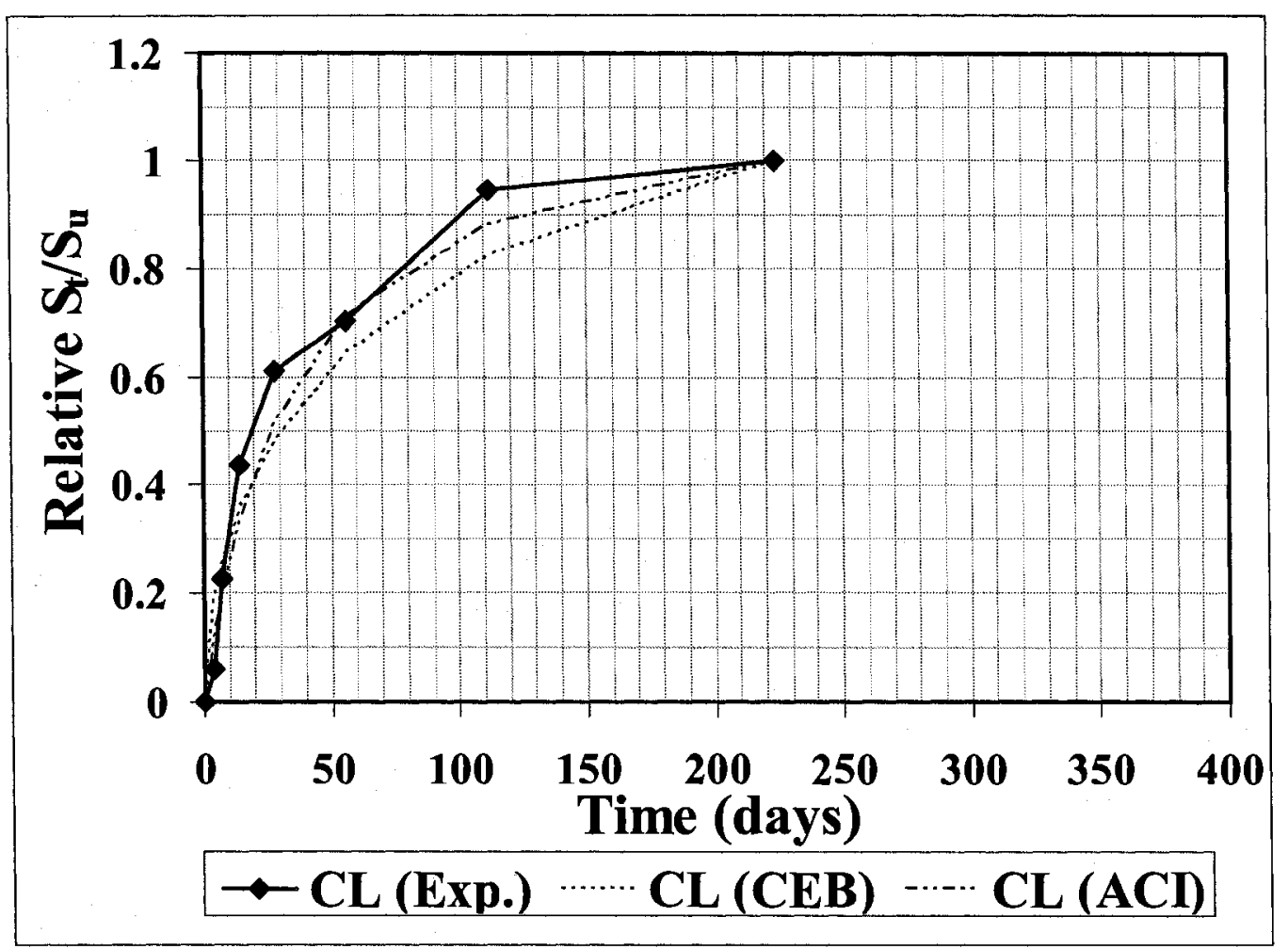

a) $\mathrm{CL}$

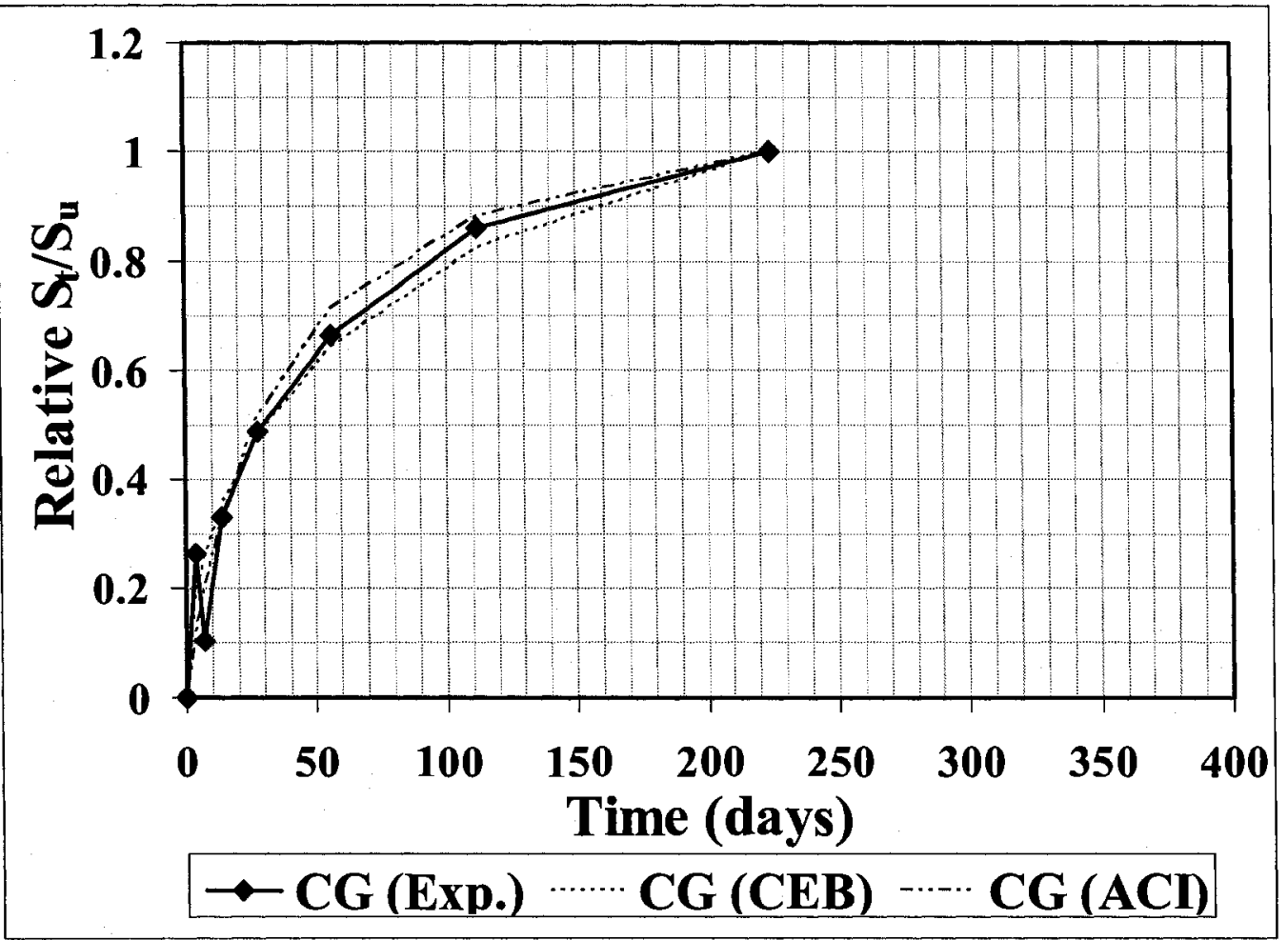

b) $\mathrm{CG}$

Figure 5.25: Normalized shrinkage strain versus time relationship based on experimental data and $\mathrm{CEB}$ and $\mathrm{ACI}$ equations for $\mathrm{CL}$ and $\mathrm{CG}$ mixes 


\section{CHAPTER 6: STRUCTURAL PERFORMANCE OF RECYCLED REINFORCED CONCRETE BEAMS:}

\section{FLEXURE}

\subsection{General}

A substantial amount of research work has been done in the past on the flexural capacity of conventional reinforced concrete (RC) beams. Therefore, their flexural failure mechanisms are well-understood and are comprehensively incorporated in design codes. However, there is not adequate information available about the flexural behaviour of RC beams made with RAC. Although the majority of the investigations generally have reported larger deflections and wider cracks, the results regarding the ultimate flexural strength of RRC beams are contradictory. Therefore, there is a need to develop information about the recycled reinforced concrete (RRC) beams to ensure proper use of this material in structural applications.

It is worthy to mention that the flexural behaviour of RC members (made with RAC or NAC) is highly dependant on the compressive stress-strain relation of the concrete and on the bond behaviour and strength of the steel reinforcement embedded in it. The stress strain relation is required to evaluate the true behaviour of the section and to provide the information required for calculating the so-called rectangular stress block parameters at failure, which are used in most flexural design codes. Therefore, the flexural response of RRC members is expected to be dependant on the stress-strain relationship of RAC, which was comprehensively investigated in Chapters 4 and 5 . Another basic requirement in reinforced concrete construction is that there be adequate means for transfer of the 
force in the reinforcement to the surrounding concrete. This phenomenon, known as the bond behaviour, will be investigated in this Chapter.

The major objective of this chapter is: (1) to study the bond behaviour of concrete produced with RCA, and (2) to investigate the flexural behaviour of RRC beams. The details and results of both the bond and the flexural performance tests of RRC beams will be presented in the following sections.

\subsection{Bond Specimens}

As sated in Chapter 2, all of the existing investigations on the bond behaviour of RAC have been carried out using the direct pullout tests. Since the concrete around the bar in this test mainly is in compression, it is opposite of what is happening in a flexural member, where the concrete around the bars is mainly under tension. The compression around a bar is commonly known to prevent cracks that would normally form in the concrete when in tension, and as a result, the direct pullout test gives higher bond strength than that observed in actual beams. Due to the inaccurate results of the direct pullout test, another test method will be employed. Beam-end bond test is an alternative test method to the direct pullout test. In a beam-end bond test the concrete around the bar is in tension, which more accurately represents the state of stress in the concrete surrounding the tensile steel reinforcement. Therefore, there is a need to generate information about the bond strength of steel reinforcement embedded in RAC by using the so-called beam-end bond test. Information about this phenomenon is particularly scant in the available literature. 


\subsubsection{Mix Proportions}

To investigate the effect of aggregate type (RCA and NA) and mix proportioning method (conventional versus the proposed EMV method) on the bond performance of steel rebars embedded in RAC, three mix types were prepared for each RCA source using only Type 10 Portland cement without any fly ash or bfs. The three mix type included:

1. RAC made with $100 \%$ coarse RCA proportioned by the conventional method (CM and $\mathrm{CV}$ mixes).

2. NAC made with $100 \%$ coarse NA and proportioned by the conventional method (CL and CG mixes).

3. RAC mixes proportioned by the EMV method (EM and EV mixes), comprising a blend of coarse NA and RCA.

Two deformed bars of size No.15 and No. 30 were selected to study the effect of bar size on the bond performance of RAC.

The bond tests were carried out following the procedure described in ASTM A944-99 (ASTM, 1999). The bond investigation was conducted on $230 \times 500 \times 600 \mathrm{~mm}$ prismatic specimens. The specimens were demolded and moist cured until the age of 7 days using a plastic covering sheet and wet burlap; thereafter, they were stored in an air-dry condition until the day of the testing. Three standard concrete cylinders $(100 \times 200 \mathrm{~mm})$ were prepared in accordance with Practice ASTM C 192/C 192M-02 (ASTM, 2003) using a representative sample of the concrete used to make the test specimen. The concrete cylinders were cured adjacent to and in the same manner as the test specimens. Three concrete cylinders $(100 \times 200 \mathrm{~mm})$ were also cast to evaluate the compressive strength of each mix at the age of 28 days. The mix proportions of each mix, whose fresh and 
hardened properties are presented in Table 6.1, are similar to the corresponding mix proportions used to make the stress-strain test specimens (Table 4.5).

Although the loading procedure and measurement techniques used in these tests are standardized, and their details can be found in the corresponding references, in the following, a brief description of the test is provided.

\subsubsection{Test Setup}

The beam-end bond test setup is shown in Figure 6.1. The test frame was designed to have sufficient capacity to prevent yielding of its various components, and it was ensured that the applied tensile load remains collinear with the axis of the steel reinforcing bar during the test.

A compression reaction plate (20 mm thick, $100 \mathrm{~mm}$ wide and $294 \mathrm{~mm}$ long) was placed at a distance of $400 \mathrm{~mm}$ from the tensile fibre of the bond specimen. The function of this plate was the uniform distribution of the horizontal reaction force of the testing system needed to balance the tensile load in the steel reinforcing bar. This set-up basically simulates the compression zone of the beams under flexure.

Displacements of the loaded and free ends of the steel reinforcing bar were measured with respect to the loaded and free surfaces, respectively, of the concrete using linear potentiometers (LP) with minimum accuracy as required by ASTM A944-99.

Since the free end of the test bar was embedded inside the RAC, its steel tube was placed in RAC and butted against the test bar to provide access to its free end for measuring slip during the test. Therefore, a spring mechanism was designed specifically for this purpose, comprising a spring loaded threaded rod placed inside the steel tube. A compressive force was applied to the threaded rod by compressing the spring. In this manner, it was ensured 
that the free end of the test bar remained in contact with the rod. The free end displacement of the test bar was assumed equal to the free end displacement of the spring loaded threaded rod. To prevent the slippage of the bar end during the test, hydraulic grips were used.

\subsubsection{Details of Bond Specimens}

The test specimen consisted of the reinforcing bar cast in a $230 \times 500 \times 600 \mathrm{~mm}$ block of reinforced concrete as illustrated in Fig. 6.2. The specimen was reinforced by four closed stirrups oriented parallel to the sides of the specimen and two flexural steel reinforcing bars parallel to the test bar, as shown in Fig. 6.2. The embedded length $\left(\mathrm{L}_{b}\right)$ and the cover thicknesses from the centre of the test bar $\left(\mathrm{C}_{1}\right)$ and the two flexural bars $\left(\mathrm{C}_{2}\right)$ for the different test specimens are presented in Table 6.2. It should be noted that each mix in these tables is identified with a unique designation. The following summarizes the designation:

- First letter E or C: designates a mix proportioned by the EMV or the conventional mix proportioning method.

- Second letter M, V, L or G: designates a mix made with RCA-MO, RCA-VA, natural limestone or natural gravel.

- Number 15 or 30: designates the nominal diameter of the test bar.

For instance, the test specimens made with $100 \%$ RCA-MO, proportioned by the conventional method and containing test bar No.30 is designated as CM-30.

Two polyvinyl chloride (PVC) pipes were used as bond breakers to control the bonded length of the bar and to avoid a localized cone-type failure of the concrete at the loaded end of the specimen. The free end of the test bar was butted against a hollow steel conduit 
to provide access to the free end for measuring slip during the test. The closed stirrups were fabricated from Grade 400 No. 10 bars for test bar No. 15 and No. 15 bars for test bar No. 30. The mechanical properties of the test bars are presented in Table 6.3.

A tensile load was applied to the test bar by means of a 60 -ton hydraulic ram, as illustrated in Figure 6.1. The loading rate was kept at $20 \mathrm{kN} / \mathrm{min}$ and $10 \mathrm{kN} / \mathrm{min}$ for No.30 and No.15 test bars, respectively, to avoid failure in less than 3 min after the start of the test. Data, including the applied load and displacement readings, were recorded for every test by using a data acquisition system and a personal computer.

The following section presents the experimental observations and results of the bond tests.

\subsubsection{Experimental Observations}

Table 6.4 presents the bond strength and failure type of all the bond test specimens. The

bond strength $\left(\tau_{b}\right)$ was calculated based on the bond failure load and the surface area of the test bar embedded in concrete as follows:

$$
\tau_{b}=\frac{P}{\pi d_{b} \cdot L_{b}}
$$

where $d_{b}$ is the nominal diameter of the test bar, and $L_{b}$ is the embedded length of the test bar in concrete.

During one test, a problem occurred with the hydraulic grip system while testing specimen EM-30, hence its results could not be fully traced and therefore specimen was discarded. Furthermore, all of the bond test specimens with test bar size No.15, except CM-15, did not fail in bond, but they failed in tension failure prior to bond failure. 
In Table 6.4, the bond strength value of each specimen is normalized by the square root

of the compressive strength of its concrete $\left(\tau_{b} / \sqrt{f_{c}^{\prime}}\right)$ in order to investigate the effect of mix proportioning method, aggregate type, mix type, and bar size on the bond strength of RAC mixes. The normalized form is often used in codes and design standards.

\subsubsection{Effect of Mix Proportioning Method}

Generally, the bond strength of RAC mixes proportioned by the conventional method was found lower than those of RAC mixes proportioned by the proposed EMV method and the companion NAC. On the other hand, the bond strength of RAC mixes proportioned by the EMV method was comparable to those of the NAC.

In the bond specimens made of concrete proportioned by the conventional method and containing bar size No.30 (CM-30 and CV-30), the $\tau_{b} / \sqrt{f_{c}^{\prime}}$ values were about $24 \%$ lower than those of companion CL-30 and CG-30 specimens. On the other hand, $\tau_{b} / \sqrt{f_{c}^{\prime}}$ value of EV-30 specimen proportioned by the proposed EMV method was only $6 \%$ lower than that of the companion CG-30.

Since in all of the bond specimens involving bar No.15 (except CM-15), the bar failed in tension prior to bond failure, the actual bond strength in these specimens could not be measured, but the average bond stress corresponding to the failure load is indicative of the bond strength that can be achieved. In other words, the bond strength in these specimens was higher than the ultimate strength of the corresponding bar $\left(\tau_{b} \phi f_{u}\right)$. Generally, in the CM-15 proportioned by the conventional method, the $\tau_{b} / \sqrt{f_{c}^{\prime}}$ value was at least $34 \%$ and $8 \%$ lower than those of the EM-15 (proportioned by the proposed EMV method) and the companion CL-15, respectively. 
Since all of the factors affecting the bond properties of concrete (w/c ratio, test bar size, concrete cover over test bar, curing condition, etc.) in this study were kept identical in different mix types, the mix proportioning method can be identified as the main reason for the lower bond strength of the RAC mixes proportioned by the conventional method compared to those proportioned by the EMV method. In other words, the lower TNA volume, or higher TM volume, in the RAC mixes proportioned by the conventional method decrease the bond capacity due to less interlocking of the ribs on the deformed test bar with the surrounding concrete.

Figures 6.3 and 6.4 illustrate the effect of mix proportioning method on the bond stress variation with the loaded-end displacement and the free-end slip, respectively. Generally, the rate of bond stress variation with the end displacements for all specimens involving bar size No.30 was found to be similar regardless of the mix type. In other words, there was no significant difference observed in the bond behaviour of RAC and NAC, irrespective of aggregate or mix type. Although in the specimens involving bar size No.15, the rate of bond stress increase with loaded-end displacement was found to be basically the same, regardless of the mix type, there were some differences observed in their rate of bond stress increase with the free-end slip. This is mostly attributed to the fact that most of the bond specimens with bar size No. 15 did not fail in bond and the test bar failed in tension. Therefore, the illustrated bond-displacement curves do not represent the full bond behaviour of the corresponding specimens.

\subsubsection{Effect of Test Bar Size}

According to Table 6.4, for all mix types the bond strengths of specimens containing bar

No.15 are generally higher than those involving bar No.30. The $\tau_{b} / \sqrt{f_{c}^{\prime}}$ values of CM-15 
and CV-15 specimens are $35 \%$ and at least $28 \%$ higher than those of CM-30 and CV-30 specimens, while that of EV-15 is at least $41 \%$ higher than the corresponding value of EV-30. Furthermore, the $\tau_{b} / \sqrt{f_{c}^{\prime}}$ values of CL-15 and CG-15 are at least $12 \%$ and $9 \%$ higher than those of CL-30 and CG-30, respectively. This is in conformity with the requirements of CSA A23.3-04 (clause 12.2.4) standard in which allows a reduction factor of 0.8 for the minimum required development length of deformed bars in tension $\left(\mathrm{k}_{1}=0.8\right)$ for bar sizes smaller than No.20. In other words, the CSA A23.3-04 standard recognizes the bar size effect by requiring $20 \%$ less development length for bars smaller than No.20.

Figure 6.5 illustrates the effect of bar size on the bond stress variation with loaded-end displacement and free-end slip. Generally speaking, the bar size did not have a noticeable effect on the bond behaviour at either the loaded or free-end of the test bar, irrespective of the mix type. However, as stated earlier, the recorded bond strength of bar No. 15 was found to be greater than that of bar No.30. It is of course recognized.

\subsubsection{Effect of Aggregate Type}

Figure 6.6 illustrates the effect of aggregate type on the bond stress variation with both loaded-end displacement and free-end slip for all the test specimens. Generally speaking, the type of aggregate was not found to be a major factor affecting the bond behaviour of either RAC or NAC mixes, irrespective of the bar size. The plateau portion of the curves for bar No. 15 is due to the yielding and subsequent tension failure of the bar and the free end undergoes relatively small slip. 


\subsubsection{Failure Modes}

Figures 6.6 and 6.7 illustrate the front and top view of the cracking patterns for all the bond test specimens involving bar No.30. Generally, there was no significant difference observed between the cracking patterns of different bond specimens. In other words, the aggregate type and mix proportioning method did not have any significant effect on the failure type and cracking pattern. Since most of the specimens involving bar No.15 failed in tension failure rather than bond, no cracking was observed in them.

\subsection{Flexural Beams}

To the writer's knowledge, in all of the previous investigations carried out on the flexural behaviour of reinforced recycled concrete (RRC) beams, the RAC has been proportioned based on the conventional method. However, it was demonstrated in Chapters 4 and 5 that this type of RAC generally suffers from some inferior mechanical properties such as lower elastic modulus, relatively lower bond strength and stress-strain relationship characteristic. The lower elastic modulus of the conventional RAC is the key factor responsible for the reported larger deflections of RRC beams compared to conventional reinforced concrete beams. Furthermore, the RRC beams used in previous studies were basically designed to be under-reinforced, in which steel yielding preceded concrete crushing. This is the main reason why using conventional RAC instead of normal concrete has been reported not to have significant effect on the ultimate strength of RRC beams. It is well known that the concrete strength has relatively small effect on the ultimate flexural strength of under-reinforced concrete members.

As verified in Chapter 4, the RAC mixes proportioned by the proposed EMV method do not suffer from the aforementioned inferiorities (e.g., lower elastic modulus). Therefore, 
it is expected that RRC beams made of RAC proportioned by the EMV method would not experience larger deflections or lower ultimate flexural strength compared to conventional RC beams made with NAC. To verify this, an extensive experimental study was carried out. Further details of this study are presented in the following section.

\subsubsection{Mix Proportions}

To investigate the flexural behaviour of RC beams made with RAC, two mix types were prepared for each RCA source using only Type 10 Portland cement without fly ash or bfs:

1. NAC mixes made with $100 \%$ coarse NA proportioned by the conventional method (CL and CG mixes).

2. RAC mixes proportioned by the EMV method (EM and EV mixes) containing a blend of coarse RCA and NA.

The proportions of each mix are similar to the corresponding proportions of the mixes used to investigate the compressive stress-strain relationship of RAC in Chapter 4 (see Table 4.5), but some slight changes were made to the dosages of water reducing and air entraining admixtures for air content and slump adjustments. Table 6.5 presents the mix proportions of the two types of mixes used in flexural RRC beams. Observe that no beams were made from RAC mixes designed by the conventional mix proportioning method because the beams made of the NAC mixes in this part of the study serve as the control.

\subsubsection{Preparation of Mixes}

In this part of the study, it was decided to cast all of the flexural and shear beams for each RCA source simultaneously using a truck mixer. The reason for this was twofold: (1) to 
save time, resources and energy by avoiding multiple usage of pan mixer for casting different beams; and (2) to provide a unique RAC in all the flexural and shear specimens for each RCA source thus minimizing potential variations in RAC mixes due to quality control and other random variations.

Since the number of the control beams made with normal aggregate concrete and consequently the required amount of NAC was small, it was not practical to use a truck mixer. Therefore, a pan mixer was used to cast the control beams. In this case, the same procedure as described in Chapter 3 was used for the preparation of the ingredients and the concrete mixing.

Figures 6.9 and 6.10 illustrate some of the material preparation and concrete mixing stages.

After the mixing process, all RRC beams ( 13 beams per truck mixer) were cast and cured following the procedures described in ASTM C31/C 31M-03a (ASTM, 2003). The beams were kept moist until the age of 7 days after casting by means of plastic covering sheets and wet burlaps and were demolded at the age of 14 days after casting. Thereafter, they were stored in an air-dry and indoor condition waiting for testing. Figure 6.11 presents some of the beams during casting and curing stages.

\subsubsection{Material Properties}

\subsubsection{Concrete}

In order to evaluate the flexural behaviour and strength of the RRC beams, six cylindrical specimens $(100 \times 200 \mathrm{~mm})$ were prepared and cured in a moist room for 28 -days to determine their compressive ( 3 specimens) and splitting tensile strengths ( 3 specimens). Three cylindrical specimens $(150 \times 300 \mathrm{~mm})$ were also prepared and cured in a moist 
room for the determination of the elastic modulus at the age of 28 days. Nine extra concrete cylinders were also prepared and cured adjacent to and in the same manner as the beams to evaluate the compressive strength (three $100 \times 200$ cylinders), splitting tensile strength (three $100 \times 200$ cylinders) and elastic modulus (three $150 \times 300$ cylinders) at the time of testing of the beams. Tables 6.6 presents the summary of the fresh and hardened properties of the RAC mixes used in the test beams.

\subsubsection{Reinforcement}

All of the steel reinforcements used in the flexural tests were deformed Grade 400 bars in accordance with the requirements of CAN/CSA G30.18. Figure 6.12 illustrates the typical stress-strain relationship of these rebars as obtained through the tests in the current study.

\subsubsection{Details of RRC Beams}

All of the beams were designed in accordance with the requirements of the CSA Standard A23.3-04. The test variables comprised three levels of reinforcement. In order to assess the performance of RRC beams under flexure over the full range of reinforcement ratios, three reinforcement ratios (i.e. minimum, average, and maximum) were selected. The minimum and maximum reinforcement ratios were calculated according to the existing CSA A23.3-04 provisions for conventional concrete. The beams were designed to ensure flexural failure prior to shear failure. Three beams for each RCA source (proportioned according to the proposed mix proportioning procedure in Chapter 4) were tested, i.e. one beam for each longitudinal reinforcement ratio. An extra beam was also constructed for each RCA source to study the effect of compression reinforcement on the flexural behaviour of RRC beams. 
As illustrated in Figure 6.13, all the test beams were rectangular, $200 \mathrm{~mm}$ wide and 350 to $390 \mathrm{~mm}$ deep (effective depth of $304 \pm 3 \mathrm{~mm}$ ) with nominal shear span/depth (a/d) ratio of 2.70 , and span length of $2.2 \mathrm{~m}$. In order to avoid premature bond failure, each bar was bent at its ends in the form of $180^{\circ}$ hook in accordance with the requirements of CSA Standard A23.3-04.

In addition to the four RRC beams for each RCA source, two companion control beams were made for each source. The control beams were made of conventional concrete made with $100 \%$ coarse natural aggregates of the same type as the OVA of the companion RRC beams. One of the control beams had only tension reinforcement (singly reinforced) with average reinforcement ratio. Such a reinforcement ratio would be typically used in practical applications. The second control beam was doubly reinforced, containing both tensile and compressive reinforcement. Note that in compliance with the prevailing custom in reinforced concrete design, the level of reinforcement can be expressed as the ratio of actual reinforcement in a section to the balanced reinforcement of the same section. The balanced reinforcement being the amount of reinforcement required to ensure concurrent failure of the concrete in compression and the yielding of steel in tension. In practice this ratio is typically 0.3 to 0.5 for beams. A ratio of greater than one implies over-reinforced, which is normally avoided to prevent brittle failure. Therefore, a total of twelve beams were tested to investigate the flexural behaviour of RRC beams and Table 6.7 presents their details. Unfortunately, time and resource limitations did not permit testing of replicate specimens.

Each beam is designated with an ID as shown in Table 6.7. The following summarizes the designation: 
- First letter F: designates flexure.

- Second letter E or C: designates the EMV or the conventional mix proportioning method.

- Third letter M, V, L or G: designates a mix made with RCA-MO, RCA-VA, natural limestone or natural gravel.

- Fourth letter Min, Av, Max, or CMP: designates a beam with minimum, average, or maximum tension reinforcement ratios, or beam with compression steel.

For instance, the flexural beams made with RCA-MO, proportioned by the EMV method with average tension reinforcement ratio is designated as FEM-Av.

\subsubsection{Instrumentation}

\subsubsection{Strain Gauges}

In order to investigate the flexural behaviour of RRC beams, one needs to know the strain values of concrete and steel reinforcement at different locations. Towards this end, several strain gauges were installed on concrete and longitudinal reinforcements at several locations. The N11-FA-5-120-11 and N11-FA-30-120-11 strain gauges provided by Showa Measuring Instruments were used for measuring the strain in the steel reinforcement and concrete, respectively. The grid pattern of all strain gauges was chosen to be uniaxial since only the strain in the longitudinal direction of the beams was of interest. All strain gauges had a resistance of $120 \mathrm{Ohms.}$ The lengths of the gauges used to measure the concrete and steel reinforcement strains were 30 and $5 \mathrm{~mm}$, respectively.

Figure 6.14a illustrates the arrangement of the concrete and steel reinforcement strain gauges, where C and LS designate the concrete and longitudinal steel strain gauges, respectively. Four different locations were chosen for longitudinal steel strain 
measurements: (1) at distance $d$ from face of the support (LS1 and LS7); (2) under the point loads (LS3 and LS5); (3) at midspan (LS4); and (4) between point loads and face of supports (LS2 and LS6). For longitudinal reinforcements, one strain gauge was installed at each location on the bottom side of the closest bar to the underside of the formwork to avoid any damage during casting procedures. For concrete strain measurements, two different locations were selected: (1) at midspan (C2); and (2) between point loads and face of supports ( $\mathrm{C} 1$ and $\mathrm{C} 3)$. One strain gauge was installed at each location on top fibre of the section. Note that in the doubly reinforced beams (with compression steel), there were no concrete and steel strain gauges installed in the east shear span (no C3, LS5, LS6 and LS7), for the strain variation along these beams was assumed to be symmetric.

All of the strain gauges were installed in accordance with the installation guide recommended by Vishay Micro-Measurements (2006).

Figure 6.14d illustrates some of the strain gauges installed on the steel reinforcement and the concrete surfaces.

\subsubsection{Linear Potentiometers}

Beam deflection was measured using HLP190 and 150 linear potentiometers (LP) provided by Penny and Giles company. Figure $6.14 \mathrm{~b}$ illustrates the arrangement of LP gauges along the beams where T refers to the LP gauge. Deflection was measured at three different locations: (1) under the point loads (T2 and T4); (2) at midspan (T3); and (3) between point loads and the centreline of the supports (T1 and T5). At each location, two LP's were installed which were connected together by a rigid rod. The rigid rod was clamped to the bottom surface of the beam by means of an elastic string looping over the top surface of the beam (Figure 6.14c). By applying the load on the beam, the beam 
deforms and the rod moves downward, and consequently the two LP's move and record the deflections below the two opposite faces of the beam. The actual deflection at each point along the beam is assumed to be the average of the readings recorded by the two parallel LP's. All of the LP's on each side of the beam were connected to a rigid bar secured to the laboratory strong floor.

\subsubsection{Test Setup}

All the beams were tested in the test frame shown in Figure 6.15a. This particular frame is equipped with a $1000 \mathrm{KN}$ MTS actuator controlled by a MTS 458.10 Microconsole controller (Figure 6.15b), which is supplied by a hydraulic pump. The MTS closed-loop servo-controlled-hydraulic actuator could apply tension/compression loads up to 1000 $\mathrm{KN}$ and downward/upward displacements up to $250 \mathrm{~mm}$ to the beams. Furthermore, it is capable of operating in both load and stroke control modes.

The actuator applied the force through a steel spreader beam under stroke control. The spreader beam was connected to the actuator through a ball-and-socket joint with full rotation freedom and allowing for a wide range of adjustments to properly position the spreader beam on the test beam (Figure 6.15c). The spreader beam rested on the specimen at two points by means of two heavy duty rocker-and-roller assemblies each located $300 \mathrm{~mm}$ from the midspan of the beams (Figure 6.15d). The rocker-and-roller assembly mainly consists of two parts: (1) three cylindrical rollers placed between two middle and top plates providing a translation degree of freedom for the spreader beam; and (2) a conical rocker located on the spreader plate $(150 \times 200 \mathrm{~mm})$ over the beam providing a rotational degree of freedom for the spreader beam about the conical rocker axis. There were some wedges provided on the surface of both the conical rocker and the 
spreader plate to avoid any relative slippage and translation during the loading process of the beam. The trapezoidal shape of the spreader plate was specifically designed to avoid any contact between the spreader plate and the rocker-and roller assembly due to the curvature of the deflected beam during the test procedure.

The beam was simply supported by means of two rocker-and roller assemblies similar to that used under the spreader beam: one acting as a roller-support providing both translational and rotational movements, and another acting as a pinned support providing only rotational movement. In order to restrain the translational degree of freedom in the pinned support, the top and middle plates in the roller-and-rocker assembly (Figure $6.15 \mathrm{e})$ were fixed to each other by means of stopper plates.

In order to avoid any uneven distribution of the loads under the point loads and reaction forces, a hydrostone mixture was prepared and applied to the corresponding surfaces to fill any cavity and smoothen the bumps. Hydrostone is a kind of gypsum cement which is considerably harder than regular plaster. The hydrostone powder was mixed with water in a Ziploc bag and placed under the spreader plate and beams 24 hours prior to the test.

The data acquisition system HP3497A was used and both displacement and concomitant load values were measured every 10 seconds. The loading rate was set at $0.01 \mathrm{~mm} / \mathrm{sec}$.

\subsubsection{Flexural Behaviour of RRC Beams}

Table 6.8 presents the observed moment and deflection values of test beams at cracking, yielding and ultimate states. In addition, the value of the moment corresponding to the crushing of the top fibre of the beam $\left(M_{\text {crush }}\right)$ is also shown. Note that the crushing and ultimate moments in singly reinforced beams are the same, while the crushing moment of a doubly reinforced beam is smaller than its ultimate moment. In order to evaluate the 
performance of the flexural beams under customary service loads, their maximum deflection at $40 \%$ of their failure loads $\left(\delta_{s}\right)$ are also presented in the same table.

During the tests, the cracking load $\left(P_{c r}\right)$ was recorded as soon as the first flexural crack (usually between two point loads) was observed by the naked eye. The formation of a single flexural crack did not necessarily affect the overall moment of inertia sufficiently to result in a noticeable change in the deflections measured by LVDT's and the subsequent change in the slope of the load-deflection curve. The observed cracking moment $\left(M_{c r}\right)$ is calculated as

$$
M_{c r}=0.5 P_{c r} \times a+\frac{\left(\gamma_{c} \times b \times h\right) \times L_{n}{ }^{2}}{8}
$$

where $a$ is the shear span of the beam, $\gamma_{c}$ is the hardened density of the concrete, $b$ is the section width, $h$ is the section height, and $L_{n}$ is the net beam span, measured from centre to centre of the supports.

The observed yielding load $\left(P_{y}\right)$ was obtained by using the data from the strain variation of steel gauges installed on the tensile reinforcement. The load at which the maximum strain in the steel reached the known yield strain of the rebar, is considered the yield load. Consequently the yielding moment $\left(\mathrm{M}_{\mathrm{y}}\right)$ is calculated as

$$
M_{y}=0.5 P_{y} \times a+\frac{\left(\gamma_{c} \times b \times h\right) \times L_{n}{ }^{2}}{8}
$$

During the tests, the crushing load $\left(P_{\text {crush }}\right)$ was recorded as soon as concrete crushing at top fibre between the point loads was observed. The corresponding crushing moment $\left(M_{c r u s h}\right)$ is calculated as

$$
M_{\text {crush }}=0.5 P_{\text {crush }} \times a+\frac{\left(\gamma_{c} \times b \times h\right) \times L_{n}{ }^{2}}{8}
$$


The observed ultimate load $\left(P_{u}\right)$ is the maximum recorded load during the test, and the corresponding ultimate moment $\left(M_{u}\right)$ is:

$$
M_{u}=0.5 P_{u} \times a+\frac{\left(\gamma_{c} \times b \times h\right) \times L_{n}^{2}}{8}
$$

Since all of the section properties (effective depth and width) and material properties (concrete compressive strength and steel yield stress) were kept constant in this study, the main factors which might influence the flexural behaviour of the beams are: (1) the longitudinal tensile reinforcement ratio, (3) the presence of the longitudinal compression steel, and (3) the concrete type (RAC or NAC).

In the following sections, firstly the failure modes and cracking patterns of these beams will be presented, followed by the discussion of each of the preceding factors.

\subsubsection{Failure Modes}

Figures 6.16 and 6.17 illustrate the cracking patterns of all the FEM and FEV beams at failure, respectively. In these figures, the inclined lines and dark regions near the top fibres represent the cracks and the crushed zones, respectively. In all of the beams with minimum and average tension reinforcement ratios (FEM-Min, FEM-Av, FCL-Av, FEVMin, FEV-Av, and FCG-Av) and the beams with compression steel (FEM-CMP, FCLCMP, FEV-CMP and FCG-CMP), firstly the longitudinal tension steel reached its yield strength, followed a little later by the concrete crushing, a ductile mode of failure called tension failure. In the beams with maximum longitudinal reinforcement ratio (FEM-max and FEV-max), firstly the concrete reached its maximum capacity followed shortly after by the yielding of the longitudinal tensile reinforcements and final failure of the beam. This mode of failure was relatively brittle and is often referred to as compression failure. These modes of failure are well-established for conventional reinforced concrete beams 
and based on the current results the flexural behaviour of RRC beams is not affected by the presence of RAC. This is true for both under- and over-reinforced beams. Any differences that were observed were minor with no practical significance.

\subsubsection{Effect of Tension Reinforcement}

Figure 6.18 compares the cracking, yielding and ultimate moment values of the beams made with RCA-MO and RCA-VA reinforced with different amounts of longitudinal tension reinforcement. Generally, the cracking moment was not found too sensitive to the longitudinal tension reinforcement ratio. Since all of the beams were cast simultaneously, the slight increase in cracking moment values can be mainly attributed to the larger depth of the beams with higher reinforcement ratios (Table 6.7). However, the yielding and ultimate moments of the beams increased dramatically as the tension reinforcement ratio increased. Since the section properties (the effective depth and width) and the material properties (concrete compressive strength and steel yield strength) were kept constant for all these beams, the above differences may be mainly attributed to the relative quantity of the tension steel in each beam. It is noteworthy to mention that in this study all of the beams were designed to fail in flexure in a tension failure mode. In other words, the steel was expected to reach its yield strength before concrete reaching its maximum capacity. Therefore, the steel content had a major effect on the ultimate capacity of the beams.

Figure 6.19 illustrates the moment-deflection curves of the flexural beams at different longitudinal tension reinforcement ratios. Generally, the ductility, expressed as the ratio of deflection at failure to deflection at yield, decreased as the tension steel content increased. In other words, the beams with lower steel content exhibited greater post yield deformation. From Table 6.8, it can be seen that the midspan deflections of FEM-Min 
and FEV-Min beams at failure are 3.43 and 3.26 times their yield deflections compared to 1.51 and 1.52 for beams FEM-Av and FEV-Av, respectively.

On the other hand, the midspan deflections of FEM-Max and FEV-Max beams at failure were 0.89 and 0.58 times those at yielding of the tension reinforcement respectively, which signifies the compression failure of these beams. In fact, the concrete reached its maximum capacity before the steel yielded, and consequently the concrete in the compression zone of the beam at midspan crushed and the load dropped immediately. This can be clearly observed in the moment-deflection curves by noticing the very steep descending branch of the curves immediately after the peak load. However, after the tension reinforcement yielded, the beam continued to carry some load, but with a steep rate of descent (see Fig.6.19).

Figure 6.20 illustrates the observed deflection curves at different load stages (i.e. before cracking, at cracking, after cracking, and at $40 \%, 60 \%, 80 \%$ and $100 \%$ of the ultimate load) for the singly reinforced beams with different longitudinal tension reinforcement ratios as recorded by the linear potentiometers placed along each beam. As expected, all the deflection curves were found to be symmetric and tend to follow a bell curvedeflection starting slowly at first, building to a peak at midspan, and then falling at about the same rate it climbed. Generally, the beams with minimum tension reinforcement ratio experienced higher curvatures and deflections at failure than the other load beams. In other words, the beams with lower steel content exhibited greater peak deflections and curvatures at failure.

Figure 6.21 illustrates the variation of the concrete compression strain along the beam for the singly reinforced beams with different longitudinal tension reinforcement ratios 
obtained from the recorded strains by the gauges along the beams. Generally, the variation of the concrete compression strain along all the beams was almost symmetric at the different load stages. As expected, the concrete compression strain along the beams increased by increasing the load, and the maximum concrete compression strain always occurred at midspan at all load levels. In the beams with minimum tension reinforcement, the variation of the concrete strain along the beams was very small up to $80 \%$ of the failure load. However, at failure, the concrete compression strain at midspan dramatically increased. In other words, the beams with lower steel content exhibited greater rate of increase in compression strain at midspan than the beams with higher reinforcement ratio as the approached their ultimate capacity.

Figure 6.22 illustrates the recorded variation of the longitudinal tension reinforcement strain along the beam for the singly reinforced beams with different longitudinal tension reinforcement ratios. Generally, the variation of the tension steel strain along all the beams was not found to be symmetric, but the maximum strain always occurred either at midspan or under the point loads at all load levels (except VA-EMV-Max). According to Fig.6.22, it can also be observed that the tension reinforcement strain near the supports (LS1 or LS7) was generally higher than that in the middle of the shear span (LS2 or LS6) or sometimes even higher than that under point loads (LS3 or LS5). This may be mainly due to presence of shear cracks and local bond failure near the supports.

\subsubsection{Effect of Compression Steel}

Generally, the presence of the compression steel increased the ultimate strength of the FEM-CMP and FEV-CMP beams compared to those of the FEM-Max and FEV-Max beams (11\% and 9\% respectively). In FEM-Max and FEV-Max beams, after concrete 
reached its maximum capacity, the load dropped immediately and the beams failed shortly after concrete crushing. However, in FEM-CMP and FEV-CMP beams, the addition of the compression steel had a twofold effect: (1) resulting in a decrease in the depth of the compression zone, and correspondingly leading to an increase in the tension reinforcement strain and a more ductile behaviour, and (2) sufficiently strengthening the compression zone to let the tensile reinforcement reach its yield strength before the concrete crushing. This is the main reason for the higher ultimate strengths and more ductility of FEM-CMP and FEV-CMP beams compared to those of FEM-Max and FEVMax beams.

Figure 6.23 shows the variation of the moment with midspan deflection for the beams with and without compression steel. The presence of the compression steel noticeably enhanced the performance of the RRC beams by increasing their ductility.

In FEM-max and FEV-max, firstly the concrete reached its maximum capacity and almost immediately after the longitudinal tensile reinforcements yielded and the beams failed. However, in FEM-CMP and FEV-CMP beams, the presence of the compression steel prevented the concrete from reaching its maximum capacity before tension yielding of the reinforcement, which in turn allowed the load to continue to increase up to failure and the beams exhibited a ductile behaviour. In other words, the presence of the compression steel changed the failure from compression to tension mode.

From Table 6.8, it can also be seen that the midspan deflections of FEM-Max and FEVMax beams at failure are 0.89 and 0.56 times those at yielding (confirming their compression failure mode), compared to 7.88 and 2.91 for FEM-CMP and FEV-CMP beams, respectively. This can also be observed in: (1) the moment-deflection curves by 
noticing the very long plateau of the moment-deflection curves of FEM-CMP and FEVCMP beams, and (2) the deflection curves at different load stages by noticing the high peak deflection and curvature of FEM-CMP and FEV-CMP beams (see Fig.6.24).

Figure 6.25 illustrates the variation of the concrete compression strain along the beam for the doubly reinforced beams obtained from the recorded strains along the beams at different load stages. Similarly to the singly reinforced beams, the variation of the concrete compression strain along all the beams increased as the load increased (except MO-EMV-CMP), and the maximum concrete compression strain always occurred at midspan at all load levels.

Figure 6.26 illustrates the recorded variations of the longitudinal tension reinforcement strain along the beam for the doubly reinforced beams with different longitudinal tension reinforcement ratios obtained. Generally, the variation of the tension steel strain along all the beams was not found to increase with increased load, and the maximum strain always occurred either at midspan or under the point loads at all load levels (except VA-EMVCMP). As mentioned earlier and for the same reasons, once again the tension reinforcement strain near the supports (LS1 or LS7) was generally higher than that in the middle of the shear span (LS2 or LS6) or sometimes even higher than that under the point loads (LS3 or LS5).

\subsubsection{Effect of Concrete Type}

In this section, the effect of concrete type on the ultimate flexural strength and flexural performance of the test beam will be presented. Figure 6.27 illustrates the cracking, yielding and ultimate moment values of the different RC beams made with the four concrete types in Table 6.6. 
Generally, the yielding and ultimate moment values of the RC beams made of EM and EV mixes are higher than those made of $\mathrm{CL}$ and $\mathrm{CG}$. According to Table 6.8, the ultimate moment values of FEM-Av and FEV-Av are $8 \%$ and $5 \%$ higher than those of FCL-Av and FCG-Av beams, and those of FEM-CMP and FEV-CMP beams are $7 \%$ and $8 \%$ higher compared to those of FCL-CMP and FCG-CMP beams, respectively. According to Table 6.8, the cracking moment of FEM-Av and FEV-Av are $28 \%$ and $21 \%$ lower than those of control FCL-Av and FCG-Av beams, respectively, while they are $16 \%$ lower and $26 \%$ higher for the FEM-CMP and FEV-CMP beams compared to the FCL-CMP and FCG-CMP beams, respectively.

Figure 6.28 illustrates the moment-deflection curves of the flexural beams made of the four concrete types in Table 6.6. Generally, the moment-deflection curves of all the beams were similar regardless of the concrete or aggregate type. According to Figure 6.28d, it can be observed that the flexural behaviour of FEM-CMP and FEV-CMP beams are more ductile compared to the control beams FCL-CMP and FCG-CMP, respectively. According to Figures 6.20 and 6.24, the deflection curves of all the beams at all load levels were also practically similar, regardless of the concrete or aggregate type. Furthermore, no major difference was observed among the beams with different concrete or aggregate types insofar as the concrete compression strain variation is concerned (Figs.21 and 25).

In fact, the similar flexural behaviour of these beams made with the different concrete and aggregate types and with different amounts of tensile reinforcement, with or without compression steel, verifies the applicability of the general theory of flexure for normal concrete to RAC proportioned by the proposed EMV method. It also verifies the concept 
of the proposed EMV mix proportioning method in which it is contended that the TM or TNA volumes are the key factors controlling different properties of RAC.

\subsubsection{Serviceability}

Assuming $40 \%$ of the ultimate load as the customary level service loads, the maximum deflection of each beam was obtained from its load-deflection curve, and is shown in Table 6.8.

According to Table 6.8, the $\delta_{s}$ value of beam FEM-Av is $33.3 \%$ lower than that of FCLAv, while that of FEV-Av is $10 \%$ higher compared to the corresponding value for FCGAv. However, the $\delta_{s}$ values in FEM-CMP and FEV-CMP beams are $6 \%$ and $5 \%$ higher than those in FCL-CMP and FCG-CMP, respectively. Observe that this is partially due to the higher service loads in RRC beams compared to those of control RC beams. Despite these differences, all of the midspan deflection values at service load $\left(\delta_{s}\right)$ are within the maximum permissible deflection $\left(l_{n} / 480=4.6 \mathrm{~mm}\right)$ recommended by CSA A23.3-04 (clause 9.8.5.3) for the beams supporting or attached to non-structural elements likely to be damaged by large deflections.

Figures 6.29 and 6.30 illustrate the cracking spacing at service load for FEM and FEV beams and their corresponding control beams, respectively. Generally, the RC beams made with RAC experienced slightly smaller cracker spacing compared to those made of NAC. Unfortunately, due to the presence of the potentiometers and other instrumentation, crack widths could not be measured, but visual observations did not show any significant difference among the crack widths of the beams. 


\subsection{Summary}

- The bond strength of RAC mixes proportioned by the conventional method was found to be lower than that of companion NAC. In contrast, the bond strength of RAC mixes proportioned by the EMV method was comparable to that of companion NAC. The lower TNA volume or higher TM volume in RAC proportioned by the conventional method appears to decrease the bond capacity due to less interlocking of the ribs on the deformed test bars with the surrounding concrete.

- The bond strength values of all the specimens with bar size No. 15 were found to be higher than those specimens with bar No.30, irrespective of mix proportioning method and aggregate source.

- Despite the lower bond strength of specimens made of conventional RAC and/or containing bar No.30, the overall bond behaviours of all the specimens were almost identical, regardless of the mix proportioning method, aggregate source (RCA-MO or RCA-VA, and NL or NG), or concrete type (RAC or NAC).

- There was no major difference observed between the failure modes of RRC and conventional RC beams under flexure. Similar failure modes were observed for all the RRC beams regardless of the RCA source.

- Despite slightly smaller crack spacing in RRC beams compared to conventional RC beams, there was no significant difference observed among their cracking patterns at failure.

- The cracking moment of the RC beams, with or without compression steel and made with RAC, was generally smaller than cracking moment of the companion beams made with NAC. 
- The ultimate flexural strengths of all RRC beams were always higher than those of the conventional RC beams for both RCA-MO and RCA-VA sources, regardless of tension or compression steel content.

- Generally, the addition of compression steel enhanced the performance of the RRC beams containing maximum tension reinforcement by changing the compression failure mode to tension mode and increasing their ductility. This is in compliance with the observed behaviour of conventional RC beams.

- There was no difference observed between the flexural behaviour of RRC and conventional RC beams, which validates the applicability of the general flexural theory of conventional RC to RRC.

- The midspan deflections of all the RRC and RC beams at service load were found to be within the maximum permissible values recommended by CSA A23.3-04 for elements supporting or attached to non-structural elements likely to be damaged by large deflections. 
Table 6.1: Fresh and hardened properties of bond specimens

\begin{tabular}{|c|c|c|c|c|c|c|}
\hline \multirow{2}{*}{$\begin{array}{c}\text { Mix } \\
\text { ID }\end{array}$} & \multicolumn{2}{|c|}{ Fresh Properties } & \multicolumn{2}{c|}{$\begin{array}{c}\mathrm{f}_{\mathrm{c}} \\
(\mathrm{MPa})\end{array}$} & \multirow{2}{*}{$\begin{array}{c}\text { Hardened } \\
\gamma_{\mathrm{c}} \\
\left(\mathrm{kg} / \mathrm{m}^{3}\right)\end{array}$} \\
\cline { 2 - 6 } & $\begin{array}{c}\text { Slump } \\
(\mathrm{mm})\end{array}$ & $\begin{array}{c}\text { Air } \\
\text { Content } \\
\%\end{array}$ & $\begin{array}{c}\text { Fresh } \\
\gamma_{\mathrm{c}} \\
\left(\mathrm{kg} / \mathrm{m}^{3}\right)\end{array}$ & 28-days & Test date & \\
\hline CM & 75 & 5.9 & 2298 & 43.9 & 48.5 & 2290 \\
\hline EM & 95 & 6.4 & 2341 & 42.8 & 36.9 & 2333 \\
\hline CL & 185 & 5.9 & 2333 & 37.1 & 38.0 & 2308 \\
\hline CV & 25 & 5 & 2378 & 45.9 & 49.0 & 2348 \\
\hline EV & 60 & 4.5 & 2398 & 49.1 & 43.5 & 2364 \\
\hline CG & 220 & 6.4 & 2347 & 33.8 & 35.9 & 2308 \\
\hline
\end{tabular}

Table 6.2: Details of bond specimens

\begin{tabular}{|c|c|c|c|c|c|}
\hline Specimen ID & Test bar & Flexural bar & $\begin{array}{c}\mathbf{L}_{\mathbf{b}} \\
(\mathbf{m m})\end{array}$ & $\begin{array}{c}\mathbf{C}_{\mathbf{1}} \\
(\mathbf{m m})\end{array}$ & $\begin{array}{c}\mathbf{C}_{\mathbf{2}} \\
(\mathbf{m m})\end{array}$ \\
\hline CM-30 & No. 30 & No. 25 & 262 & 70 & 50 \\
\hline EM-30 & No. 30 & No. 25 & 250 & 70 & 50 \\
\hline CL-30 & No. 30 & No. 25 & 280 & 70 & 50 \\
\hline CM-15 & No. 15 & No. 10 & 279 & 60 & 45 \\
\hline EM-15 & No. 15 & No. 10 & 261 & 60 & 45 \\
\hline CL-15 & No. 15 & No. 10 & 320 & 60 & 45 \\
\hline CV-30 & No. 30 & No. 25 & 263 & 70 & 50 \\
\hline EV-30 & No. 30 & No. 25 & 260 & 70 & 50 \\
\hline CG-30 & No. 30 & No. 25 & 265 & 70 & 50 \\
\hline CV-15 & No. 15 & No. 10 & 287 & 60 & 45 \\
\hline EV-15 & No. 15 & No. 10 & 274 & 60 & 45 \\
\hline CG-15 & No. 15 & No. 10 & 291 & 60 & 45 \\
\hline
\end{tabular}

Table 6.3: Mechanical properties of steel bars used for bond test

\begin{tabular}{|c|c|c|c|c|c|c|c|}
\hline $\begin{array}{c}\text { Bar } \\
\text { Size }\end{array}$ & $\begin{array}{c}\text { Diameter } \\
(\mathbf{m m})\end{array}$ & $\begin{array}{c}\text { Cross- } \\
\text { sectional } \\
\text { area } \\
\left(\mathbf{m m}^{2}\right)\end{array}$ & $\begin{array}{c}\text { Yield } \\
\text { stress } \\
(\mathbf{M P a})\end{array}$ & $\begin{array}{c}\text { Ultimate } \\
\text { Strength } \\
\mathbf{( M P a})\end{array}$ & $\begin{array}{c}\text { Elastic } \\
\text { modulus } \\
\mathbf{( G P a )}\end{array}$ & $\begin{array}{c}\text { Elongation } \\
\mathbf{( \% )}\end{array}$ & $\begin{array}{c}\text { Yield } \\
\text { strain } \\
(\%)\end{array}$ \\
\hline No. 8 & 8 & 50 & 530 & 596 & 192 & 4.44 & 0.276 \\
\hline No. 10 & 11.3 & 100 & 473 & 572 & 178 & 11.50 & 0.266 \\
\hline No. 15 & 16 & 200 & 407 & 642 & 175 & 10.01 & 0.232 \\
\hline No. 20 & 19.5 & 300 & 418 & 466 & 166 & 10.82 & 0.252 \\
\hline No. 25 & 25.2 & 500 & 431 & 669 & 176 & 14.29 & 0.246 \\
\hline No. 30 & 29.9 & 700 & 449 & 733 & 184 & 14.43 & 0.244 \\
\hline
\end{tabular}


Table 6.4: Summary of bond tests results

\begin{tabular}{|c|c|c|c|}
\hline Specimen ID & $\begin{array}{c}\text { Bond strength, } \tau_{b} \\
\text { (MPa) }\end{array}$ & $\frac{\tau_{b}}{\sqrt{f_{c}^{\prime}}}$ & Failure Type \\
\hline CM-30 & 6.01 & 0.86 & Bond failure \\
\hline EM-30 & - & - & - \\
\hline CL-30 & 6.95 & 1.13 & Bond failure \\
\hline CM-15 & 8.10 & 1.16 & Bond failure \\
\hline EM-15. & 810.68 & 71.76 & Bar faifure \\
\hline cr-15. & 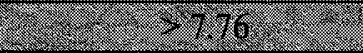 & 1.26 & Bar failure \\
\hline CV-30 & 6.29 & 0.90 & Bond failure \\
\hline EV-30 & 7.36 & 1.12 & Bond failure \\
\hline CG-30 & 7.13 & 1.19 & Bond failure \\
\hline$C V-15$ & 88.02 & -1.15 & Bar failure \\
\hline$E V-15$ & 310.35 & 3.57 & Bar failure \\
\hline CG-15 & $20=37.81$ & 281.30 & Bar atlure \\
\hline
\end{tabular}

Table 6.5: Mix proportions of flexural RRC and control beams

\begin{tabular}{|c|c|c|c|c|c|c|c|c|}
\hline \multirow{3}{*}{ Beam ID } & \multirow{3}{*}{$\begin{array}{c}\text { RCA } \\
\text { Content } \\
\%\end{array}$} & \multicolumn{7}{|c|}{ Mix Proportions $\left(\mathrm{kg} / \mathrm{m}^{3}\right)$} \\
\hline & & \multirow{2}{*}{ Water } & \multirow[t]{2}{*}{ Cement } & \multirow[t]{2}{*}{ Sand } & \multicolumn{2}{|c|}{$\begin{array}{c}\text { Coarse } \\
\text { Aggregate }\end{array}$} & \multirow[t]{2}{*}{ WRA } & \multirow{2}{*}{$\mathbf{A E}$} \\
\hline & & & & & RCA & NA & & \\
\hline FEM & 63.5 & 151 & 335 & 630 & 720 & 414 & 1005 & 53 \\
\hline FCL & 0 & 193 & 430 & 808 & 0 & 835 & None & 86 \\
\hline FEV & $\overline{74.3}$ & 161 & 358 & 645 & 813 & 281 & 1433 & 68 \\
\hline FCG & 0 & 191 & 424 & 763 & 0 & 900 & None & 85 \\
\hline
\end{tabular}

Table 6.6: Fresh and hardened properties of flexural RRC and control beams

\begin{tabular}{|c|c|c|c|c|c|c|c|c|c|c|}
\hline \multirow{3}{*}{$\begin{array}{c}\text { Mix } \\
\text { ID }\end{array}$} & \multirow{2}{*}{\multicolumn{3}{|c|}{ Fresh Properties }} & \multicolumn{6}{|c|}{ Hardened properties } & \multirow{3}{*}{$\begin{array}{c}\text { Hardened } \\
\gamma_{\mathrm{c}} \\
\left(\mathrm{kg} / \mathrm{m}^{3}\right)\end{array}$} \\
\hline & & & & \multicolumn{2}{|c|}{$\mathrm{f}_{\mathrm{c}}(\mathrm{MPa})$} & \multicolumn{2}{|c|}{$\mathrm{E}_{\mathrm{c}}(\mathrm{GPa})$} & \multicolumn{2}{|c|}{$\mathrm{f}_{\mathrm{c}}(\mathrm{MPa})$} & \\
\hline & $\begin{array}{l}\text { Slump } \\
(\mathrm{mm})\end{array}$ & $\begin{array}{c}\text { Air } \\
\text { Content } \\
\% \\
\end{array}$ & $\begin{array}{c}\text { Fresh } \\
\gamma_{\mathrm{c}} \\
\left(\mathrm{kg} / \mathrm{m}^{3}\right)\end{array}$ & $\begin{array}{c}28 \\
\text { days }\end{array}$ & $\begin{array}{l}\text { Test } \\
\text { date }\end{array}$ & $\begin{array}{c}28 \\
\text { days }\end{array}$ & $\begin{array}{l}\text { Test } \\
\text { date }\end{array}$ & $\begin{array}{c}28 \\
\text { days }\end{array}$ & $\begin{array}{l}\text { Test } \\
\text { date }\end{array}$ & \\
\hline EM & 96 & 6.4 & 2341 & 41.6 & 36.9 & 29.8 & 24.6 & 3.4 & 2.8 & 2333 \\
\hline CL & 185 & 5.9 & 2333 & 37.1 & 38 & 30.3 & 24.5 & 3.2 & 3.0 & 2308 \\
\hline EV & 60 & 4.5 & 2398 & 49.1 & 43.5 & 31.8 & 27.1 . & 3.7. & 3.4 & 2364 \\
\hline CC & 220 & 6.45 & 2347 & 33.8 & 35.9 & 30.5 & $\sqrt{27.9}$ & 33 & 3.2 & 2308 \\
\hline
\end{tabular}


Table 6.7: Structural details of RRC beams tested in flexure

\begin{tabular}{|c|c|c|c|c|c|c|}
\hline Beam ID & a/d & $\begin{array}{c}\text { h } \\
(\mathrm{mm})\end{array}$ & $\begin{array}{c}\mathrm{d} \\
(\mathrm{mm})\end{array}$ & $\begin{array}{c}\mathbf{A}_{\mathbf{s}} \\
\left(\rho_{\mathrm{s}} \%\right)\end{array}$ & $\mathbf{A}^{\prime}{ }_{s}$ & $\begin{array}{c}\mathbf{L} \\
(\mathbf{m m})\end{array}$ \\
\hline FEM-Min & 2.63 & 350 & 304 & 3 No. $10(0.493)$ & 2 No. 10 & 2600 \\
\hline FEM-AY & 2.66 & 375 & 301 & $2 \mathrm{No} .20+3 \mathrm{No} .15(1.991)$ & $2 N 0910$ & 2600 \\
\hline $\mathrm{FC}$ & 2.66 & 375 & 301 & $2 \mathrm{No} 0.20+3 \mathrm{No} .15(1.991)$ & $2 \mathrm{No.10}$ & 2600 \\
\hline FEM-Max & 2.61 & 390 & 307 & 2 No. $25+2$ No.25 (3.256) & 2No.10 & 2760 \\
\hline EES & 2.65 & 385 & 302 & $2 \mathrm{No} .25+2 \mathrm{No} .25(3.310)$ & $2 \mathrm{No} .25$ & 2700 \\
\hline FCL-CMP & 2.65 & 385 & 302 & $2 \mathrm{No} 0.25+2 \mathrm{No} .25(3.310)$ & $2 \mathrm{No} .25$ & 2700 \\
\hline FEV-Min & 2.63 & 350 & 304 & 3No.10 (0.493) & 2 No. 10 & 2600 \\
\hline FEY-AY & 2.66 & 313 & 301 & $2 \mathrm{No} .20+3 \mathrm{No}: 15(1.991)$ & $2 \mathrm{No} .10$ & 2600 \\
\hline FCC-AV & 2.66 & 375 & 301. & $2 \mathrm{No} .20+3 \mathrm{No} .15(1.991)$ & $2 N 010$ & 2600 \\
\hline FEV-Max & 2.61 & 390 & 307 & $2 \mathrm{No} .25+2 \mathrm{No} .25(3.256)$ & $2 \mathrm{No} .10$ & 2760 \\
\hline REV-cMP: & 2.65. & 385 & 302 & $2 \mathrm{No} .25+2 \mathrm{No} .25(3.310)$ & $2 N 0.25$ & 2700 \\
\hline & & & & & & 2700 \\
\hline
\end{tabular}

Note: $\mathrm{a}=$ shear span, $\mathrm{d}=$ beam effective depth, $\mathrm{h}=$ beam height, $\mathrm{A}_{\mathrm{s}}=$ total area of tension steel, $\mathrm{A}^{\prime}{ }_{\mathrm{s}}=$ total area of compression steel

Table 6.8: Summary test results for RRC beams under flexure

\begin{tabular}{|c|c|c|c|c|c|c|c|c|c|}
\hline \multirow[t]{2}{*}{ Beam ID } & \multicolumn{4}{|c|}{$\begin{array}{c}\text { Experimental moment } \\
(\mathrm{KN}-\mathrm{m})\end{array}$} & \multicolumn{4}{|c|}{$\begin{array}{c}\text { Experimental midspan } \\
\text { deflection } \\
(\mathrm{mm})\end{array}$} & \multirow{2}{*}{$\begin{array}{r}\varepsilon_{\mathrm{cu}} \\
\times 10^{-3}\end{array}$} \\
\hline & $\mathbf{M}_{\mathbf{c r}}$ & $\mathbf{M}_{\mathbf{y}}$ & & $\mathbf{M}_{\mathbf{u}}$ & $\delta_{\text {cr }}$ & $\delta_{s}$ & $\boldsymbol{\delta}_{\mathbf{y}}$ & $\boldsymbol{\delta}_{\mathbf{u}}$ & \\
\hline FEM-Min & 13.0 & 38.4 & 6.0 & 46.0 & 0.26 & 1.54 & 8.0 & 27.4 & 2.369 \\
\hline TEM-AV & 13.8 & 123.4 & 149.2 & 149.2 & 0.07 & 1.70 & 8.0 & 12.1 & 3.330 \\
\hline FCL" & 19.2 & 106.0 & 142.7 & 142.7 & 0.35 & 2.55 & 6.2 & 11.5 & 2.666 \\
\hline FEM-Max & 21.1 & 210.6 & 221.9 & 221.9 & 0.06 & 3.04 & 11.9 & 10.6 & 1.763 \\
\hline ILM CMP & 24.7 & 193.0 & 219.2 & 246.1 & 0.31 & 3.62 & 8,9 & 70.1 & 3.426 \\
\hline FCL-CMP & 29.5 & 160.0 & 217.5 & 229.1 & 0.03 & 3.40 & 9.2 & 51.0 & \\
\hline FEV & 16.2 & 39.9 & 46.7 & 46.7 & 0.39 & 1.04 & 8.9 & 29.0 & 2.750 \\
\hline FEV-AY & 15.2 & 136.6 & 150.2 & 150.2 & 0.36 & 2.67 & -7.5 & 11.4 & 3.056 \\
\hline $\operatorname{LCCAx}$ & 19.2 & 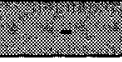 & 139.1 & 139.1 & 0.23 & 2.43 & $\sqrt{2-2}$ & 12.8 & 2.967 \\
\hline FEV-Max & 13.4 & 207.5 & 225.2 & 225.2 & 0.12 & 2.63 & 15.7 & 9.1 & 2.150 \\
\hline IEV-CMP & 29.1 & 220.2 & 221.1 & 245.7 & 0.41 & 3.67 & 212 & 61.7 & 0.578 \\
\hline FrCEAMP & 23.0 & 158.9 & 2072 & 226.5 & 0.00 & 3.5 & 7.4 & 487 & 1.62 \\
\hline
\end{tabular}

Note: $\mathrm{M}=$ Moment; subscript $c r, s, y$ and $u$ denote cracking, service, yield and ultimate, $\delta=$ deflection, $\varepsilon_{\mathrm{cu}}=$ maximum concrete compressive strain measured at ultimate 


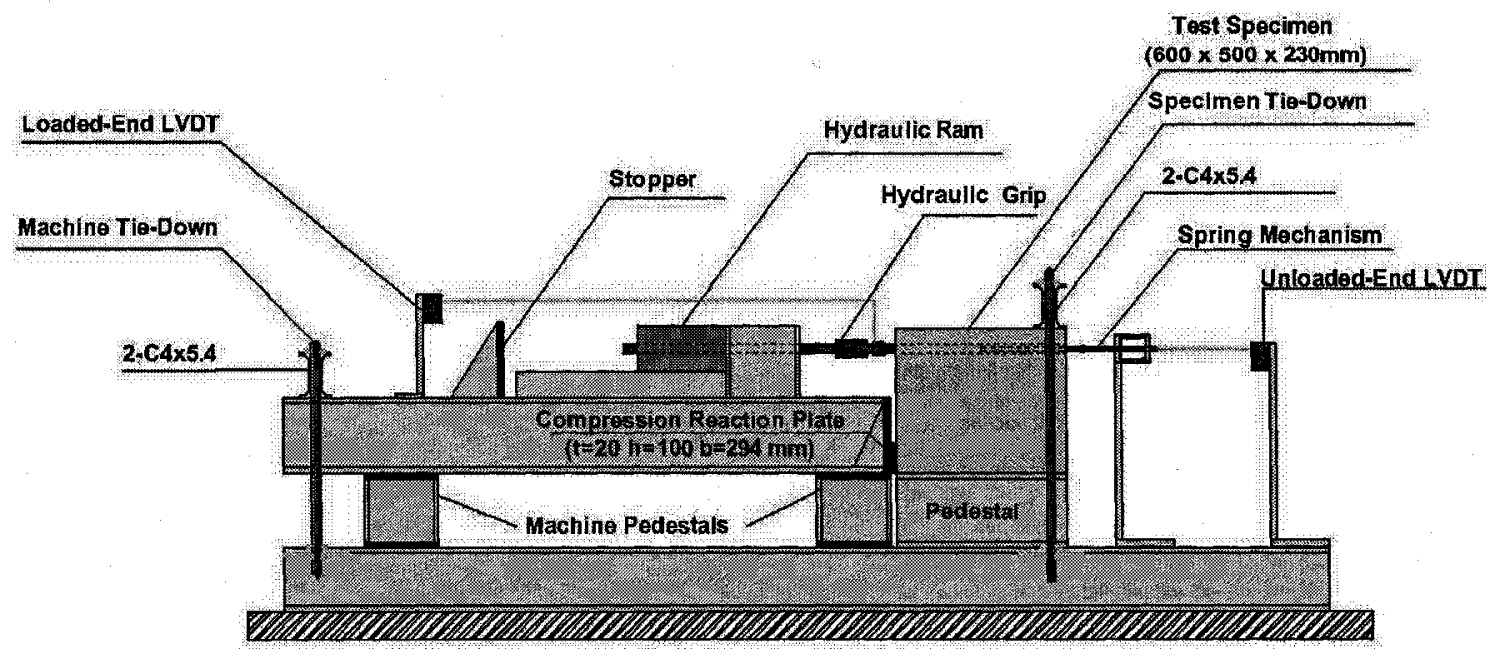

Laboratory Floor

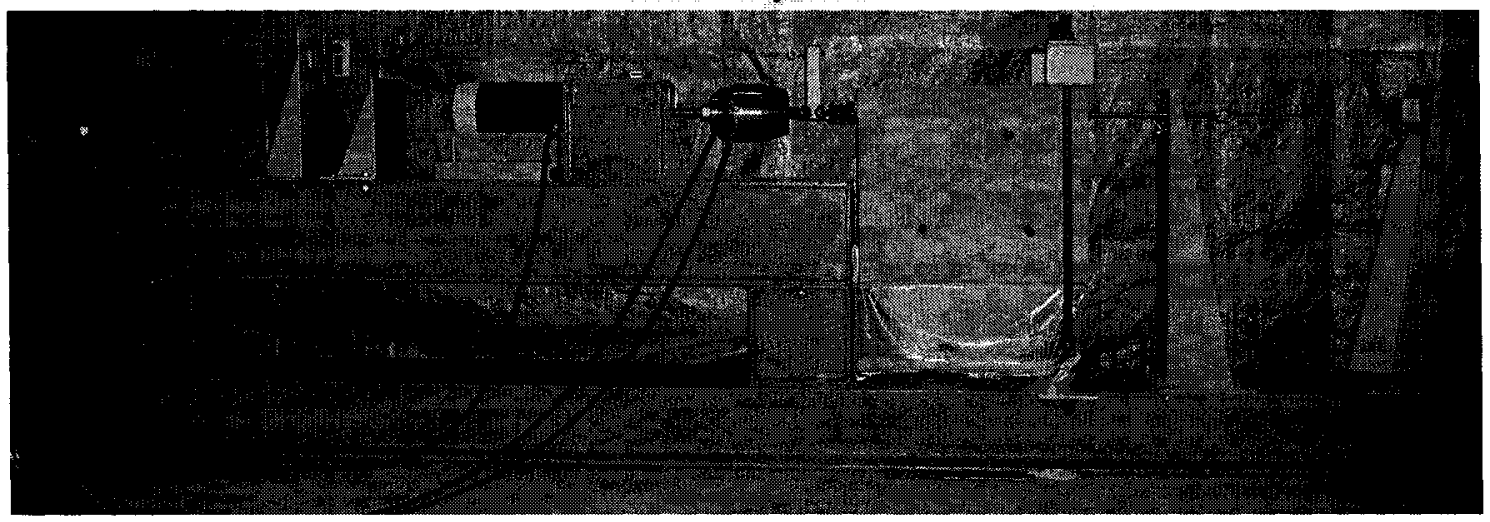

Figure 6.1: Schematic of beam-end bond test setup 


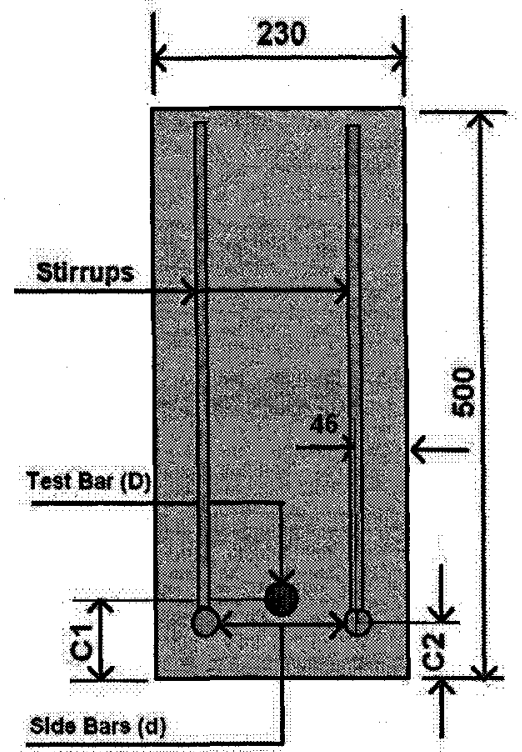

Typical Section

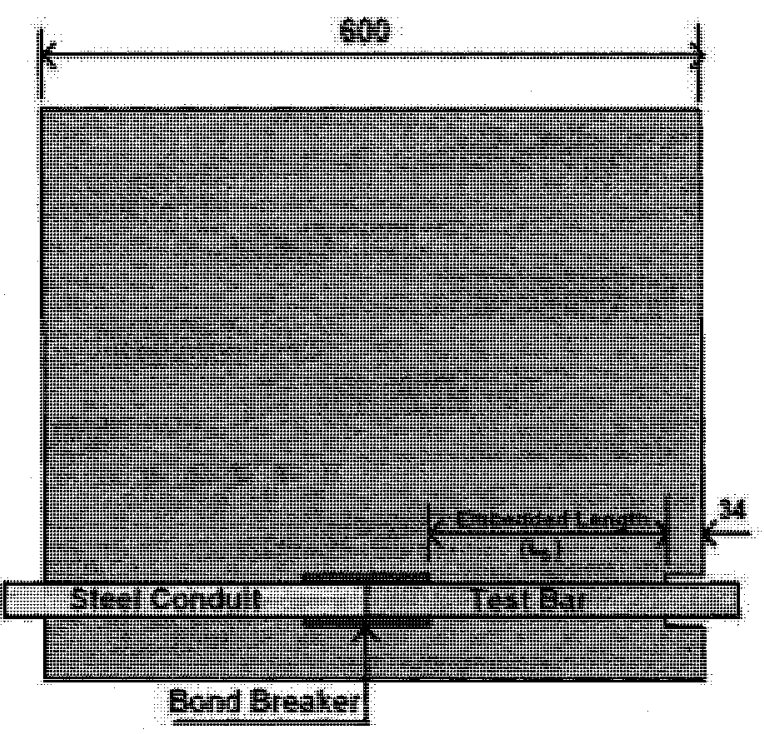

Front View

Figure 6.2: Details of bond specimen 

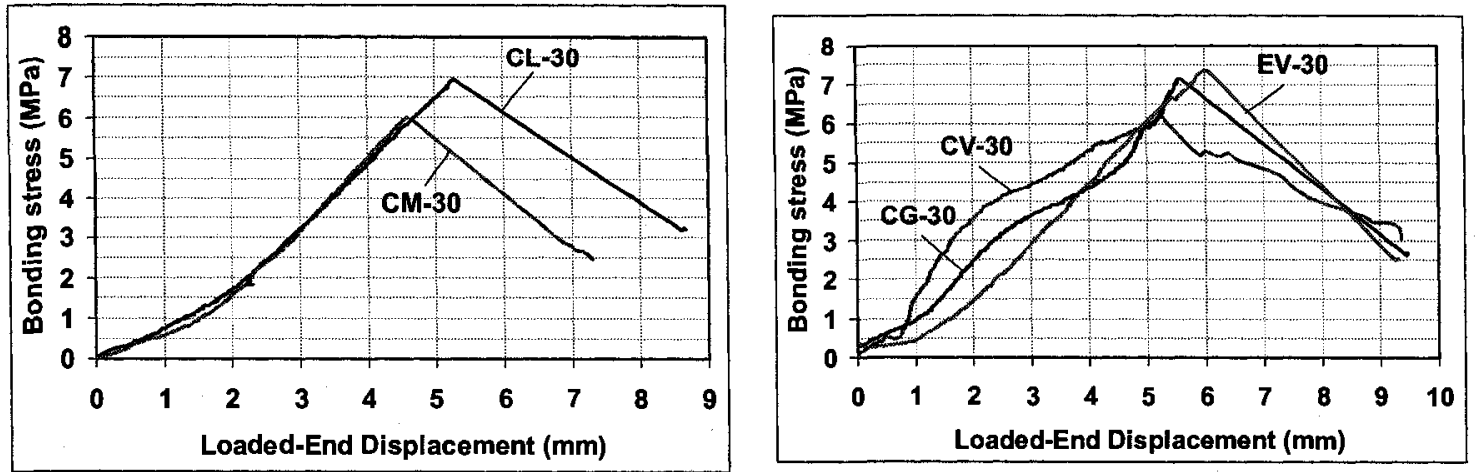

a) Bar size No.30
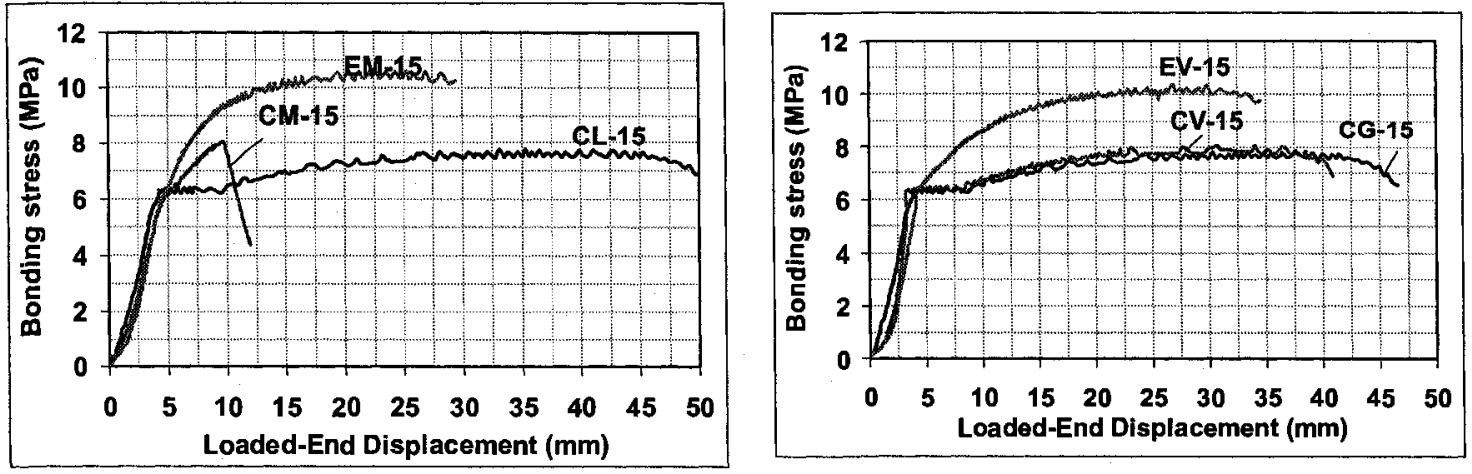

b) Bar size No.15

Figure 6.3: Effect of mix proportioning method on the bond stress loaded-end response
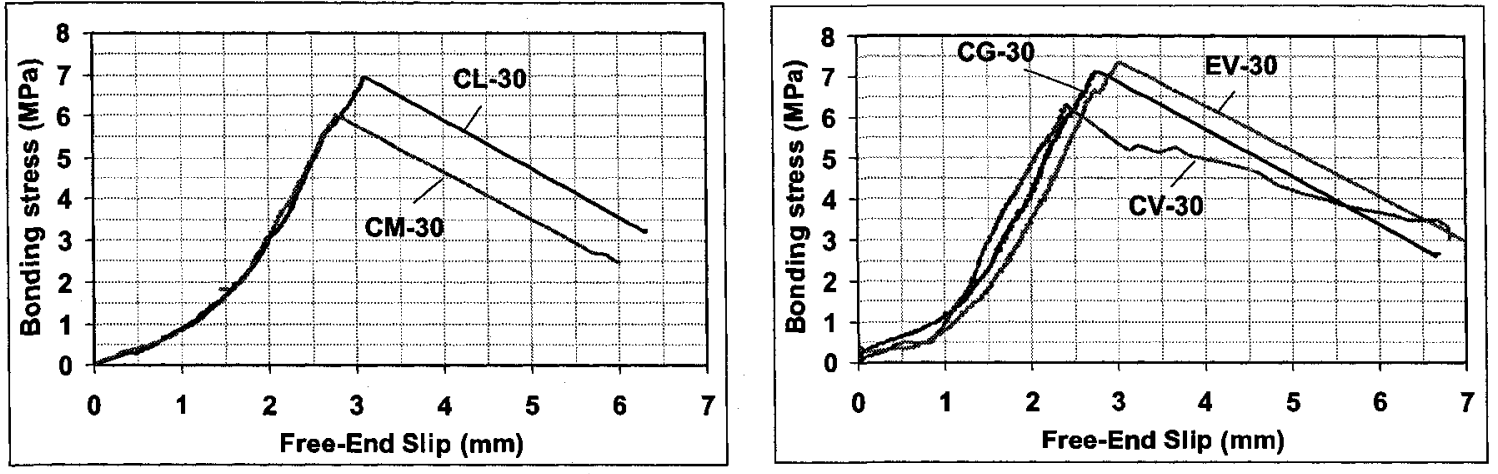

a) Bar size No.30
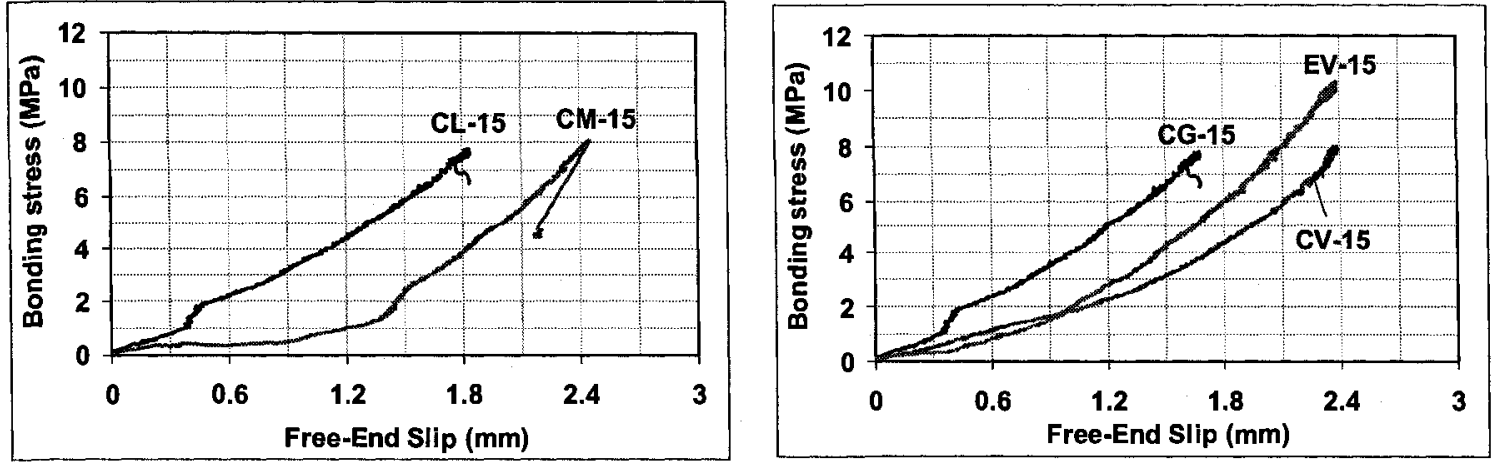

b) Bar size No.15

Figure 6.4: Effect of mix proportioning method on the bond stress-free end slip response 


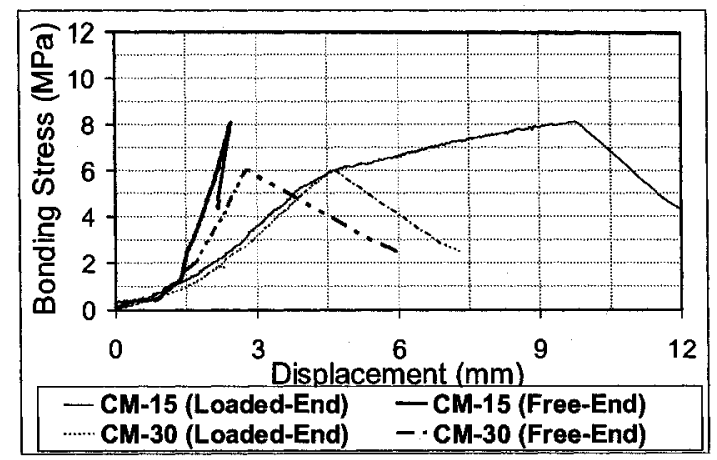

a) $\mathrm{CM}$

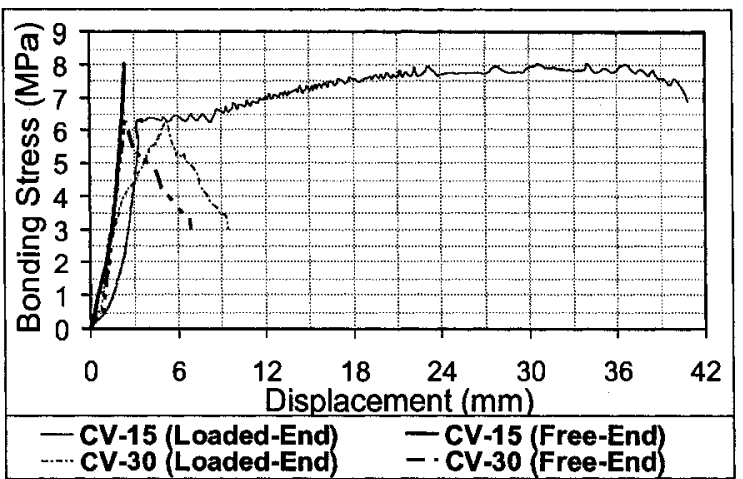

b) $\mathrm{CV}$

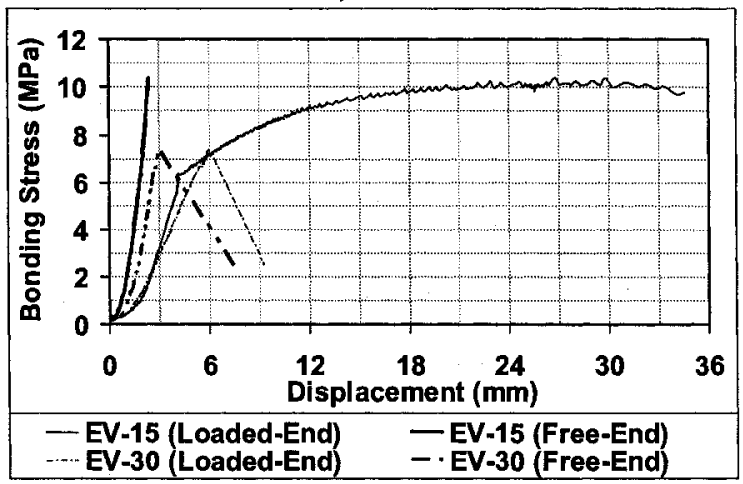

c) $\mathrm{EV}$

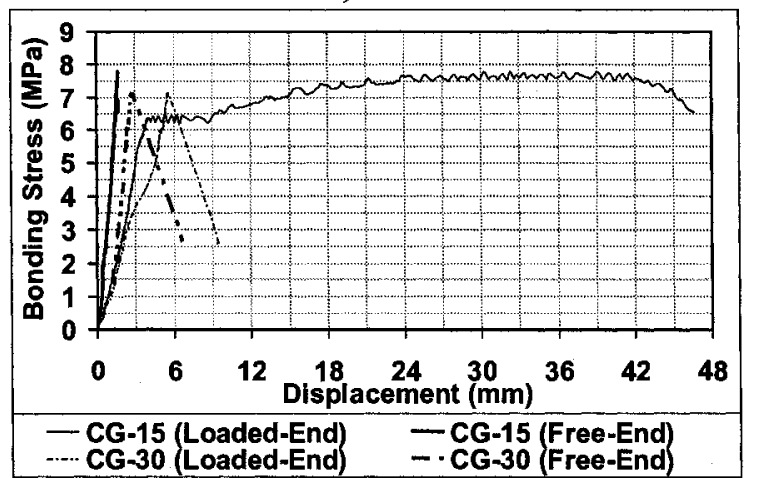

e) $\mathrm{CG}$

d) $\mathrm{CL}$ - CL-15 (Free-End)
- CL-30 (Free-End)

ffect of bar size on the bond stress variation with loaded and free-end displacements 


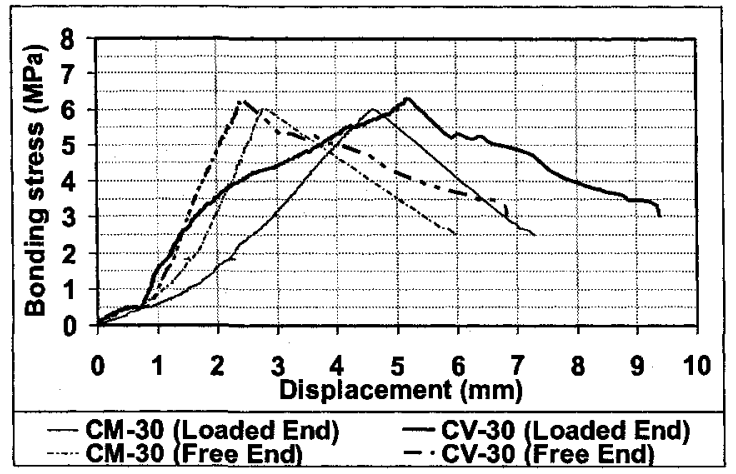

Bar size \#30

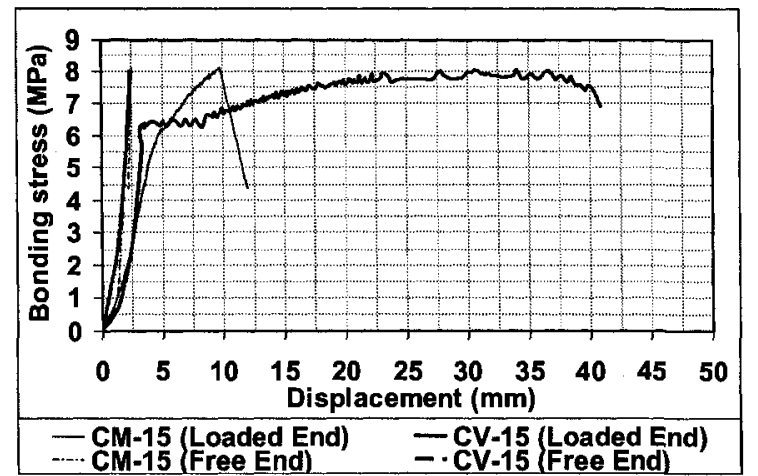

Bar size \#15

a) $\mathrm{CM}$ and $\mathrm{CV}$

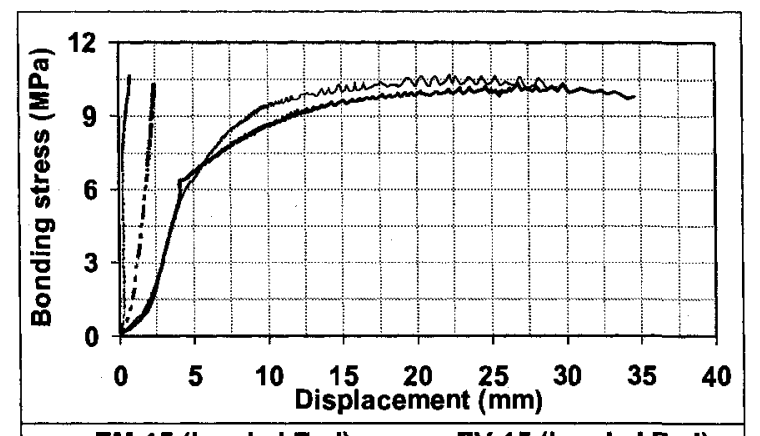

- EM-15 (Loaded End) - EV-15 (Loaded End) EM-15 (Free End) $-\cdot E V-15$ (Free End)

b) EM and EV

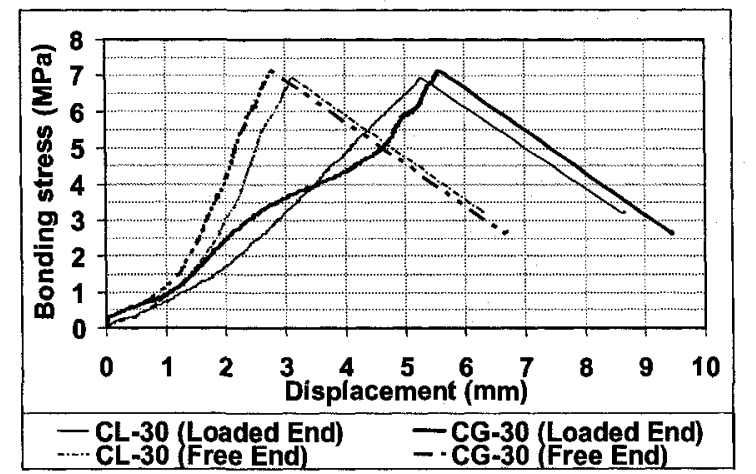

Bar size \#30

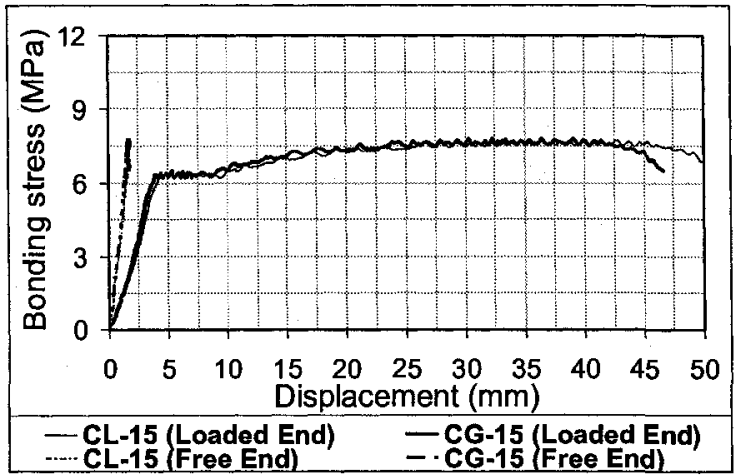

Bar size \#15

c) CL and CG

Bar size \#15

Figure 6.6: Effect of aggregate type on the bond stress variation with loaded and freeend displacements 

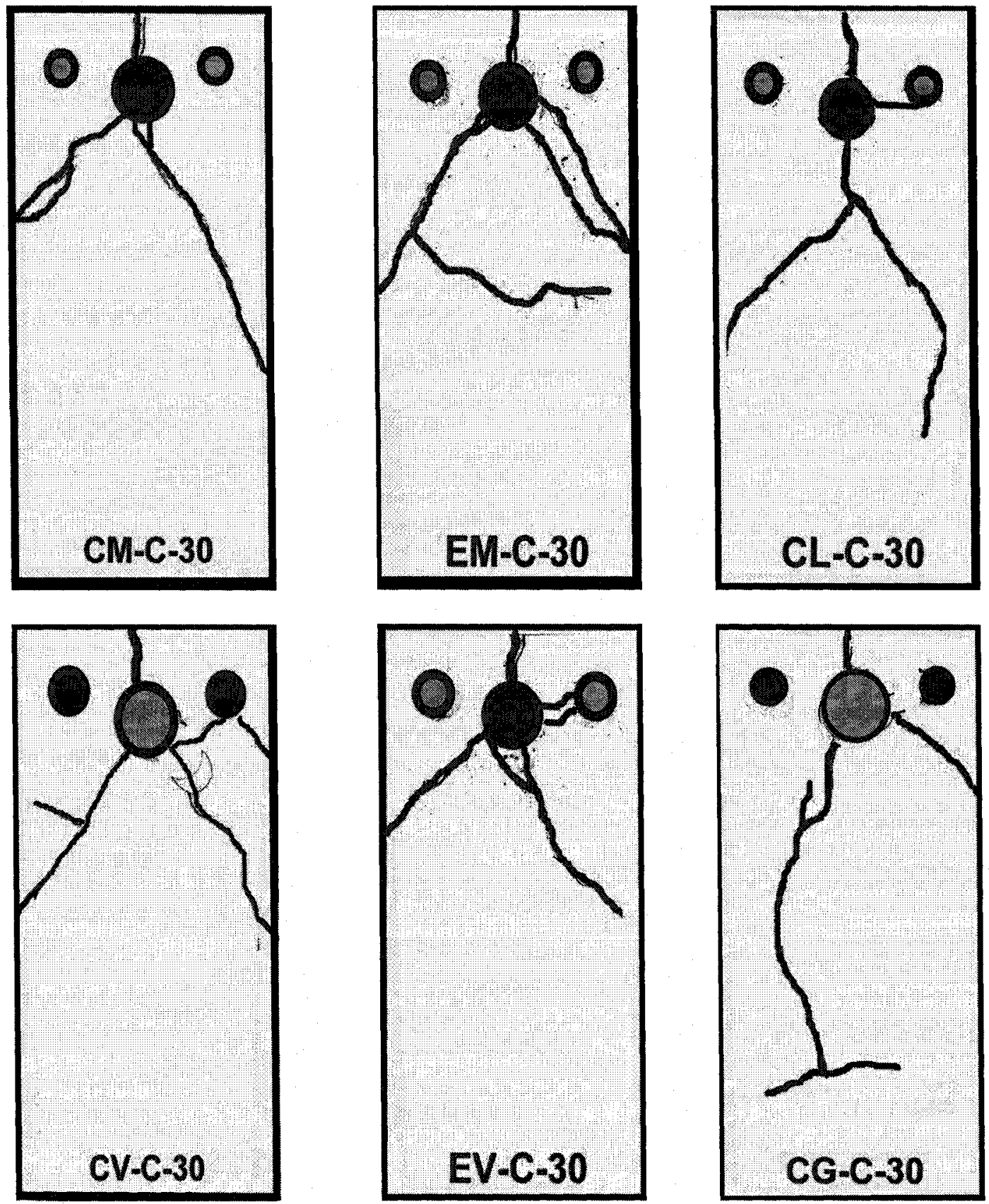

Figure 6.7: Cracking patterns of bond specimens using bar No.30: Front view 

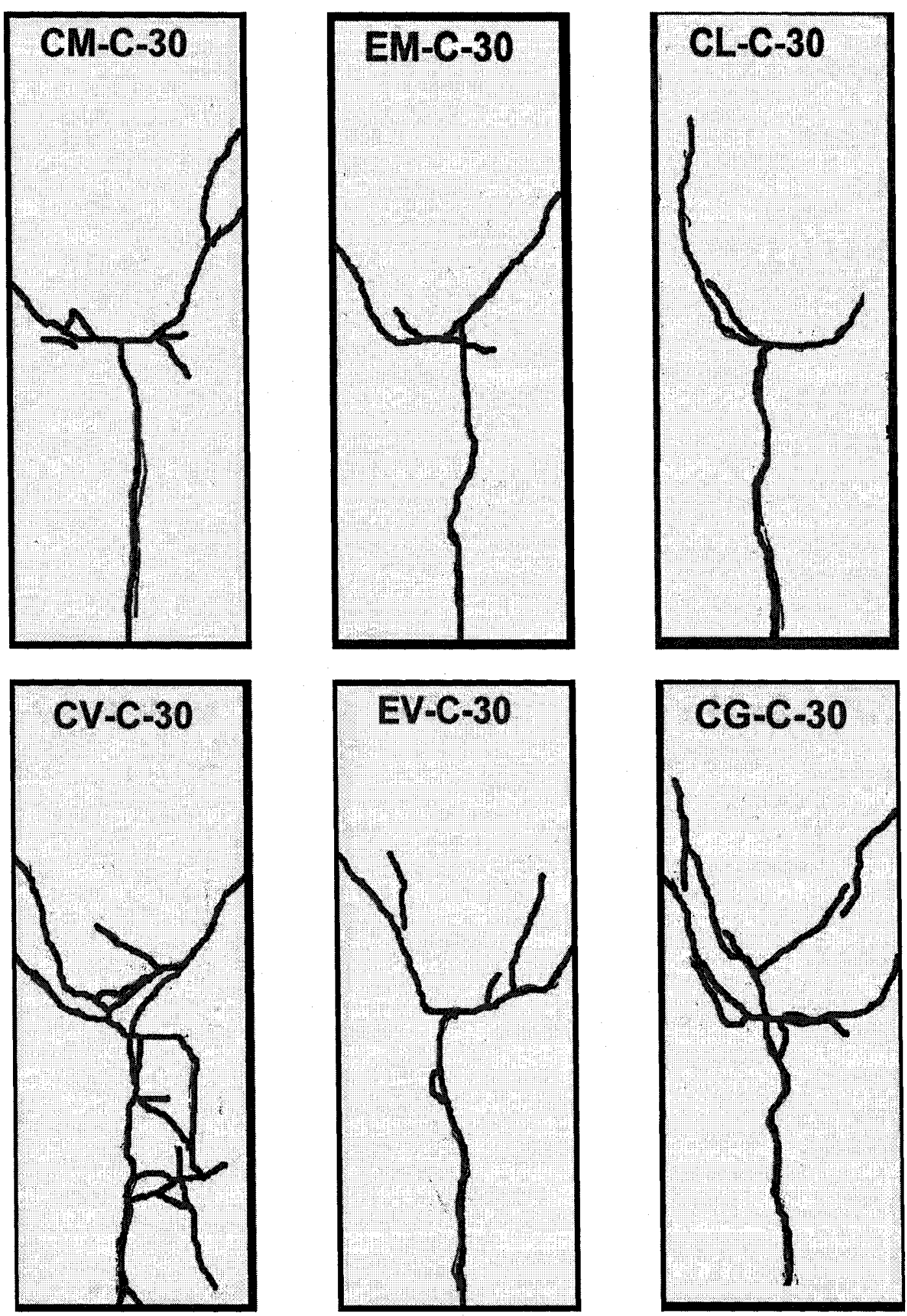

Figure 6.8: Cracking patterns of bond specimens using bar No. 30: Top view 


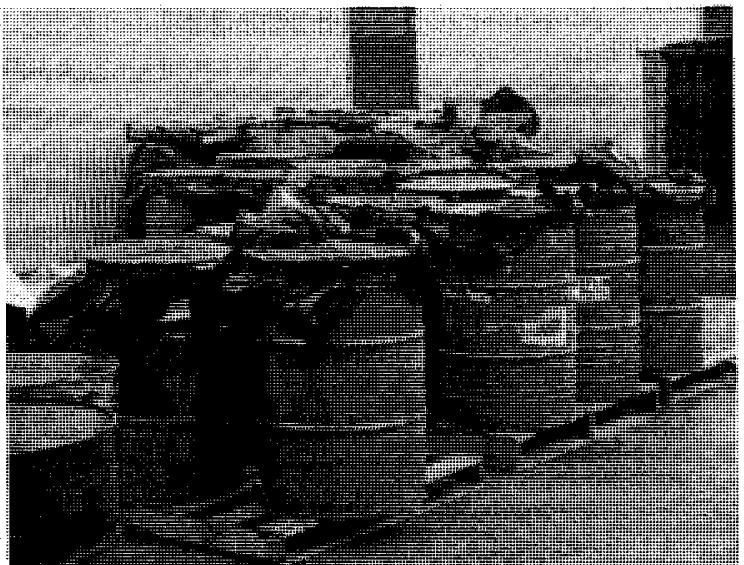

a) Covered drums of fine/coarse Aerredestes

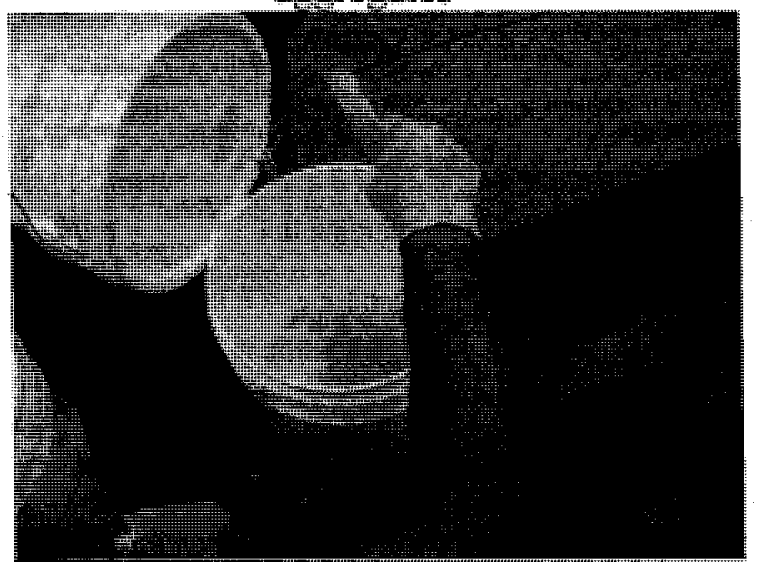

c) Air-entraining admixture solution

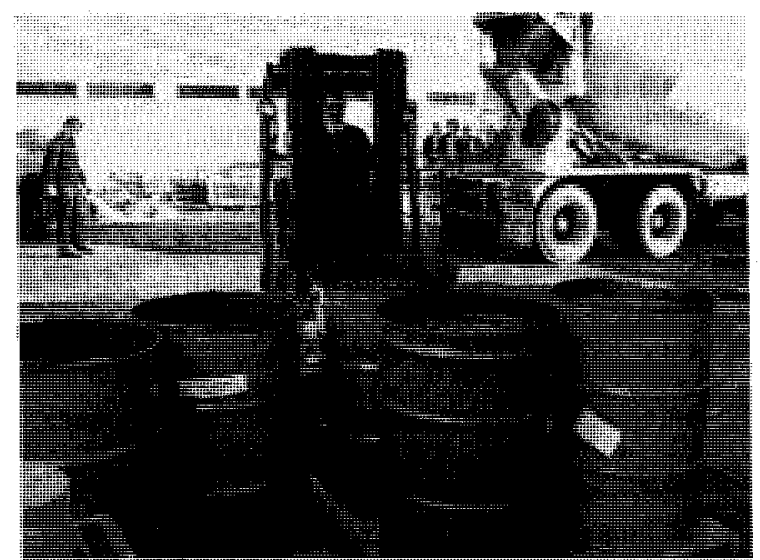

e) Taking fine aggregate to truck mixer

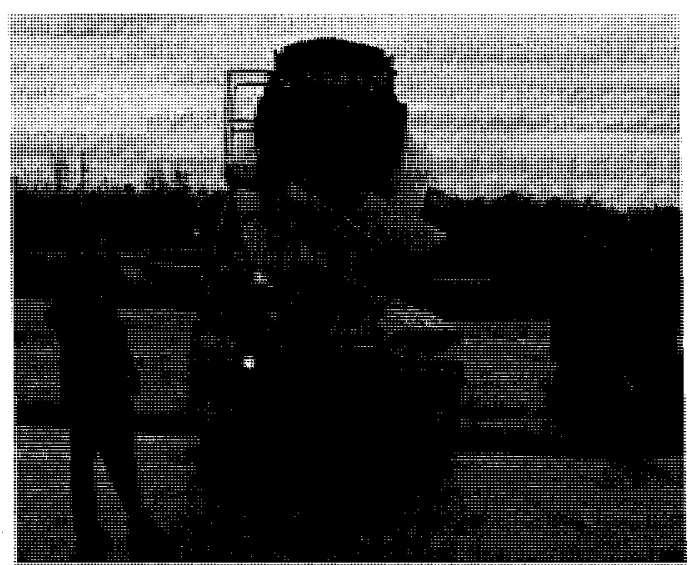

b) Truck mixer

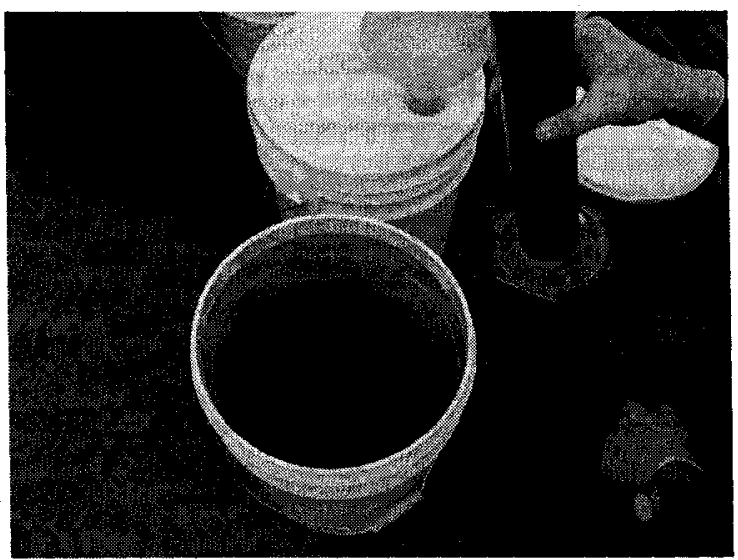

d) Water reducing admixture to water paits

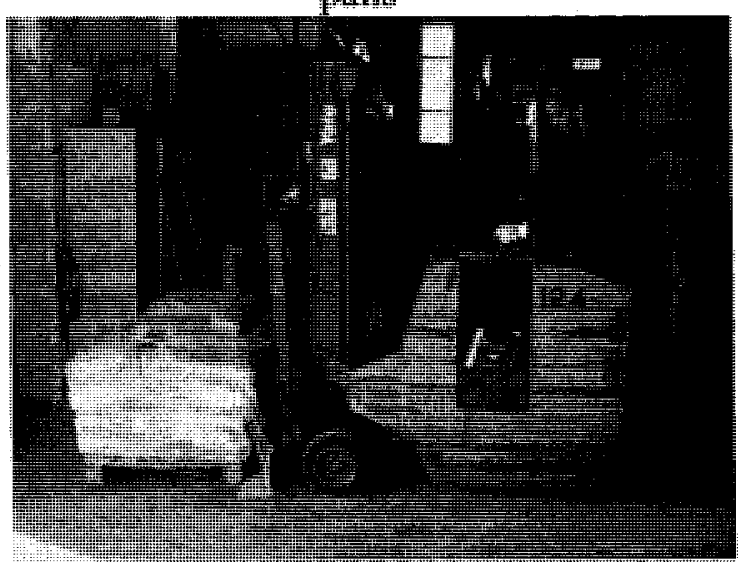

f) Taking cement bags to truck mixer Figure 6.9: Preparation of materials prior to mixing 


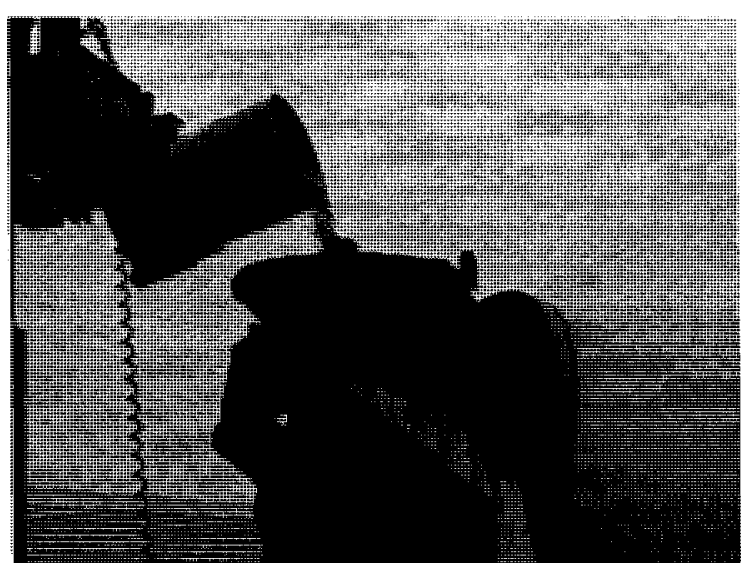

a) Loading the truck mixer with coarse and fine ageregates

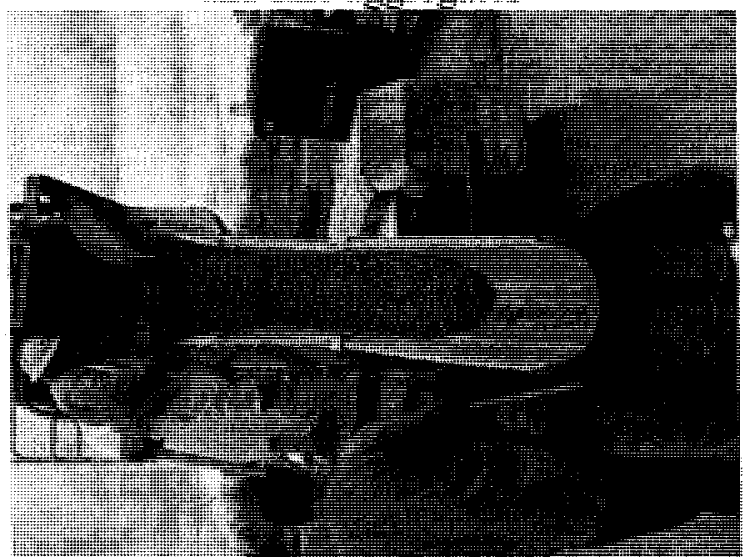

c)Taking concrete for slump and air content tests

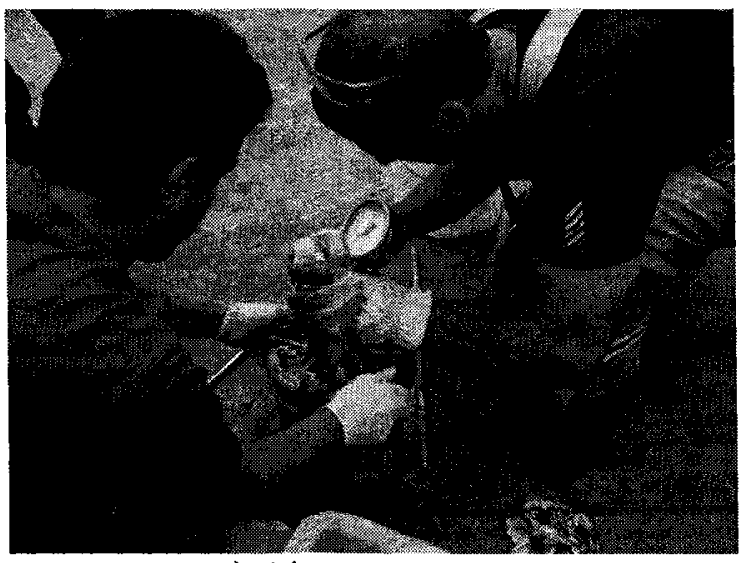

e) Air content test

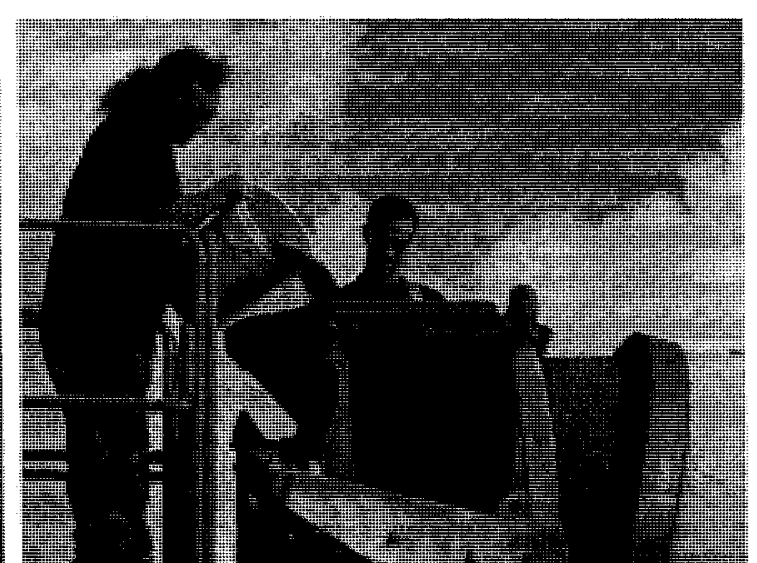

b) Loading the cement and water to truck mixer

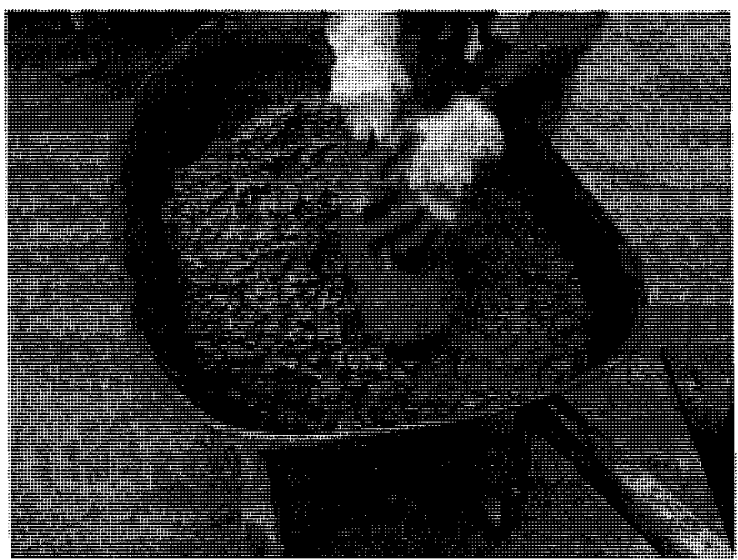

d) Fresh RAC

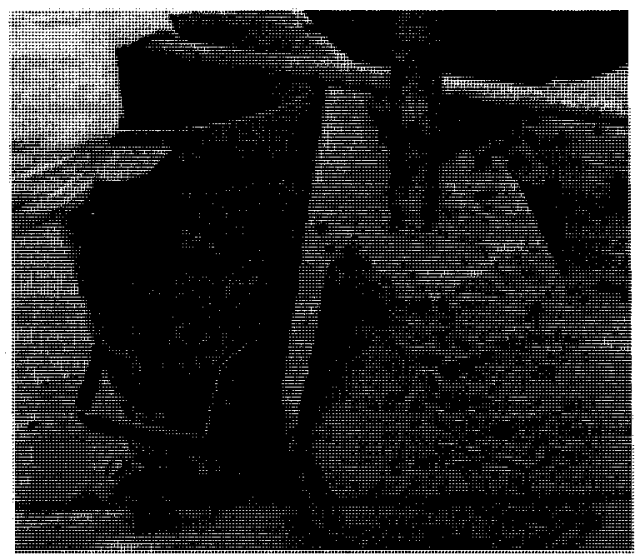

f) Slump test

Figure 6.10: Concrete mixing procedure 


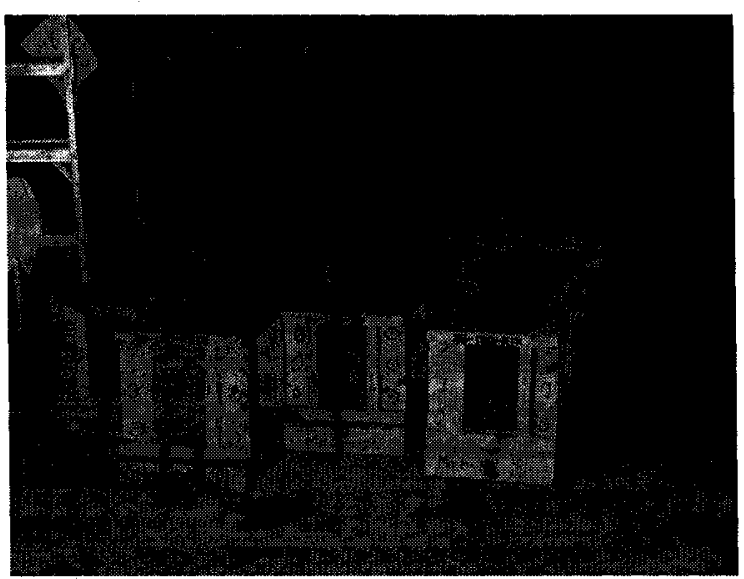

a) The fabricated steel reinforcement cages inside wooden formork

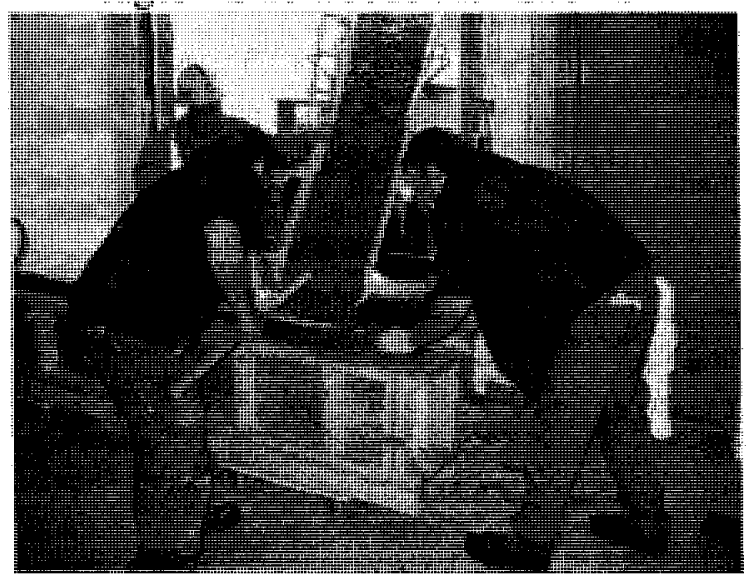

c) Casting the beams

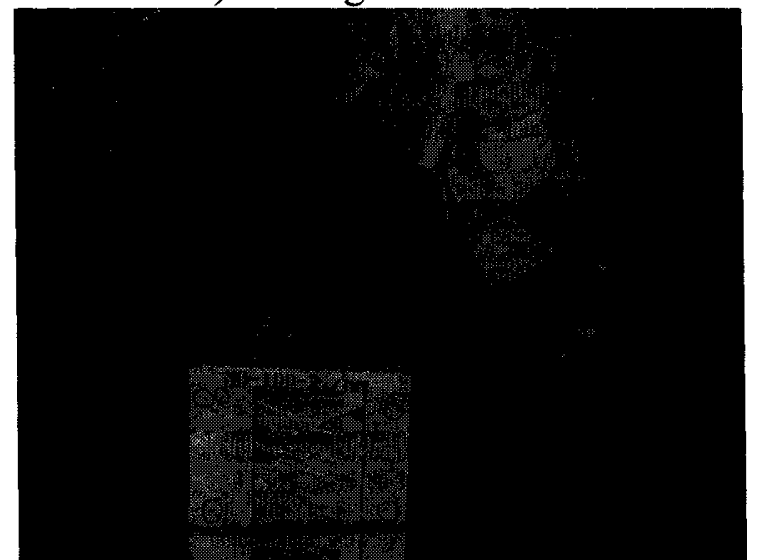

e) Finishing the top surface of the beams

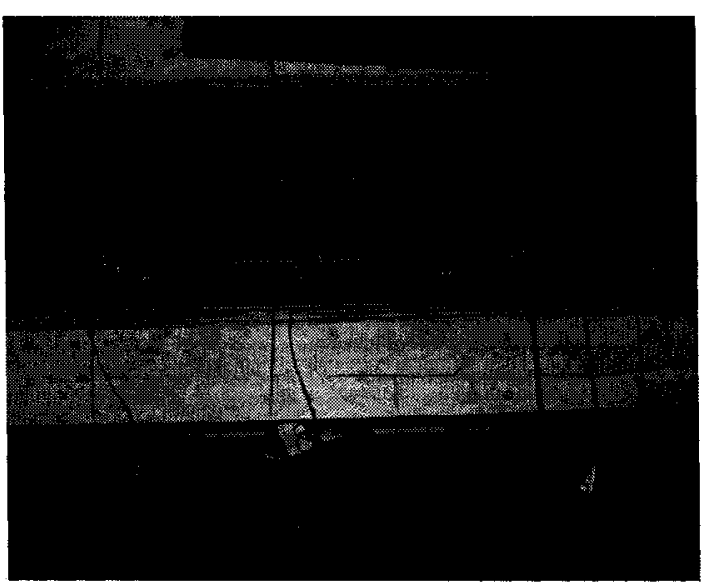

b) Reinforcement cage inside formwork

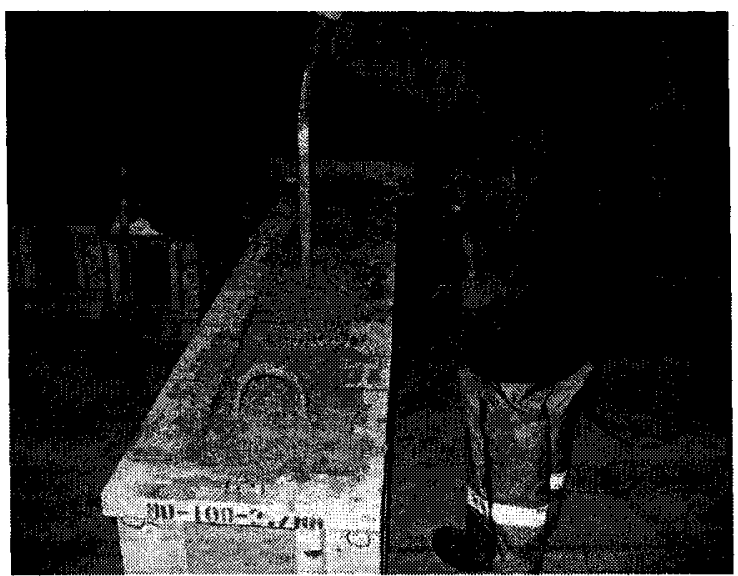

d) Compacting the fresh RAC

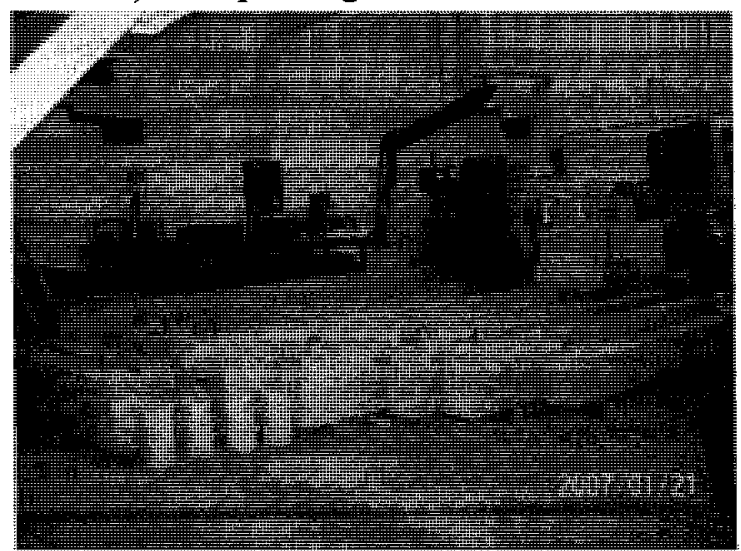

f) Curing the beams after demolding Figure 6.11: Casting and curing procedures of beams 


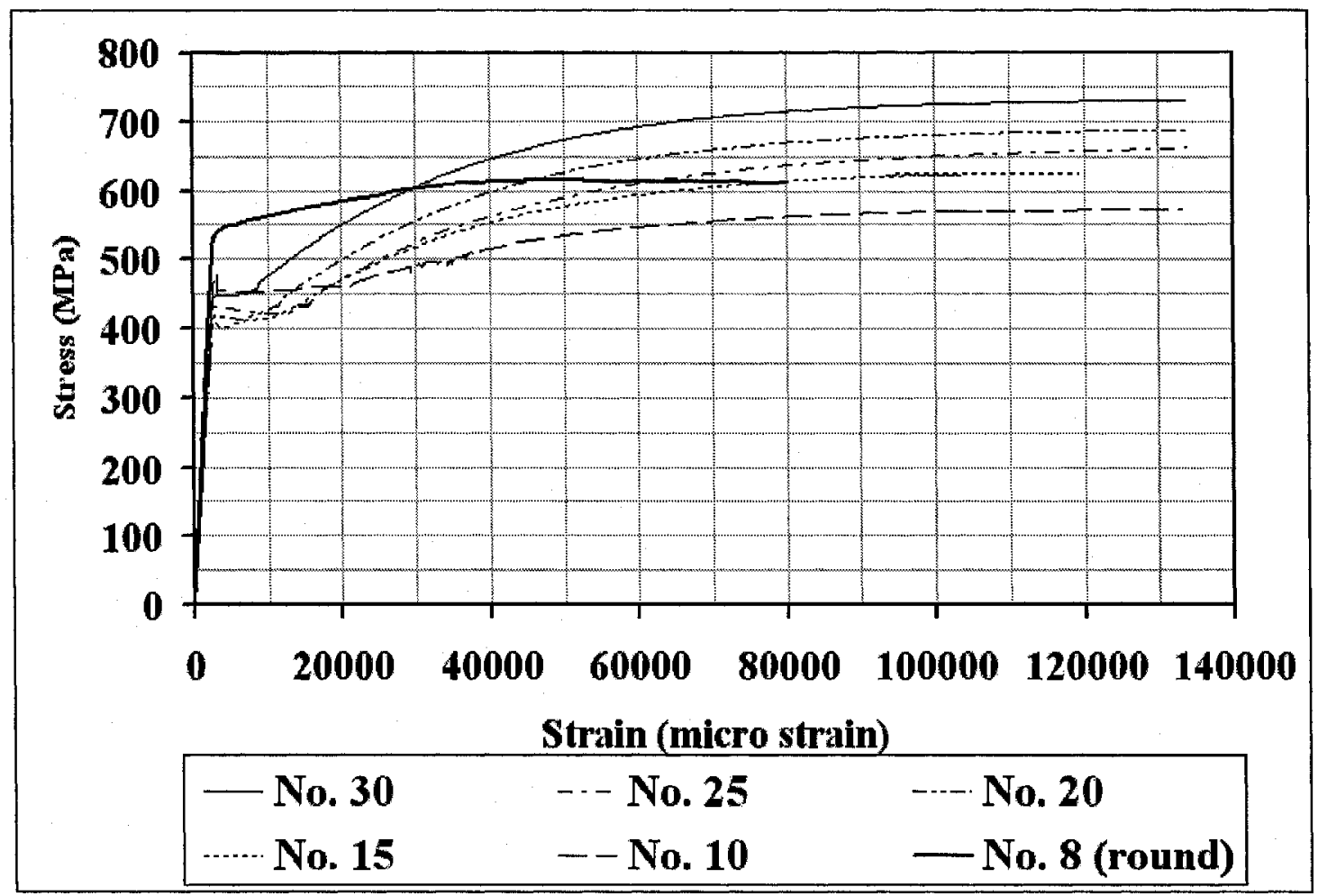

Figure 6.12: Typical stress-strain relationship of steel reinforcement bars

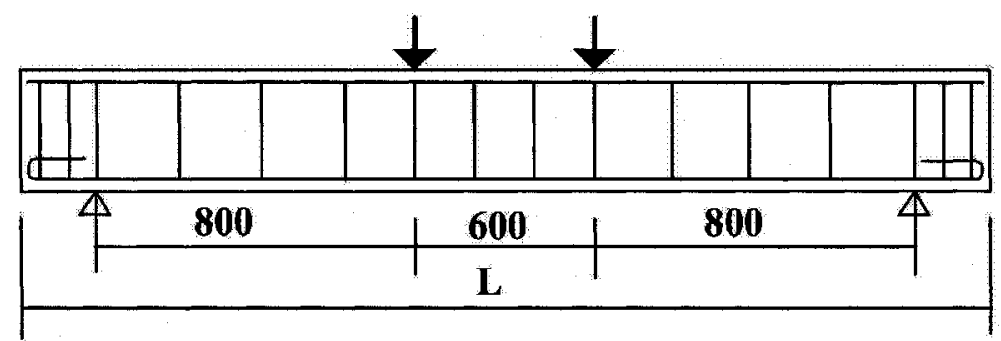

a) Elevation

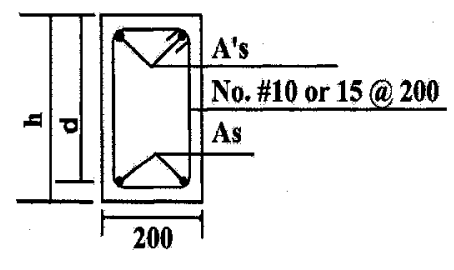

b) Section

Figure 6.13: Typical dimensions and reinforcement details of the beams tested in flexure 


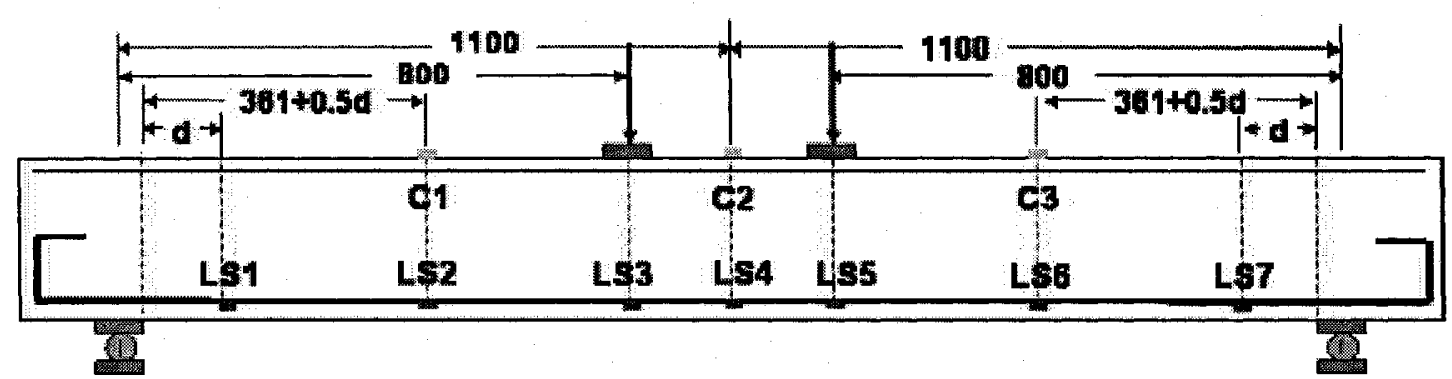

a) Strain gauge instrumentation

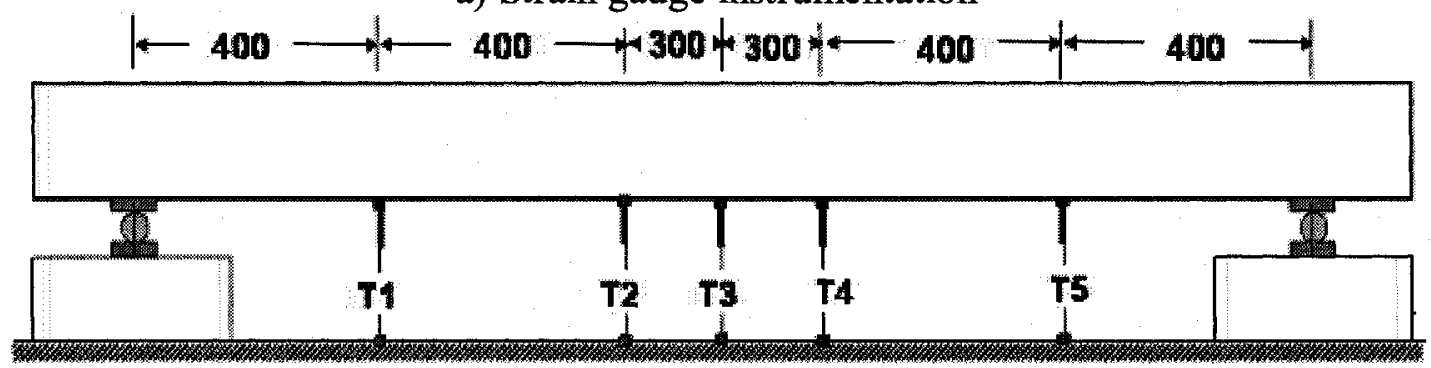

b) LP instrumentation

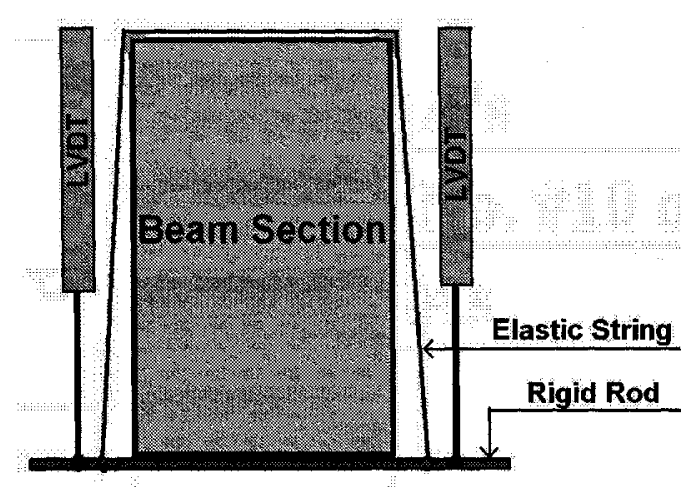

c) Connection of two LP's at each deflection measurement location

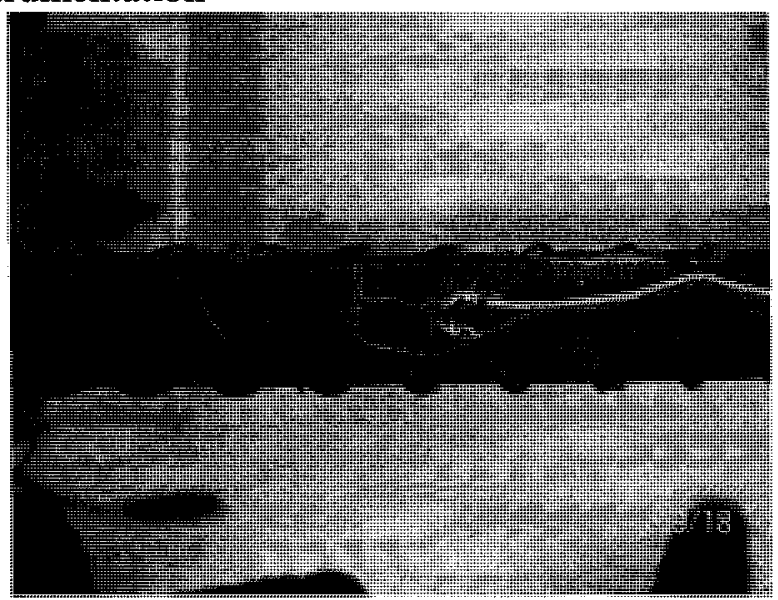

d) Installed strain gauge on steel reinforcement

Figure 6.14: Schematic instrumentation employed to test the beams under flexure 


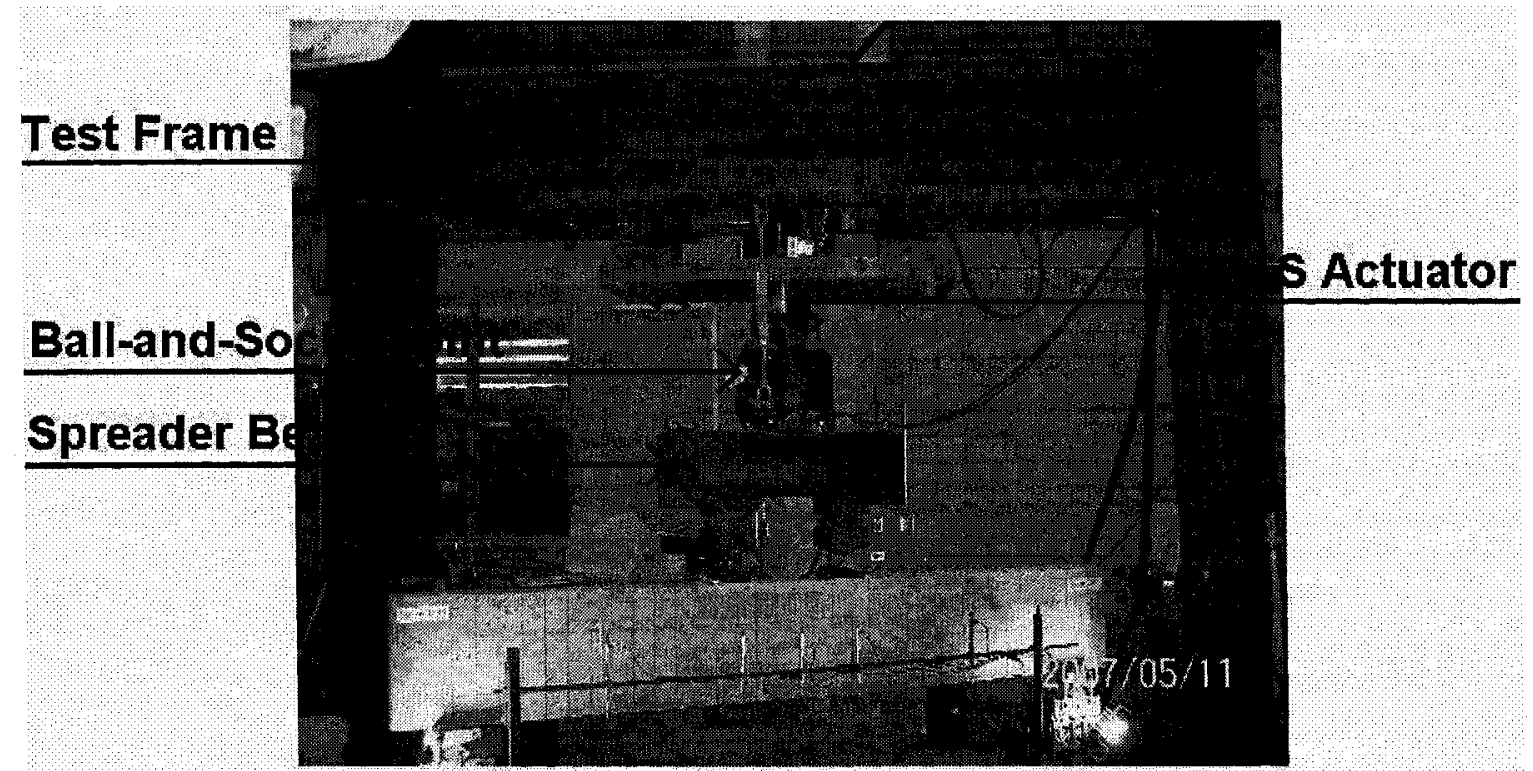

a) Test setup
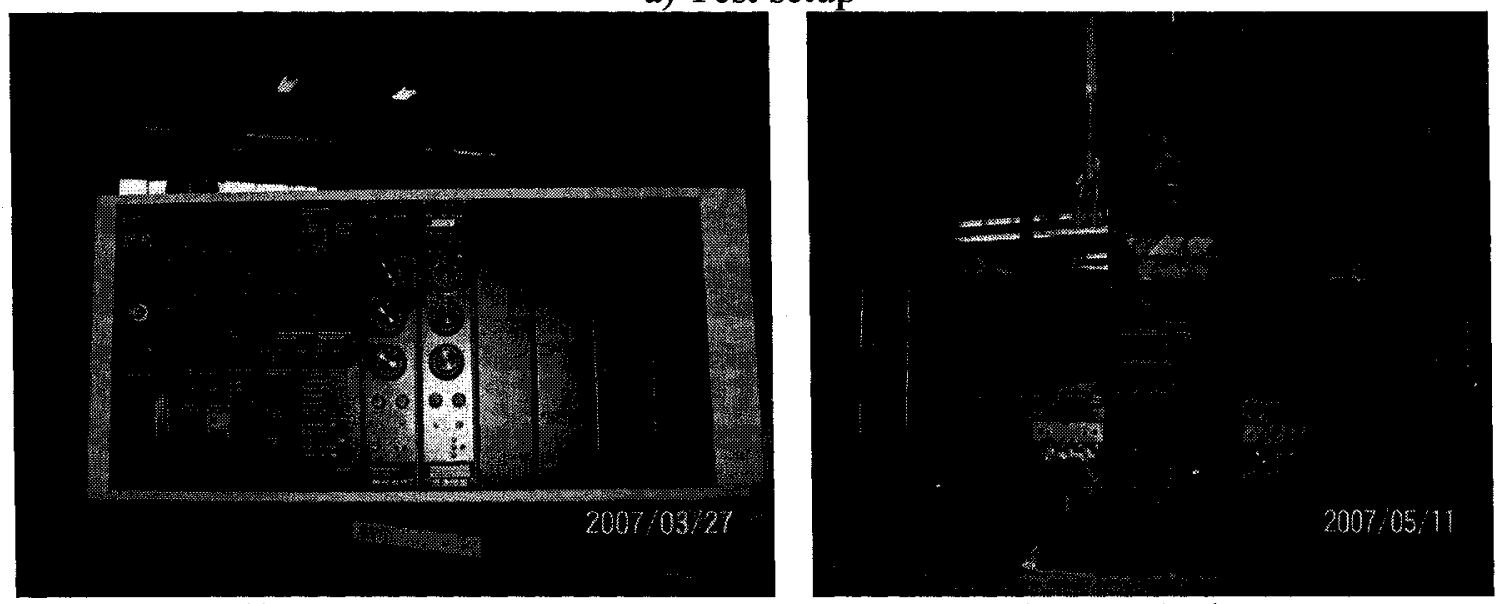

b) MTS controller

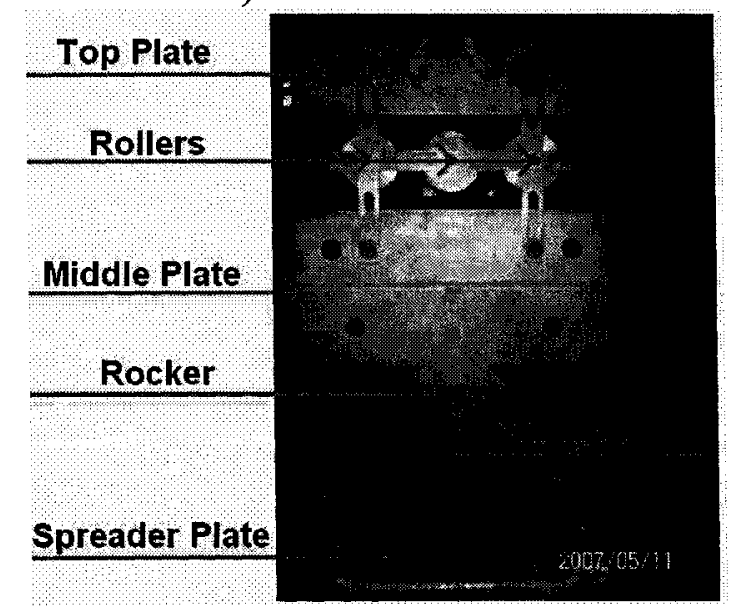

c) Spreader beam

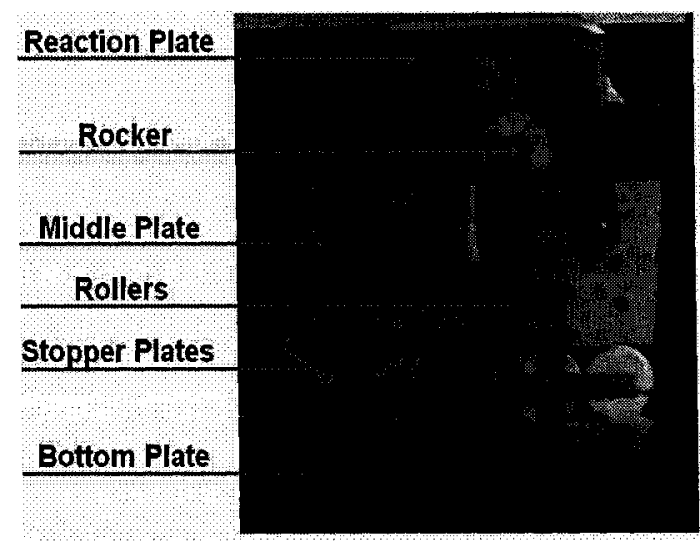

c) Rocker-and-roller assembly under spreader beam

e) Pinned support

Figure 6.15: Loading test setup 


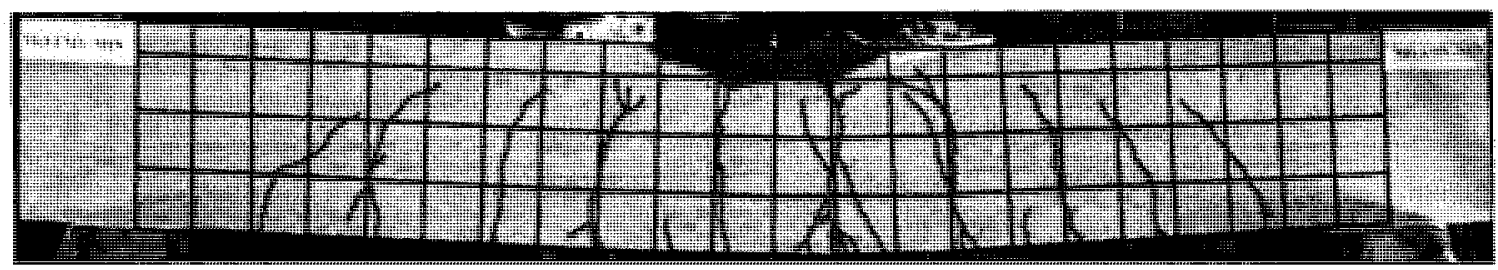

a) FEM-Min

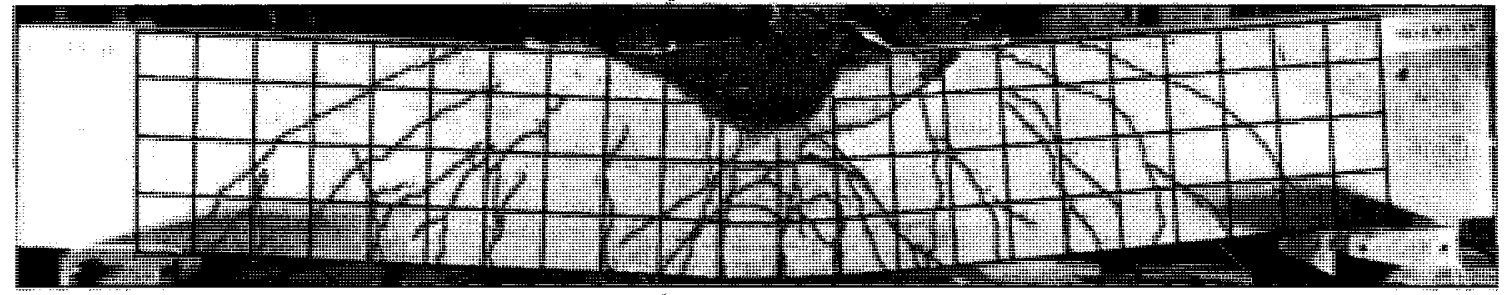

b) $\mathrm{EMM}-\mathrm{Ay}$

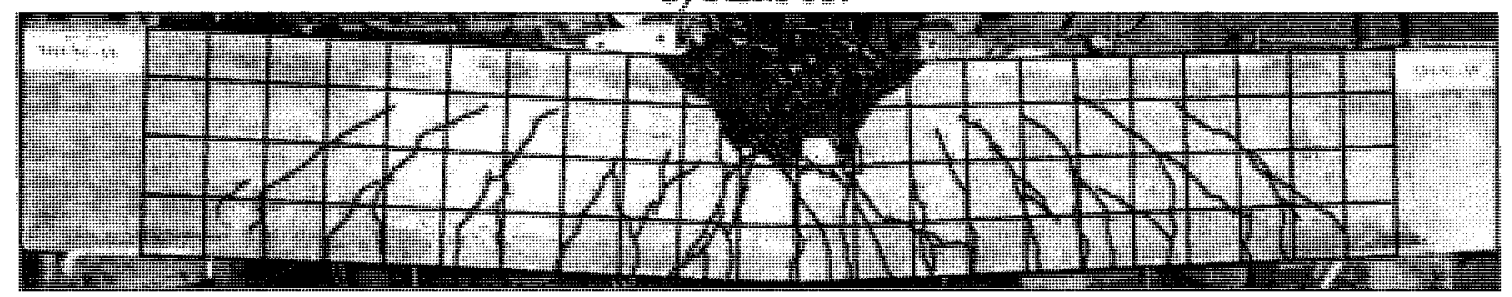

c) FCL-Av

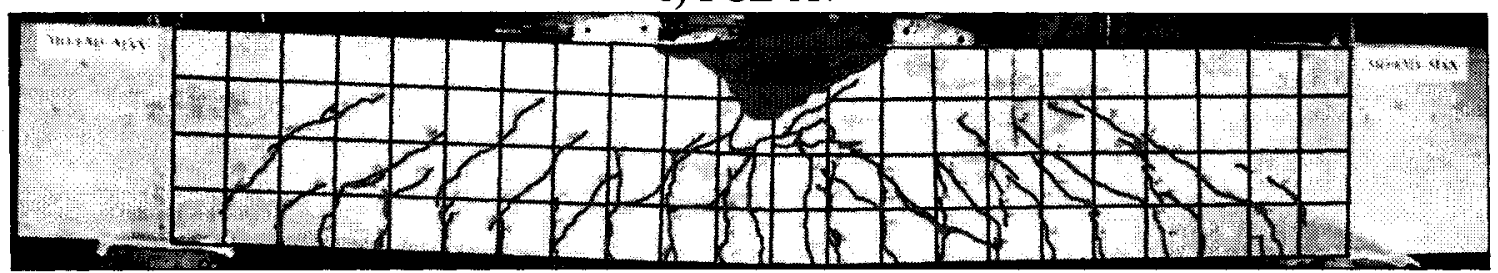

d) FEM-Max

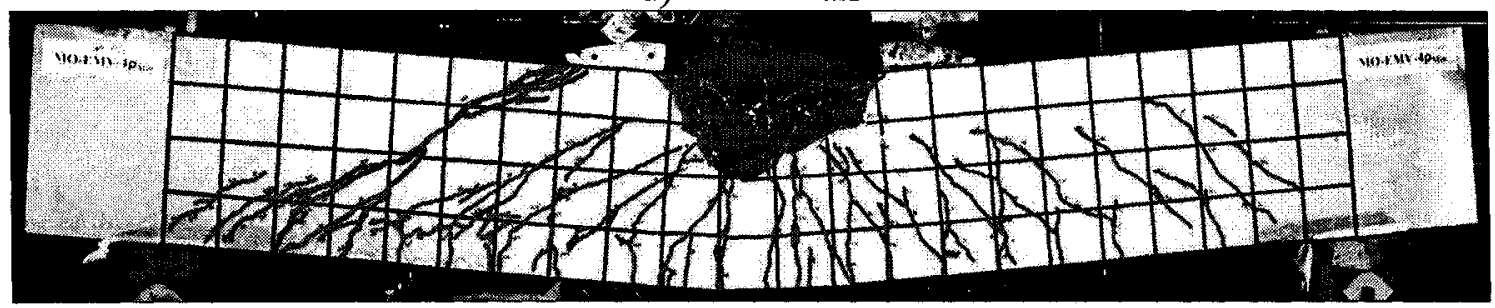

a TEM-CME

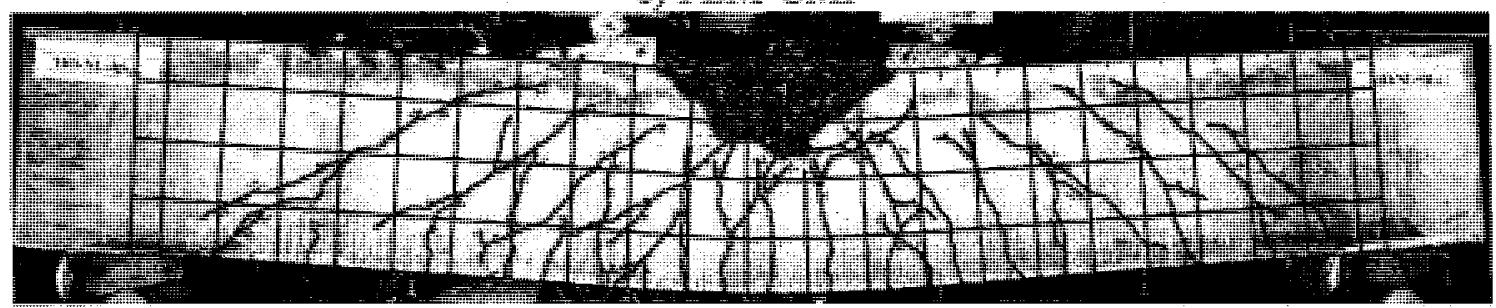

f) FCL-CMP

Figure 6.16: Typical crack patterns of FEM and control FCL beams 


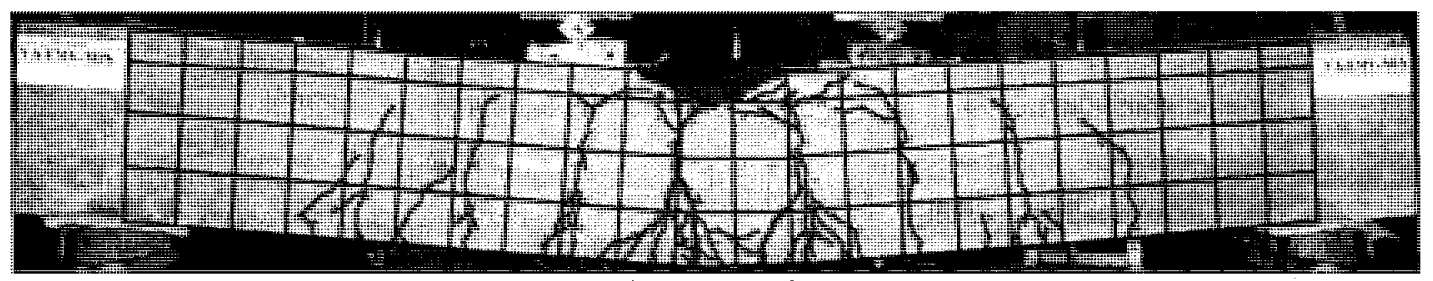

a) FEV-Min

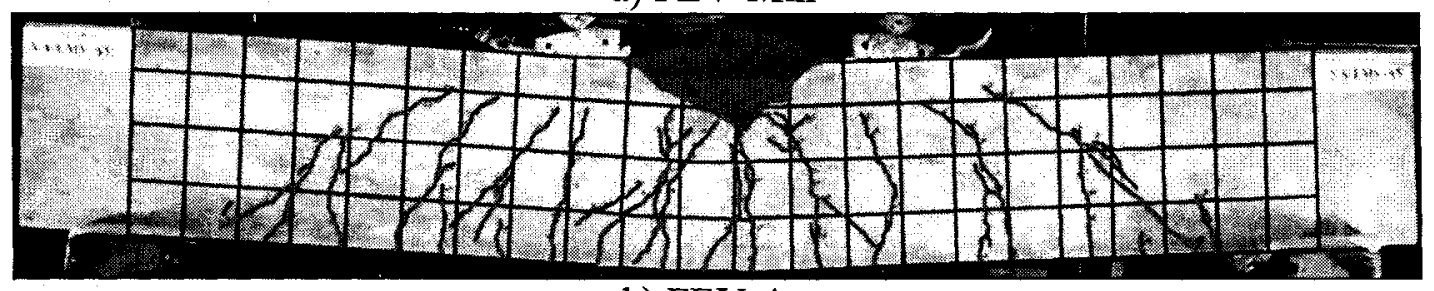

b) FEV-Av

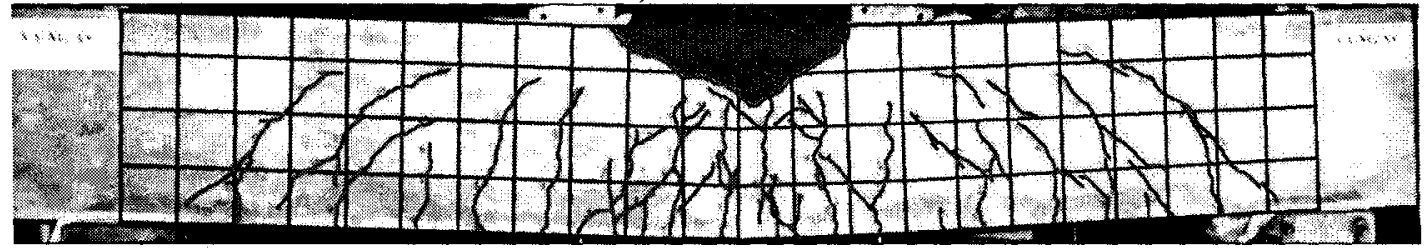

c) FCG-Av

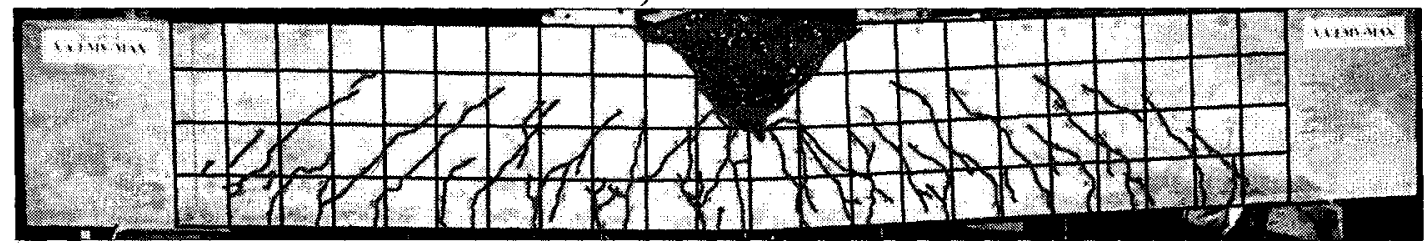

d) FEV-Max

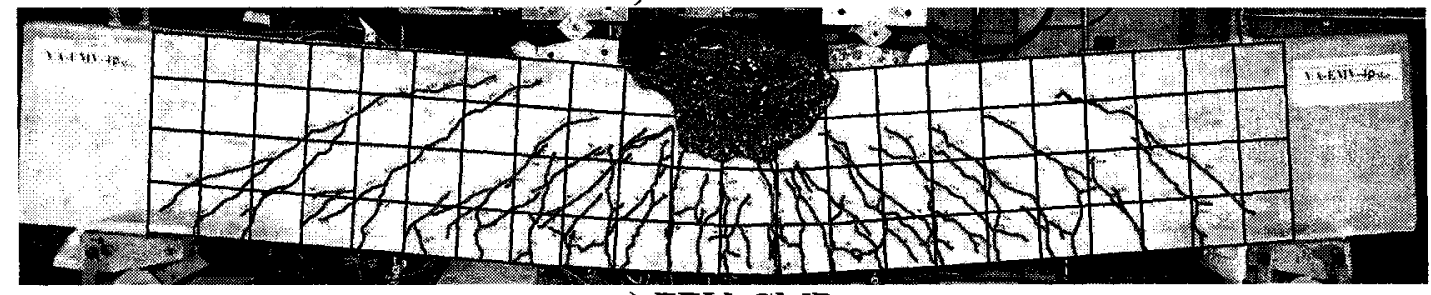

e) FEV-CMP

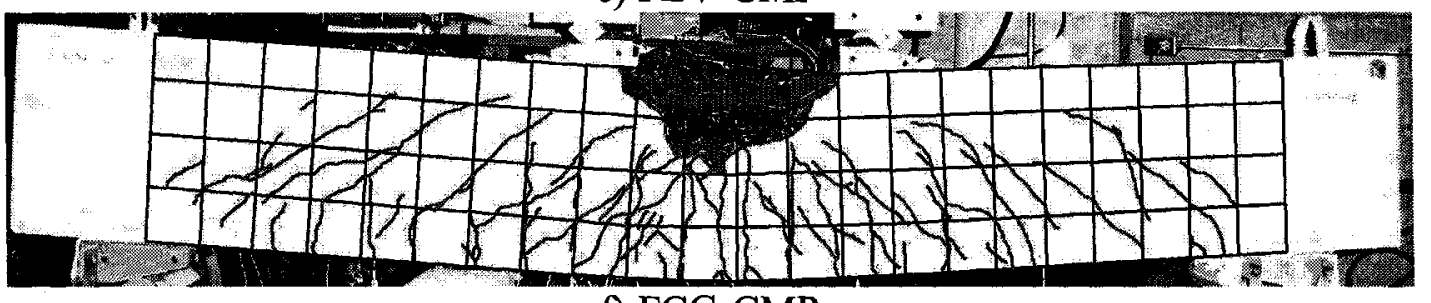

f) FCG-CMP

Figure 6.17: Typical crack patterns of FEV and control FCG beams 


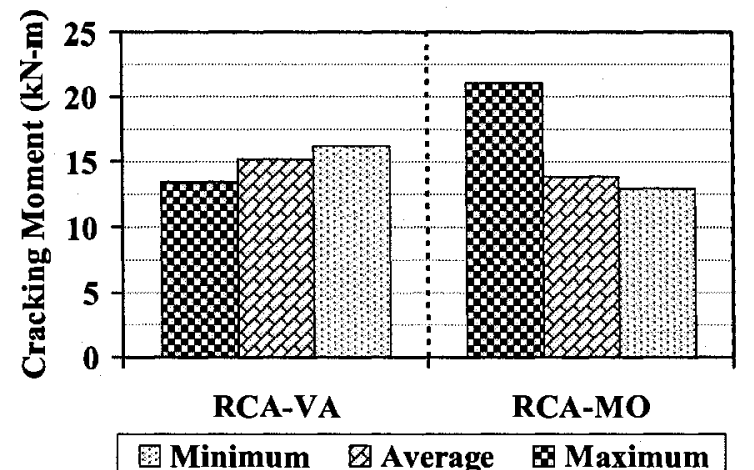

a) Cracking Moment

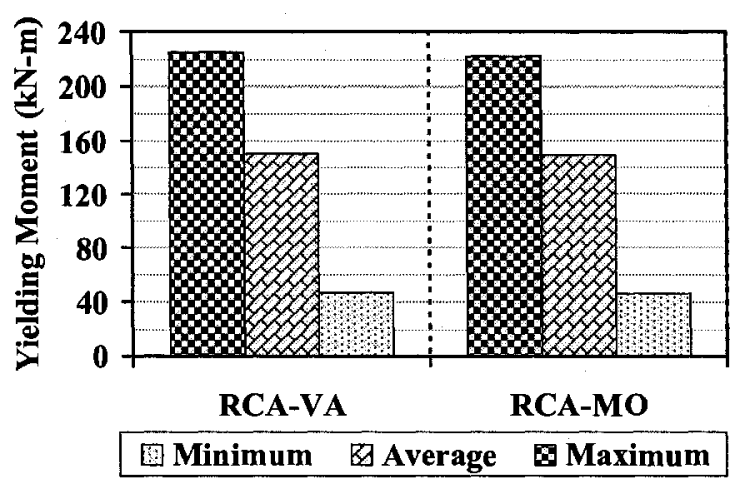

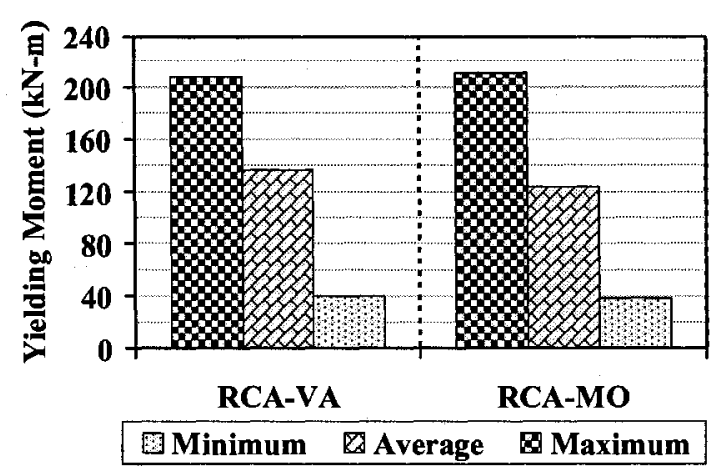

b) Yielding Moment

c) Ultimate moment

Figure 6.18: Effect of longitudinal tension reinforcement ratio on:

a) cracking moment, b) yielding moment, and c) ultimate moment strengths 


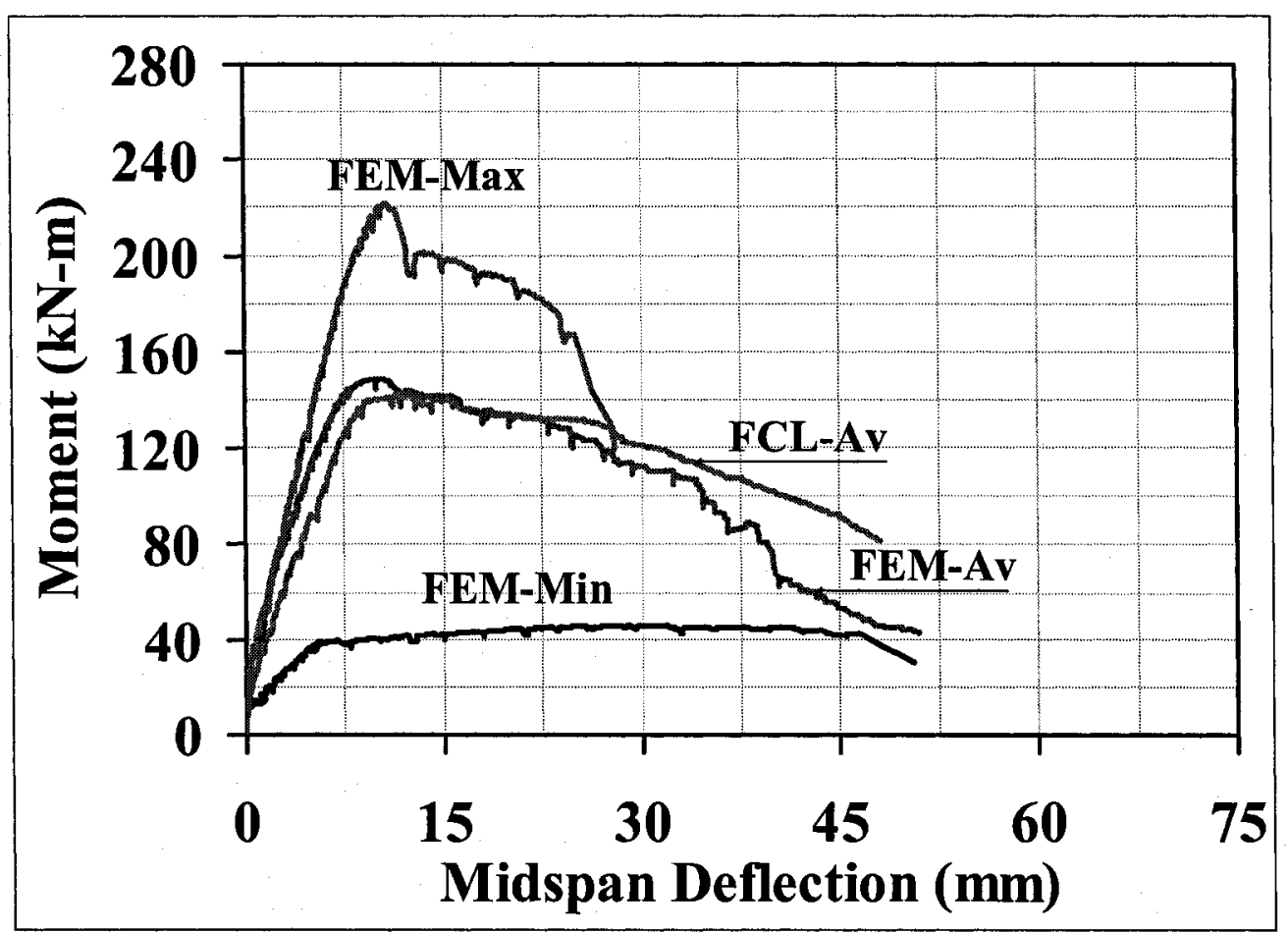

a) MO-Beams

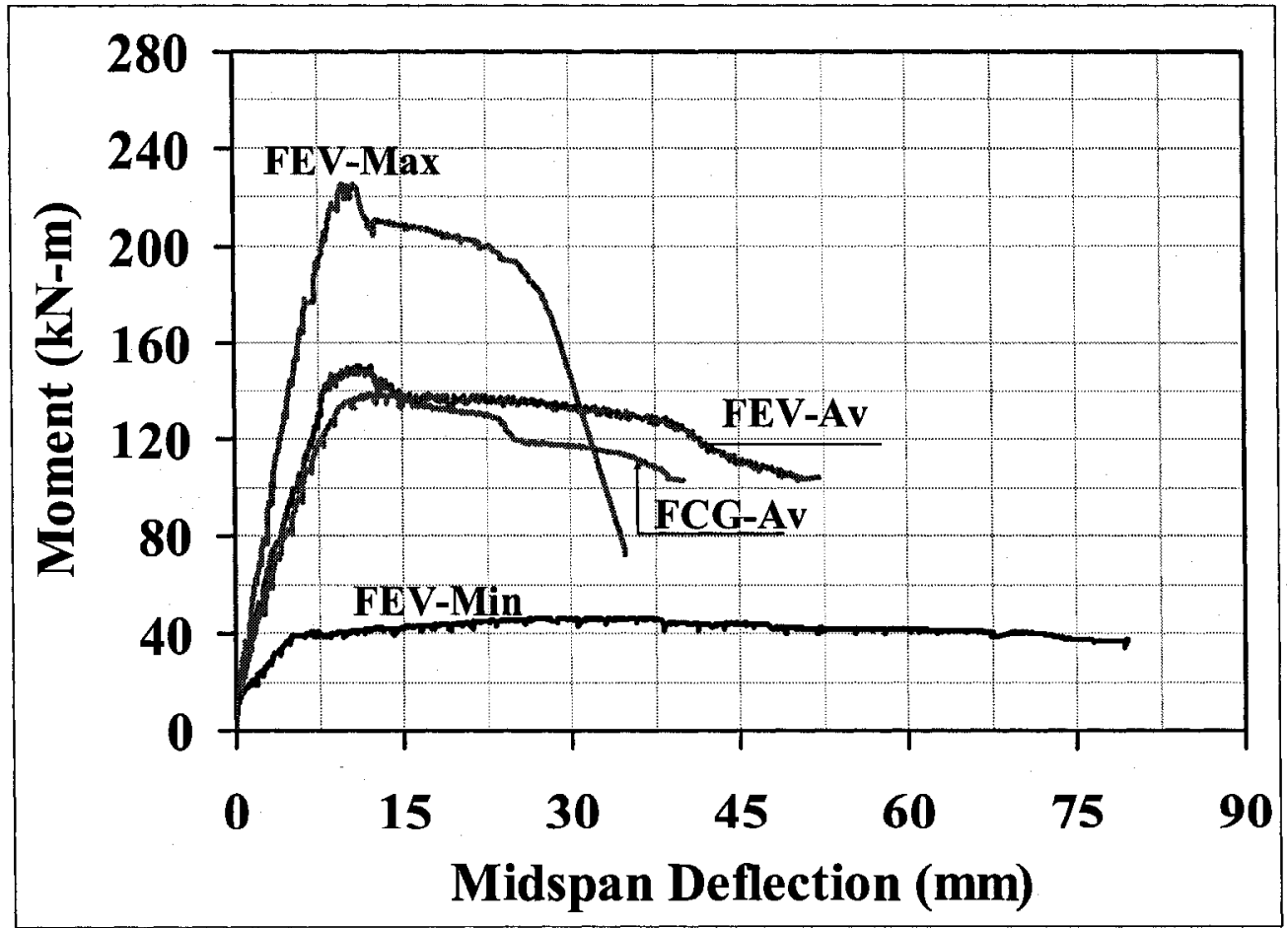

b) VA-Beams

Figure 6.19: Effect of longitudinal tension reinforcement ratio on the moment- deflection response of RRC beams 


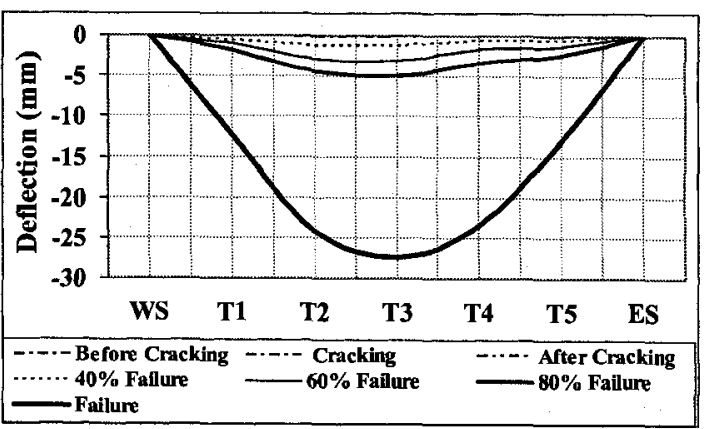

a) MO-EMV-Min

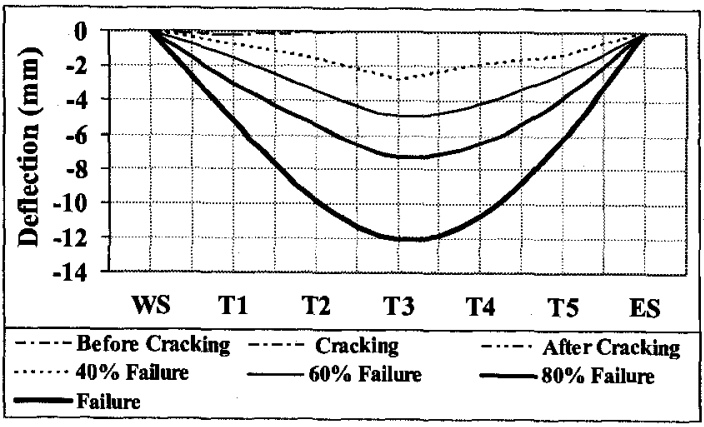

c) $\mathrm{MO}-\mathrm{EMV}-\mathrm{Av}$

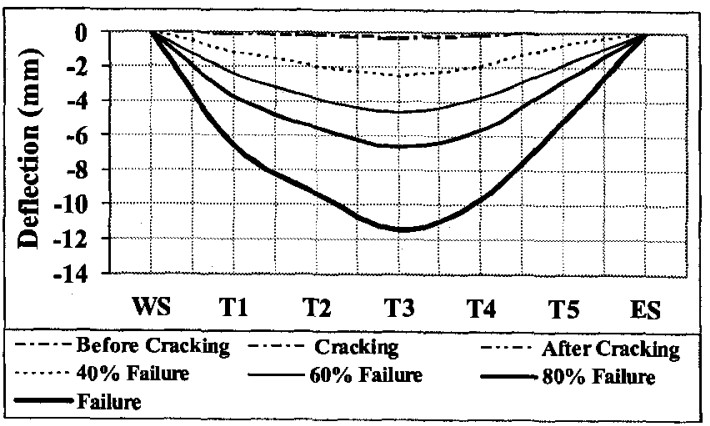

e) MO-NL-Av

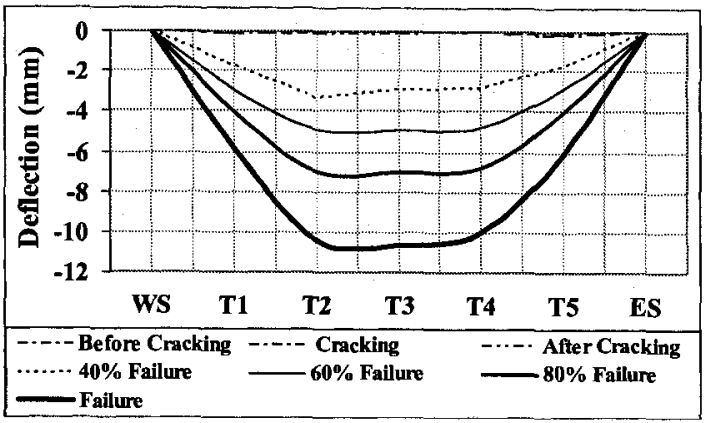

g) MO-EMV-Max

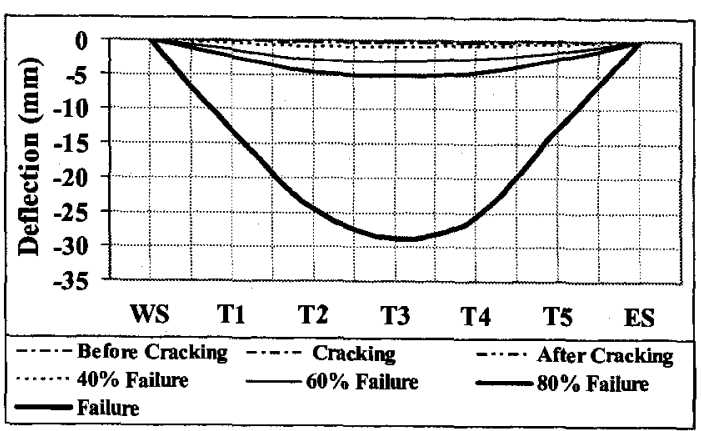

b) VA-EMV-Min

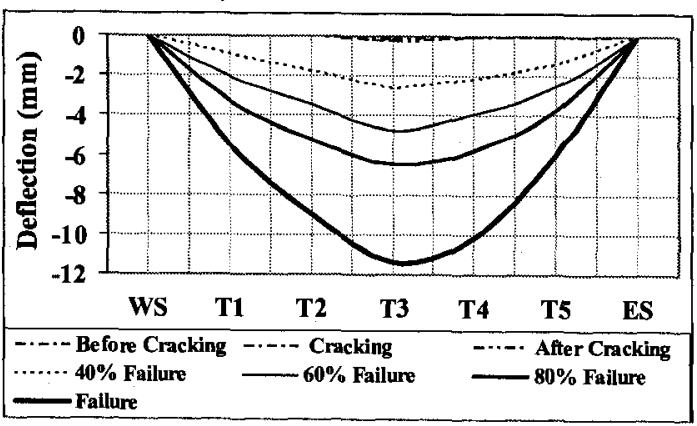

d) VA-EMV-Av

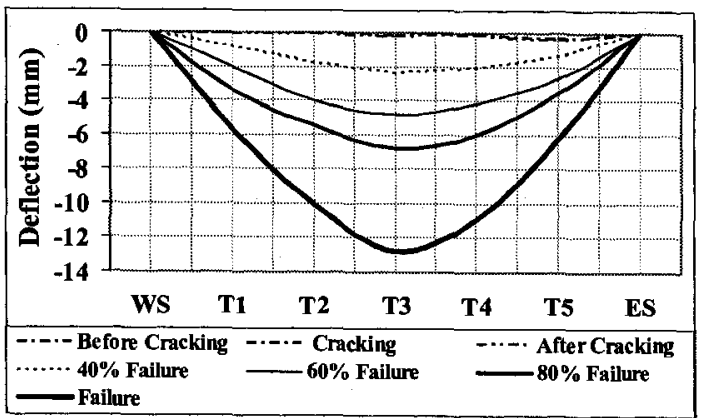

f) VA-NG-Av

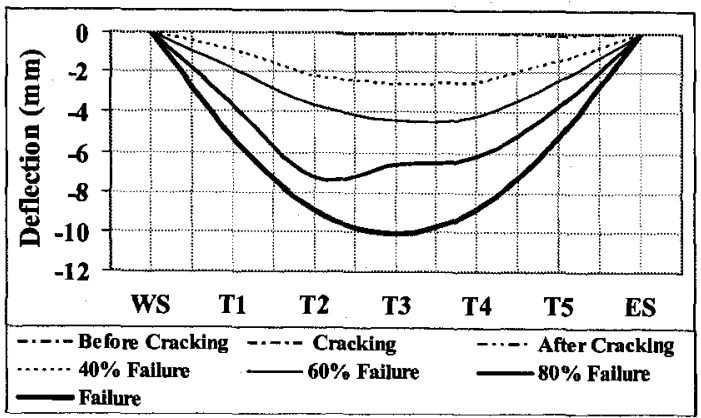

h) VA-EMV-Max

Figure 6.20: Deflection curve of singly reinforced beams at different load levels 


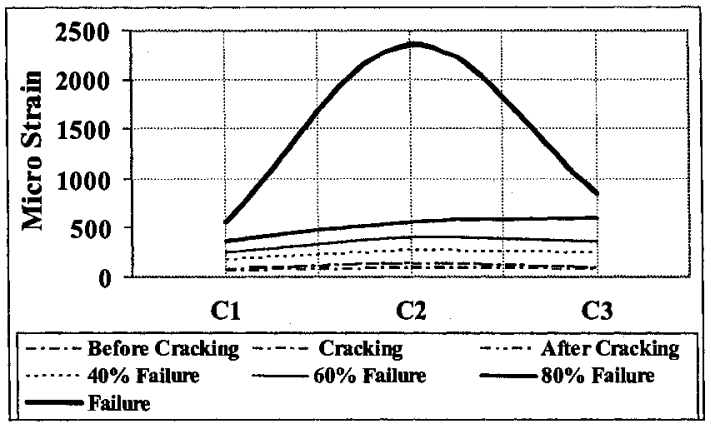

a) MO-EMV-Min

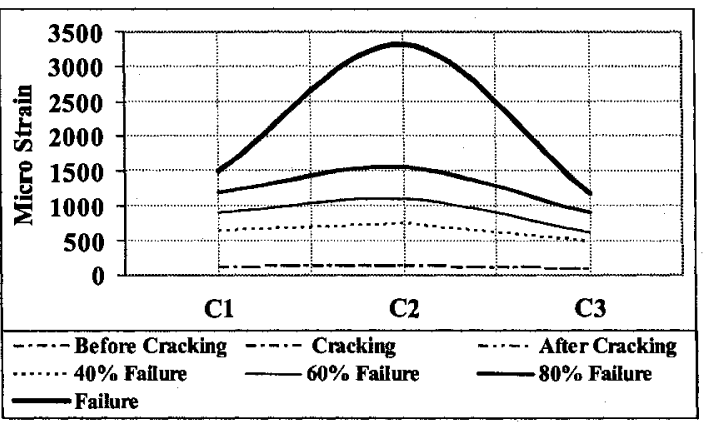

c) MO-EMV-Av

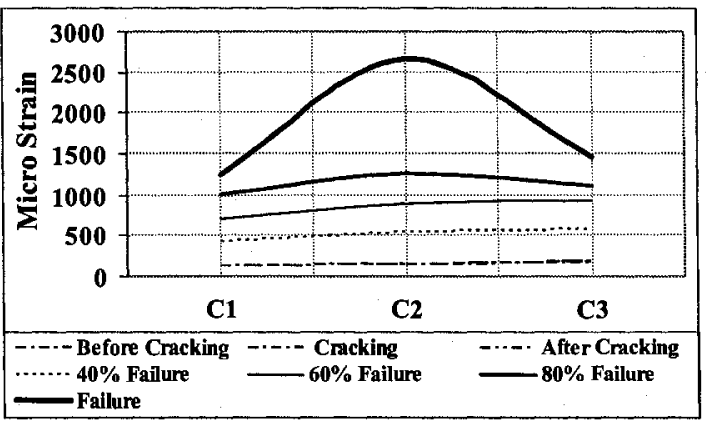

e) MO-NL-Av

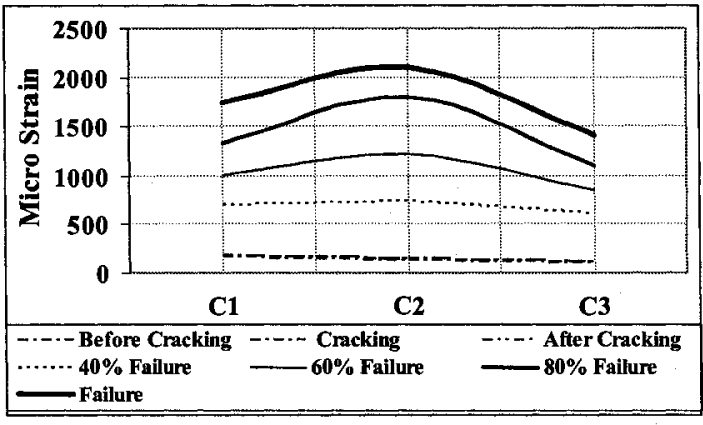

g) MO-EMV-Max

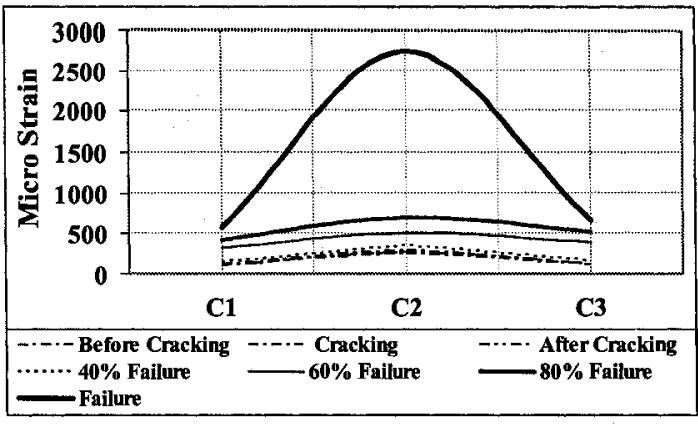

b) VA-EMV-Min

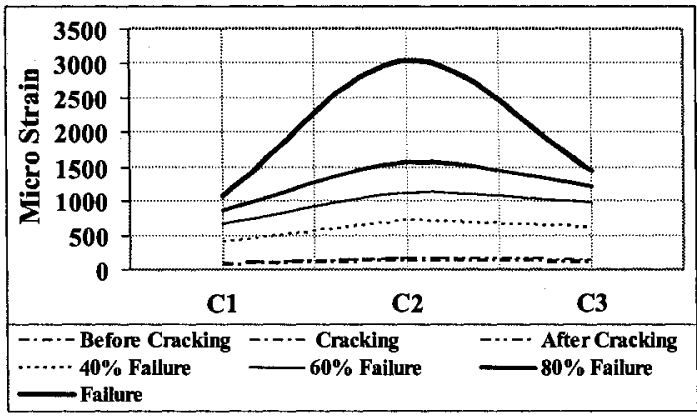

d) VA-EMV-Av

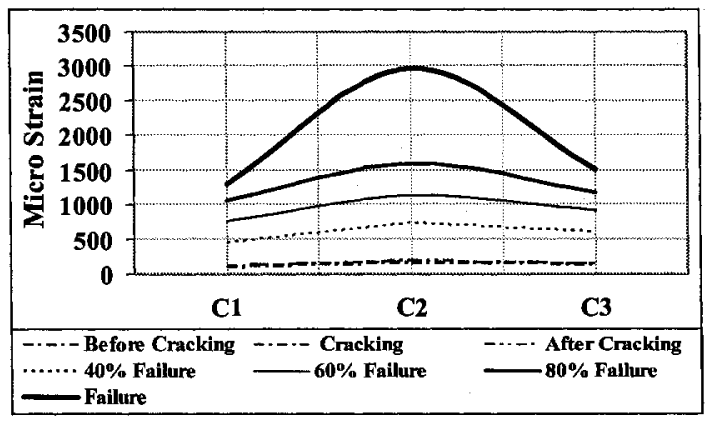

f) VA-NG-Av

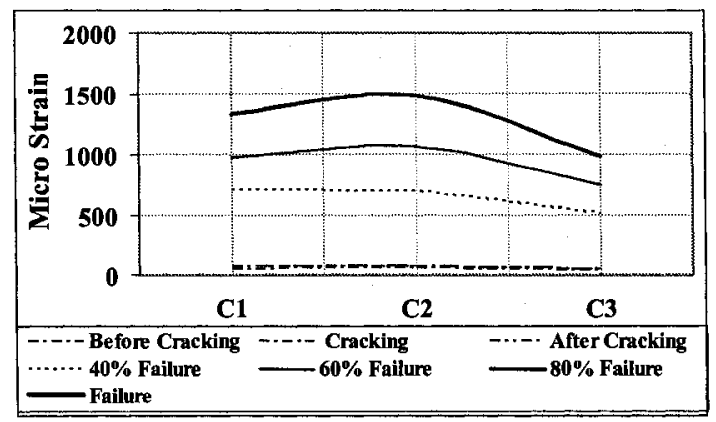

h) VA-EMV-Max

Figure 6.21: Variation of concrete compression strain along singly reinforced beams at different load levels 


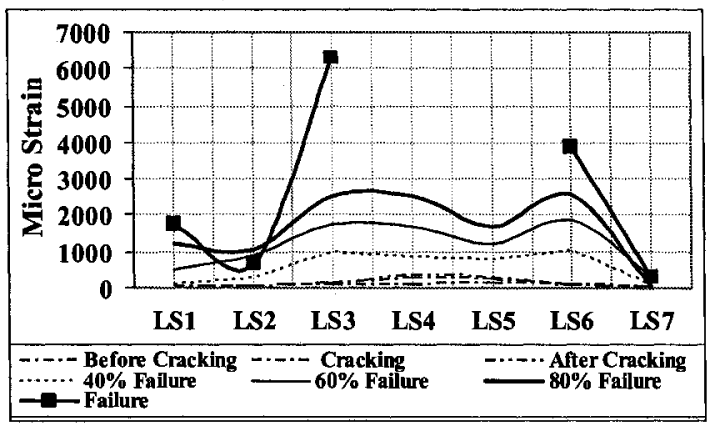

a) MO-EMV-Min

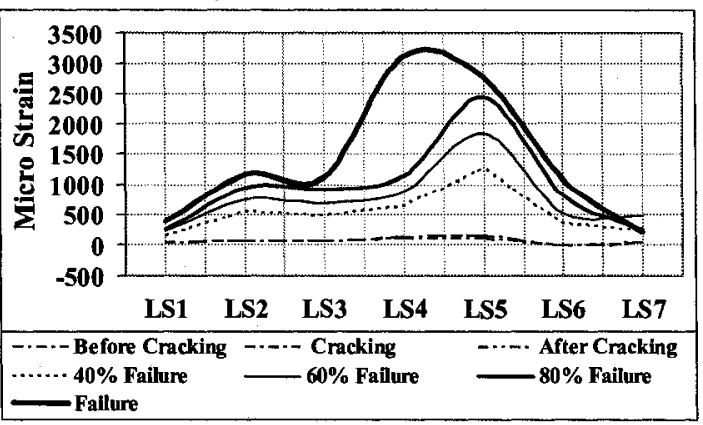

c) MO-EMV-Av

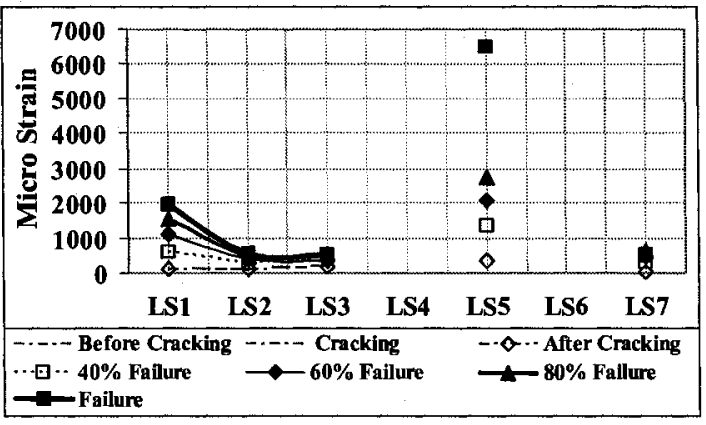

e) $\mathrm{MO}-\mathrm{NL}-\mathrm{Av}$

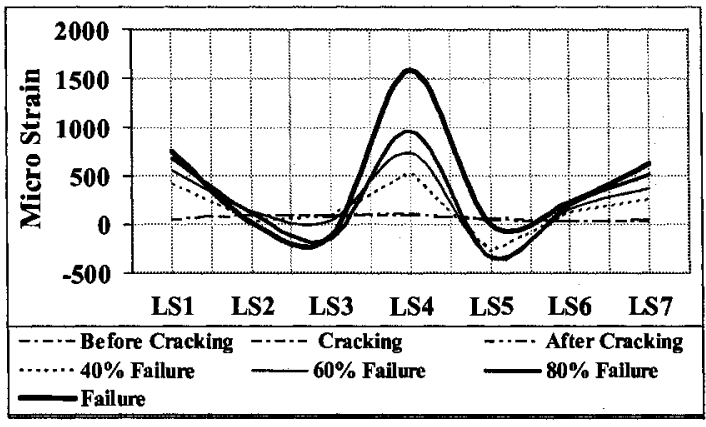

g) MO-EMV-Max

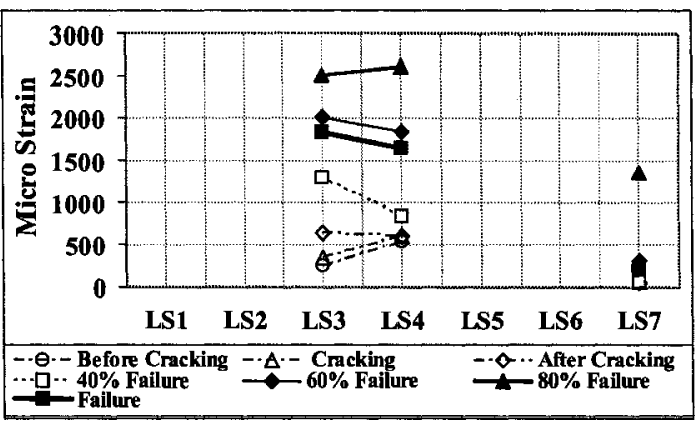

b) VA-EMV-Min

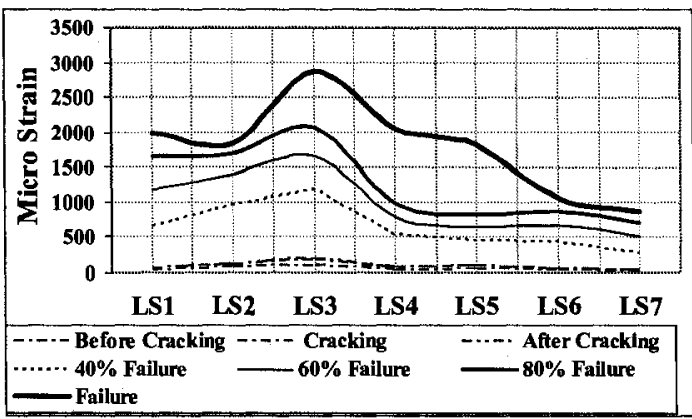

d) VA-EMV-Av

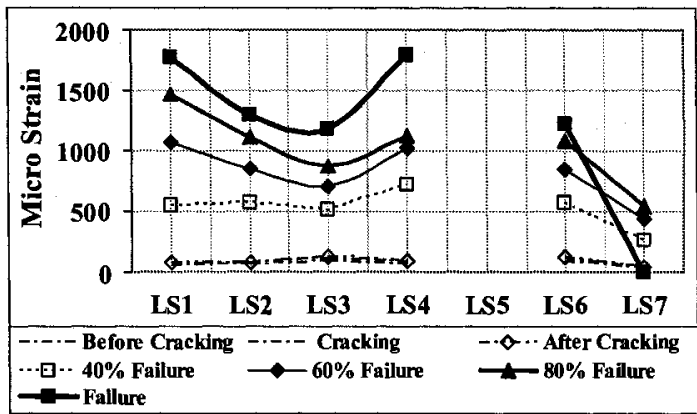

f) VA-NG-Av

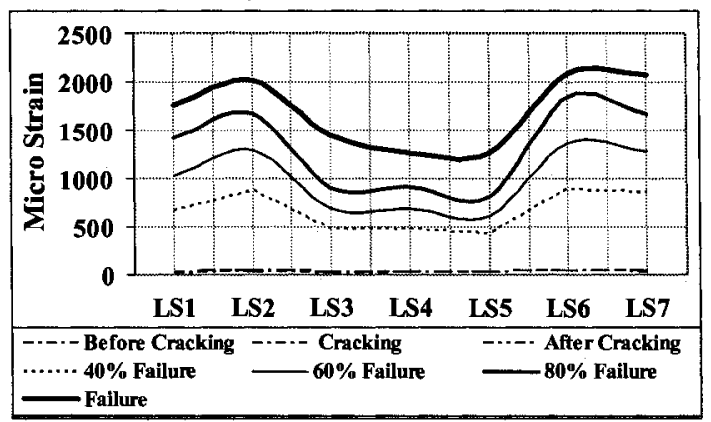

h) VA-EMV-Max

Figure 6.22: Variation of longitudinal steel strain along singly reinforced beams at different load levels 


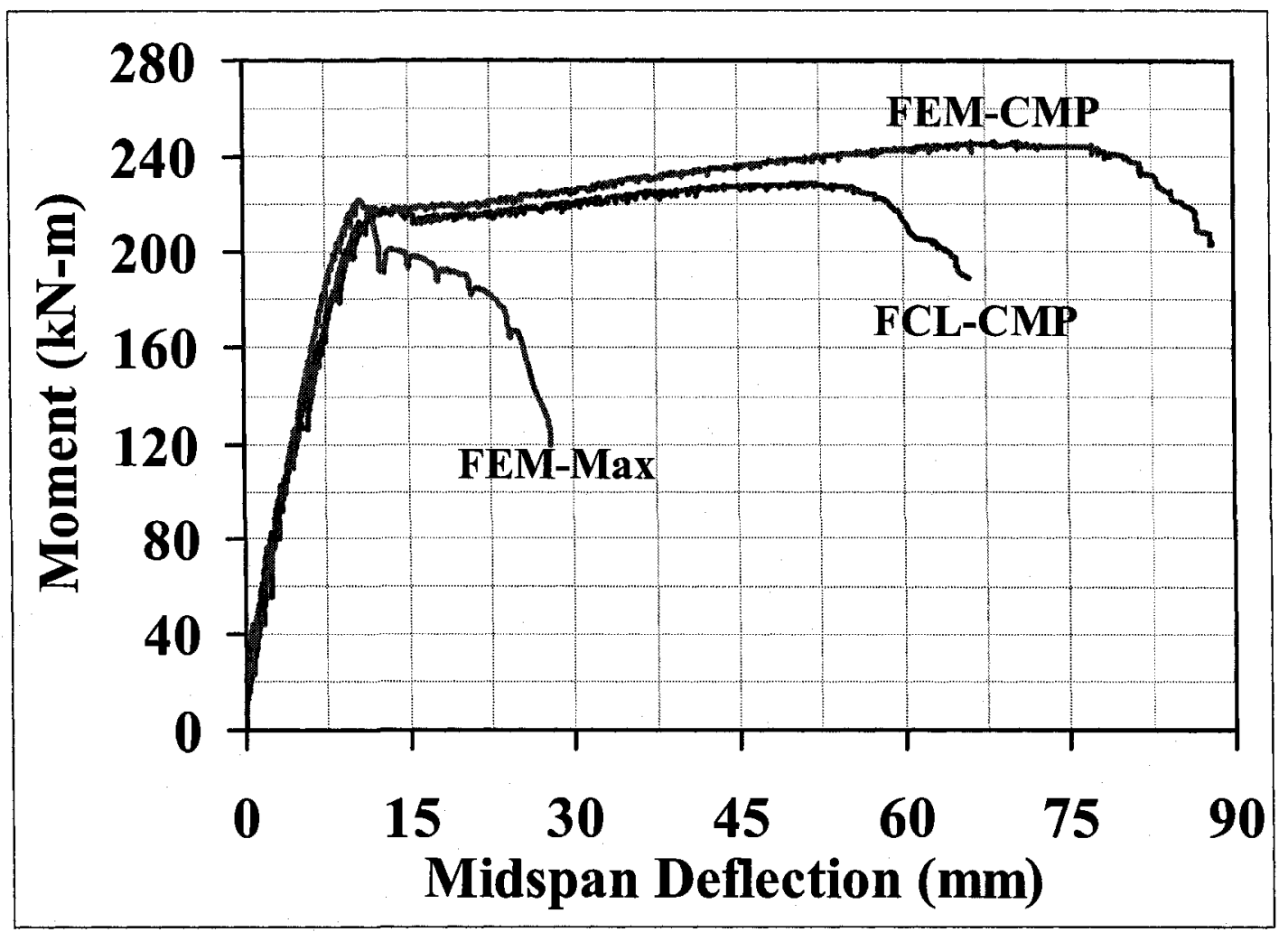

a) MO-Beams

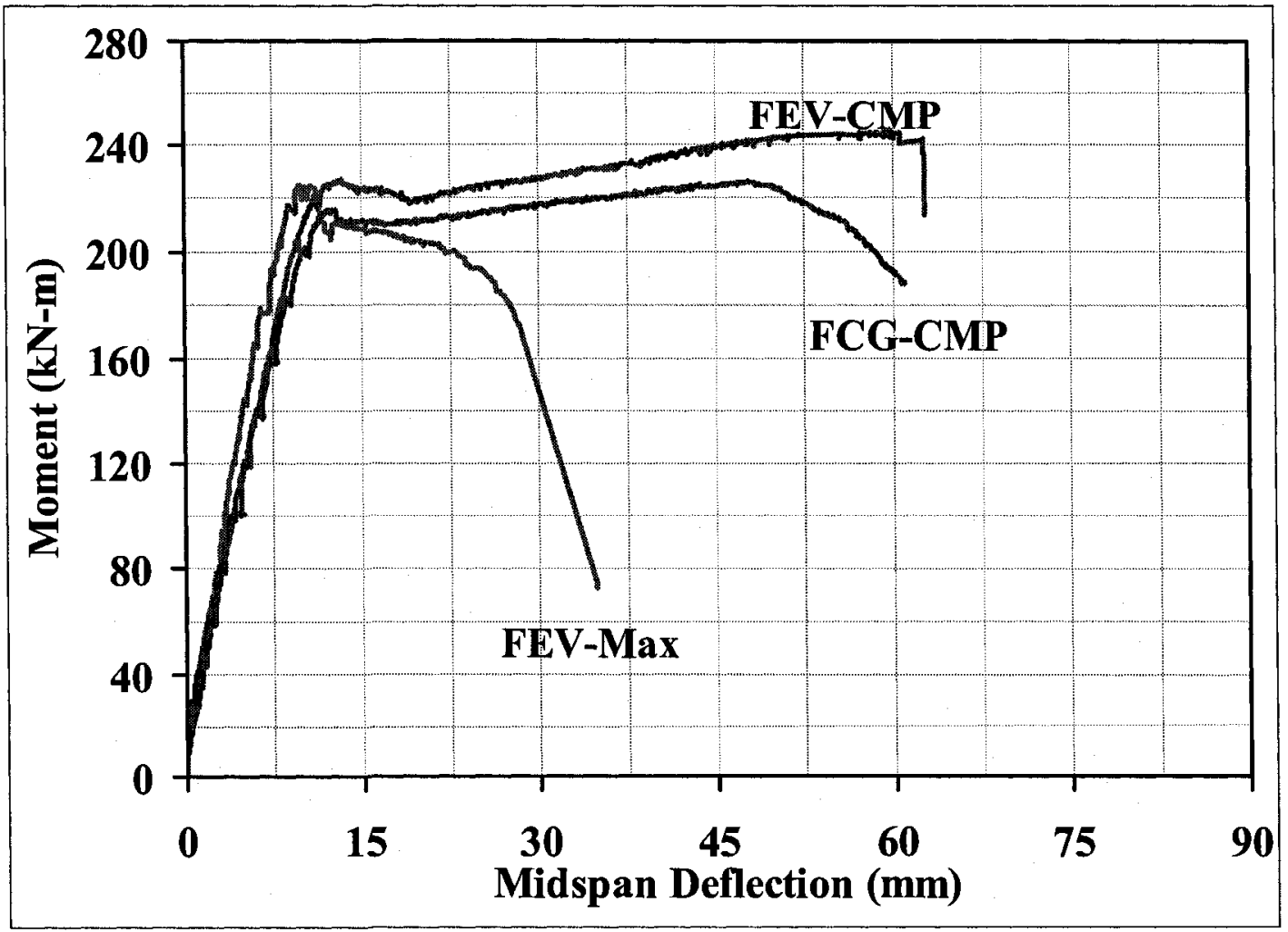

b) VA-Beams

Figure 6.23: Effect of compression steel on flexural behaviour of RRC beams 


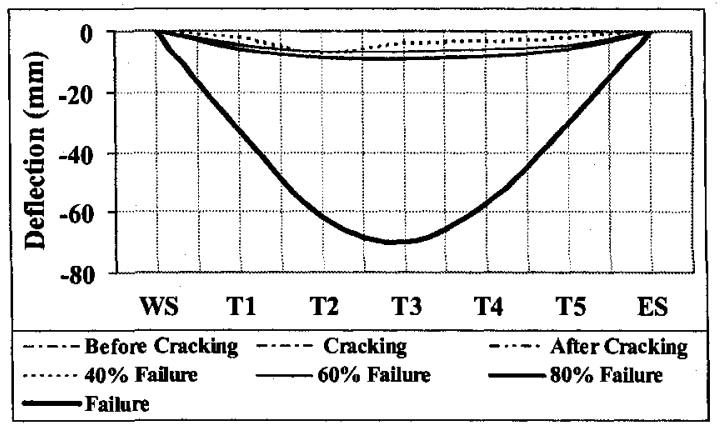

a) MO-EMV-CMP

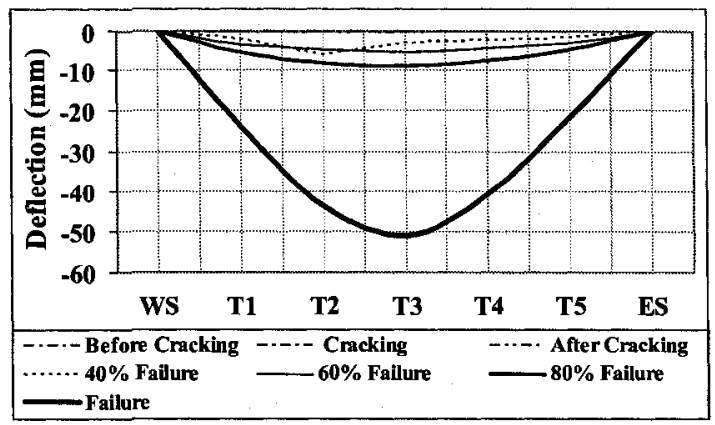

c) MO-NL-CMP

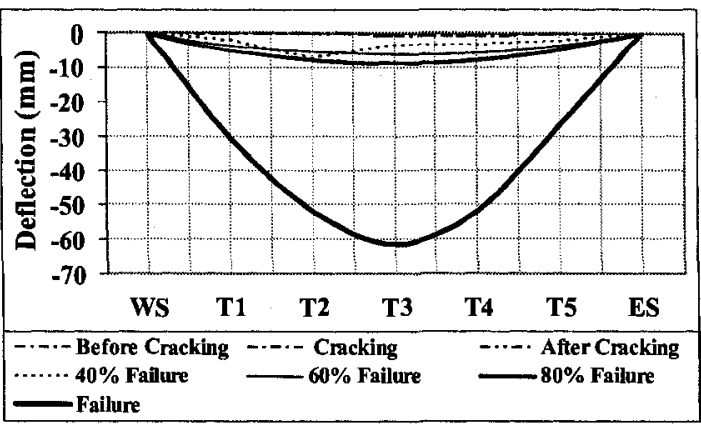

b) VA-EMV-CMP

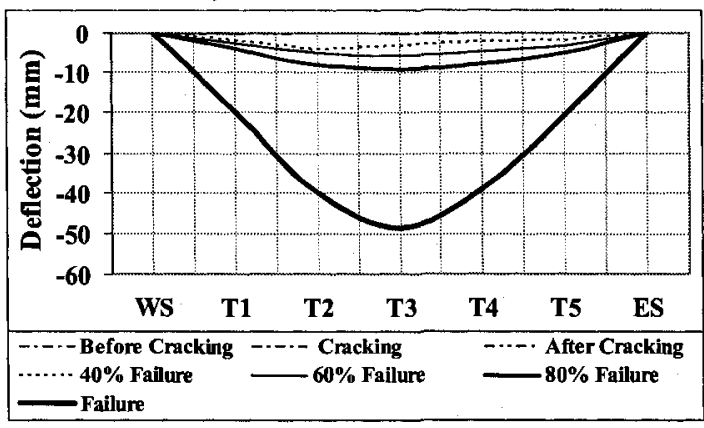

d) VA-NG-CMP

Figure 6.24: Deflection curve of doubly reinforced beams at different load levels

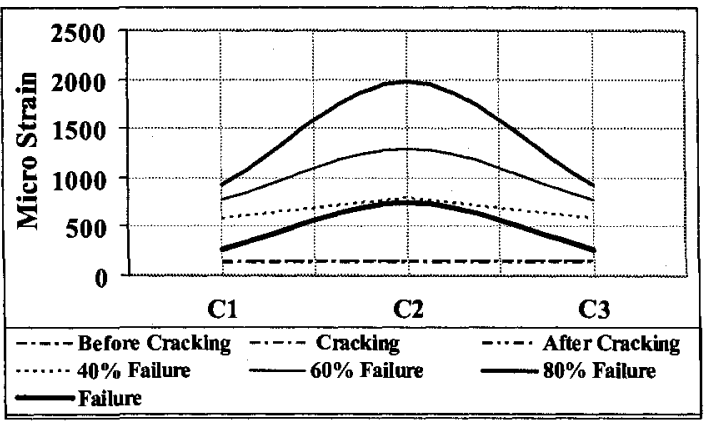

a) MO-EMV-CMP

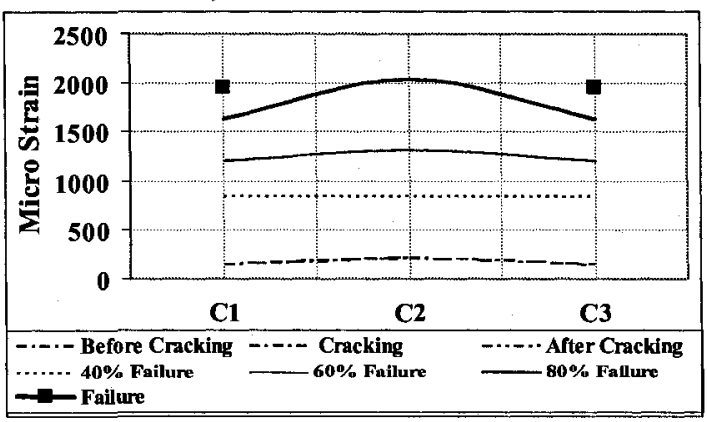

c) MO-NL-CMP

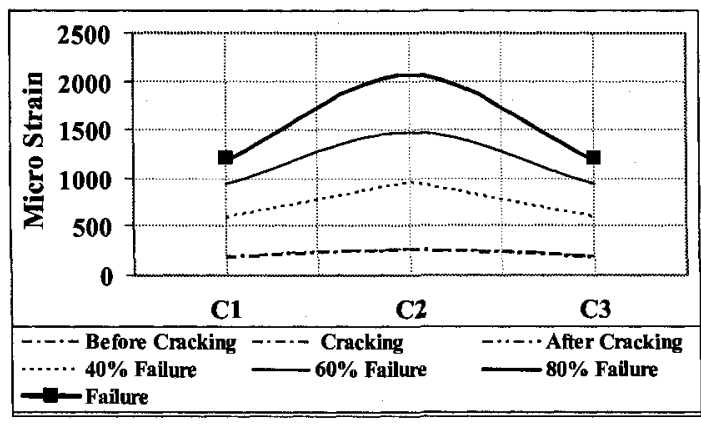

b) VA-EMV-CMP

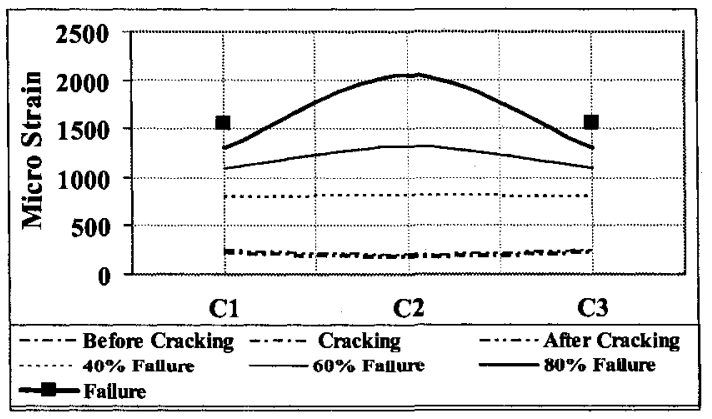

d) VA-NG-CMP

Figure 6.25: Variation of concrete compression strain along doubly reinforced beams at different load levels 


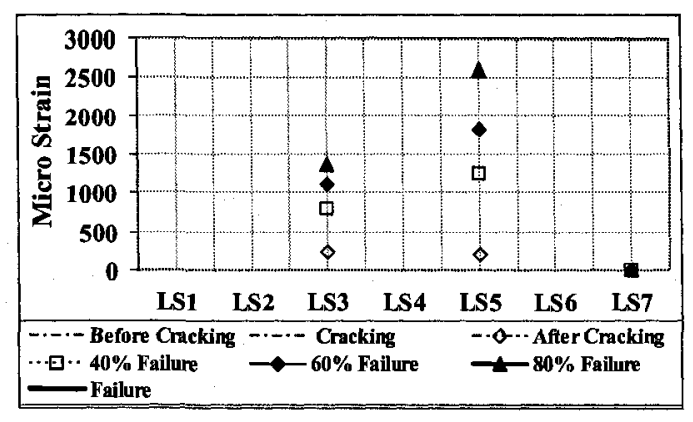

a) MO-EMV-CMP

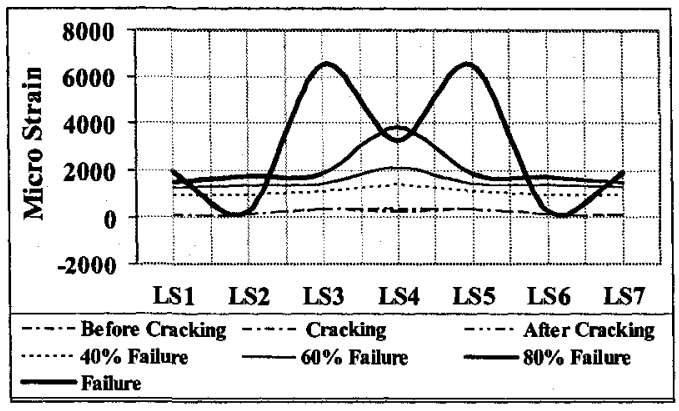

c) MO-NL-CMP

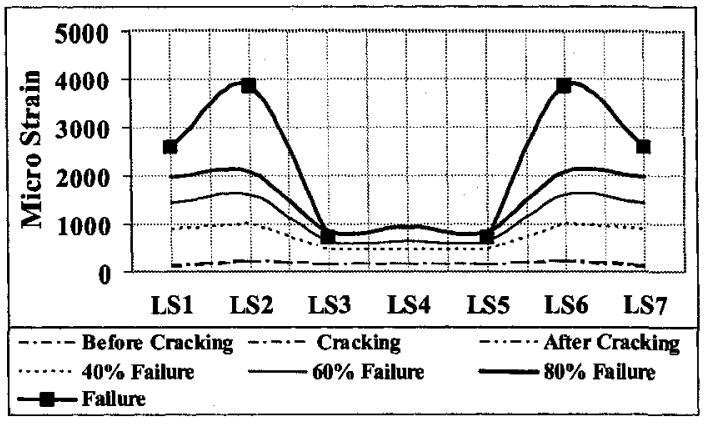

b) VA-EMV-CMP

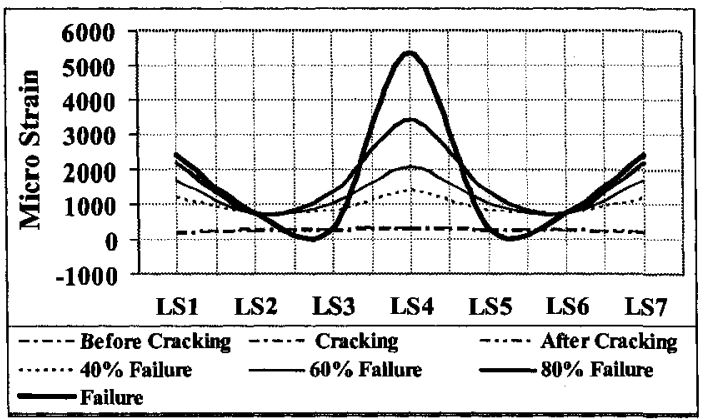

d) VA-NG-CMP

Figure 6.26: Variation of longitudinal steel strain along doubly reinforced beams at different load levels

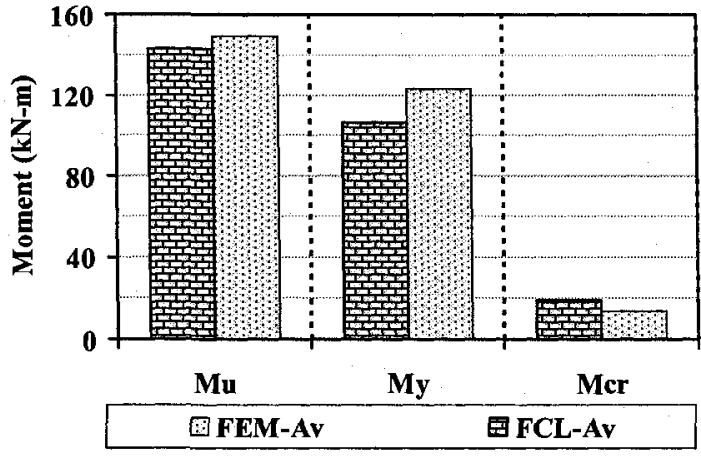

a) FEM-Av and FCL-Av

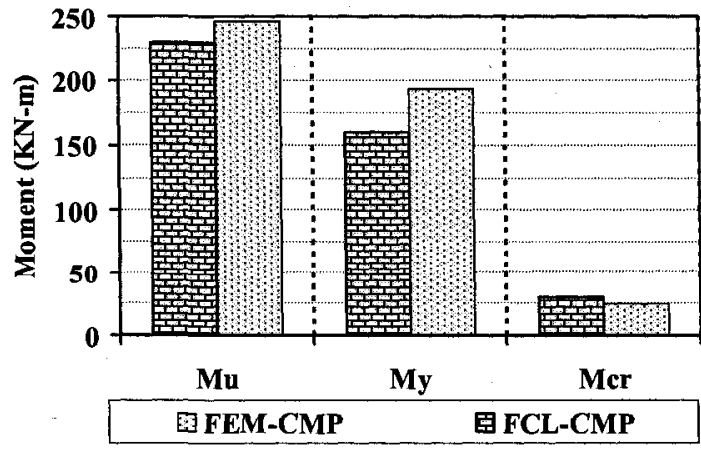

c) FEM-CMP and FCL-CMP

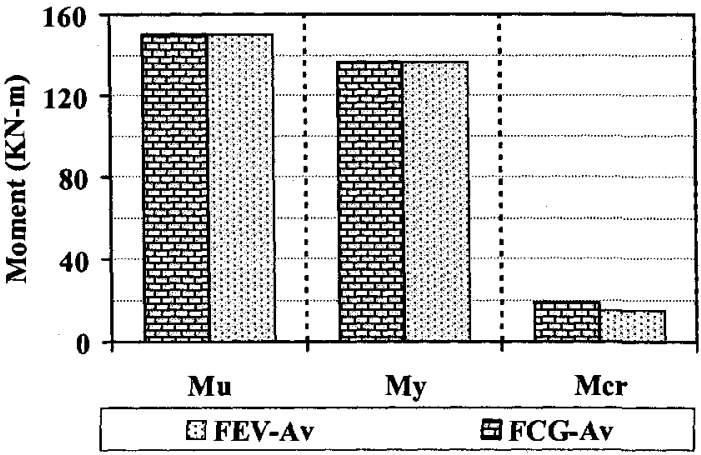

b) FEV-Av and FCG-Av

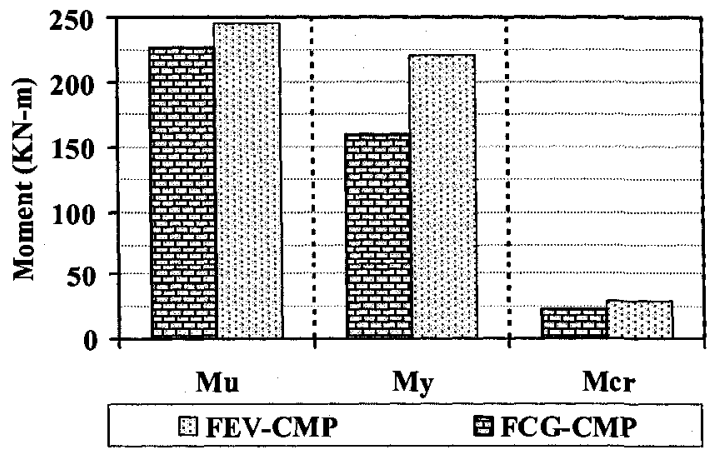

d) FEV-CMP and FCG-CMP

Figure 6.27: Effect of concrete type on cracking, yield and ultimate moments of beams 


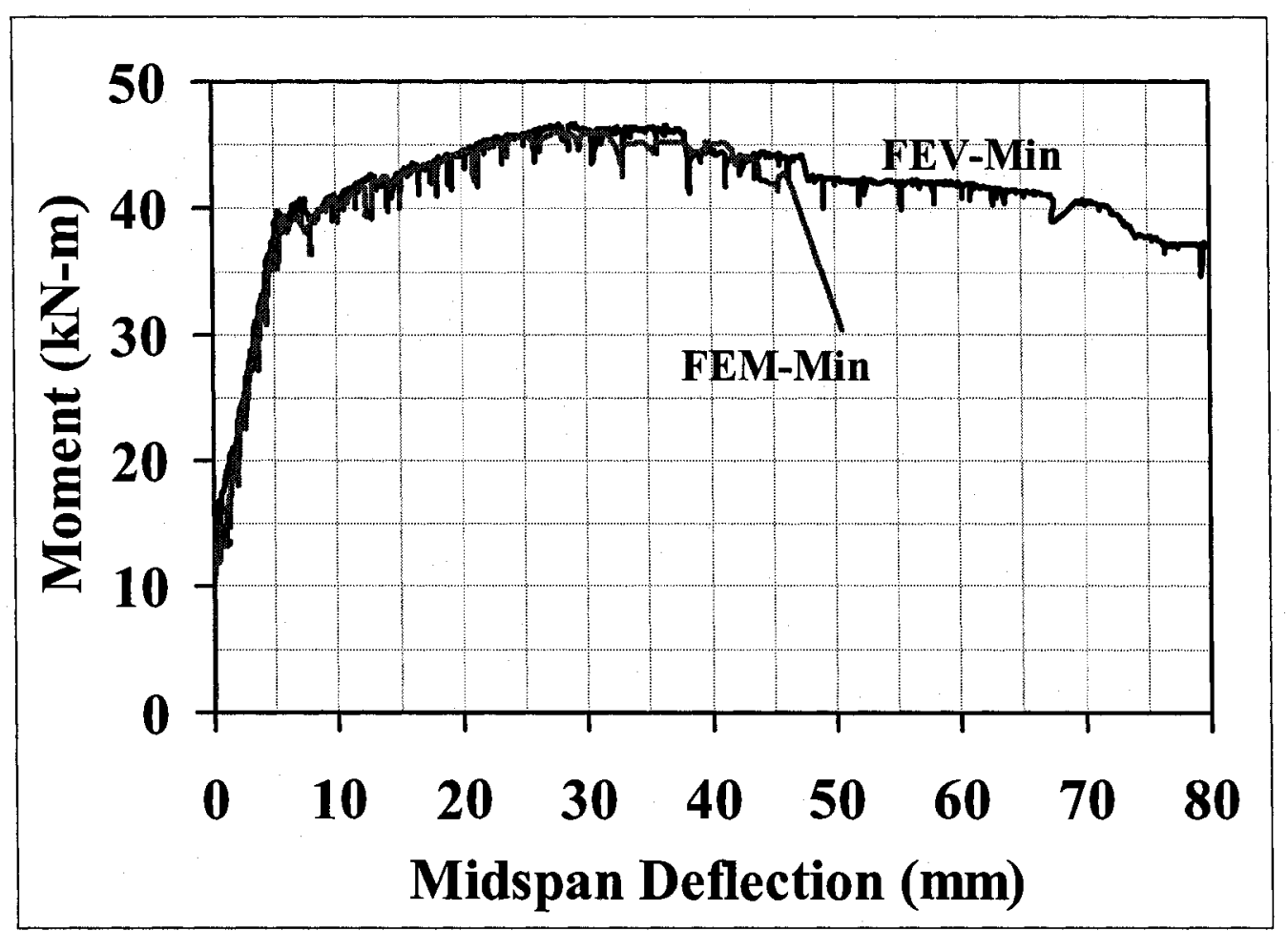

a) Minimum tension reinforcement

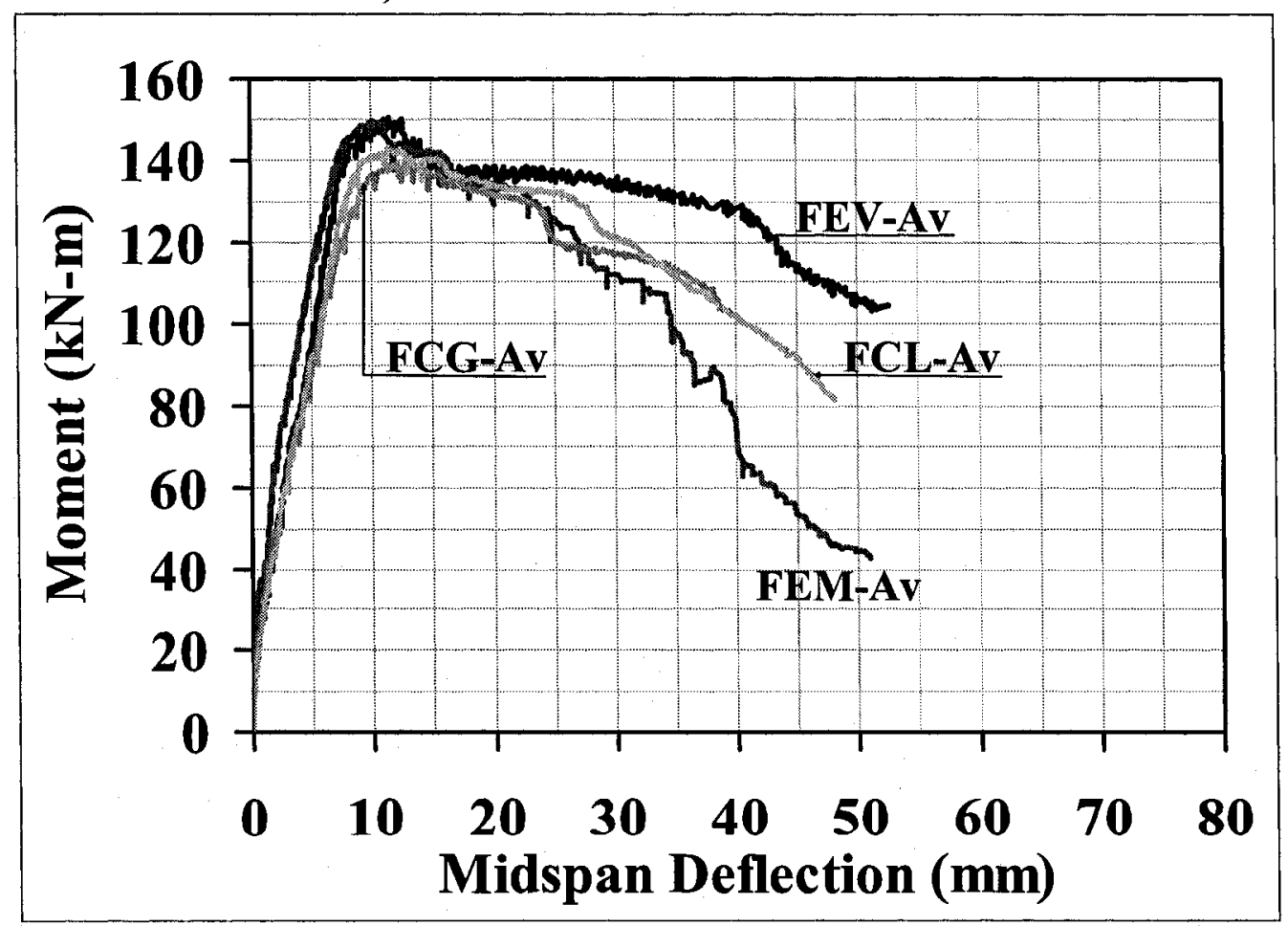

b) Average tension reinforcement

Figure 6.28: Effect of concrete type on flexural behaviour of RRC beams 


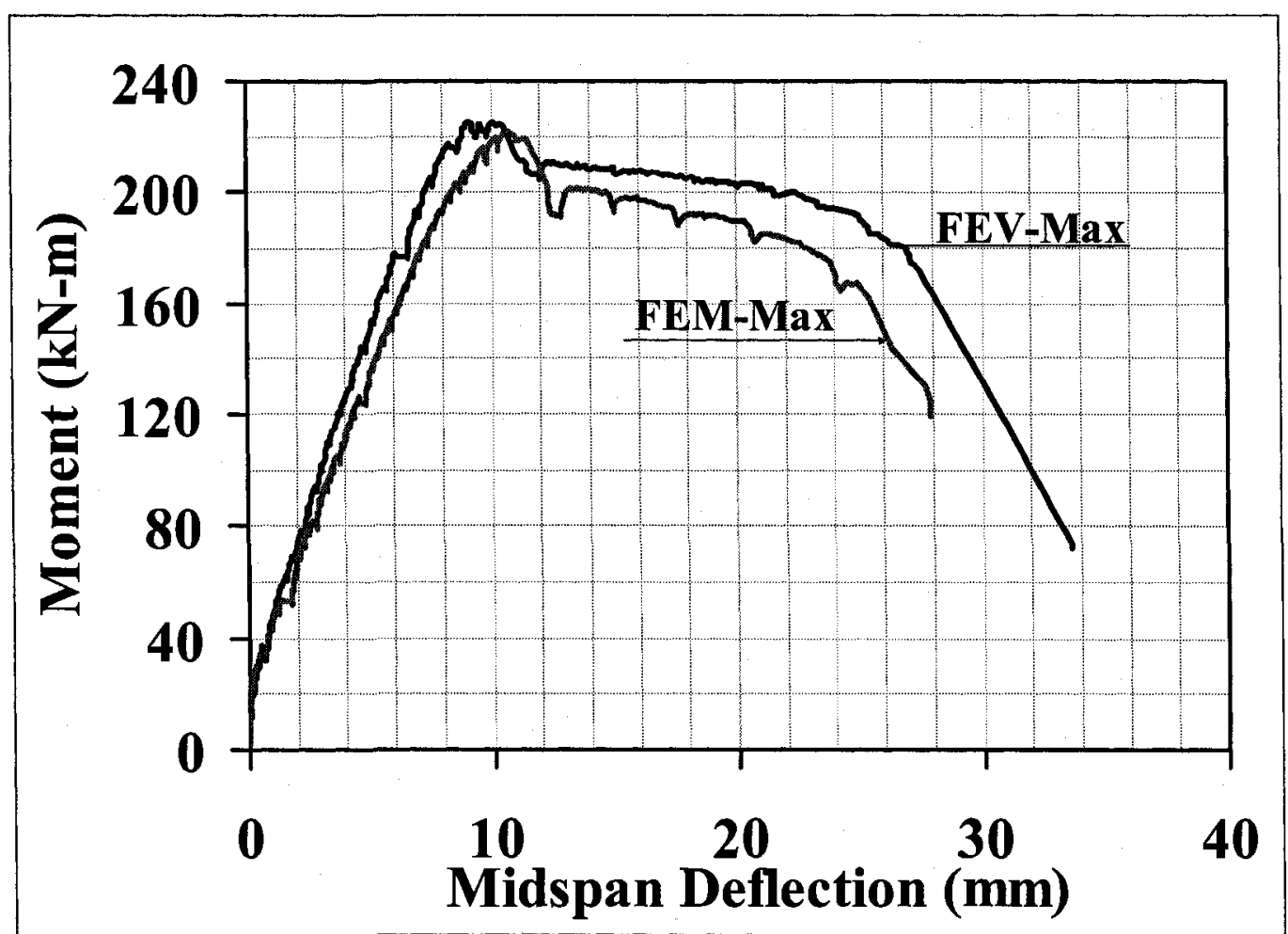

a) Maximum tension reinforcement

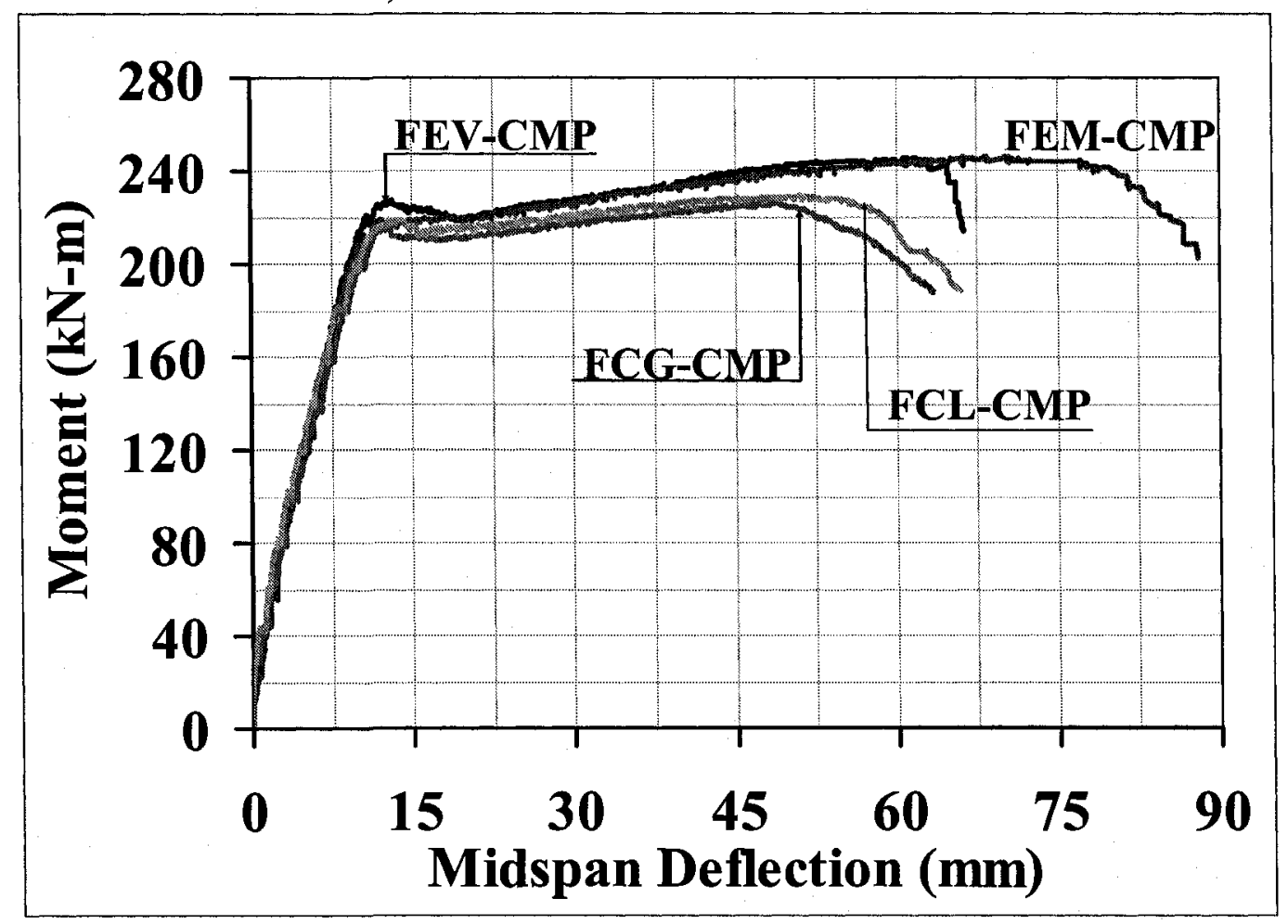

b) Compression reinforcement

Cont. Figure 6.28: Effect of concrete type on flexural behaviour of RRC beams 


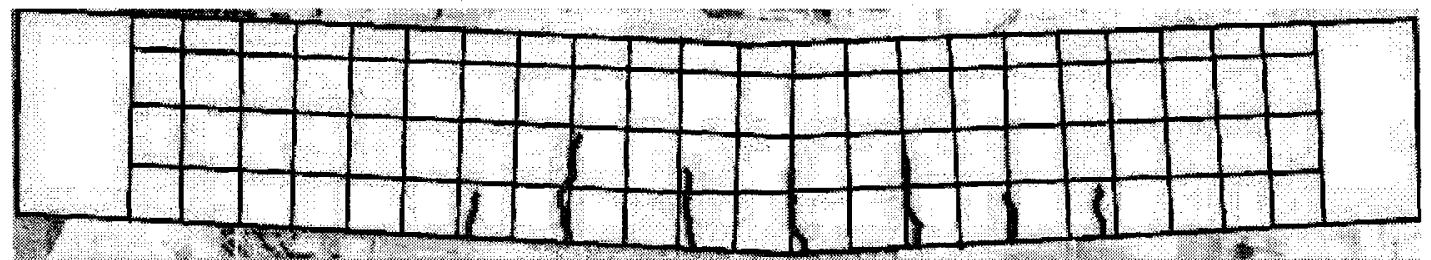

a) WM-Min

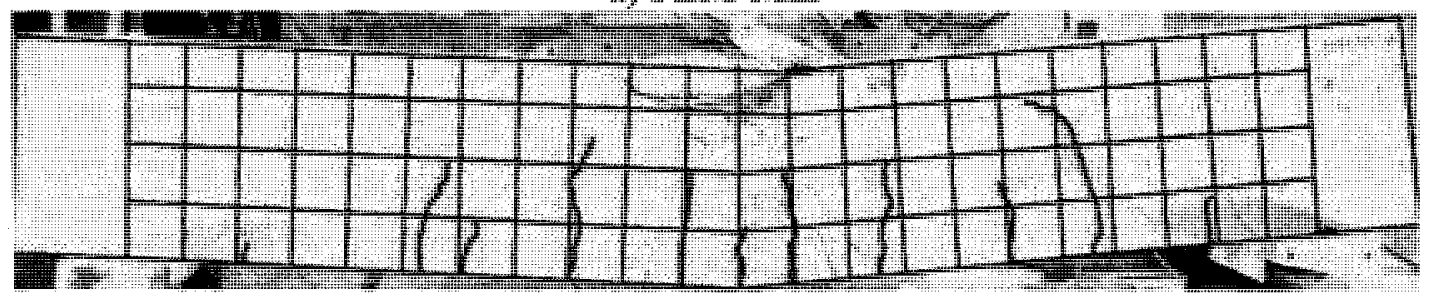

b) FEM-Av

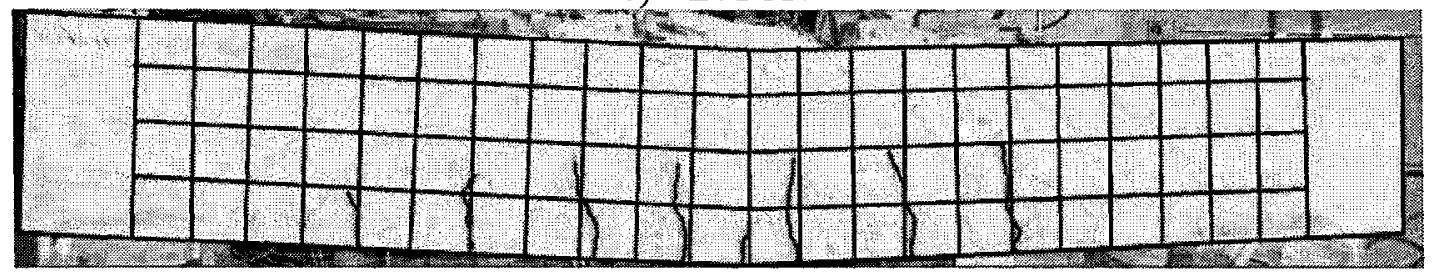

c) FCL-Av

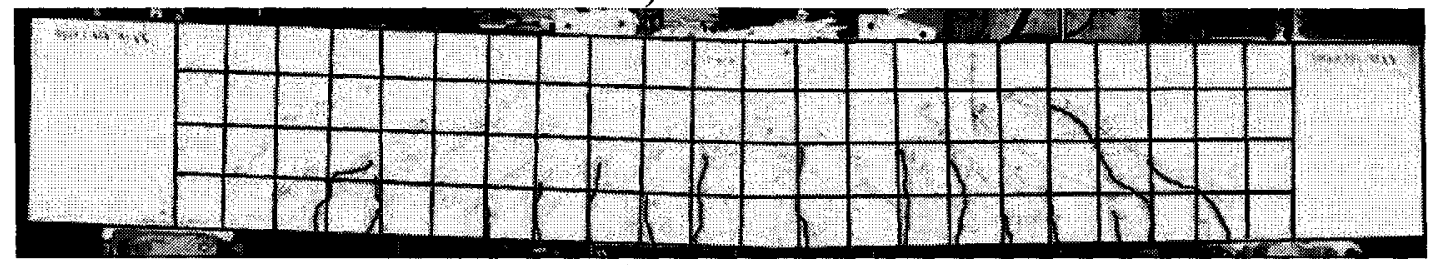

d) FEM-Max

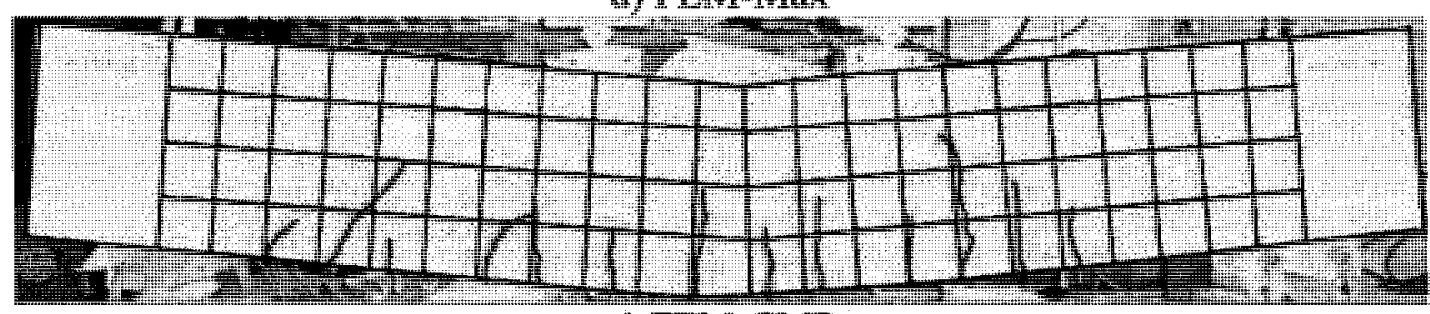

b) $\mathrm{FEM} M \mathrm{CMP}$

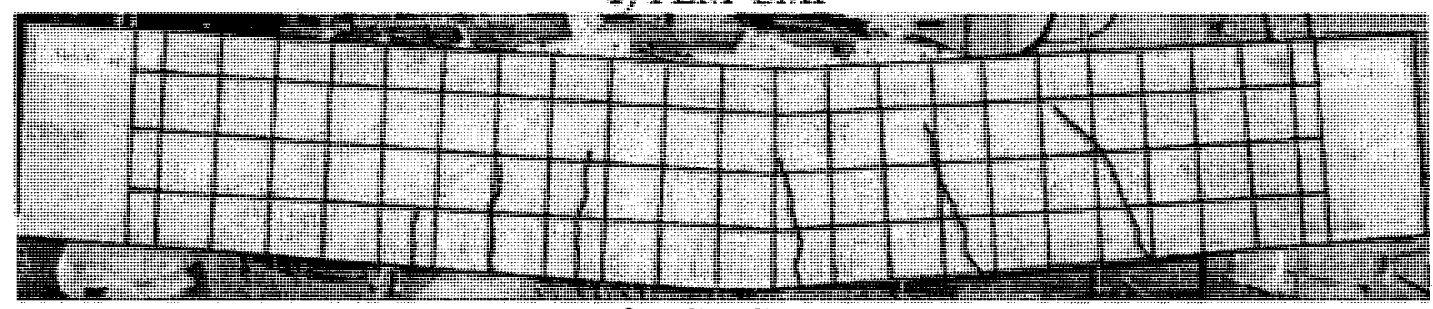

f) FCL-CMP

Figure 6.29: Typical crack spacing of FEM and control FCL beams at service load 


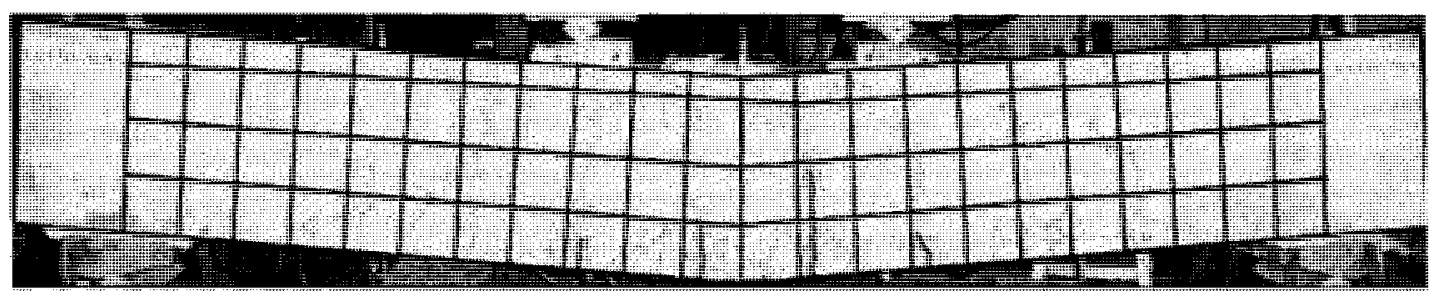

a) FEV-Min

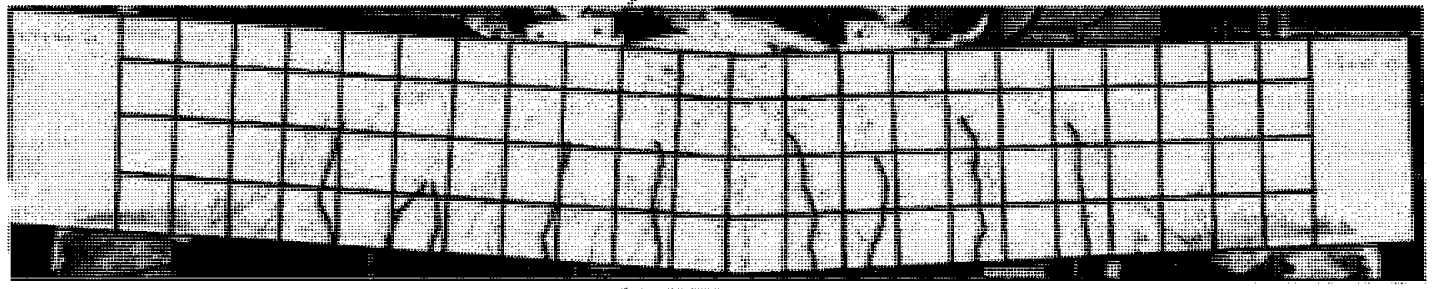

b) IEV-Ay

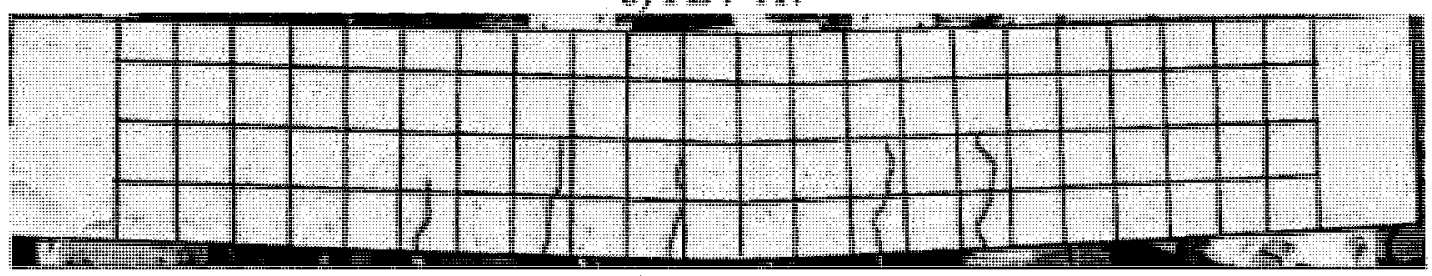

c) FCG-Av

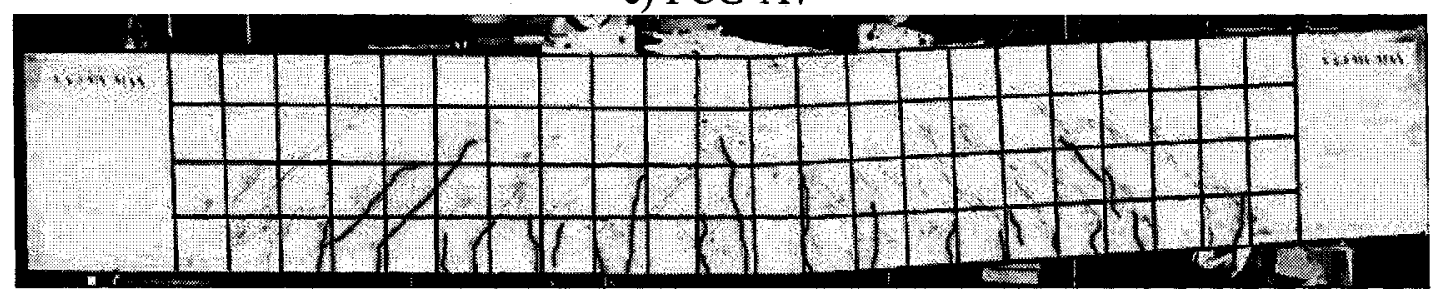

6) FEV-Max

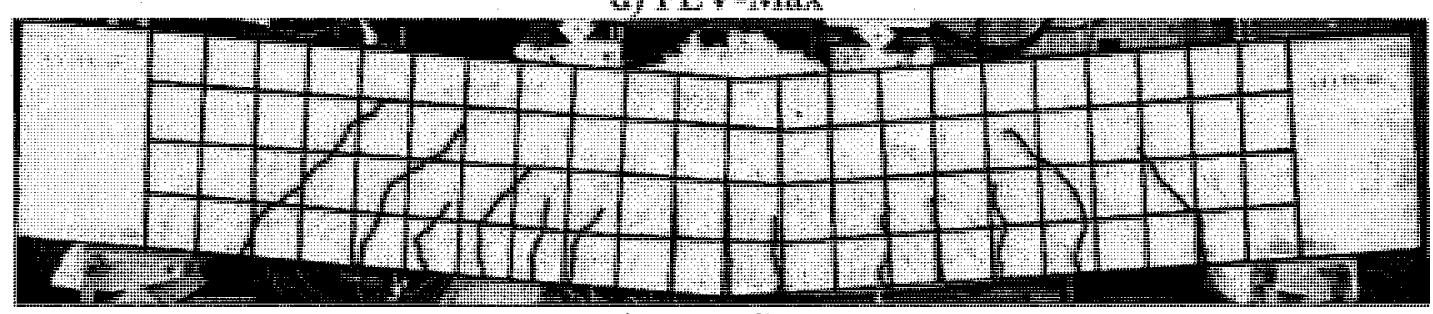

e) FEV-CMP

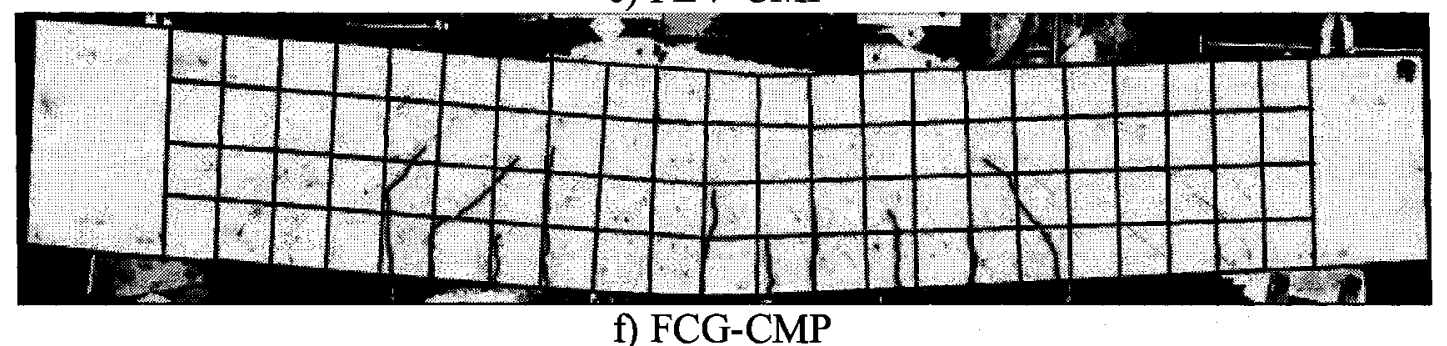

Figure 6.30: Typical crack spacing of FEV and control FCG beams at service load 


\section{CHAPTER 7: STRUCTURAL PERFORMANCE OF RECYCLED REINFORCED CONCRETE BEAMS: SHEAR}

\subsection{General}

A substantial amount of research on shear in conventional reinforced concrete has been carried out over the last 50 years. Today, the shear resistance mechanisms of reinforced concrete are well known and the effect of the various material and geometric parameters on the shear strength of reinforced concrete members is understood. On the other hand, due to lack of adequate research results, it is not evident whether reinforced recycled concrete (RRC) members have the same shear behaviour as conventional reinforced concrete (RC) members. While intuitively one expects the same behaviour, the contribution of the various resistance mechanisms to the total shear resistance may not be the same. In particular, the contribution of the aggregate interlock mechanism and the uncracked concrete to the shear resistance of RAC may not be the same as in conventional concrete because these mechanisms are strongly dependent on concrete properties.

According to existing literature (Chapter 2), the majority of the investigations generally have reported similar shear cracking patterns and failure modes in conventional RC and RRC beams, but practically always lower diagonal cracking load and concrete shear strength $\left(v_{c}\right)$ has been reported. Furthermore, smoother crack interface in RRC beams and consequently less ductile shear behaviour has been reported compared to conventional RC beams. In fact, some of theses characteristics may be expected since in 
all of the previous investigations, the RAC was proportioned based on the conventional method. As the conventional RAC generally contains higher total mortar and lower total natural coarse aggregate compared to normal concrete (Chapters 3 and 4), statistically one would expect fewer aggregate particles to intercept the diagonal cracks, which in turn will decrease the roughness of the crack interface and the effectiveness of the aggregate interlock mechanism in RRC beams. Based on this hypothesis, it is expected that RRC beams made of RAC proportioned by the EMV method will not experience lower ultimate shear strength compared to conventional RC beams made with NAC because the method ensures equal amount of coarse natural aggregate in the two types of concrete. To verify this, an extensive experimental study was carried out. Concrete mixes proportioned by the EMV method were used to cast a number of beams. Since some of the key parameters which affect the shear resistance of reinforced concrete members are the shear span to depth ratio, the beam size and the amount of transverse reinforcement, this chapter will mainly focus on studying these parameters.

Since the RRC beams used in flexural and shear tests were cast simultaneously, the mix proportions as well as the fresh and hardened properties of RAC used in the shear tests were the same as those used in flexural tests presented in Chapter 6 (see Tables 6.5 and 6.6). Similarly, the steel reinforcement of these beams had basically the same strength and stress-strain characteristics as reinforcement used in the flexural tests (see Figure 6.11 in Chapter 6).

Since the number of the control beams made with normal aggregate concrete and consequently the required amount of NAC was small, a pan mixer was used to cast the control beams in two different batches. Table 7.1 presents the summary of the fresh and 
hardened properties of both NAC batches used in control RC beams presented in this chapter. For convenience, in this table, the fresh and hardened properties of the RAC mixes presented in Table 6.8 are also recapped.

\subsection{Details of Beams}

All of the shear beams were designed in accordance with the requirements of the CSA A23.3-04 code.

\subsubsection{Beams without Shear Reinforcement}

It is well known that the shear span/depth ratio has a significant effect on the shear strength of concrete beams. A reduction in this quantity normally increases the shear strength (ASCE-ACI, 1998). Hence, in the present study different shear span to depth (a/d) ratios $(1.5,2,2.7$, and 4$)$ were selected in order to investigate the effect of a/d ratio on the shear behaviour and strength of beams made of RAC without shear reinforcement. The a/d ratios were selected to study the concrete contribution to the shear resistance of short, intermediate, and slender beams. The beams were designed to fail in shear according to CSA Standard A23.3-04 procedures for conventional concrete. Four beams for each RCA source, with their concrete mix proportioned by the proposed mix design method, were tested, i.e. one beam for each $a / d$ ratio. As illustrated in Figure 7.1a, all the test beams are rectangular having width of $200 \mathrm{~mm}$ and depth of 350 to $375 \mathrm{~mm}$ (effective depth of $305 \pm 5 \mathrm{~mm}$ ). For each RCA source, one control beam made of conventional concrete and having $a / d=2.7$ was tested to compare its shear behaviour with those of the companion RAC beams. Considering the two types of RCA, a total of ten beams were tested to investigate the effect of a/d ratio on the shear behaviour of RRC beams. 
It is recognized by the CSA Standard A23.3-04 that larger beams without shear reinforcement have lower shear strength than otherwise similar smaller beams. This is the so-called size-effect. In order to study the size effect on the shear strength of RRC beams without shear reinforcement, four beams with total depths of $250,375,450$, and $550 \mathrm{~mm}$, and a constant $a / d$ ratio of 2.70 were tested. These results for these beams will be used to check the applicability of Equation (11-9) in Clause 11.3.6.3 of CSA A23.3-04 to RRC members. Once again to study the size effect, a total of ten beams were tested, five beams per RCA type, including one control beam. As illustrated in Figure 7.1a, all the rectangular beams are $200 \mathrm{~mm}$ wide by 250 to $550 \mathrm{~mm}$ deep. The control beams made of conventional concrete were $375 \mathrm{~mm}$ deep.

Table 7.2 presents the details of the beams used to investigate the shear performance of RRC beams without shear reinforcement. It should be noted that each beam in this table is identified with a unique designation. The following notation is used in these designations:

- Part 1:S (shear).

- Part 2: Type of concrete EM or EV (RAC proportioned by the EMV method and made with RCA-MO or RCA-VA); and CL or CG (control beams produced with NAC made of natural limestone or natural river gravel).

- Part 3: 1.50N, 2.0N, 2.70N, or 4.0N (nominal a/d ratios of 1.5, 2.0, 2.7, or 4.0 with N signifying no shear reinforcement) and $\mathrm{L}, \mathrm{M}, \mathrm{H}$, or HH (beam depth classification: Low (250 mm), Medium (375 mm), High (450 mm), very High (550 mm)). 


\subsubsection{Beams with Shear Reinforcement}

To study the behaviour of RRC beams with shear reinforcement, a number of important variables were considered. The test variables comprised three shear reinforcement ratios: (1) no shear reinforcement, (2) three times the minimum shear reinforcement, and (3) six times the minimum shear reinforcement. All shear reinforcement ratios were chosen to be less than the maximum shear reinforcement ratio required by CSA A23.3-04 for normal concrete. In order to satisfy the requirements of CSA A23.3-04 (clause 11.3.8.1) regarding the maximum stirrup spacing, the round bar size No.8 was used to ensure the desired shear reinforcement ratios. The minimum shear reinforcement ratio was calculated according to the CSA Standard A23.3-04 for conventional or normal concrete. For each RCA source, three beams were tested, i.e. one beam for each shear reinforcement ratio. The concrete for these beams was proportioned by the EMV method. An additional beam was designed and tested for each RCA source. The latter beams were reinforced with deformed No.10 bars as stirrups and they had shear reinforcement equal to six times the minimum shear reinforcement. As illustrated in Figure 7.1b, once again all the beams were rectangular $200 \mathrm{~mm}$ wide, and from 375 to $385 \mathrm{~mm}$ deep (effective depth of $305 \pm 4 \mathrm{~mm}$ ) with nominal shear span/depth $(a / d)$ ratio of 2.70 and span length of $2.2 \mathrm{~m}$. In addition, a control beam for each RCA source was tested to investigate the shear behaviour of normal RC beams with six times the minimum shear reinforcement. Therefore, a total of ten beams were used to investigate the shear behaviour of RRC beams with shear reinforcement. Table 7.3 presents the details of these beams. It should be noted that each beam in this table is identified with a unique designation. The following notation is applied: 
- Part 1: S (shear).

- Part 2: EM or EV (RAC proportioned with EMV method and produced with RCAMO or RCA-VA); and CL or CG (control beams produced with NAC made of natural limestone or natural river gravel).

- Part 3: NS, 3S, or 6S (using no shear reinforcement, three times, or six times of minimum shear reinforcement).

- Part 4: $\mathrm{R}$ or $\mathrm{D}$ (using smooth round bars or deformed bars for shear reinforcement).

To ensure shear failure in a designated location, all the shear beams, with or without shear reinforcement, were designed to fail in their west shear span by providing higher shear reinforcement in their east shear span. The amount of shear reinforcement in east shear span was increased by either halving the stirrup spacing or increasing the shear reinforcement size compared to the west span.

In order to avoid any unexpected bond failure of the longitudinal reinforcement bars prior to shear failure, they were provided with $180^{\circ}$ standard hook in accordance with the specifications of CSA A23.3-04. Furthermore, all the stirrups had a $135^{\circ}$ hook with minimum extension length specified by CSA A23.3-04 code. All the beams were simply supported and were tested in four point bending.

\subsection{Instrumentation}

\subsubsection{Strain Gauges}

In order to investigate the shear behaviour of RRC beams, one needs to know the strain values of concrete and steel reinforcement at different locations. Towards this end, strain gauges were installed on concrete and on the shear and longitudinal reinforcement at several locations. The N11-FA-5-120-11 and N11-FA-30-120-11 strain gauges provided 
by Showa Measuring Instruments were used for measuring the strain in the steel reinforcement and concrete, respectively. Figure $7.2 \mathrm{a}$ and $7.2 \mathrm{~b}$ illustrate the arrangement of the strain gauges for shear beams without and with shear reinforcement, respectively. In this figure, C, LS and TS designate the concrete, longitudinal steel and transverse steel strain gauges, respectively. Four different locations were chosen for the longitudinal steel strain measurement: (1) at distance $d$ from the face of the support in the west shear span; (2) under the point loads; (3) at midspan; and (4) between west point load and the face of the support in the west shear span. For longitudinal reinforcement, one strain gauge was installed at each location on the bottom of the bar closest to the underside of the formwork to avoid any damage during casting. For transverse reinforcement, two strain gauges were installed at each location (one at midheight of each leg of the stirrup). In shear beams without shear reinforcement, the shear reinforcement located between the east point load and the face of the support at east shear span was also instrumented by strain gauges to measure its deformation. For concrete strain measurements, two different locations were selected: (1) at midspan (C2); and (2) between the west point load and the face of the support in the west shear span. (C1). One strain gauge was installed at each location on the top fibre of the section.

\subsubsection{Linear Potentiometers (LPs)}

In order to capture the load-deflection response of the RRC beams, several linear potentiometers were installed at different locations along each beam. Figure 7.3 illustrates the typical arrangement of the Linear Potentiometers (LP). 


\subsubsection{Linear Variable Displacement Transducers (LVDTs)}

One of the main mechanisms contributing to the shear resistance of reinforced concrete members is aggregate interlock, which is a function of the diagonal crack width, the roughness of the crack interface, and the nominal maximum aggregate size used. Since in the majority of the previous research (Chapter 2) wider cracks, smoother crack interface, and less ductile behaviour for RRC beams compared to conventional RC beams have been reported, it is important to investigate these phenomena for the current beams because they are made of RAC designed by the EMV method. To gain insight into the aggregate interlock mechanism, it was decided to instrument the shear beams with a rosette of three LVDTs (ST1, ST2 and ST3) arranged as illustrated in Figure 7.4. The length of the LVDTs and the location of the rosette were chosen to cross the anticipated major diagonal crack.

\subsection{Shear Performance of RRC Beams without Shear Reinforcement}

Since the main variables in studying the shear performance of RRC beams were the a/d ratio and the size of the beams, the results of this study will be presented in two separate subsections.

\subsubsection{Effect of $a / d$ Ratio}

\subsubsection{Failure Modes}

Figure 7.5 illustrates the cracking patterns of both SEM and SEV beams for different $a / d$ ratios as well as those of the control beams SCL and SCG with a/d ratio of 2.70. In these figures, the dark inclined lines show the major diagonal cracks leading to shear failure; the light lines represent the minor shear and flexural cracks; and the dark region near the top of the beam between the point loads indicate the crushed concrete zone. 
After inclined cracking, the RRC beams with small a/d ratios (i.e., 1.5 and 2.0 ) behaved akin to a tied arch carrying the load by direct compression, and exhibited considerable reserve capacity. On the other hand, the RRC beams with larger $a / d$ ratios (i.e., 2.7 and 4.0) resisted the shear by so-called beam action and consequently they failed at or shortly after the formation of the major diagonal crack.

The details of the failure modes are presented for different $a / d$ ratios as follows:

- $a / d=1.5$

In the SEM-1.5N beam, after the formation of inclined cracks in the west shear span at about $143 \mathrm{kN}$, the beam behaved similar to a tied-arch, carrying the load by direct compression from the point of loading to the support, which contributed to its considerable shear capacity. By increasing the load, the diagonal crack extended further into the compression zone and horizontally along the tension reinforcement. It also branched into a secondary crack propagating toward the bottom of the beam. Due to the excessive extension of the inclined crack into the compression zone, the available area of concrete in the vicinity of the west load point became too small to be able to resist the compression force and crushed at $P_{\mathrm{u}}=371 \mathrm{kN}$ in a shear-compression failure mode and in a relatively ductile manner.

In SEV-1.5N beam, after flexure-shear cracking occurred $\left(\mathrm{P}_{\mathrm{u}} \approx 127 \mathrm{kN}\right)$ in the west shear span, it extended further into the compression zone and propagated as a secondary crack toward the bottom of the beam. However, the beam eventually failed at $P_{u}=388 \mathrm{kN}$ in flexure in a tension failure mode and in a very ductile manner. Both of these beams failed at significantly higher loads than predicted by available design standards. 
- $a / d=2.0$

In both SEM-2.0N and SEV-2.0N beams, once the inclined cracking occurred in the west shear span at about 162 and $123 \mathrm{kN}$, respectively, the beams quickly transformed to a tied arch and resisting further increase in load by this mechanism. As the load increased, the crack advanced further into the compression zone. It again formed a secondary branch moving along the longitudinal reinforcement and then bending towards the end hook in the latter reinforcement. Failure eventually occurred in the west shear span at $P_{u}=336$ and $355 \mathrm{kN}$ in SEM-2.0N and SEV-2.0N beams, respectively. The failure was caused by anchorage failure of the tension reinforcement, and it can be characterized as a brittle and a shear-tension failure mode.

- $\quad a / d=2.7$

In both SEM-2.7N and control SCL-2.7N beams, the inclined flexure-shear cracks occurred in the west shear span at approximately 102 and $131 \mathrm{kN}$, respectively, mainly as extension of vertical flexural cracks, propagated diagonally towards the compression face and as a secondary crack toward the tension reinforcement, and horizontally progressed along the tension reinforcement. After excessive propagation of the cracks, the beam action mechanism was not capable of load redistribution in SEM-2.7N and control SCL$2.7 \mathrm{~N}$ beams similar to those with smaller $a / d$ ratios, and failed at west shear span in a diagonal tension failure mode at $\mathrm{P}_{\mathrm{u}}=204$ and $181 \mathrm{kN}$ in a brittle way, respectively.

Similarly, in SCG-2.7N, the flexure-shear crack occurred at approximately $185 \mathrm{kN}$ in the west shear span and quickly propagated diagonally towards the compression face and concurrently towards and subsequently along the tension reinforcement. The beam 
eventually failed at $\mathrm{P}_{\mathrm{u}}=296 \mathrm{kN}$ in a brittle manner in the west shear span in a diagonal tension failure mode.

Due to an error in the fabrication of the steel reinforcement cages in SEV-2.7N, its west shear span was mistakenly reinforced with stirrups, and therefore the beam failed in flexure rather than shear.

- $a / d=4.0$

In SEM-4.0N, several flexural cracks formed initially. However, at approximately 160 $\mathrm{kN}$, an inclined crack formed in the west shear span and very quickly propagated towards both the compression face and the tension reinforcement, and immediately after the beam failed in a brittle manner in a diagonal tension failure mode at $\mathrm{P}_{\mathrm{u}}=161 \mathrm{kN}$.

In SEV-4.0N, the inclined flexure-shear cracks occurred in the west shear span at about $131 \mathrm{kN}$ mainly as extension of vertical flexural cracks. After formation of these cracks, they propagated diagonally upward to the compression face and downward to the tension reinforcement. Again the failure was brittle and it occurred in the west shear span in a diagonal tension mode at $\mathrm{P}_{\mathrm{u}}=205 \mathrm{kN}$.

Considering the ratio of the failure load to the inclined cracking load as an indicator of ductility, SEM-1.5N had the highest ductility ratio of 2.59 compared to the $2.07,2.00$, and 1.0 for beams SEM-2.0N, SEM-2.7N and SEM-4.0N, respectively. Similarly, SEV$1.5 \mathrm{~N}$ had a ductility ratio of at least 3.06 compared to the 2.89 and 1.56 for beams SEM$2.0 \mathrm{~N}$ and SEM-4.0N, respectively. Furthermore, beam SEM- $2.7 \mathrm{~N}$ had a ductility ratio of 2.00 , which is much higher than the1.38 ratio for control beam SCL-2.7N.

Figure 7.7 illustrates the variation of the longitudinal steel strain at distance $d$ from the face of the west support (LS1) with load for beams SEM and SEV and the control beams 
SCL and SCG at different $a / d$ ratios. According to this figure, the longitudinal steel reinforcement near the west support has yielded in both beams SEM-1.5N and SEV$1.5 \mathrm{~N}$. This is mainly attributable to the arch action at low a/d ratios which generally requires a substantial horizontal tension, i.e. tie force, to be effective. This tension is provided by the longitudinal tension reinforcement. This also can be observed in Table 7.4 which presents the measured strains of both the longitudinal and shear reinforcements as well as the concrete at different locations of shear beams at failure load.

\subsubsection{Ultimate Shear Strength}

Figure 7.6a and 7.6b illustrate the normalized shear stress resistance values $\left(v_{c} /{\sqrt{f^{\prime}}}_{c}\right)$ of beams SEM and SEV for different $a / d$ ratios, respectively. Note that the $a / d$ value of each beam is indicated by the number in its designation. In compliance with standard practice in concrete design standards, the $v_{c}$ values of all the beams were normalized by the square root of the compressive strength of its concrete on the day of the test. The shear stress resistance (provided by concrete) was calculated as follows:

$$
v_{c}=\frac{V_{u}}{b \times d_{v}}
$$

where $V_{u}$ is the ultimate shear force acting on the beam, $b$ is the section width, and $d_{v}$ is the effective shear depth (taken as the greater of $0.9 d$ and $0.72 h$, where $d$ and $h$ are the beam effective depth and overall height, respectively). The ultimate shear $\left(V_{u}\right)$ was calculated as

$$
V_{u}=0.5 P_{u}+\frac{\left(\gamma_{c} \times b \times h\right) \times L_{n}}{2}
$$

where $P_{u}$ is the failure load of the beam, $\gamma_{c}$ is the hardened density of the concrete, and $L_{n}$ is the net beam span, i.e. from centre to centre of its supports. 
Note that the $v_{c} / \sqrt{f^{\prime}}$, values increased with decreasing a/d ratio. This is mainly owing to the more efficient arch action mechanism after the formation of inclined cracks in beams with lower a/d ratio. In other words, the available strength from arch action mechanism is greatly dependant on the intensity of the diagonal compression stress, which itself is a function of the thrust line inclination or $a / d$ ratio. According to Fig.7.6a, the $v_{c} / \sqrt{f_{c}^{\prime}}$ values of beams SEM-1.50N, SEM-2.0N, SCL-2.7N and SEM-2.7N are $9 \%$, $24 \%, 102 \%$, and $128 \%$ higher than that of SEM-4.0N. Furthermore, the $v_{c} / \sqrt{f_{c}^{\prime}}$ values of beams SEV-1.50N and SEV-2.0N are $68 \%$ and $88 \%$ higher than that of SEV- $4.0 \mathrm{~N}$ (Figure 7.6b). Note that the actual $v_{c} / \sqrt{f^{\prime}}$ c value for SEV-1.5N may be higher than that given in Figure 7.6b since it failed in flexure rather than in shear. This is indicated by the arrow at the top of the SEV-1.5N bar chart.

According to Figure 7.6a, the $v_{c} / \sqrt{f^{\prime}}{ }_{c}$ value of SEM-2.7N is also $14 \%$ higher than that of control SCL-2.7N made with natural limestone. This finding is in contrary to the lower shear strength of few reported RRC beams in the literature (Chapter 2), which may be ascribed to the application of the EMV method in this study by contrast to the conventional mix proportioning method used in the previous studies. This observation again supports the validity of concept of equivalent total mortar (EMV) mix proportioning method. In fact, by providing equivalent total natural aggregate volume in RAC and the companion NAC, due to the lower nominal maximum OVA size in the beam SEM-2.7N compared to the nominal maximum NA size in SCL-2.7N, the number of the natural aggregate particles (NA and OVA) on the surfaces of the inclined crack in SEM-2.7N may have been higher than that in SCL-2.7N, which would have increased the 
roughness of the crack interface, and consequently resulted in the higher contribution of the aggregate interlocking mechanism to the shear resistance of beam SEM-2.7N.

\subsubsection{Shear Performance}

Figure 7.8a shows the variation of $v_{c} / \sqrt{f^{\prime}}{ }_{c}$ versus midspan deflection for different a/d ratios in beams SEM and SEV.

Generally, the slope of the curves tends to decrease with increasing the $a / d$ ratio in both the beams SEM and SEV. This can be mainly attributed to the effect of the arch action. At high a/d ratios, after the formation of the major inclined crack, the stiffness of the beam dramatically drops (SEM-4.0N and SEV-4.0N beams) while at low a/d ratios (SEM-1.5N and SEV-1.5N), after the inclined crack formation, the beam quickly transforms to a tied arch and redistributes the load, which prevents the loss of stiffness due to beam action breakdown.

Figure $7.8 \mathrm{~b}$ presents the variation of $v_{c} / \sqrt{f^{\prime}}{ }_{c}$ with the deformation measured by the LVDT crossing the inclined crack in the west shear span (ST1) in beams SEM and SEV. According to this figure, all of the curves exhibit an initial linear elastic portion, a falling portion (i.e., segment in which the strain increases with load drop), a hardening segment in which the load again increases with increased deformation, and finally another softening segment in which the load drops with increased deformation until failure occurred.

The initial linear elastic portion represents the behaviour of the beam before inclined crack formation. The first observed drop in the load is due to the formation of the diagonal crack which causes a reduction in the stiffness of the beam. The extent of the falling portion appears to be generally a function of the $a / d$ ratio of the beam. Deep 
beams with lower $a / d$ ratio have a much shorter falling segment than the long beams with higher $a / d$ ratio. Since beams with larger $a / d$ (i.e. 2.7 or higher) rely mainly on beam action for shear resistance, their shear stiffness is more noticeably affected by the formation of the diagonal cracks.

The extent of hardening following the initial softening is also a function of the $a / d$ ratio. Deep beams with lower $a / d$ ratio experience more noticeable hardening compared to the long beams with higher $a / d$ ratio. At higher $a / d$ ratios, after the inclined crack formation, the load drops slightly, but owing to the aggregate interlock mechanism, it climbs up again until diagonal tension failure occurs.

Figure 7.9 illustrates the variation of the concrete compression strain along the beams at different load stages for different $a / d$ ratios as recorded by the gauges along the beams. As expected, all the midspan concrete compression strains (C2) increased as the load increased, and it always occurred at midspan at all load levels. However, in the beams with the $a / d$ ratio less than 4.0 and failing in shear, the concrete compression strain in the shear span (C1) started to drop after about $40 \%$ of the failure load. This may be attributed to the formation of diagonal cracking and the contribution of arch action and redistribution of internal forces across the beam section in the shear span. Observe that in the beams with high a/d ratios of 4.0, due to the low contribution of arch mechanism and subsequent shear failure of the beams after formation of diagonal cracking, no drop in the strains in the shear span occurred.

Figure 7.10 illustrates the variation of the longitudinal tension reinforcement recorded strain along the beam for the beams with different $a / d$ ratios. Generally, the variation of the tension steel strain along all the beams tended to increase with increasing load, and 
the maximum strain generally occurred either at midspan or under the point loads at all load levels (except VA-EMV-2.7N). According to Fig.7.10, it can also be observed that the tension reinforcement near the supports of the beams with $a / d=1.5$ (LS1) has yielded and is the highest compared to the other beams with higher a/d ratios, while for the beams with $a / d=4.0$, it was found to be the lowest. This can be mainly attributed to the greater contribution of arch action and consequently the effective role of tension reinforcement serving as tie in the tied arch to which the beam transforms after formation of diagonal cracking. The arch mechanism is enhanced by bond failure and can be very effective in resisting increased load, provided the tension reinforcement is well anchored at its ends as was the case for in present beams.

Figures 7.11 and 7.12 illustrate the effect of aggregate type on the $v_{c} / \sqrt{f^{\prime}}{ }_{c}$ variation with midspan deflection and diagonal deformation for different a/d ratios. Generally, similar behaviour is exhibited by the beams SEM and SEV for the same a/d ratio, regardless of the aggregate type.

According to Figures $7.11 \mathrm{c}$ and $7.12 \mathrm{c}$, beam SEM-2.7N beam is slightly less stiff compared to beam SCL-2.7N, but it is more ductile. This again contradicts previous findings in the literature where RRC beams are reported to be less ductile. By using the proposed mix proportioning method, both crack interface roughness and aggregate interlock mechanism are enhanced due to the higher number of particles on the inclined crack surfaces in SEM-2.7N compared to SCL-2.7N.

\subsubsection{Serviceability}

Due to three main reasons, viz. appearance, leakage, and corrosion, crack width is one of the major serviceability criteria in design codes. Assuming $40 \%$ of the failure load as the 
customary level of the service load in beams, the diagonal crack width in the present RRC beams was obtained using the deformation recorded by the LVDT crossing the inclined crack (see ST1 in Fig.7.4) at the above load level. Of course, the deformation measured by ST1 also includes some elastic deformations. By ignoring these elastic strains as being small relative to the crack movement, crack width values of $0.19,0.05$, $0.06,0.01$ and $0.03 \mathrm{~mm}$ were calculated for beams SEM-1.5N, SEM-2.0N, SEM-2.7N, SCL-2.7N and SEM-4.0N, respectively. Similarly, crack width values of $0.22,0.00,0.04$ and $0.02 \mathrm{~mm}$ were determined for beams SEV-1.5N, SEV-2.0N, SCG-2.7N and SEV$4.0 \mathrm{~N}$, respectively. Note that the higher crack width in the beams with lower $a / d$ ratios is mainly due to their higher ultimate shear resistance, and their correspondingly higher service loads. Similarly, the higher crack width in SEM-2.7N compared to control SCL$2.7 \mathrm{~N}$ beam is partially due to the higher failure load and consequently relatively higher service load for the former beam.

Note that all of the above crack width values at service load are well below the maximum crack width of 0.4 and $0.33 \mathrm{~mm}$ for interior and exterior exposures recommended by the CSA Standard A23.3-04.

\subsubsection{Size Effect}

\subsubsection{Failure Modes}

Figure 7.13 exhibits the cracking patterns of both the SEM and SEV beams with different overall depths as well as those of control beams SCL-M and SCG-M with an overall depth of $375 \mathrm{~mm}$.

All of the RRC beams failed in diagonal tension, except beam SEV-L which failed in flexure. In these beams, after the formation of a major inclined crack, it propagated 
diagonally towards the compression face of the beam. In the RRC beams with small and medium depth values, respectively, (effective depth of 200 and $300 \mathrm{~mm}$ ), the aggregate interlock mechanism and dowel action were capable of sustaining the increased load after the inclined crack formation. Upon further increase of the load, eventually these beams failed in diagonal tension. On the other contrary, the RRC beams with larger depth (effective depth of 400 and $500 \mathrm{~mm}$ ) were not capable of load redistribution after the formation of inclined cracking, and they failed shortly after. It must be stated that the diagonal crack in the present discussion refers to the major or fatal crack that eventually led to failure and not to the smaller inclined cracks.

In the followings, the behaviour of each beam is discussed in more detail.

\section{- Effective depth $\mathrm{d}=200 \mathrm{~mm}$}

In SEM-L beam, after the formation of the inclined flexure-shear cracking in the west shear span at approximately $102 \mathrm{kN}$, it quickly propagated towards the compression face. At about $145 \mathrm{kN}$, a new diagonal crack suddenly formed, it propagated as a secondary crack toward the tension reinforcement and horizontally along the tension reinforcement. After extensive propagation of the cracks, the beam action mechanism was not capable of load redistribution and the beam failed in a brittle manner in the west shear span due to diagonal tension failure at $\mathrm{P}_{\mathrm{u}}=177 \mathrm{kN}$.

In SEV-L, the inclined crack formed in the west shear span at $\mathrm{P}=112 \mathrm{kN}$ mainly as an extension of the vertical flexural cracks near the tension face of the beam. At $P=127 \mathrm{kN}$, a new diagonal crack suddenly formed and propagated diagonally from the west support toward the west point load. It also propagated as a secondary crack toward the tension 
reinforcement. However, the beam eventually failed at $P_{u}=243 \mathrm{kN}$ in flexure in a tension failure mode and in a very ductile manner.

\section{- Effective depth $d=300 \mathrm{~mm}$}

The failure modes and cracking patterns of beams SEM-M, SCL-M, SEV-M and SCG-M were described in Section 7.4.1.1. The current beams, although designated differently for convenience, are one and the same as beams SEM-2.7N, SCL-2.7N, SEV-2.7N and SCG$2.7 \mathrm{~N}$, respectively.

\section{- Effective depth $\mathrm{d}=400 \mathrm{~mm}$}

In beams SEM-H and SEV-H, the inclined flexure-shear cracks occurred in the west shear span at approximately 150 and $180 \mathrm{kN}$, respectively, and quickly propagated diagonally towards both the compression face and the tension reinforcement. A secondary diagonal crack also formed in each beam and propagated toward and subsequently along the tension reinforcement moving in the direction of the support. After extensive diagonal cracking, the beam resistance mechanism was exhausted and was not capable of sustaining the load, therefore they failed in a brittle manner in the west shear span in a diagonal tension failure mode at $P_{u}=193$ and $217 \mathrm{kN}$, respectively.

\section{- Effective depth $\mathrm{d}=500 \mathrm{~mm}$}

In beam SEM-HH, the flexure-shear cracking initiated in the west shear span at $\mathrm{P}=181$ $\mathrm{kN}$. At $\mathrm{P}=202 \mathrm{kN}$, suddenly a new diagonal crack formed crossing from top to bottom of the beam and concurrently propagating along the tension reinforcement. This crack immediately resulted in a brittle diagonal tension failure at $\mathrm{P}_{\mathrm{u}}=202 \mathrm{kN}$.

In beam SEV-HH, after the formation of the inclined flexure-shear cracks at $\mathrm{P}=164 \mathrm{kN}$, it quickly propagated diagonally towards both the compression face and the tension 
reinforcement as load increased. The crack branched into a secondary crack moving toward and along the tension reinforcement in the direction of the support. It failed brittly in a diagonal tension failure mode at $\mathrm{P}_{\mathrm{u}}=231 \mathrm{kN}$.

It is clear from the above description and the crack patterns in Fig.7.13 that except for beams SEV-L and SEV-M all the other beams in this group failed in shear in a diagonal tension failure mode. This is the expected failure mode for beams without shear reinforcement and having adequate width. Consequently, the shear failure mode of beams made of RAC is no different than that beams made of natural aggregate concrete.

\subsubsection{Ultimate Shear Strength}

Figures $7.14 \mathrm{a}$ and $7.14 \mathrm{~b}$ illustrate the normalized shear stress resistance values $\left(v_{c} / \sqrt{f^{\prime}}{ }_{c}\right)$ of beams SEM and SEV, respectively, for different beam sizes, i.e. high, medium, and low height. Generally, the $v_{c} /{\sqrt{f^{\prime}}}_{c}$ values decrease with increasing size. This can be ascribed to less efficient aggregate interlock and dowel resistance after the formation of inclined cracking in larger beams. The available resistance due to aggregate interlock and dowel actions are dependant on the maximum distance between the layers of distributed longitudinal reinforcement. This is the main reason for clause 11.3.6.3 of CSA A23.3-04 which allows using equivalent crack spacing parameter $\left(s_{z e}\right)$ instead of $d_{v}$ in the presence of using minimum required skin reinforcements along the beam height. According to Figure 7.14a, the $v_{c} / \sqrt{f^{\prime}}{ }_{c}$ values of beams SEM-H, SEM-M and SEM-L are $19 \%, 53 \%$, and $102 \%$ higher than that of SEM-HH. Furthermore, the $v_{c} / \sqrt{f_{c}^{\prime}}$ values of SEV-H and SEV-L beams are 17\% and 143\% higher than that of SEV-HH (Figure 7.14b). Note that the actual $v_{c} / \sqrt{f^{\prime}}{ }_{c}$ value for beam SEV-L may be higher than that 
given in Figure $7.14 \mathrm{~b}$ since it failed in flexure rather than shear. This is indicated by the arrow at the top of the SEV-L bar chart.

Finally, according to Figure $7.14 \mathrm{a}$, the $v_{c} /{\sqrt{f^{\prime}}}_{c}$ value of SEM-M is $14 \%$ higher than that of control SCL-M made with natural limestone.

\subsubsection{Shear Performance}

Figure 7.15a shows the variation of $v_{c} / \sqrt{f^{\prime}}{ }_{c}$ versus midspan deflection for different sizes of beams SEM and SEV. Generally, the post-cracked stiffness tends to decrease with increasing depth. This can be partly attributed to the higher efficiency of the aggregate interlock mechanism at smaller depths. The beams with the largest depth (SEM-HH and SEV-HH), experienced dramatic decline in stiffness after the formation of the inclined shear crack. On the contrary, in the shallower beams (SEM-L and SEV-L), due to the effective aggregate interlock, shear deformations were restrained and they did not suffer the same level of decline in their stiffness.

Figure $7.15 \mathrm{~b}$ illustrates the variation of the $v_{c} / \sqrt{f^{\prime}}{ }_{c}$ in the above beams with the deformation measured by the LVDT crossing the major inclined crack in the west shear span (ST1) at nearly $90^{\circ}$. All of the curves exhibit an initial ascending linear portion, followed by an immediate descending branch in some cases. In other cases a yield plateau, followed by a hardening response is observed. The sudden drop in load caused by the formation of the major inclined shear crack while the more gentle reduction is due to the development of flexural cracks.

The load corresponding to the point where a sudden decline in the load is observed is the diagonal cracking load. The severity of the decline in the load is generally a function of the depth of the beam. The beams with smaller depth have a gentler falling branch than 
those with larger depth. In fact, this response can be predicted based on the basic principles of mechanics. Where the flexural stiffness of a beam is a function of the moment of inertia of its cross-section, its shear stiffness is a function of its cross-sectional area. In large size beams, the advent of diagonal cracking leads to a larger reduction in its shear stiffness relative to its flexural stiffness, consequently to more deflection and to the loss of shear capacity.

The hardening portion of the curve was also found to be a function of the beam depth. In beams with smaller depth, this branch is more noticeable compared to the beams with larger depth.

Figure 7.16 illustrates the variation of the recorded concrete compression strain along the beams with different depths. As expected, all the midspan concrete compression strains (C2) increased with increasing load, and the maximum concrete compression strain always occurred at midspan. However, in the beams with $\mathrm{d}=200 \mathrm{~mm}$ (MO-EMV-2.7L and VA-EMV-2.7L) the concrete compression strain in the shear span (C1) started to fall off after about $40 \%$ of the failure load. This may be attributed to the formation of diagonal cracking and the high contribution of aggregate interlocking mechanism and redistribution of internal forces. Observe that in the beams with the larger depth values of 400 and $500 \mathrm{~mm}$, due to lower contribution of aggregate interlocking mechanism and subsequent shear failure of the beams after formation of diagonal cracking, no drop in the strains in the shear span occurred.

Figure 7.17 illustrates the variation of the longitudinal tension reinforcement strain along the beam for the beams with different depths. Generally, the variation of the tension steel strain along all the beams tended to increase with increasing load, and the maximum 
strain generally occurred either at midspan or under the point loads at all load levels (except VA-EMV-2.7M). Observe that some higher strain values in the tension steel near support occurred compared to that in the middle of the shear span. Again this may be attributed to the occurrence of bond failures near the supports and the contribution of the longitudinal steel to the beam shear resistance.

Figures 7.18 and 7.19 show the effect of aggregate type on the $v_{c} /{\sqrt{f^{\prime}}}_{c}$ variation with midspan deflection and diagonal deformation, respectively. Comparing in Fig.7.19a the behaviour of the two shallow beams made of RCA from Montreal and Vancouver, beams SEM-L and SEV-L, we observe that beam SEV-L carries $20 \%$ higher shear before its sudden failure due to the crushing of the concrete at the section of maximum moment. The crack pattern of SEV-L also shows that the diagonal crack having advanced to practically the top fibre of the beam, thus shear failure may have been imminent at the same time as the flexural failure. The higher strength of SEV-L is somewhat unexpected because beams made with crushed stone generally exhibit higher shear strength than those made with river gravel.

Comparing the behaviour of medium depth beams SEM-M and SCL-M in Fig.7.19b, we observe that SEM-M has higher shear strength and greater ductility than the control beam SCL-M. Although SEM-M failed in shear, it has a very ductile response, which is not the typical characteristic of reinforced concrete beam failing in shear. Thus, a RRC beam made of concrete designed by the EMV method could exhibity a highly ductile shear failure.

Figures $7.18 \mathrm{c}$ and $7.18 \mathrm{~d}$ compare the shear behaviour of the bigger size beams made of two types of RCA. For these beams, the aggregate type does not seem to have any 
significant effect on their shear strength or ductility. This may be partly due to the fact that the size effect becomes dominant for larger size beams and the aggregate type becomes less important.

\subsubsection{Serviceability}

Assuming the service load to be $40 \%$ of the ultimate load, the diagonal crack width values under service load were determined to be $0.16,0.06,0.01,0.02$ and $0.00 \mathrm{~mm}$ for beams SEM-L, SEM-M, SCL-M, SEM-H and SEM-HH, respectively. Similarly, the crack widths of $0.28,0.04,0.03$ and $0.00 \mathrm{~mm}$ were determined for beams SEV-L, SCG$\mathrm{M}, \mathrm{SEV}-\mathrm{H}$ and SEV-HH, respectively. Note that the higher crack width in beams with lower $d$ values is mainly due to the higher ultimate shear resistance, and correspondingly higher service load for these beams. Generally, all the crack width values for service load are well below the maximum crack width of 0.4 and $0.33 \mathrm{~mm}$ for interior and exterior exposures recommended by CSA Standard A23.3-04.

\subsection{Shear Performance of RRC Beams with Shear Reinforcement}

\subsubsection{Failure Modes}

Figures 7.20 and 7.21 illustrate the cracking patterns of the SEM and SEV beams with different shear reinforcement ratios as well as those of control beams SCL-6S-R and SCG-6S-R.

Generally, the presence of the shear reinforcement did not fundamentally change the previously described failure modes for RRC beams without shear reinforcement. The shear reinforcement mainly enhanced the beam action by (1) improving the contribution of dowel action by providing a support for longitudinal tension reinforcement, (2) enhancing the aggregate interlock mechanism by limiting the diagonal crack opening, and 
(3) preventing the bond deterioration after splitting cracks development in anchorage zones because of dowel action ad anchorage forces.

In all RRC beams (except SEM-6S-R, SCL-6S-R, SEV-3S-R, SEV-6S-R and SCG-6S$\mathrm{R})$, after the formation of the inclined shear-flexure crack, it propagated up towards the compression face and down toward the tension reinforcement as the load increased. After yielding of the stirrups crossing the diagonal crack, unrestricted widening of the diagonal crack occurred, which resulted in loss of the aggregate interlock mechanism and the eventually brittle failure of the beam in the west shear span in a diagonal tension failure mode.

Beams SEM-6S-R, SCL-6S-R, SEV-3S-R, SEV-6S-R and SCG-6S-R unexpectedly failed in flexure when the load reached, respectively, $\mathrm{P}=613,570,466,612$ and $564 \mathrm{kN}$. Figures $7.22 \mathrm{a}$ and $7.22 \mathrm{~b}$ illustrate the variation of the shear reinforcement strain in the west shear span (TS2) with load for beams SEM and SEV with different shear reinforcement ratios. From the figure, the yielding of some of the stirrups in those RRC beams that failed in shear is quite evident. In other cases, either the strain gauges failed before the end of the test or did not provide reliable data. It must be pointed out that the strain in the stirrups can be highly variable due to the unpredictability of the diagonal crack location and the relative distance of the strain gauge on the stirrup from the crack. For this reason, it is more rational to estimate the concrete contribution to the shear resistance of the beam from tests on concrete beams without shear reinforcement. By subtracting the concrete contribution from the total shear resistance of beams with shear reinforcement, the shear reinforcement contribution can be estimated. This will be further discussed in the following chapter. 


\subsubsection{Ultimate Shear Strength}

Figures $7.23 \mathrm{a}$ and $7.23 \mathrm{~b}$ illustrate the ultimate shear stress resistance values $\left(v_{u}\right)$ of beams SEM and SEV with different shear reinforcement ratios, respectively. The ultimate shear stress was calculated as

$$
v_{u}=\frac{V_{u}}{b \times d_{v}}
$$

Note that $v_{u}$ consists of two parts: (1) the shear stress resistance provided by concrete $\left(v_{c}\right)$, and (2) the shear stress resistance provided by shear reinforcement $\left(v_{s}\right)$.

As expected, $v_{u}$ increased with increasing shear reinforcement ratio. Note in particular the difference between the ultimate shear resistance of the beam without shear reinforcement, beam SEM-NS, and the other beams with shear reinforcement. Based on Figure 7.23a, the $v_{u}$ values for beams SEM-3S-R and SEM-6S-D are $65 \%$ and $228 \%$ higher than that of beam SEM-NS. Since both SEM-6S-R and SCL-R failed in flexure, their actual ultimate shear stress resistance must be higher as indicated by the arrows in their bar graphs. Similarly, since all SEV-NS, SEV-3S-R, SEV-6S-R, and SCG-6S-R failed in flexure, their actual ultimate shear stress resistance must be greater than the values shown in Fig.7.23b. It must be pointed out that all of these beams were designed to have $50 \%$ higher flexural strength than their shear strength based on prevailing methods of design for regular concrete. It is clear from these test results that the shear design methods are much more conservative than the flexural design methods. This conservatism is the main reason for the premature flexural failure of many of these beams. This also indicates that using the existing design methods for normal concrete to design RRC beams would be not only safe, but also conservative. 


\subsubsection{Shear Performance}

Figure 7.24a illustrates the shear-midspan deflection response for the beams with different shear reinforcement ratios. Unlike the RRC beams with no shear reinforcement, there is no major difference observed between the shear-midspan deflections of the SEM and SEV beams for different shear reinforcement ratios. This is basically due to the beneficial effects of stirrups on the aggregate interlock and dowel mechanisms by preventing unrestricted widening of diagonal cracks. In other words, in the beams with no shear reinforcement (SEM-NS), after the formation of the inclined crack, the stiffness of the beam quickly dropped due to crack widening and it resulted in loss of aggregate interlock mechanism. On the contrary, in RRC beams with shear reinforcement, after the inclined crack occurrence, the stirrups crossing the diagonal crack prevented the unrestricted crack widening and the aggregate interlock mechanism breakdown, therefore, the loss of stiffness was postponed till yielding of the stirrups.

Note that the slight differences in the slope of the shear-midspan deflection curves are mainly due to the slightly higher rigidity of the beams with higher shear reinforcement ratios due to either their higher section depth and/or higher steel content. The plateau of the curves of beams SEM-6S-R and SEV-6S-R beams are due to their ductile flexural failures. Beam SEM-6S-D was on the verge of flexural failure since the concrete at its top fibre at midspan reached its maximum capacity after tension steel yielded. However, eventually it suddenly failed in shear which is indicated by the noticeable plateau in its load-midspan deflection curve. Notice the clear difference between the ductile flexural failures and the brittle shear failures by observing the amount of maximum deflection at failure for each beam. 
Figure $7.24 \mathrm{~b}$ shows the variation of the shear force with the diagonal deformation in beams SEM and SEV with different transverse shear reinforcement ratios. All of the curves exhibit yielding and variable amounts of plastic deformation, depending on the amount of shear reinforcement. Strictly speaking, the plotted deformations are a combination of crack opening/sliding displacements and material strains.

The initial linear portion in these curves mainly represents beams behaviour before the inclined crack formation. The load at the start of the plateau, referred to as the diagonal cracking load, is mainly a function of the concrete properties. The length of the plateau was found to be generally a function of the shear reinforcement ratio of the beam. The beams with no stirrups had the longest plateau while those with the highest shear reinforcements had the shortest plateau. This is expected because the stirrups crossing the diagonal crack will limit its deformations. Similarly, the extent of the deformation of the plateau is also a function of the shear reinforcement ratio. In beams with higher shear reinforcement content, a more noticeable hardening segment is noticed compared to the beams with no stirrups or wider stirrup spacing. The slope of this segment is indicative of the shear reinforcement contribution to the total shear resistance.

Note that the effectiveness of the shear reinforcement in preventing the crack-widening generally decreases as the stirrup crossing over the diagonal crack yields. This is clearly evident from Figure 7.25, which illustrates the variation of the shear reinforcement and the concrete contributions to the total shear resistance of the beams failing in shear. In this figure, $\mathrm{V}_{\mathrm{s} 1}$ and $\mathrm{V}_{\mathrm{s} 2}$ denote the shear resistance provided by the stirrups located at TS1 and TS2 (see Fig.7.2). According to Fig.7.25, it can also be observed that the increasing rate of stress in the stirrups is not necessarily linear. In other words, the contribution of 
the aggregate interlock mechanism to the overall shear capacity is not constant, and as the strain in the stirrups approaches the yielding strain, the diagonal crack starts widening. After the stirrup yields, the crossing stirrups over the diagonal crack can not prevent the unrestricted crack widening and eventually the aggregate interlock mechanism breaks down, and the beam fails. This is quite evident by observing the plateau portion of the $V_{s}$ variation before failure in Fig.7.25.

Figures 7.26 and 7.27 illustrate the effect of aggregate type on the shear-midspan deflection and shear-diagonal deformation response of the test beams.

It may be revealed from the earlier discussion and with reference to Figs.7.20 and 7.21 beams SEM-3S-R and SEV-3S-R both failed in flexure, but from their crack patterns one could clearly see that the major diagonal crack in SEM-3S-R spans the entire height of the beam whereas in beam SEV-3S-R it has not reached the top fibre at failure. Considering the above observation and the shear-deflection responses of these beams in Fig.7.26a, it can be concluded that beam SEM-3S-R failed in shear while beam SEV-3S$\mathrm{R}$ failed in flexure. Since shear failure is normally brittle, the shear-deflection curves in Fig.7.26a agree with the expected failure modes.

Although beam SEV-3S-R which is made of RCA from Vancouver, exhibited higher shear strength than its counterpart made from the RCA from Montreal, the same did not happen when one compares the shear-midspan deflection curves of beams SEM-6S-D and SEV-6S-D in Fig.7.26b. Here the reverse happened, for the SEM beam exhibited higher strength and ductility compared to the SEV beam. Due to lack of any systematic trend in the relative response of the SEM and SEV beams, it may be concluded that at 
least for the two RCA types used in this study, the RCA type or source does not have a significant effect on the shear strength of RRC beams.

When comparing the shear-midspan deflection behaviour of the beams made with RCA with those made of natural aggregates, as in Fig.7.26c, we observe little difference between the shear responses of the two types of beams. Thus, overall, the aggregate type does not have a significant effect on the shear strength of reinforced concrete beams. However, this statement is only true when comparing aggregates with a certain minimum strength. If the aggregate strength falls below the specified minimum value, it will affect the shear strength. This fact is already recognized by reinforced concrete design standards which assume the shear strength of concrete made with lightweight aggregates to be lower than the concrete made of normal density aggregates.

The observations made with respect to the type of aggregate on the midspan deflection also apply to its effect on diagonal deformation and diagonal cracking load as can be seen in Fig.7.27a to 7.27c. In general, the results in these figures show that aggregate type does not significantly affect the diagonal cracking load or deformations.

Figure 7.28 illustrates the variation of the recorded concrete compression strain along the beams with different shear reinforcement ratios. As expected, all the midspan concrete compression strains (C2) increased with increasing load, and at all load levels the maximum concrete compression strain occurred at midspan. However, in the beams which failed in shear, for the reasons explained earlier, the concrete compression strain in the shear span (C1) started to fall off before failure.

Figure 7.29 illustrates the variation of the recorded longitudinal tension reinforcement strain along the beams with different shear reinforcement ratios. Generally, the variation 
of the tension steel strain along all the beams increased as the load increased, and at all load levels the maximum strain generally occurred either at midspan or under the point loads (except VA-EMV-6S-R).

\subsubsection{Serviceability}

If we assume the service load to be $40 \%$ of the failure load for each of these beams, diagonal crack width values of $0.06,0.18,0.42,0.23$ and $0.42 \mathrm{~mm}$ were determined for beams SEM-NS, SEM-3S-R, SEM-6S-R, SCL-6S-R and SEM-6S-D, respectively, under service conditions. Similarly, crack width values of $0.06,0.27,0.53$ and $0.66 \mathrm{~mm}$ were determined for beams SEV-3S-R, SEV-6S-R, SCG-6S-R and SEV-6S-D at their respective service loads. Note that the higher crack width in beams with higher shear reinforcement ratios is mainly due to the higher ultimate shear resistance, and correspondingly higher service loads of these beams. Since beams SEM-6S-R and SCL6S-R beams failed in flexure rather than shear, the higher crack widths in SEM-6S-R compared to control SCL-6S-R beam is not necessarily due to the presence of RAC, rather it is highly dependant on the stirrup strain level and on the steel mechanical properties. Some of the above crack widths exceed the current limits specified by the CSA Standard A23.3-04, but this is mainly due to the fact that the actual shear strength of these beams significantly exceeds their design strength. Although the CSA Standard makes no specific reference to shear crack width, the conservative shear design provisions may indirectly limit shear crack width under service loads.

\subsection{Summary}

- There was no major difference observed between the failure modes, cracking patterns and shear performance of RRC beams and conventional RC beams. Generally, the RRC 
beams had higher shear stress resistance $\left(v_{c}\right)$ and were found more ductile after formation of diagonal cracking compared to conventional RC beams. This once again validates the concept of equivalent total mortar or total natural aggregate volume in the proposed mix proportioning method.

- The shear strength of RRC beams had a tendency to increase by decreasing the a/d ratio for both RCA-MO and RCA-VA sources. This is mainly due to the contribution of arch action mechanism at lower $a / d$ ratios, which has been well known for conventional $\mathrm{RC}$ beams.

- The shear strength of RRC beams had a tendency to increase by decreasing the overall depth of the beams made of both RCA-MO and RCA-VA sources, which is well known in conventional RC beams as size effect. This is mainly due to the less effectiveness of aggregate interlock mechanism at higher depth values.

- The ultimate shear strength of RRC beams had a tendency to increase by increasing the shear reinforcement ratios of beams made of both RCA-MO and RCA-VA sources. This is mainly due to the contribution of the shear reinforcement by providing an additional shear stress resistance $\left(v_{s}\right)$ in RRC beams.

- Despite slightly higher diagonal crack widths in RRC beams compared to RC beams at a/d=2.7, all observed crack widths in RRC beams at different $a / d$ ratios and depth values were below the maximum crack width recommended by CSA A23.3-04.

- At identical a/d ratio, compressive strength and depth values, the effect of aggregate type was found negligible on shear performance of RRC beams at different $a / d$ and shear reinforcement ratios and depth values. 
g

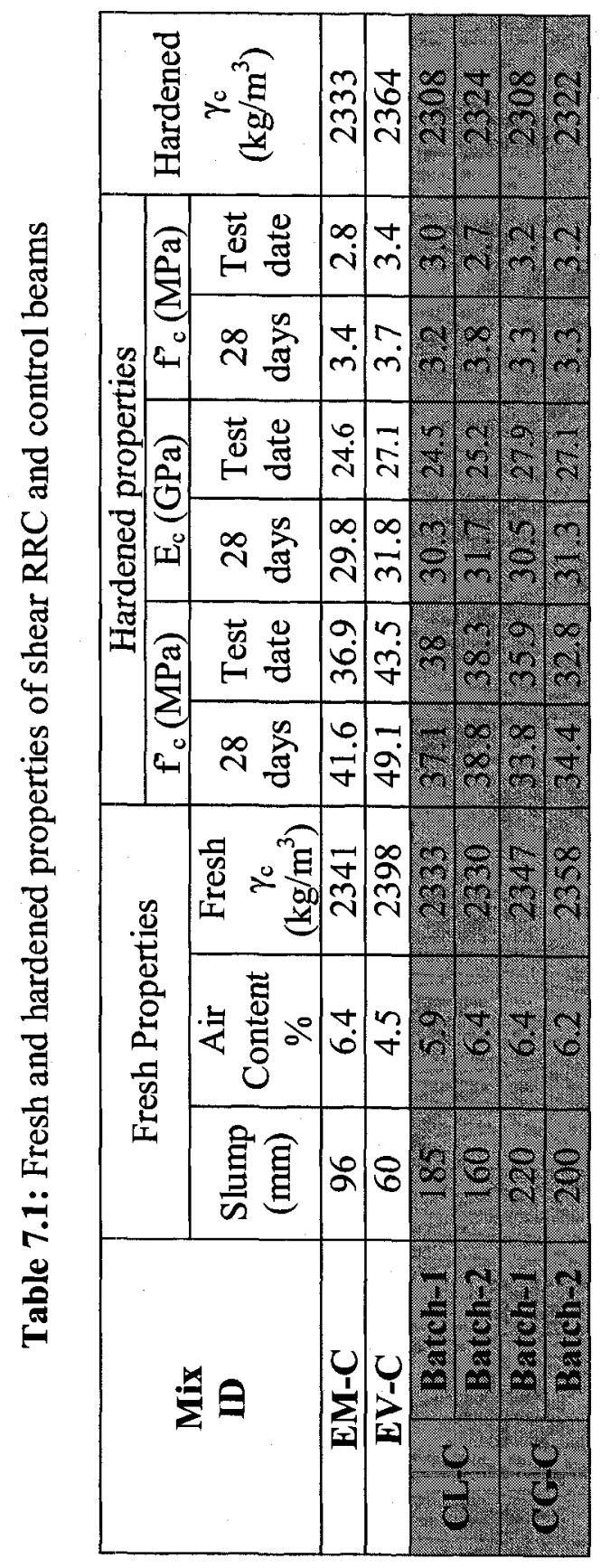


Table 7.2: Structural details of shear RRC beams without shear reinforcement

\begin{tabular}{|c|c|c|c|c|c|c|c|c|}
\hline \multirow{2}{*}{\multicolumn{2}{|c|}{ Beam ID }} & & \multicolumn{4}{|c|}{ Dimensions (mm) } & \multirow{2}{*}{$\mathbf{A}_{\mathbf{s}}$} & \multirow{2}{*}{$\mathbf{s}(\mathbf{m m})$} \\
\hline & & & $\mathbf{h}$ & $\mathbf{d}$ & $\mathbf{a}$ & $\mathbf{L}$ & & \\
\hline \multirow{10}{*}{ 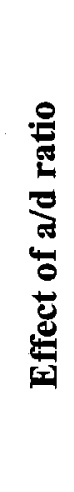 } & SEM-1.5N & 1.50 & 350 & 300 & 450 & 1900 & 2 No. 20 & 150 \\
\hline & SEM-2.0N & 2.00 & 350 & 300 & 600 & 2200 & 3 No. 20 & 200 \\
\hline & SEM-2.7N & 2.59 & 375 & 309 & 800 & 2600 & 3No.15+2No.15 & 200 \\
\hline & SCL-2.7N & 2.59 & 375 & 309 & 800 & 2600 & 3No.15+2No.15 & 200 \\
\hline & SEM-4.0N & 3.93 & 375 & 305 & 1200 & 3400 & $3 \mathrm{No} .20+2 \mathrm{No} .20$ & 200 \\
\hline & SEV-1.5N & 1.50 & 350 & 300 & 450 & 1900 & 2 No. 20 & 150 \\
\hline & SEV-2.0N & 2.00 & 350 & 300 & 600 & 2200 & 3 No. 20 & 200 \\
\hline & SEV-2.7N & 2.59 & 375 & 309 & 800 & 2600 & 3No.15+2No.15 & 200 \\
\hline & SCG-2.7N & 2.59 & 375 & 309 & $\overline{800}$ & 2600 & $3 \mathrm{No} .15+2 \mathrm{No} .15$ & 200 \\
\hline & SEV-4.0N & 3.93 & 375 & 305 & 1200 & 3400 & $3 \mathrm{No} .20+2 \mathrm{No} .20$ & 200 \\
\hline \multirow{10}{*}{ 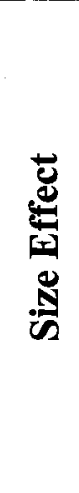 } & SEM-L & 2.69 & 250 & 201 & 540 & 2080 & $2 \mathrm{No} .20+1$ No. 15 & 135 \\
\hline & SEM-M & 2.59 & 375 & 309 & 800 & 2600 & 3No.15+2No.15 & 200 \\
\hline & SCL-M & 2.59 & 375 & 309 & 800 & 2600 & 3No.15+2No.15 & 200 \\
\hline & SEM-H & 2.73 & 450 & 381 & $\overline{1040}$ & 3180 & $2 \mathrm{No} .25+2 \mathrm{No} .15$ & 200 \\
\hline & SEM-HH & 2.73 & 550 & 476 & 1300 & 3700 & $2 \mathrm{No} .25+2 \mathrm{No} .20$ & 200 \\
\hline & SEV-L & 2.69 & 250 & 201 & 540 & 2080 & $2 \mathrm{No} .20+1 \mathrm{No} .15$ & 135 \\
\hline & SEV-M & 2.59 & 375 & 309 & 800 & 2600 & $3 \mathrm{No} .15+2 \mathrm{No} .15$ & 200 \\
\hline & SCG-M & 2.59 & 375 & 309 & 800 & 2600 & 3No.15+2No.15 & 200 \\
\hline & SEV-H & 2.73 & 450 & 381 & 1040 & 3180 & 2 No. $25+2$ No. 15 & 200 \\
\hline & SEV-HH & 2.73 & 550 & 476 & 1300 & 3700 & $2 \mathrm{No} .25+2 \mathrm{No} .20$ & 200 \\
\hline
\end{tabular}


Table 7.3: Structural details of shear RRC beams with shear reinforcement

\begin{tabular}{|c|c|c|c|c|c|c|c|c|}
\hline \multirow{2}{*}{$\begin{array}{c}\text { Beam } \\
\text { ID }\end{array}$} & \multirow[t]{2}{*}{$\mathbf{a} / \mathbf{d}$} & \multicolumn{3}{|c|}{$\begin{array}{c}\text { Dimensions } \\
\text { mm) }\end{array}$} & \multirow{2}{*}{$\mathbf{A}_{\mathbf{s}}$} & \multirow[t]{2}{*}{ A's } & \multirow{2}{*}{\multicolumn{2}{|c|}{$A_{v}\left(\rho_{w}, \%\right)$}} \\
\hline & & h & d & $\mathbf{L}$ & & & & \\
\hline \multirow{2}{*}{ SEM-NS } & \multirow{2}{*}{2.59} & \multirow{2}{*}{375} & \multirow{2}{*}{309} & \multirow{2}{*}{2600} & \multirow{2}{*}{$\begin{array}{l}\text { 3No.15+ } \\
\text { 2No.15 }\end{array}$} & \multirow{2}{*}{2 No. 10} & west & No stirrups $(0.0)$ \\
\hline & & & & & & & east & $00(1.0)$ \\
\hline \multirow{2}{*}{ SEM-3S } & \multirow{2}{*}{2.61} & \multirow{2}{*}{375} & \multirow{2}{*}{306} & \multirow{2}{*}{2600} & \multirow{2}{*}{$\begin{array}{c}\text { 2No. } 20+ \\
3 \text { No. } 20\end{array}$} & \multirow{2}{*}{2 No. 10} & west & $(0.25)$ \\
\hline & & & & & & & east & \\
\hline \multirow[t]{2}{*}{ SEM-OSRR } & \multirow{2}{*}{$\frac{2.65}{2.65}$} & 385 & 302 & 2700 & $\begin{array}{l}2 \mathrm{No} .25 \mathrm{t} \\
2 \mathrm{No} 0.25 \\
\end{array}$ & $2 \mathrm{No.25}$ & $\frac{\text { west }}{\text { east }}$ & $\frac{\text { No.8@100(0.5) }}{\text { No.10@100(1.0) }}$ \\
\hline & & 385 & 302 & 2700 & $\begin{array}{l}2 \mathrm{No}, 25 \mathrm{t} \\
2 \mathrm{No} 0.25\end{array}$ & & $\frac{\text { west }}{\text { east: }}$ & $\frac{N 0.8 @ 100(0.5)}{N 0.10 @ 100(1.0)^{2}}$ \\
\hline \multirow{2}{*}{ SEM-6SD } & \multirow{2}{*}{2.66} & \multirow{2}{*}{385} & \multirow{2}{*}{301} & \multirow{2}{*}{2700} & 2 No. $30+$ & \multirow{2}{*}{ 2No.30 } & west & $(0.50)$ \\
\hline & & & & & & & east & $0(1.0)$ \\
\hline SEV-NS & 2.59 & 375 & 309. & & $\begin{array}{r}3 \mathrm{No} .15+ \\
2 \mathrm{No} .15 \\
\end{array}$ & & $\frac{\text { west }}{\text { east }}$ & $\frac{\text { No stirrups }(0.0)}{\text { No.10@200(1.0) }}$ \\
\hline & 2.61 & 375 & & 2600 & $\begin{array}{l}2 \mathrm{No.20+} \\
3 \mathrm{No} .20\end{array}$ & & $\frac{\text { West }}{\text { east }}$ & $\frac{\mathrm{No} .8 @ 200(0.25)}{\mathrm{No} .10 @ 200(0.5)}$ \\
\hline SIEV- & 2.65 & 385 & 302 & 2700 & & $2 \mathrm{No} .25$ & west & No.8@100 $(0.5)$ \\
\hline & & & & & & & east & \\
\hline SCG-6SR & 2.65 & 385 & 302 & 2700 & 2No. 25+ & 2No. 25 & west & $(0.5)$ \\
\hline & & & & & & & east & $\frac{(1.0)}{(0.50)}$ \\
\hline SEV-6SD & 2.66 & 385 & 301 & 2700 & $2 \mathrm{No} .25$ & 2 No. 30 & $\frac{\text { West }}{\text { east }}$ & $\frac{N 0.10 @, 200(0.50)}{\text { No.10@100(1.0) }}$ \\
\hline
\end{tabular}


Table 7.4: The measured strains in longitudinal and shear reinforcements and concrete at failure load in shear RRC beams

\begin{tabular}{|c|c|c|c|c|c|c|c|c|c|}
\hline \multirow{2}{*}{ Beam ID } & $\boldsymbol{\varepsilon}_{\mathbf{y}}$ & \multicolumn{6}{|c|}{ Measured strains at failure load $\left(\times \mathbf{1 0}^{-3}\right)$} \\
\cline { 3 - 10 } & $\left(\times \mathbf{1 0}^{-3}\right)$ & $\boldsymbol{\varepsilon}_{\mathbf{c} 2}$ & $\boldsymbol{\varepsilon}_{\mathbf{c 1}}$ & $\boldsymbol{\varepsilon}_{\text {LS1 }}$ & $\boldsymbol{\varepsilon}_{\mathbf{L S} 2}$ & $\boldsymbol{\varepsilon}_{\text {LS3 }}$ & $\boldsymbol{\varepsilon}_{\text {LS4 }}$ & $\boldsymbol{\varepsilon}_{\text {LS5 }}$ & $\boldsymbol{\varepsilon}_{\text {TS1 }}$ \\
\hline SEM-1.5N & 2.52 & 3.60 & 0.75 & 4.53 & 2.01 & 6.67 & 2.72 & 3.04 & 0.54 \\
\hline SEM-2.0N & 2.52 & 1.46 & 0.75 & 1.18 & 2.28 & - & 2.53 & 1.11 & 0.53 \\
\hline SEM-2.7N & 2.32 & 1.02 & 0.95 & 1.70 & 0.49 & 1.92 & 0.72 & 1.95 & 0.68 \\
\hline SCL-2.7N & 2.32 & - & 0.53 & 2.05 & 1.63 & 1.58 & 1.83 & - & 0.28 \\
\hline SEM-4.0N & 2.52 & 1.13 & 0.67 & 0.66 & 1.37 & 1.75 & 0.87 & 1.32 & 0.39 \\
\hline SEY-1.5N & 2.52 & 5.24 & 1.72 & 8.37 & 3.39 & 6.99 & 4.28 & & 1.97 \\
\hline SEV-2.0N & 2.52 & 1.45 & 0.54 & 1.15 & 0.80 & 1.35 & 1.22 & 0.93 & 1.35 \\
\hline SEV-2.9N & 2.92 & 2.90 & 0.96 & 2.33 & 3.58 & 2.31 & 2.37 & 0.26 & 0.71 \\
\hline SCG-2.7N & 2.32 & 1.95 & 0.45 & 2.78 & 2.18 & 8.68 & 2.90 & - & 0.89 \\
\hline SEV-4.0N & 2.52 & 1.11 & 0.58 & 0.55 & 1.78 & - & 0.67 & 1.74 & 0.50 \\
\hline SEM-L & 2.32 & 1.19 & 0.72 & 1.35 & 1.16 & 1.55 & 0.00 & 0.72 & 1.40 \\
\hline SEM-M & 2.32 & 1.02 & 0.95 & 1.70 & 0.49 & 1.92 & 0.72 & 1.95 & 0.68 \\
\hline SCL-M & 2.32 & 0.83 & 0.53 & 2.05 & 1.63 & 1.58 & 1.83 & 0.00 & 0.28 \\
\hline SEM-H & 2.46 & 0.89 & 0.63 & 1.18 & 2.28 & 0.00 & 2.53 & 1.11 & 0.53 \\
\hline SEM-HH & 2.46 & 0.70 & 0.37 & 0.47 & -0.11 & 1.35 & 0.00 & 0.88 & 0.28 \\
\hline SEV-L & 2.32 & 2.70 & 0.23 & 0.00 & 1.83 & 0.00 & 5.82 & 6.13 & 0.89 \\
\hline SEV-M & 2.32 & 2.90 & 0.96 & 2.33 & 3.58 & 2.31 & 2.37 & 0.26 & 0.71 \\
\hline SCG-M & 2.32 & 1.95 & 0.45 & 2.78 & 2.18 & 8.68 & 2.90 & 0.00 & 0.89 \\
\hline SEV-H & 2.46 & 0.78 & 0.59 & 0.57 & 1.63 & 1.84 & 0.00 & 1.10 & 0.23 \\
\hline SEV-HH & 2.46 & 0.61 & 0.56 & 1.07 & 1.18 & 1.38 & 1.37 & 1.04 & 0.31 \\
\hline
\end{tabular}

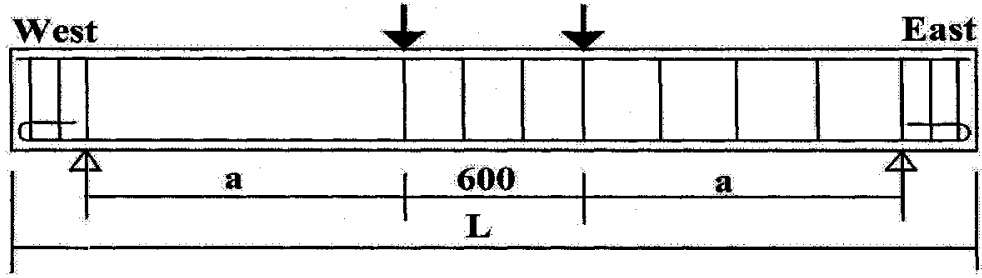

a) without shear reinforcement

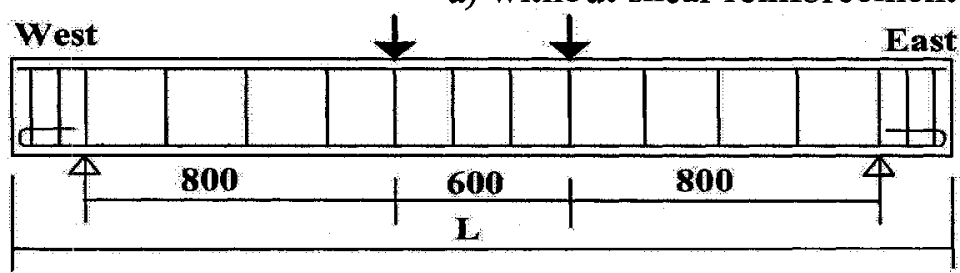

b) with shear reinforcement
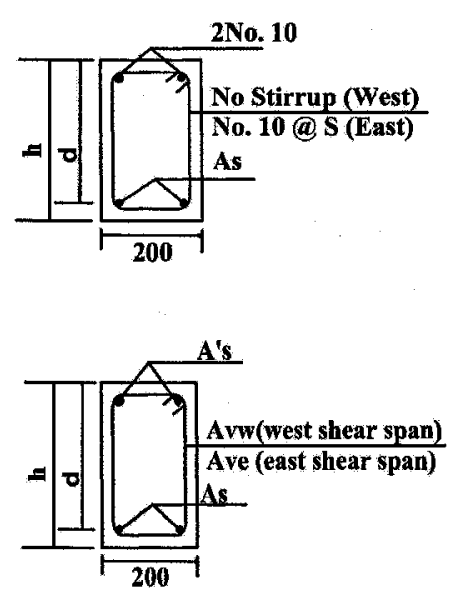

Figure 7.1: Details of shear beams 


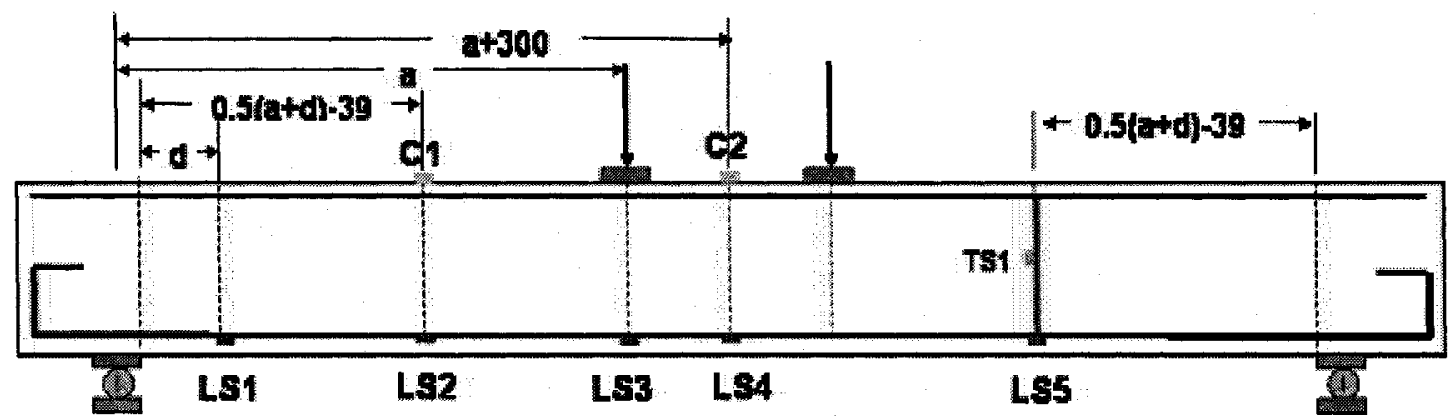

a) without shear reinforcement

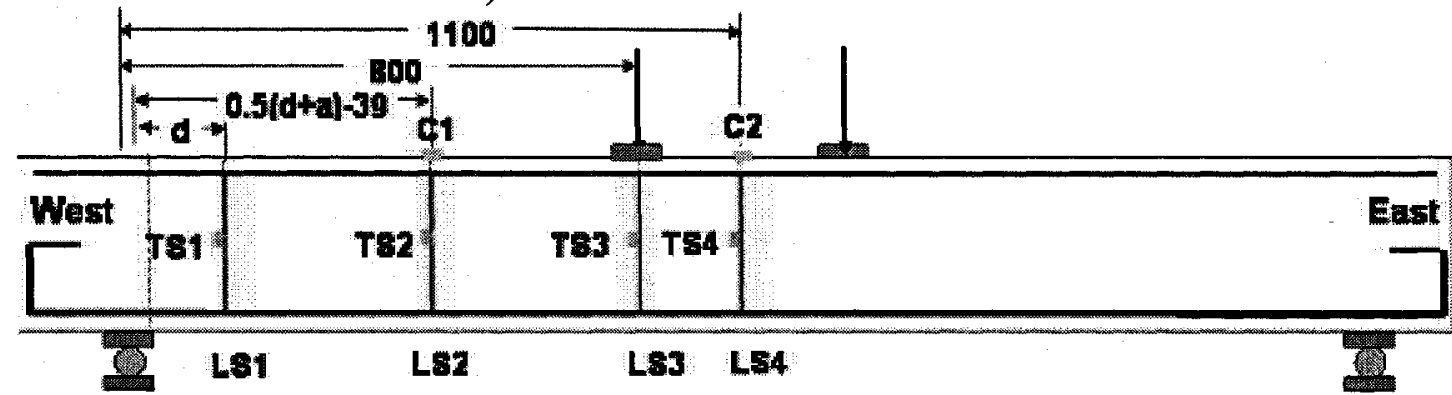

b) with shear reinforcement

Figure 7.2: Schematic arrangement of strain gauges in shear beams

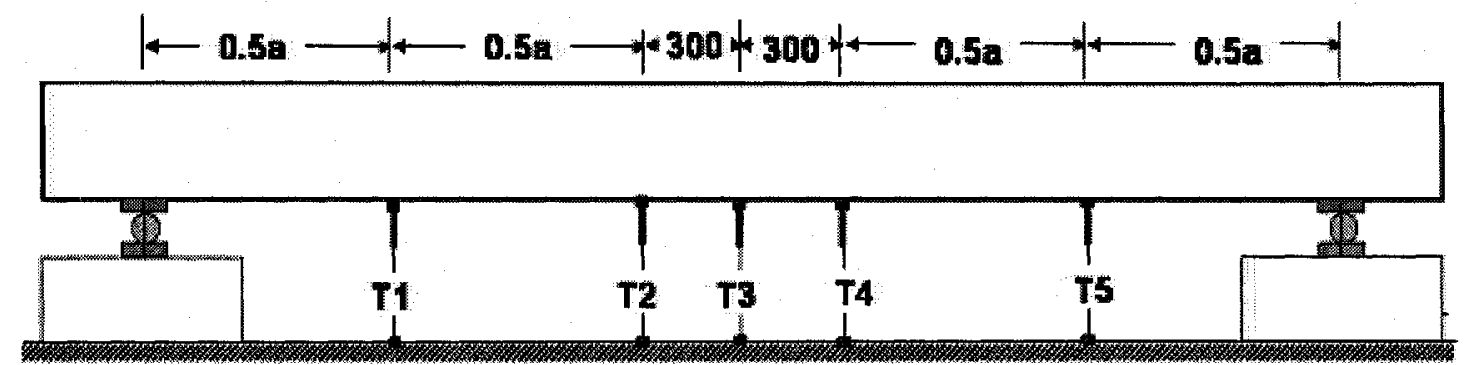

Figure 7.3: Schematic arrangement of LPs for deflection measurements

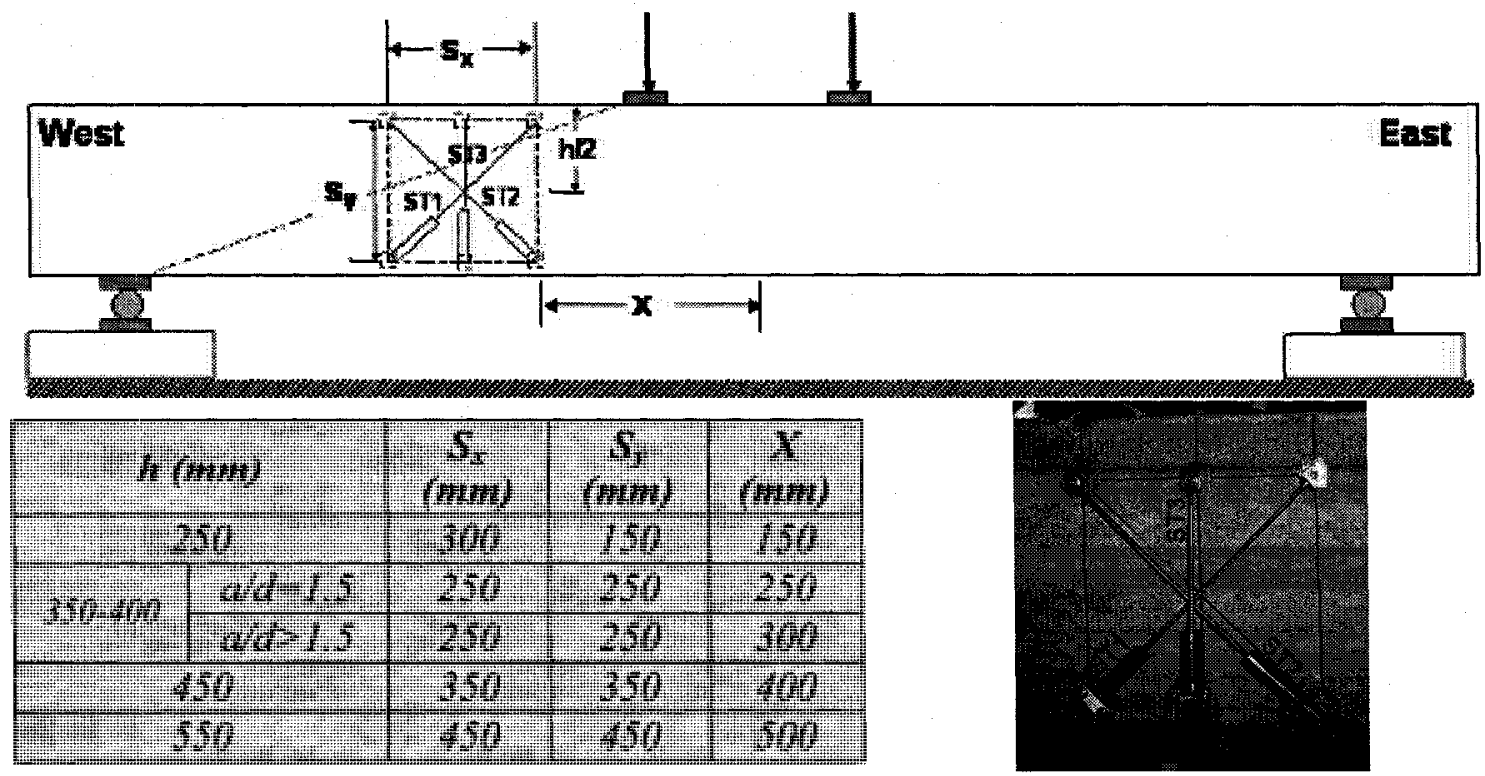

Figure 7.4: Schematic arrangement of side LVDTs for shear deformation measurements 

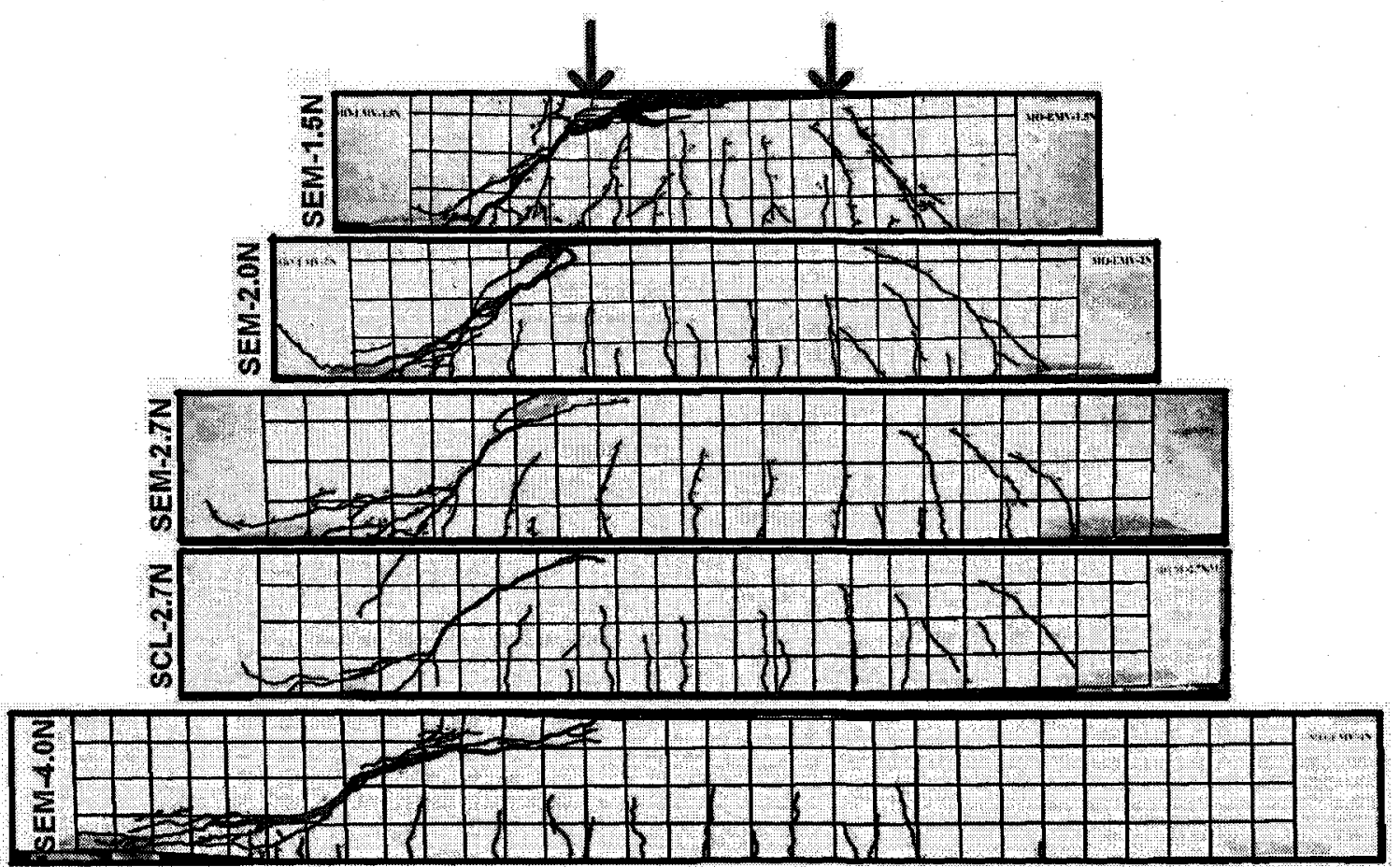

a) SEM and SCL beams

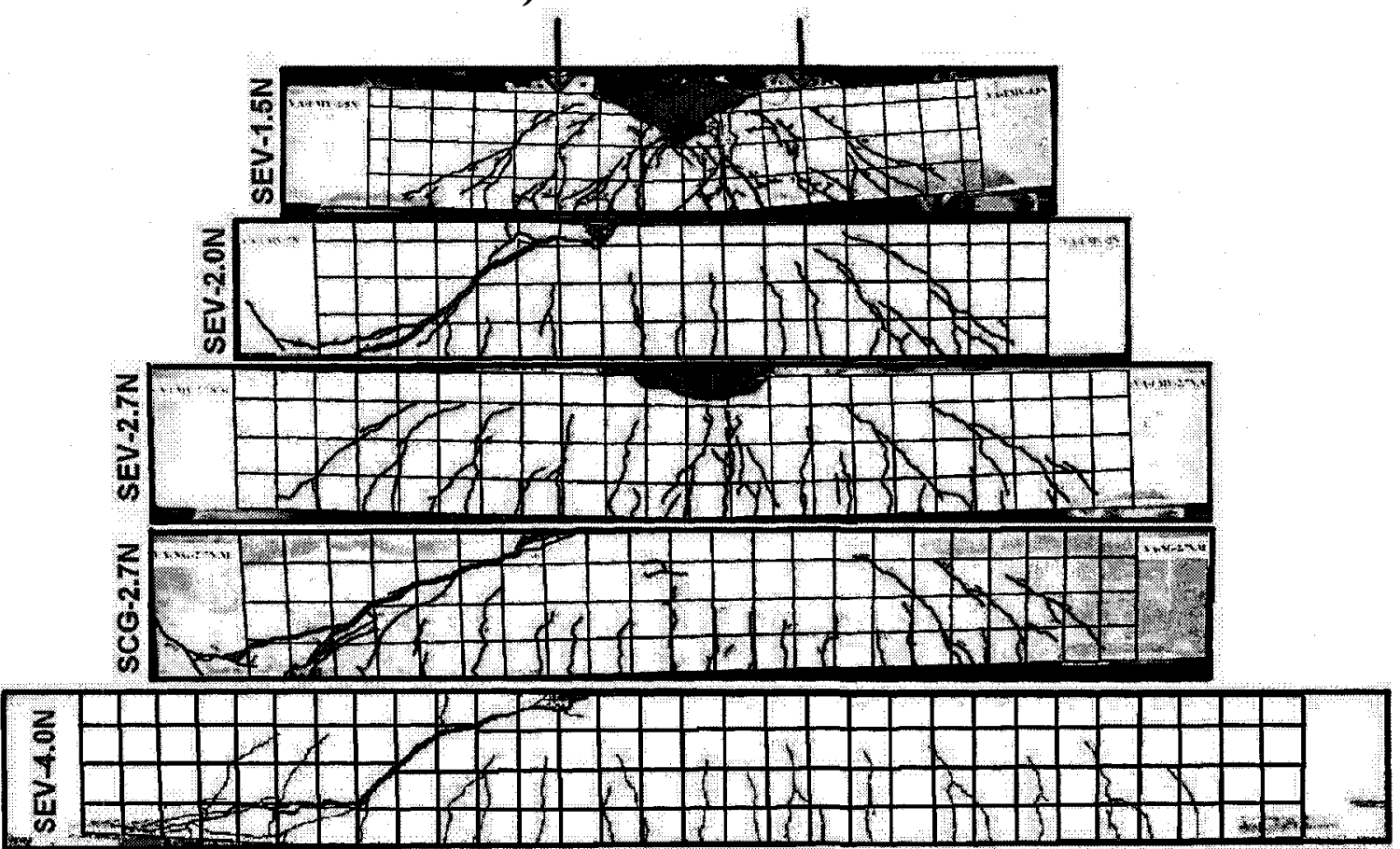

a) SEV and SCG beams

Figure 7.5: Typical crack patterns of RRC shear beams 


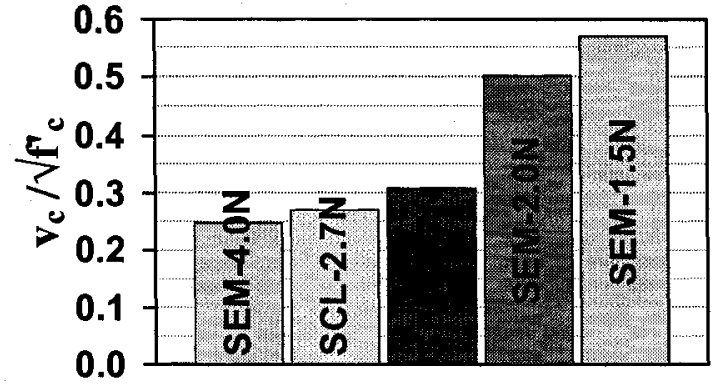

a) SEM and control SCL beams

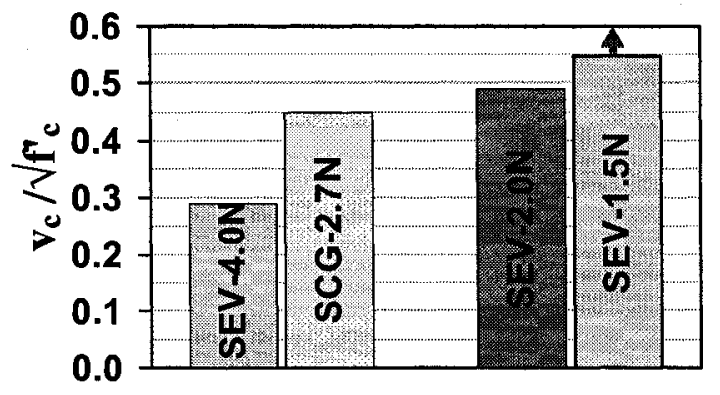

b) SEV and control SCG beams Figure 7.6: Experimental ultimate shear stress values for different $\mathrm{a} / \mathrm{d}$ ratios 

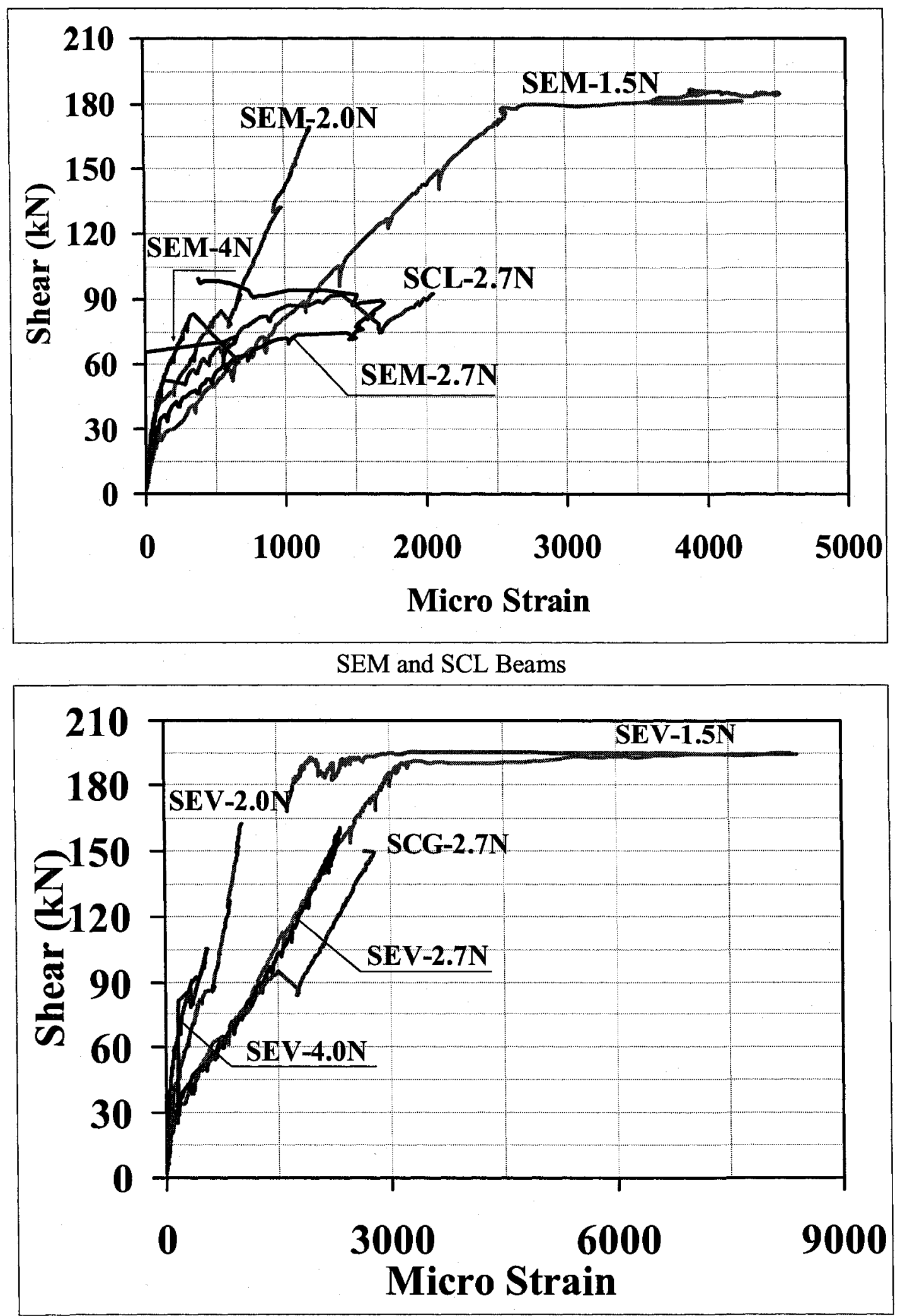

SEV and SCG Beams

Figure 7.7: The effect of shear span ratio on longitudinal steel strain near support (LS1) 

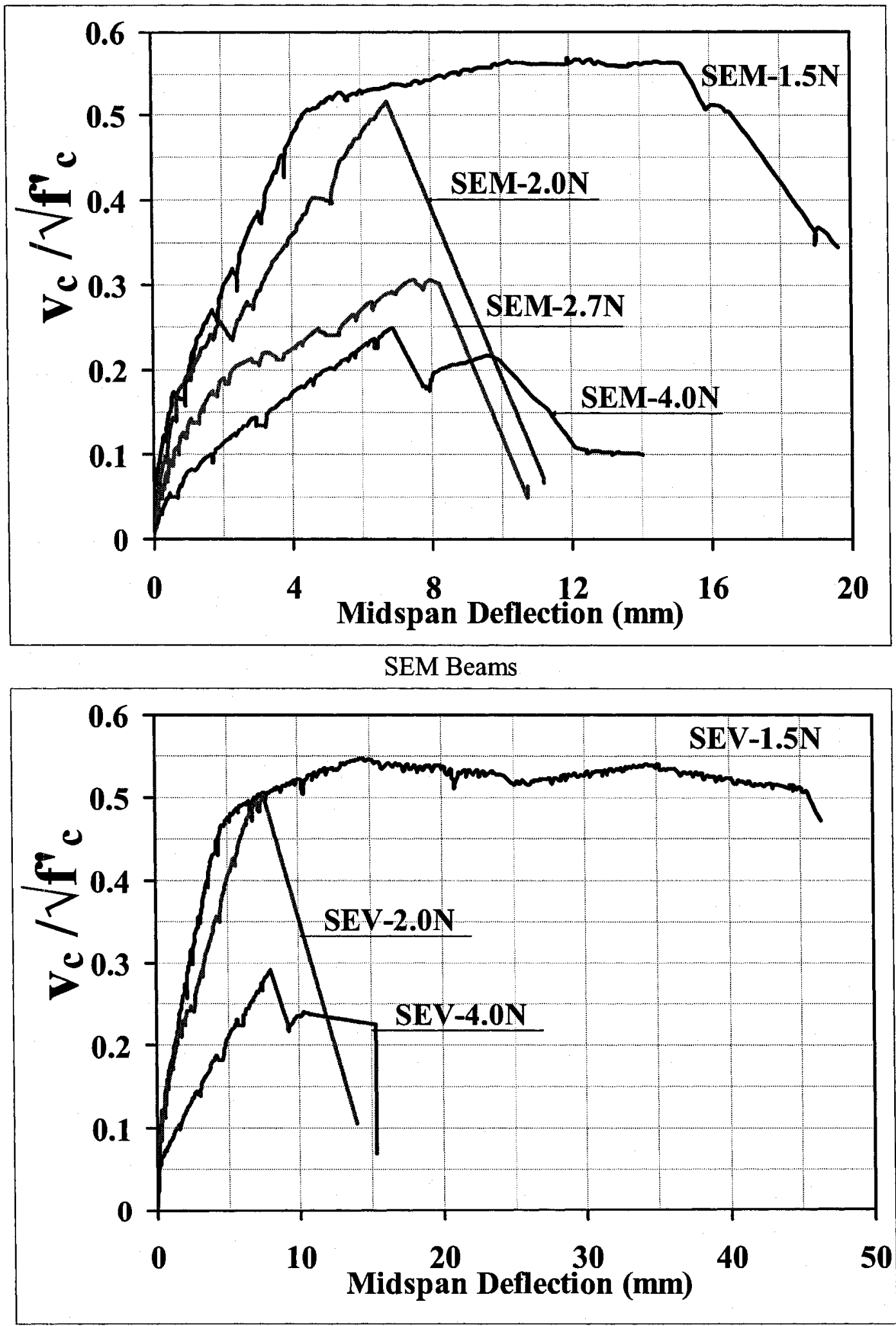

SEV Beams

a) Normalized shear stress strength-midspan deflection response

Figure 7.8: Effect of a/d ratio on shear behaviour of RRC beams 

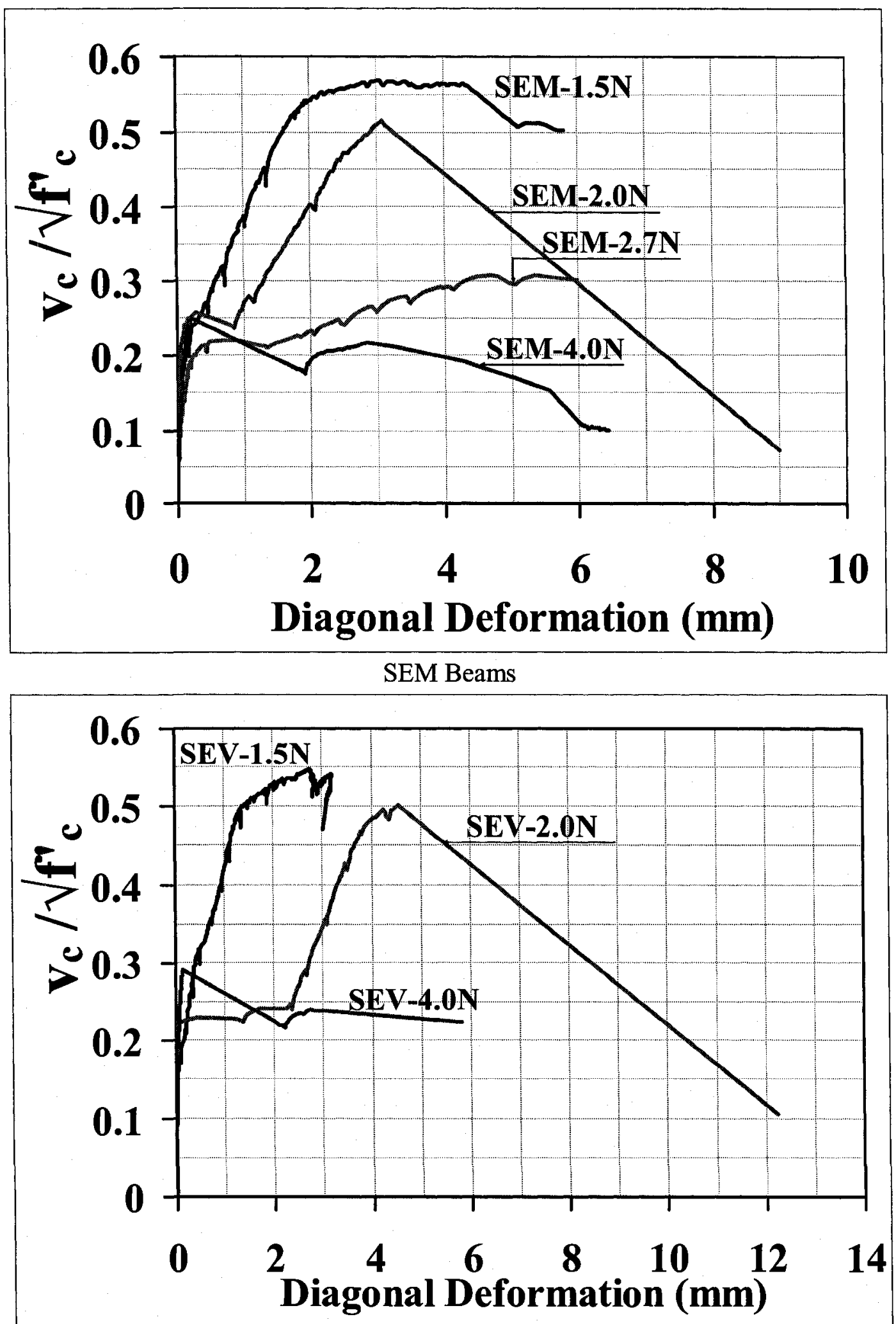

SEV Beams

b) Normalized shear stress strength -diagonal deformation response

Cont. Figure 7.8: Effect of a/d ratio on shear behaviour of RRC beams 


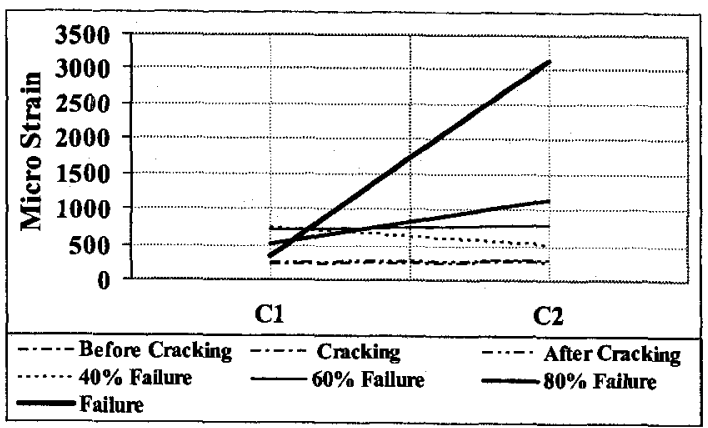

a) $\mathrm{MO}-\mathrm{EMV}-1.5 \mathrm{~N}$

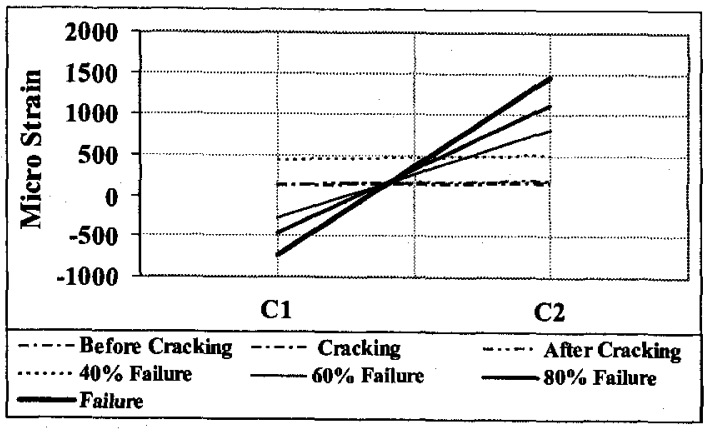

c) $\mathrm{MO}-\mathrm{EMV}-2 \mathrm{~N}$

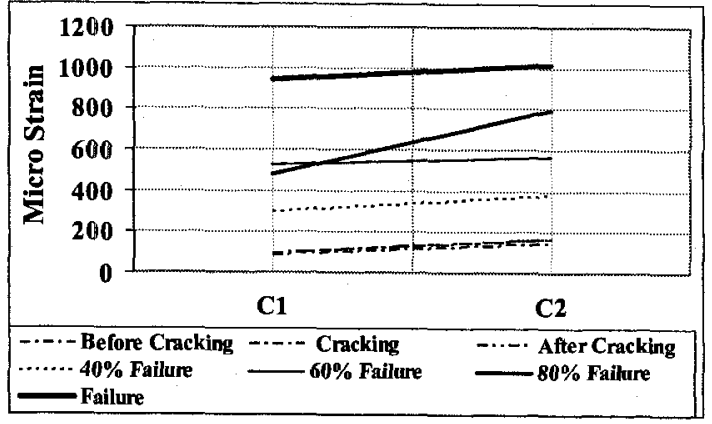

e) $\mathrm{MO}-\mathrm{EMV}-2.7 \mathrm{~N}$

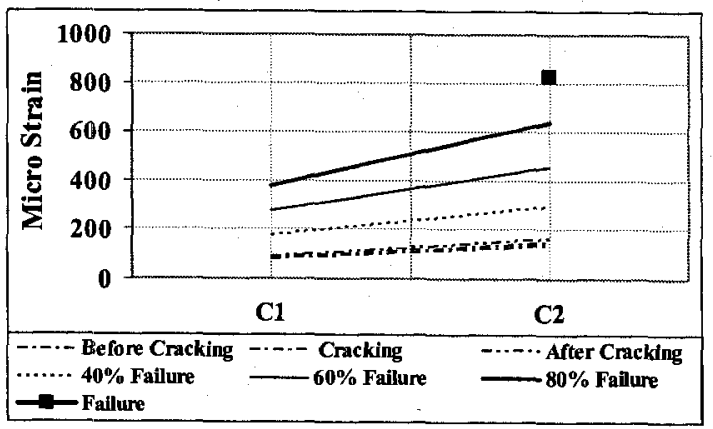

g) MO-NL-2.7N

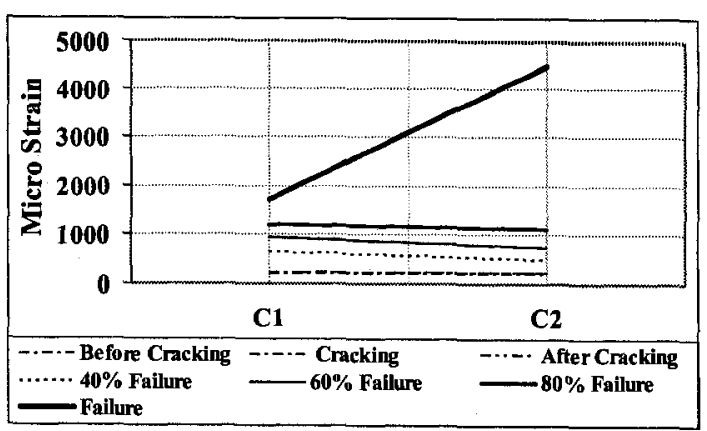

b) VA-EMV-1.5N

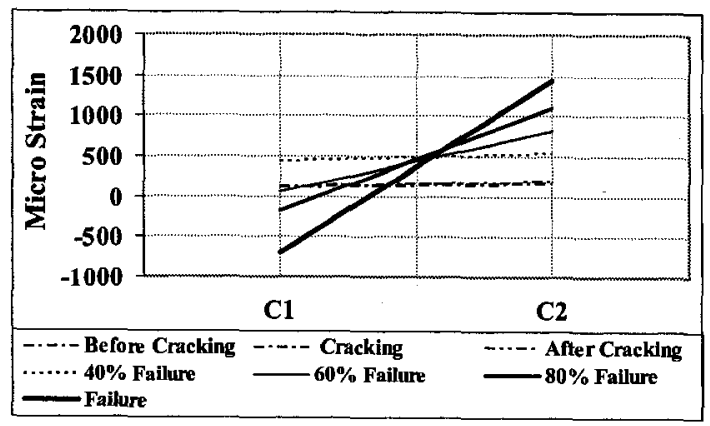

d) VA-EMV-2N

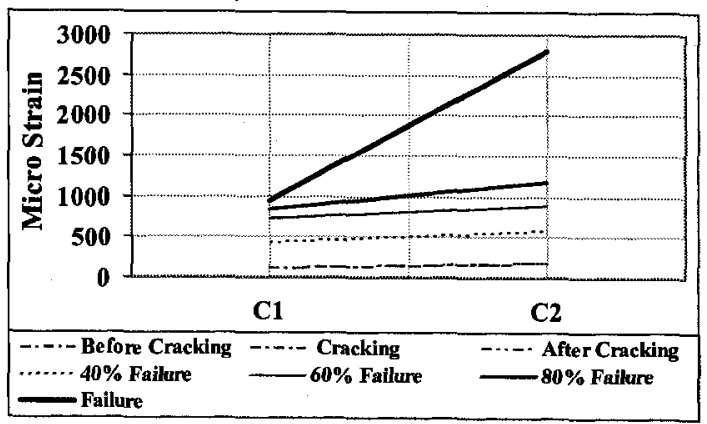

f) VA-EMV-2.7N

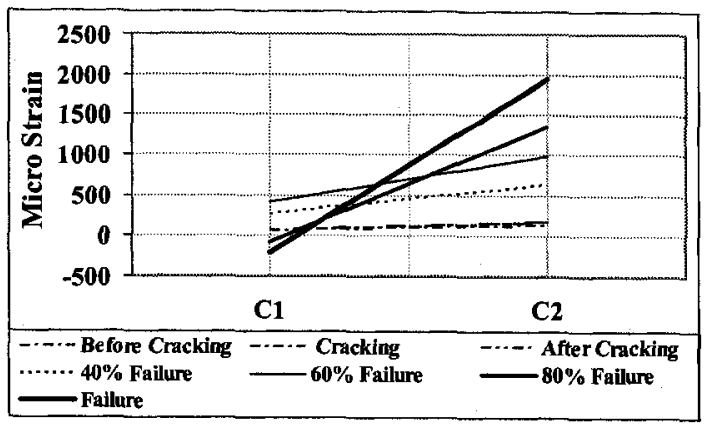

h) VA-NG-2.7N

Figure 7.9: Variation of concrete compression strain along the beams for different $a / d$ ratios 


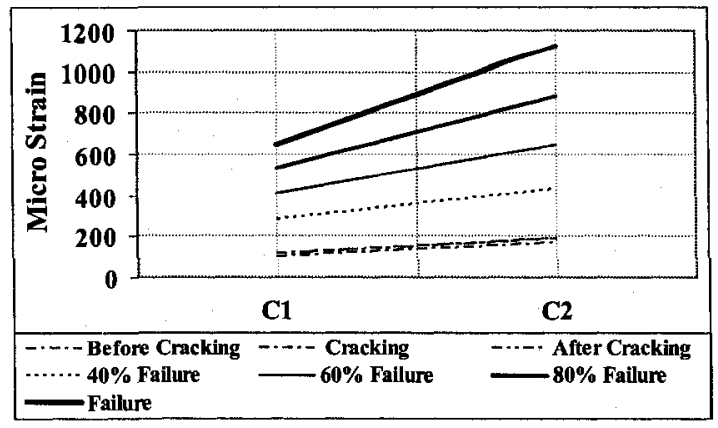

i) $\mathrm{MO}-\mathrm{EMV}-4 \mathrm{~N}$

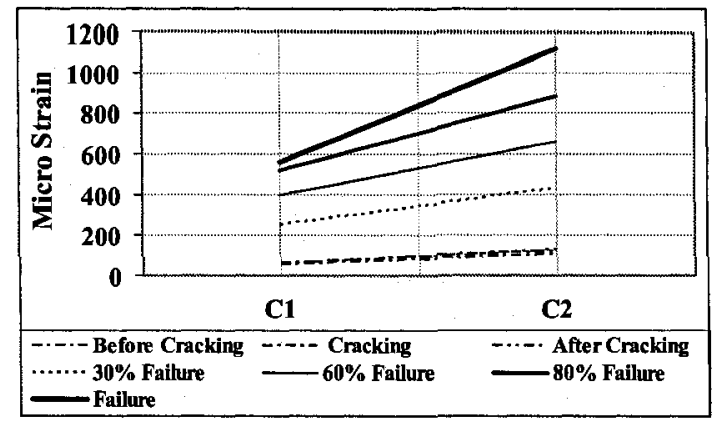

j) VA-EMV-4N

Cont. Figure 7.9: Variation of concrete compression strain along the beams for different $\mathrm{a} / \mathrm{d}$ ratios

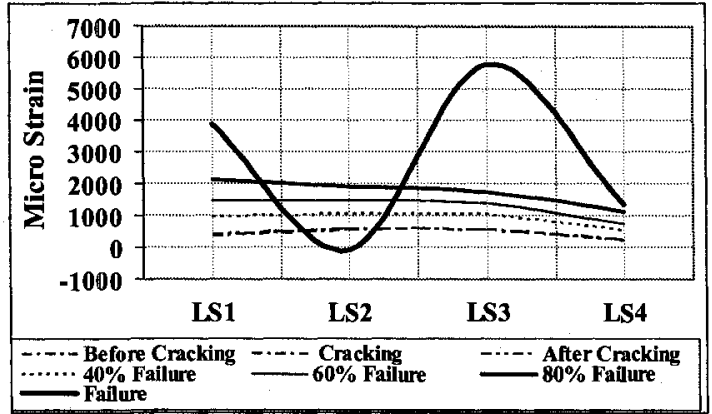

a) MO-EMV-1.5N

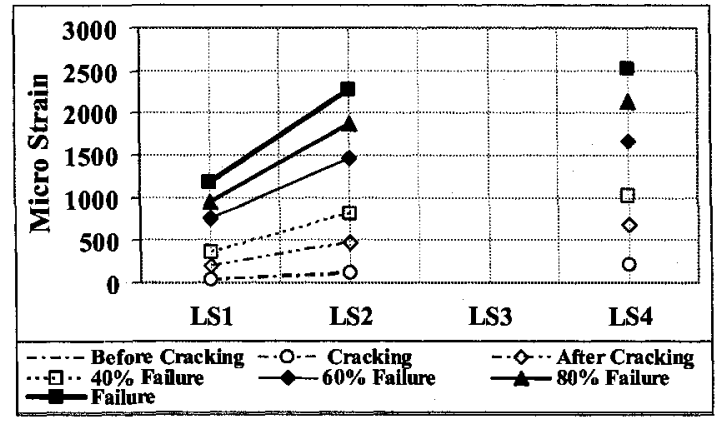

c) MO-EMV-2N

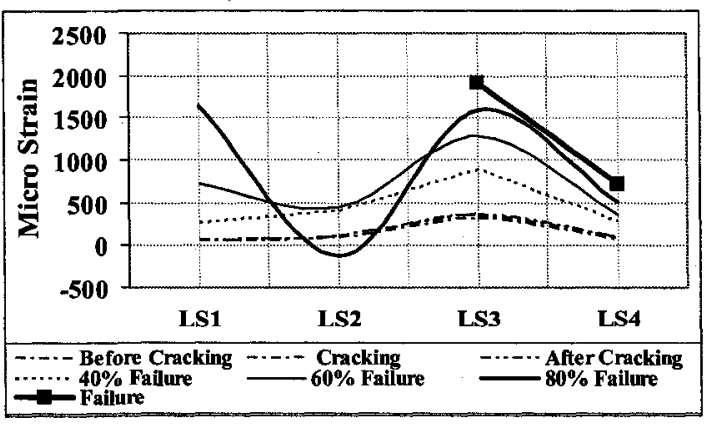

e) MO-EMV-2.7N

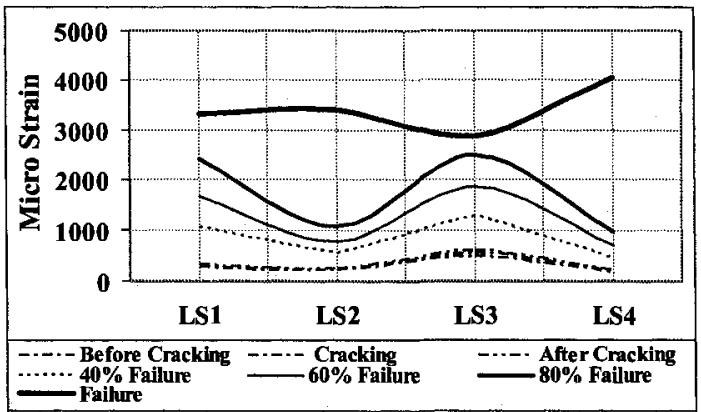

b) VA-EMV-1.5N

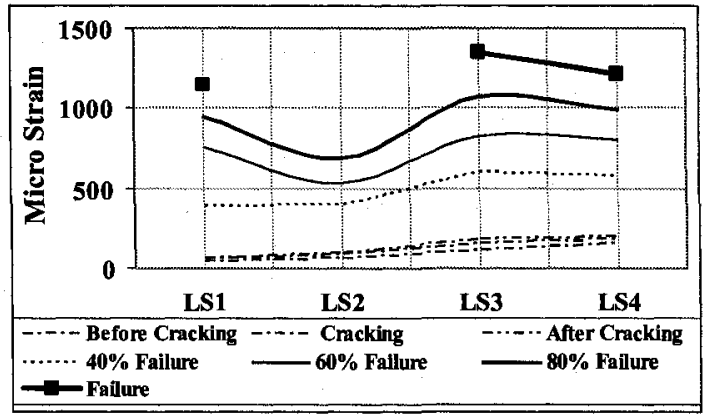

d) VA-EMV-2N

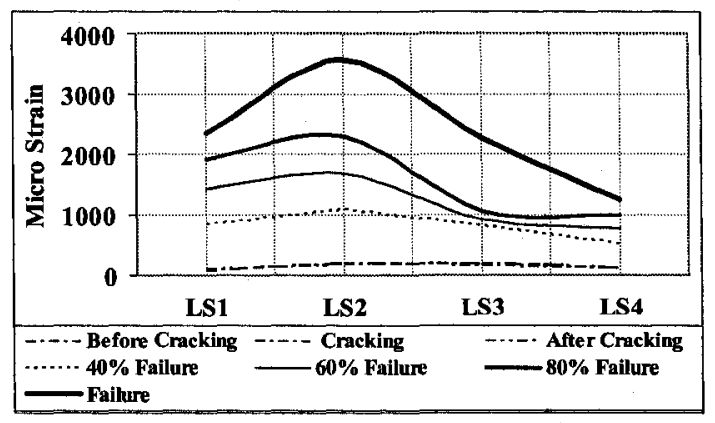

f) VA-EMV-2.7N

Figure7.10: Variation of longitudinal steel strain along beam for different $\mathrm{a} / \mathrm{d}$ ratios 


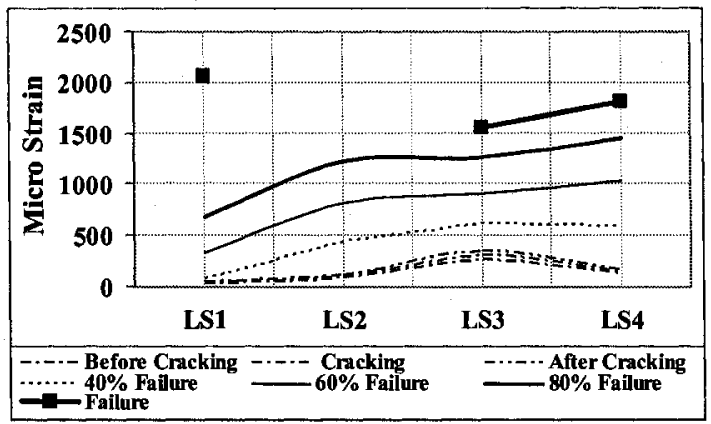

g) $\mathrm{MO}-\mathrm{NL}-2.7 \mathrm{~N}$

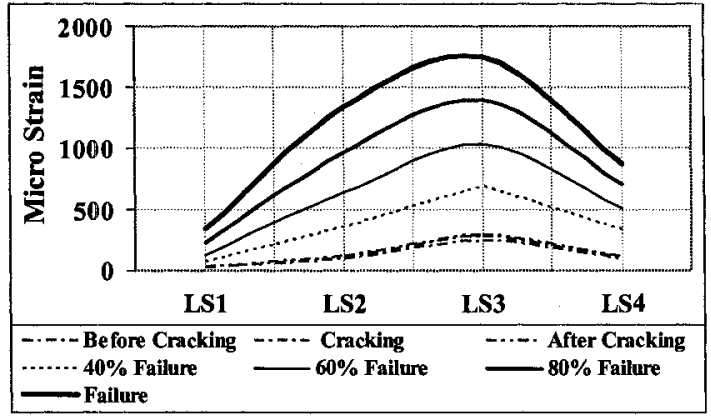

i) MO-EMV-4N

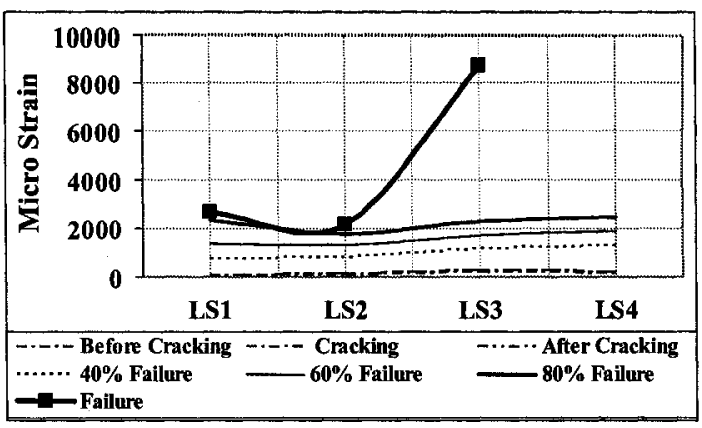

h) VA-NG-2.7N

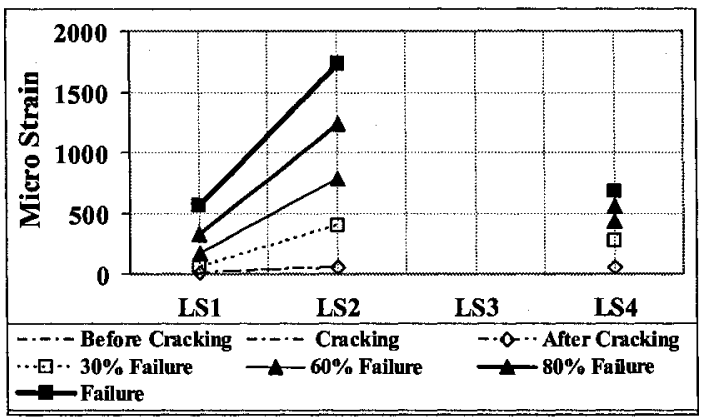

j) VA-EMV-4N

Cont.Figure7.10: Variation of longitudinal steel strain along beam for different $\mathrm{a} / \mathrm{d}$ ratios 


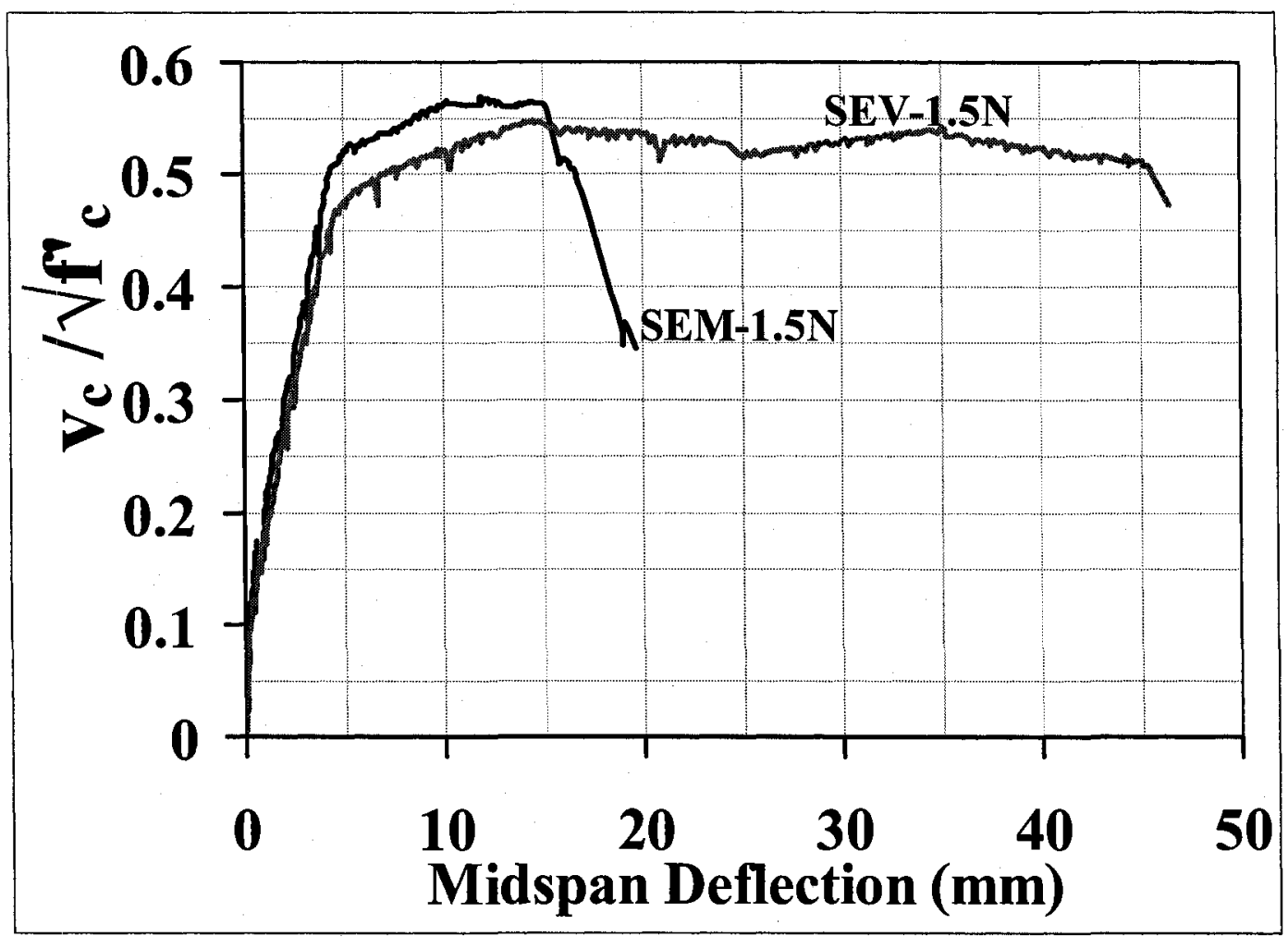

a) $\mathrm{a} / \mathrm{d}=1.5$

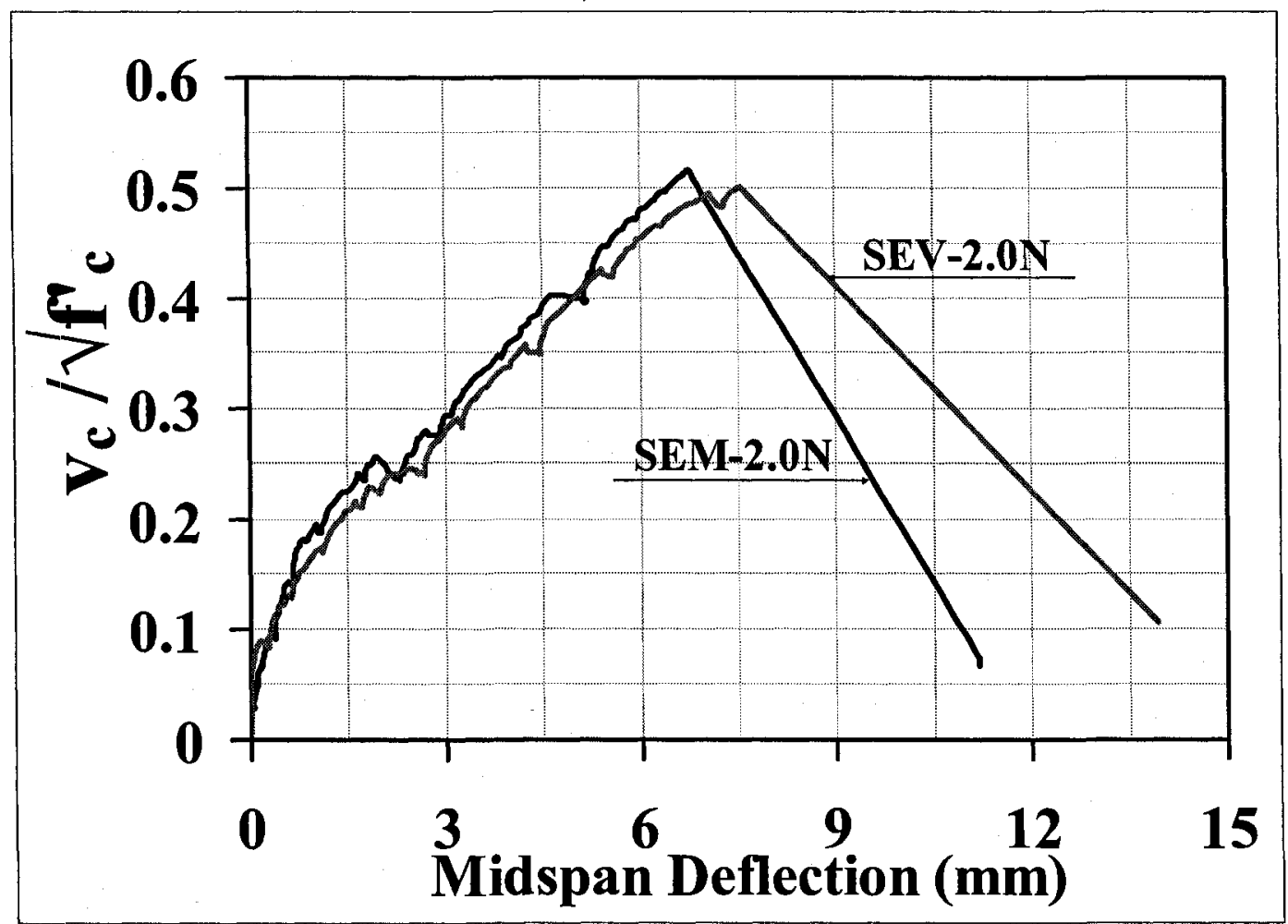

b) $\mathrm{a} / \mathrm{d}=2$

Figure 7.11: Effect of aggregate type on normalized shear stress resistance-midspan deflection response of $\mathrm{RRC}$ beams for different $\mathrm{a} / \mathrm{d}$ ratios 


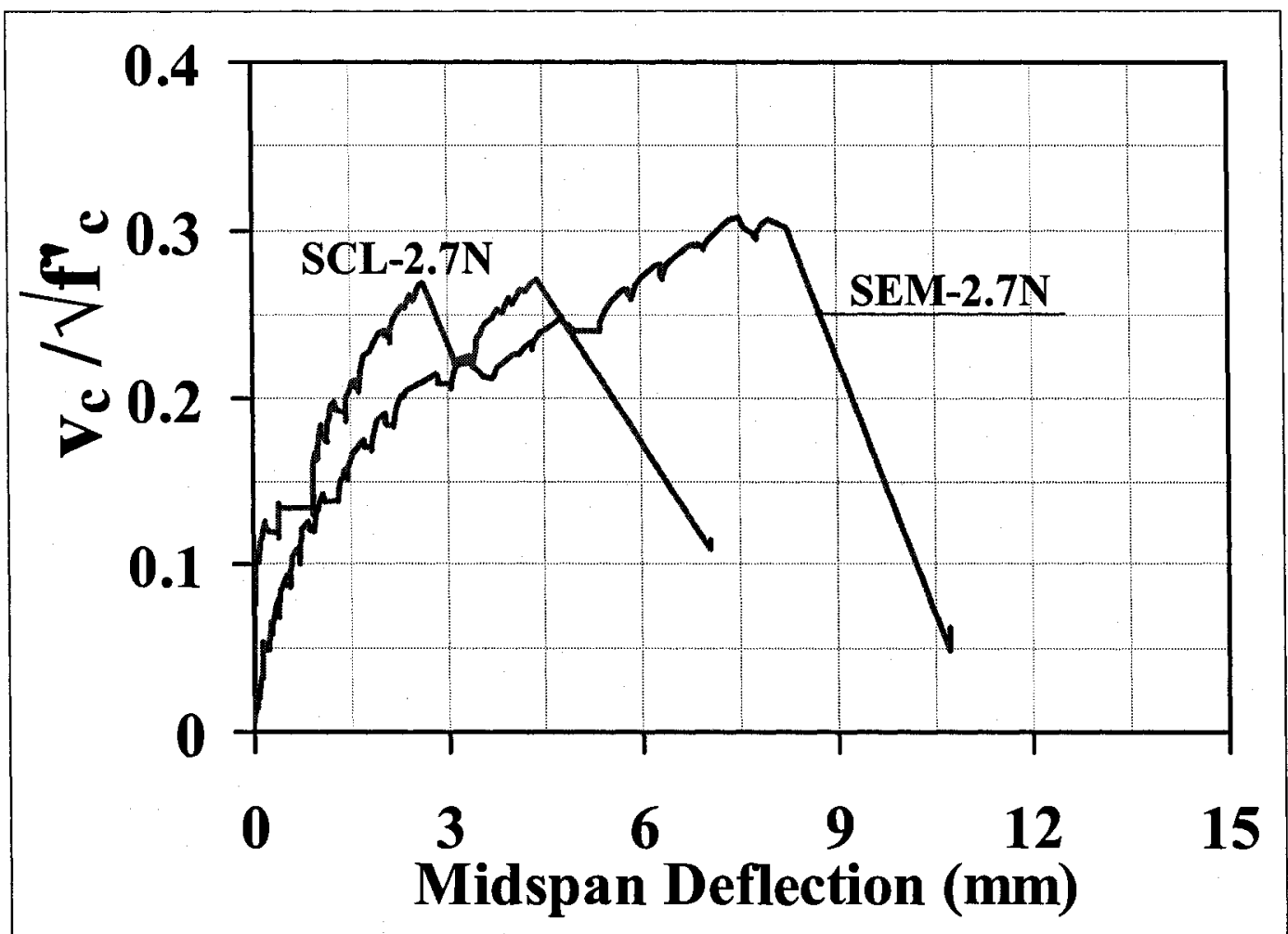

c) $\mathrm{a} / \mathrm{d}=2.7$

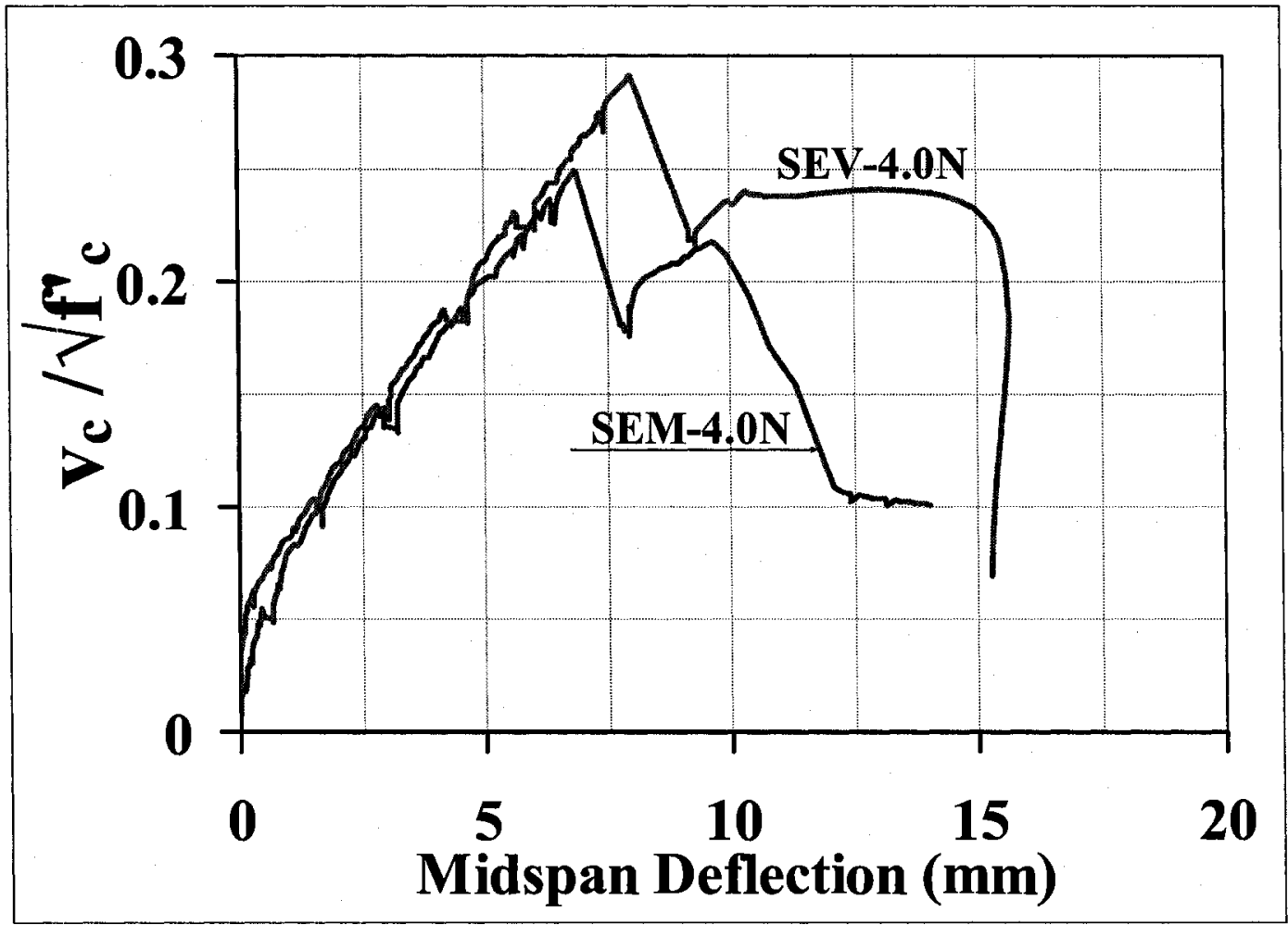

d) $\mathrm{a} / \mathrm{d}=4$

Cont. Figure 7.11: Effect of aggregate type on normalized shear stress resistancemidspan deflection response of RRC beams for different $\mathrm{a} / \mathrm{d}$ ratios 


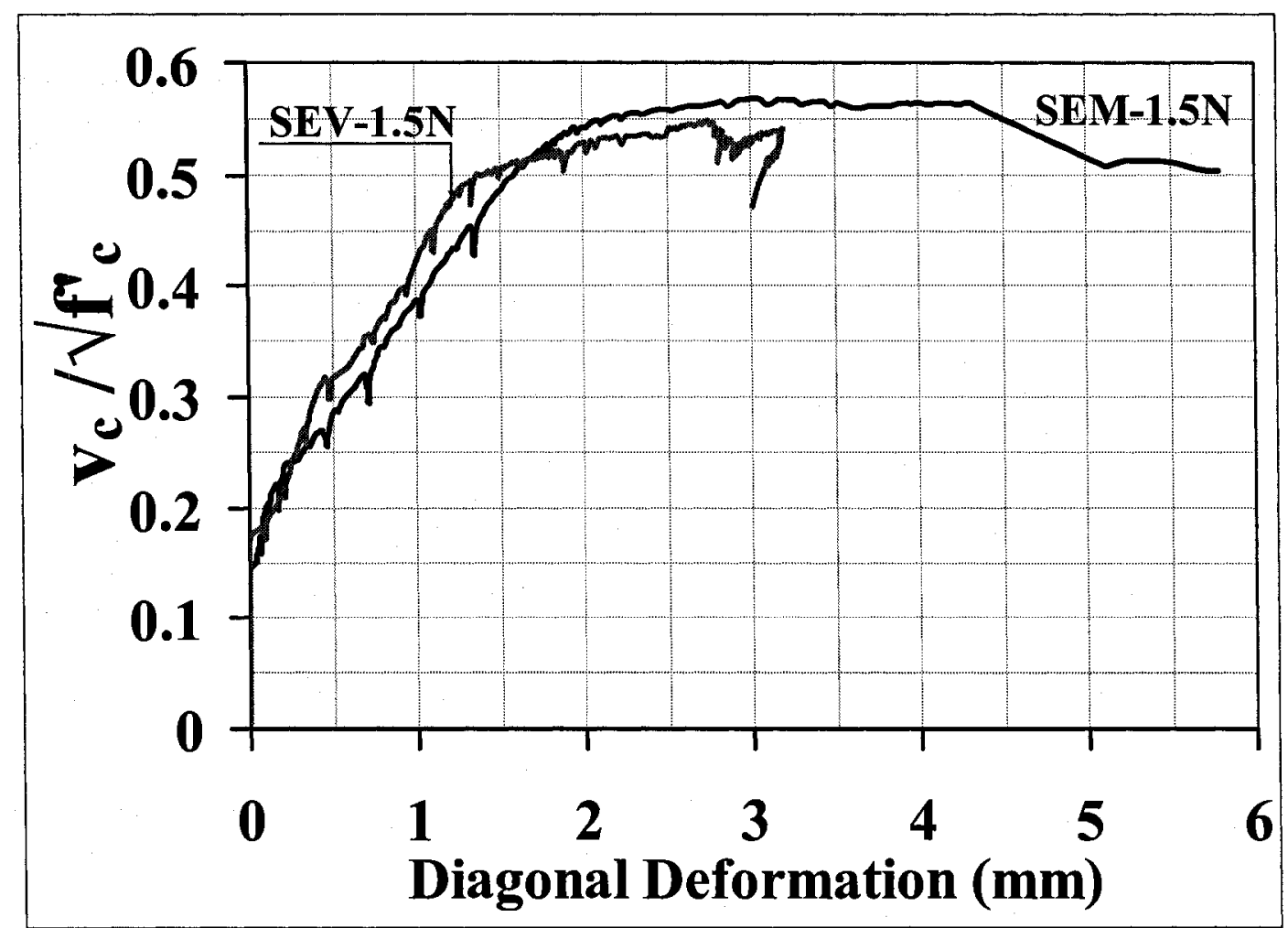

a) $\mathrm{a} / \mathrm{d}=1.5$

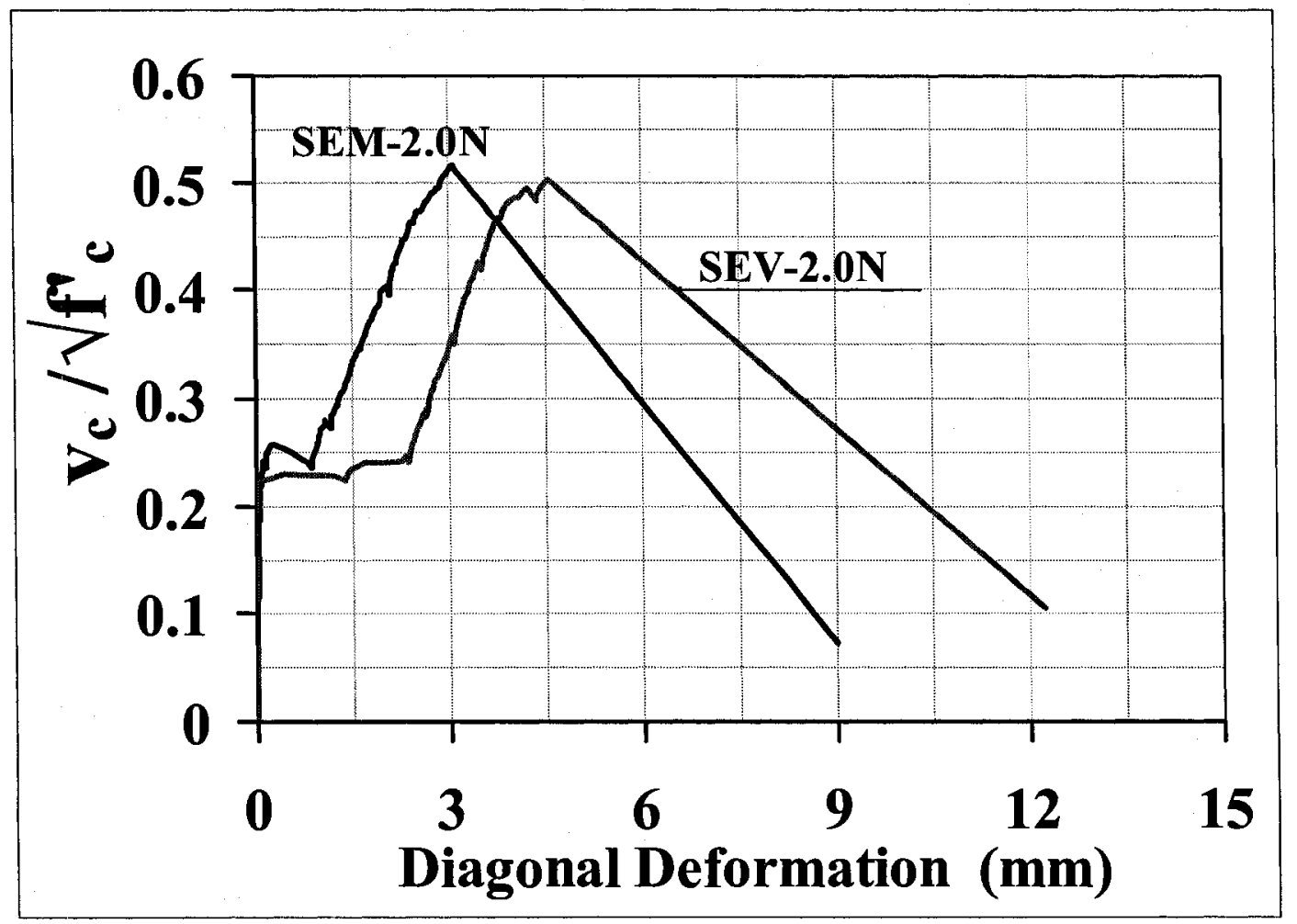

b) $\mathrm{a} / \mathrm{d}=2$

Figure 7.12: Effect of aggregate type on normalized shear stress resistance-diagonal deformation response of RRC beams at different a/d ratios 


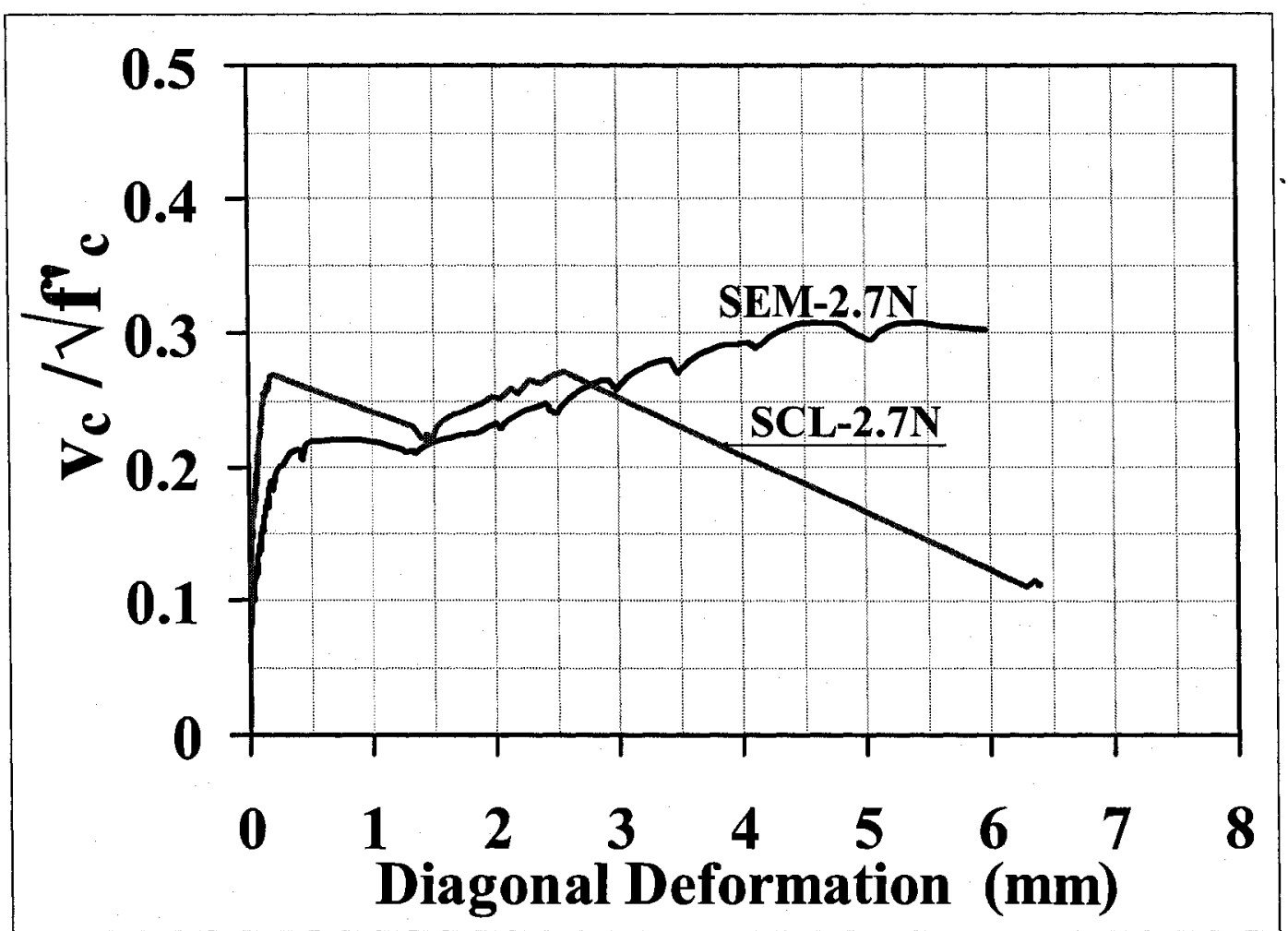

c) $\mathrm{a} / \mathrm{d}=2.7$

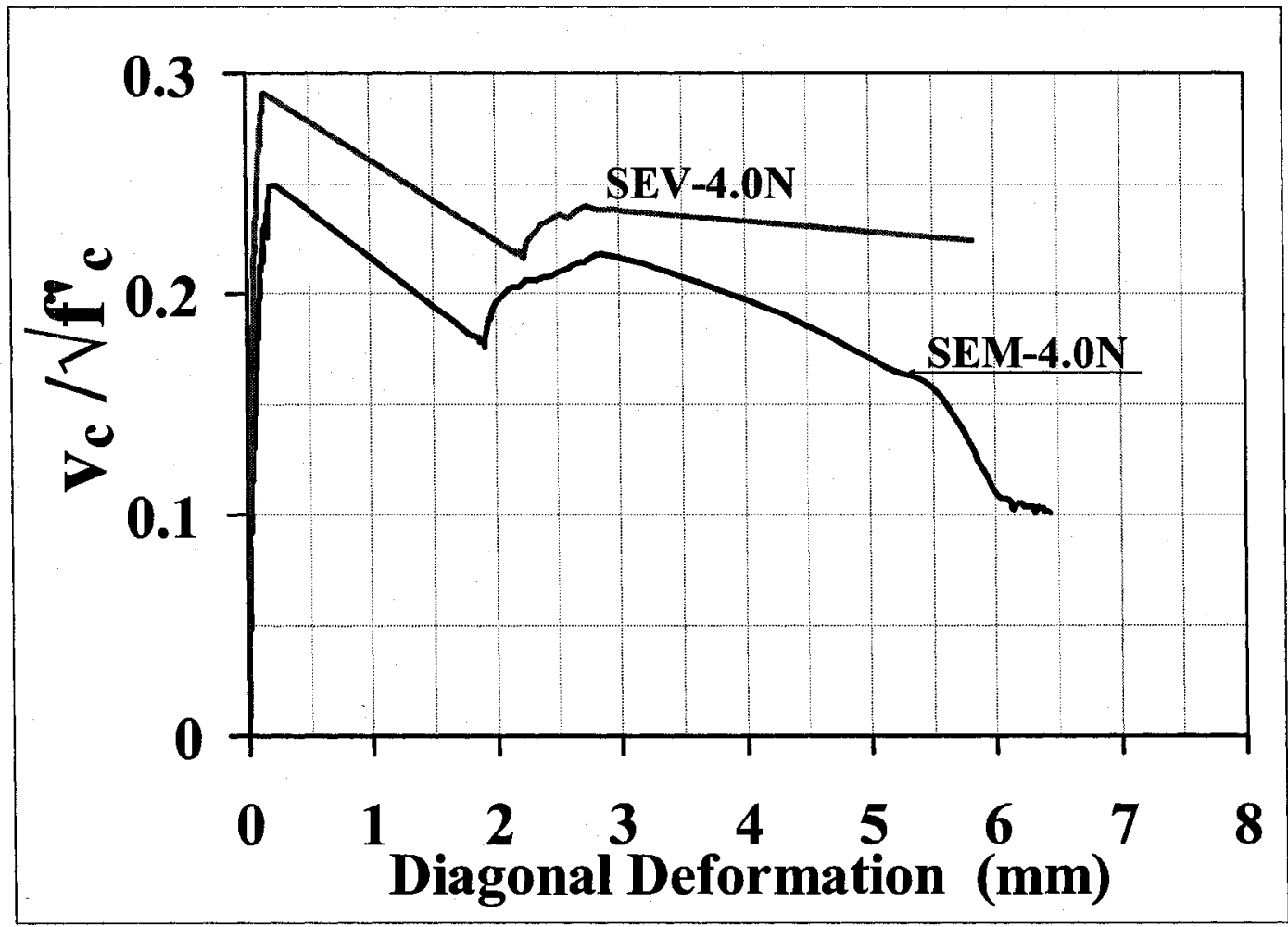

d) $a / d=4$

Cont. Figure 7.12: Effect of aggregate type on normalized shear stress resistancediagonal deformation response of RRC beams at different $\mathrm{a} / \mathrm{d}$ ratios 


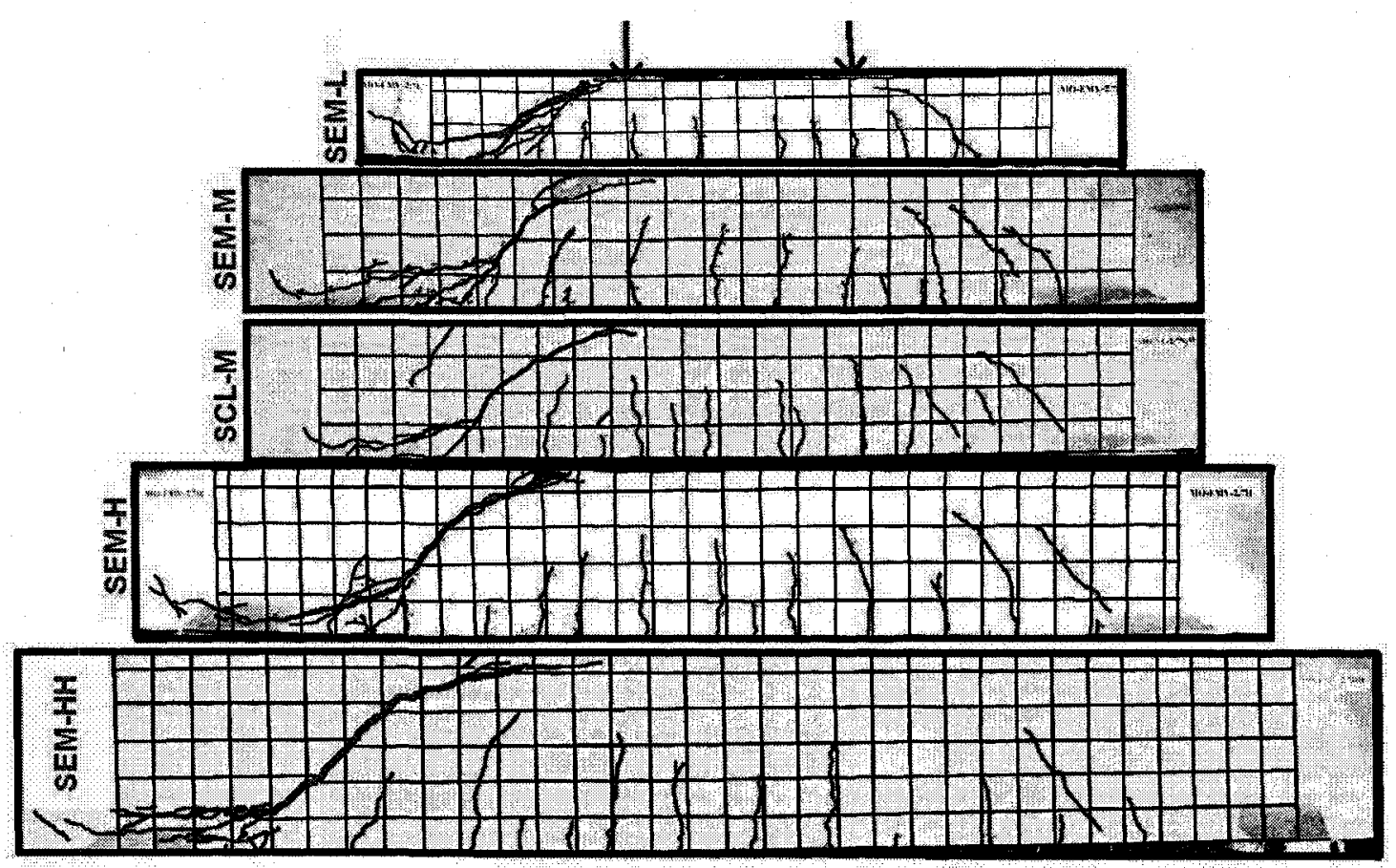

a) SEM and SCL beams

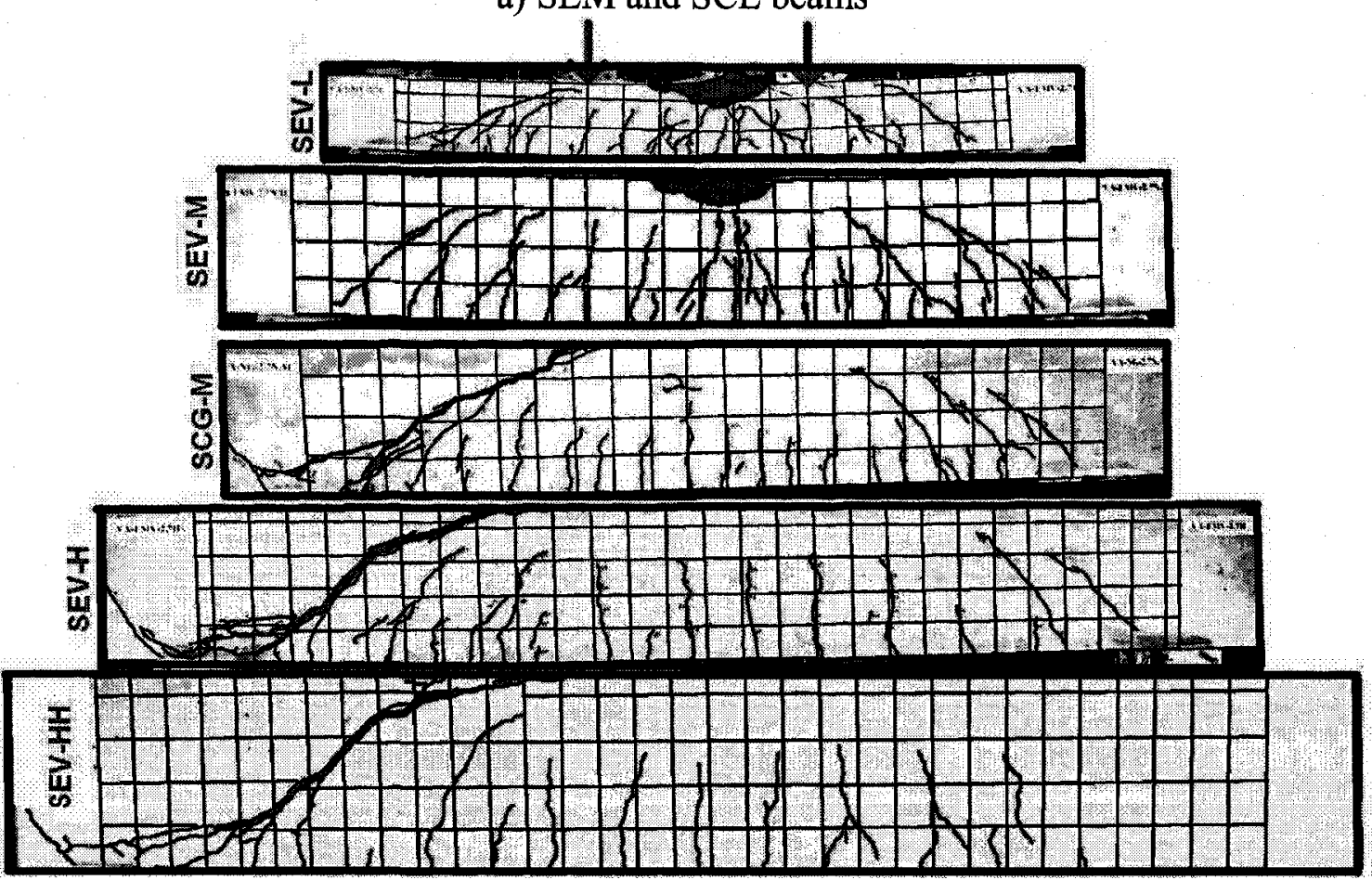

b) SEV and SCG beams

Figure 7.13: Typical crack patterns of shear RRC beams 


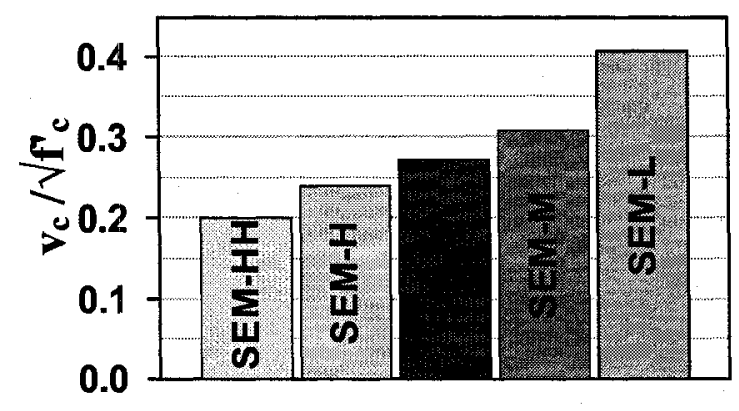

a) SEM and control SCL beams

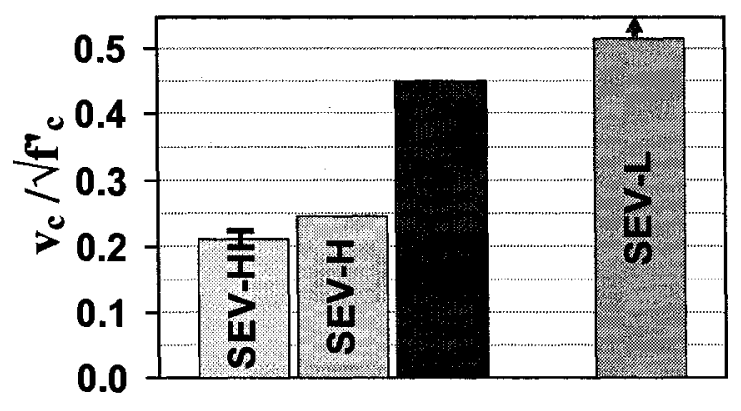

b) SEV and control SCG beams Figure 7.14: Experimental nominal shear strength of RRC beams for different size beams 


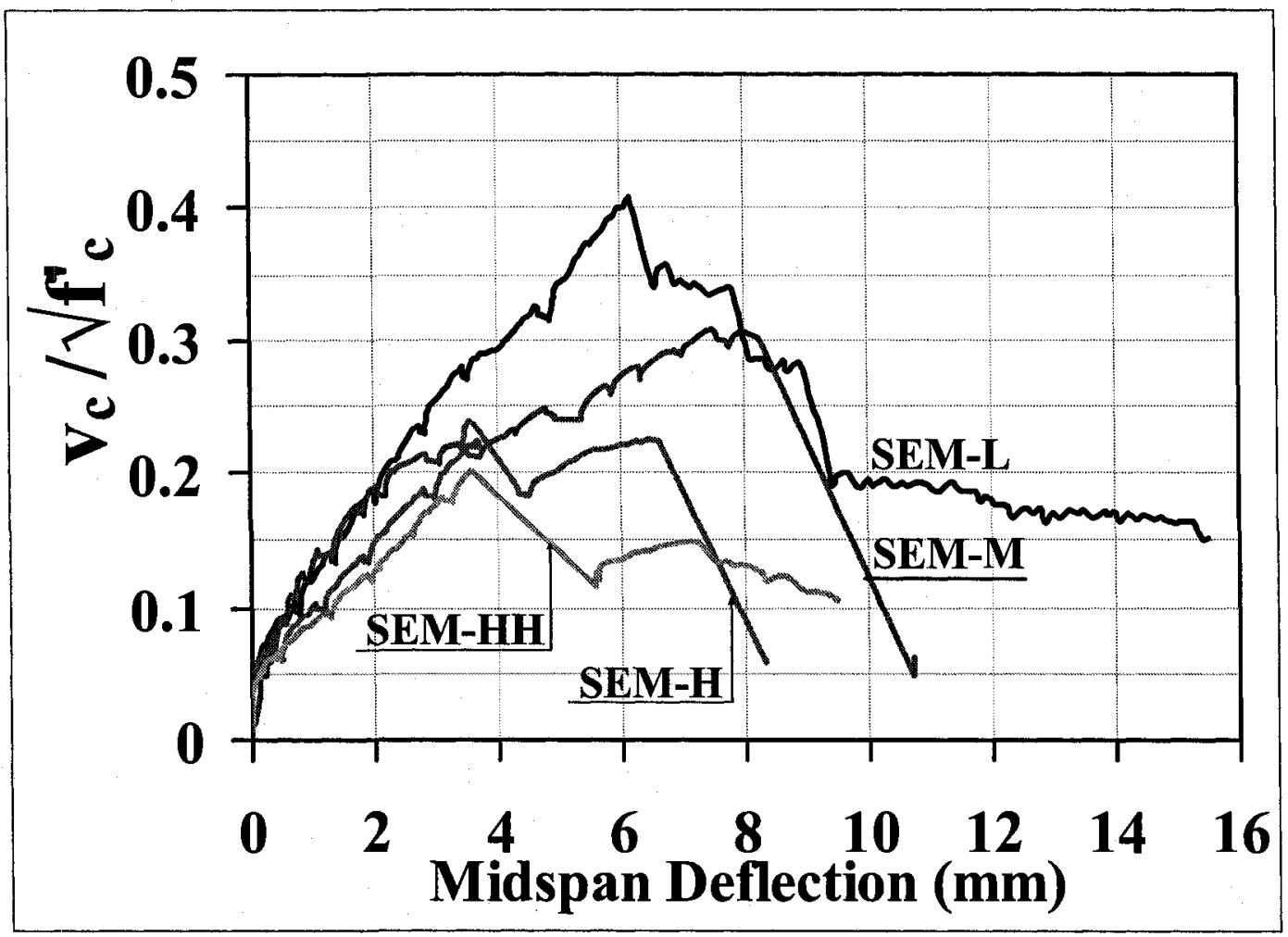

SEM beams

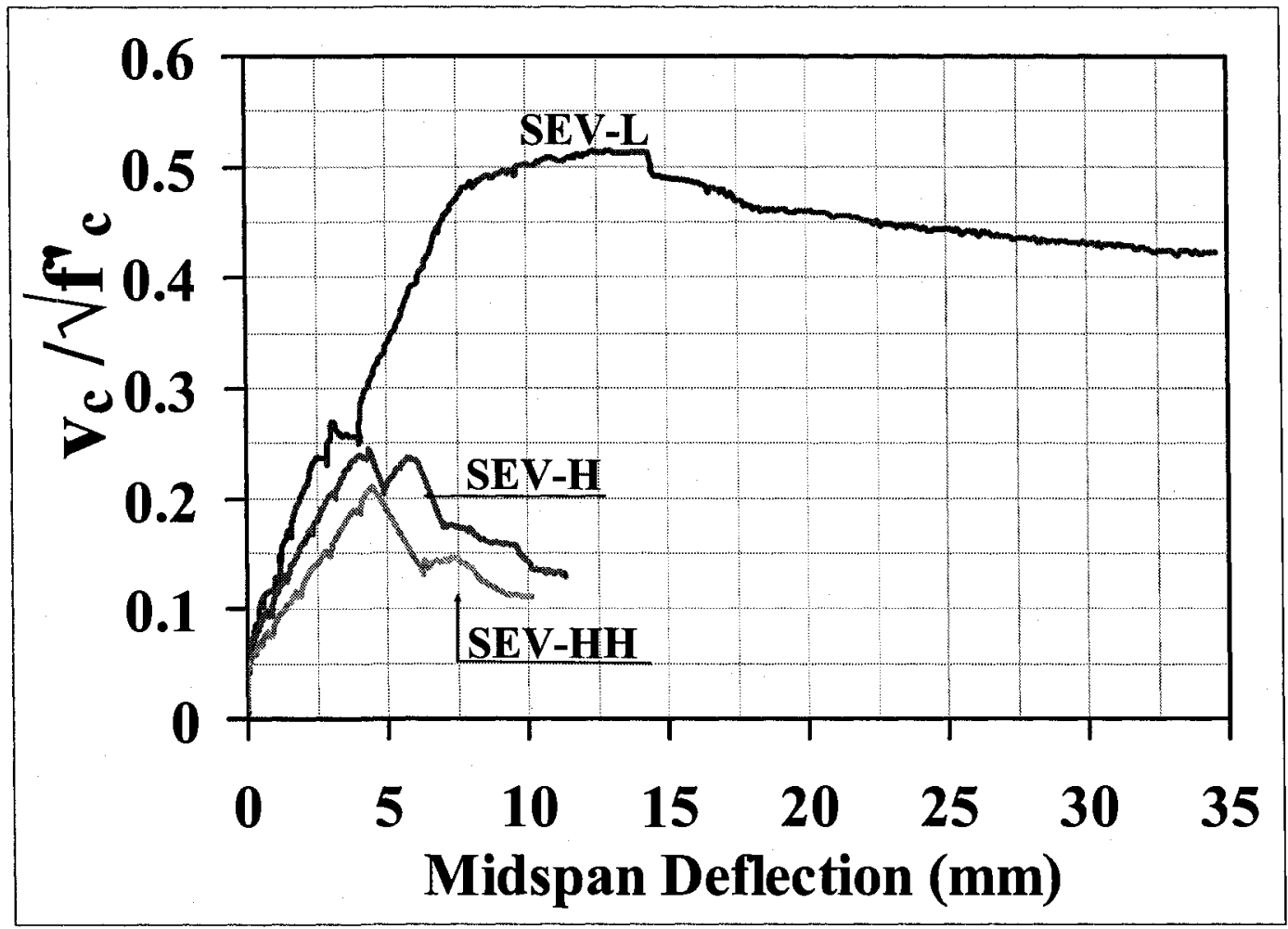

SEV beams

a) Normalized shear stress resistance-midspan deflection response

Figure 7.15: Size effect on shear behaviour of RRC members 

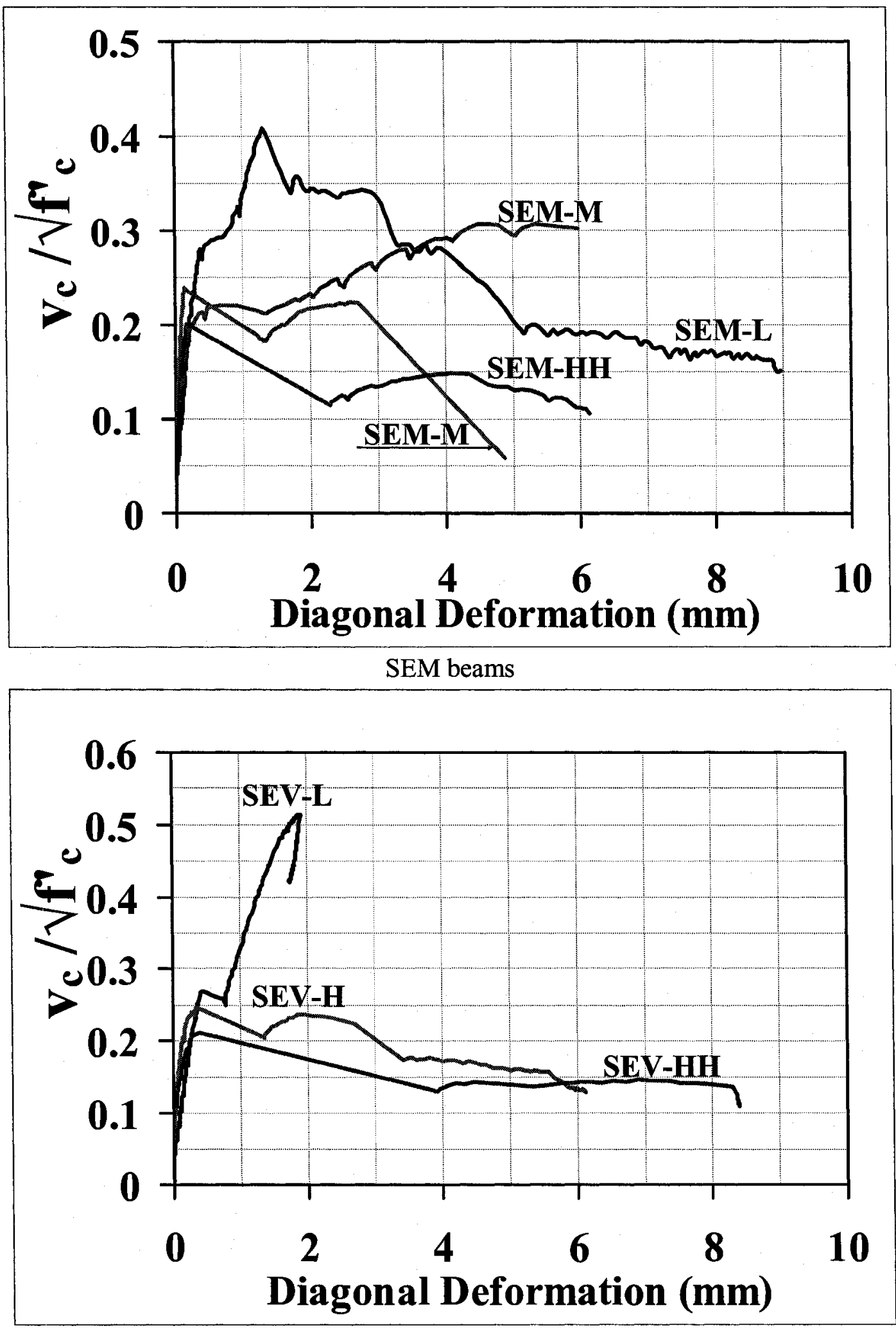

SEV beams

b) Normalized shear stress resistance-diagonal deformation response Cont. Figure 7.15: Size effect on shear behaviour of RRC members 


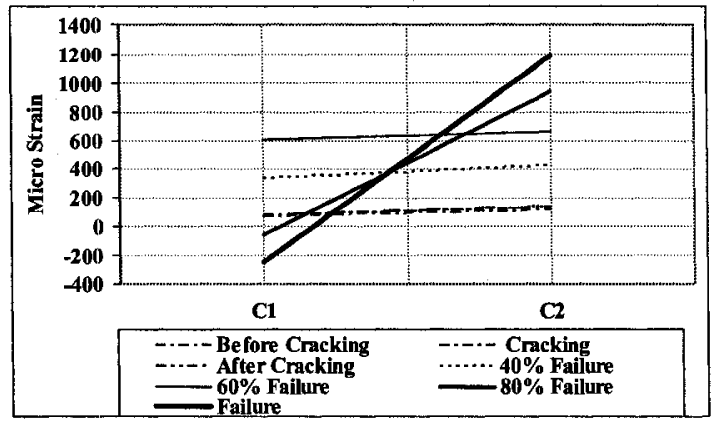

a) MO-EMV-2.7L

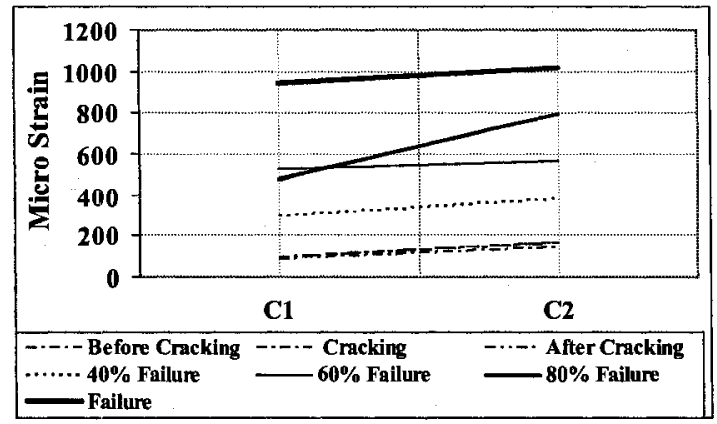

e) MO-EMV-2.7M

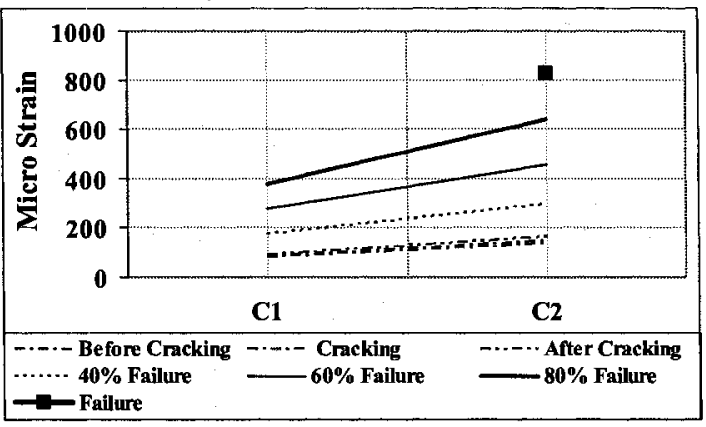

g) MO-NL-2.7M

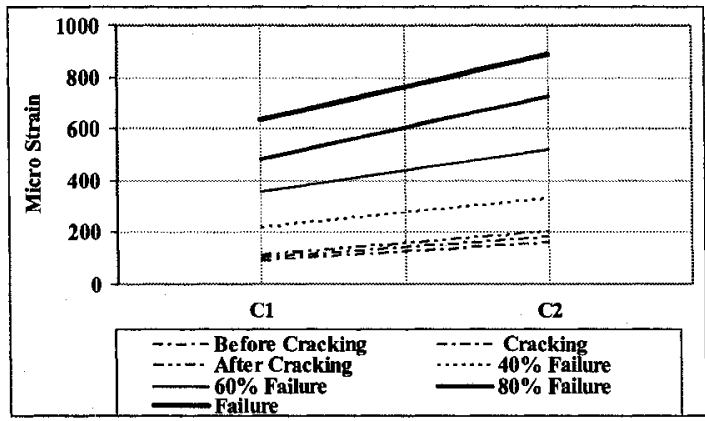

g) MO-EMV-2.7H

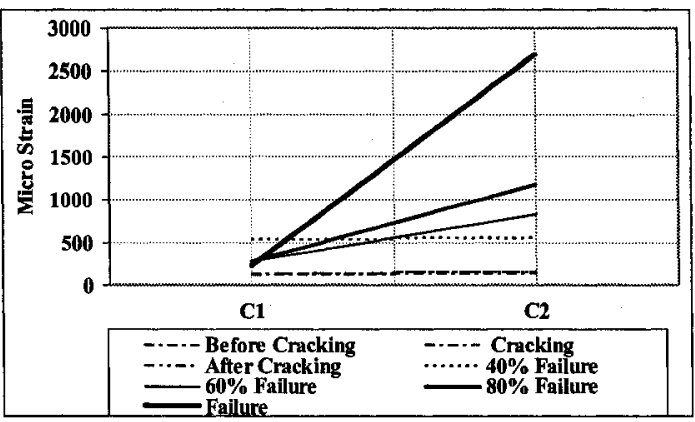

b) VA-EMV-2.7L

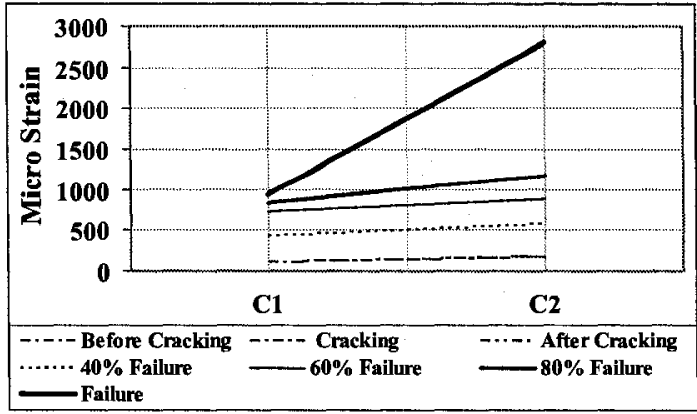

f) VA-EMV-2.7M

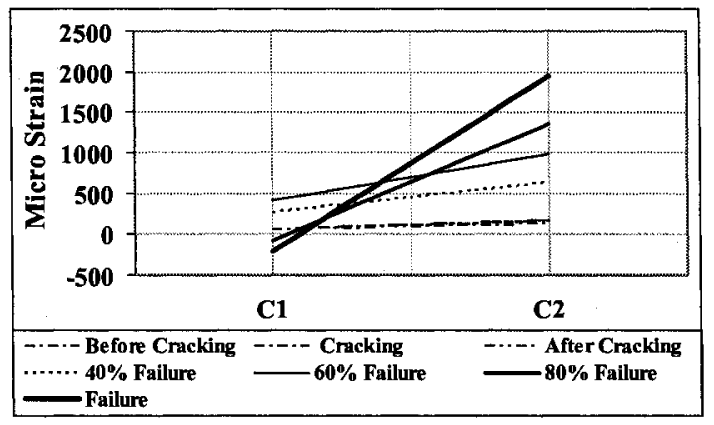

h) VA-NG-2.7M

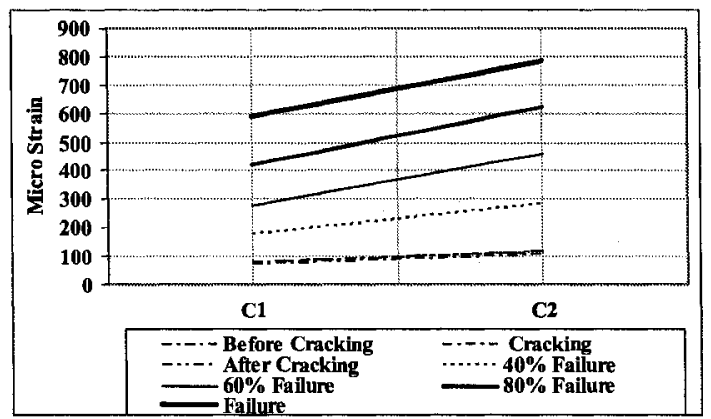

h) VA-EMV-2.7H

Figure 7.16: Variation of concrete compression strain along beam for different size beams 


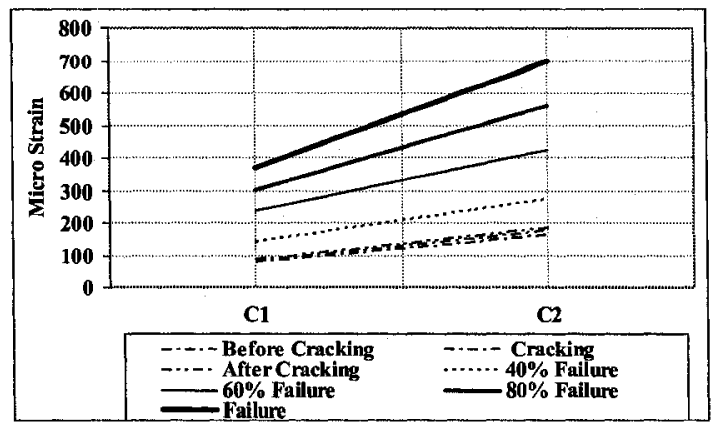

i) MO-EMV-2.7HH

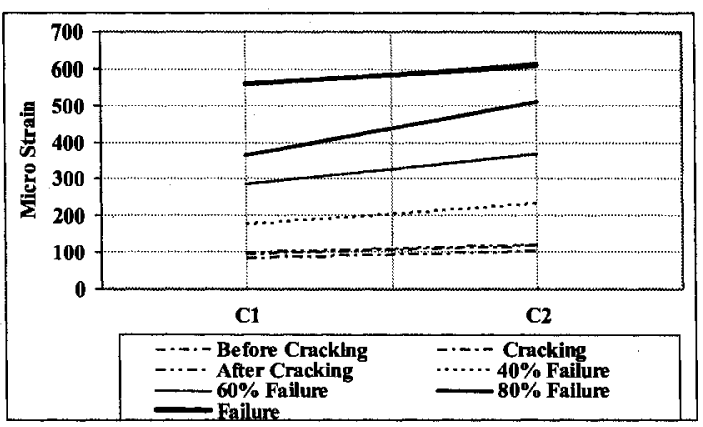

j) VA-EMV-2.7HH

Cont. Figure 7.16: Variation of concrete compression strain along beam for different size beams

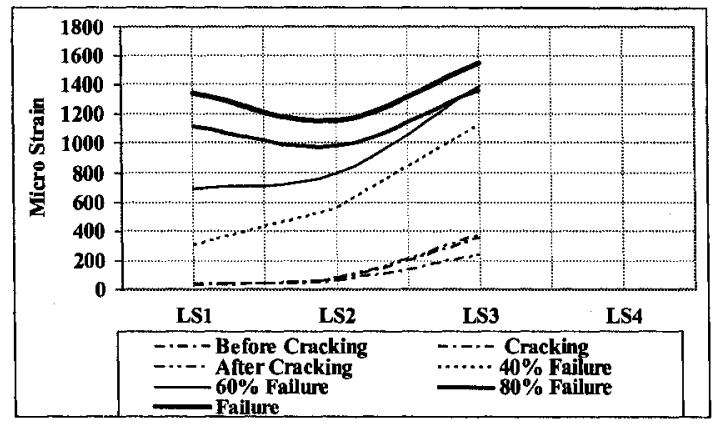

a) MO-EMV-2.7L

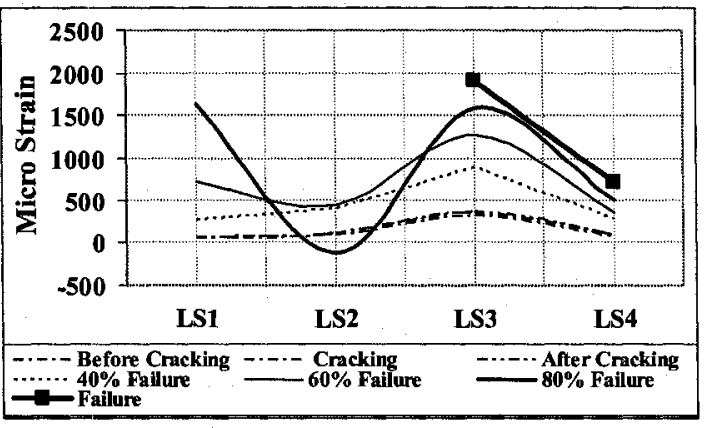

e) MO-EMV-2.7M

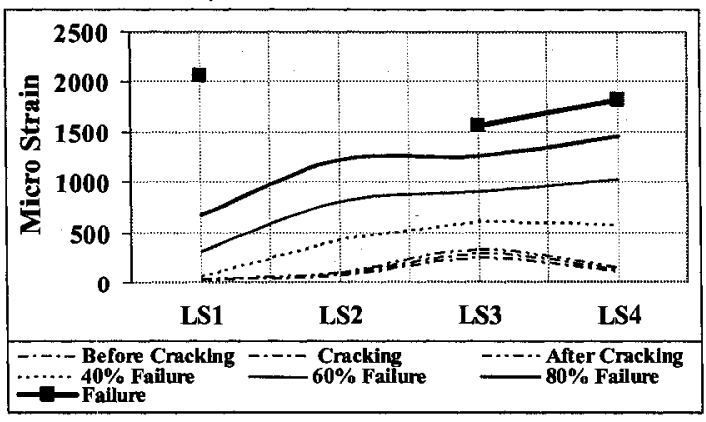

g) MO-NL-2.7M

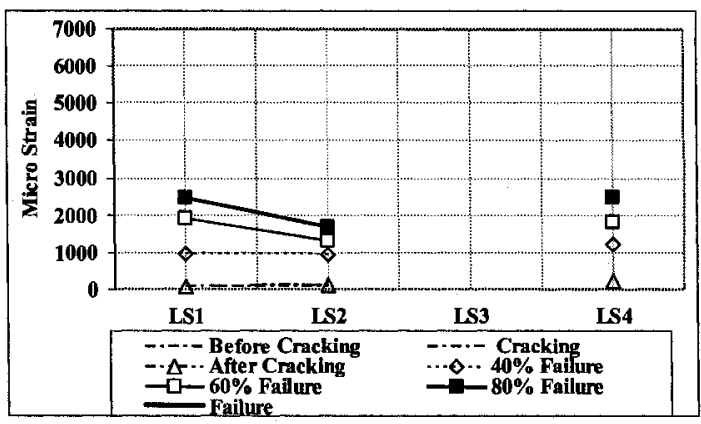

b) VA-EMV-2.7L

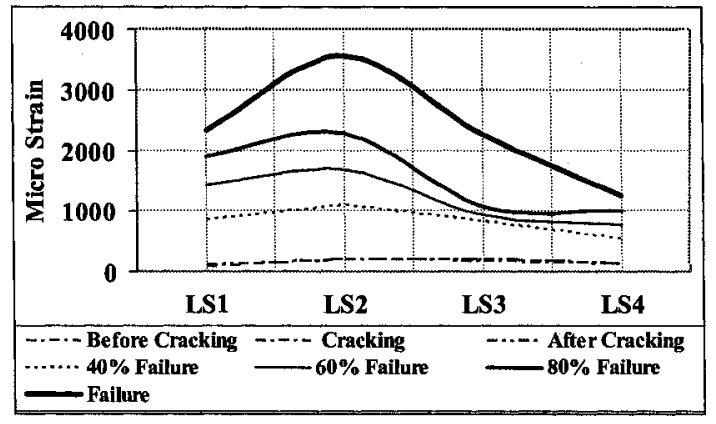

f) VA-EMV-2.7M

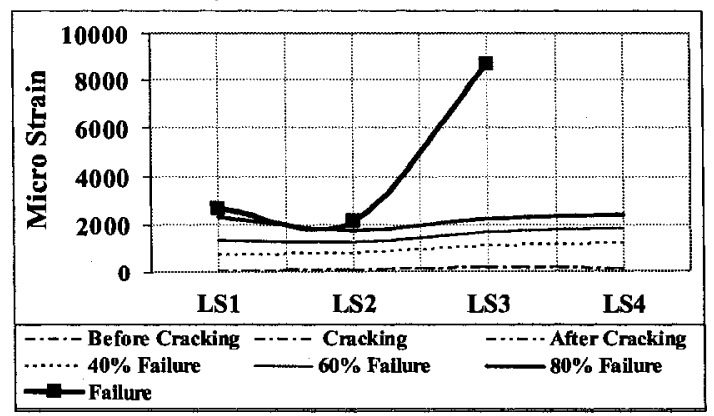

h) VA-NG-2.7M

Figure 7.17: Variation of longitudinal steel strain along beam for different size beams 


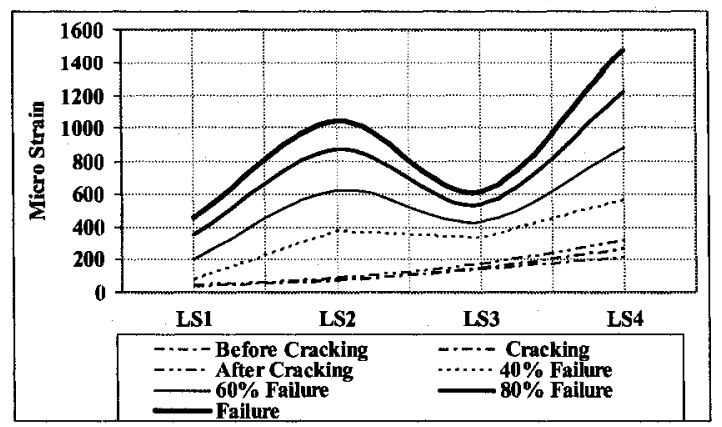

g) MO-EMV-2.7H

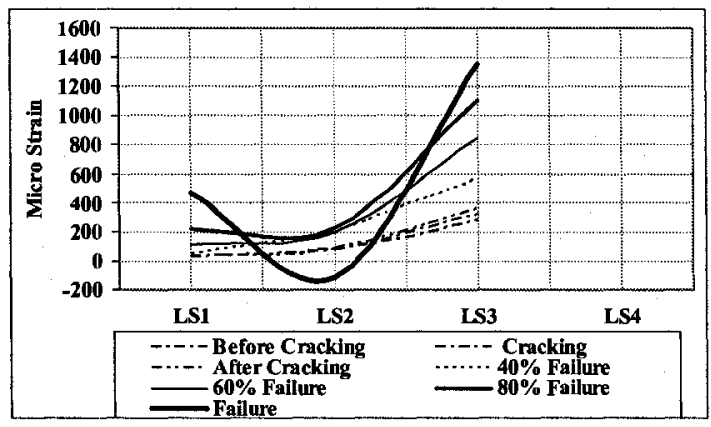

i) $\mathrm{MO}-\mathrm{EMV}-2.7 \mathrm{HH}$

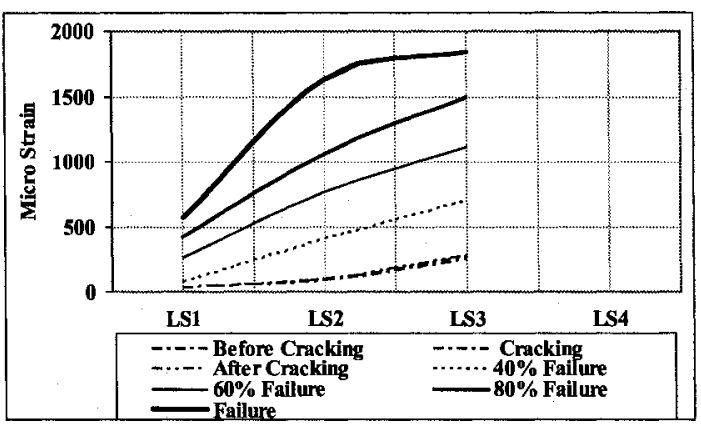

h) VA-EMV-2.7H

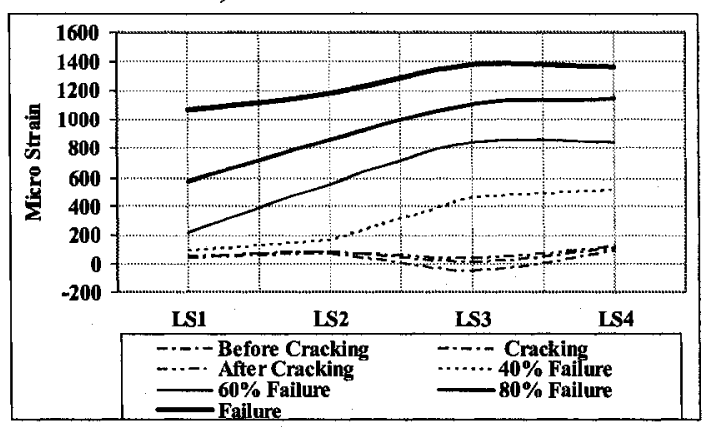

j) VA-EMV-2.7HH

Cont. Figure 7.17: Variation of longitudinal steel strain along beam for different size beams 


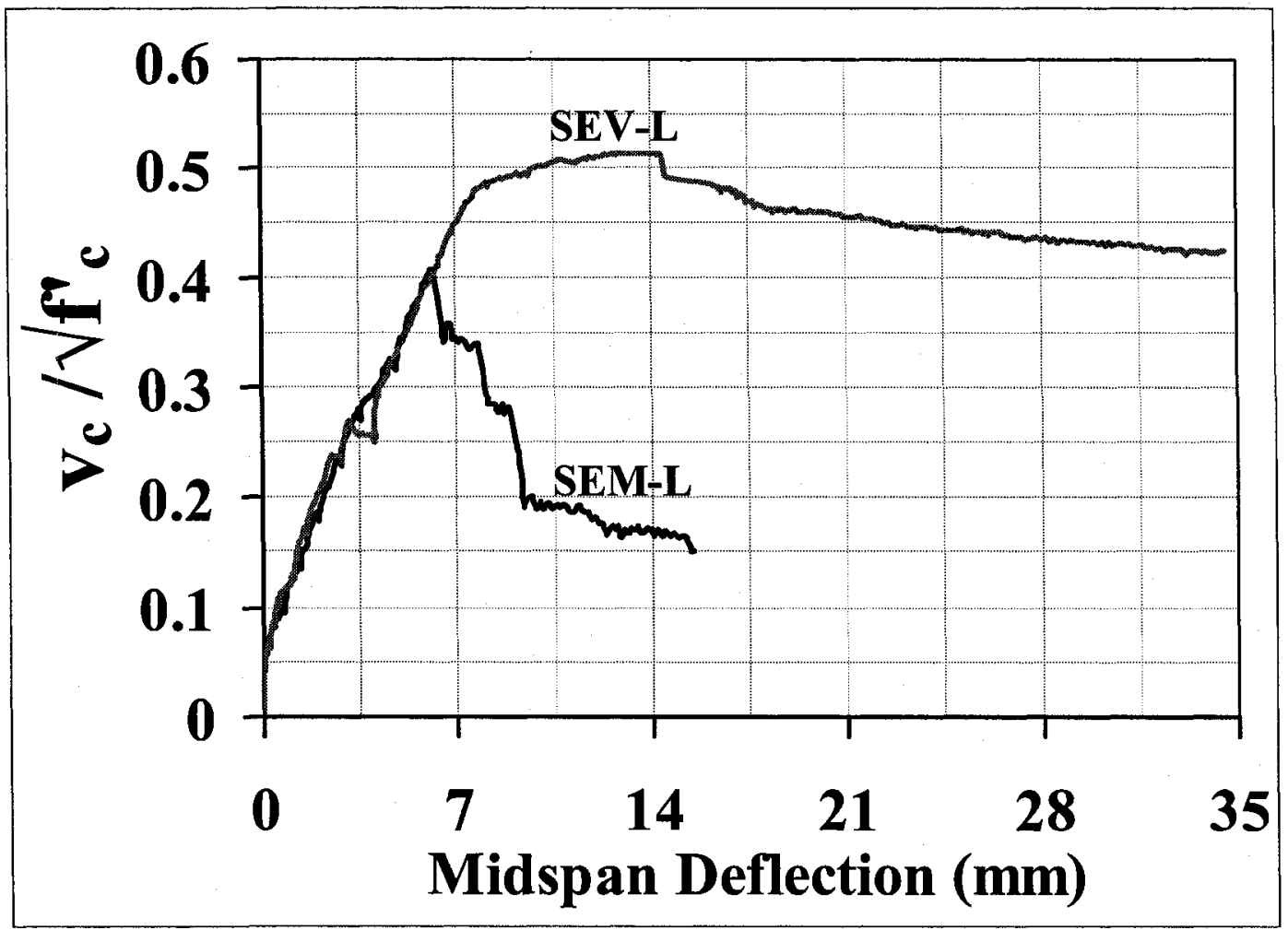

a) $\mathrm{d}=200 \mathrm{~mm}$

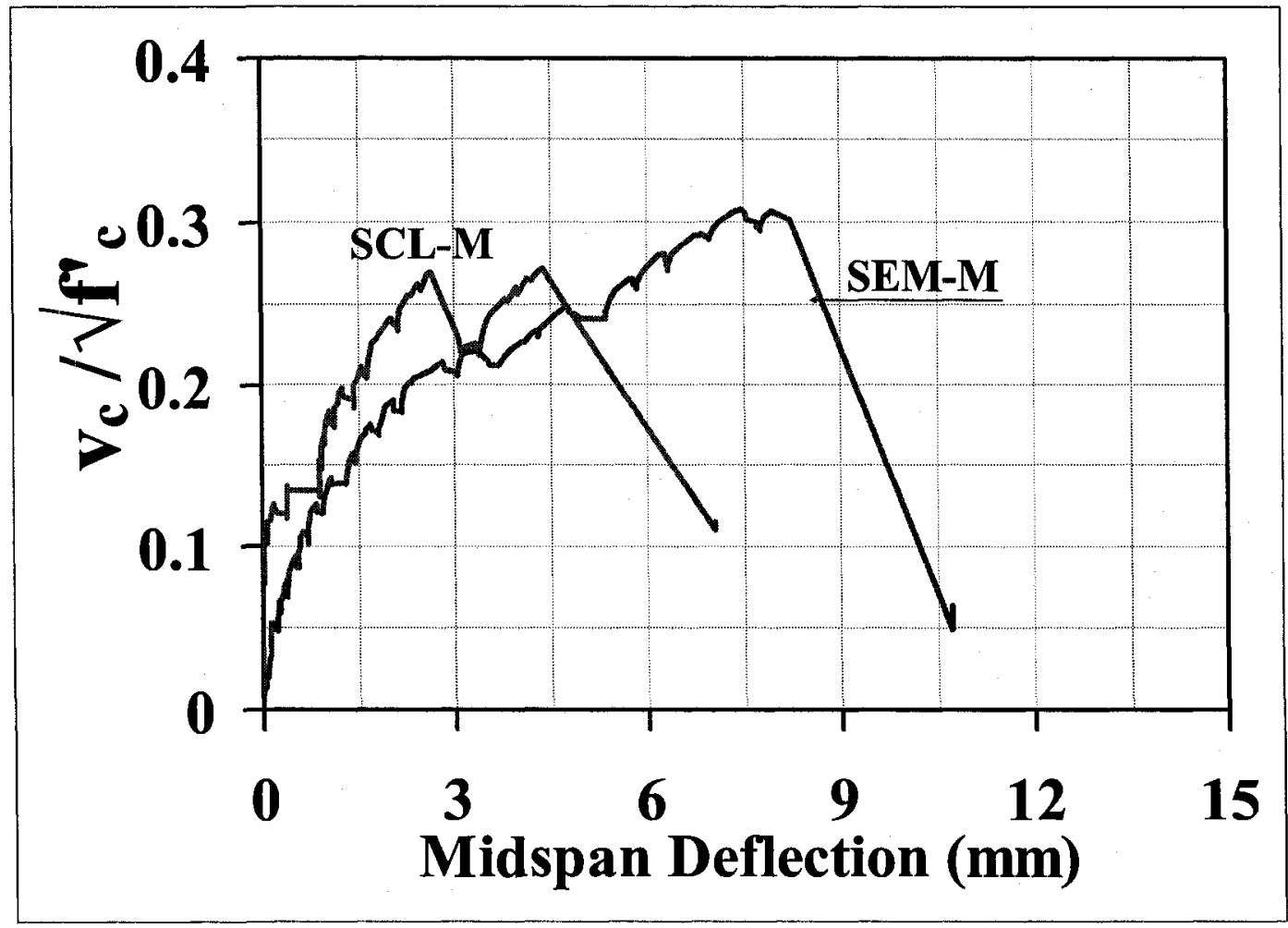

b) $\mathrm{d}=300 \mathrm{~mm}$

Figure 7.18: Effect of aggregate type on normalized shear stress -midspan deflection response of $\mathrm{RRC}$ beams with different depths 


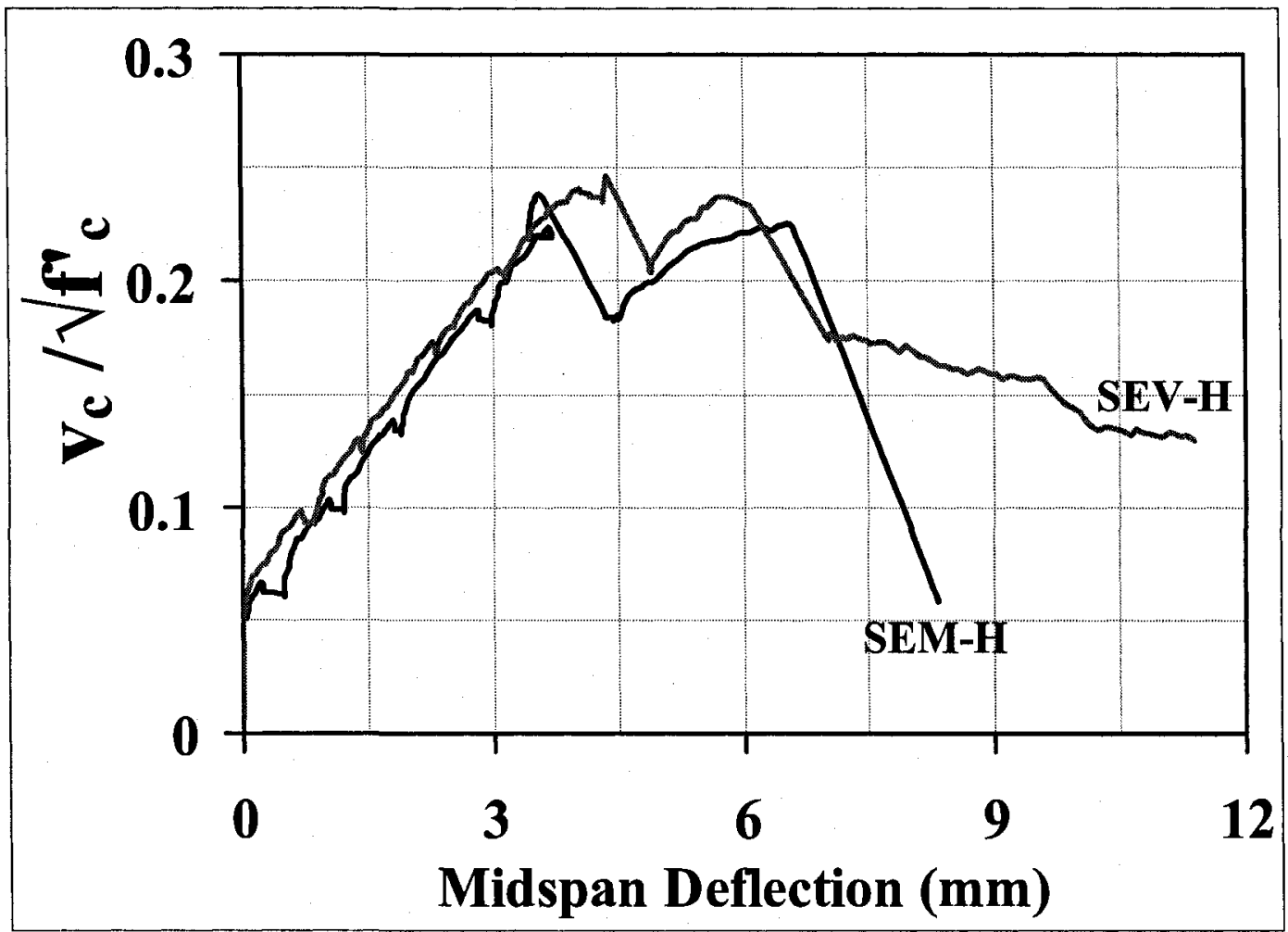

c) $\mathrm{d}=400 \mathrm{~mm}$

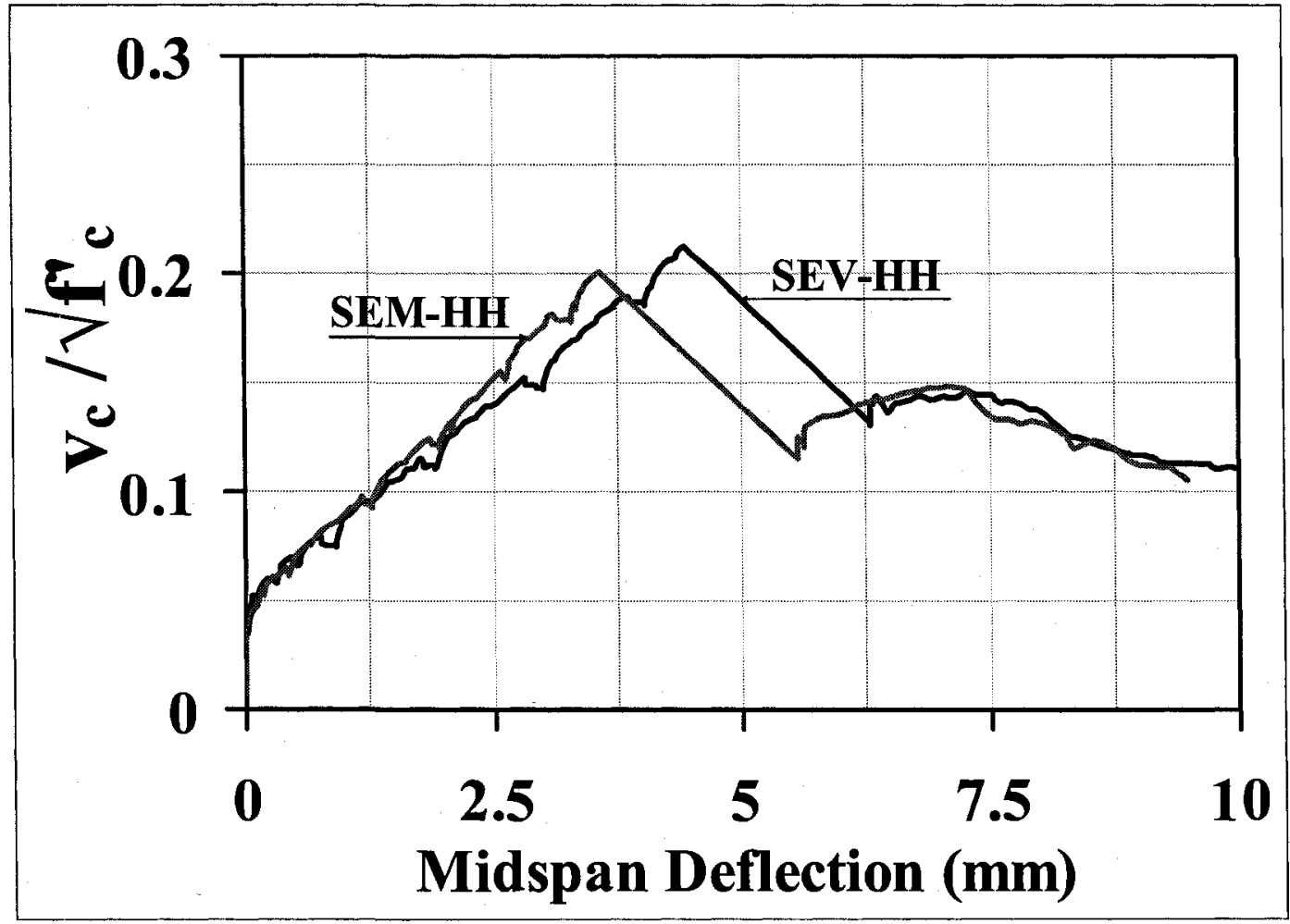

d) $\mathrm{d}=500 \mathrm{~mm}$

Cont. Figure 7.18: Effect of aggregate type on normalized shear stress -midspan deflection response of RRC beams with different depths 


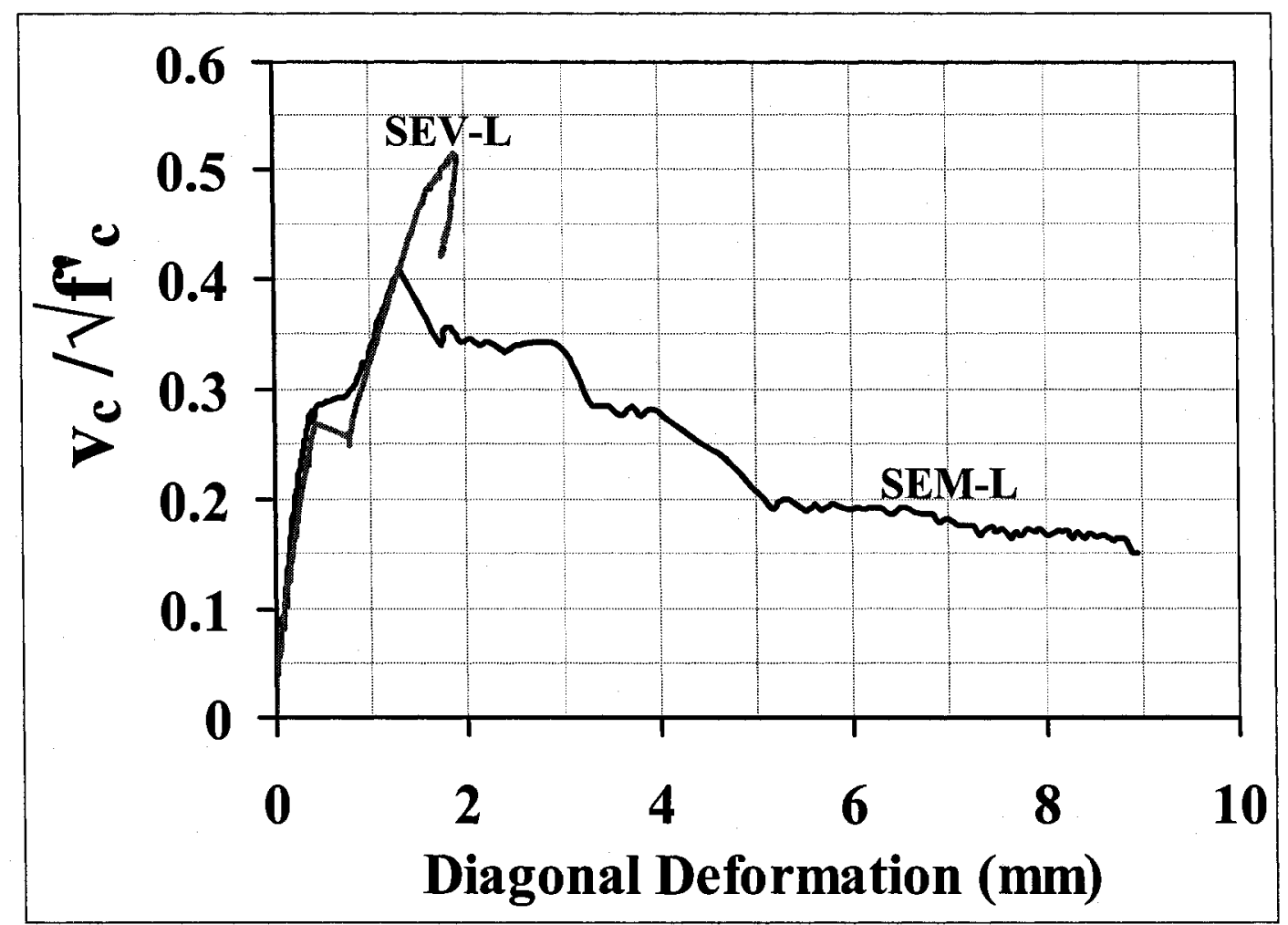

a) $\mathrm{d}=200 \mathrm{~mm}$

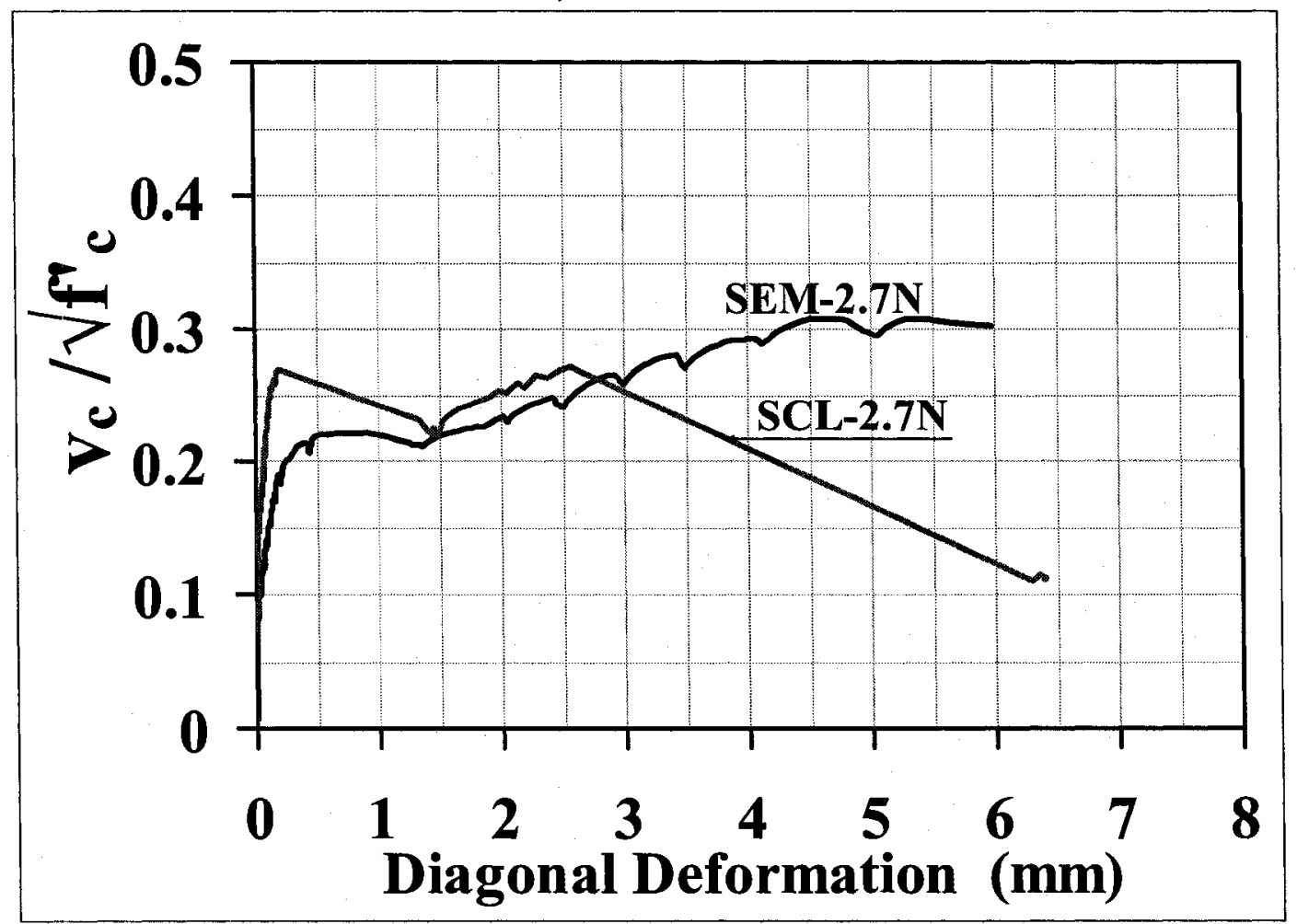

b) $\mathrm{d}=300 \mathrm{~mm}$

Figure 7.19: Effect of aggregate type on normalized shear stress -diagonal deformation response of RRC beams with different depths 


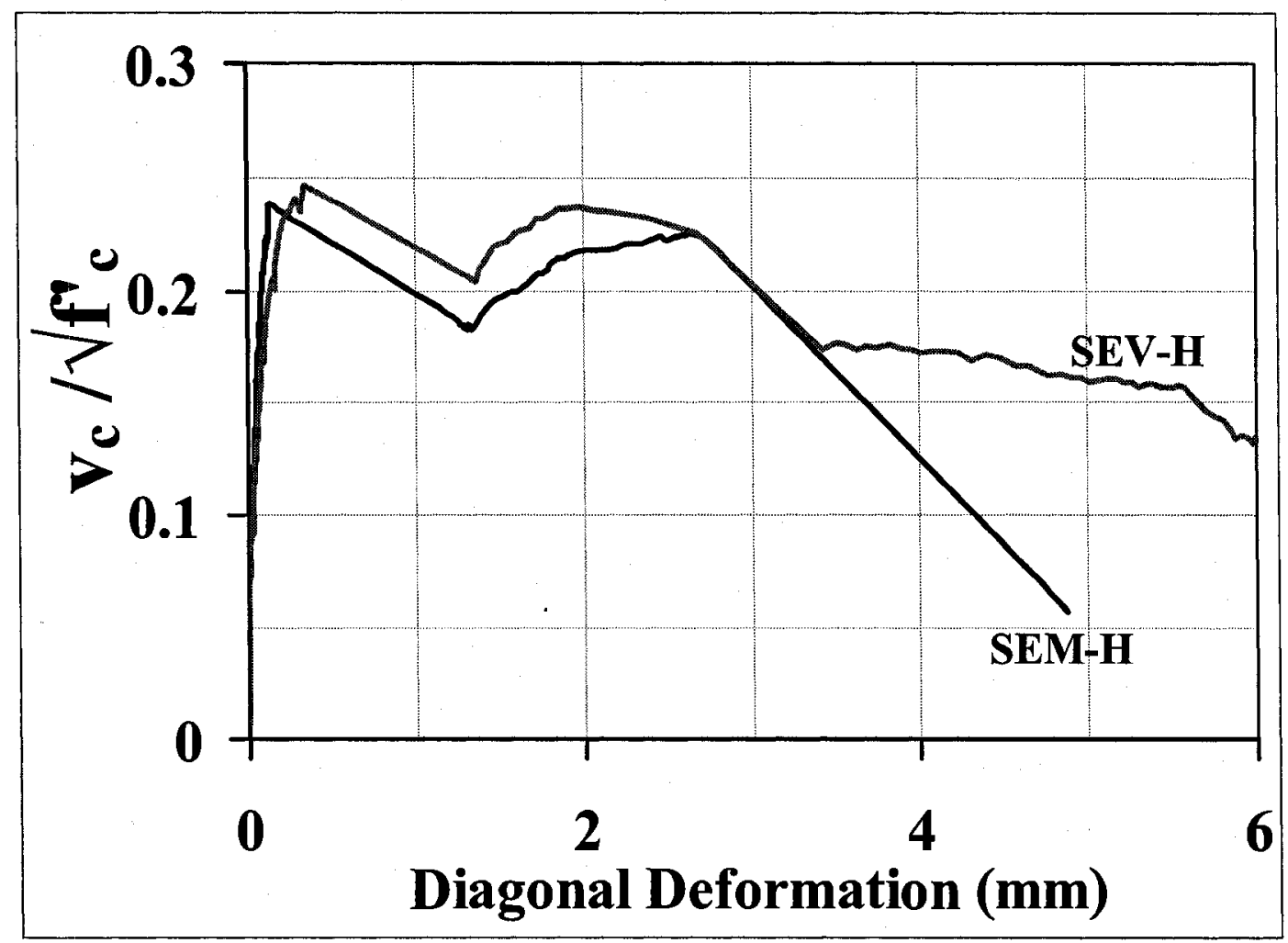

c) $\mathrm{d}=400 \mathrm{~mm}$

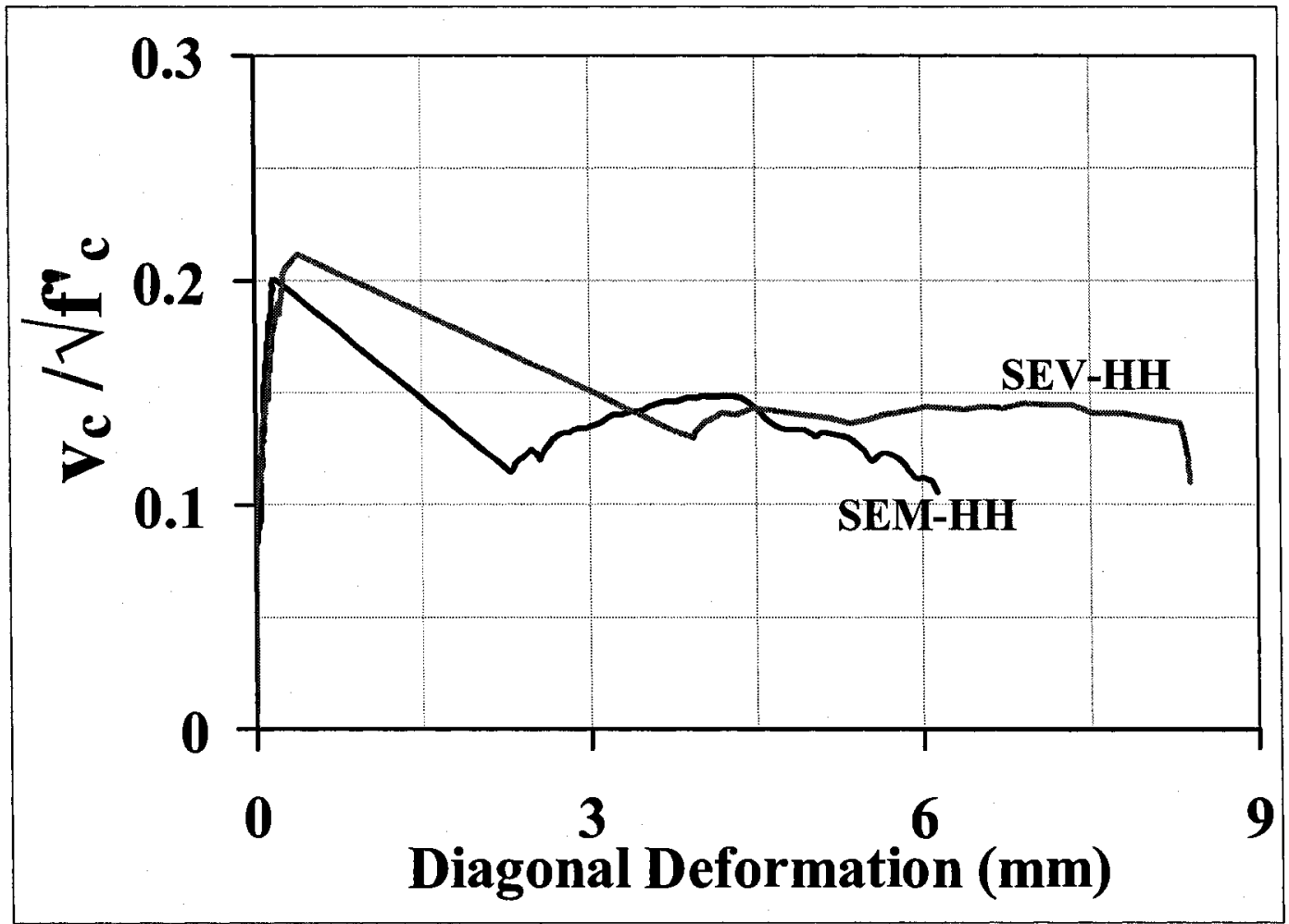

d) $\mathrm{d}=500 \mathrm{~mm}$

Cont. Figure 7.19: Effect of aggregate type on normalized shear stress -diagonal deformation response of RRC beams with different depths 


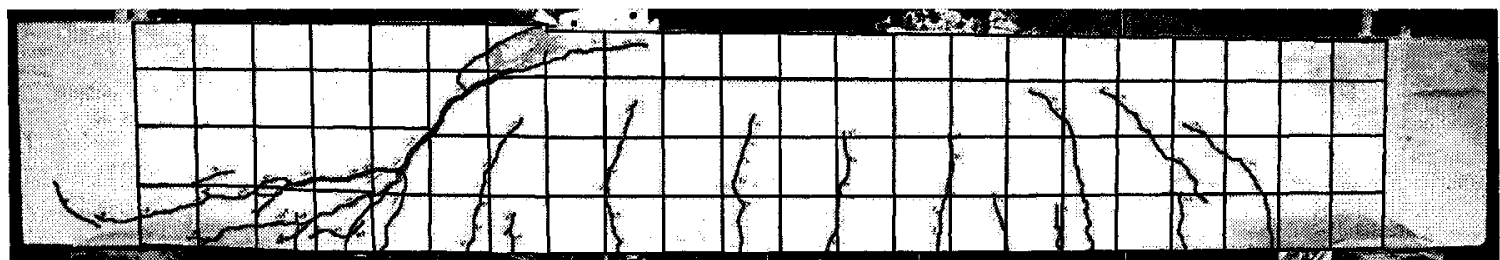

a) SEM-NS

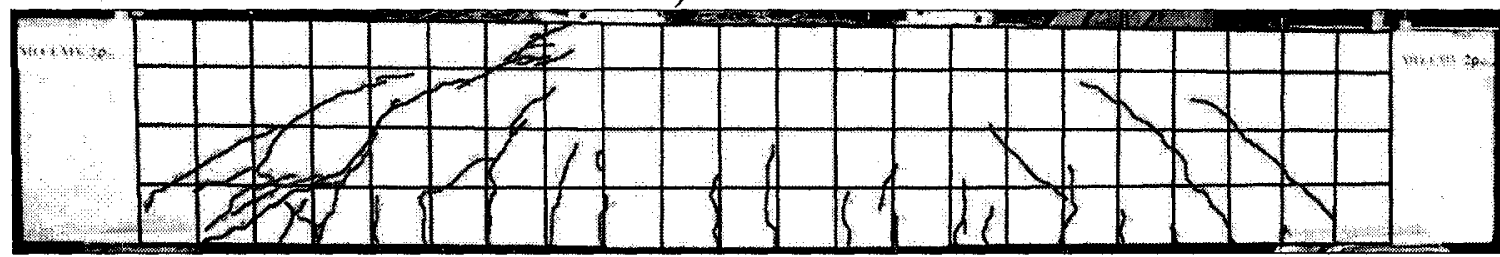

b) SEM-3S-R

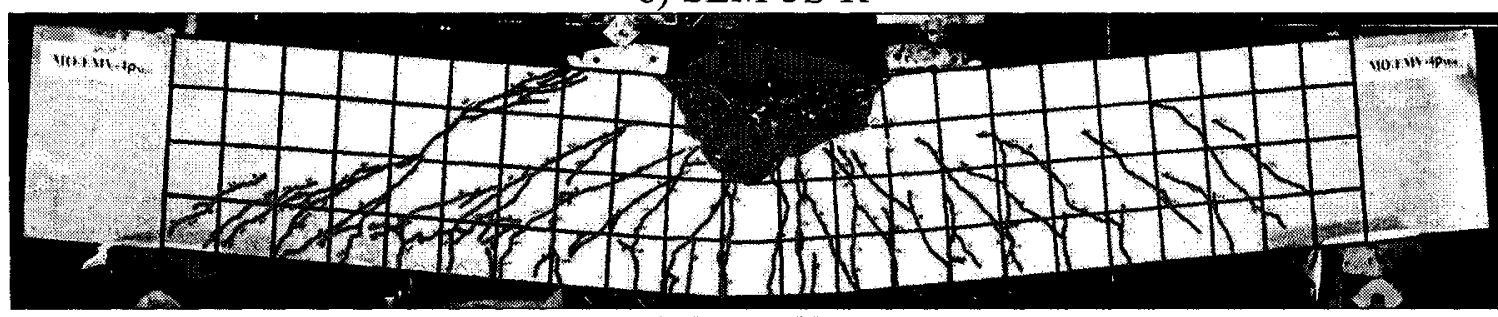

c) SEM-6S-R

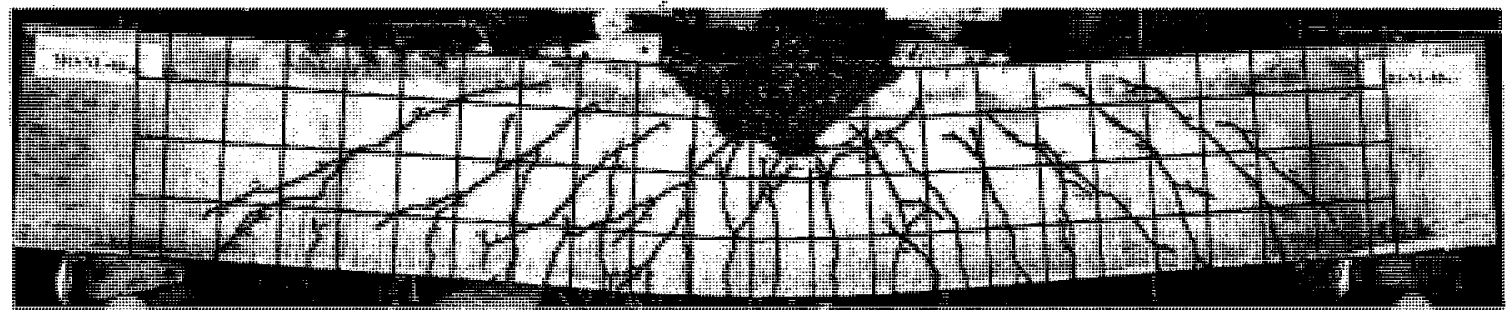

d) SCL-6S-R

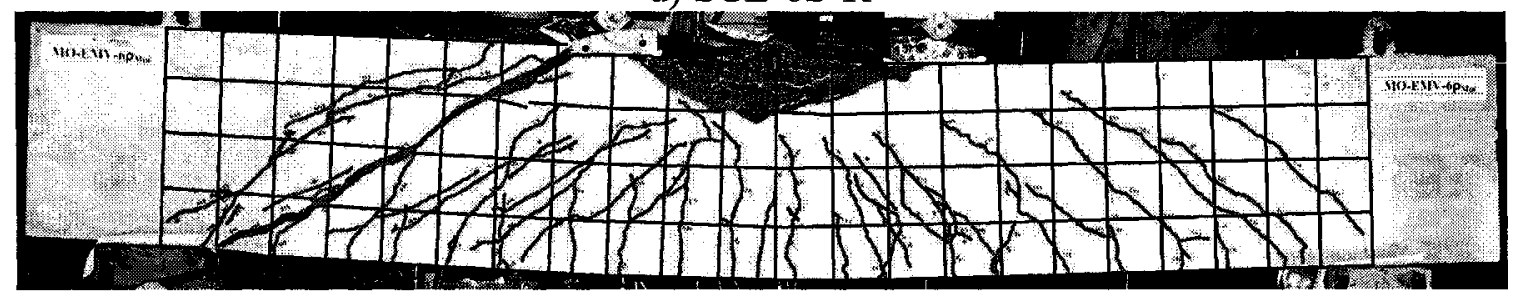

e) SEM-6S-D

Figure 7.20: Crack patterns of SEM and SCL beams with different shear reinforcement ratios 


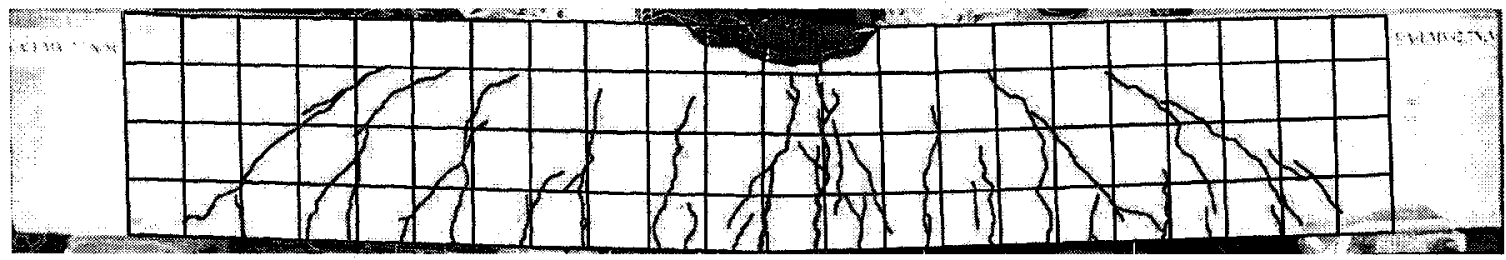

a) SEV-NS

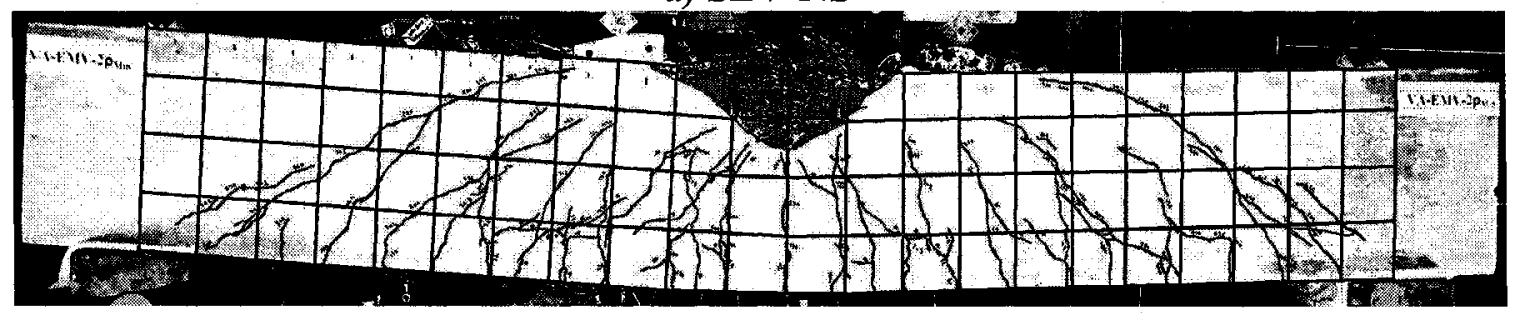

b) SEV-3S-R

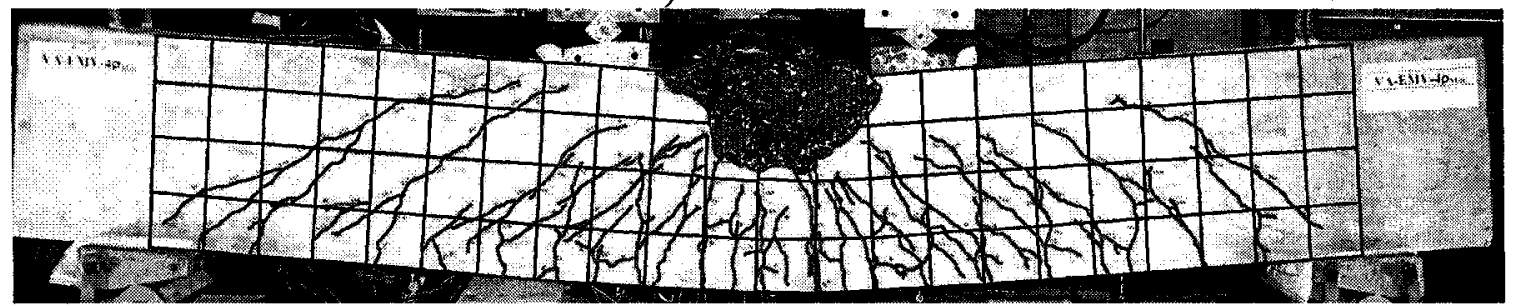
c) SEV-6S-R

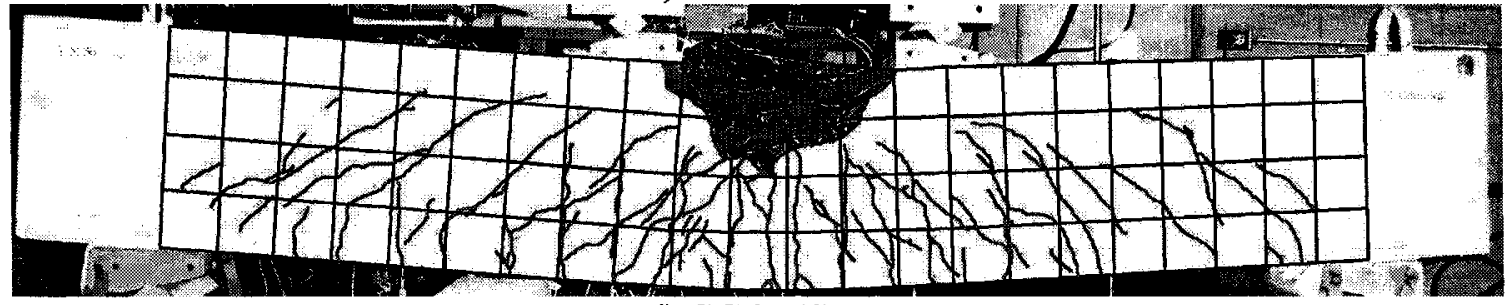

d) SCG-6S-R

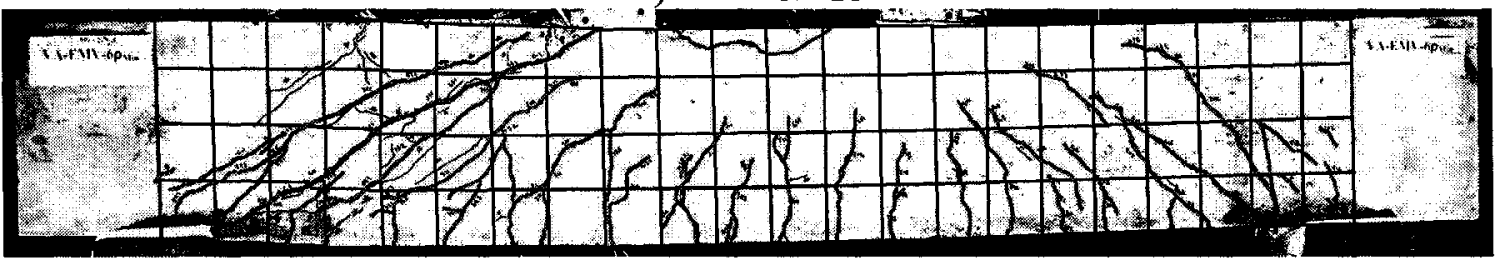

e) SEV-6S-D

Figure 7.21: Crack patterns of SEV and SCG beams with different shear reinforcement ratios 

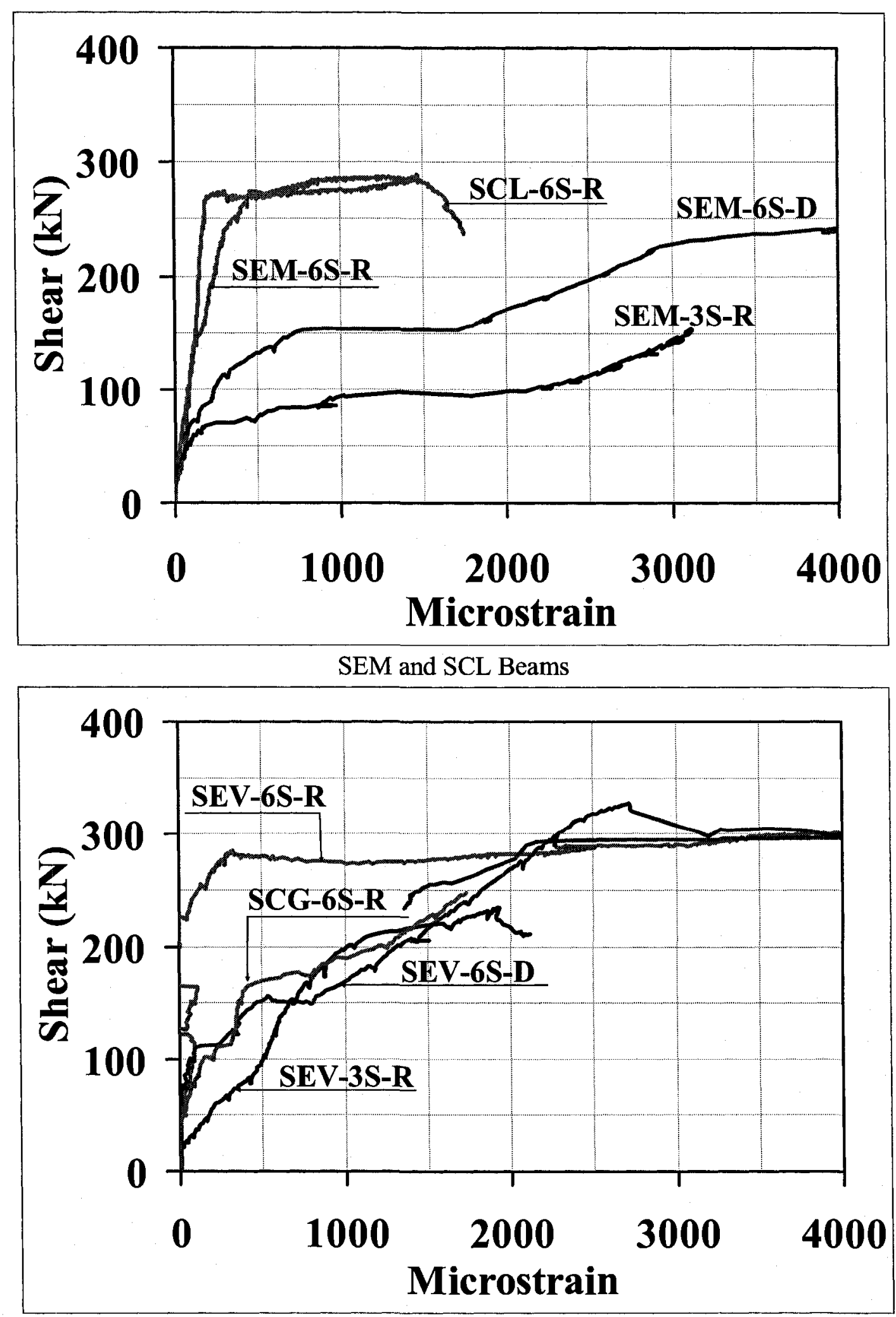

SEV and SCG Beams

Figure 7.22: The effect of shear reinforcement ratio on shear reinforcement strain in the west shear span (TS2) 


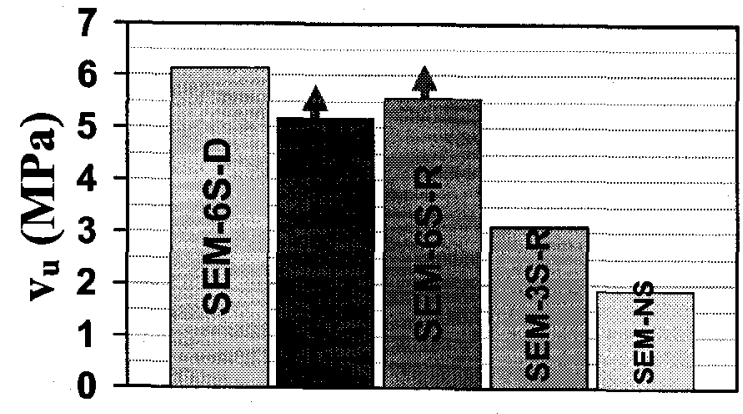

a) SEM and SCL beams

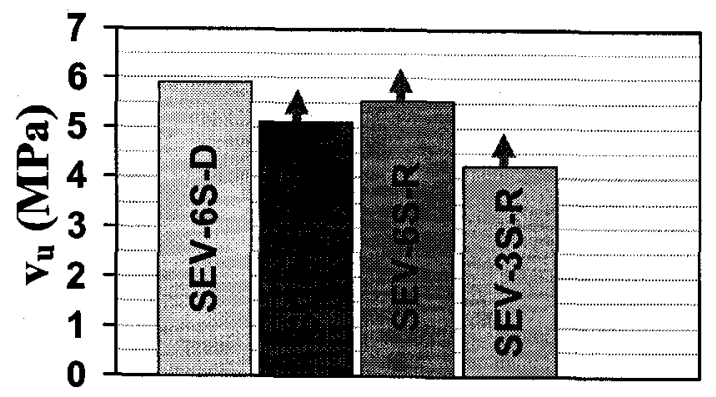

b) SEV and SCG beams

Figure 7.23: Experimental ultimate shear stress resistance values 

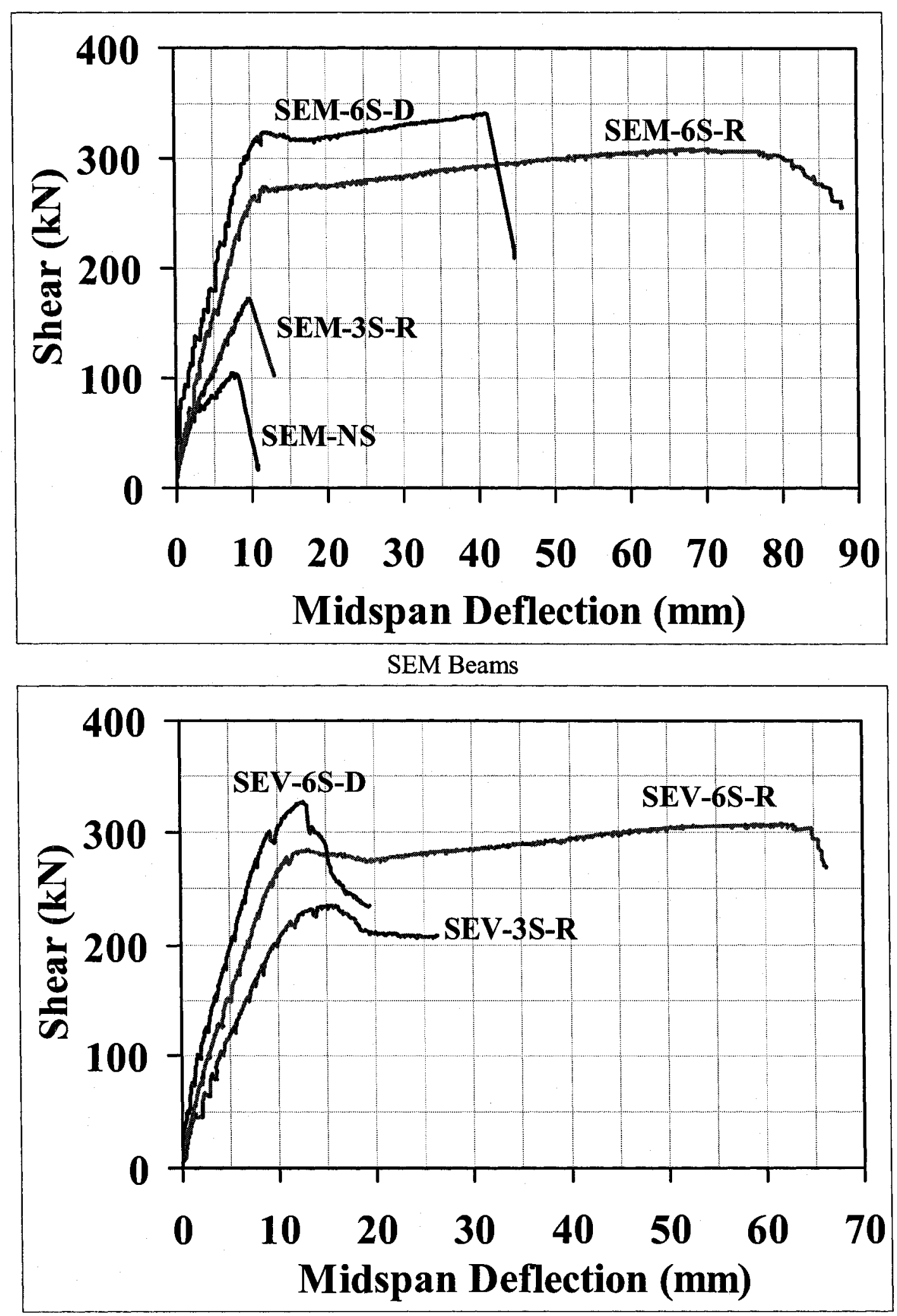

SEV Beams

a) Ultimate shear-midspan deflection response

Figure 7.24: Effect of shear reinforcement ratio on shear behaviour of RRC members 

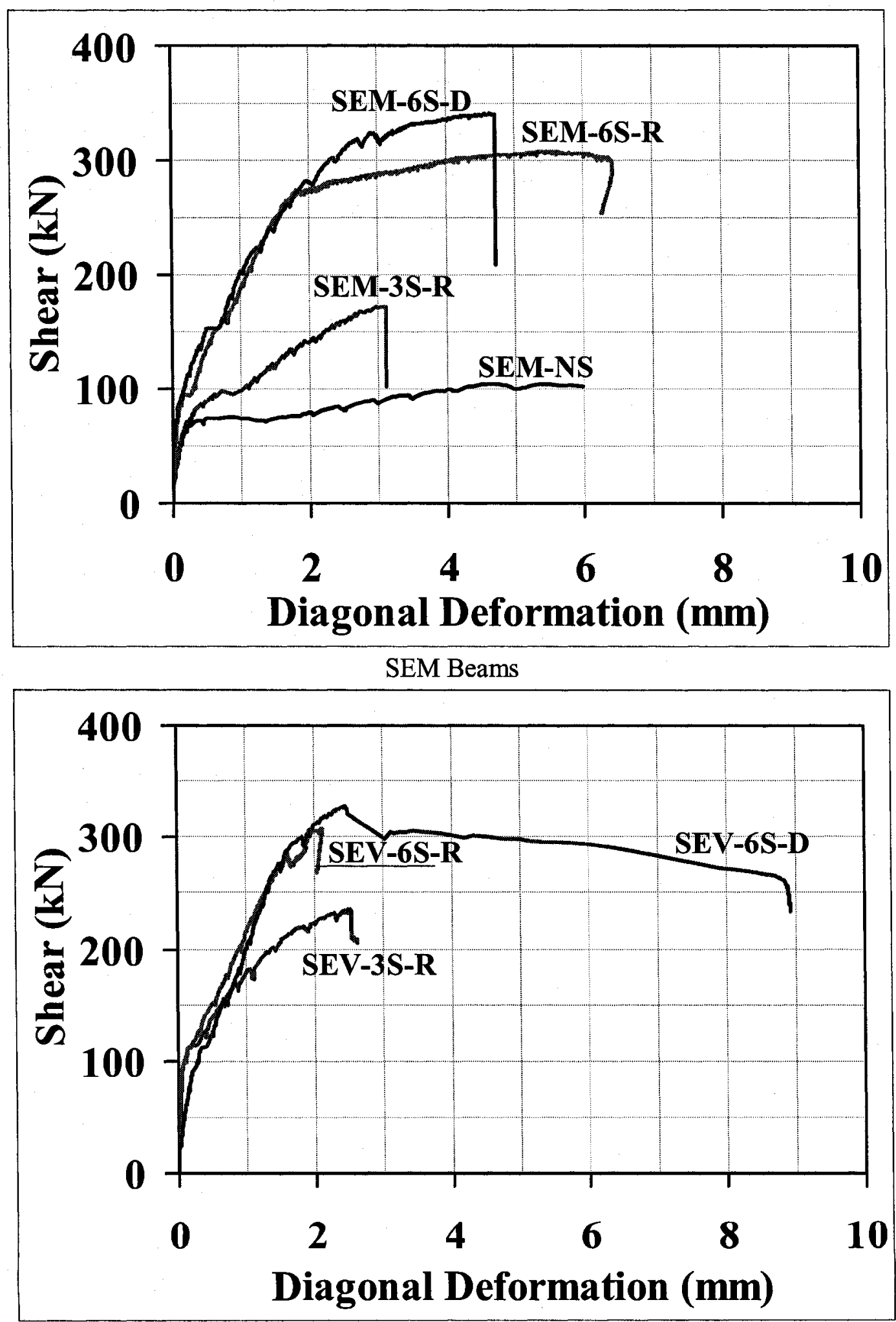

SEV Beams

b) Ultimate shear-diagonal deformation response

Cont. Figure 7.24: Effect of shear reinforcement ratio on shear behaviour of RRC members 


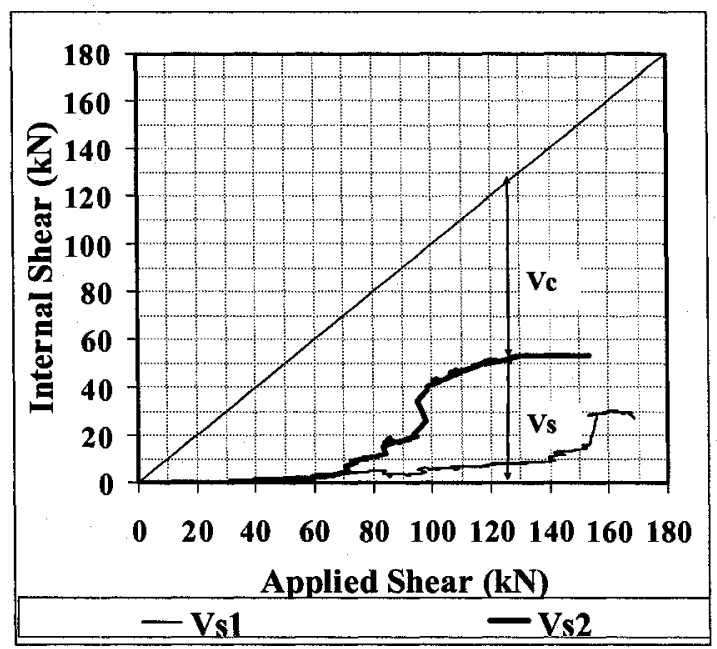

a) MO-EMV-3S-R

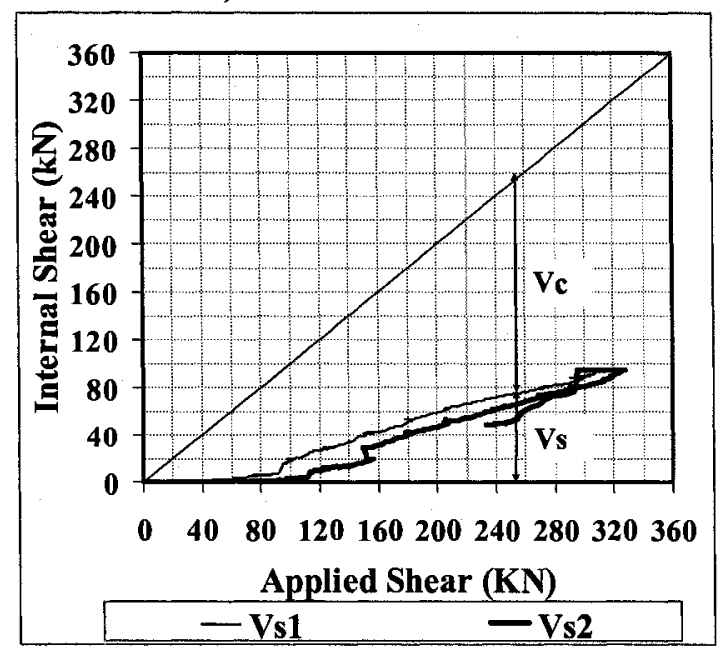

c) VA-EMV-6S-D

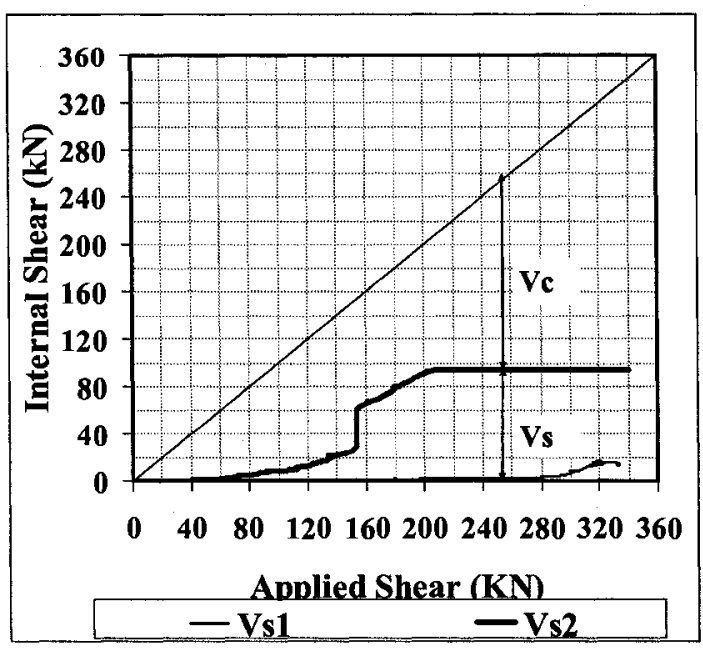

b) MO-EMV-6S-D

Figure 7.25: Variation of Shear reinforcement and concrete contribution to shear resistance along beams 


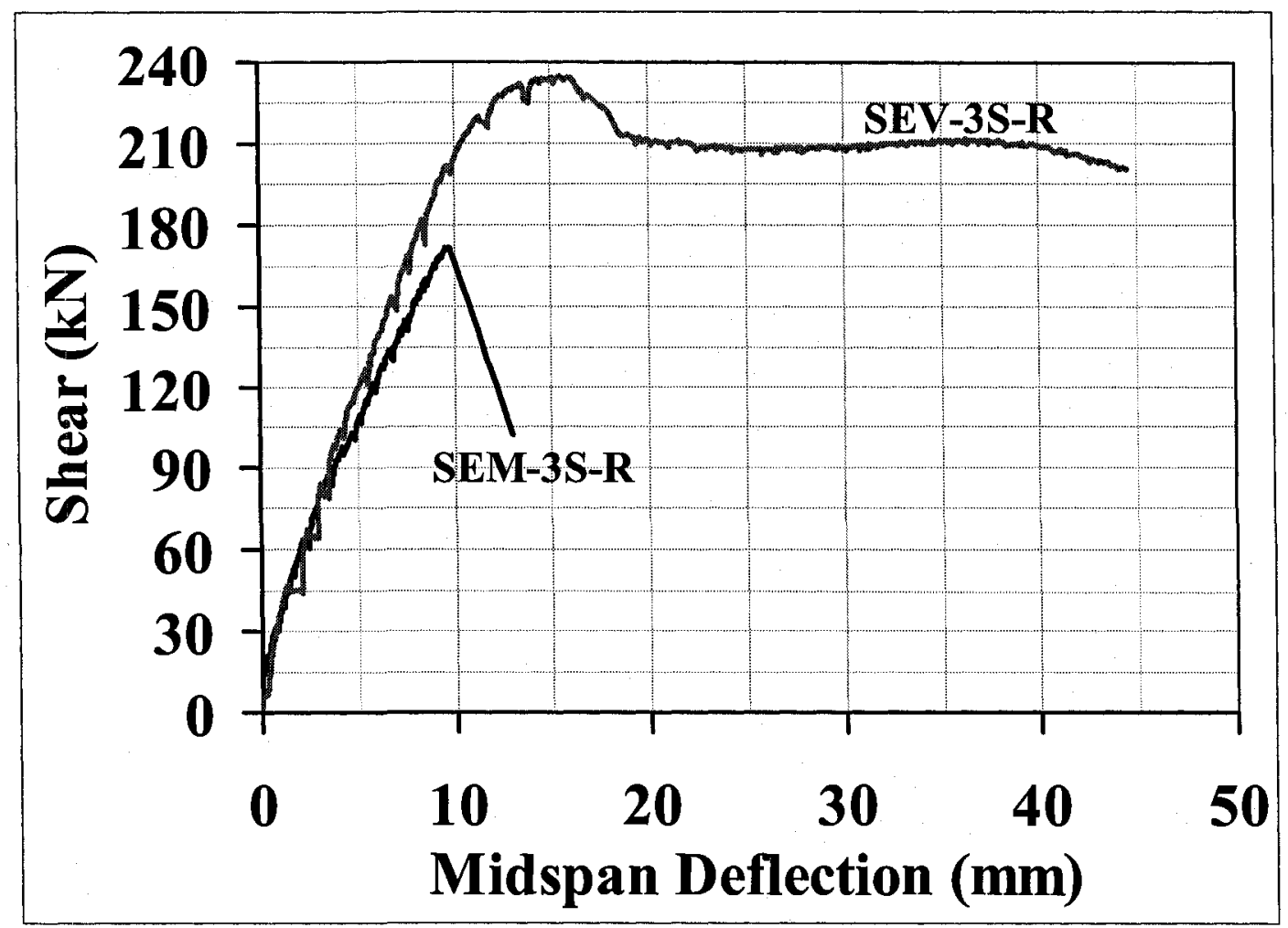

a) SEM and SEV-3S-R

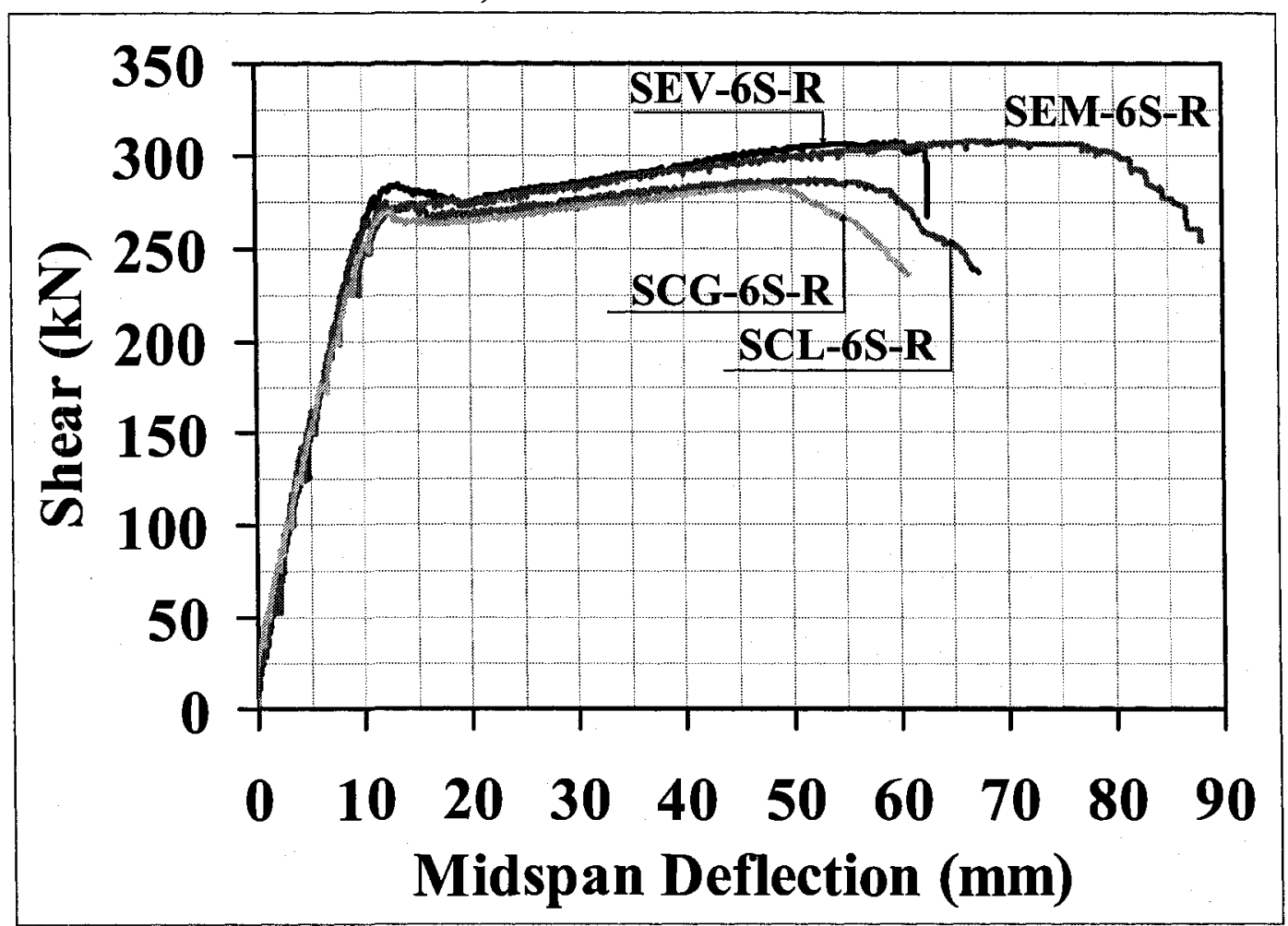

b) SEM, SEV, SCL and SCG-6S-R

Figure 7.26: Effect of aggregate type on shear-midspan deflection response of RRC beams with different shear reinforcement ratios 


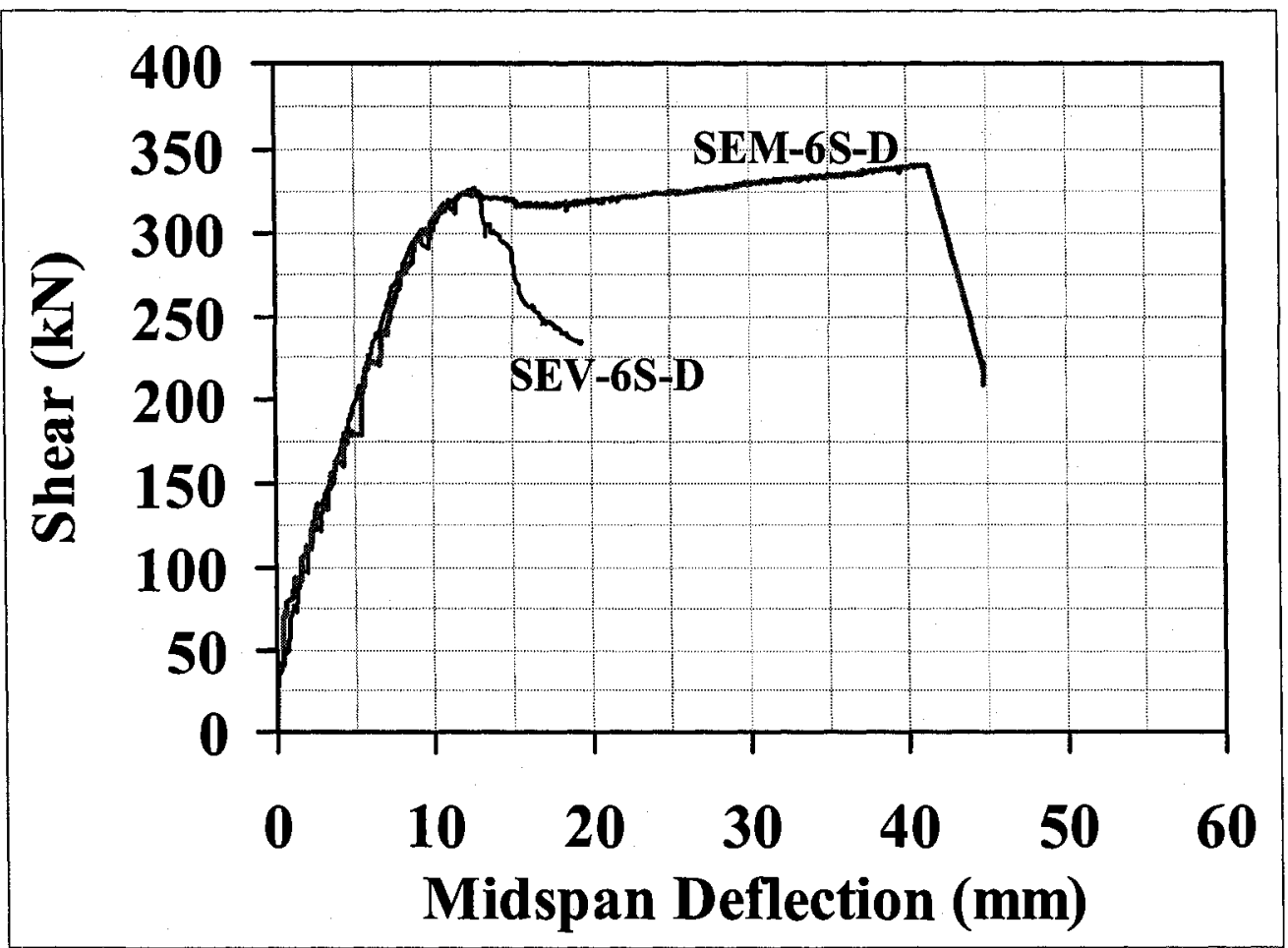

c) SEM and SEV-6S-D

Cont. Figure 7.26: Effect of aggregate type on shear-midspan deflection response of RRC beams with different shear reinforcement ratios

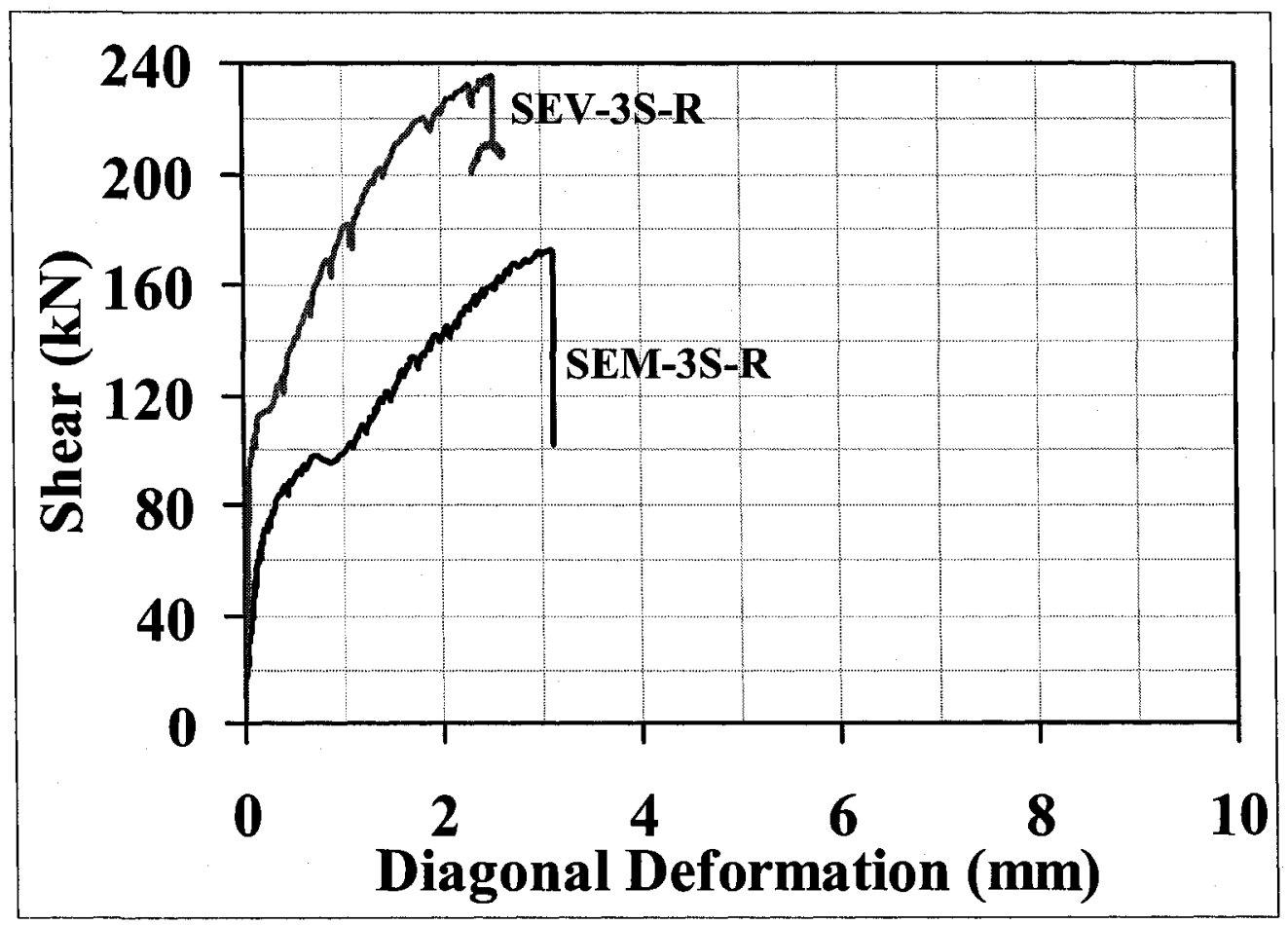

a) SEM and SEV-3S-R

Figure 7.27: Effect of aggregate type on shear-diagonal deformation response of RRC beams with different shear reinforcement ratios 


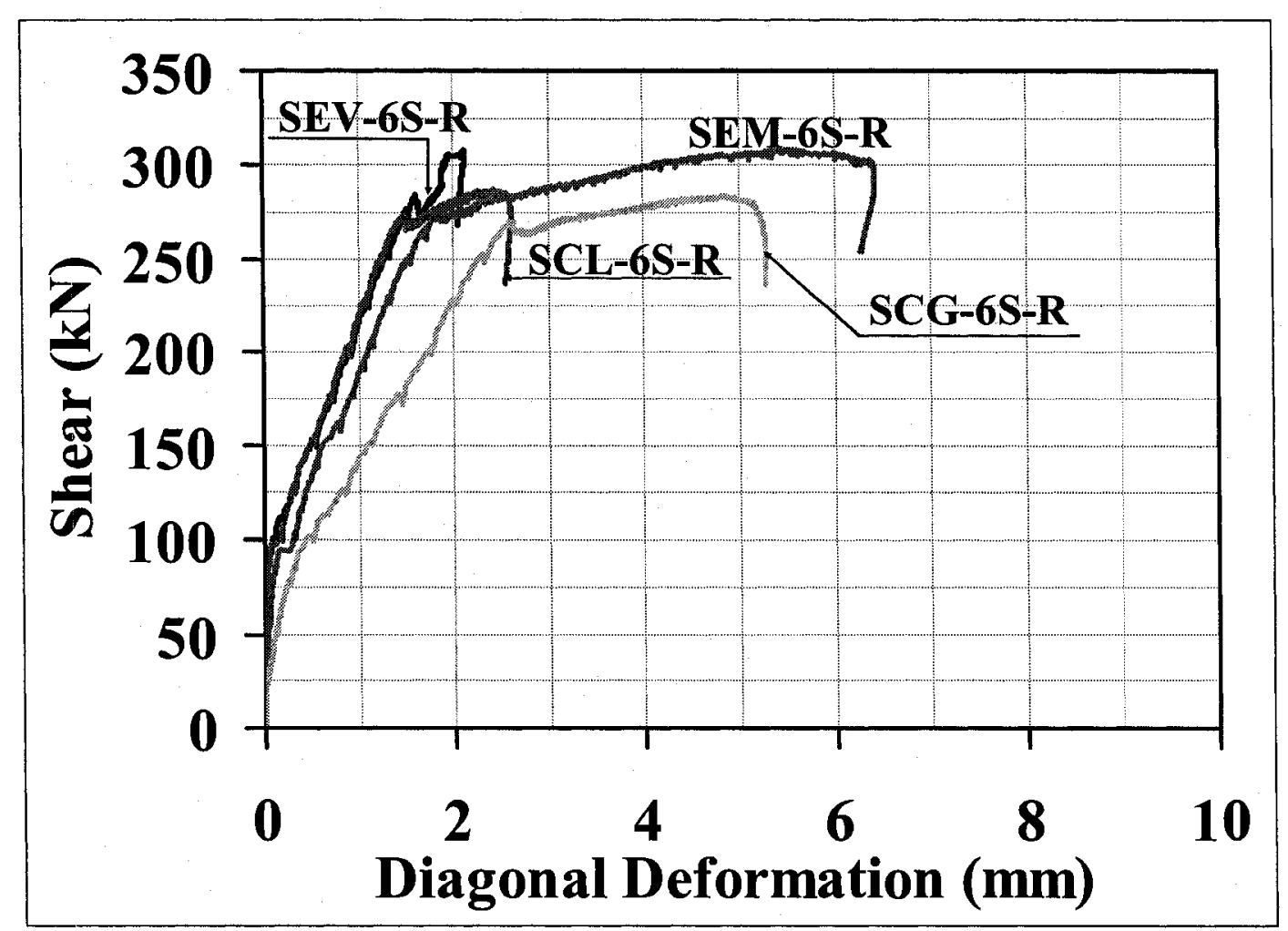

b) SEM, SEV, SCL and SCG-6S-R

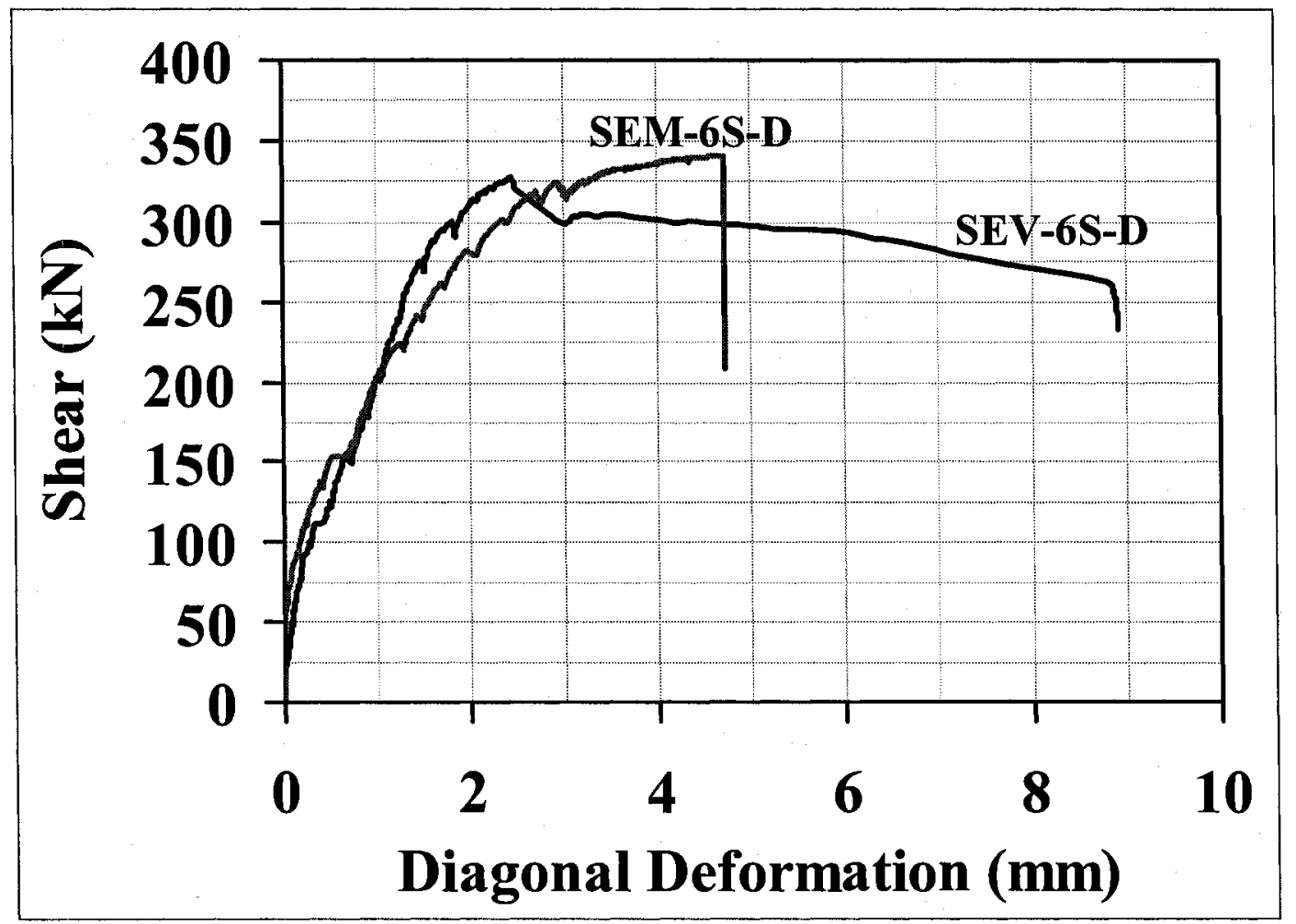

c) SEM and SEV-6S-D

Cont. Figure 7.27: Effect of aggregate type on shear-diagonal deformation response of $\mathrm{RRC}$ beams with different shear reinforcement ratios 


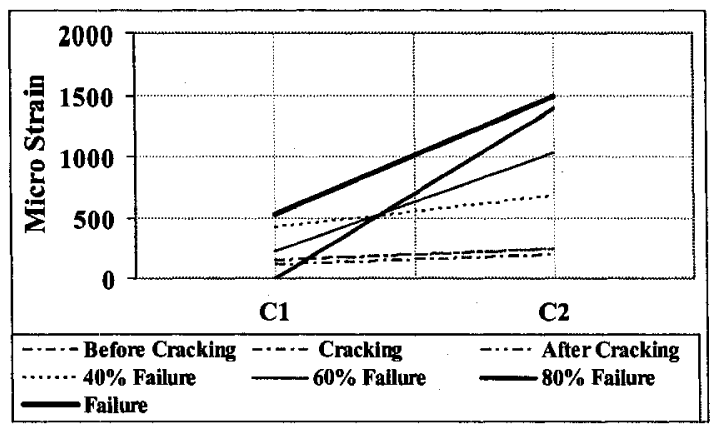

a) MO-EMV-3S-R

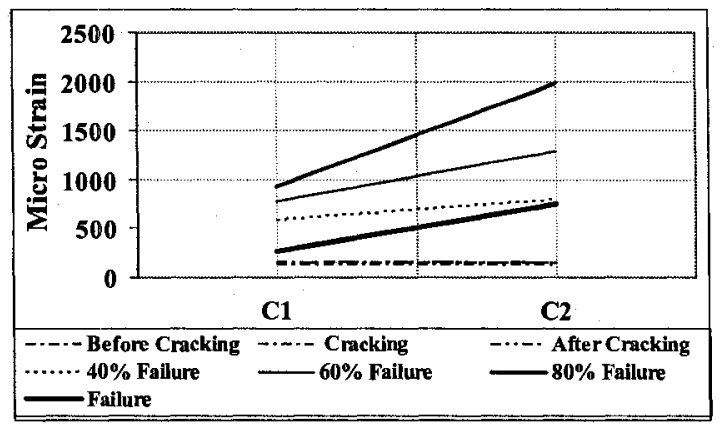

c) MO-EMV-6S-R

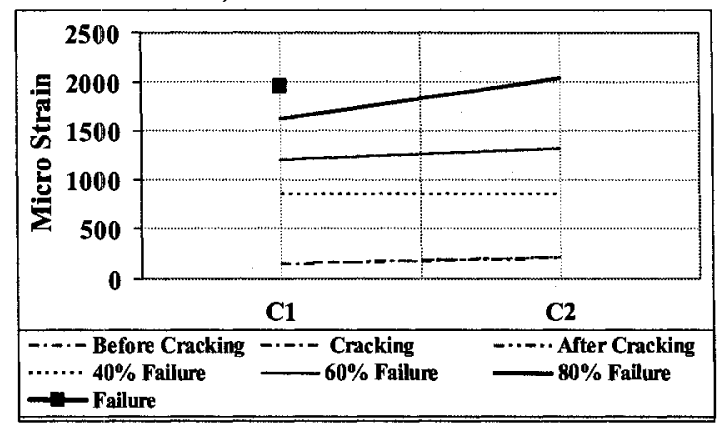

e) MO-NL-6S-R

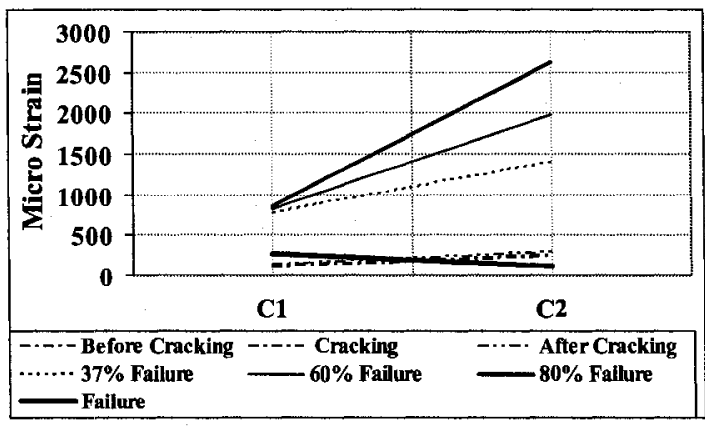

g) MO-EMV-6S-D

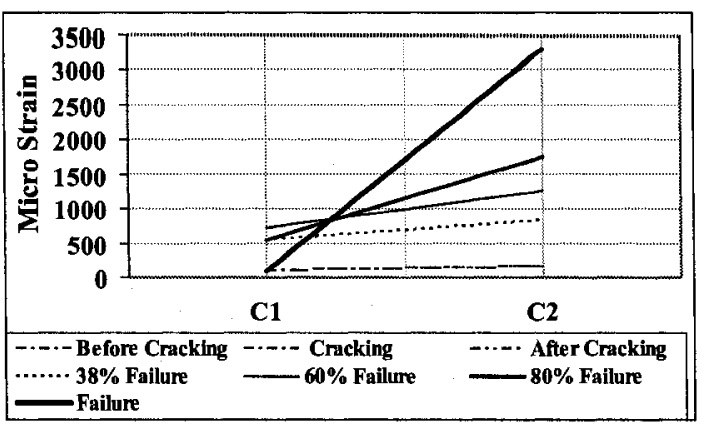

b) VA-EMV-3S-R

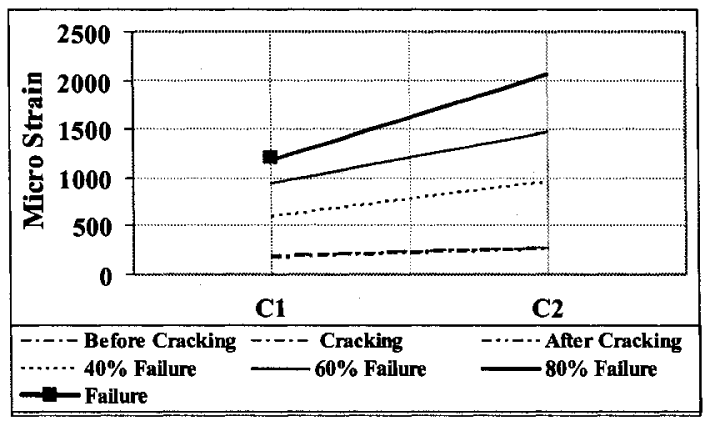

d) VA-EMV-6S-R

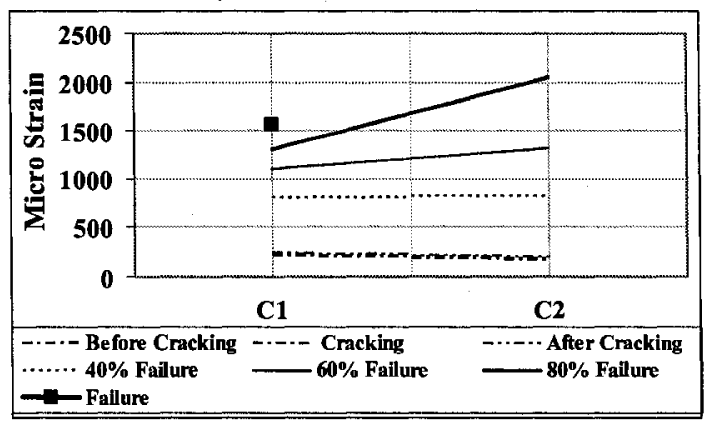

f) VA-NG-6S-R

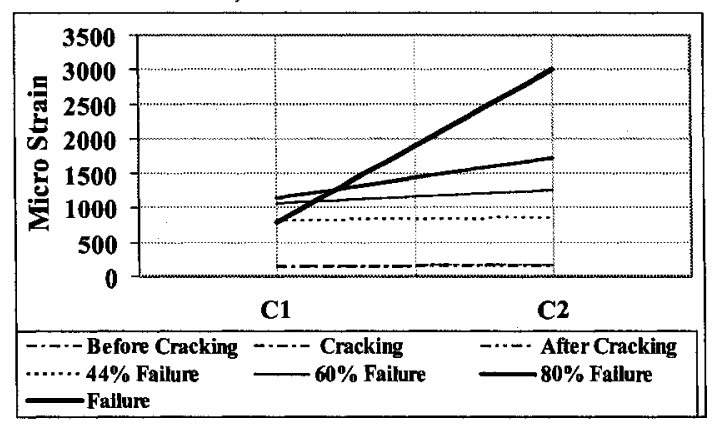

h) VA-EMV-6S-D

Figure 7.28: Variation of concrete compression strain along beam with different shear reinforcement ratios 


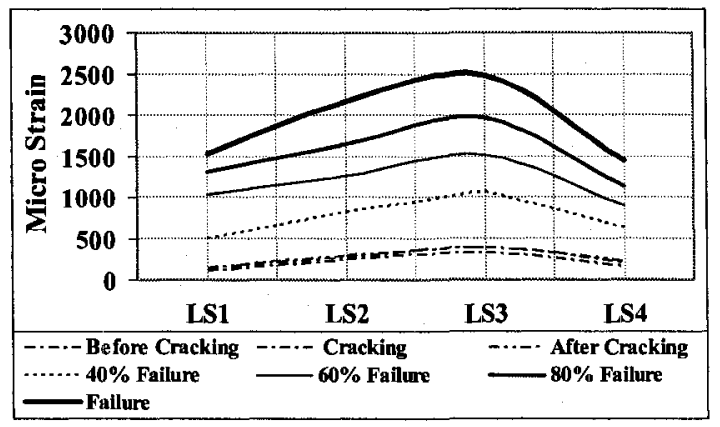

a) MO-EMV-3S-R

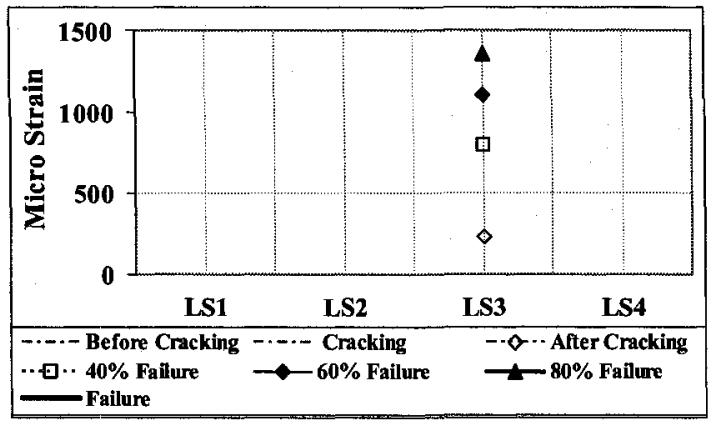

c) MO-EMV-6S-R

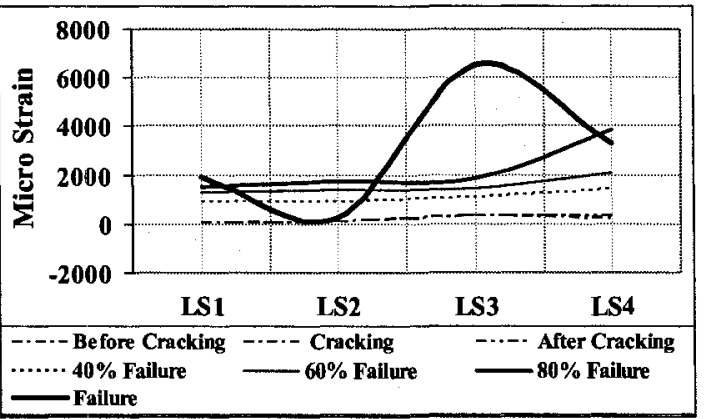

e) MO-NL-6S-R

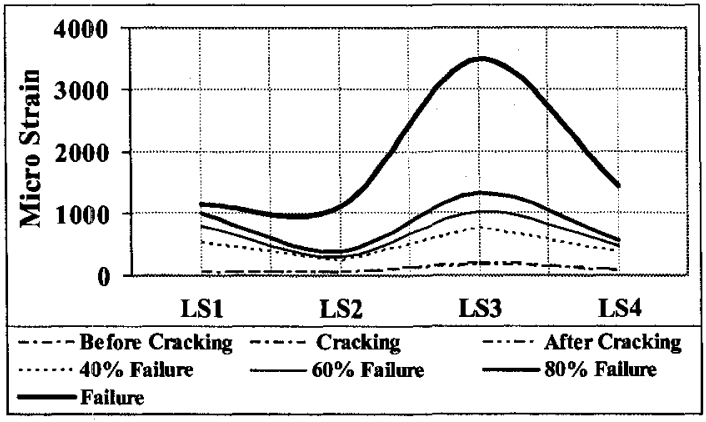

g) MO-EMV-6S-D

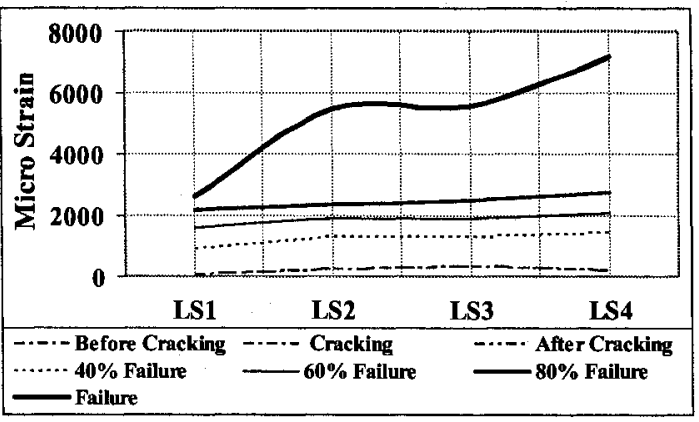

b) VA-EMV-3S-R

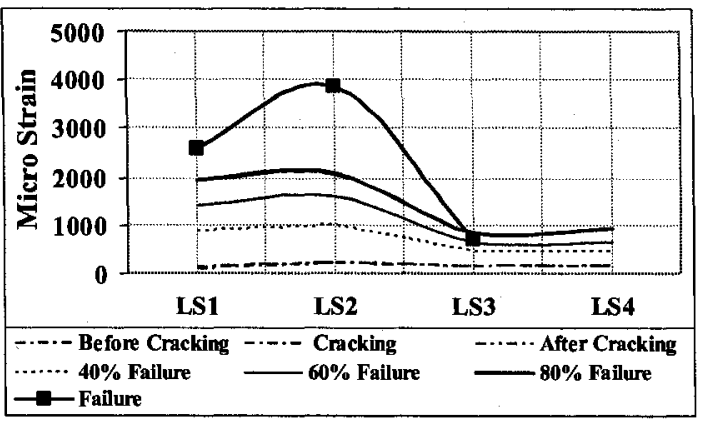

d) VA-EMV-6S-R

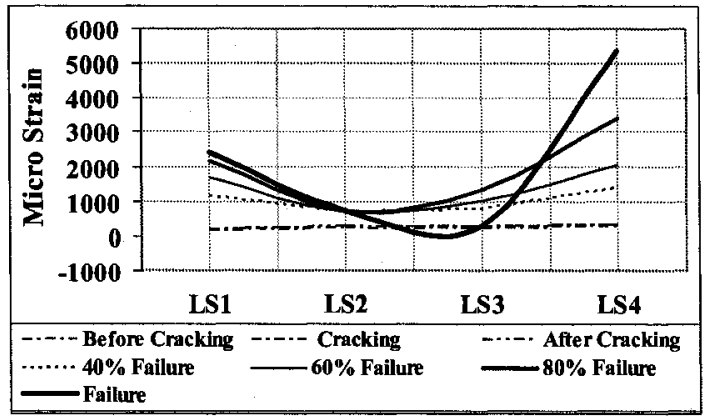

f) VA-NG-6S-R

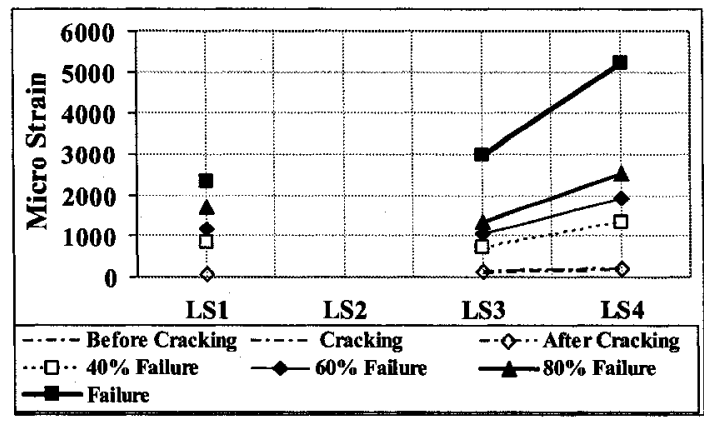

h) VA-EMV-6S-D

Figure 7.29: Variation of longitudinal steel strain along beam with different shear reinforcement ratios 


\section{CHAPTER 8: ANALYSIS OF THE EXPERIMENTAL}

\section{DATA: ULTIMATE FLEXURAL AND SHEAR} STRENGTHS OF RRC BEAMS

\subsection{General}

According to existing literature presented in the state of the art in Chapter 2, the applicability of the existing design methods for calculating the ultimate flexural strength of conventional RC members to RRC beams have been generally demonstrated; however, several issues have been raised with regard to the applicability of the existing empirical equations for calculating the concrete contribution to the shear resistance of $\mathrm{RC}$ members $\left(v_{c}\right)$ to RRC beams, especially at higher $a / d$ ratios. This has been mainly explained in terms of the less effective aggregate interlock mechanism in RRC compared to RC beams. The writer believes that the reason for the latter is mainly due to the application of conventional mix proportioning method in previous investigations. Since the proportioning of the RAC by the proposed EMV method resulted in both higher ultimate flexural and shear strengths in all RRC beams compared to their RC counterparts (control beams), and since the main difference between previous studies and the current investigation of RRC beams behaviour is the RAC mix design method, it is clear that it is not the presence of RAC that results in lower shear strength. Hence, the current design may be fully appreciable to RRC beams, be it shear or flexural design. This issue will be discussed in detail in this chapter through comparison of the current experimental results with prediction of existing theoretical/empirical methods. 
The major objectives of this chapter are as follows:

1. Evaluating the applicability of the CSA Standard A23.3-04 for calculating the cracking moment of RC beams to RRC beams: Towards this, firstly clauses 8.6 .4 is used to calculate the modulus of rupture $\left(f_{r}\right)$ for predicting the cracking moment $\left(M_{c r}\right)$ of the beams. Secondly, the experimental splitting tensile strength $\left(f_{s t}\right)$ is used instead of modulus of rupture for calculating $M_{c r}$.

2. Evaluating the applicability of the recommended empirical equations by CSA A23.304 (clause 10.1.7) for calculating the equivalent rectangular stress block distribution parameters $\left(\alpha_{1}\right.$ and $\left.\beta_{1}\right)$ of RC beams to RRC beams. In this section, the $\alpha_{1}$ and $\beta_{1}$ values are calculated based on CSA A23.3-04 as well as based on the some available theoretical compressive stress-strain relationship of concrete as described in Chapter 2 and Chapter 5. The results are compared with the $\alpha_{1}$ and $\beta_{1}$ values calculated by using the actual stress-strain curves obtained in the current investigation.

3. Evaluating the applicability of the prevailing flexure analysis method for predicting the flexural strength of RC beams to RRC beams by (1) using the $\alpha_{1}$ and $\beta_{1}$ values based on CSA Standard A23.3-04, the actual concrete stress-strain relationships and theoretical stress-strain relations, and (2) using the actual stress-strain properties of steel and concrete and applying first principles based on satisfying the strain compatibility and equilibrium conditions.

4. Predicting the yielding moment of RRC beams by using the actual stress-strain properties of steel and concrete and satisfying the strain compatibility and equilibrium conditions. 
5. Evaluating the applicability of the recommended empirical equations of CSA Standard A23.3-04 (clause 9.8.2.3) for calculating the immediate deflections of RC beams to RRC beams. The immediate deflections of RRC beams at $40 \%$ of their corresponding failure load $\left(\delta_{s}\right)$ are predicted by (1) using the elastic deflection equations and the effective moment of inertia calculated based on clause 9.8.2.3 of CSA Standard A23.3-04, and (2) using the moment-curvature response of the beams obtained from full stress-strain response of RRC beams in step 4, and the results are compared with the corresponding experimental data.

6. Evaluating the applicability of the recommended empirical equations of CSA Standard A23.3-04 (clause 11.3.6.3 and 11.3.6.4) for calculating the concrete contribution to the shear strength $\left(v_{c}\right)$ of RC beams to the RRC beams without shear reinforcement. In addition, CSA A23.3-04 method, the ACI-318 code methods and the empirical equations described in Chapter 2 (Table 2.14) will also be used to calculate $v_{c}$ and the results will be compared.

7. Evaluating the applicability of the recommended empirical equations of CSA Standard A23.3-04 (clause 11.3.6.3 and 11.3.6.4) and ACI-318 (Eq.11.3 and 11.5) for calculating the ultimate shear strength $\left(v_{u}\right)$ of $\mathrm{RC}$ beams to RRC beams by comparing the predicted results with the experimental data..

\subsection{Cracking Moment $\left(M_{c r}\right)$}

Table 8.1a presents the observed and predicted cracking moments ( $M_{c r}^{\text {obsvd. }}$ and $\left.M_{c r}^{\text {pred. }}\right)$ of the RRC beams made of RCA-MO and/or natural limestone (NL). The cracking moment $\left(M_{c r}\right)$ was predicted based on: (1) the modulus of rupture $\left(f_{r}\right)$ using clauses 8.6.4 of 
CSA A23.3-04, and (2) the observed splitting tensile strength $\left(f_{s t}\right)$. The observed cracking moment was calculated using the observed cracking load based on visual inspections during the test rather than solely the load-midspan deflection curves. Generally, the $M_{c r}^{\text {obsvd. }} / M_{c r}^{\text {pred. }}$ values based on clause 8.6.4 of CSA A23.3-04 ( $f_{r}$-based) range from 0.84 to 1.60 with an average of 1.18 and standard deviation of 0.21 , while they range from 1.10 to 2.21 with an average of 1.53 and standard deviation of 0.28 using the observed splitting tensile strength values $\left(f_{s t}\right.$-based).

According to range of $M_{c r}^{\text {obsvd. }} / M_{c r}^{\text {pred. }}$ values, it is observed that the predicted cracking moments using clause 8.6.4 of CSA A23.3-04 ( $f_{r}$-based) are conservative for all the SEM and SCL beams except FEM-Min, FEM-Av and FEM-2.7L beams with $\frac{M_{c r}^{\text {obsid. }}}{M_{c r}^{\text {pred. }}}$ values of $0.88,0.84$ and 0.97 , respectively. By using $f_{s t}$ values instead of $f_{r}$, the $M_{c r}^{\text {pred. }}$ values are conservative for all the SEM beams. This is more evident in Figure 8.1a in which one can see that all of $M_{c r}^{\text {pred. }}$ values using the observed splitting tensile strength values are less than the corresponding $M_{c r}^{\text {obsvd. }}$ values.

Table $8.1 \mathrm{~b}$ presents the observed and predicted cracking moments ( $M_{c r}^{\text {obsvd. }}$ and $M_{c r}^{\text {pred. }}$ ) for RRC beams made of RCA-VA and/or NG. The range of $M_{c r}^{\text {obsvd. }} / M_{c r}^{\text {pred. }}$ values based on $f_{r}$ values according to CSA A23.3-04 (clause 8.6.4) is from 0.71 to 1.48 with an average of 1.12 and standard deviation of 0.22 , while the same varies from 0.84 to 1.74 with an average of 1.30 and standard deviation of 0.26 by using the observed $f_{s t}$ values.

According to the preceding results, it is observed that the predicted cracking moments based on the modulus of rupture $\left(f_{r}\right.$-based) are conservative for all the SEV beams 
except beams FEV-Av, FEV-Max, and FEV-2.7N, for which the $M_{c r}^{\text {obsvd. }} / M_{c r}^{\text {pred. }}$ values are $0.81,0.71$, and 0.93 , respectively. By using the $f_{s t}$ values instead of $f_{r}$, the $M_{c r}^{\text {pred. }}$ values noticeably improved and the corresponding $M_{c r}^{\text {pred. }}$ values were still found to be conservative for all the SEV beams except FEV-Av, and FEV-Max with $\frac{M_{c r}^{\text {obsvd. }}}{M_{c r}^{\text {pred. }}}$ values of 0.96 and 0.84 , respectively. Note that the unconservatism of CSA A23.3-04 (clause 8.6.4) is not necessarily due to the presence of RCA because it also overestimates the beam SCG-2.7N which is made of conventional concrete $\left(M_{c r}^{o b s v d .} / M_{c r}^{\text {pred. }}\right.$ value of 0.81 ). This is likely due to the effect of aggregate angularity on the tensile strength of concrete which is not considered in clause 8.6.4 of CSA A23.3-04. In other words, less mechanical bonding strength between the round OVA and RM in RCA-VA compared to the bonding strength between crushed OVA and RM in RCA-MO may have resulted in lower tensile strength and corresponding lower cracking moment in SEV and SCG beams compared to SEM and SCL beams. This effect is more evident in Figure $8.1 \mathrm{~b}$ which illustrates the predicted versus observed cracking moments for beams SEV and SCG based on splitting tensile strength and modulus of rupture values of the concrete in each group of beams.

It should be pointed out that the CSA Standard A23.3-04 method for predicting the cracking moment of a beam is known to be accurate within $\pm 20 \%$ for normal concrete (Ramsey et al., 1979). The present results for RRC beams fall within that range; therefore, one could apply the existing CSA recommendations for deflection calculation to RRC beams. 


\subsection{Equivalent Rectangular Stress Block parameters $\left(\alpha_{1}\right.$ and $\left.\beta_{I}\right)$}

The CSA Standard A23.3-04 allows the use of equivalent uniform concrete stress distribution instead of the actual nonlinear stress distribution (clause 10.1.7) in the compression zone of a flexural member to calculate its ultimate flexural strength. The equivalent uniform or rectangular stress block width and height parameters are designated as $\alpha_{1}$ and $\beta_{l}$. These parameters are a function of the compressive stress-strain relationship of concrete.

According to the results in Chapter 4, there was no major difference between the stressstrain relationship of RAC (proportioned by the proposed EMV method) and the companion NAC, but the strain corresponding to the peak stress in the RAC was found to be slightly higher compared to that in NAC. Therefore, it is essential to examine the applicability of CSA A23.3-04 (clause 10.1.7) for calculating the $\alpha_{1}$ and $\beta_{1}$ parameters of conventional concrete to RAC.

In this section, the equivalent rectangular stress block parameters, called $\alpha$ and $\beta$ are derived based on the actual stress-strain curves (Chapter 4) as well as empirical stressstrain curves proposed as described in Chapter 5. Since the CSA A23.3-04 assumes a maximum strain of 0.0035 at the extreme concrete compression fibre at failure, the latter equivalent stress block parameters will be derived by terminating the descending branch of the pertinent stress-strain curve up to a maximum strain of 0.0035 . The equivalent $\alpha$ and $\beta$ values were calculated as follows (Park and Paulay, 1975):

$$
\begin{aligned}
& \alpha=\frac{1}{f_{c}^{\prime} \cdot \varepsilon} \int_{0}^{\varepsilon} \sigma \cdot d \varepsilon \\
& \beta=1-\frac{\int_{0}^{\varepsilon} \sigma \cdot \varepsilon \cdot d \varepsilon}{\int_{0}^{\varepsilon} \sigma \cdot d \varepsilon}
\end{aligned}
$$


Note that the $\alpha$ and $\beta$ values can be calculated at any loading stage up to failure $\left(0 \leq \varepsilon \leq \varepsilon_{c u}\right)$. However, in order to compare these stress block parameters with $\alpha_{1}$ and $\beta_{1}$ parameters in CSA A23.3-04, the maximum strain is limited to 0.0035. Based on equivalent rectangular stress block parameter concept, the compressions force $(C)$ acting on the section at ultimate state and the corresponding ultimate flexural resistance $\left(M_{r}\right)$ can be simply calculated as:

$$
\begin{aligned}
C & =\alpha \cdot f_{c}^{\prime} \cdot b \cdot c \\
M_{r} & =C \cdot(d-\beta \cdot c)
\end{aligned}
$$

where $c$ is the depth of the neutral axis.

In the CSA Standard A23.3-04, $C$ and $M_{r}$ are calculated as

$$
\begin{aligned}
& C=\alpha_{1} \cdot f_{c}^{\prime} \cdot b \cdot\left(\beta_{1} \cdot c\right) \\
& M_{r}=C \cdot\left(d-0.5 \beta_{1} \cdot c\right)
\end{aligned}
$$

For comparison purposes, the $\alpha_{1}$ and $\beta_{1}$ parameters in the CSA Standard A23.3-04 were converted to equivalent $\alpha_{C S A}$ and $\beta_{C S A}$ parameters by using Eq. 8.3 to 8.6 as follows:

$$
\begin{gathered}
\alpha_{C S A}=\alpha_{1} \cdot \beta_{1} \\
\beta_{C S A}=0.5 \beta_{1}
\end{gathered}
$$

Table 8.2 presents the predicted over observed $\alpha$ and $\beta$ values ( $\alpha_{\text {pred. }} / \alpha_{\text {obsvd. }}$ and $\beta_{\text {pred. }} / \beta_{\text {obsvd. }}$ ) values based on CSA A23.3-04 and different existing models. The observed and predicted $\alpha$ and $\beta$ values presented in the table are the average of three repeat test samples for which the stress-strain properties were presented in Chapter 5 (Figures 5.17 and 5.18). The range of $\alpha_{\text {pred. }} / \alpha_{\text {obsvd. }}$ values for the RAC mixes are $0.97-1.02,0.74-0.83,0.98-1.07,0.87-1.03$, and $0.97-1.05$ based on CSA A23.33-04 and stress-strain relationships proposed by Smith and Young (1955), Desayi and 
Krishnan (1964), Hognestad (1951) and modified Thorenfeldt et al. (Collins and Mitchell (1990)), while the range of $\beta_{\text {pred. }} / \beta_{\text {obsvd. }}$ values are between 1.01-1.08, 0.84-0.96, 0.91$1.00,0.93-1.06$, and $0.97-1.03$, respectively.

According to these values, it can be observed that the recommended equations by CSA A23.3-04 are still valid for all RAC mixes regardless of the mix proportioning method (conventional or EMV method). This reveals why the previous studies have found the theory of flexure for conventional RC beams to be applicable to RRC beams.

Figure 8.2 illustrates the observed and predicted $\alpha$ and $\beta$ values based on CSA A23.3-04 and the various stress-strain relationships. Generally, the predicted $\alpha$ and $\beta$ values using the stress-strain relationships proposed by the modified Thorenfeldt et al. are found to be in closest agreement with the observed values, while those based on the Smith and Young relationship agree the least.

\subsection{Ultimate Flexural Strength $\left(M_{u}\right)$}

In this section, the ultimate flexural strength of RRC beams are calculated based on $\alpha$ and $\beta$ values described in the preceding section and are compared with the corresponding experimental values. The ultimate flexural strength of RRC beams are also predicted by developing their moment-curvature relationships based on their observed stress-strain relationships obtained from three repeat samples. Thus, the predicted ultimate flexural strength value of each beam is based on the average of three values calculated using three stress-strain relationships for the concrete in each beam (Figures 5.17 and 5.18).

The detailed procedure for developing the moment-curvature of RRC beams is presented in Appendix C. The full moment-curvature response of each beam was obtained by writing a macro in the Microsoft Excel program which was capable of performing the 
detailed procedure, and finding the moment and curvature corresponding to each point on the observed stress-strain curves. The ultimate flexural strength is the peak value on the moment-curvature curve.

Tables $8.3 \mathrm{a}$ and $8.3 \mathrm{~b}$ present the ratio of the observed over the predicted ultimate flexural strength $\left(M_{u}^{\text {obsvd. }} / M_{u}^{\text {pred. }}\right)$ of the FEM and the FCL beams as well as of the FEV and of FCG beams, respectively. The ultimate flexural strengths of the RRC beams were also predicted by using CSA Standard A23.3-04 method and the different existing stress-strain relationships.

With reference to the above tables, the $M_{u}^{\text {obsvd. }} / M_{u}^{\text {pred. }}$ ratio is $1.07-1.22,1.04-1.15,1.12-$ $1.44,1.07-1.22,1.06-1.23$, and 1.07-1.22 based on observed stress-strain curves, CSA , and the empirical stress-strain relationships proposed by Smith and Young, Desayi and Krishnan, Hognestad, and modified Thorenfeldt et al., respectively. By performing a full response analysis based on the observed stress-strain curves (Appendix C), the $M_{u}^{\text {obsvd. }} / M_{u}^{\text {pred. }}$ ratio was found to be 1.06-1.19.

Based on these ratios, it can be observed that the CSA flexural design provisions are still applicable to RRC beams. Furthermore, the $M_{u}^{\text {obssd. }} / M_{u}^{\text {pred. }}$ ratios calculated based on $\alpha$ and $\beta$ values obtained from different observed and predicted stress-strain curves are all within the same range as those based on the full response analysis. This is also evident from Figure 8.3 which illustrates the observed and predicted ultimate flexural strength values of the RRC beams based on CSA method and on different empirical stress-strain relationships. 


\subsection{Yielding Moment $\left(M_{y}\right)$}

The observed yielding load $\left(P_{y}\right)$ was determined by gleaning the data from the strain gauges bonded to the longitudinal steel reinforcement and finding the load corresponding to commencement of yielding (see Table 6.3). Then, the observed yielding moment $\left(M_{y}\right)$ was obtained as follows:

$$
M_{y}=0.5 P_{y} \times a+\frac{\left(\gamma_{c} \times b \times h\right) \times L_{n}^{2}}{8}
$$

where $a$ is the shear span of the beam, $\gamma_{c}$ is the hardened density of the concrete, $b$ is the section width, $h$ is the section height, and $L_{n}$ is the net beam span.

Furthermore, according to the developed moment-curvature of the beams (described in Appendix C), the yielding moment can be found by knowing the actual yield strain of the steel reinforcement from Table 6.3.

According to Tables $8.3 \mathrm{a}$ and $8.3 \mathrm{~b}$, the range of $M_{y}^{\text {obsvd. }} / M_{y}^{\text {pred. }}$ values for different RRC beams was found to be $0.94-1.36$ based on the full response analysis procedure.

\subsection{Immediate Deflection $\left(\delta_{s}\right)$}

In this section, the immediate midspan deflections of RRC beams at $40 \%$ of their corresponding failure load $\left(\delta_{s}\right)$ are predicted using three different methods:

1. Using the elastic deflection equations and the effective moment of inertia calculated based on clause 9.8.2.3 of CSA Standard A23.3-04:

$$
I_{e}=I_{c r}+\left(I_{g}-I_{c r}\right)\left(\frac{M_{c r}}{M_{a}}\right)^{3} \leq I_{g}
$$


where $I_{e}$ is the effective moment of inertia, $I_{g}$ is the gross moment of inertia, $M_{c r}$ is the cracking moment and $M_{a}$ is the applied maximum moment corresponding to the load for which deflection is calculated.

2. Using the elastic deflection equations and the effective moment of inertia calculated based on Eurocode-2 (2002) for deformed bars and first loading:

$$
I_{e}=\frac{I_{c r} \cdot I_{g}}{\left(\frac{M_{c r}}{M_{a}}\right)^{2} \cdot I_{c r}+\left[1.0-\left(\frac{M_{c r}}{M_{a}}\right)^{2}\right] \cdot I_{g}} \leq I_{g}
$$

3. Using the moment-curvature response as described earlier.

Table 8.4 presents the ratio of the observed to the predicted midspan deflections of RRC beams at $40 \%$ of their corresponding failure loads $\left(\delta_{s}^{\text {obsvd. }} / \delta_{s}^{\text {pred. }}\right)$. This ratio for the different RRC beams varies over the range of $0.34-0.72,0.87-1.46$, and $0.88-1.19$ using the moment-curvature, CSA A23.3-04 and EC2-02 methods, respectively. According to these values, it can be observed that predicted deflections based on the moment curvature method are much higher than the observed values. This can be mainly attributed to neglecting tension stiffening, i.e. the contribution of the uncracked segments between the cracked sections along the beam to the overall rigidity of the beam.

The tension-stiffening phenomenon has been indirectly taken into account in CSA A23.304 and EC2-02 methods by assuming a uniform moment of inertia, the so-called effective moment of inertia (Eq.8.10 and 8.11).

The range of $\delta_{s}^{\text {obssd. }} / \delta_{s}^{\text {pred. }}$ values for the flexural beams with minimum, average and maximum longitudinal reinforcement ratios were $1.38-1.46,0.89-0.90$ and $0.87-0.96$, respectively, with the predicted values calculated based on the CSA method. The 
corresponding values for the same beams based on the EC2-02 were $0.96-0.97,0.91$ and $0.88-0.97$, respectively. Furthermore, the range of $\delta_{s}^{\text {obsvd. }} / \delta_{s}^{\text {pred. }}$ values for flexural beams with compression steel based on the CSA A23.3-04 and EC2-02 predicted values were $1.08-1.14$ and 1.09-1.15, respectively.

According to Table 8.4, there is no major difference observed the $\delta_{s}^{\text {obsvd. }} / \delta_{s}^{\text {pred. }}$ values of conventional RC and RRC beams. This verifies the applicability of both the CSA A23.304 and the EC2-02 methods to RRC members. Note that the slight over-prediction of the deflection of beams in FEM-Min, FEV-Min, FEM-CMP and FEV-CMP by both the CSA and EC2-02 is not necessarily due to presence of RCA in RRC beams since predicted deflection of beams FCL-CMP and FCG-CMP, made of conventional concrete, are underestimated by both the CSA and EC methods $\left(\delta_{s}^{\text {obsvd. }} / \delta_{s}^{\text {pred. }}=1.13\right.$ and 1.17 based on CSA and 1.14 and 1.19 based on EC2-02 methods for beams FCL-CMP and FCG-CMP). According to the range of $\delta_{s}^{\text {obsvd. }} / \delta_{s}^{\text {pred. }}$ values, it can be observed that both the CSA A23.3-04 and EC2-02 methods predict the deflection of RRC beams with average and maximum longitudinal reinforcement ratios reasonably well $(4-13 \%$ higher than the observed values). However, for the beams with minimum longitudinal reinforcement ratio, the CSA predicted values are $38-46 \%$ lower than observed values, while those predicted by EC2-02 were almost equal to the observed values $(2-3 \%$ higher than observed values). The reason for this is twofold:

1. The high level of tension-stiffening included in CSA A23-04 is not realistic for the beams with low longitudinal reinforcement ratios due to early yielding of steel and consequently rapid widening of cracks and loss of tension-stiffening practically immediately after cracking moment. 
2. In CSA method, the effective moment of inertia is assumed to vary between the cracked and gross moment of inertia depending on the level of the applied maximum moment, while in the EC2-02 method, the curvature is assumed to vary between the fully cracked and uncracked curvatures of the section. Note that the reduction of the concrete elastic modulus at higher levels of load is not generally considered in either CSA A23.304 or EC2-02 methods. Overall, the EC2-02 method gives better results.

\subsection{Ultimate Shear Strength}

\subsubsection{Shear Strength without Shear Reinforcement $\left(v_{c}\right)$}

\subsubsection{Shear Strength variation with $a / d$}

Tables 8.5 and 8.6 present the ratio of the observed to the predicted ultimate shear resistance $\left(v_{c}^{\text {obsvd. }} / \nu_{c}^{\text {pred. }}\right)$ of the SEM and SEV beams with different a/d ratios using a number of empirical equations. From Table 8.5, it can be observed that for the SEM beams the ranges of the $v_{c}^{\text {obsvd. }} / v_{c}^{\text {pred. }}$ are 3.14-1.38, 3.08-1.26, 3.07-1.35, 3.58-1.57, $1.57-1.00,3.43-1.20,2.31-1.27,2.72-1.22,2.54-0.9,2.26-0.9,2.29-0.77$, and 2.86-1.00 based on the empirical equations proposed by CSA A23.3-04 (simplified method), CSA A23.3-04 (general method), ACI-318 (Eq.11.3), ACI-318 (Eq.11.5), Zsutty, EHE, Gastebled and May, CEB-FIP, Bažant and Yu (Eq.1), Bažant and Yu (Eq.2), Bažant and $\mathrm{Yu}(\mathrm{Eq} .3)$, and EC2-02, respectively.

Similarly, from Table 8.6 the $v_{c}^{\text {obsvd. }} / v_{c}^{\text {pred. }}$ values of the SEV beams are 3.03-1.61, 3.06$1.51,2.96-1.57,3.45-1.83,1.57-1.21,3.40-1.44,2.28-1.52,2.69-1.47,2.55-1.10,2.27-$ 1.09, 2.21-0.90, and 2.83-1.20 using the empirical equations proposed by CSA A23.3-04 (simplified method), CSA A23.3-04 (general method), ACI-318 (Eq.11.3), ACI-318 
(Eq.11.5), Zsutty, EHE, Gastebled and May, CEB-FIP, Bažant and Yu (Eq.1), Bažant and $\mathrm{Yu}(\mathrm{Eq} .2)$, Bažant and Yu (Eq.3), and EC2-02, respectively.

According to the preceding results, it can be seen that with the exception of Bažant and Yu methods, which give unconservative results in some cases, all the other methods give a conservative estimate of the actual strength of these beams. For low ald values, the estimates given by all the methods, except Zsutty's method, are highly conservative and are off by over $200 \%$ in many cases. For $a / d \geq 2.7$, the extent of the conservatism decreases. There is not a significant difference between the ACI and CSA methods, but for $a / d \geq 2.7$, the CSA general method yields more accurate results than the ACI method. However, the best prediction of the shear strength of these beams is given by Zsutty's equation. Its predicted values are consistently better than all the other methods, irrespective of the a/d ratio. This remarkable given that this method has existed for a long time and despite its consistently good accuracy, has not been adopted by any standard.

The reason for the inability of both the ACI and CSA methods to accurately predict the shear strength of beams with $a / d$ values less than 2.7 is that they both rely on beam action only rather than beam and/or arch action for estimating the shear strength of reinforced concrete members. It is well known that for $a / d \leq 2.7$, arch action begins to dominate the shear strength of concrete beams without shear reinforcement.

As stated earlier, for the present beams both the $\mathrm{ACI}$ and CSA A23.3-04 equations gave a conservative estimate of their strength. Han et al. (2001) reported that the ACI simplified method over-estimated the actual shear strength of their beams and consequently they concluded that RRC beams have lower shear strength than conventional RC beams. Since they used the conventional mix design method to design their RAC mixes, their 
conclusions can not be generalized. The high shear strength values of the current RRC beams can be attributed to the application of the EMV method to their mix design. Thus RRC beams whose concrete mixes have been designed by the EMV method would have equal or even higher shear strength than their companion beams made with conventional concrete. This can be easily confirmed by comparing the ultimate shear strength of SEM2.7N and SCL-2.7N. In this case, the RRC beam has higher shear strength than its companion NAC beam.

According to Figure 8.4, it can be observed that the empirical equations of CSA A23.304 (simplified method), ACI-318 (Eq.11.3), EHE, CSA A23.3-04 (general method), Gastebled-May and CEB-FIP always give the most conservative results for all a/d ratios, while the Zsutty, Bažant-Yu and EC2-02 give relatively more accurate results for all a/d ratios, except for $a / d=4.0$. For $a / d$ ratio of 4.0 , all the three equations proposed by Bažant-Yu are found to give unconservative results for beam SEM-4.0N, while their first equation was also found to give unconservative results for beam SEV-4.0N.

For $a / d$ ratios of 1.5 , the Zsutty (1971) equation was found to be remarkably accurate with $v_{c}^{\text {obsvd. }} / v_{c}^{\text {pred. }}$ value of 1.35 and 1.33 for SEM and SEV beams, respectively, compared to other equations whose predictions ranged from 3.58 (ACI-318: Eq.11.5) to 2.21 (Bažant and Yu: Eq.2). Similarly, for a/d ratio of 2.0, Zsutty (1971) gave the most precise accurate prediction with a $v_{c}^{\text {obsvd. }} / v_{c}^{\text {pred. }}$ value of 1.57 for both SEM and SEV beams compared to other equations whose predictions ranged from 3.25 (ACI-318: Eq.11.5) to 1.77 (Bažant and Yu: Eq.3). For a/d ratios of 2.7 and 4, the Zsutty's and EC02 equations were both very accurate. 


\subsubsection{Shear Strength for Different Beam Depths (Size Effect)}

Tables 8.7 and 8.8 present the ratio of the observed to the predicted ultimate shear resistance $\left(v_{c}^{\text {obsvd. }} / v_{c}^{\text {pred. }}\right)$ of the SEM and SEV beams with different depths using various available empirical equations. From Table 8.7, it can be observed that the $v_{c}^{\text {obsvd. }} / v_{c}^{\text {pred. }}$ values of SEM beams have the following ranges: $2.09-1.25,1.90-1.11$, $2.19-1.09,2.56-1.27,1.55-0.82,1.77-1.12,1.51-1.18,1.71-1.09,1.36-0.94,1.29-0.9$, 1.26-0.86, and 1.48-0.94 when their predicted strengths are obtained using the empirical equations recommended by the CSA A23.3-04 (simplified method), CSA A23.3-04 (general method), ACI-318 (Eq.11.3), ACI-318 (Eq.11.5), Zsutty, EHE, Gastebled and May, CEB-FIP, Bažant and Yu (Eq.1), Bažant and Yu (Eq.2), Bažant and Yu (Eq.3), and Eurocode-2, respectively. Similarly, from Table 8.8 the $v_{c}^{\text {obsvd. }} / v_{c}^{\text {pred. }}$ values of SEV beams have the following ranges: $2.64-1.31,2.47-1.19,2.78-1.14,3.23-1.33,2.02-0.88$, $2.30-1.22,1.96-1.27,2.22-1.18,1.77-1.03,1.68-0.99,1.54-0.90$, and 1.92-1.01 when using the empirical equations recommended by the CSA A23.3-04 (simplified method), CSA A23.3-04 (general method), ACI-318 (Eq.11.3), ACI-318 (Eq.11.5), Zsutty, EHE, Gastebled and May, CEB-FIP, Bažant and Yu (Eq.1), Bažant and Yu (Eq.2), Bažant and Yu (Eq.3), and Eurocode-2, respectively.

Based on the preceding results, we can observe that all the available methods used in this study give conservative estimate of the actual shear strength of these beams, except for the Bažant and Yu and Zsutty's equations which over-estimate the strength of the larger size beams with depths of 450 and $550 \mathrm{~mm}$. It may be also noticed that the $v_{c}^{\text {obsvd. }} / \nu_{c}^{\text {pred. }}$ values decrease as depth increases regardless of the empirical equation type 
and for both SEM and SEV beams. In other words, the predicted shear strength values based on all empirical equations are more conservative for shallower beams. This is mainly due to higher contribution of the aggregate interlock mechanism to the shear resistance in shallower beams.

According to range of the $v_{c}^{\text {obsvd. }} / v_{c}^{\text {pred. }}$ values using different empirical equations recommended by different researchers and/or standards, it can be observed that the ACI318 (Eq.11.5) method resulted in the most conservative estimate of the shear strength for all depth values, in both SEM and SEV beams, compared to the other methods. This is quite evident in Figure 8.5.

It is apparent that the Zsutty's equation does not account for size effect. The reason is that this equation was derived based on statistical analysis of large amount of experimental data, but the data was obtained from the test results of beams whose depths were practically always less than $400 \mathrm{~mm}$. Consequently, in its current form, it does not apply to large size beams.

The CSA Standard, on the other hand, has specific provisions for size effect, therefore its predicted values reflect the reduction in nominal shear strength with increased size. The ACI method does not specifically consider the size effect, but since it is highly conservative for shallow beams, it is less conservative for larger size beams. Obviously, if the beam depth were to exceed $0.5 \mathrm{~m}$, it may yield unconservative results. Therefore, at present, it is recommended that the existing CSA shear design provisions be applied to RRC beams without any changes. It is recognized that they give conservative results, but this conservatism equally applies to RRC beams. 


\subsubsection{Shear Strength of Beams with Shear Reinforcement $\left(v_{u}\right)$}

Table 8.9 presents the ratio of the observed to the predicted ultimate shear resistance $\left(v_{u}^{\text {obsvd. }} / v_{u}^{\text {pred. }}\right)$ of the SEM and SEV beams with different shear reinforcement ratios using the recommended CSA A23.3-04 and ACI-318 equations. Both the simplified and the general methods of CSA A23.3-04 predict reasonably well the shear strength of both the SEM and the SEV beams for all shear reinforcement ratios.

According to Table 8.9, it can be observed that the $v_{u}^{\text {obsvd. }} / v_{u}^{\text {pred. }}$ values of SEM beams vary as $1.04-1.71,1.18-1.58,1.20-1.67$, and $1.28-1.94$ when their strengths are predicted by the CSA A23.3-04 (simplified method), CSA A23.3-04 (general method), ACI-318 (Eq.11.3), and ACI-318 (Eq.11.5), respectively. Similarly, the $v_{u}^{\text {obsvd. }} / v_{u}^{\text {pred. }}$ values of SEV beams vary as $1.06-1.38,1.23-1.57,1.30-1.59$, and $1.35-1.70$ based on the CSA A23.3-04 (simplified method), CSA A23.3-04 (general method), ACI-318 (Eq.11.3), and ACI-31 8 (Eq.11.5), respectively. Note that the actual $v_{u}^{\text {obsvd. }} / v_{u}^{\text {pred. }}$ values of SEM-6S-R, SEV-6S-R, SCL-6S-R, SCG-6S-R, and SEV-3S-R in Table 8.9 may be much higher since they failed in flexure rather than shear.

According to the $v_{u}^{\text {obsvd. }} / v_{u}^{\text {pred. }}$ values in Table 8.9 , it can be observed that ACI-318 (Eq.11.5) and ACI-318 (Eq.11.3) resulted in more conservative estimate of the ultimate shear strength of these beams for all shear reinforcement ratios compared to the simplified and general methods of CSA A23.3-04. This is further highlighted in Figure 8.6. The slight differences between the $v_{u}^{\text {obsvd. }} / v_{u}^{\text {pred. }}$ values based on Eq.11.3 and 11.5 of ACI-318 are due to the different $v_{c}$ values given by the latter equations since the 
assumed angle of inclination of diagonal stresses to the longitudinal axis of the member $(\theta)$ in ACI-318 is assumed to be 45 degrees in both methods. On the other hand, the

slight difference between the $v_{u}^{\text {obsvd. }} / v_{u}^{\text {pred. }}$ values based on simplified and general methods of CSA A23.3-04 is due to both different $\nu_{c}$ values and different $\theta$ values given by the simplified and general methods.

Once again it is important to emphasize that the current CSA A23.3-04 shear design provisions can be applied to RRC beams without any modification, provided that the RAC from which the RRC beams are made is designed by the EMV method. Consequently, there is no need for developing new design provisions for RRC members. The conservative nature of the code equations is independent of the type of the concrete.

\subsubsection{Summary}

- Despite overestimating the cracking moment values of some RRC beams, the CSA A23.3-04 equation for prediction of cracking moment of RC beams (clause 8.6.4) is applicable to RRC beams. However, using the observed splitting tensile strength instead of the predicted modulus of rupture based on CSA A23.3-04 (clause 8.6.4) gave generally more conservative estimate of the cracking moment of both SEM and SEV beams.

- Generally, the CSA A23.3-04 (clause 10.1.7) method for predicting the equivalent rectangular stress block parameters ( $\alpha$ and $\beta$ ) of conventional concrete was found to be applicable to all RAC mixes regardless of mix proportioning method.

- The CSA A23.3-04 flexural design provisions for conventional RC members were found to be applicable to both the FEM and the FEV beams with different longitudinal tension reinforcement ratios, with or without compression steel. 
- There was no major difference observed between the predicted ultimate flexural strength values of both the FEM and the FEV beams when using the equivalent $\alpha$ and $\beta$ values based on the CSA A23.3-04 or on the actual and empirical stress-strain curves.

- Both the CSA A23.3-04 and the EC2-02 methods for predicting the immediate deflections of conventional RC members were found to be applicable to RRC beams. However, EC2-02 was to give more accurate results for beams with small longitudinal tension reinforcement ratios.

- Both the simplified and the general methods of CSA A23.3-04 and ACI-318 were found to give conservative estimate of the shear strength of both the SEM and the SEV beams for all $\mathrm{a} / \mathrm{d}$ ratios and depth values with or without shear reinforcement.

- Other empirical equations for predicting the concrete contribution to the shear resistance of beams such as the Gastebled and May, EHE, CEB-FIP and EC2-02, also yielded conservative estimate of the shear strength of both the SEM and the SEV beams for all a/d ratios and depth values.

- The proposed equations of Bažant and Yu were found to be applicable to RRC beams with a/d ratios up to 2.7 and overall depth values of up to $375 \mathrm{~mm}$.

- The proposed equation by Zsutty was found to yield the most estimate of the shear strength of the current test beams and for all $a / d$ ratios (especially at lower $a / d$ ratios). However, it was found to give unconservative results for the RRC beams with large depths, i.e. 450 and $550 \mathrm{~mm}$.

- There is no need to develop new design method for RRC beams because the current CSA A23.3-04 provisions apply to them, provided the RAC used in the construction of these members is designed by the EMV method. 
Table 8.1a: Predicted and observed cracking moments of RRC beams made of RCA-MO and/or NL

\begin{tabular}{|c|c|c|c|c|c|}
\hline \multirow{3}{*}{ Beam ID } & \multicolumn{3}{|c|}{$\mathbf{M}_{\mathrm{cr}}(\mathrm{kN}-\mathrm{m})$} & \multirow{2}{*}{\multicolumn{2}{|c|}{$M_{c r}^{o b s v d .} / M_{c r}^{p r e d .}$}} \\
\hline & \multicolumn{2}{|c|}{ Predicted } & \multirow{2}{*}{ Experimental } & & \\
\hline & $f_{r}$-based & $f_{s t}$-based & & $f_{r}$-based & $f_{s t}$-based \\
\hline FEM-Min & 14.8 & 11.4 & 13.0 & 1.14 & 0.88 \\
\hline FEM-Av & 16.4 & 12.6 & 13.8 & 1.10 & 0.84 \\
\hline FCL-Av & 17.5 & 14.1 & 19.2 & 1.36 & 1.10 \\
\hline FEM-Max & 17.3 & 13.3 & 21.1 & 1.59 & 1.22 \\
\hline FEM-CMP & 18.1 & 13.9 & 24.7 & 1.77 & 1.36 \\
\hline FCL-CMP & 18.5 & 13.3 & 29.5 & 2.21 & 1.60 \\
\hline SEM-3S-R & 18.2 & 14.0 & 25.9 & 1.85 & 1.42 \\
\hline SEM-6S-D & 18.0 & 13.9 & 20.0 & 1.44 & 1.11 \\
\hline SEM-1.5N & 14.9 & 11.5 & 18.5 & 1.61 & 1.23 \\
\hline SEM-2.0N & 15.0 & 11.5 & 19.2 & 1.67 & 1.28 \\
\hline SEM-2.7N & 17.2 & 13.2 & 17.4 & 1.32 & 1.01 \\
\hline SCL-2.7N & 17.4 & 15.0 & 25.8 & 1.72 & 1.48 \\
\hline SEM-4.0N & 17.3 & 13.3 & 21.1 & 1.59 & 1.22 \\
\hline SEM-2.7L & 7.6 & 5.9 & 7.4 & 1.26 & 0.97 \\
\hline SEM-2.7H & 24.8 & 19.1 & 26.8 & 1.41 & 1.08 \\
\hline SEM-2.7HH & 37.1 & 28.5 & 42.2 & 1.48 & 1.14 \\
\hline \multicolumn{4}{|c|}{ Average } & 1.53 & 1.18 \\
\hline \multicolumn{4}{|c|}{ Standard Deviation } & 0.28 & 0.21 \\
\hline
\end{tabular}


Table 8.1b: Predicted and observed cracking moments of RRC beams made of RCA-VA and/or NG

\begin{tabular}{|c|c|c|c|c|c|}
\hline \multirow{3}{*}{ Beam ID } & \multicolumn{3}{|c|}{$M_{\mathrm{er}}(\mathrm{kN}-\mathrm{m})$} & \multirow{2}{*}{\multicolumn{2}{|c|}{$M_{c r}^{\text {obsvd. }} / M_{c r}^{\text {pred. }}$}} \\
\hline & \multicolumn{2}{|c|}{ Predicted } & \multirow{2}{*}{ Experimental } & & \\
\hline & $f_{r}$-based & $f_{s t}$-based & & $f_{r}$-based & $\mathbf{f}_{\mathrm{st}}$-based \\
\hline FEV-Min & 16.1 & 13.6 & 16.2 & 1.19 & 1.01 \\
\hline FEV-Av & 18.7 & 15.9 & 15.2 & 0.96 & 0.81 \\
\hline FCG-Av & 17.0 & 15.2 & 19.2 & 1.26 & 1.13 \\
\hline FEV-Max & 18.9 & 16.1 & 13.4 & 0.84 & 0.71 \\
\hline FEV-CMP & 19.7 & 16.7 & 29.1 & 1.74 & 1.48 \\
\hline FCG-CMP & 17.1 & 16.0 & 23.0 & 1.44 & 1.35 \\
\hline SEV-3S-R & 19.7 & 16.8 & 25.1 & 1.50 & 1.27 \\
\hline SEV-6S-D & 19.6 & 16.6 & 24.0 & 1.44 & 1.22 \\
\hline SEV-1.5N & 16.2 & 13.8 & 19.4 & 1.41 & 1.19 \\
\hline SEV-2.0N & 16.3 & 13.8 & 19.3 & 1.39 & 1.18 \\
\hline SEV-2.7N & 18.7 & 15.8 & 17.5 & 1.10 & 0.93 \\
\hline SCG-2.7N & 17.0 & 15.4 & 13.8 & 0.90 & 0.81 \\
\hline SEV-4.0N & 18.8 & 15.9 & 24.8 & 1.56 & 1.32 \\
\hline SEV-2.7L & 8.3 & 7.0 & 8.8 & 1.25 & 1.06 \\
\hline SEV-2.7H & 27.0 & 22.9 & 27.4 & 1.20 & 1.01 \\
\hline SEV-2.7HH & 40.3 & 34.2 & 55.3 & 1.62 & 1.37 \\
\hline \multicolumn{4}{|c|}{ Average } & 1.30 & 1.12 \\
\hline \multicolumn{4}{|c|}{ Standard Deviation } & 0.26 & 0.22 \\
\hline
\end{tabular}

Table 8.2: Predicted over observed equivalent stress block parameters for CM-C, EM-C and CL-C mixes

\begin{tabular}{|c|c|c|c|c|c|c|c|}
\hline \multicolumn{2}{|c|}{ Models } & \multicolumn{7}{|c|}{ Mix Type } \\
\cline { 3 - 8 } \multicolumn{1}{|c|}{ Observed } & $\boldsymbol{\alpha}$ & 0.6486 & 0.6947 & 0.7579 & 0.6704 & 0.6849 & 0.7221 \\
\hline & $\boldsymbol{\beta}$ & 0.3939 & 0.4185 & 0.4227 & 0.3896 & 0.3884 & 0.4375 \\
\hline \multirow{4}{*}{$\alpha_{\text {pred. }}$} & $\mathbf{C S A}$ A23.3-04 & 1.02 & 1.02 & 0.91 & 0.97 & 0.98 & 0.98 \\
\cline { 2 - 8 } & Smith-Young & 0.82 & 0.77 & 0.71 & 0.83 & 0.80 & 0.74 \\
\cline { 2 - 8 }$\alpha_{\text {obsvd. }}$ & Desayi-Krishnan & 0.99 & 1.04 & 1.02 & 0.98 & 0.98 & 1.07 \\
\cline { 2 - 8 } & $\mathbf{H o g n e s t a d}$ & 0.96 & 0.98 & 0.98 & 0.89 & 0.87 & 1.03 \\
\cline { 2 - 8 } & Thorenfeldt et al. & 1.05 & 0.99 & 0.84 & 0.97 & 0.97 & 0.97 \\
\hline \multirow{3}{*}{$\beta_{\text {pred. }}$} & CSA A23.3-04 & 1.08 & 1.06 & 1.03 & 1.08 & 1.10 & 1.01 \\
\cline { 2 - 8 } & Smith-Young & 0.93 & 0.87 & 0.87 & 0.95 & 0.96 & 0.84 \\
\cline { 2 - 8 }$\beta_{\text {obsvd. }}$ & Desayi-Krishnan & 0.95 & 0.91 & 0.97 & 0.98 & 1.00 & 0.94 \\
\cline { 2 - 8 } & Hognestad & 0.94 & 0.94 & 0.95 & 0.97 & 1.06 & 0.93 \\
\cline { 2 - 8 } & Thorenfeldt et al. & 0.97 & 1.00 & 1.10 & 1.03 & 1.03 & 1.03 \\
\hline
\end{tabular}




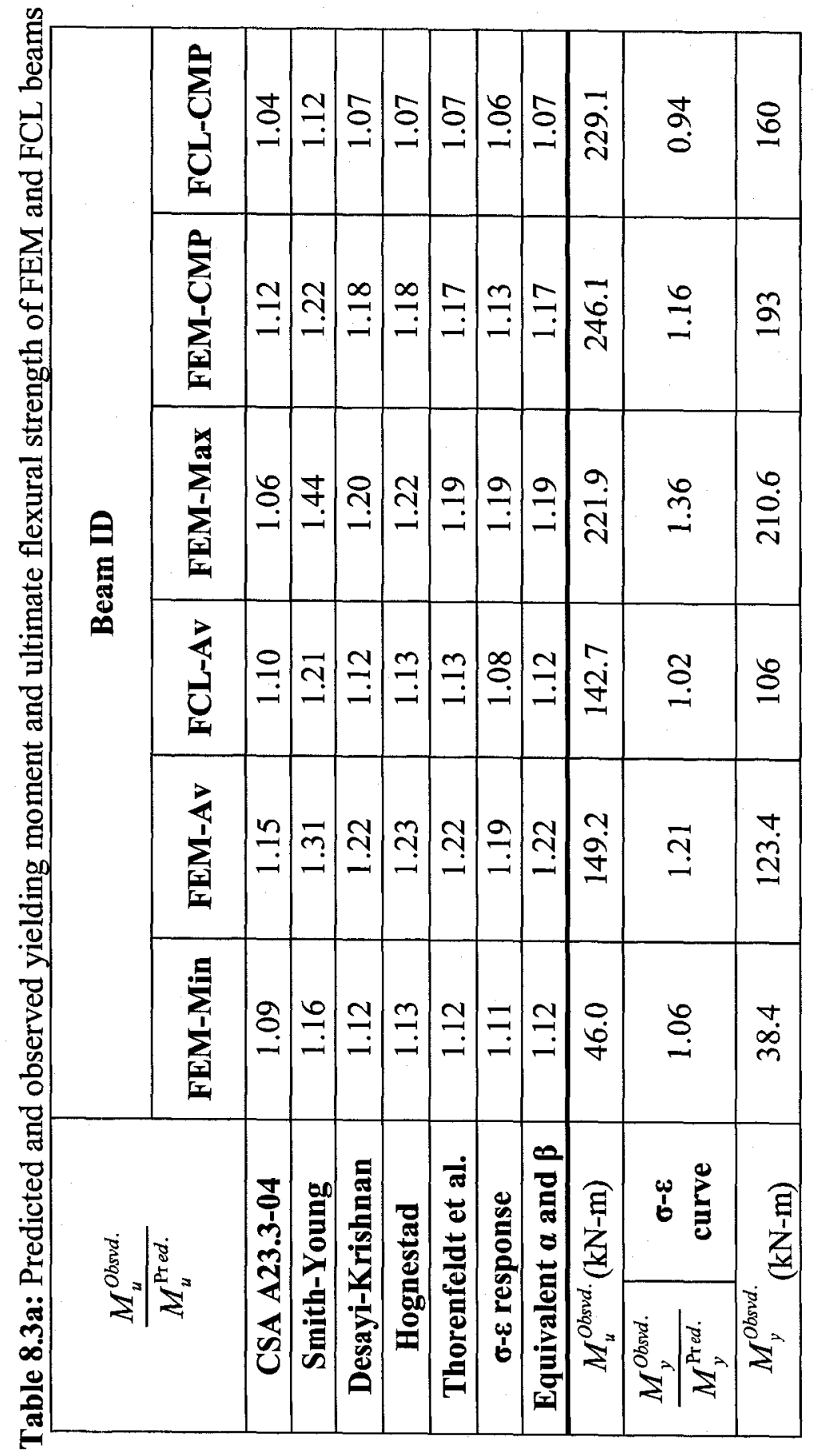


Table 8.3b: Predicted and observed yielding moment and ultimate flexural strength of FEV and FCG beams

\begin{tabular}{|c|c|c|c|c|c|c|c|}
\hline \multirow{2}{*}{\multicolumn{2}{|c|}{$\frac{M_{u}^{\text {Obsid. }}}{M_{u}^{\text {Pred. }}}$}} & \multicolumn{6}{|c|}{ Beam ID } \\
\hline & & FEV-Min & FEV-Av & FCG-Av & FEV-Max & FEV-CMP & FCG-CMP \\
\hline \multicolumn{2}{|c|}{ CSA A23.3-04 } & 1.09 & 1.14 & 1.08 & 1.04 & 1.11 & 1.04 \\
\hline \multicolumn{2}{|c|}{ Smith-Young } & 1.10 & 1.23 & 1.09 & 1.22 & 1.18 & 1.13 \\
\hline \multicolumn{2}{|c|}{ Desayi-Krishnan } & 1.08 & 1.19 & 1.06 & 1.13 & 1.16 & 1.07 \\
\hline \multicolumn{2}{|c|}{ Hognestad } & 1.09 & 1.20 & 1.06 & 1.16 & 1.16 & 1.08 \\
\hline \multicolumn{2}{|c|}{ Thorenfeldt et al. } & 1.08 & 1.19 & 1.09 & 1.13 & 1.15 & 1.07 \\
\hline \multicolumn{2}{|c|}{$\sigma-\varepsilon$ response } & 1.11 & 1.19 & 1.16 & 1.14 & 1.17 & 1.11 \\
\hline \multicolumn{2}{|c|}{ Equivalent $\alpha$ and $\beta$} & 1.07 & 1.19 & 1.08 & 1.13 & 1.15 & 1.07 \\
\hline \multicolumn{2}{|c|}{$M_{u}^{\text {Obssd. }}(\mathrm{kN}-\mathrm{m})$} & 46.7 & 150.2 & 139.1 & 225.2 & 245.7 & 226.5 \\
\hline$\frac{M_{y}^{\text {Obsrd. }}}{M_{y}^{\text {Pred. }}}$ & $\begin{array}{c}\sigma-\varepsilon \\
\text { curve }\end{array}$ & 1.10 & 1.30 & - & 1.29 & 1.31 & 0.96 \\
\hline \multicolumn{2}{|c|}{$M_{y}^{\text {Obssid. }}(\mathrm{kN}-\mathrm{m})$} & 39.9 & 136.6 & - & 207.5 & 220.2 & 158.9 \\
\hline
\end{tabular}


Table 8.4: Mid span deflections of flexural RRC beams

\begin{tabular}{|c|c|c|c|c|}
\hline \multirow{2}{*}{ Beam ID } & \multirow{2}{*}{$\begin{array}{l}\delta_{s}^{\text {Obsvd. }} \\
(\mathbf{m m})\end{array}$} & \multicolumn{3}{|c|}{$\frac{\delta_{s}^{\text {obsvd. }}}{\delta_{s}^{\text {Pred. }}}$} \\
\hline & & $\begin{array}{l}\text { Moment- } \\
\text { Curvature }\end{array}$ & $\begin{array}{c}\text { CSA } \\
\text { A23.3-04 }\end{array}$ & $\begin{array}{r}\text { EC2- } \\
91\end{array}$ \\
\hline FEM-Min & 1.54 & 0.72 & 1.38 & 0.96 \\
\hline FEM-Av & 1.70 & 0.34 & 0.90 & 0.91 \\
\hline FCL-Av & 2.55 & 0.55 & 0.88 & 0.89 \\
\hline FEM-Max & 3.04 & 0.62 & 0.96 & 0.97 \\
\hline FEM-CMP & 3.62 & 0.67 & 1.08 & 1.09 \\
\hline FCL-CMP & 3.40 & 0.69 & 1.13 & 1.14 \\
\hline FEV-Min & 1.04 & 0.57 & 1.46 & 0.97 \\
\hline FEV-Av & 2.67 & 0.57 & 0.89 & 0.91 \\
\hline FCG-Av & 2.43 & 0.50 & 0.90 & 0.91 \\
\hline FEV-Max & 2.63 & 0.53 & 0.87 & 0.88 \\
\hline FEV-CMP & 3.67 & 0.65 & 1.14 & 1.15 \\
\hline FCG-CMP & 3.51 & 0.61 & 1.17 & 1.19 \\
\hline
\end{tabular}

Table 8.5: Ratio of observed to predicted ultimate shear resistance of SEM and SCL beams with different $a / d$ ratios

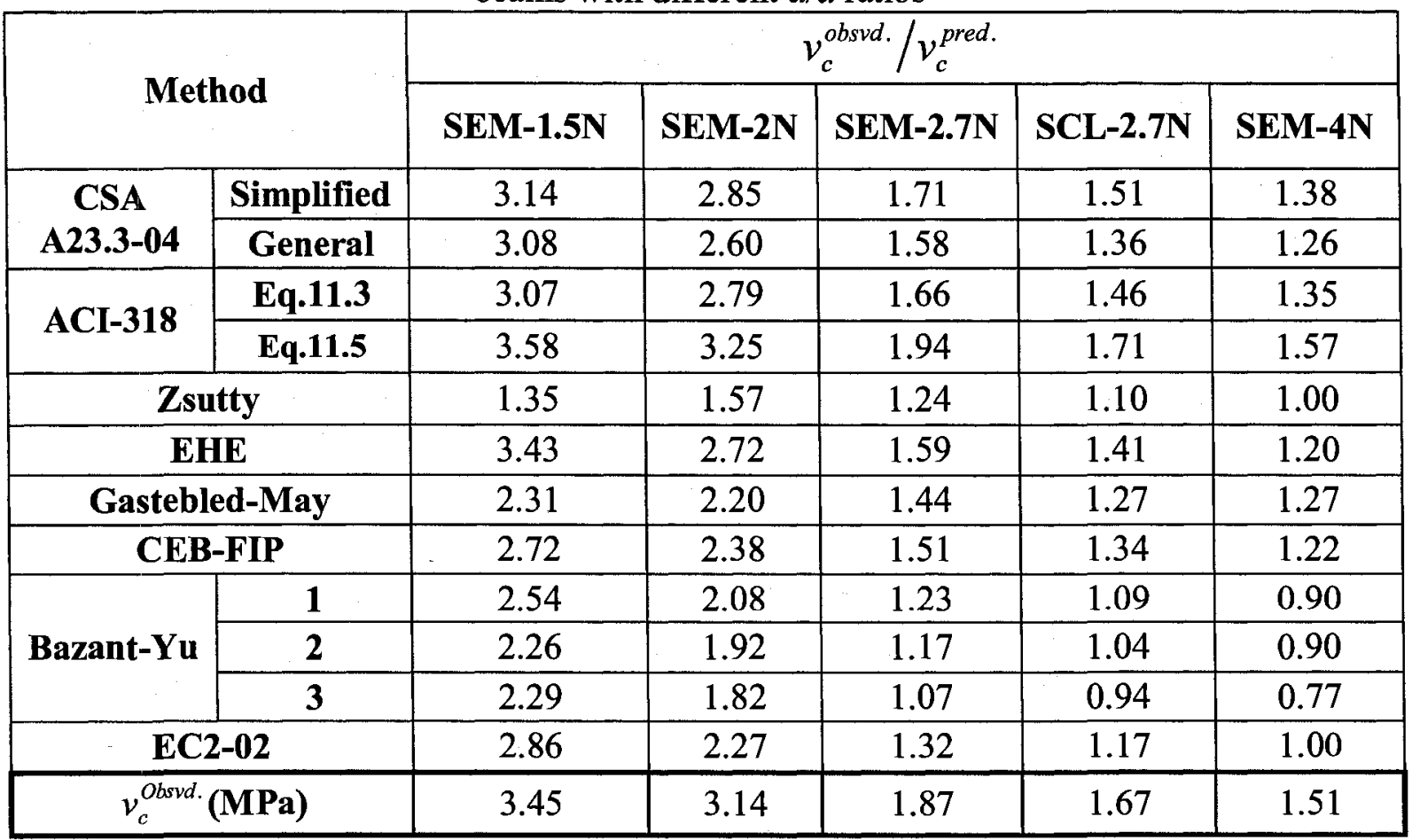


Table 8.6: Ratio of observed to predicted ultimate shear resistance of SEV and SCG beams with different $a / d$ ratios

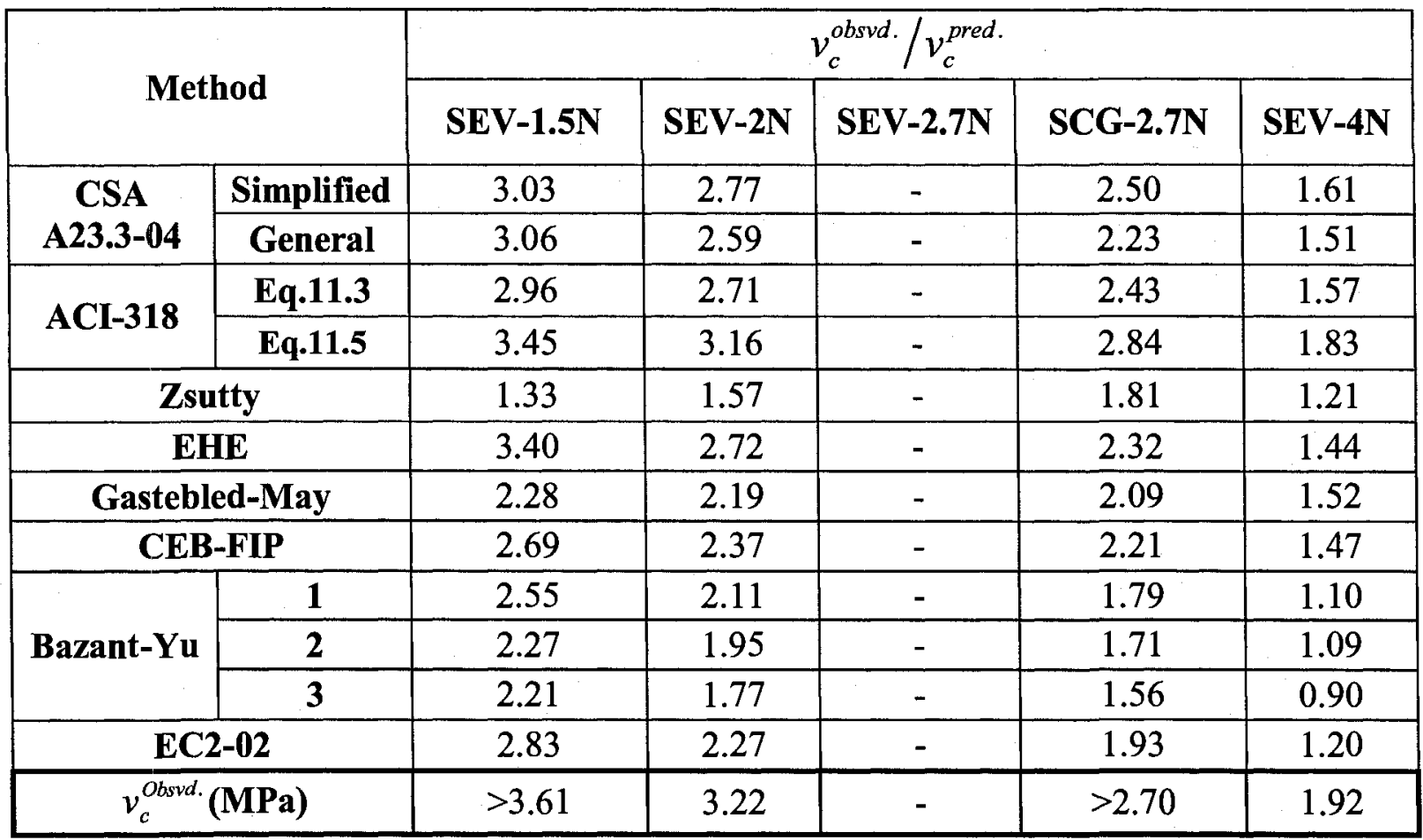

Table 8.7: Ratio of observed to predicted ultimate shear resistance of SEM and SCL beams with different depths

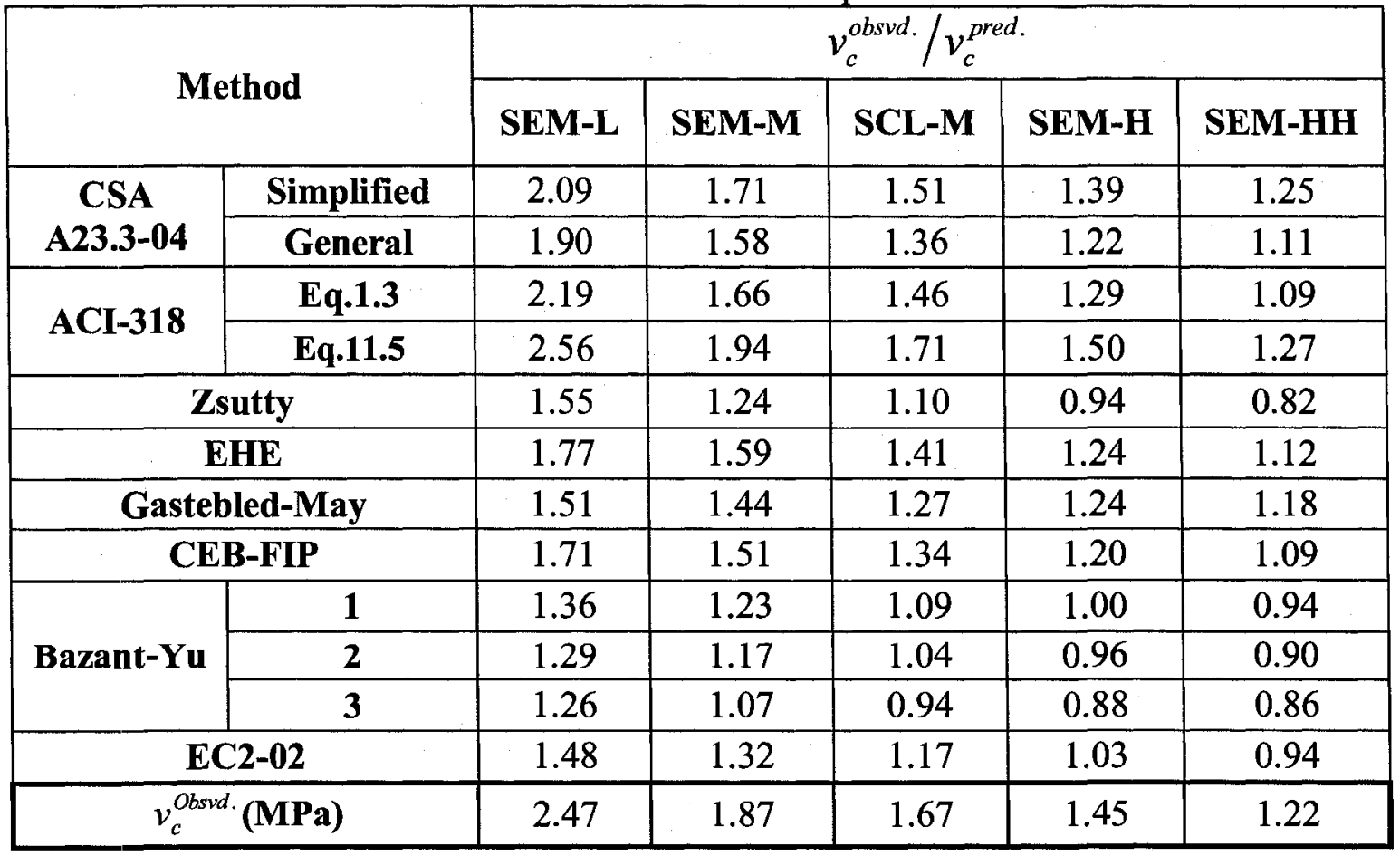


Table 8.8: Ratio of observed to predicted ultimate shear resistance of SEV and SCG beams with different depths

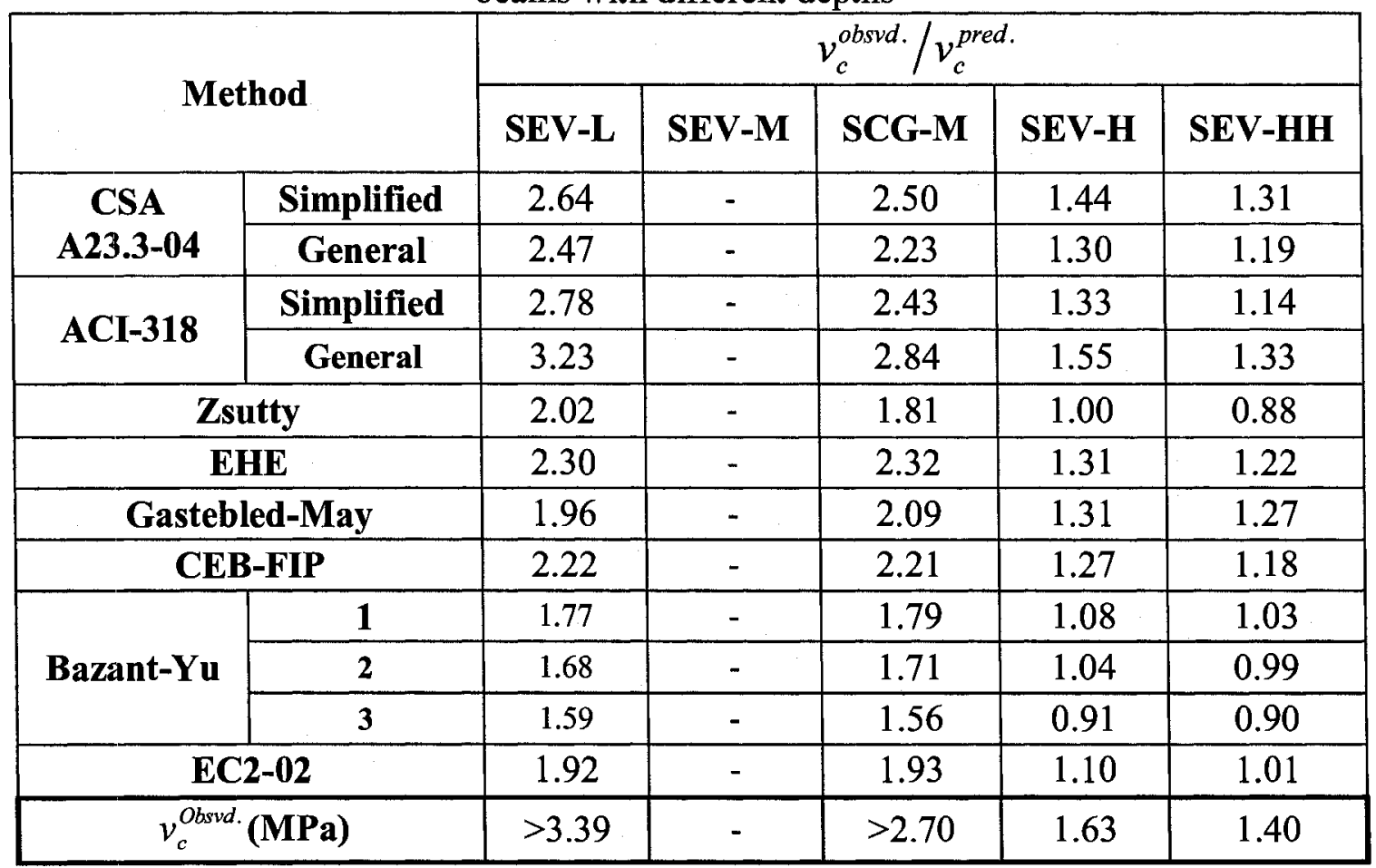

Table 8.9: Comparison of observed shear strength of RRC beams with their predicted strengths by the CSA and ACI methods

\begin{tabular}{|c|c|c|c|c|c|}
\hline \multirow{3}{*}{ Beam ID } & \multicolumn{4}{|c|}{$V_{u}^{\text {obsvd. }} / V_{u}^{\text {pred. }}$} & \multirow[b]{3}{*}{$\begin{array}{c}V_{u}^{o b s v d .} \\
(\mathbf{k N})\end{array}$} \\
\hline & \multicolumn{2}{|c|}{ CSA-A23.3-04 } & \multicolumn{2}{|c|}{ ACI-318 } & \\
\hline & Simplified & General & Simplified & General & \\
\hline SEM-NS & 1.71 & 1.58 & 1.66 & 1.94 & 103.9 \\
\hline SEM-3S & 1.04 & 1.18 & 1.20 & 1.28 & 171.9 \\
\hline SEM-6SR & 1.14 & 1.32 & 1.39 & 1.45 & $>308.3$ \\
\hline SCL-6SR & 1.06 & 1.22 & 1.29 & 1.35 & $>287.0$ \\
\hline SEM-6SD & 1.37 & 1.56 & 1.67 & 1.74 & 340.8 \\
\hline SEV-NS & - & - & - & - & - \\
\hline SEV-3SR & 1.38 & 1.57 & 1.59 & 1.70 & $>235.0$ \\
\hline SEV-6SR & 1.12 & 1.30 & 1.36 & 1.42 & $>307.8$ \\
\hline SCG-6SR & 1.06 & 1.23 & 1.30 & 1.35 & 283.8 \\
\hline SEV-6SD & 1.29 & 1.47 & 1.56 & 1.64 & 327.4 \\
\hline \begin{tabular}{l|l} 
Average \\
\end{tabular} & \multicolumn{2}{|c|}{\begin{tabular}{|l|}
1.24 \\
\end{tabular}} & \multicolumn{2}{|c|}{1.45} & \begin{tabular}{l|l}
.54 &
\end{tabular} \\
\hline St. Dev. & 0.2 & & 0.1 & & 22 \\
\hline
\end{tabular}




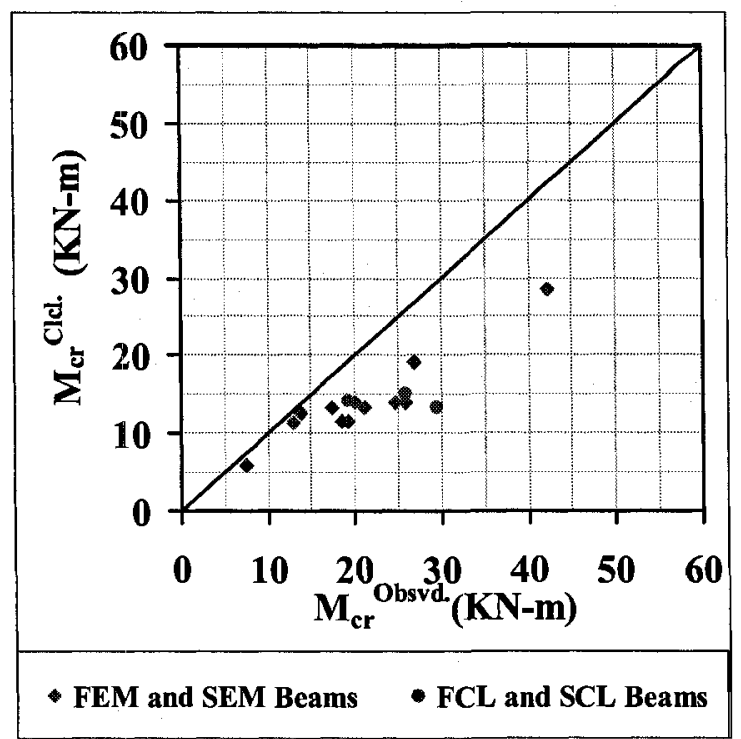

a) $f_{\text {st }}$ based

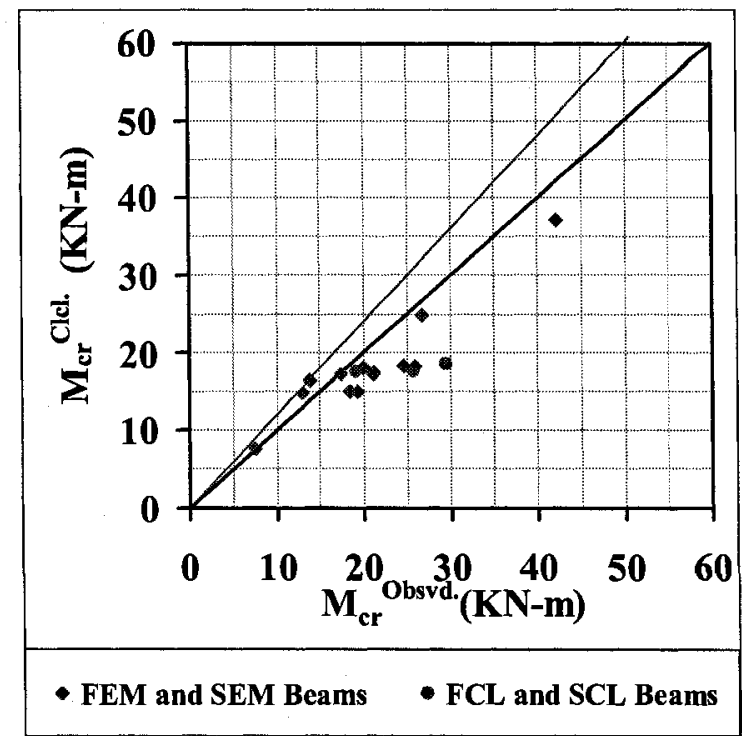

b) $f_{\mathrm{r}}$-based

Figure 8.1a: Observed versus calculated cracking moments of SEM and FEM beams

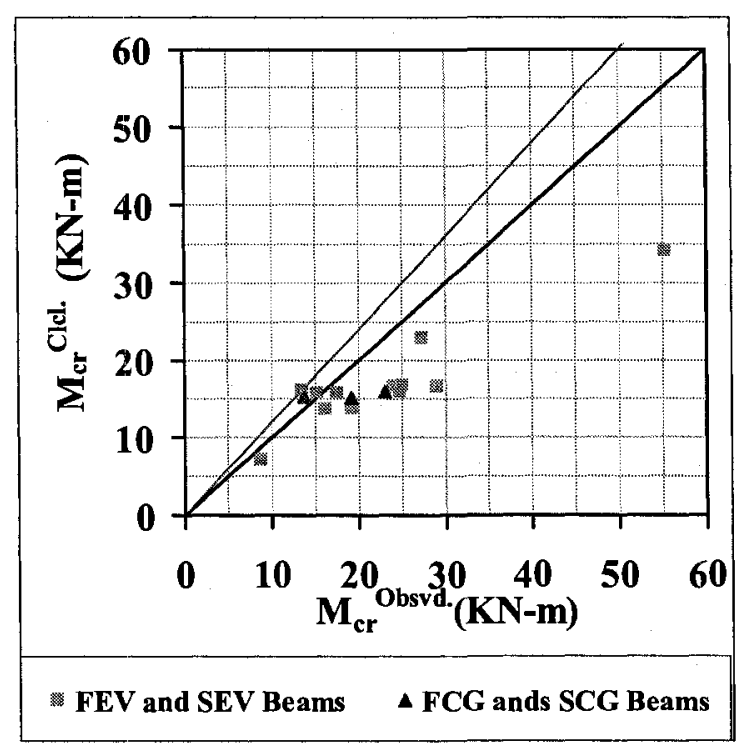

a) $f_{\text {st }}$-based

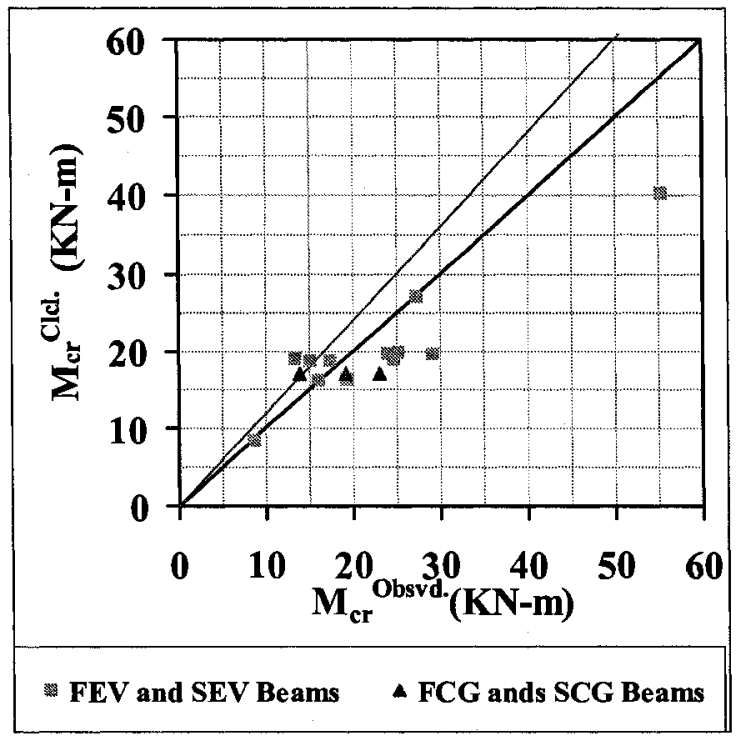

b) f $\mathrm{f}_{\mathrm{r}}$-based

Figure 8.1b: Observed versus calculated cracking moments of SEV and FEV beams 

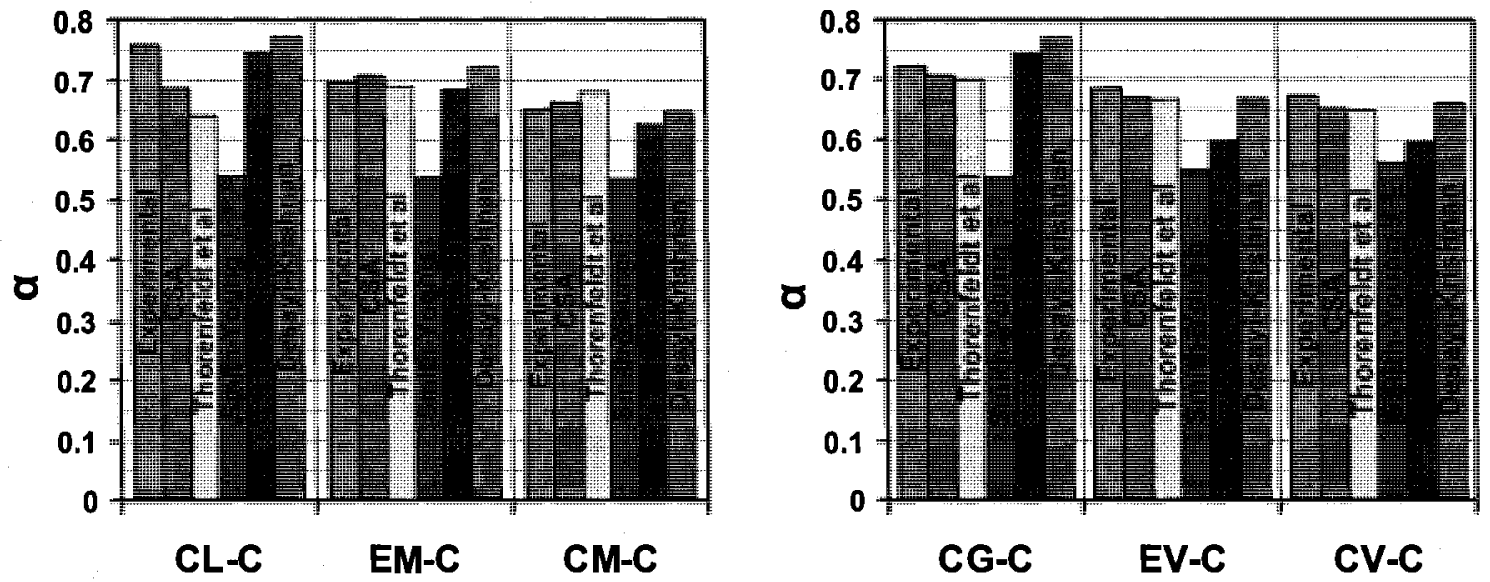

a) Equivalent $\alpha$ values
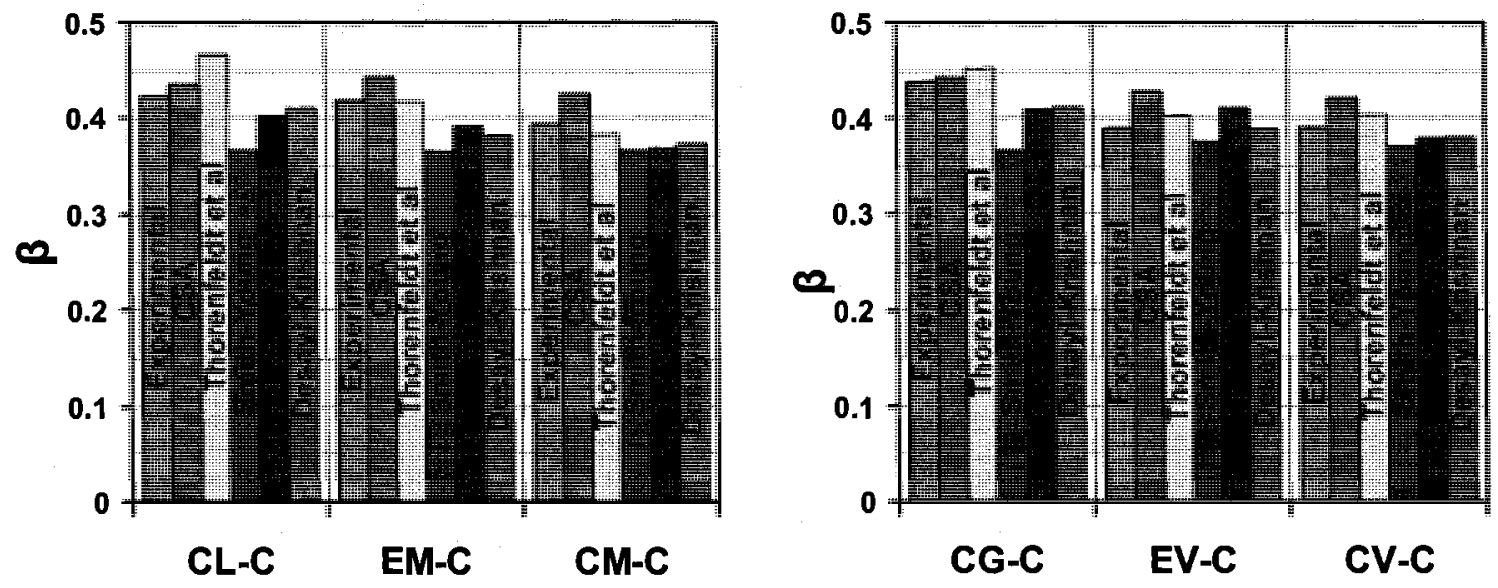

b) Equivalent $\beta$ values

Figure 8.2: Calculated and observed equivalent stress block parameters 


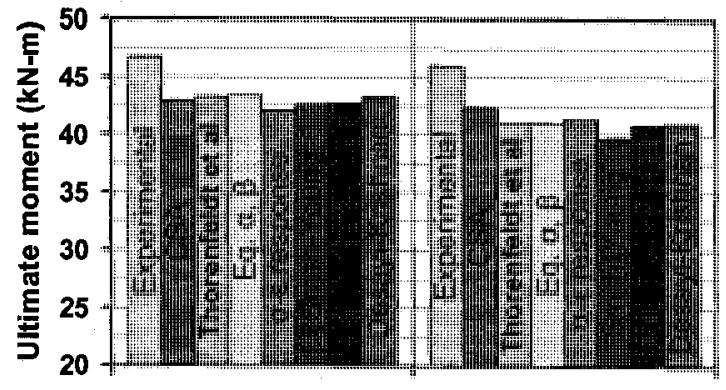

FEV-Min

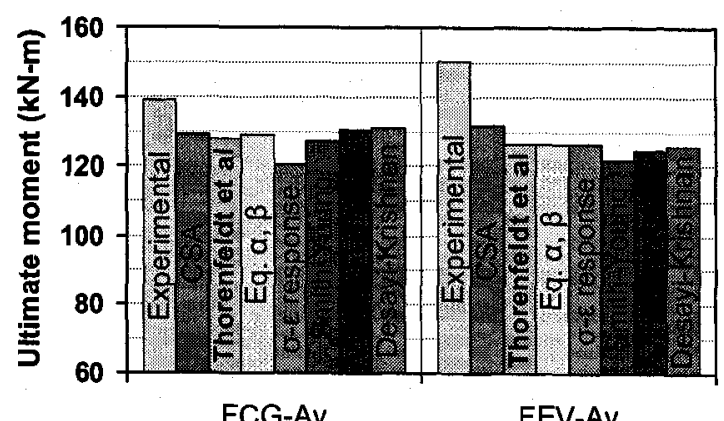

FCG-AV

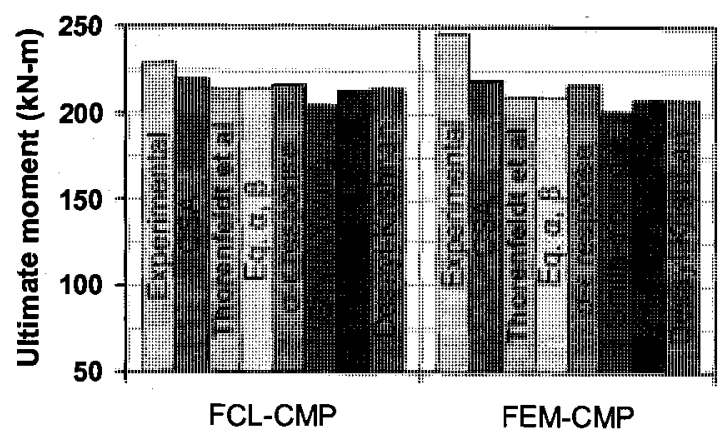

FCL-CMP

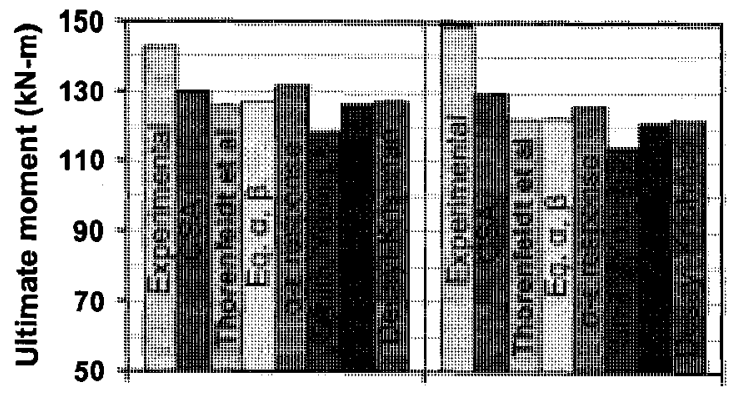

FCL-Av

FEM-Av

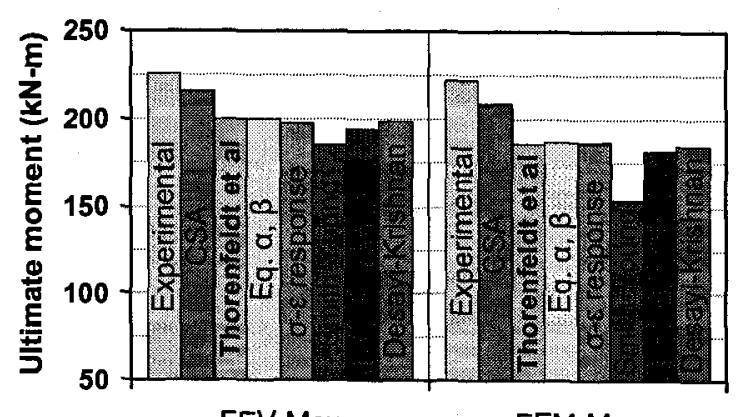

FEV-Max

FEM-Max

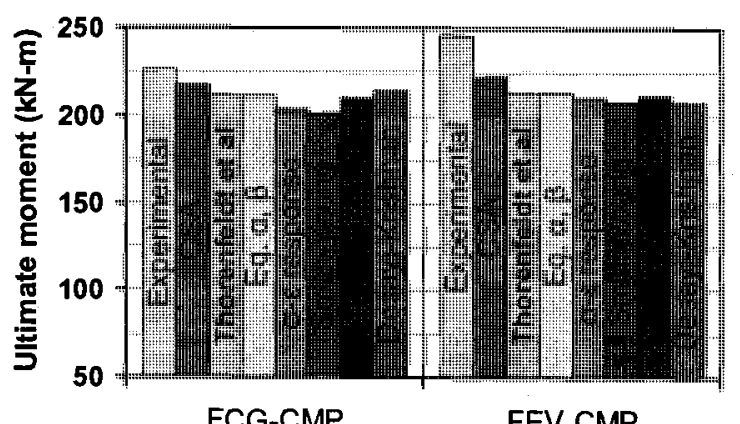

FEV-CMP

Figure 8.3: Experimental and predicted ultimate flexural strength of RRC beams 


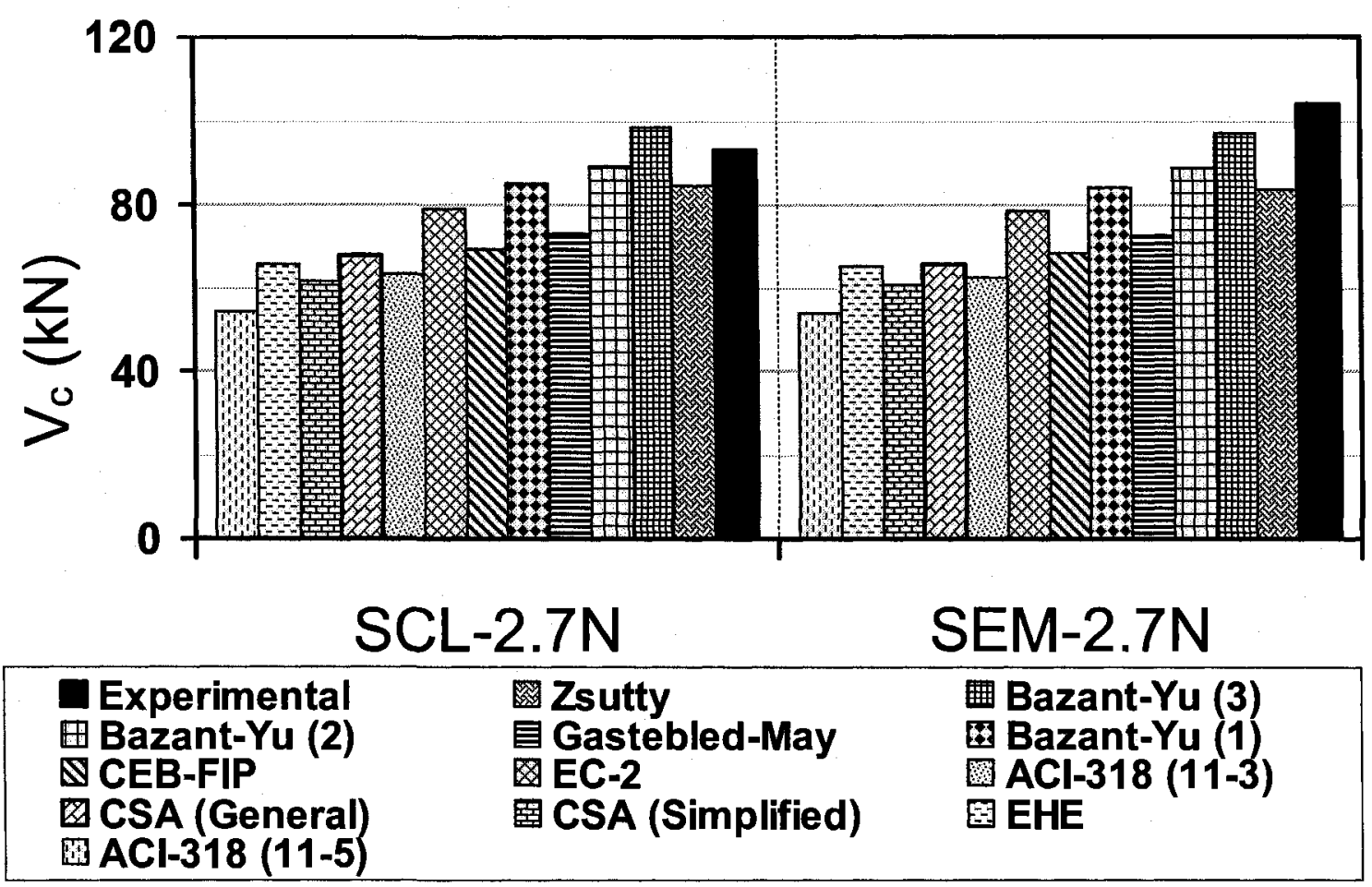

Figure 8.4c: Experimental and predicted ultimate shear strength of SEM and SCL beams with $a / d=2.7$

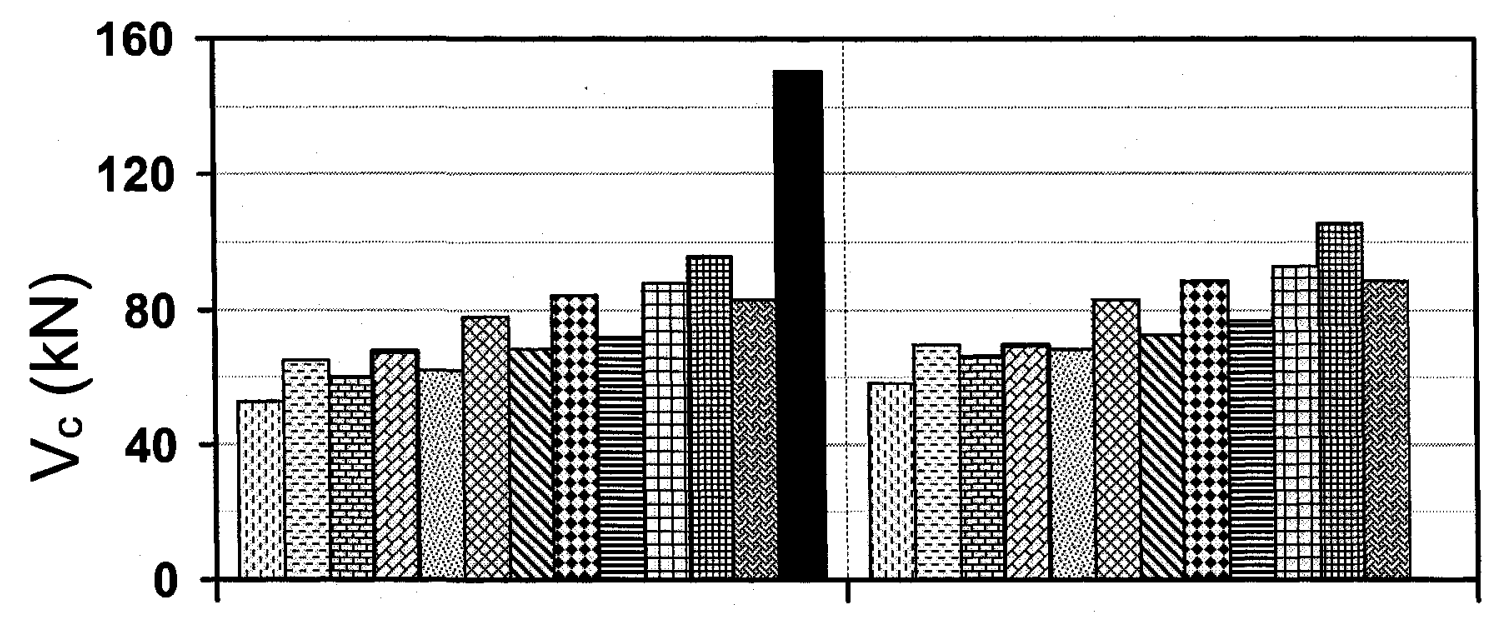

SCG-2.7N SEV-2.7N

\begin{tabular}{|c|c|c|}
\hline $\begin{array}{l}\text { Experimental } \\
\text { Bazant-Yu (2) } \\
\text { B CEB-FIP } \\
\text { CSA (General) } \\
\text { ACl-318 (11-5) }\end{array}$ & $\begin{array}{l}\text { Zsutty } \\
\text { 圈 Gastebled-May } \\
\text { EC-2 } \\
\text { 甶 CSA (Simplified) }\end{array}$ & $\begin{array}{l}\text { Bazant-Yu (3) } \\
\text { 国 Bazant-Yu (1) } \\
\text { 圈 ACl-318 (11-3) } \\
\text { 园 EHE }\end{array}$ \\
\hline
\end{tabular}

Figure 8.4d: Experimental and predicted ultimate shear strength of SEV and SCG beams with $a / d=2.7$ 


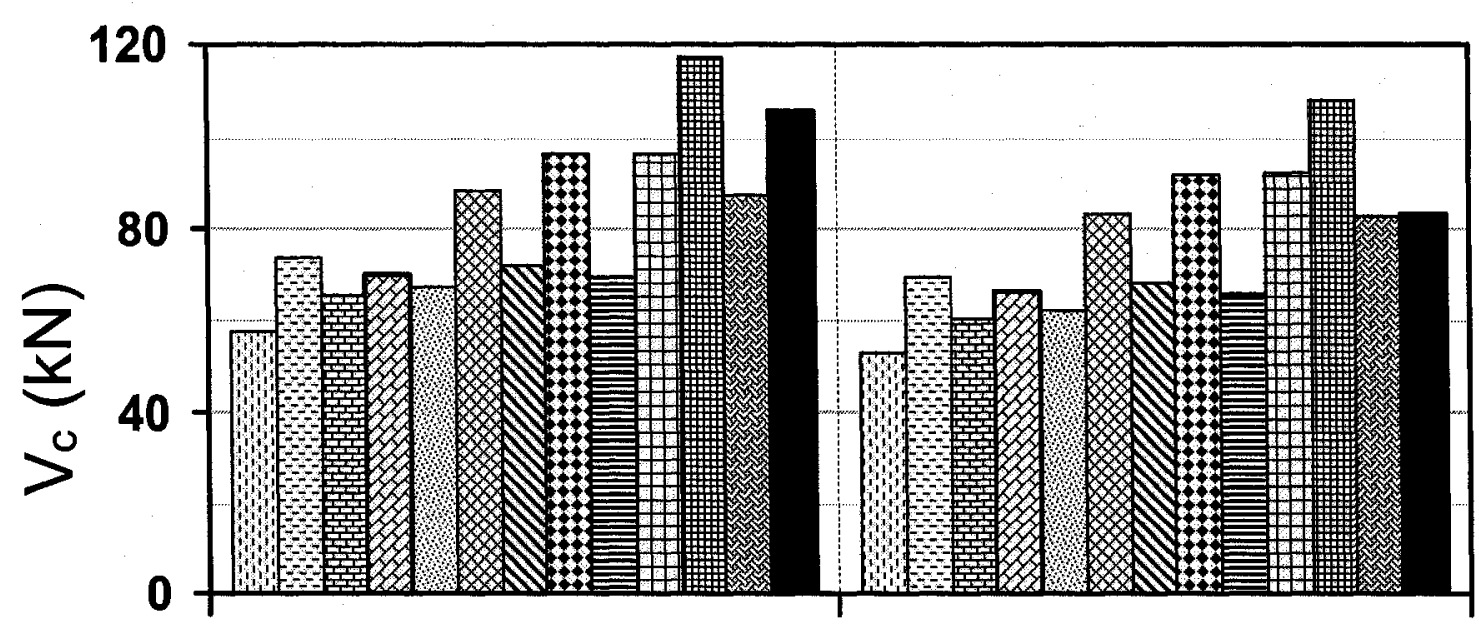

SEV-4.0N SEM-4.0N

\begin{tabular}{|c|c|c|}
\hline $\begin{array}{l}\text { Experimental } \\
\text { Bazant-Yu (2) } \\
\text { B CEB-FIP } \\
\text { Q CSA (General) } \\
\text { ACI-318 (11-5) }\end{array}$ & $\begin{array}{l}\text { Zsutty } \\
\text { 图 Gastebled-May } \\
\text { EC-2 } \\
\text { 国 CSA (Simplified) }\end{array}$ & $\begin{array}{l}\text { Bazant-Yu (3) } \\
\text { Bazant-Yu (1) } \\
\text { ACI-318 (11-3) } \\
\text { : EHE }\end{array}$ \\
\hline
\end{tabular}

Figure 8.4e: Experimental and predicted ultimate shear strength of RRC beams with $a / d=4.0$

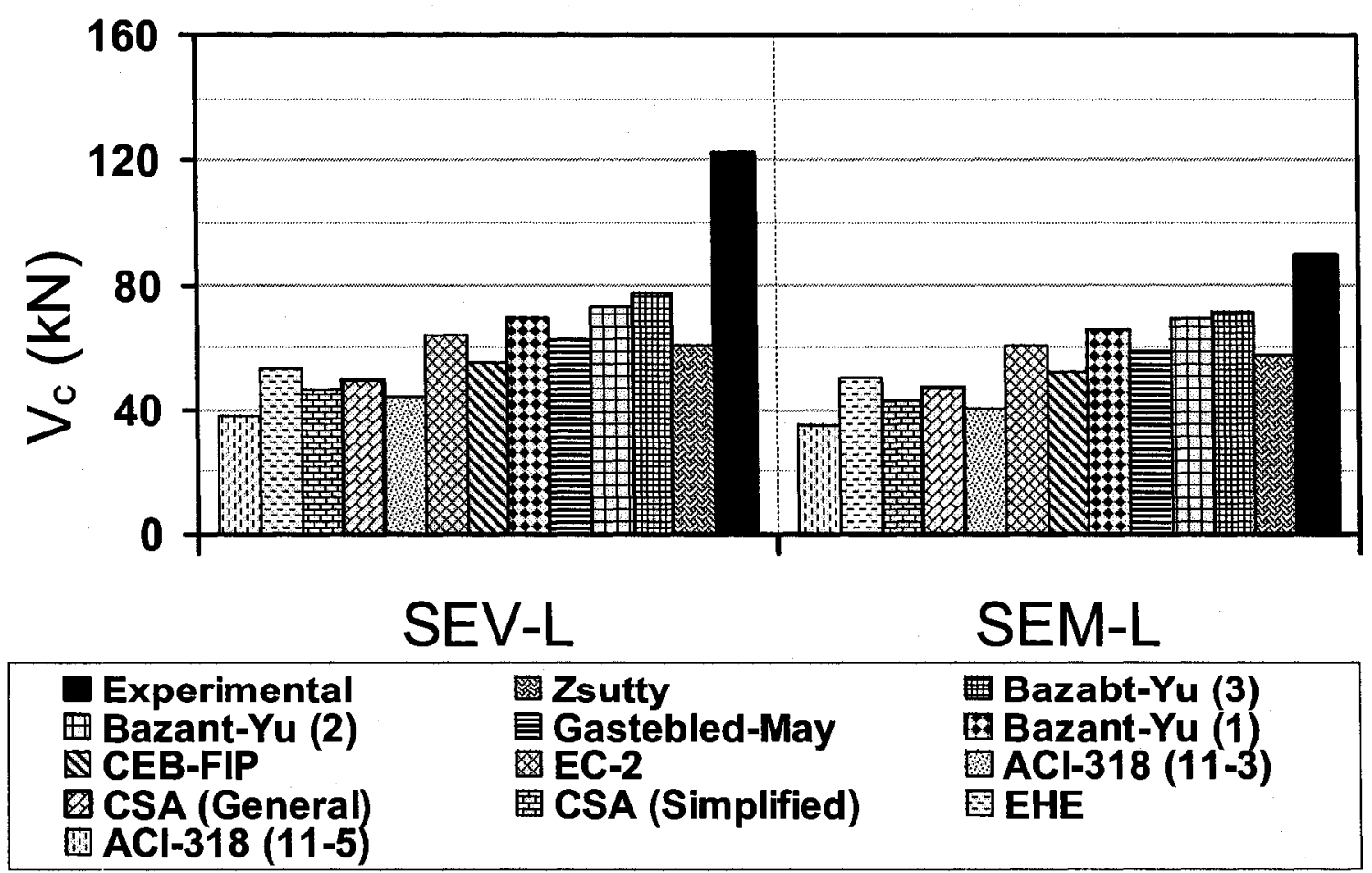

Figure 8.5a: Experimental and predicted ultimate shear strength of RRC beams with $d=200 \mathrm{~mm}$ 


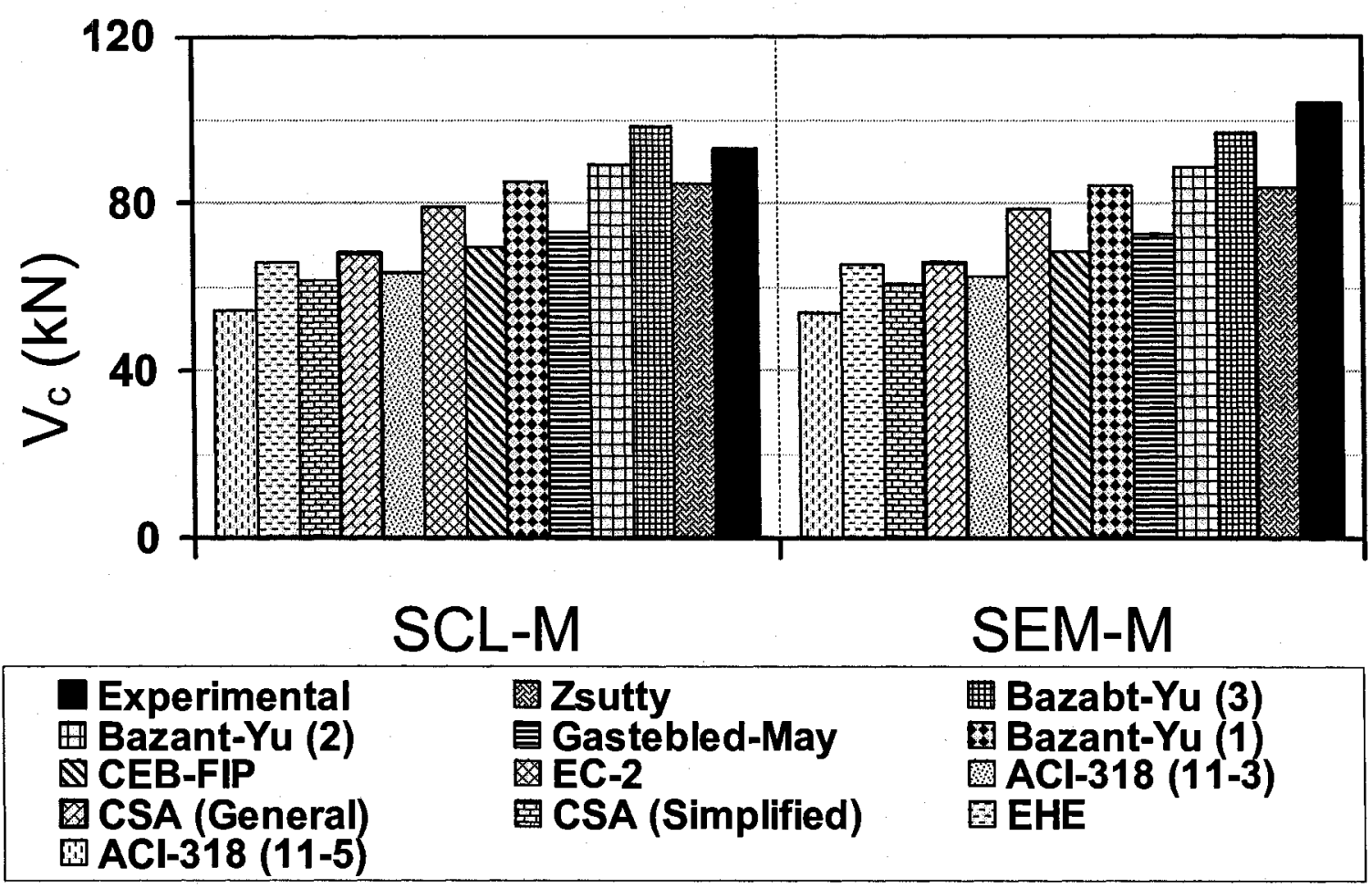

Figure 8.5b: Experimental and predicted ultimate shear strength of SEM and SCL beams with $d=300 \mathrm{~mm}$

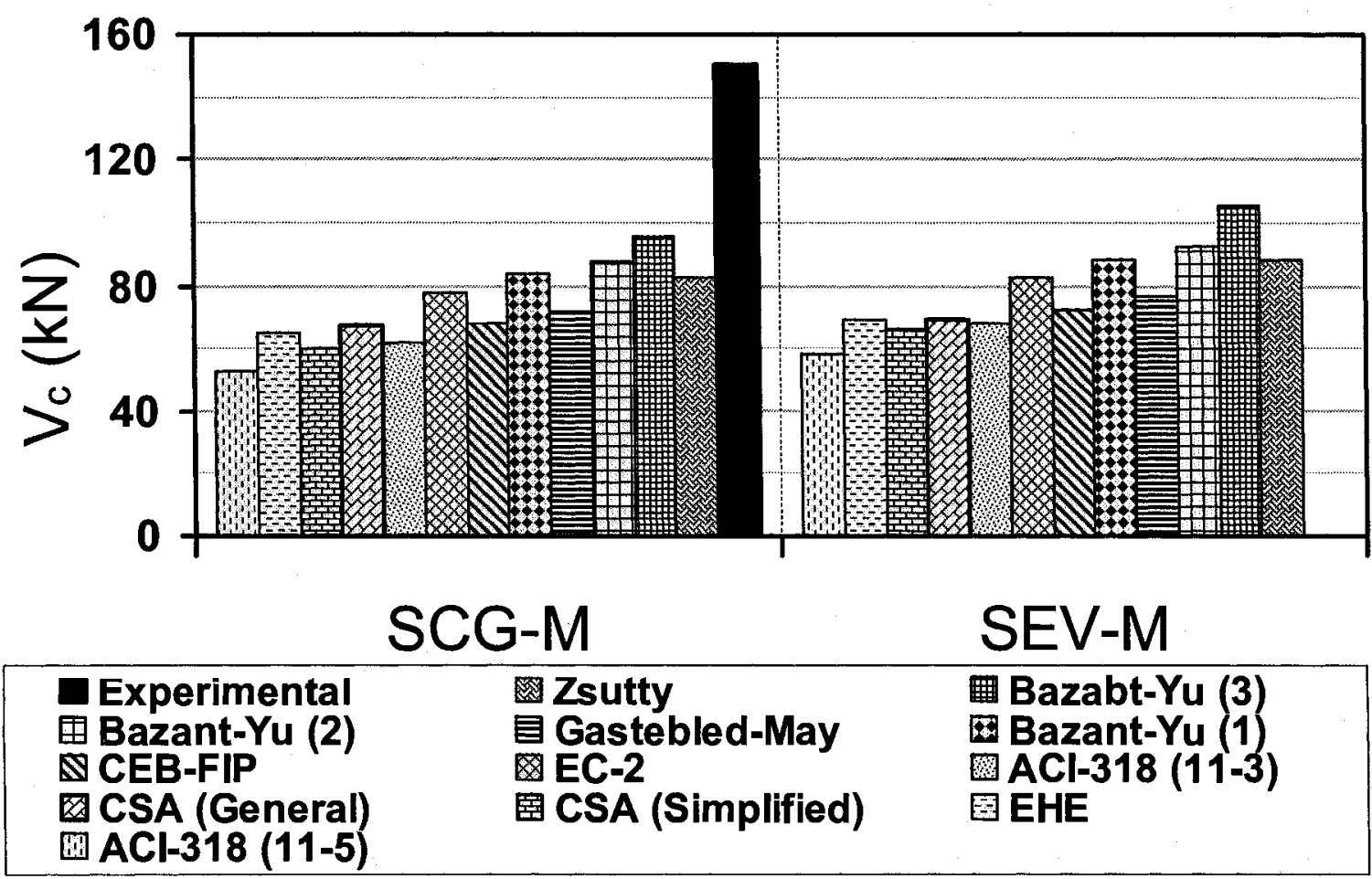

Figure 8.5c: Experimental and Predicted ultimate shear strength of SCG and SEV beams with $d=300 \mathrm{~mm}$ 


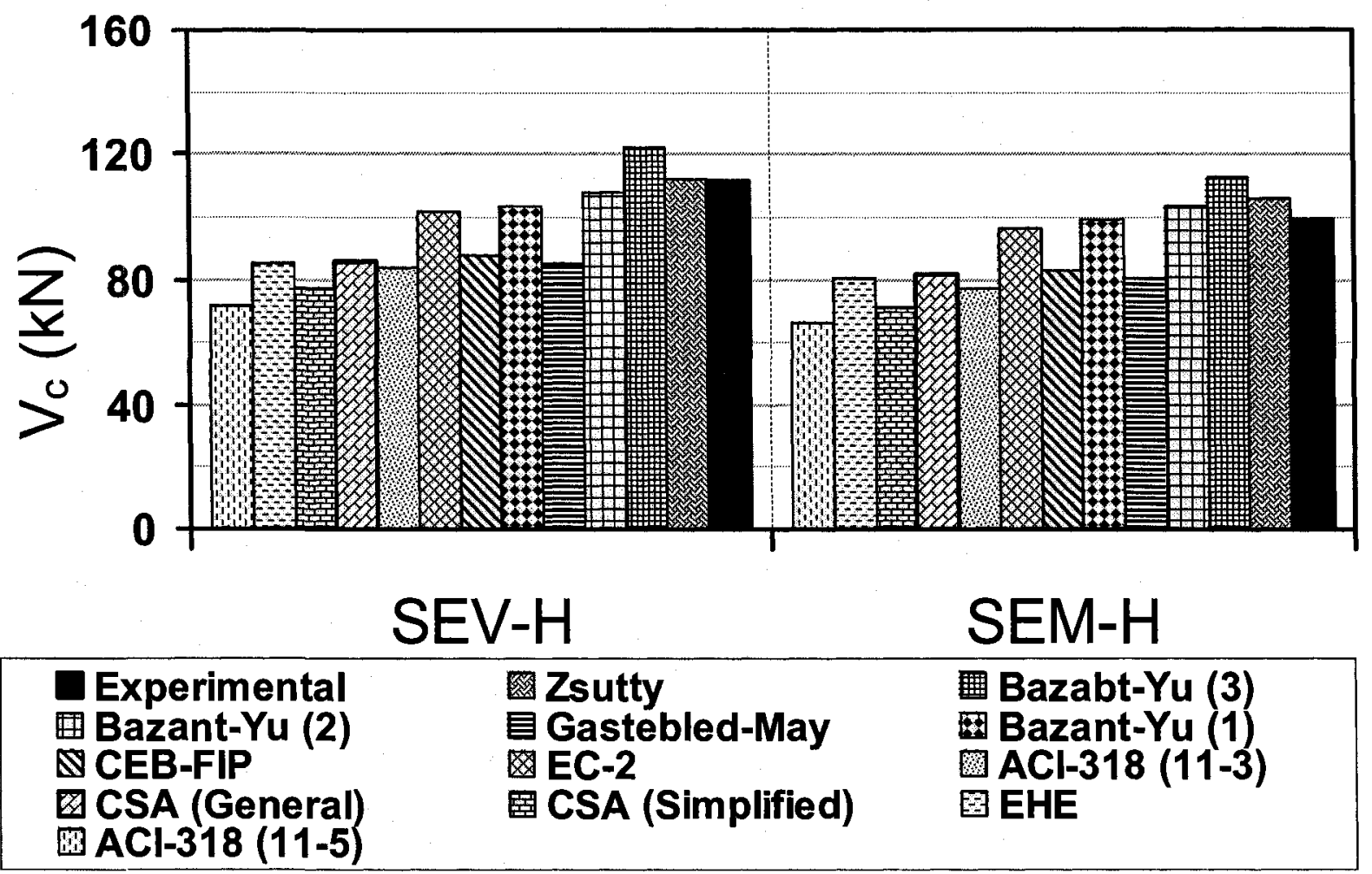

Figure 8.5d: Experimental and predicted ultimate shear strength of RRC beams with $d=400 \mathrm{~mm}$

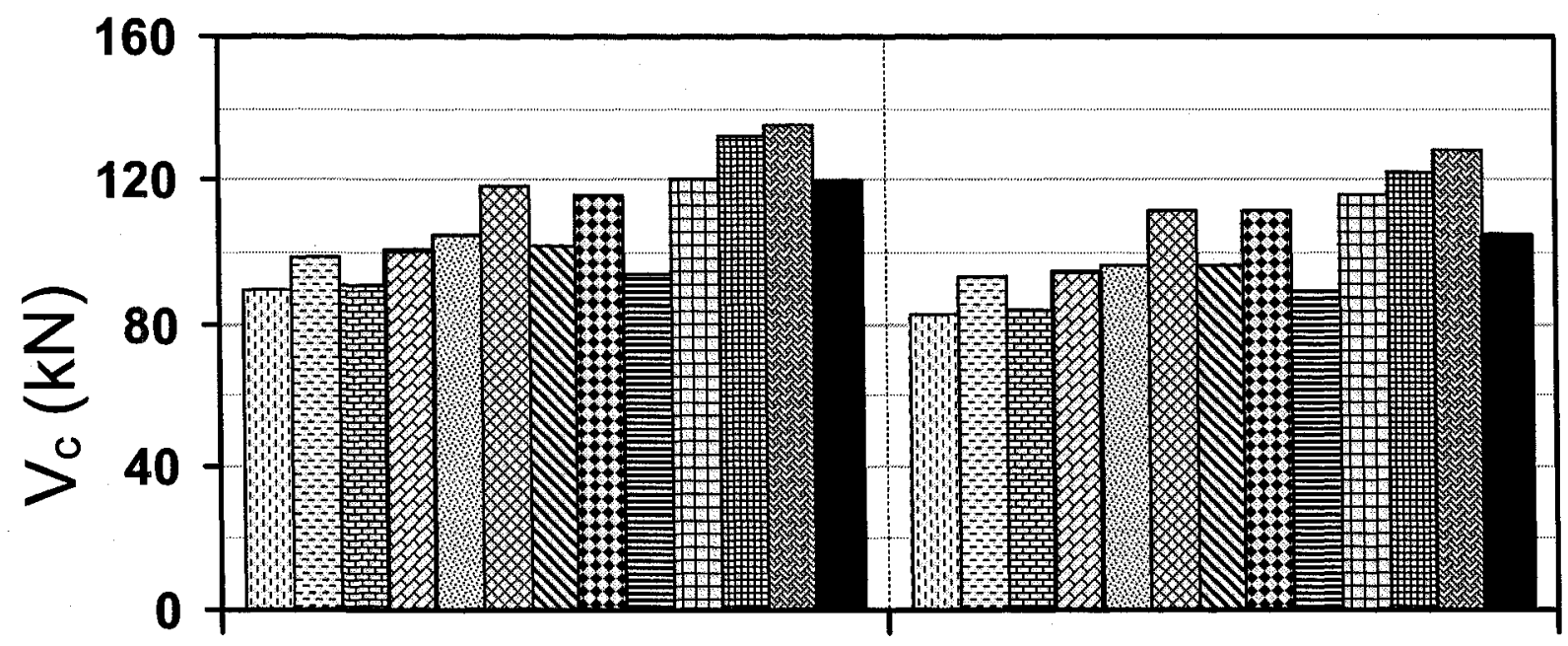

\section{SEV-HH SEM-HH}

\begin{tabular}{|c|c|c|}
\hline $\begin{array}{l}\text { Experimental } \\
\text { 田Bazant-Yu (2) } \\
\text { CEB-FIP } \\
\text { CSA (General) } \\
\text { ACl-318 (11-5) }\end{array}$ & $\begin{array}{l}\text { Zsutty } \\
\text { 图 Gastebled-May } \\
\text { EC-2 } \\
\text { CSA (Simplified) }\end{array}$ & $\begin{array}{l}\text { 嘈 Bazabt-Yu (3) } \\
\text { 国 Bazant-Yu (1) } \\
\text { ACl-318 (11-3) } \\
\text { 圈 EHE }\end{array}$ \\
\hline
\end{tabular}

Figure 8.5e: Experimental and predicted ultimate shear strength of RRC beams with $d=500 \mathrm{~mm}$ 


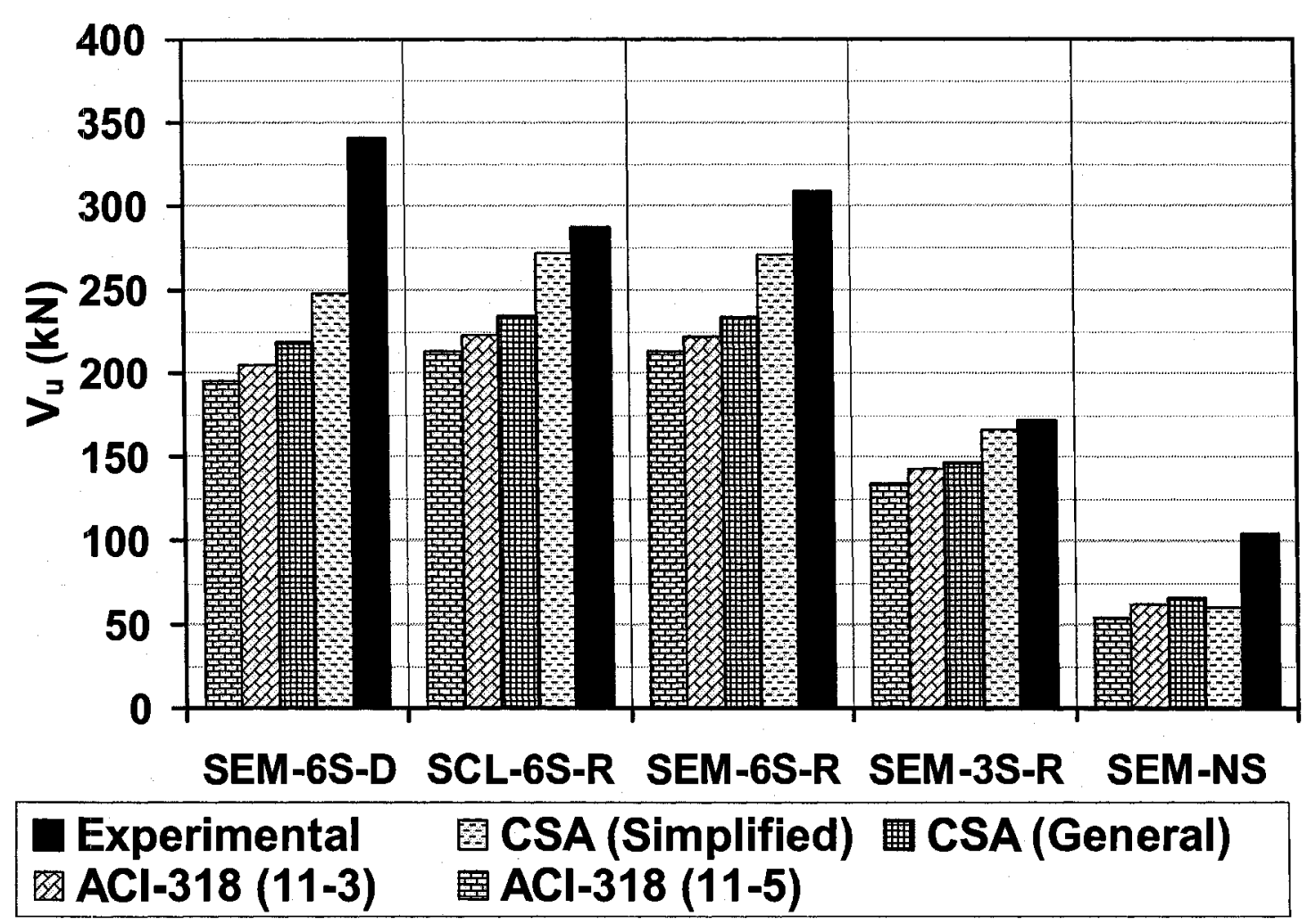

a)MO-Beams

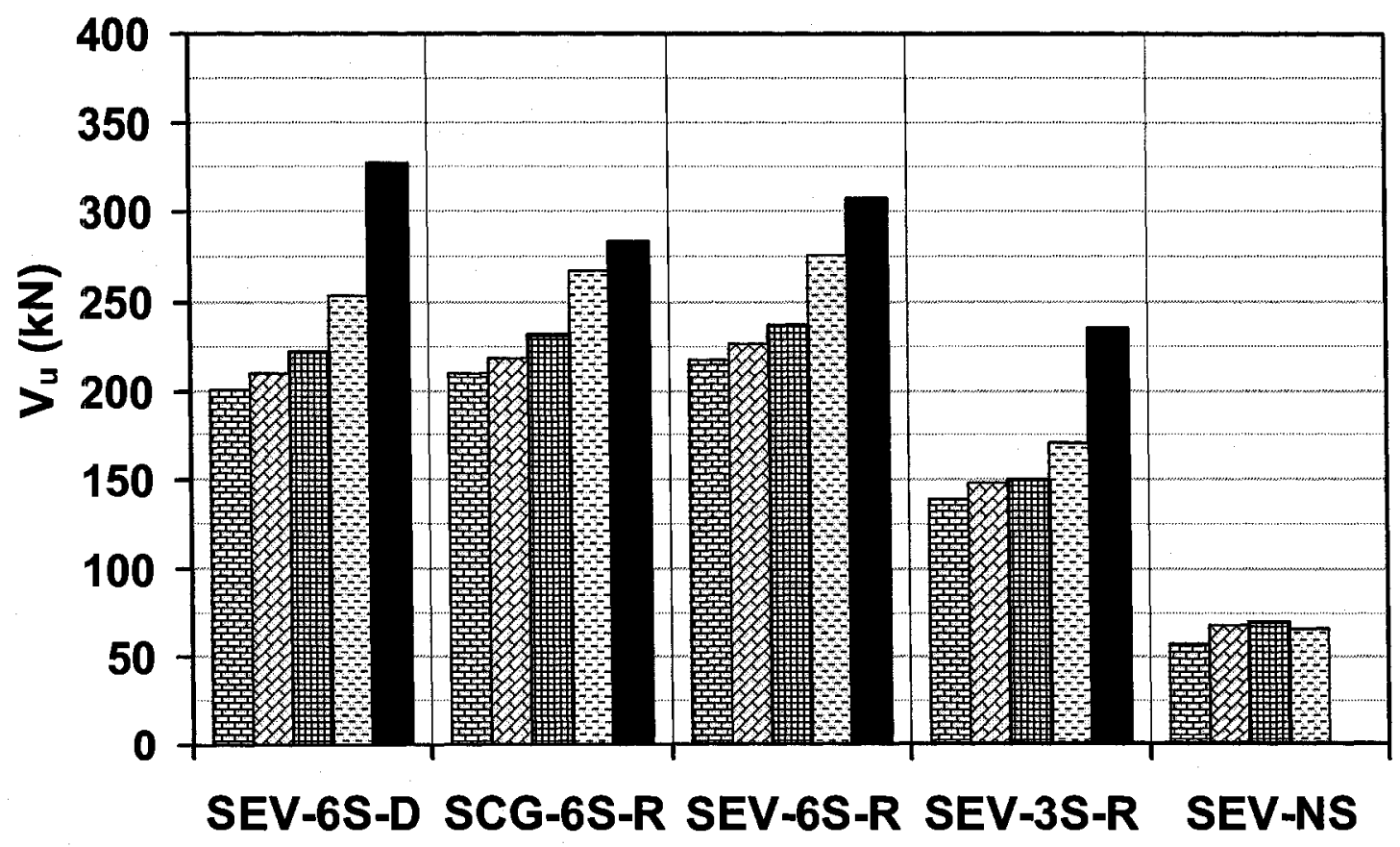

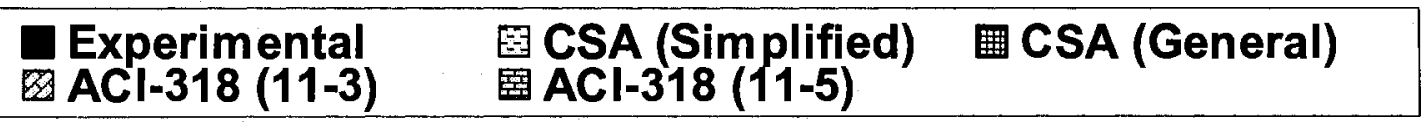

b)VA-Beams

Figure 8.6: Experimental and predicted shear resistance of beams with stirrups 


\section{CHAPTER 9: CONCLUSIONS AND FUTURE WORK}

\subsection{Summary}

The results of a comprehensive study dealing with the structural performance of steel reinforced recycled concrete (RRC) members are presented in this thesis. The research program was planned to fill the identified gaps in the existing literature on the subject and to provide data which would help to remove the fundamental barriers to the use of RAC as structural-grade concrete in the construction industry.

The current study focused on the short- and long-term behaviour of RAC as a new material and on the flexural and shear behaviour of reinforced RRC beams. As stated in the state of the art (Chapter 2), one of the main concerns about the use of RCA is the preconceived notion of its inferiority (i.e., lower fresh and hardened densities, lower elastic modulus, higher creep and shrinkage, etc.) compared to conventional concrete. In this study it was discovered that RAC is not inherently inferior, but the inferiority emanates from using improper concrete mix proportioning method. Therefore, a new concrete mix proportioning method was developed and verified experimentally. It was also determined based on extensive testing that from the structural point of view RAC is not fundamentally different from conventional concrete and that methods of structural design developed for conventional concrete could be used to design RRC structures, provided the mechanical properties of RAC are duly accounted for.

The other major findings of the study are encompassed by the following detailed conclusions. 


\subsection{Conclusions}

- The main factor responsible for the reported inferiorities of RAC is the improper mix proportioning method used in the past rather than factors such as RCA content, residual mortar content, and residual mortar quality, as reported by previous investigators. In other words, in the conventional mix proportioning method, RCA is simply considered as a one-phase material (solid coarse aggregate), and no consideration is given to the volume of the attached residual mortar. Therefore, the concrete produced in this manner has a larger total mortar content (i.e., residual mortar plus new mortar) compared to a similar concrete made entirely with natural aggregates.

- The w/c ratio is the main factor affecting the slump of RAC mixes, followed by the angularity and surface roughness characteristics of the RCA.

- Increase of the RCA content might result in lower or higher slump depending on the angularity and surface roughness characteristics of the RCA compared to NA. For instance, the less angular RCA-MO compared to NL led to higher slump in RAC at higher RCA contents, while the more angular RCA-VA compared to NG, mainly resulted in lower slump at higher RCA content. This completely explains the contradictory findings of the previous studies in the literature regarding the effect of RCA content on slump.

- The w/c ratio and air content are the main factors affecting the compressive and splitting tensile strength of RAC. The RCA content is not a major factor affecting the compressive and splitting tensile strength of RAC due to better quality of RM compared to NM. 
Based on the results of the preliminary study, a new mix proportioning method was developed and presented in Chapter 4, whose main premise is maintenance of equivalent total mortar volume in RAC and the companion NAC. RAC proportioned on this basis will not suffer from the aforementioned inferiorities originated. To verify the method, a comprehensive research program was carried out, comprising the following tasks:

- Evaluation of the fresh properties (the slump and fresh density), short term properties (hardened density, compressive strength, elastic modulus, and stress-strain relationship) and long term properties (creep and shrinkage) of RAC mixes proportioned according to the proposed EMV method.

- Investigation of the bond performance of deformed bars in RAC, the flexural performance of steel reinforced recycled concrete (RRC) beams with different longitudinal reinforcement ratios, and the shear performance of RRC beams by studying the effects of $a / d$ ratio, shear reinforcement ratio and beam size (size effect).

- Development of different models for fresh and hardened density, elastic modulus, compressive strength, creep and shrinkage of RAC, and their experimental verification.

- Evaluation of the applicability of existing empirical equations, developed originally for NAC, to (1) predict the elastic modulus, splitting tensile strength, stress-strain properties, creep and shrinkage of RAC; and (2) predict the cracking moment, ultimate flexural and shear strengths, and immediate deflection of RRC beams.

The following sections provide the principal conclusions of the above investigations. 


\subsubsection{Evaluation of the Proposed Mix Proportioning Method}

\subsubsection{Short-Term Properties}

- Mixes designed by the EMV method generally resulted in comparable or higher fresh and hardened density and elastic modulus values in RAC mixes, for both RCA sources, compared to those of the companion NAC mixes. On the other hand, the RAC mixes proportioned by conventional method experienced lower fresh and hardened density and elastic modulus values than those of the companion NAC mixes. However, the mix proportioning method did not have major effect on the compressive strength of RAC mixes because strength is mostly dependant on the strength of mortar and the quality of bond and interface between aggregates and mortar rather than total mortar volume. Note that the cement and water contents in RAC mixes proportioned by the conventional and EMV methods were identical.

- For the same cement and water contents, regardless of RCA source and SCM's addition, higher slump values were observed for all RAC mixes proportioned by the EMV method compared to those proportioned by the conventional method.

\subsubsection{Stress-Strain Relationship}

- The mix proportioning method and the aggregate-mortar bond and interface quality were the most important factors affecting the ascending branch and the value of strain corresponding to peak stress in the stress-strain curves of RAC mixes. In the case of RCA-MO, the mix proportioning method was the dominant factor because applying the EMV method resulted in similar stress-strain behaviour, but in slightly higher strain value corresponding to peak stress for RAC mixes compared to the companion NAC mixes. However, for RCA-VA, the quality of aggregate-mortar interface and bond were found to 
be the more dominant factors. These factors resulted in slightly smoother ascending branch and higher strain value corresponding to peak stress in RAC compared to NAC.

- Based on the shape of the descending branch of their stress-strain curves, the RAC mixes proportioned by the EMV method were found to be generally more ductile compared to mixes proportioned by conventional method with or without RCA.

\subsubsection{Long-Term Properties}

- Generally, the RAC mixes proportioned by conventional method experienced higher creep compared to the companion NAC mixes. However, for mixes designed by the EMV method, the creep of RAC mixes were comparable to, or even lower, than those of the companion NAC mixes.

- Despite the slightly higher drying shrinkage values at early ages, generally the RAC mixes proportioned by the EMV method had comparable or lower shrinkage values at later ages than the companion NAC while for RAC mixes proportioned by the conventional method, the shrinkage values were always higher.

\subsubsection{Bond Performance}

- Generally, the bond strength of all RAC mixes proportioned by the conventional method was lower than those of companion NAC for both bar sizes and RCA sources, while those proportioned by the EMV method showed comparable or higher bond strength compared to those of companion NAC. The lower TNA volume or higher TM volume in RAC proportioned by the conventional method may decrease the bond strength due to a reduction in the number of potential sites where the lugs of the rebars could interlock with natural aggregates in the surrounding concrete. 
- Irrespective of mix proportioning method and aggregate, the bond strength of No. 15 bar was always higher than that of No.30 bar.

- Despite the lower bond strength of specimens made of RAC proportioned by the conventional method and/or specimens containing larger bar size (No.30), the overall bond behaviour was found to be independent of the mix proportioning method, aggregate type (RCA-MO or RCA-VA, and NL or NG), and concrete type (RAC or NAC).

\subsubsection{Flexural Performance}

- There was no major difference observed between the failure modes of flexural RRC and conventional RC beams. Furthermore, similar failure modes were observed for all flexural RRC beams regardless of RCA source.

- Despite slightly smaller crack spacing in RRC beams compared to conventional RC beams, there was no significant difference observed between the corresponding cracking patterns at failure.

- Despite the slightly lower cracking moment in FEM-CMP compared to FCL-CMP, there was no major difference observed between the cracking moments of the flexural RC beams made with RCA compared to those made with NAC.

- The ultimate flexural strength of the RRC beams, with or without compression steel, was always higher than those of conventional RC beams for both RCA sources.

- The cracking moment of the RC beams, with or without compression steel and made with RAC, was generally lower than the corresponding moment of the companion NAC beams. 
- Generally, the RCA source and type of aggregate did not have any major effect on the load-midspan deflection response of RRC beams with or without compression steel. This confirms the applicability of the general flexural theory of conventional RC beams to RRC beams.

- The midspan deflections at service load for all RRC and RC beams tested in flexure were found to be within the maximum permissible values recommended by CSA A23.304 for members supporting or attached to non-structural elements likely to be damaged by large deflections.

\subsubsection{Shear Performance}

- There was no major difference observed between the failure modes, cracking patterns and shear performance of RRC beams and conventional RC beams. Generally, in the RRC beams the concrete contribution to shear resistance $\left(v_{c}\right)$ was found to be higher and the behaviour to be more ductile after the formation of diagonal cracking compared to conventional RC beams. Note that the RRC beams made of RAC proportioned by the conventional method have been reported by others to have lower ultimate shear strength and to be less ductile compared to conventional RC beams.

- Irrespective of the RCA source, the shear strength of RRC beams increased with decrease in the $a / d$ ratio. This is mainly due to the higher contribution of arch action mechanism at lower a/d ratios, a phenomenon that is well known for conventional RC beams.

- Irrespective of the RCA source, the shear strength of RRC beams increased due to decrease of the overall depth of the beam. This is due to the so-called size effect and the diminishing effectiveness of aggregate interlock mechanism at higher depths. 
- Irrespective of the RCA source and as expected, the ultimate shear strength of RRC beams consistently increased with increase of their shear reinforcement ratio. This is primarily due to the higher contribution of the shear reinforcement $\left(v_{s}\right)$ to their shear resistance.

- Despite slightly larger maximum diagonal crack widths in RRC beams compared to $\mathrm{RC}$ beams at $a / d=2.7$, all observed crack widths in RRC beams at different $a / d$ ratios and depth values were below the maximum crack width specified by CSA A23.3-04.

- Generally, the RCA source and aggregate type did not have any major effect on the load-midspan deflection and load-diagonal deformation response of RRC beams at identical $a / d$ ratios, depth values, and shear reinforcement ratios.

\subsubsection{Developed Models for Short and Long Term Properties of RAC}

- The predicted fresh and hardened densities of RAC using the proposed phase-based model (Eq.5.10) agree well with the corresponding experimental data.

- The predicted fresh and hardened densities of RAC using either of the proposed mixture-based models (Eq.5.16 or 5.18) were more accurate compared to the predicted values using the phase-based models. Furthermore, Eq.5.16 was mainly applicable to RAC mixes proportioned by the conventional method while Eq.5.18 was applicable to all RAC mixes regardless of the mix proportioning method.

- Irrespective of the mix proportioning method, the predicted elastic modulus of RAC using the proposed phase-based models (Eq.5.26 to 5.30) agreed well with the experimental values for all RAC mixes.

- The predicted elastic modulus of RAC using either of the proposed mixture-based models (Eq.5.31 to 5.35 or Eq.5.36 to 5.40) were in good agreement with the 
experimental data. Furthermore, Eqs.5.31 to 5.35 were mainly applicable to RAC mixes proportioned by the conventional method, while Eqs.5.36 to 5.40 were applicable to all RAC mixes proportioned by either method.

- The predicted compressive strength of RAC using the proposed EMV models (Eq.5.47 to 5.52) agreed well with the corresponding experimental values provided by Hansen and Narud (1983). However, in case of high strength RAC made of low strength RCA, the model for light-weight-aggregate (Eq.5.54a) gave more accurate prediction.

- Both EMV and LWA models revealed that a high-strength RAC could be produced even if a lower strength RCA is, provided a high quality NM is utilized.

- The predicted creep and shrinkage of RAC using the proposed Eq.5.60 and 5.67 were in relatively good agreement with the experimental values of all RAC mixes proportioned by the conventional or the EMV method.

\subsubsection{Evaluation of Existing Empirical Equations}

\subsubsection{Short and Long Term Properties of RAC}

- Regardless of the w/c ratio, source of RCA, RCA content and air-content, the empirical equation of ACI 318-05 for the splitting tensile strength was found applicable to RAC.

- The simple empirical equations of ACI 318-05 and CSA A23.3-04 (clause 8.6.2.3) were found to be overestimating the elastic modulus of RAC mixes proportioned by the conventional method, while for RAC mixes proportioned by the EMV method, the empirical equations gave reasonable results.

- The general empirical equations of ACI 363 and CSA A23.3-04 (clause 8.6.2.2) were found applicable to all RAC mixes regardless of the mix proportioning method. 
- Generally, the recommended expressions by Gardner, Nassif et al., and Tomasawa et al. were all found applicable to RAC. However, the AIJ equation was valid mostly for those RAC mixes with compressive strength of $36 \mathrm{MPa}$ or less, as recommended by AIJ. The Euocode-2 equation was found unconservative for all RAC mixes regardless of the mix proportioning method.

- Compared to other empirical equations, the equation proposed by Thorenfeldt et al. (modified by Collins and Mitchell (1990)) was found to fit better the ascending branch of the compressive stress-strain curve for all NAC and RAC mixes regardless of the mix proportioning method. On the other hand, the descending branch of the curve for most of the RAC and NAC mixes was captured relatively better by the Smith-Young model (1955).

- For all RAC and NAC mixes, irrespective of aggregate type or mix proportioning method, the normalized creep coefficient and drying shrinkage strain variation with time predicted by both the CEB and the ACI empirical equations agreed well with the corresponding experimental values.

- Neither the ACI nor the CEB empirical equation for creep and shrinkage of normal concrete considers the effect of RCA content or residual mortar volume on creep and shrinkage of RAC. By applying the proposed residual mortar coefficients of $K_{R M}$ and $S_{R M}$ to the empirical equations of $\mathrm{ACI}$ and $\mathrm{CEB}$, they were found to be applicable to RAC mixes proportioned by conventional or EMV method.

\subsubsection{Ultimate Flexural Strength}

- Despite some overestimated cracking moment values in some RRC beams, generally the CSA A23.3-04 proposed equation for prediction of cracking moment for $\mathrm{RC}$ beams 
(clause 8.6.4) was found to be applicable to RRC beams. However, using observed splitting tensile strength instead of predicted modulus of rupture values based on CSA A23.3-04 (clause 8.6.4) generally gave more conservative estimate of the cracking moment in both SEM and SEV beams.

- Generally, the CSA A23.3-04 (clause 10.1.7) expressions for predicting the equivalent rectangular stress block parameters $(\alpha$ and $\beta$ ) for conventional concrete were found applicable to all RAC mixes regardless of mix proportioning method.

- The CSA A23.3-04 flexural design provisions for conventional RC members were found applicable to all RRC beams regardless of their longitudinal tension reinforcement ratio, RCA sources, and compression steel content.

- Generally, there was no major difference observed between the predicted ultimate flexural strength values of RRC beams when using different equivalent $\alpha$ and $\beta$ values based on CSA A23.3-04 or actual and empirical stress-strain curves.

- Both CSA A23.3-04 and Eurocode2 standards for predicting the immediate deflections of conventional RC members were found applicable to RRC beams. However, Eurocode2 (2002) was found more accurate in flexural beams at low longitudinal tension reinforcement ratios.

\subsubsection{Ultimate Shear Strength}

- Generally, both the simplified and the general methods of CSA A23.3-04 and ACI318 were found applicable to both SEM and SEV beams for all a/d ratios and depth values with or without shear reinforcement. 
- Similarly, all empirical equations proposed by Gastebled and May, EHE, CEB-FIP and Eurocode- 2 were found applicable to all RRC beams for all a/d ratios and depth values.

- The equations of Bažant and Yu were applicable to all RRC beams with $a / d$ ratios up to 2.7 and overall depth not exceeding $375 \mathrm{~mm}$.

- Zsutty's equation generally gave the most accurate prediction of concrete contribution to shear resistance for $a l l \mathrm{a} / \mathrm{d}$ ratios (especially at lower $a / d$ ratios). However, for RRC beams with depth of $450 \mathrm{~mm}$ or $550 \mathrm{~mm}$, its results were unconservative.

\subsection{Recommendations for Future Work}

- Although two RCA sources used in this study (RCA-MO and RCA-VA) had significantly different mechanical and physical properties, and the results of this study can be extrapolated to other RCA sources, it is beneficial to expand the study to cover other types of RCA from different sources, especially those made of low-strength concrete.

- Evaluation of EMV mix proportioning method applicability to RAC mixes made of both coarse and fine RCA.

- Evaluation of the EMV mix proportioning method for mixes with different $w / c$ ratios is recommended.

- Although the EMV method can be used for different RCA contents without producing inferior concrete compared to NAC, it is useful to evaluate its validity for RCA contents closer to the maximum possible values, and more specifically in special concretes such as zero slump concrete, cast stone masonry, etc. 
- Although in this study the lower OVA size in RRC beams compared to the maximum NA size in RC beams did not affect the ultimate shear strength values, it is useful to investigate the shear behaviour of RRC beams made of RAC with a nominal maximum OVA size equal to the NA.

- Due to both time and resource limitations, the control RC beams did not include all longitudinal and shear reinforcement ratios, a/d ratios and depth values as in the companion RRC beams. Therefore, it would be useful to compare the flexural and shear performance of RRC beams with control RC beams at different longitudinal and shear reinforcement ratios, a/d ratios and depth values.

- Due to limited RCA available for this study, the range of $a / d$ ratios and depth were 1.5-4.0 and 250-550 mm, respectively. While the results of this study can be extrapolated to other $a / d$ ratios and depth values, it would be useful to expand the study to cover other a/d ratios and especially greater depths.

- To be able to use RCA in a wide range of structures, the behaviour of RRC columns and slabs, particularly, the direct and punching shear behaviour of slabs, as well as the performance of prestressed RRC members, need be investigated. 


\section{REFERENCES}

Abbas, A., Fathifazl, G., Isgor, O.B., Razaqpur, A.G., Fouriner, B., and Foo, S. $2006 a$. Environmental Benefits of Green Concrete. Climate Change Conference, Ottawa, Ontario.

Abbas, A., Fathifazl, G., Isgor, O.B., Razaqpur, A.G., Fournier, B., and Foo, S. 2006 b. Effect of Residual Mortar Content on the Properties of Recycled Aggregate: Experimental Study, Submitted to the ACI Structural and Materials Journals in October 2006.

Abbas, A., Fathifazl, G., Isgor, O.B., Razaqpur, A.G., Fournier, B., and Foo, S. 2006c. Effect of Residual Mortar Content on the Properties of Recycled Aggregate: Rational Study, Submitted to the ACI Structural and Materials Journals in October 2006.

Abbas, A., Fathifazl, G., Isgor, O.B., Razaqpur, A.G., Fournier, B., and Foo, S. $2006 \mathrm{~d}$. Proposed Method for Determining the Residual Mortar Content of Recycled Concrete Aggregates, CSCE 2006 Annual Conference, May 23-26, 2006, Calgary, Alberta, Canada.

Abbas, A., Fathifazl, G., Isgor, O.B., Razaqpur, A.G., Fournier, B., and Foo, S. 2007 a. Qualification of Recycled Concrete Aggregate for Use in Structural Grade Concrete, Sustainable Construction Materials and Technologies Conference, June 11-13, 2007, Coventry, United Kingdom.

Abbas, A., Fathifazl, G., Isgor, O.B., Razaqpur, A.G., Fournier, B., and Foo, S. $2007 b$. Proposed Method for Determining the Residual Mortar Content of Recycled Concrete Aggregates" Submitted to the ASTM Journal in February 2007. 
Abbas, A., Fathifazl, G., Fournier, B., Isgor, O.B., Razaqpur, A.G., and Foo, S. 2007c. Quantification of the Residual Mortar Content in Recycled Concrete Aggregates by Image Analysis, 11th Euoseminar on Microscopy Applied to Building Materials, June $5-7,2007$.

Abbas, A. 2007. Durability of Green Concrete as a Structural Material," Ph.D. Dissertation, Carleton University, Ottawa, Canada , 411 pp.

ACI Committee 209. 1982. Prediction of Creep, Shrinkage and Temperature Effects in Concrete Structures. Designing for Creep and Shrinkage in Concrete Structures. ACI Publication SP-76, American Concrete Institute, Detroit, pp. 193-300.

ACI Committee 211, 1997. Standard Practice for Selecting Proportions for Normal, Heavyweight and Mass Concrete, ACI 211.1-97, American Concrete Institute, Farmington Hills, Michigan.

ACI Committee 318. 2005. Building Code Requirements for Structural Concrete. ACI 318-05, Farmington Hills, M.I.

ACI Committee 363. 1997. State-of-the-Art Report on High-Strength Concrete, ACI 363R-92, Farmington Hills, M.I., p. 55.

ASCE-ACI Committee 426. 1973. The Shear Strength of Reinforced Concrete Members. Journal of the Structural Division, ASCE, 99(ST6): 1091-1187.

ASCE-ACI Task Committee 445. 1998. Recent Approaches to Shear Design of Structures. Journal of the Structural Engineering, ASCE, 124(12): 1375-1417. ASTM. 1979. Standard Definitions of Terms Relating to Concrete and Concrete Aaggregates. American Standards for Testing and Materials, ASTM 125-79a, Philadelphia. 
ASTM. 1982. Standard Specification for Concrete Aggregates. American Standards for Testing and Materials, ASTM C 33-82, Philadelphia.

ASTM. 1993. Length Change of Hardened Hydraulic-Cement Mortar and Concrete ASTM C 157-93.

ASTM. 1994. Creep of Concrete in Compression ASTM C 512-87 (Reapproved 1994).

ASTM. 1994. Test for Compressive Strength of Cylindrical Concrete Specimens ASTM C 39-94.

ASTM. 1994. Test for Static Modulus of Elasticity and Poisson's Ratio of Concrete in Compression ASTM C 469-94.

ASTM. 1996. Test for Splitting Tensile Strength of Cylindrical Concrete Specimens ASTM C 496-96.

ASTM. 1999. Standard Test Method for Comparing Bond Strength of Steel Reinforcing Bars to Concrete Using Beam-End Specimens ASTM A 944-99 (Reapproved 2004).

ASTM . 2003. Concrete and Concrete Aggregates. Annual Book of American Society for Testing and Materials, Volume 4.02, ASTM, Philadelphia.

Ajdukiewicz, A., and Kliszczewicz, A. 2002. Influence of Recycled Aggregates on Mechanical Properties of HS/HPC. Cement and Concrete Composites, 24 (2): 269279.

Alaejos, P., Sanchez, M., Dapena, E.,Vazquez, E., Barra, M., Eteberria, M., Mari, A., Agullo, L., Martinez, F., Fonteboa, B., Polanco, A., Franciso,G., Aleza, F., Parra, J.L., and Burón, M. 2004. Draft of Spanish Regulations for the Use of Recycled Aggregate in the Production of Structural Concrete. Conference on the Use of 
Recycled Materials in Building and Structures, Barcelona, Spain. Available at: http://congress.cimne.upc.es/rilem04/frontal/papers.htm.

Bažant, Z.P., and Yu, Q. 2005. Designing Against Size Effect on Shear Strength of Reinforced Concrete Beams Without Stirrups. Journal of Structural Engineering, 113(12): $1877-1885$.

Buck A. D., 1976. Recycled Concrete. Report No. 2: Additional Investigations, Miscellaneous Paper No. C-72-14(2), U. S. Army Engineer Waterways Experiment Station, Vicksburg.

Bureau de normalization du Quebec (BNQ) 2002. Classification and Characteristic of Recycled Material from Concrete Construction Debris Coated with Bitumen and Bricks. Recycle material specifications. Quebec.NQ2560-600/2002.

Buyle-Bodin F., and Hadjieva-Zaharieva, R. 2002. Influence of Industrially Produced Recycled Aggregates on Flow Properties of Concrete. Materials and Structures/Materiaux et Constructions, 35 (252): 504-509.

Canadian Standard Association (CSA). 2004. Design of Concrete Structures, CSA Standard A23.3-04, Rexdale, Ontario.

Chen H. J., Yen T., and Chen K.H. 2003. Use of Building Rubbles as Recycled Aggregates. Cement and Concrete Research, 33 (1): 125-132(8).

Collins, R. J. 1994. The Use of Recycled Aggregates in Concrete. Building Research Establishment.

Collins, M.P., and Kuchma, D. 1999. How Safe Are Our Large, Lightly Reinforced Concrete Beams, Slabs, and Footings. ACI Structural Journal, 96: 482-490. 
Comitè Euro-International du Bèton, CIB-FIP Model Code 1990, Thomas Telford Services Ltd. London, 1993, 437 pp.

Comité Européen de Normalisation (CEN) (2005), Eurocode 2: Design of Concrete.

Corinaldesi, V., and Moriconi, G. 2001. Role of Chemical and Mineral Admixtures on Performance and Economics of Recycled Aggregate Concrete, ACI Special Publications, vol. 199, no. 2, p 869-884.

Corinaldesi, V., Tittarelli, F., Coppola, L., and Moriconi, G. 2001. Feasibility and Performance of Recycled Aggregate in Concrete Containing Fly Ash for Sustainable Buildings. ACI Special Publications, 202: 161-180.

Corinaldesi V., Giuggiolini M., and Moriconi G. 2002. Use of Rubble from Building Demolition in Mortars. Waste Management,. 22 (8): 893-899(7).

Dantu, P. 1958. Etude des contraintes dans les milieux hétérogénes: Applications au Béton, Annales Inst. Technicque du Bâtiment et des Travaux Publics 11 (121): 55-98.

De Juan, M.S., and Gutiérrez, P.A. 2004. Influence of Recycled Aggregate Quality on Concrete Properties. Conference on the use of the Recycled Materials in Buildings and Structures, Barcelona, Spain.

De Oliveira, M. B., Vazquez, E. 1996. Influence of Retained Moisture in Aggregates from Recycling on the Properties of New Hardened Concrete. Waste Management, $16(1-3): 113-117$.

De Pauw. 1983. Recycling of Demolished Reinforced Concrete. Carlo (Belgian Building Research Inst, Belgium), Motteu, Henri National Swedish Institute for Building Research, pp. 97-106. 
Desayi, P. and Krishnan, S. 1964. Equation for the Stress-Strain Curve of Concrete, ACI Journal, Proc. 61: 345-350.

De Vries, P. 1993. Concrete Recycled. Concrete (London), 27 (3): 9-13.

Dhir, R.K., Limbachiya, M.C., and Leelawat, T. 1999. Suitability of Recycled Concrete Aggregate for Use in BS 5328 Designated Mixes. Proceedings of the Institution of Civil Engineers, Structures and Buildings, Vol. 134 (3), pp. 257-274.

Digest 433. 1998. Recycled Aggregates, BRE, CI/SFB p (T6).

Digest 1 Research Information. 2005. Recycled Concrete Aggregate. Concrete, pp. 27289.

Discovery Economic Consulting Province of British Columbia (DECPBC), Ministry of Environment, Lands and Parks, May 2001.Using Tax Shifting and Tax Incentives to Promote the Deconstruction/Renovation Industry. [Online]. Available: http://www.dec.bc.ca/resources/tax_shifting_incentives.pdf [Retrieved January 2006].

Environmental Council of Concrete Organization (ECCO). 1999. Recycling Concrete and Masonry. [Online]. Available: http://ecco.org/. [August, 2004].

Environment Canada, Government Canada, 2006. [online]. Available: http://www.ec.gc.ca/press/2001/010711 b e.htm, July issue [cited 20 January 2006]. European Committee for Standardization. 2002. Eurocode 2: Design of Concrete Structures, Part 1: General rules and rules for buildings, revised final draft.

Frondistou-Y. S. 1977. Waste Concrete as Aggregate for New Concrete. ACI Journal, 373-376. 
Fumoto, T., and Yamada, M. 2003. Strength and Drying Shrinkage of Concrete Used Recycled Aggregate. Memoirs of Faculty of Engineering Osaka City University, 44: 79-82.

Gardner N.J. 2000. Design Provisions for Shrinkage and Creep of Concrete. ACI, SP194, The Adam Neville Symposium: Creep and Shrinkage - Structural Design Effects, pp. 101-134.

Gastebled, O.J., and May L.M. 2001. Mechanics Model Applied to Shear Failure of Reinforced Concrete Beams without Stirrups. ACI Structural Journal, 98(2): 184-190. George, J.V and Michael, N. 2001. Waste Streams from Building Construction and Demolition, with a Specific Focus on Concrete Reuse and Recycling Report Submitted to Public Works Government Services Canada, Gatineau, Quebec, Canada. Gomez Soberon, J.M.V. 2002a. Shrinkage of Concrete with Replacement of Aggregate with Recycled Concrete Aggregate. ACI Special Publications, 209: 475-496.

Gomez Soberon, J.M.V. 2002b. Creep of Concrete with Substitution of Normal Aggregate by Recycled Concrete Aggregate. ACI Special Publications, 209: 461-474. Gomez Soberon, J. M. V. 2002c. Porosity of Recycled Concrete with Substitution of Recycled Concrete Aggregate: An Experimental Study. Cement and Concrete Research, 32 (8): 1301-1311.

Gomez Soberon, J. M. V. 2003. Relationship between Gas Adsorption and the Shrinkage and Creep of Recycled Aggregate Concrete. Cement, Concrete and Aggregates, 25 (2): $42-48$. 
Gonzalez B., and Martinez F. 2004. Shear Strength of Concrete with Recycled Aggregates. Conference on the Use of Recycled Materials in Building and Structures, Barcelona, Spain.

Han, B.C., Yun, H.D., and Chung, S.Y. 2001. Shear Capacity of Reinforced Concrete Beams Made with Recycled-Aggregate. ACI Special Publications, 200: 503-516.

Hansen, T. C., and Narud, H. 1983. Strength of Recycled Concrete Made from Crushed Concrete Coarse Aggregate. Concrete International: Design and Construction, 5 (1): 79-83.

Hansen, T. C., and Boegh E. 1985. Elasticity and Drying Shrinkage of RecycledAggregate Concrete. Journal of the American Concrete Institute, 82 (5): 648-652.

Hansen, T. C. 1986. Recycled Aggregates and Recycled Aggregate Concrete Second State-of-the-art Report Developments 1945-1985, Materials and Structures, 111: 201246.

Hansen, T. C. 1992. Recycling of Demolished Concrete and Masonry, RILEM, Report No. 6, Published by E \& FN Spon.

Hognestad, E. 1951. A Study of Combined Bending and Axial Load in Reinforced Concrete Members. University of Illinois Engineering Experimental Station, Bulletin Series No. 299, pp128.

Intergovernmental Panel on Climate Change (IPCC), Government of Canada, 2005. [Online].

Available: www.climatechange.gc.ca/english/climate_change/factsheet_en.pdf [cited 20 January 2006]. 
Kani, G.N.G. 1967. How Safe Are Our Large Reinforced Concrete Beams. Journal ACI, 64: $128-141$.

Katz, A. 2003. Properties of Concrete Made with Recycled Aggregate from Partially Hydrated Old Concrete. Cement and Concrete Research, 33 (5): 703-711.

Katz, A. 2004. Treatments for the Improvement of Recycled Aggregate. Journal of Materials in Civil Engineering, 16 (6): 597-603.

Kheder, G. F., and Al-Windawi, S. A. 2005. Variation in Mechanical Properties of Natural and Recycled Aggregate Concrete as Related to the Strength o their Binding Mortar, Journal of Materials and Structures, 38: 701-709.

Kikuchi, M., Yasunaga, A., and Ehara, K. 1994. Total Evaluation of Recycled Aggregate and Recycled Concrete. Proceeding of $3^{\text {rd }}$ International RILEM Symposium Demolition Reuse Concrete Masonry, pp. 367-377.

Kiuchi, T. 2001. A Study on the Recycle Concrete by Using Type III Recycled Coarse Aggregate. Memoirs- Faculty of Engineering Osaka City University, 42: 31-40.

Kiuchi, T., and Horiuchi, E. 2003. An Experimental Study on Recycle Concrete by using High Quality Recycled Coarse Aggregate. Memoirs of Faculty of Engineering Osaka City University, 44: 37-44.

Lamond, J. F., Campbell Sr., R. L., Campbell, T. R., Cazares, J. A., Giraldi, A., Halczak, W., Hale Jr., H. C., Jenkins, N. J.T., Miller, R., Seabrook, P. T. 2002. Removal and Reuse of Hardened Concrete. ACI Materials Journal, 99(3): 300-325.

Leonhardt, F. 1965. Reducing the Shear Reinforcement in Reinforced Concrete Beams and Slabs. Magazine of Concrete Research, 17(53): 187-198. 
Limbachiya, M.C., Leelawat, T., and Dhir, R.K. 2000. Use of Recycled Concrete Aggregate in High-strength Concrete. Materials and Structures/Materiaux et Constructions, 33 (233): 574-580.

Lin, Y. H., Tyan, Y. Y., Chang, T. P., and Chang, C. Y. 2004. An Assessment of Optimal Mixture for Concrete Made with Recycled Concrete Aggregates. Cement and Concrete Research, 34 (8): 1373-1380.

Loo, Y. H., Sri Ravindrarajah, R., and Tam, C. T. 1987. Recycled Concrete as Fine and Coarse Aggregates in Concrete. Magazine of Concrete Research, 39 (141): 214-220.

Lydon, F. D.1982. Concrete Mix Design, $2^{\text {nd }}$ edition, Applied Science Publisher, London and New York.

Malhotra V. M. 1976. Use of Recycled Concrete as a New Aggregate. Report 79-18, Canada Centre for Mineral and Energy Technology, Ottawa, Canada.

Mandal, S., Chakarborty, S., and Gupta, A. 2002. Some Studies on Durability of Recycled Aggregate Concrete. Indian Concrete Journal, 76 (6): 385-388.

Maruyama, M. Sogo, T. Sogabe, R. Sato, K. Kawai. 2004a. Flexural Properties of Reinforced Recycled Concrete Beams. Conference on the Use of Recycled Materials in Building and Structures, Barcelona, Spain.

Maruyama, M. Sogo, T. Sogabe, R. Sato, K. Kawai. 2004b. Shear Behaviour of Reinforced Recycled Concrete Beams. Conference on the Use of Recycled Materials in Building and Structures, Barcelona, Spain.

Mehta P.K, and Monterio P.J.M. 2006. Concrete, Microstructure, Properties, and Materials, third edition, The McGraw-Hill companies, Inc. 
Mehta, P.K. 2001.Reducing the Environmental Impact of Concrete. Concrete International ,61-66.

Merlet, J.D., and Pimienta, P. 1994. Mechanical and Physico-Chemical Properties of Concrete Produced with Coarse and Fine Recycled Concrete Aggregates. Proceeding of $3^{\text {rd }}$ International RILEM Symposium Demolition Reuse Concrete Masonry, pp. 343-353.

Mesbah, H.A., and Buyle-Bodin, F. 1999. Efficiency of Polypropylene and Metallic Fibres on Control of Shrinkage and Cracking of Recycled Aggregate Mortars. Construction and Building Materials, 13: 439-447.

Mindess, S., Young, J.F. 1981. Concrete. Prentice-Hall, INC., Englewood Cliffs, New Jersey.

Mishulovich, A., 2003. Used Concrete/Construction Debris in Portland Cement Manufacturing. Portland Cement Association Research and Development Report Serial No. 2635.

Mitchell, D., and Collins, M.P. 1974. Diagonal Compression Field Theroy- A Rational Model for Structural Concrete in Pure Torsion. ACI journal, 71: 396-408.

Mitchell, D., and Collins, M.P. 1990. Prestressed Concrete Structures.Prentice Hall, Englewood Cliffs, N.J., pp 776.

Mörsch, E. 1902. Concrete-Steel Construction. McGrow-Hill, Newyork (English translation by E.P. Goodrich of Der Eisenbetobou, $1^{\text {st }}$ ed., 1902)

MOTH, 1999.Construction Aggregates Sector Trends. British Columbia Ministry of Transport and Highways. 
Mukherjee, D., Chakraborty, S., and Bhattacharya, P.G. 2003. A Statistical Study on Compressive Strength of Recycled Concrete, Journal of the Institution of Engineers (India): Civil Engineering Division, 83: 219-220.

Nagataki, S., Gokce, A., and Saeki, T. 2000. Effects of Recycled Aggregate Characteristics on Performance Parameters of Recycled Aggregate Concrete. ACI Special Publications, 192 (1): 51-72.

Nagataki, S., Gokce, A., Saeki, T., and Hisada, M. 2004. Assessment of Recycling Process Induced Damage Sensitivity of Recycled Concrete Aggregates. Cement and Concrete Research, 34 (6): 965-971.

Nassif, H.H., Najm, H. and Suksawang N. 2005. Effect of Pozzolanic Materials and Curing Methods on the Elastic Modulus of HPC. Cement and Concrete Composites, 27 (6): 661-670.

Neville, A. M. 1995. Properties of Concrete. Longman Group Limited, Essex

Neville, A. M. 1970. Creep of Concrete: Plain, Reinforced, and Prestressed. North Holland Publishing Company, Amsterdam.

Nisbet, M., Venta, G., and Foo, S. 2001. Waste Streams from Building Construction and Demolition with a Specific Focus on Concrete Reuse and Recycling. Building Construction and Demolition Waste: Concrete Reuse and Recycling, PW\&GS, Canada.

Nishibayashi, S., Yamura, K., and Hayashi, A. 1985. Elastic-Plastic Properties and Durability of Concrete Made From Recycled Coarse Aggregate Prepared by Crushing Concrete. Transaction of the Japan Concrete Institute, 6: 127-132. 
Nishiura, N., Kasamatsu, T., Miyashita, T., and Tanaka, R. 2000. Experimental Study on Half-Precast Beams with Recycled Aggregate Concrete. Transactions of the Japan Concrete Institute, 22: 353-360.

Nishiura, N., Kasamatsu, T., Miyashita, T., and Tanaka, R. 2001. Experimental Study of Recycled Aggregate Concrete Half-Precast Beams with Lap Joints. Transactions of the Japan Concrete Institute, 23: 295-302.

Niwa, J., Yamada, K., Yokozawa, K. and Okamura, H. 1986. Revaluation of the Equation for Shear Strength of Reinforced Concrete Beams without Web Reinforcement, Proceedings of JSCE, 372:167-176.

Nixon P.J. 1977. Recycled Concrete as an Aggregate for Concrete. A review, RILEM TC-37-DRC, Materials and Structures (RILEM), 65: 371-378.

Noguchi, T., and Tamura, M., 2001. Concrete Design Towards Complete Recycling. Structural Concrete, 2 (3): 155-167.

Ontario Aggregate Resources Corporation (OARC). 2002. Mineral Aggregates of Ontario Statistical Update, 2002 [Online]. Available: http://www.toarc.com/publications_statistics.asp [Retrieved December 2005].

Ong, K. C. G., Sri Ravindrarajah, R. 1987. Mechanical Properties and Fracture Energy of Recycled-Aggregate Concretes. Society for Experimental Mechanics Inc, 150-158.

Otsuki, N., Miyazato, S., and Yodsudjai, W. 2003a. Influence of Recycled Aggregate on Interfacial Transition Zone, Strength, Chloride Penetration and Carbonation of Concrete. Journal of Materials in Civil Engineering, 15 (5): 443-451. 
Otsuki, N., Yodsudjai, W., Nishida, T., and Yamane, H. 2003b. Developed Method for Measuring Flexural Strength and Modulus of Elasticity of Micro-Regions in Normal and Recycled Aggregate Concretes. Magazine of Concrete Research, 55 (5): 439-448.

Panagapko, D. 2003. Canadian Minerals Yearbook, Natural Resources Canada.

Park R., and Paulay, T. 1975. Reinforced Concrete Structures. Wiley-interscience publication, pp. 271-300.

Poon, C.S., Shui, Z.H., Lam, L., Fok, H., and Kou, S.C. 2004a. Influence of Moisture States of Natural and Recycled Aggregates on the Slump and Compressive Strength of Concrete. Cement and Concrete Research, 34 (1): 31-36.

Poon, C.S., Shui, Z.H., and Lam, L. 2004b. Effect of Microstructure of ITZ on Compressive Strength of Concrete Prepared with Recycled Aggregates. Construction and Building Materials, 18 (6): 461-468.

Popovics, S. 1998. Strength and Related Properties of Concrete: A Quantitative Approach, John and Wiley and Sons Inc.

Ramamurthy, K., and Gumaste, K.S. 1998. Properties of Recycled Aggregate Concrete. Indian Concrete Journal, 72 (1): 49-53.

Ramsey, R., Mirza, A., and MacGregor, J.G. 1979. Variability in Deflections of Reinforced Concrete Beams. ACI Journal Proceedings, 76 (8): 897-918.

Rashwan, M. S., and AbouRizk, S. 1997. Properties of Recycled Concrete. Concrete International, 19 (7): 56-60.

Ray, S.P., and Venkateswarlu, B. 1991. Recycled Aggregate Concrete. Journal of Structural Engineering, 18 (2): 67-75. 
RILEM Recommendation. 1994. "Specifications for Concrete with Recycled Aggregates." Materials and Structures 27, pp. 557-559.

Ritter, W. 1899. Die Bauweise Henneibique Scweizerische Bauzeitung, 33(7): 59-61.

Ryu, J.S. 2002a. An Experimental Study on the Effect of Recycled Aggregate on Concrete Properties. Magazine of Concrete Research, 54 (1): 7-12.

Ryu, J.S. 2002b. Improvement on Strength and Impermeability of Recycled Concrete Made from Crushed Concrete Coarse Aggregate. Journal of Materials Science Letters, 21 (20): 1565-1567.

Sagoe-Crentsil, K.K., Brown, T., and Taylor, A.H. 2001. Performance of Concrete Made with Commercially Produced Coarse Recycled Concrete Aggregate. Cement and Concrete Research, 31 (5): 707-712.

Sakata, K., and Ayano T. 2000. Improvement of Concrete with Recycled Aggregate. ACI Special Publications, 192 (2): 1089-1108.

Salem, R. M., and Burdette, E. G. 1998. Role of Chemical and Mineral Admixtures on Physical Properties and Frost-Resistance of Recycled Aggregate Concrete. ACI Materials Journal, 95 (5): 558-563.

Salem, R. M., Burdette, E. G., and Jackson, N. M. 2003. Resistance to Freezing and Thawing of Recycled Aggregate Concrete. ACI Materials Journal, 100 (3): 216-221. Sandler, K. 2003. Analyzing What's Recyclable in C\&D Debris. [Online]. Available:http://yosemite.epa.gov/oar/globalwarming.nsf/uniquekeylookup/BMOE5 XLKDG/\$File/Analyzing_C_D_Debris.pdf [Retrieved April 2007]. 
Santos, J. R., Branco, F., and Brito, J. 2004. Mechanical Properties of Concrete with Coarse Recycled Aggregates. Structural Engineering International, 14 (3): 213 215(3).

Shayan, A., and Xu, A. 2003. Performance and Properties of Structural Concrete made with Recycled Concrete Aggregate. ACI Materials Journal, 100(5): 371-380.

EHE-99. 1999. Spanish Instruction of Structural Concrete. AENOR (Ed), Madrid, III:1355.

Smith, G.M. and Young, L.E. 1955. Ultimate Theory in Flexure by Exponential Function. Journal of the American Concrete Institute, 52 (3): 349-359.

Sri Ravindrarajah, R. 1987.Utilization of Waste Concrete for New Construction. Conservation and Recycling, 10 (2-3): 69-74.

Tamura, H., Nishio, A., Ohashi, J., and Imamoto, K. 2001. High Quality Recycled Aggregate Concrete (HiRAC) Processed by Decompression and Rapid Release. ACI Special Publications, 200: 491-502.

Taschereau, D., 2001. Moving Mountains. Solid Waste and Recycling, Toronto, Canada: 3-6.

Tavakoli, M., and Soroushian, P. 1996a. Strengths of Recycled Aggregate Concrete Made Using Field-Demolished Concrete as Aggregate. ACI Materials Journal, 93 (2): $182-190$.

Tavakoli, M., and Soroushian, P. 1996b. Drying Shrinkage Behavior Of Recycled Aggregate Concrete, Concrete International - Design and Construction, 18 (11): 5861. 
Thorenfeldt, E., Tomaszewicz, and Jensen, J.J. 1987. Mechanical Properties of HighStrength Concrete and Application in Design. Proceedings of the Symposium " Utilization of High-Strength Concrete", Stavanger, Norway, Tapir, Trondheim, pp $149-159$.

Tomosawa, F. and Noguchi, T. 1993. Relationship Between Compressive Strength and Modulus of Elasticity of High-Strength Concrete. Proceedings of the $3^{\text {rd }}$ International Symposium on the Utilization of High-Strength Concrete, pp. 1254-1274.

Tomozo S. 1983. Recycled Concrete. National Swedish Institute for Building Research, pp. 251-262.

Topcu, I. B., and Guncan, N. F. 1995. Using Waste Concrete as Aggregate. Cement and Concrete Research, 25 (7): 1385-1390.

Topcu, I.B. 1997. Physical and Mechanical Properties of Concretes Produced with Waste, Cement and Concrete Research, 27 (12): 1817-1823.

Topcu, I. B., and Sengel, S. 2004. Properties of Concretes Produced with Waste Concrete Aggregate. Cement and Concrete Research, 34 (8): 1307-132.

United Nation. 1992. Report of the United Nations Conference on Environment and Development, Annex I: Rio Declaration on Environment and Development. Rio de Janeiro, Brazil.3-14 June, 1992.

Vecchio, F.J., and Collins, M.P. 1986. The Modified Compression Field Theory for Reinforced Concrete Elements Subjected to Shear. ACI Journal proceedings, 83(2): 219-231.

Vishay Micro Measurements. 2006. M-Bond 200 Installations: Gage Installation Techniques. [Online]. Available: 
http://www.Vishay.com/brands/measurements_group/guide/ib/b127/127Cl.htm. [October, 2006].

Wainwright, P.J., Trevorrow, A., Yu, Y., and Wang, Y. 1994. Modifying the Performance of Concrete Made with Coarse and Fine Recycled Concrete Aggregates. Proceeding of $3^{\text {rd }}$ International RILEM Symposium Demolition Reuse Concrete Masonry, pp. 319.

Wilson, A., $2003 . \quad$ [Online]. Available: www.cement.ca/cement.nsf/e/5707B2CB7E10E24585256E1C007D7C9D?Open Document [2004, August].

Winfield, M.S., Taylor, A. (2005). Replacing the Load: The Need for an Aggregates Conservation Strategy for Ontario, Report Published by Pembina Institute for Appropriate Development, ISBN 0-921719-71-x.

Xiao, J., Li, J., and Zhang, C. 2004. Mechanical Properties of Recycled Aggregate Concrete under Uniaxial Loading. Proceedings of the International Conference on Restoration, Recycling and Rejuvenation Technology for Engineering and Architecture Application, pp. 323-330.

Xiao, J., Sun, Y., and Fulkner, H. 2006. Structural Performance of Frame Structures with Recycled Aggregate Concrete. Engineering Structures, No. 28, pp. 1-8.

Yagishita, F., Sano, M., and Yamada, M. 1994. Behaviour of Reinforced Concrete Beams Containing Recycled Aggregate. Proceeding of $3^{\text {rd }}$ International RILEM Symposium Demolition Reuse Concrete Masonry, pp. 331-342. 
Yamasaki, J., and Tatematsu, K. 1998. Strength and Freeze-Thaw Resistance Properties of Concrete Using High-Quality Recycled Aggregate. Transactions of the Japan Concrete Institute, 20: 45-52.

Yanagi, K., Hisaka, M., and Kasai, Y. 1994. Physical Properties of Recycled Concrete Using Recycled Coarse Aggregate Made of Concrete with Finishing Materials. Proceeding of $3^{\text {rd }}$ International RILEM Symposium Demolition Reuse Concrete Masonry, pp. 379-390.

Zsutty T.C. 1968. Beam Shear Strength Prediction by Analysis of Existing Data. ACI Structural Journal, 65: 943-951.

Zsutty T.C. 1971. Shear Strength Prediction for Separate Categories of Simple Beam Tests. ACI Structural Journal, 68: 138-143. 


\section{APPENDIX A: DETERMINATION OF RESIDUAL MORTAR SPEC IFIC GRAVITY: DIRECT METHOD}

\section{Scope}

This test method covers the testing of residual mortar to determine its specific gravity and absorption capacity. This test is a slightly modified version of ASTM C 128-97 (ASTM, 2003), the standard test method for absorption capacity and specific gravity for fine aggregates.

\section{Apparatus}

2.1. Balance: A balance or scale sensitive to $0.1 \mathrm{~g}$ or less and accurate within $0.1 \%$ of the sample mass over the range required for the test.

2.2. Le Chatelier flask: As described in test method ASTM C 188-95 (ASTM, 2003).

2.3. Sieves: With square openings and with suitable sizes to prepare the test sample as in Section 3.

2.4. Oven: Oven capable of maintaining a temperature of $110 \pm 5{ }^{\circ} \mathrm{C}$.

2.5. Magnifier.

2.6. Tweezer.

\section{Preparation of Test Specimens}

3.1. Crush RCA particles passing no. $1 / 2$ in. and retaining on $3 / 8$ in. by a Jaw Crusher machine.

3.2. Sieve the crushed RCA and keep the RCA particles passing sieve No. \# 4 and retaining on No. \# 8 .

3.3. Separate the residual mortar particles by visual inspection using a magnifier. 
For each sample prepare $45 \pm 5 \mathrm{~g}$. Prepare at least three samples.

\section{Procedure}

4.1. Dry the RM samples in an oven (at $110 \pm 5^{\circ} \mathrm{C}$ ) to constant weight, and cooling in air at room temperature for 1 to 3 hours.

4.2. Fill the flask initially with water to a point on the stem between 0 and $1-\mathrm{ml}$ mark. Record the initial water level in the flask $\left(\mathrm{R}_{1}\right)$.

4.3. Immerse the oven-dry RM sample in the flask and leave it for $24 \pm 4$ hours to achieve SSD condition, and cover the flask with the stopper to avoid any evaporation. Then roll the flask in an inclined position to dislodge the entrapped air.

4.4. Record the final water level in the flask 24 hours after immersing RM samples $\left(\mathrm{R}_{2}\right)$.

4.5. Remove the wet RM samples from the flask and roll it in a large absorbent cloth until all visible films of water are removed.

4.6. Recording the SSD weight of the samples $\left(W_{S S D}{ }^{R M}\right)$.

4.7. Dry the RM samples in an oven (at $110 \pm 5^{\circ} \mathrm{C}$ ) to constant weight, and cooling in air at room temperature for 1 to 3 hours.

4.8. Record the weight of oven-dry RM samples $\left(W_{O D}{ }^{R M}\right)$.

\section{Calculation}

5.1. Calculate the SSD specific gravity of $\mathrm{RM}\left(S G_{S S D}{ }^{R M}\right)$ and absorption capacity of $\mathrm{RM}\left(A C^{R M}\right)$ as follows:

$$
S G_{S S D}^{R M}=\frac{W_{S S D}^{R M}}{0.9975\left(R_{2}-R_{1}\right)}
$$




$$
A C^{R M}=\frac{W_{S S D}{ }^{R M}-W_{O D}{ }^{R M}}{W_{O D}{ }^{R M}}
$$

5.2. Calculate the bulk specific gravity of $\mathrm{RM}\left(S G_{b}{ }^{R M}\right)$ as follows:

$$
S G_{b}{ }^{R M}=\frac{S G_{S S D}{ }^{R M}}{1+A C^{R M}}
$$

5.3. Calculate the apparent specific gravity of RM $\left(S G_{a}{ }^{R M}\right)$ and porosity of RM $\left(n^{R M}\right)$ as follows:

$$
\begin{aligned}
S G_{a}{ }^{R M} & =\frac{1}{\frac{1}{S G_{b}{ }^{R M}}-\frac{A C^{R M}}{100}} \\
n^{R M} & =A C^{R M} \times S G_{b}{ }^{R M}
\end{aligned}
$$




\section{APPENDIX B: DETAILS OF EXPERIMENTAL INVESTIGATION CARRIED OUT BY HANSEN AND NARUD}

\section{Portland Cement}

A high-early strength Portland cement (similar to ASTM Type III in composition and properties) was used.

\section{Natural Aggregates (NA)}

Partly siliceous, partly calcareous marine gravel was used for the production of OC. A natural sand of glacial origin, graded in B.S. Zone 2, was used for production of both RAC and NAC.

\section{Recycled Concrete Aggregates (RCA)}

A high-strength, medium-strength, and low-strength concrete were produced from NA by varying the w/c ratio and keeping the other factors essentially identical. After 38 days of accelerated curing (to reach the highest degree of maturity possible within minimum required time), the compressive strengths of the three concretes were determined, and the concretes were passed through a laboratory jaw crusher. The crusher products were then screened and recombined into three qualities of coarse RCA, all having approximately the same grading as NA from which OC were produced $(52 \%$ of $16-32 \mathrm{~mm}, 29 \%$ of $8-16$ $\mathrm{mm}$, and $19 \%$ of $4-8 \mathrm{~mm}$ ).

Table B.1 presents the mix proportions of high, medium, and low strength $\mathrm{OC}$, which were crushed to form RCA with different qualities. All fine and coarse aggregates were 
in Saturated Surface Dry (SSD) condition. All concretes have achieved the slump values between $60-100 \mathrm{~mm}$.

The residual mortar volume (RMV) of RCA was determined by means of linear transverse method similar to ASTM C 457-71. They prepared a representative sample of each grade and size fraction mixed with red-colored cement. After hardening of samples, they cut them in slices, and polished them. Therefore, the residual mortar attached to original natural aggregate (ONA) was clearly distinguished both from ONA and red cement paste.

Table B.2 presents the physical properties of coarse NA and RCA including the specific gravity and RMV of RCA. It should be mentioned that the physical properties of aggregates presented in Table B.2 are weighted-average values calculated by the authors' of this paper based on the corresponding values of different faction sizes reported in Hansen and Narud's experimental report.

\section{Mix Proportioning}

All RAC mixes were produced with basically the same mix proportions as original concretes except that small adjustments were made due to lower specific gravity in RCA compared to NA. Table B. 3 presents the mix proportions of RAC and NAC mixes. In all RAC mixes, $10 \mathrm{~kg} / \mathrm{m}^{3}$ higher free water content was used to achieve the same slump as NAC. Cement content was increased correspondingly to maintain w/c ratio. Three types of RAC mixes were produced: high-strength, medium strength, and low strength, using each of three grades of RCA $(H, M$, and $L)$. The RAC mixes were designated as $H / H$, $\mathrm{H} / \mathrm{M}, \mathrm{H} / \mathrm{L}, \mathrm{M} / \mathrm{H}, \mathrm{M} / \mathrm{M}, \mathrm{M} / \mathrm{L}, \mathrm{L} / \mathrm{H}, \mathrm{L} / \mathrm{M}$, and $\mathrm{L} / \mathrm{L}$, where the first letter stands .4 presents the compressive strength of all RAC and NAC mixes at 14 and 38 days. 
Table B.1: The Mix Proportions of Original Concretes

\begin{tabular}{|c|c|c|c|c|c|}
\hline \multirow{2}{*}{ Mix ID } & \multirow{2}{*}{ w/c } & \multicolumn{4}{|c|}{ Mix Proportion of OC $\left(\mathbf{k g} / \mathbf{m}^{\mathbf{3}}\right)$} \\
\cline { 3 - 6 } & & Cement & Water & $\begin{array}{c}\text { Fine } \\
\text { Aggregate }\end{array}$ & $\begin{array}{c}\text { Coarse } \\
\text { Aggregate }\end{array}$ \\
\hline H & 0.4 & 410 & 165 & 545 & 1266 \\
\hline M & 0.7 & 215 & 151 & 695 & 1290 \\
\hline L & 1.2 & 138 & 165 & 951 & 1117 \\
\hline
\end{tabular}

Table B.2: Physical Properties of Natural Gravel and Recycled Coarse Aggregates

\begin{tabular}{|c|c|c|}
\hline Aggregate & SG & RMV, \% \\
\hline NA & 2.592 & 0 \\
\hline RCA (H) & 2.4499 & 40.24 \\
\hline RCA (M) & 2.4437 & 38.03 \\
\hline RCA (L) & 2.4412 & 35.9 \\
\hline
\end{tabular}

Table B.3: The Mix Proportions of RAC and NAC mixes

\begin{tabular}{|c|c|c|c|c|c|c|}
\hline \multirow{3}{*}{ Mix ID } & \multirow{3}{*}{$\mathbf{w} / \mathbf{c}$} & \multicolumn{5}{|c|}{ Mix Proportion $\left(\mathrm{kg} / \mathrm{m}^{3}\right)$} \\
\hline & & \multirow{2}{*}{ Cement } & \multirow{2}{*}{ Water } & \multirow{2}{*}{$\begin{array}{c}\text { Fine } \\
\text { Aggregate }\end{array}$} & \multicolumn{2}{|c|}{$\begin{array}{c}\text { Coarse } \\
\text { Aggregate }\end{array}$} \\
\hline & & & & & NA & RCA \\
\hline H & 0.4 & 410 & 165 & 545 & 1266 & 0 \\
\hline $\mathbf{H} / \mathbf{H}$ & 0.4 & 434.8 & 175.0 & 545.0 & 0 & 1196.6 \\
\hline H/M & 0.4 & 434.8 & 175.0 & 546.0 & 0 & 1193.6 \\
\hline $\mathrm{H} / \mathrm{L}$ & 0.4 & 434.8 & 175.0 & 547.0 & 0 & 1192.3 \\
\hline $\mathbf{M}$ & 0.7 & 215 & 151. & 695 & 1290 & 0 \\
\hline $\mathbf{M} / \mathbf{H}$ & 0.7 & 229.2 & 161.0 & 695.0 & 0 & 1219.3 \\
\hline $\mathbf{M} / \mathbf{M}$ & 0.7 & 229.2 & 161.0 & 695.0 & 0 & 1216.2 \\
\hline $\mathbf{M} / \mathbf{L}$ & 0.7 & 229.2 & 161.0 & 695.0 & 0 & 1214.9 \\
\hline L & 1.2 & 138 & 165 & 951 & 1117 & 0 \\
\hline $\mathbf{L} / \mathbf{H}$ & 1.2 & 146.4 & 175.0 & 951.0 & 0 & 1055.8 \\
\hline $\mathbf{L} / \mathbf{M}$ & 1.2 & 146.4 & 175.0 & 952.0 & 0 & 1053.1 \\
\hline $\mathbf{L} / \mathbf{L}$ & 1.2 & 146.4 & 175.0 & 953.0 & 0 & 1052.0 \\
\hline
\end{tabular}


Table B.4: The Compressive Strength of RAC and NAC mixes

\begin{tabular}{|c|c|c|}
\hline \multirow{2}{*}{ Mix ID } & \multicolumn{2}{|c|}{ Compressive Strength (MPa) } \\
\cline { 2 - 3 } & 14-days & 38-days \\
\hline H & 49.5 & 56.4 \\
\hline $\mathbf{H} / \mathbf{H}$ & 54.4 & 61.2 \\
\hline $\mathbf{H} / \mathbf{M}$ & 46.3 & 49.3 \\
\hline $\mathbf{H} / \mathbf{L}$ & 34.6 & 34.6 \\
\hline $\mathbf{M}$ & 26.2 & 34.4 \\
\hline $\mathbf{M} / \mathbf{H}$ & 27.7 & 35.1 \\
\hline $\mathbf{M} / \mathbf{M}$ & 27.0 & 33.0 \\
\hline $\mathbf{M} / \mathbf{L}$ & 23.2 & 26.9 \\
\hline $\mathbf{L}$ & 9.1 & 13.8 \\
\hline $\mathbf{L} / \mathbf{H}$ & 10.2 & 14.8 \\
\hline $\mathbf{L} / \mathbf{M}$ & 10.3 & 14.5 \\
\hline $\mathbf{L} / \mathbf{L}$ & 9.6 & 13.4 \\
\hline
\end{tabular}




\section{APPENDIX C: THE PRINCIPAL STEPS IN FULL}

\section{RESPONSE ANALYSIS OF RRC BEAMS}

1. Assuming a concrete strain value $\left(\varepsilon_{c i}\right)$ at the extreme compression fibre of the beam starting with a smallest value from the observed stress-strain relationship,

2. Assuming the position of the neutral axis $\left(c_{i}\right)$,

3. Establishing the strain profile based on steps 1 and 2,

4. Establishing the strain in the compression steel and tension steel at different layers using the strain compatibility as follows:

$$
\begin{aligned}
& \varepsilon_{s i}^{\prime}=\varepsilon_{c i} \cdot \frac{c_{i}-d_{c}^{\prime}}{c} \\
& \varepsilon_{1 s i}=\varepsilon_{c i} \cdot \frac{d_{1}-c_{i}}{c_{i}} \\
& \varepsilon_{2 s i}=\varepsilon_{c} \cdot \frac{d_{2}-c_{i}}{c_{i}}
\end{aligned}
$$

Note: If $\varepsilon_{1 s i}=\varepsilon_{y}$, then the yielding moment is equal to the resultant moment in step 8 $\left(M_{y}=M_{i}\right)$.

5. Determining equivalent stress block parameters $(\alpha$ and $\beta$ ) by integration of the observed stress-strain curve as follows:

$$
\begin{gathered}
\alpha_{i}=\frac{1}{f_{c}^{\prime} \cdot \varepsilon_{c i}} \int_{b}^{c_{c i}} \sigma \cdot d \varepsilon \\
\beta_{i}=1-\frac{\int_{c i}^{c_{i i}} \sigma \cdot \varepsilon \cdot d \varepsilon}{\int^{c_{i i}} \sigma \cdot d \varepsilon}
\end{gathered}
$$

6. Determining the forces in steel bars and the resultant concrete force as follows: 


$$
\begin{gathered}
C_{c i}=\alpha_{i} \cdot f_{c}^{\prime} \cdot b \cdot\left(\beta_{i} \cdot c_{i}\right) \\
C_{s i}=\varepsilon_{s i}^{\prime} \cdot E_{s}^{\prime} \cdot A_{s}^{\prime} \leq f_{y} \cdot A_{s}^{\prime} \\
T_{1 i}=\varepsilon_{1 s i} \cdot E_{s} \cdot A_{1 s} \leq f_{y} \cdot A_{1 s} \\
T_{2 i}=\varepsilon_{2 s i} \cdot E_{s} \cdot A_{2 s} \leq f_{y} \cdot A_{2 s}
\end{gathered}
$$

Note: In Eq.D.7 to D.9, the actual mechanical properties of the steel bar size used as the compression steel or tension steel was used.

7. Checking the equilibrium of all internal forces in step 6 as follows:

$$
\begin{gathered}
\sum C_{i}=\sum T_{i} \\
\sum C_{i}=C_{c i}+C_{s i} \\
\sum T_{i}=T_{1 i}+T_{2 i}
\end{gathered}
$$

8. Finding the resultant moment at concrete strain value of $\varepsilon_{c i}$ :

- If $\sum C_{i}=\sum T_{i}$ :

$$
M_{i}=C_{c i} \cdot\left(d_{1}-c_{i}+\beta_{i} \cdot c_{i}\right)+C_{s i} \cdot\left(d_{1}-d_{c}^{\prime}\right)+T_{2 i} \cdot\left(d_{1}-d_{2}\right)
$$

- If $\sum C_{i} \neq \sum T_{i}$, assume a new value for $c_{i}$ in step 2 and repeat step 3 to 8 .

9. Finding curvature of the beam:

$$
\phi_{i}=\frac{\varepsilon_{c i}}{c_{i}}
$$

10. Assuming the concrete strain value $\left(\varepsilon_{c i+1}\right)$ equal to another strain value on the observed stress-strain curve, and repeating steps 1 to 9 . By calculating the moment and curvatures at different strain values on the stress-strain curve, the moment curvature response of the beam can be graphed. 